\title{
Supporting Information $\mathrm{Ni} /$ chiral sodium carboxylate dual catalyzed asymmetric $O$ - propargylation
}

\author{
Xianghong Xu, Lingzi Peng, Xihao Chang, Chang Guo* \\ Hefei National Laboratory for Physical Sciences at the Microscale and Department of \\ Chemistry, University of Science and Technology of China, Hefei 230026, China \\ *e-mail: guochang@ustc.edu.cn
}

\section{CONTENTS:}

1. General Information

2. Experimental Procedure

3. Mechanistic Studies

4. Characterization of Products

5. Synthesis Procedure of Derivatization Reaction

7. References

8. ${ }^{1} \mathrm{H}$ and ${ }^{13} \mathrm{C}$ NMR Spectra

9. HPLC of Products 


\section{General Information}

Unless otherwise noted, all reagents were purchased from commercial suppliers and used without further purification. ${ }^{1} \mathrm{H}$ NMR and ${ }^{13} \mathrm{C}$ NMR spectra were recorded at $20{ }^{\circ} \mathrm{C}$ on a Bruker Advance $400 \mathrm{M}$ or $500 \mathrm{M}$ NMR spectrometers. Chemical shifts $(\delta)$ are given in ppm relative to TMS. The residual solvent signals were used as references and the chemical shifts converted to the TMS scale $\left(\mathrm{CHCl}_{3}: \delta 7.26\right.$ for proton and $\delta 77.16$ for carbon). Multiplicities were given as: s (singlet); d (doublet); t (triplet); q (quartet); dd (doublet of doublets); dt (doublet of triplets); m (multiplets); brs (broad signal). Coupling constants are reported as a $J$ value in Hz. Highresolution mass spectral analysis (HRMS) was performed on Waters XEVO G2 Q-TOF. The measurement of enantiomeric excesses was performed on Waters-Alliance (2998. Photodiode Array Detector, UV detection monitored at $254 \mathrm{~nm}$ ). Chiralpak IA, IB, IC, AD-H, OJ-H, and OD-H columns were purchased from Daicel Chemical Industries, LTD. The absolute configuration of 3ah and 3gf were assigned by the X-ray analysis. Optical rotations were determined at $589 \mathrm{~nm}$ (sodium D line) by using a Perkin-Elmer-343 polarimeter. Propargylic carbonates ( $r a c)-\mathbf{1}^{1}$ and compounds $\mathbf{2}^{2}$ were prepared according to the literature procedure.

\section{Experimental Procedure}

\subsection{General procedure for the enantioselective synthesis of 3 .}
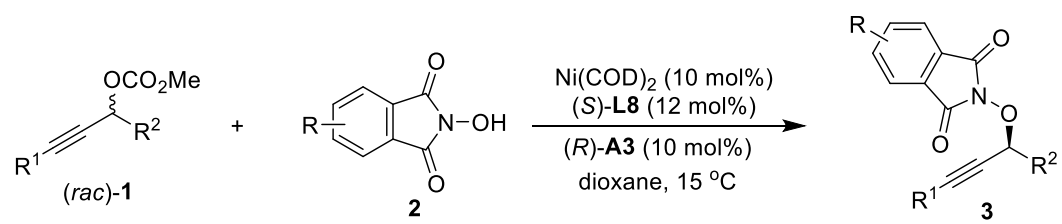

$\mathrm{Ni}(\mathrm{COD})_{2}$ (2.8 mg, $\left.0.01 \mathrm{mmol}, 10 \mathrm{~mol} \%\right)$, (S)-Cl-MeO-BIPHEP (L8) (7.8 mg, $0.012 \mathrm{mmol}, 12$ mol\%) were added into a $10 \mathrm{~mL}$ Schlenk flask equipped with a magnetic stirrer in a glove box. Then dry 1,4-Dioxane $(4.0 \mathrm{~mL})$ was added into the reaction system under argon and stirred for 30 minutes at room temperature, after which the $(R)-\mathbf{A 3}(3.9 \mathrm{mg}, 0.01 \mathrm{mmol}, 10 \mathrm{~mol} \%)$ was added into the reaction system and stirred for 30 minutes under argon. Then propargylic carbonate ( $\mathrm{rac})-\mathbf{1}(0.2$ mmol, 2.0 equiv) and compound $\mathbf{2}(0.1 \mathrm{mmol}, 1.0$ equiv) were added into the reaction mixture under argon at $15^{\circ} \mathrm{C}$. The reaction solution was stirred at $15^{\circ} \mathrm{C}$ for 12 hours to 5 days. The reaction mixture was filtered through a short pad of silica gel, and the solvent was evaporated under vacuum. The residue was purified by silica gel chromatography to afford the desired products 3 .

\subsection{General procedure for A1-A10.}

The sodium hydroxide (116.8 mg, $2.92 \mathrm{mmol}, 2.0$ equiv) was added to the solution of $(R)$-[1,1'binaphthalene]-2,2'-dicarboxylic acid (500 mg, $1.46 \mathrm{mmol}, 1.0$ equiv) in $\mathrm{DCM}^{-\mathrm{CH}_{3} \mathrm{OH}}$ (100:1, $5 \mathrm{~mL})$ at room temperature overnight. The precipitate was filtered, washed with DCM $(3 \times 3 \mathrm{~mL})$, and dried under vacuum overnight to afford the sodium binaphthyldicarboxylate $(R)$-A3 $(560$ $\mathrm{mg},>99 \%)$. The synthetic methods of other salts are similar to $(R)-\mathbf{A 3} .{ }^{1} \mathbf{H} \mathbf{~ N M R}\left(400 \mathbf{~ M H z}, \mathbf{D}_{\mathbf{2}} \mathbf{O}\right)$ $\delta 7.91-7.75(\mathrm{~m}, 2 \mathrm{H}), 7.67(\mathrm{~d}, J=8.5 \mathrm{~Hz}, 1 \mathrm{H}), 7.40-7.27(\mathrm{~m}, 1 \mathrm{H}), 7.17-6.94(\mathrm{~m}, 2 \mathrm{H}) .{ }^{13} \mathrm{C}$ 
NMR (100 MHz, $\left.\mathbf{D}_{2} \mathbf{O}\right) \delta 176.99,136.50,134.59,133.18,132.72,127.79,127.29,126.92$, 126.30, 126.22, 125.70 .

\section{Mechanistic studies}

\subsection{Study of the kinetic resolution process}

$(R)-\mathbf{1 a}$ and $(S)$-1 a was prepared according to the literatures. ${ }^{1}$

$(R)-1 \mathrm{a}: 99 \%$ ee, ${ }^{1} \mathbf{H}$ NMR (500 MHz, $\left.\mathbf{C D C l}_{3}\right) \delta 7.45-7.42(\mathrm{~m}, 2 \mathrm{H}), 7.34-7.28(\mathrm{~m}, 3 \mathrm{H}), 5.56$ $(\mathrm{q}, J=6.7 \mathrm{~Hz}, 1 \mathrm{H}), 3.82(\mathrm{~s}, 3 \mathrm{H}), 1.64(\mathrm{~d}, J=6.7 \mathrm{~Hz}, 3 \mathrm{H}) .{ }^{13} \mathbf{C}$ NMR $\left(\mathbf{1 2 5} \mathbf{~ M H z}, \mathbf{C D C l}_{3}\right) \delta$ 154.90, 131.87, 128.71, 128.27, 122.09, 86.61, 85.43, 64.92, 54.94, 21.54. ESI-MS: calculated $\left[\mathbf{C}_{12} \mathrm{H}_{12} \mathrm{O}_{3}+\mathbf{H}\right]^{+}$: 205.0859, found: 205.0866. $[\alpha]_{\mathrm{D}}^{20}=+176\left(\mathrm{c}=0.42, \mathrm{CHCl}_{3}\right)$. The product was analyzed by HPLC to determine the enantiomeric excess: 99\% ee (CHIRALPAK AD-H, hexane $/ i-\mathrm{PrOH}=95 / 5$, detector: $254 \mathrm{~nm}, \mathrm{~T}=25^{\circ} \mathrm{C}$, flow rate: $1 \mathrm{~mL} / \mathrm{min}$ ), $\mathrm{t}_{1}$ (major) $=4.45 \mathrm{~min}$, $\mathrm{t}_{2}(\operatorname{minor})=4.81 \mathrm{~min}$.

$(S)$-1a: 99\% ee, Spectral data were in agreement with $(R)$-1a reported above. $[\alpha]_{\mathrm{D}}^{20}=-176(\mathrm{c}=0.44$, $\left.\mathrm{CHCl}_{3}\right)$. The product of $(S)$-1a was analyzed by HPLC to determine the enantiomeric excess: 99\% ee (CHIRALPAK AD-H, hexane $/ i-\mathrm{PrOH}=95 / 5$, detector: $254 \mathrm{~nm}, \mathrm{~T}=25^{\circ} \mathrm{C}$, flow rate: 1 $\mathrm{mL} / \mathrm{min}), \mathrm{t}_{1}(\operatorname{minor})=4.46 \mathrm{~min}, \mathrm{t}_{2}$ (major $)=4.82 \mathrm{~min}$.

(rac)-1a

\begin{tabular}{|llll|}
\hline & \multicolumn{2}{c|}{ S A M P L E } & I N F O R M A T I O N \\
\hline \hline Sample Name: & xxh-6-62-AD-5\% & Acquired By: & System \\
Sample Type: & Unknown & Sample Set Name: & 20210623 \\
Vial: & 89 & Acq. Method Set: & $5 \%$ quanbo \\
Injection \#: & 1 & Processing Method: & 622 \\
Injection Volume: & 10.00 ul & Channel Name: & $254.0 \mathrm{~nm}$ \\
Run Time: & 6.0 Minutes & Proc. Chnl. Descr.: & 2998 PDA 254.0 nm (2998 \\
& & & \\
Date Acquired: & $6 / 23 / 2021$ 9:47:22 PM CST & & \\
Date Processed: & 10/4/2021 8:36:49 PM CST & & \\
\hline
\end{tabular}

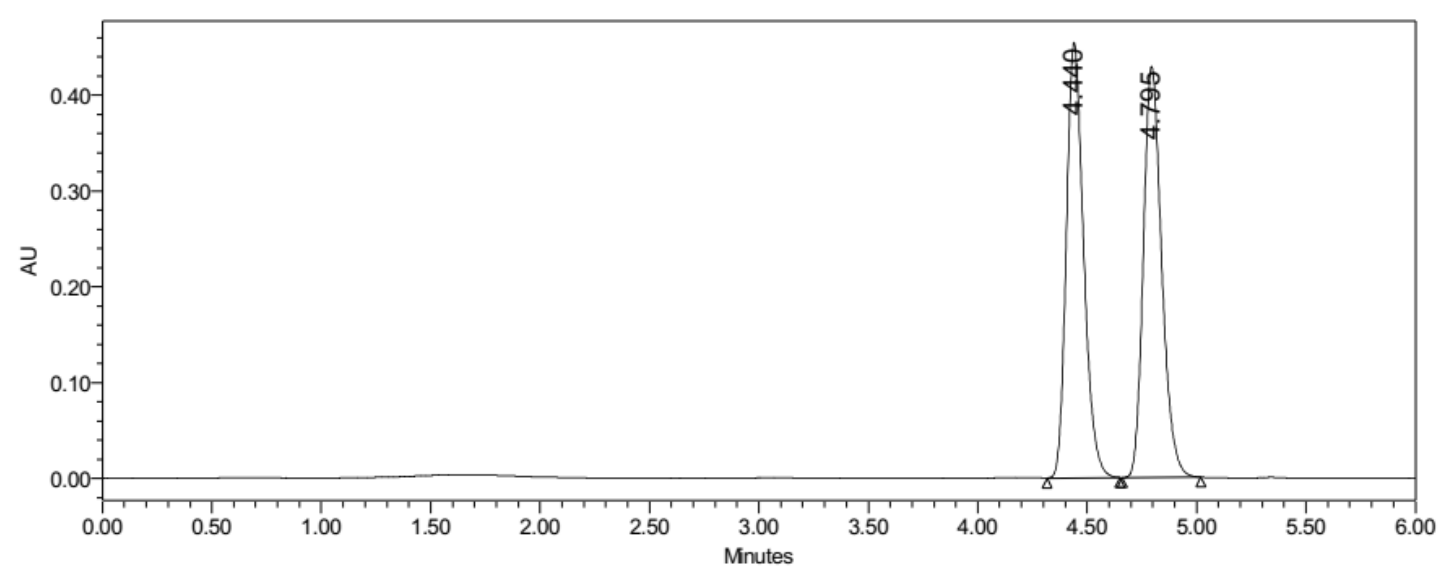

\begin{tabular}{|c|c|c|r|c|}
\hline & RT & Area & $\%$ Area & Height \\
\hline 1 & 4.440 & 2495115 & 50.00 & 454313 \\
\hline 2 & 4.795 & 2495154 & 50.00 & 429084 \\
\hline
\end{tabular}


Asy-(R)-1a

\begin{tabular}{|llll|}
\hline & \multicolumn{2}{c|}{ S A M P L E } & I N F O R M A T I O N \\
\hline \hline Sample Name: & xxh-9-148-AD-5\% & Acquired By: & System \\
Sample Type: & Unknown & Sample Set Name: & 20210622 \\
Vial: & 43 & Acq. Method Set: & $5 \%$ quanbo \\
Injection \#: & 1 & Processing Method: & 9148 \\
Injection Volume: & 10.00 ul & Channel Name: & $254.0 \mathrm{~nm}$ \\
Run Time: & 6.0 Minutes & Proc. Chnl. Descr.: & 2998 PDA 254.0 nm (2998 \\
& & & \\
Date Acquired: & 6/22/2021 4:22:04 PM CST & & \\
Date Processed: & $10 / 4 / 20218: 14: 51$ PM CST & & \\
\hline
\end{tabular}

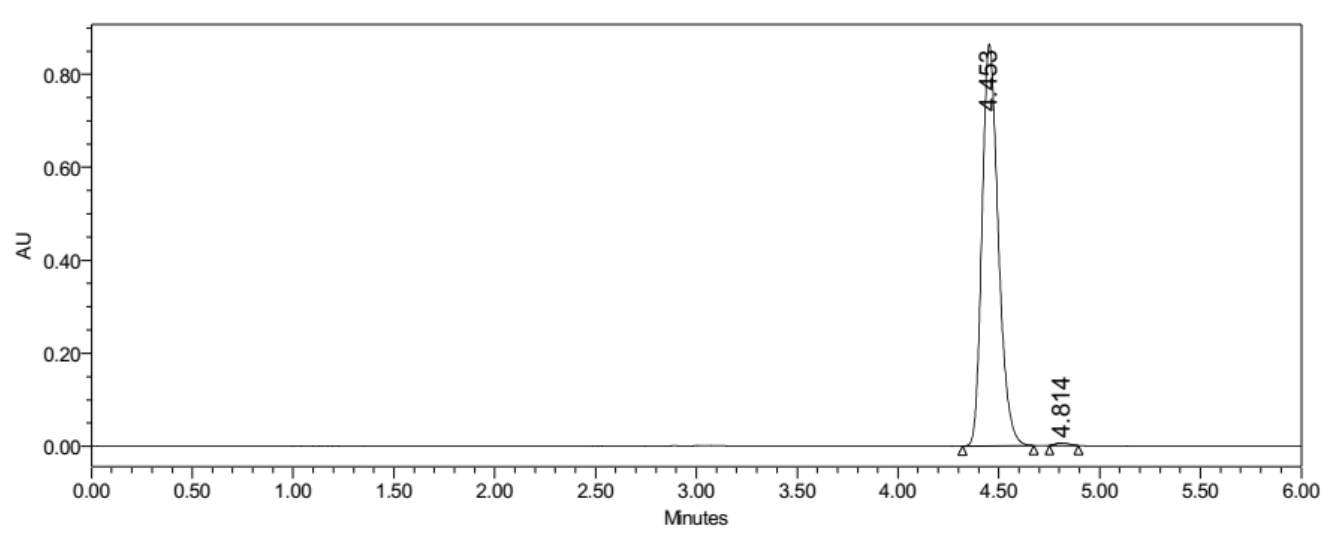

\begin{tabular}{|r|c|r|r|r|}
\hline & RT & \multicolumn{1}{c|}{ Area } & \% Area & Height \\
\hline 1 & 4.453 & 4846885 & 99.50 & 863804 \\
\hline 2 & 4.814 & 24214 & 0.50 & 5194 \\
\hline
\end{tabular}

Asy-(S)-1a

\begin{tabular}{|llll|}
\hline & \multicolumn{1}{c|}{ S A M P L E } & I N F O R M A T I O N \\
\hline \hline Sample Name: & xxh-9-149-AD-5\% & Acquired By: & System \\
Sample Type: & Unknown & Sample Set Name: & 20210622 \\
Vial: & 44 & Acq. Method Set: & $5 \%$ quanbo \\
Injection \#: & 1 & Processing Method: & 91492 \\
Injection Volume: & 10.00 ul & Channel Name: & $254.0 \mathrm{~nm}$ \\
Run Time: & 6.0 Minutes & Proc. Chnl. Descr.: & 2998 PDA 254.0 nm (2998 \\
& & & \\
Date Acquired: & $6 / 22 / 20214: 28: 44$ PM CST & & \\
Date Processed: & 10/4/2021 8:35:05 PM CST & & \\
\hline
\end{tabular}

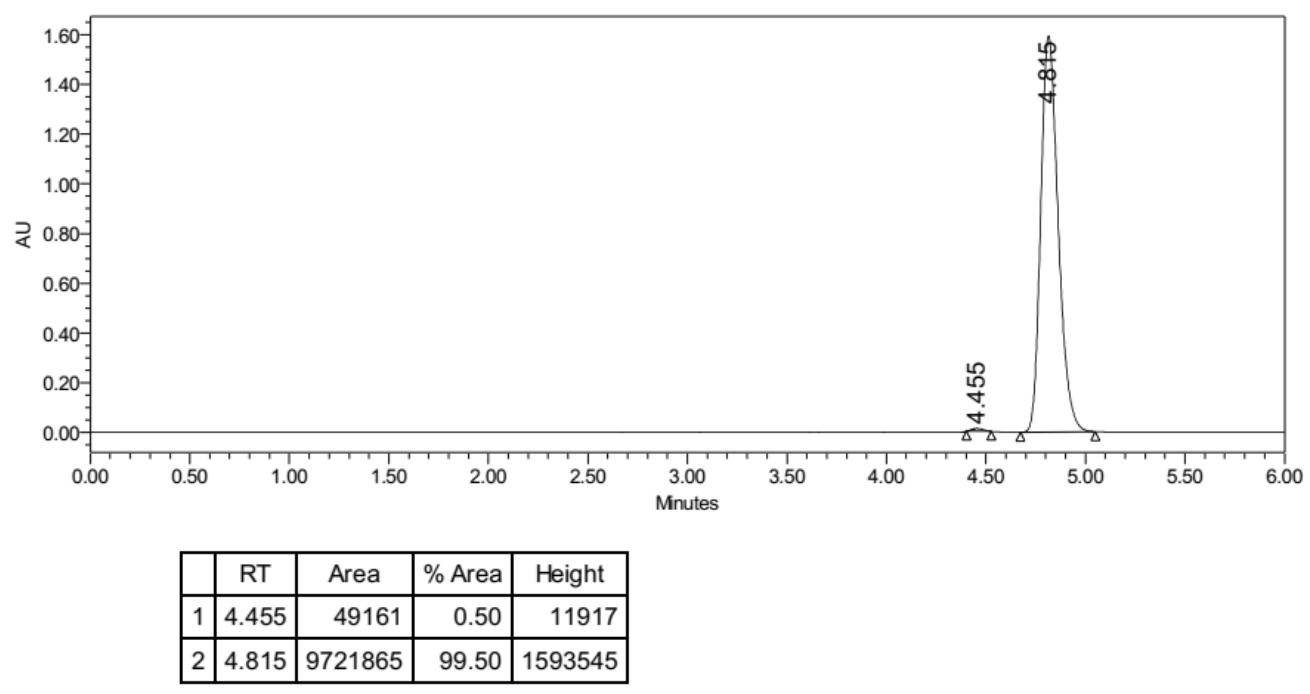


${ }^{1} \mathrm{H}$ NMR spectrum of $\mathbf{1 a}$

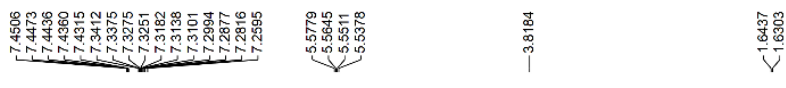

$\overbrace{\mathrm{Me}}^{\mathrm{OCO}_{2} \mathrm{Me}}$

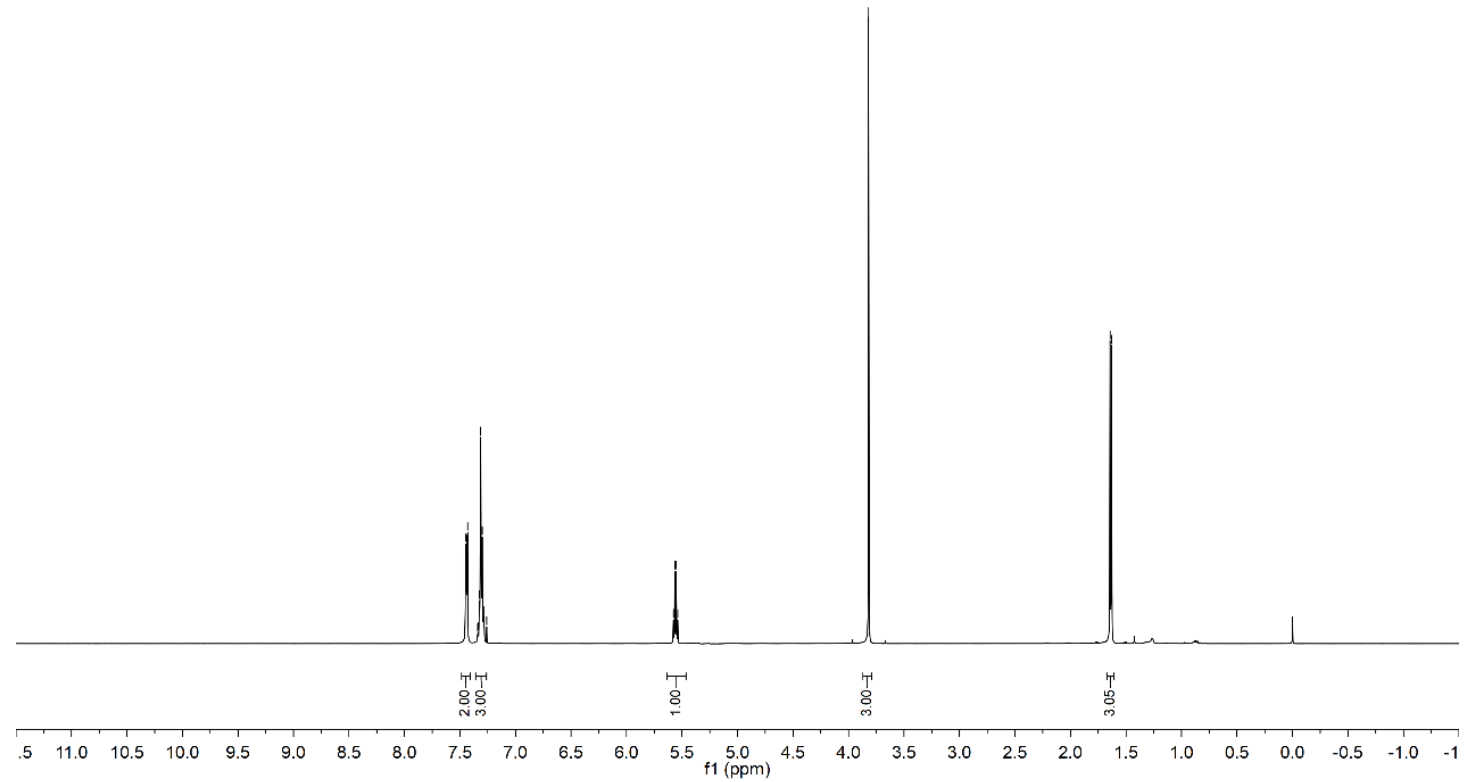

${ }^{13} \mathrm{C}$ NMR spectrum of $\mathbf{1 a}$
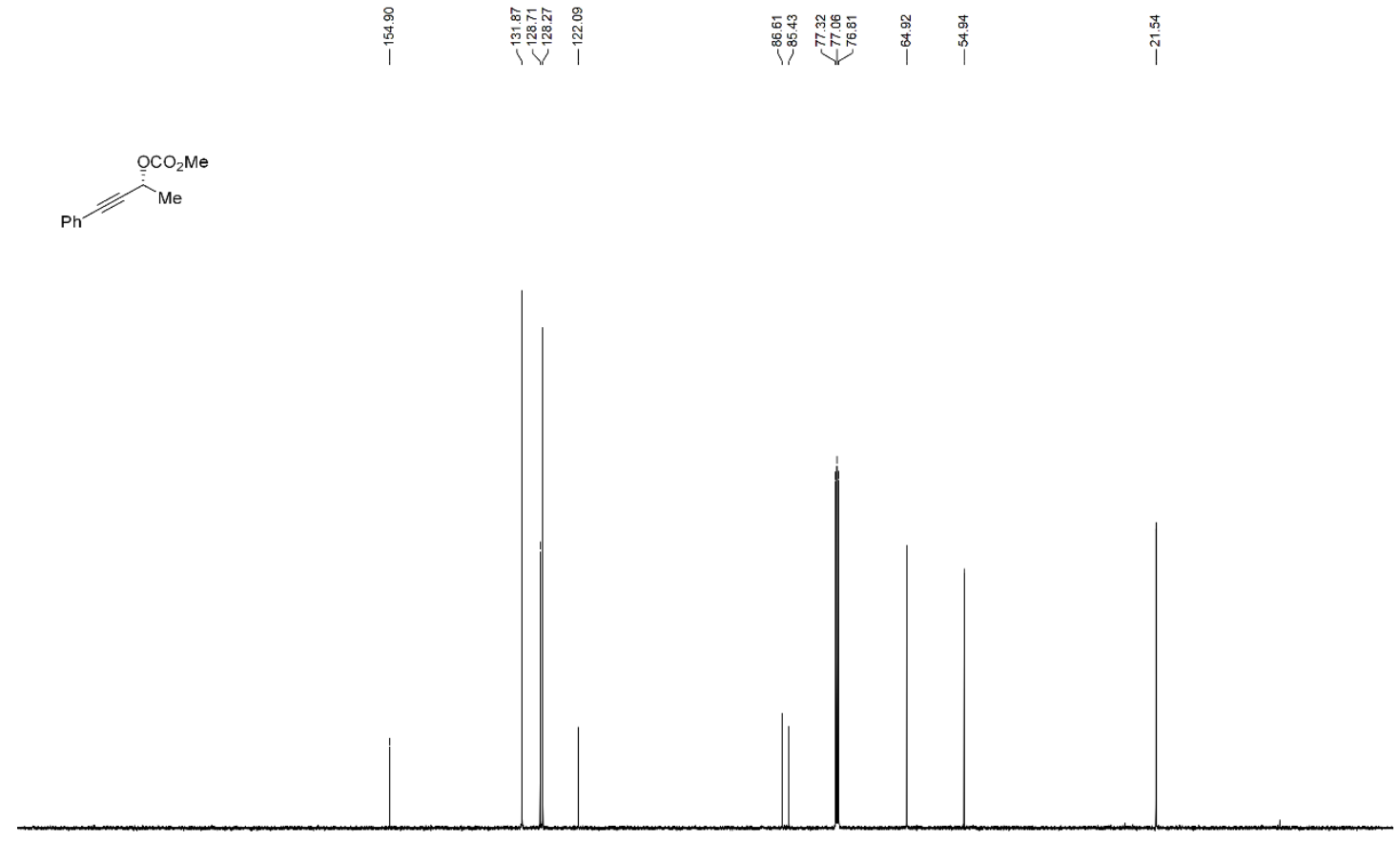

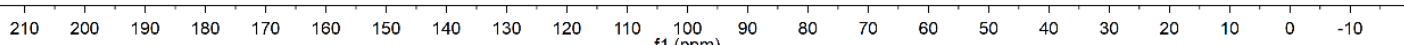


3.1.1 The reaction with $(\mathrm{rac})$-1a monitored over different reaction time

$\mathrm{Ni}(\mathrm{COD})_{2}$ (2.8 mg, $\left.0.01 \mathrm{mmol}, 10 \mathrm{~mol} \%\right)$, (S)-Cl-MeO-BIPHEP (L8) $(7.8 \mathrm{mg}, 0.012 \mathrm{mmol}, 12$ mol\%) were added into a $10 \mathrm{~mL}$ Schlenk flask equipped with a magnetic stirrer in a glove box. Then dry dioxane $(4.0 \mathrm{~mL})$ was added into the reaction system under argon and stirred for 30 minutes at room temperature, after which the $(R)-\mathbf{A 3}(3.9 \mathrm{mg}, 0.01 \mathrm{mmol}, 10 \mathrm{~mol} \%)$ was added into the reaction system and stirred for 30 minutes under argon. Then propargylic carbonate (rac)-1a $(0.2$ mmol, 2.0 equiv) and $N$-hydroxyphthalimide $2 \mathbf{a}(0.1 \mathrm{mmol}, 1.0$ equiv) were added into the reaction mixture under argon at $15{ }^{\circ} \mathrm{C}$. The reaction of (rac)-1a with $2 \mathbf{a}$ was monitored at $3 \mathrm{~h}, 6 \mathrm{~h}, 9 \mathrm{~h}$, and $12 \mathrm{~h}$. The recovered substrate $\mathbf{1 a}$ and the corresponding products 3aa were analyzed by HPLC.

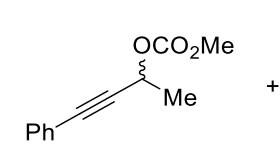

(rac)-1a

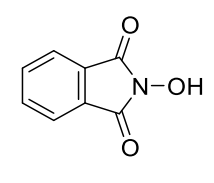

$2 a$

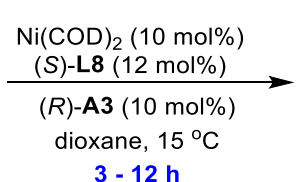

$3-12 \mathrm{~h}$

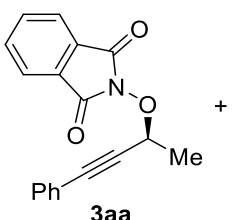

3aa

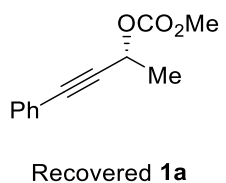

Recovered 1a

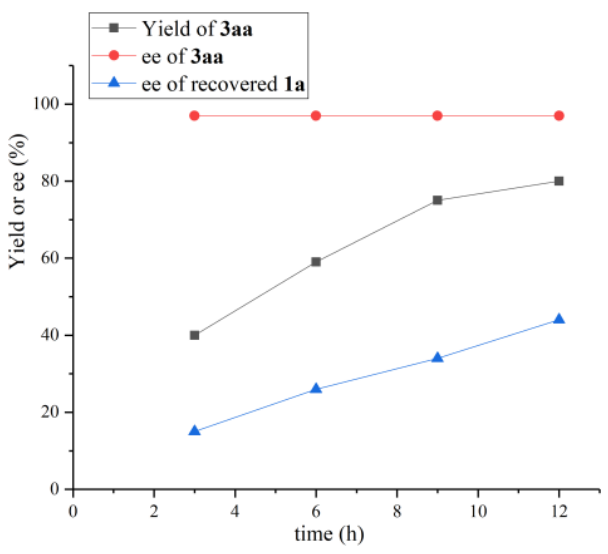

time (h) Yield of zaa ee (\%) of zaa ee (\%) of recovered 1a

$\begin{array}{llll}3 & 40 & 97(S) & 15(R)\end{array}$

$6 \quad 59 \quad 97(S) \quad 26(R)$

$9 \quad 75 \quad 97(S) \quad 34(R)$

12

80

$97(S)$

$44(R)$

Figure S1. The reaction with ( $\mathrm{rac})$-1a monitored over different reaction times

The enantioselectivity of (S)-3aa remains unchanged (97\% ee) throughout the reaction process. In addition, the propargylic carbonate ( $r a c)$-1a was resolved to its $R$-enantiomer with a significant ee.

3.1.2 The reaction with $(R)$-1a monitored over different reaction times

$\mathrm{Ni}(\mathrm{COD})_{2}(2.8 \mathrm{mg}, 0.01 \mathrm{mmol}, 10 \mathrm{~mol} \%)$, (S)-Cl-MeO-BIPHEP (L8) $(7.8 \mathrm{mg}, 0.012 \mathrm{mmol}, 12$ mol\%) were added into a $10 \mathrm{~mL}$ Schlenk flask equipped with a magnetic stirrer in a glove box. Then dry dioxane $(4.0 \mathrm{~mL})$ was added into the reaction system under argon and stirred for 30 minutes at room temperature, after which the $(R)-\mathbf{A 3}(3.9 \mathrm{mg}, 0.01 \mathrm{mmol}, 10 \mathrm{~mol} \%)$ was added into the reaction system and stirred for 30 minutes under argon. Then propargylic carbonate $(R)$-1a $(0.2$ mmol, 2.0 equiv) and $N$-hydroxyphthalimide $\mathbf{2 a}(0.1 \mathrm{mmol}, 1.0$ equiv) were added into the reaction mixture under argon at $15{ }^{\circ} \mathrm{C}$. The reaction of $(R)$-1a with $2 \mathbf{a}$ was monitored at $3 \mathrm{~h}, 6 \mathrm{~h}, 9 \mathrm{~h}$, and 12 h. The recovered substrate $\mathbf{1 a}$ and the corresponding products 3aa were analyzed by HPLC.

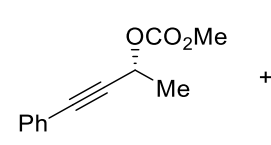

$(R)-\mathbf{a}$

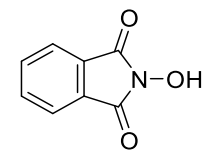

$2 a$

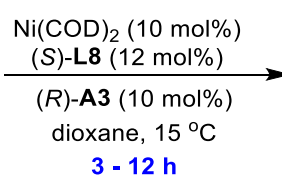

3- $12 \mathrm{~h}$

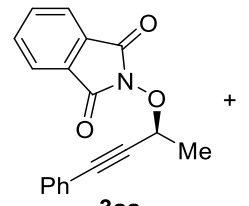

$3 a a$

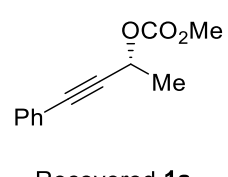

Recovered 1a 


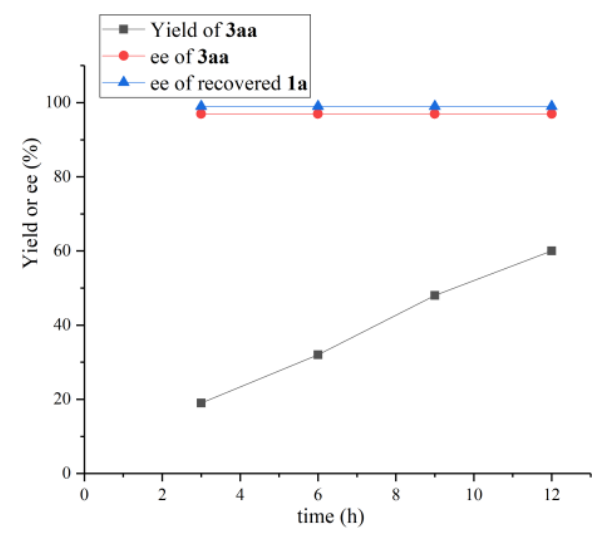

\begin{tabular}{cccc}
\hline time (h) & Yield of zaa & ee (\%) of zaa & ee (\%) of recovered 1a \\
3 & 19 & $97(S)$ & $99(R)$ \\
6 & 32 & $97(S)$ & $99(R)$ \\
9 & 48 & $97(S)$ & $99(R)$ \\
12 & 60 & $97(S)$ & $99(R)$ \\
\hline
\end{tabular}

Figure S2. The reaction with $(R)$-1a monitored over different reaction times

The enantioselectivity of $(S)$-3aa remains unchanged (97\% ee) throughout the reaction process. Furthermore, a time-course experiment revealed that no racemization of propargylic carbonate $(R)$ 1a was found. Moreover, the enantioselectivity of $(R)$-1a remains unchanged (99\% ee) throughout the reaction process.

3.1.3 The reaction with $(S)$-1a monitored over different reaction times

$\mathrm{Ni}(\mathrm{COD})_{2}(2.8 \mathrm{mg}, 0.01 \mathrm{mmol}, 10 \mathrm{~mol} \%),(S)-\mathrm{Cl}-\mathrm{MeO}-\mathrm{BIPHEP}$ (L8) (7.8 mg, $0.012 \mathrm{mmol}, 12$ mol\%) were added into a $10 \mathrm{~mL}$ Schlenk flask equipped with a magnetic stirrer in a glove box. Then dry dioxane $(4.0 \mathrm{~mL})$ was added into the reaction system under argon and stirred for 30 minutes at room temperature, after which the $(R)$-A3 $(3.9 \mathrm{mg}, 0.01 \mathrm{mmol}, 10 \mathrm{~mol} \%)$ was added into the reaction system and stirred for 30 minutes under argon. Then propargylic carbonate $(S)$-1a $(0.2$ mmol, 2.0 equiv) and $N$-hydroxyphthalimide $\mathbf{2 a}(0.1 \mathrm{mmol}, 1.0$ equiv) were added into the reaction mixture under $\arg$ on at $15^{\circ} \mathrm{C}$. The reaction of $(S)$-1a with $\mathbf{2 a}$ was monitored at $3 \mathrm{~h}, 6 \mathrm{~h}, 9 \mathrm{~h}$, and 12 h. The recovered substrate $\mathbf{1 a}$ and the corresponding products $3 \mathbf{a a}$ were analyzed by HPLC.

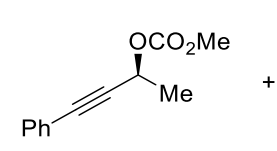

(S)-1a

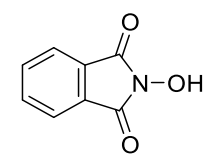

$2 a$

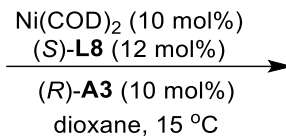

$3-12 \mathrm{~h}$

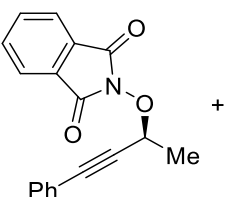

3aa

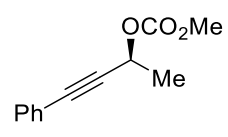

Recovered 1a

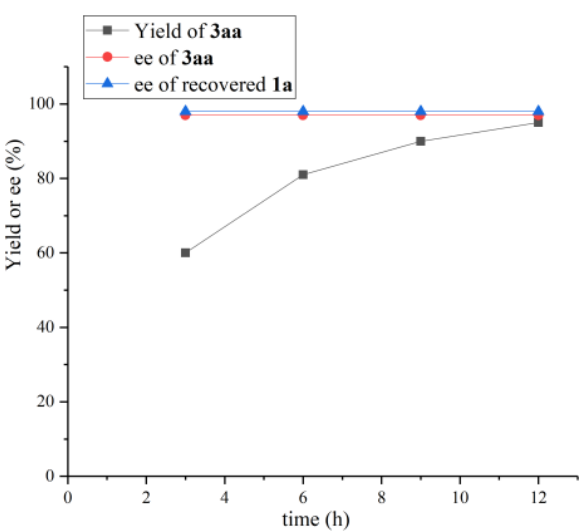

\begin{tabular}{cccc}
\hline time (h) & Yield of zaa & ee (\%) of zaa & ee (\%) of recovered 1a \\
3 & 60 & $97(S)$ & $98(S)$ \\
6 & 81 & $97(S)$ & $98(S)$ \\
9 & 90 & $97(S)$ & $98(S)$ \\
12 & 95 & $97(S)$ & $98(S)$ \\
\hline
\end{tabular}

Figure S3. The reaction with $(S)$-1a monitored over different reaction times

The enantioselectivity of $(S)$-3aa remains unchanged (97\% ee) throughout the reaction process. Furthermore, a time-course experiment revealed that no racemization of propargylic carbonate $(S)$ - 
1a was found. Moreover, the enantioselectivity of $(S)$-1a remains unchanged ( $98 \%$ ee) throughout the reaction process.

3.1.4 The reaction with (rac)-, $(R)-$, and $(S)$-1a monitored over different reaction times
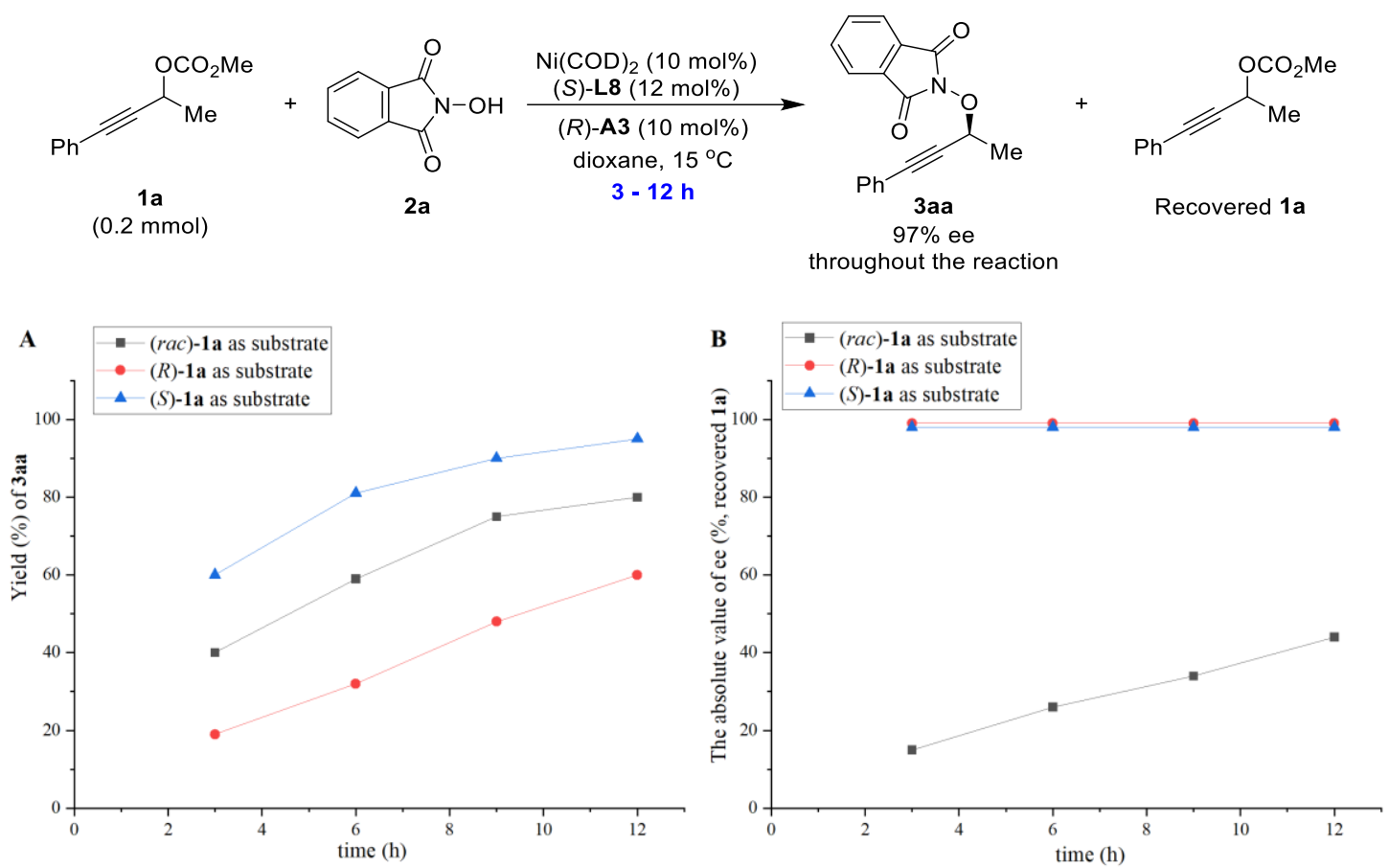

Figure S4. Time-course studies of the Ni-catalyzed $O$-APS reactions of 1 a

The propargylic carbonate $(r a c)-1 a$ was successfully resolved to its $R$-enantiomer with a significant ee. Reactions of enantiomerically pure $(S)$-1a and $(R)-\mathbf{1 a}$ were also conducted, and ee of the recovered 1a at partial conversion is essentially unchanged. In addition, the reaction rate with $(S)$-1a was faster than that with $(R)-\mathbf{1 a}$, and these results are consistent with kinetic studies of propargylic carbonate ( $r a c)-\mathbf{1 a}$.

\subsection{Study of the carboxylate additive on the Ni-catalyzed APS reactions}

Synthesis of S1-S3

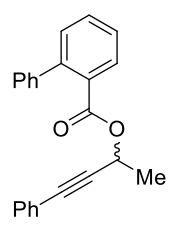

(rac)-1a"

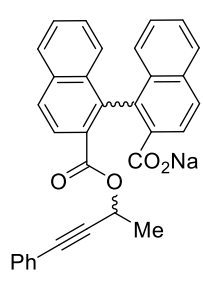

S1

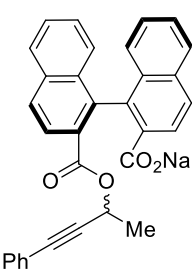

S2

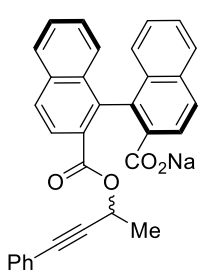

S3

A specific example for the synthesis of $\mathbf{S 1}$ :

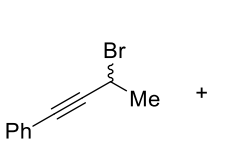

S1-I

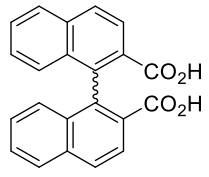

(rac)-A7
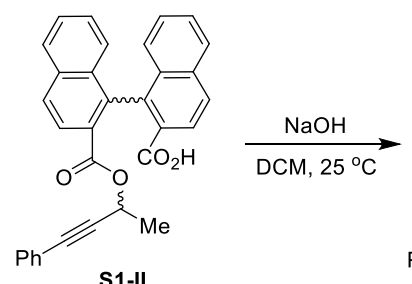

Procedure: The (rac)-A7 (1 g, $2.92 \mathrm{mmol}, 1.0$ equiv) was dissolved in DMF $(10 \mathrm{~mL})$, then $\mathrm{K}_{2} \mathrm{CO}_{3}$ 
(181 mg, $1.3 \mathrm{mmol}, 0.448$ equiv) was added to the reaction in batches. The solution was stirred at room temperature for 1 hour. The S1-I dissolved in DMF (1 mL) was slowly added to the reaction. The final solution was stirred at room temperature for 24 hours. The combined organic phase was concentrated under reduced pressure. The residue was purified by flash column chromatography to give the desired product S1-II ( $646 \mathrm{mg}, 47 \%$ yield).

The sodium hydroxide ( $40 \mathrm{mg}, 1 \mathrm{mmol}, 1.0$ equiv) was added to the solution of S1-II (470 mg, $1 \mathrm{mmol}, 1.0$ equiv) in DCM $(5 \mathrm{~mL})$ at room temperature overnight. The precipitate was filtered, washed with DCM $(3 \times 1 \mathrm{~mL})$, and dried under vacuum overnight to afford the product S1 (467 mg, 95\%). The synthetic methods of $\mathbf{S 2}$ and $\mathbf{S 3}$ are similar to $\mathbf{S 1}$.

\section{4-phenylbut-3-yn-2-yl [1,1'-biphenyl]-2-carboxylate (( $\mathrm{rac})$-1a")}

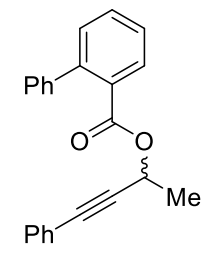

${ }^{1} \mathbf{H}$ NMR (500 MHz, $\left.\mathbf{C D C l}_{3}\right) \delta 7.87(\mathrm{~d}, J=7.7 \mathrm{~Hz}, 1 \mathrm{H}), 7.54-7.27(\mathrm{~m}, 13 \mathrm{H})$, $5.65(\mathrm{q}, J=6.7 \mathrm{~Hz}, 1 \mathrm{H}), 1.32(\mathrm{~d}, J=6.7 \mathrm{~Hz}, 3 \mathrm{H}) .{ }^{\mathbf{1 3}} \mathbf{C ~ N M R}\left(\mathbf{1 2 5} \mathbf{~ M H z}, \mathbf{C D C l}_{\mathbf{3}}\right)$ $\delta 167.62,142.67,141.27,131.88,131.36,130.81,130.73,129.96,128.57$, $128.55,128.24,128.09,127.24,127.18,122.43,87.26,84.58,76.82,61.40$, 20.96. ESI-MS: calculated $\left[\mathrm{C}_{23} \mathrm{H}_{18} \mathrm{O}_{2}+\mathbf{N a}\right]^{+}: \mathbf{3 4 9 . 1 1 9 9}$, found: $\mathbf{3 4 9 . 1 2 0 3}$

sodium 2'-(((4-phenylbut-3-yn-2-yl)oxy)carbonyl)-(1,1'-binaphthalene)-2-carboxylate (S1-II)

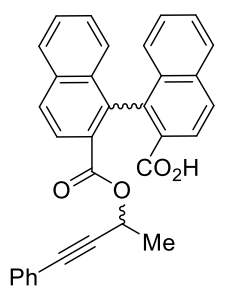

1:1 dr; Isomer A: ${ }^{1} \mathbf{H}$ NMR (500 MHz, DMSO-d 6 ) $\delta 12.46$ (br, s, 1H), $8.17-8.01$ $(\mathrm{m}, 6 \mathrm{H}), 7.61-7.49(\mathrm{~m}, 2 \mathrm{H}), 7.41-7.26(\mathrm{~m}, 7 \mathrm{H}), 7.00(\mathrm{~d}, J=8.5 \mathrm{~Hz}, 1 \mathrm{H}), 6.92(\mathrm{~d}$, $J=8.6 \mathrm{~Hz}, 1 \mathrm{H}), 5.41-5.35(\mathrm{~m}, 1 \mathrm{H}), 0.85(\mathrm{~d}, J=6.7 \mathrm{~Hz}, 3 \mathrm{H}) .{ }^{13} \mathbf{C} \mathbf{N M R}(\mathbf{1 2 5} \mathbf{~ M H z}$, DMSO-d $\boldsymbol{d}_{\boldsymbol{6}} \delta 167.95,165.75,140.54,139.40,135.06,134.78,133.01,132.91,131.88$, $129.32,129.04,128.71,128.54,128.40,128.35,128.30,128.25,127.98,127.43$, 127.32, 127.26, 126.99, 126.58, 126.15, 121.92, 87.93, 84.25, 60.79, 20.69. Isomer B:

${ }^{1}$ H NMR (500 MHz, DMSO-d 6 ) $\delta 12.46$ (br, s, 1H), $8.17-8.01$ (m, 6H), $7.61-7.49$ (m, 2H), $7.41-$ $7.26(\mathrm{~m}, 5 \mathrm{H}), 7.21-7.19(\mathrm{~m}, 2 \mathrm{H}), 6.98(\mathrm{~d}, J=8.6 \mathrm{~Hz}, 1 \mathrm{H}), 6.92(\mathrm{~d}, J=8.6 \mathrm{~Hz}, 1 \mathrm{H}), 5.41-5.35(\mathrm{~m}$, 1H), $1.00(\mathrm{~d}, J=6.7 \mathrm{~Hz}, 3 \mathrm{H}) .{ }^{13} \mathrm{C}$ NMR (125 MHz, DMSO-d 6$) \delta 168.11,165.84,140.36,139.24$, 135.02, 134.85, 133.08, 132.95, 132.91, 129.34, 128.96, 128.77, 128.54, 128.40, 128.35, 128.30, 128.25, 127.91, 127.37, 127.32, 127.26, 127.15, 126.37, 126.16, 121.79, 87.76, 84.11, 60.85, 20.92. ESI-MS: calculated $\left[\mathrm{C}_{32} \mathrm{H}_{22} \mathrm{O}_{4}+\mathrm{H}\right]^{+}$: 471.1591 , found: 471.1593 .

sodium 2'-(((4-phenylbut-3-yn-2-yl)oxy)carbonyl)-(1,1'-binaphthalene)-2-carboxylate (S1)

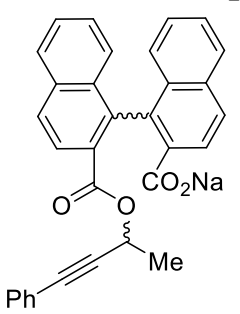
1.3:1 dr; Isomer A: ${ }^{1}$ H NMR (500 MHz, DMSO-d 6 ) $\delta 8.14(\mathrm{~d}, J=8.5 \mathrm{~Hz}, 1 \mathrm{H}), 8.04$ $(\mathrm{d}, J=2.4 \mathrm{~Hz}, 1 \mathrm{H}), 7.99-7.88(\mathrm{~m}, 4 \mathrm{H}), 7.49(\mathrm{~d}, J=7.6 \mathrm{~Hz}, 1 \mathrm{H}), 7.39-7.34(\mathrm{~m}, 5 \mathrm{H})$, $7.24-7.19(\mathrm{~m}, 2 \mathrm{H}), 7.16-7.11(\mathrm{~m}, 1 \mathrm{H}), 7.05(\mathrm{~d}, J=8.6 \mathrm{~Hz}, 1 \mathrm{H}), 6.80(\mathrm{~d}, J=8.5$ $\mathrm{Hz}, 1 \mathrm{H}), 5.33-5.28(\mathrm{~m}, 1 \mathrm{H}), 0.84(\mathrm{~d}, J=6.6 \mathrm{~Hz}, 3 \mathrm{H}) .{ }^{13} \mathrm{C}$ NMR (125 MHz, DMSOd6) $\delta 171.42,166.25,143.70,138.02,135.43,135.04,133.60,133.58,133.49,131.90$, $129.27,129.00,128.42,128.31,128.10,127.97,127.78,127.12,126.83,126.71$, $126.63,126.50,126.43,126.36,125.87,125.61,121.93,88.40,83.86,60.20,20.80$. Isomer B: ${ }^{1} \mathbf{H}$ NMR (500 MHz, DMSO-d 6$) \delta 8.21(\mathrm{~d}, J=8.5 \mathrm{~Hz}, 1 \mathrm{H}), 8.06(\mathrm{~d}, J=2.4 \mathrm{~Hz}, 1 \mathrm{H}), 7.99-$ $7.91(\mathrm{~m}, 4 \mathrm{H}), 7.51(\mathrm{~d}, J=7.4 \mathrm{~Hz}, 1 \mathrm{H}), 7.35-7.31(\mathrm{~m}, 5 \mathrm{H}), 7.22-7.19(\mathrm{~m}, 2 \mathrm{H}), 7.14-7.11(\mathrm{~m}, 1 \mathrm{H})$, $7.08(\mathrm{~d}, J=8.6 \mathrm{~Hz}, 1 \mathrm{H}), 6.78(\mathrm{~d}, J=8.5 \mathrm{~Hz}, 1 \mathrm{H}), 5.34-5.26(\mathrm{~m}, 1 \mathrm{H}), 0.93(\mathrm{~d}, J=6.6 \mathrm{~Hz}, 3 \mathrm{H}) .{ }^{13} \mathrm{C}$ NMR (125 MHz, DMSO-d 6 ) $\delta$ 171.11, 165.94, 144.30, 137.74, 135.73, 135.04, 133.58, 133.42, 133.31, $131.95,129.25,129.03,128.72,128.31,128.08,128.02,127.78,127.12,126.85,126.75,126.63,126.54$, $126.41, \quad 126.21, \quad 125.87, \quad 125.69,122.09,88.55,84.04,60.29, \quad 20.92 . \quad$ ESI-MS: calculated 
$\left[\mathrm{C}_{32} \mathrm{H}_{21} \mathrm{NaO}_{4}+\mathrm{H}\right]^{+}$: 493.1410, found: 493.1419.

${ }^{1} \mathrm{H}$ NMR spectrum of $(\mathrm{rac})-\mathbf{1 a} \mathbf{a}^{\prime}$
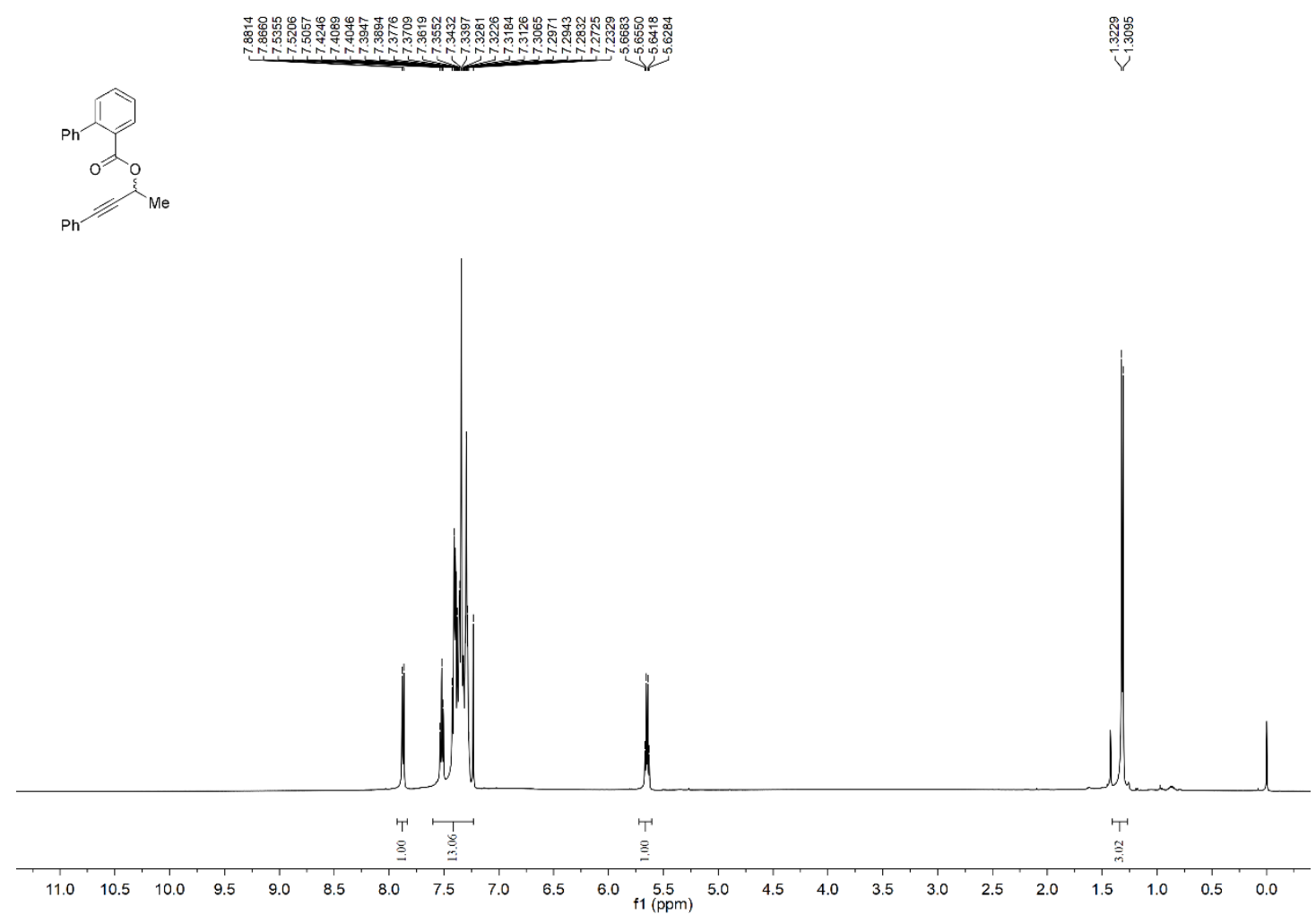

${ }^{13} \mathrm{C}$ NMR spectrum of $(\mathrm{rac})$-1a"

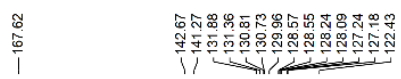

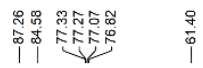
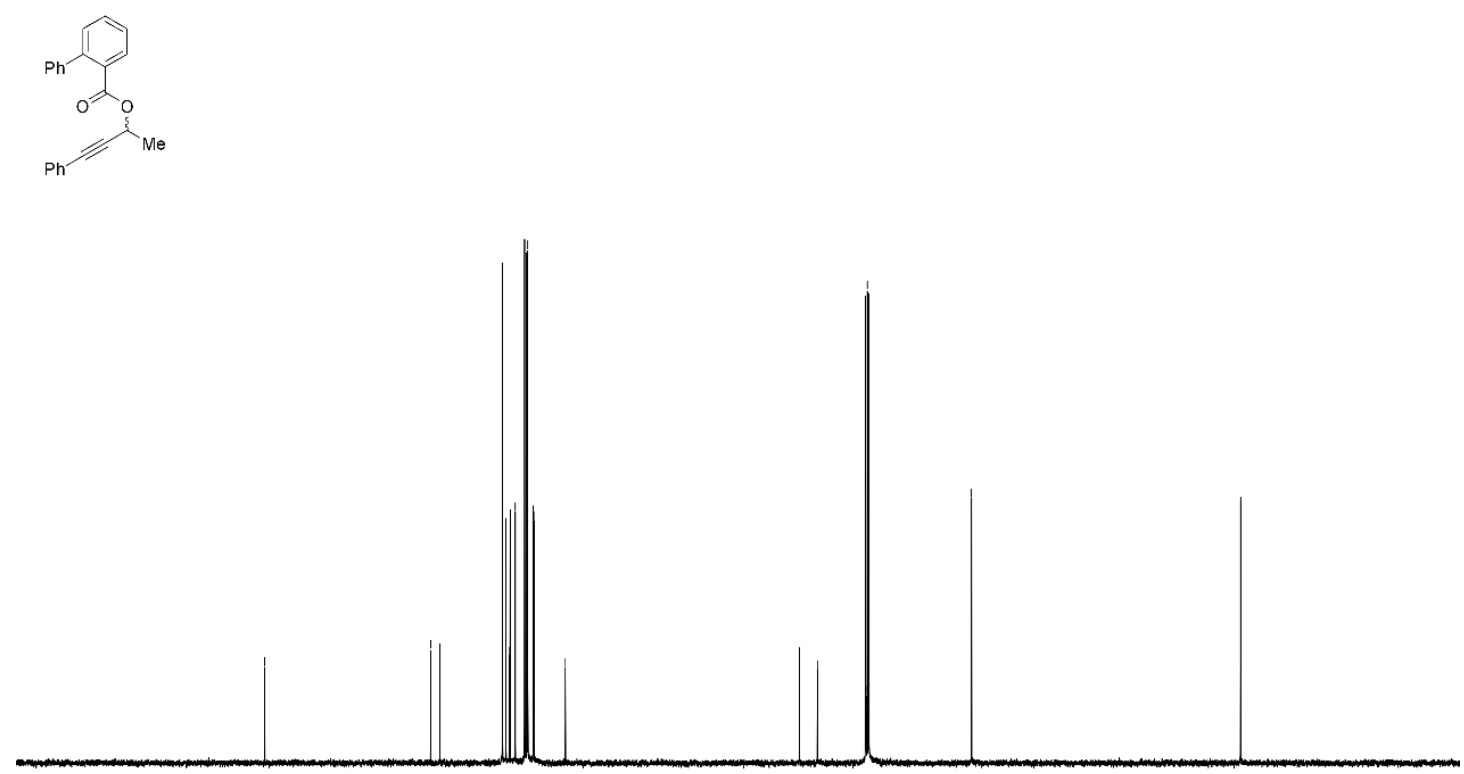

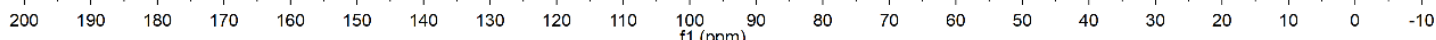


${ }^{1} \mathrm{H}$ NMR spectrum of S1-II

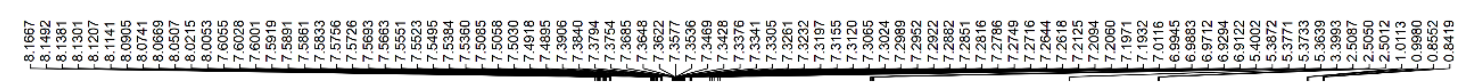
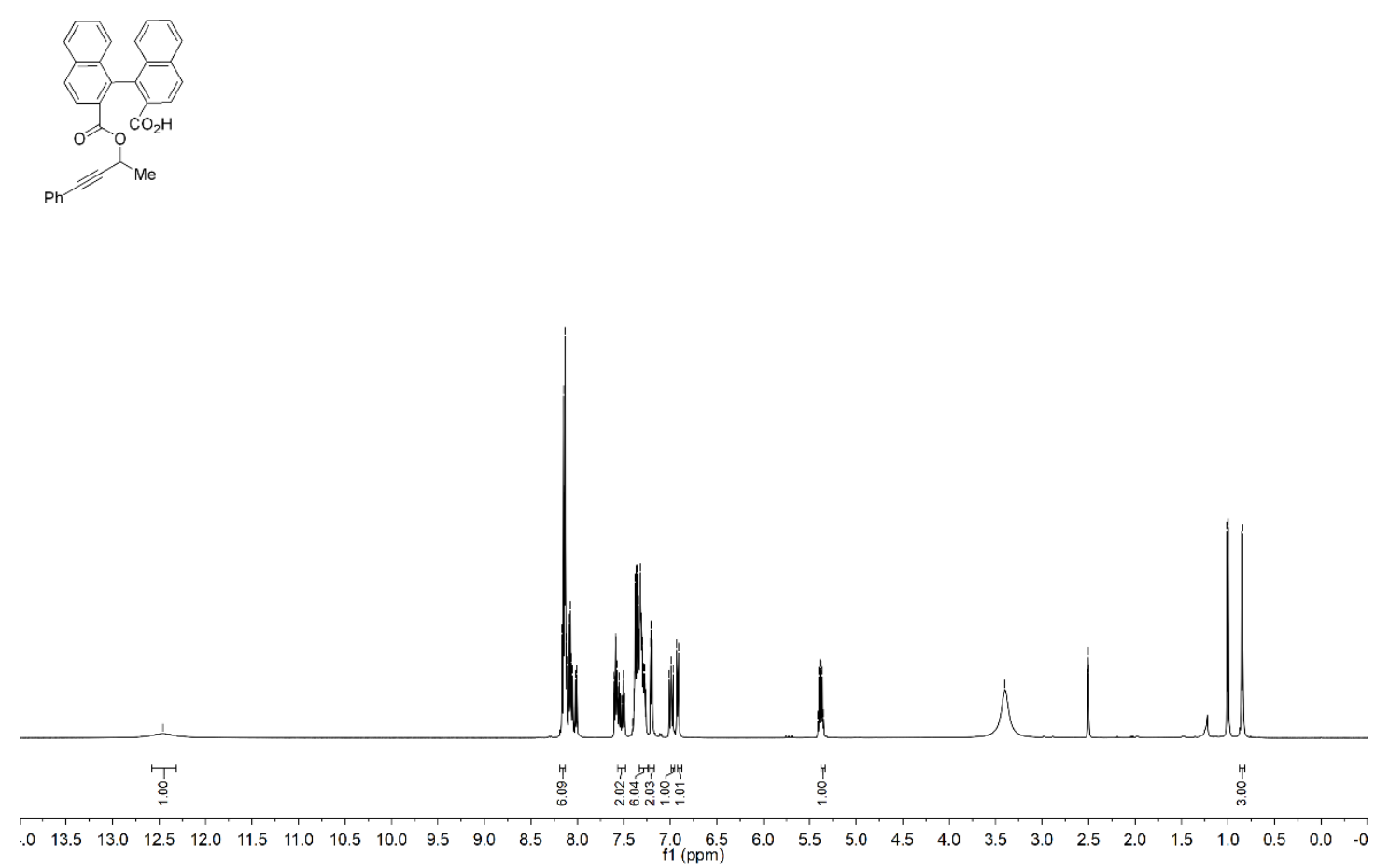

${ }^{13} \mathrm{C}$ NMR spectrum of S1-II

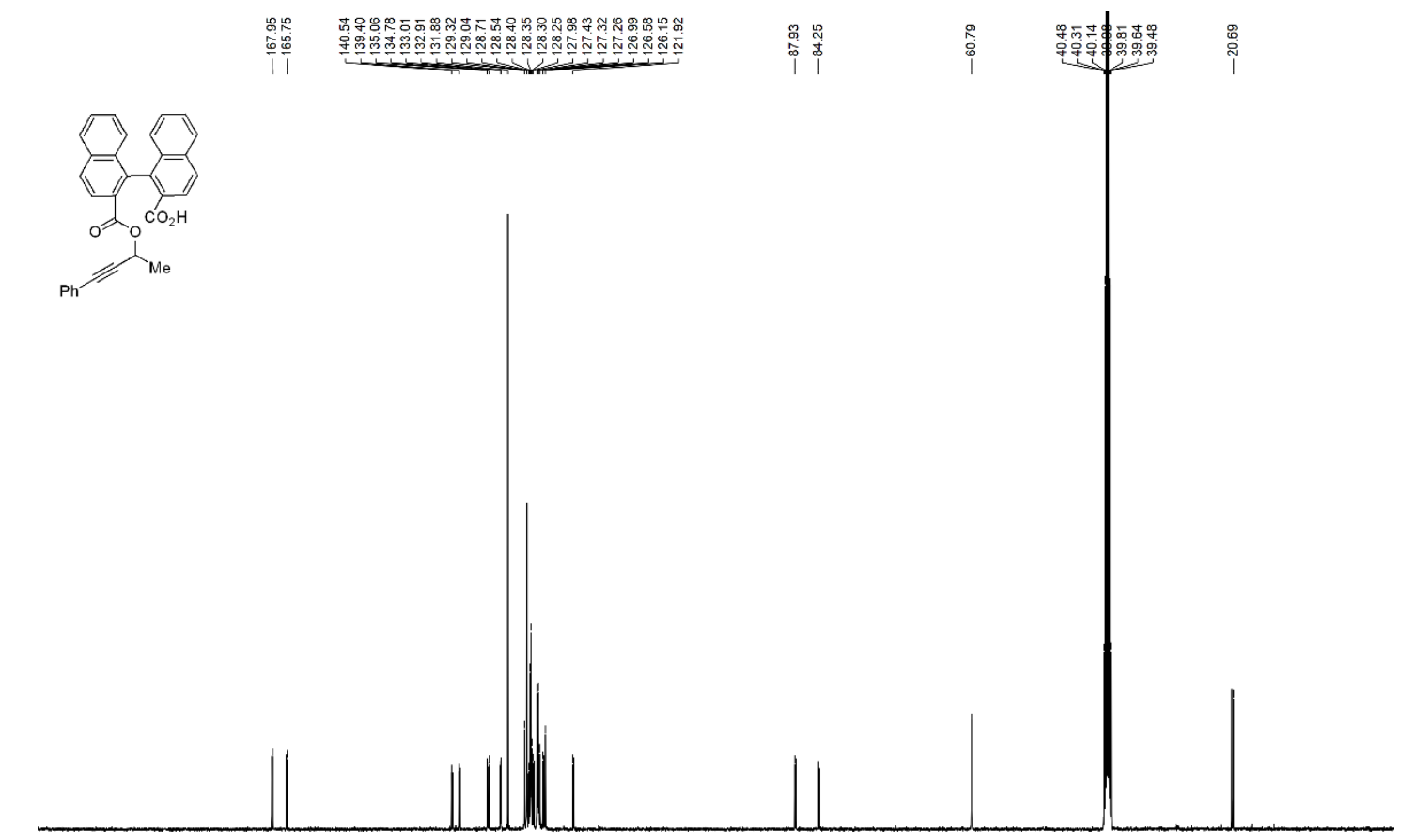

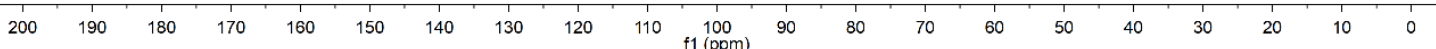




\section{${ }^{1} \mathrm{H}$ NMR spectrum of $\mathbf{S 1}$}
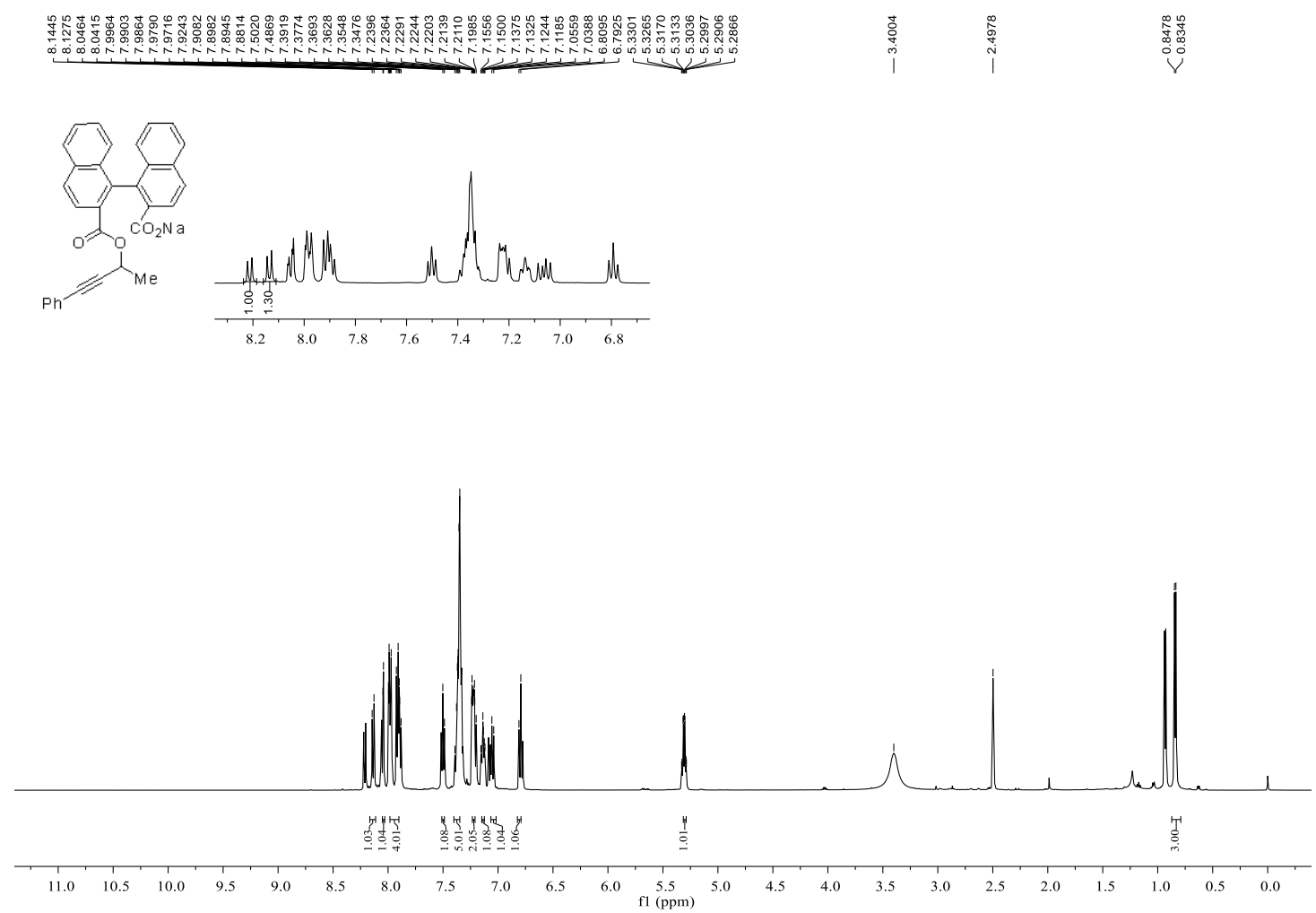

${ }^{13} \mathrm{C}$ NMR spectrum of $\mathbf{S 1}$

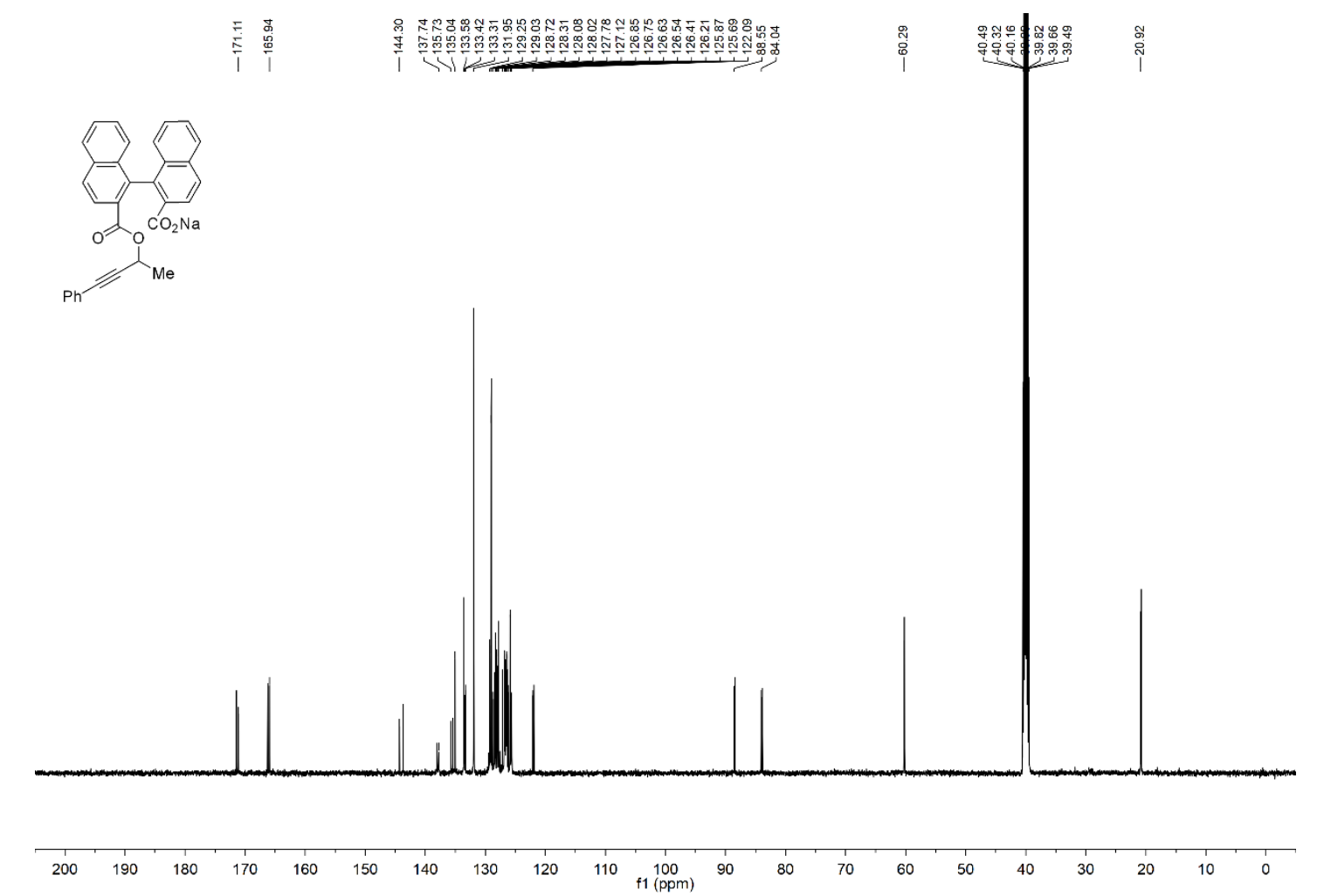


3.2.1 Ni-catalyzed asymmetric propargylic esterification
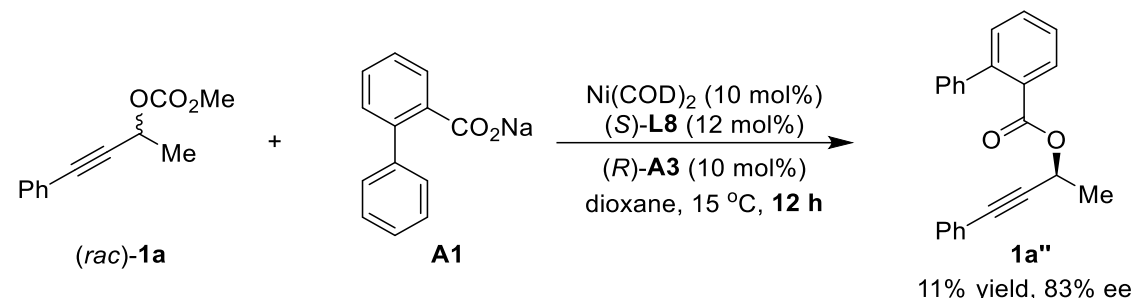

$\mathrm{Ni}(\mathrm{COD})_{2}(2.8 \mathrm{mg}, 0.01 \mathrm{mmol}, 10 \mathrm{~mol} \%),(S)-\mathrm{Cl}-\mathrm{MeO}-\mathrm{BIPHEP}(\mathbf{L 8})(7.8 \mathrm{mg}, 0.012 \mathrm{mmol}, 12$ mol\%) were added into a $10 \mathrm{~mL}$ Schlenk flask equipped with a magnetic stirrer in a glove box. Then dry dioxane $(4.0 \mathrm{~mL})$ was added into the reaction system under argon and stirred for 30 minutes at room temperature, after which the $(R)-\mathbf{A 3}(3.9 \mathrm{mg}, 0.01 \mathrm{mmol}, 10 \mathrm{~mol} \%)$ was added into the reaction system and stirred for 30 minutes under argon. Then propargylic carbonate $(\mathrm{rac})-\mathbf{1 a}(0.2$ mmol, 2.0 equiv) and sodium carboxylate $\mathbf{A 1}$ ( $0.1 \mathrm{mmol}, 1.0$ equiv) were added into the reaction mixture under argon at $15{ }^{\circ} \mathrm{C}$. The reaction solution was stirred at $15{ }^{\circ} \mathrm{C}$ for $12 \mathrm{~h}$. The reaction mixture was filtered through a short pad of silica gel, and the solvent was evaporated under vacuum. The residue was purified by silica gel chromatography to afford the desired products 1a" (11\% yield, 83\% ee). ${ }^{1} \mathbf{H}$ NMR (500 MHz, $\left.\mathbf{C D C l}_{3}\right) \delta 7.87(\mathrm{~d}, J=7.7 \mathrm{~Hz}, 1 \mathrm{H}), 7.54-7.27(\mathrm{~m}, 13 \mathrm{H}), 5.65$ $(\mathrm{q}, J=6.7 \mathrm{~Hz}, 1 \mathrm{H}), 1.32(\mathrm{~d}, J=6.7 \mathrm{~Hz}, 3 \mathrm{H}) .{ }^{13} \mathbf{C} \mathbf{N M R}\left(\mathbf{1 2 5} \mathbf{M H z}, \mathbf{C D C l}_{3}\right) \delta 167.62,142.67$, $141.27,131.88,131.36,130.81,130.73,129.96,128.57,128.55,128.24,128.09,127.24$, $127.18,122.43,87.26,84.58,76.82,61.40,20.96$. The product was analyzed by HPLC to determine the enantiomeric excess: $83 \%$ ee (CHIRALPAK AD-H, hexane/ $i$-PrOH $=70 / 30$, detector: $254 \mathrm{~nm}, \mathrm{~T}=25^{\circ} \mathrm{C}$, flow rate: $1 \mathrm{~mL} / \mathrm{min}$ ), $\mathrm{t}_{1}$ (minor) $=4.36 \mathrm{~min}, \mathrm{t}_{2}$ (major) $=4.75 \mathrm{~min}$.

The reaction of propargylic carbonate ( $\mathrm{rac}$-1a with sodium carboxylate $\mathbf{A 1}$ under the standard reaction condition led to $1 \mathbf{a}^{\prime \prime}$ in $11 \%$ yield and $83 \%$ ee.

3.2.2 The $O$-APS reactions with propargylic esters as the substrate

Table S1. The $O$-APS reactions with (rac)-1a and S1-S3

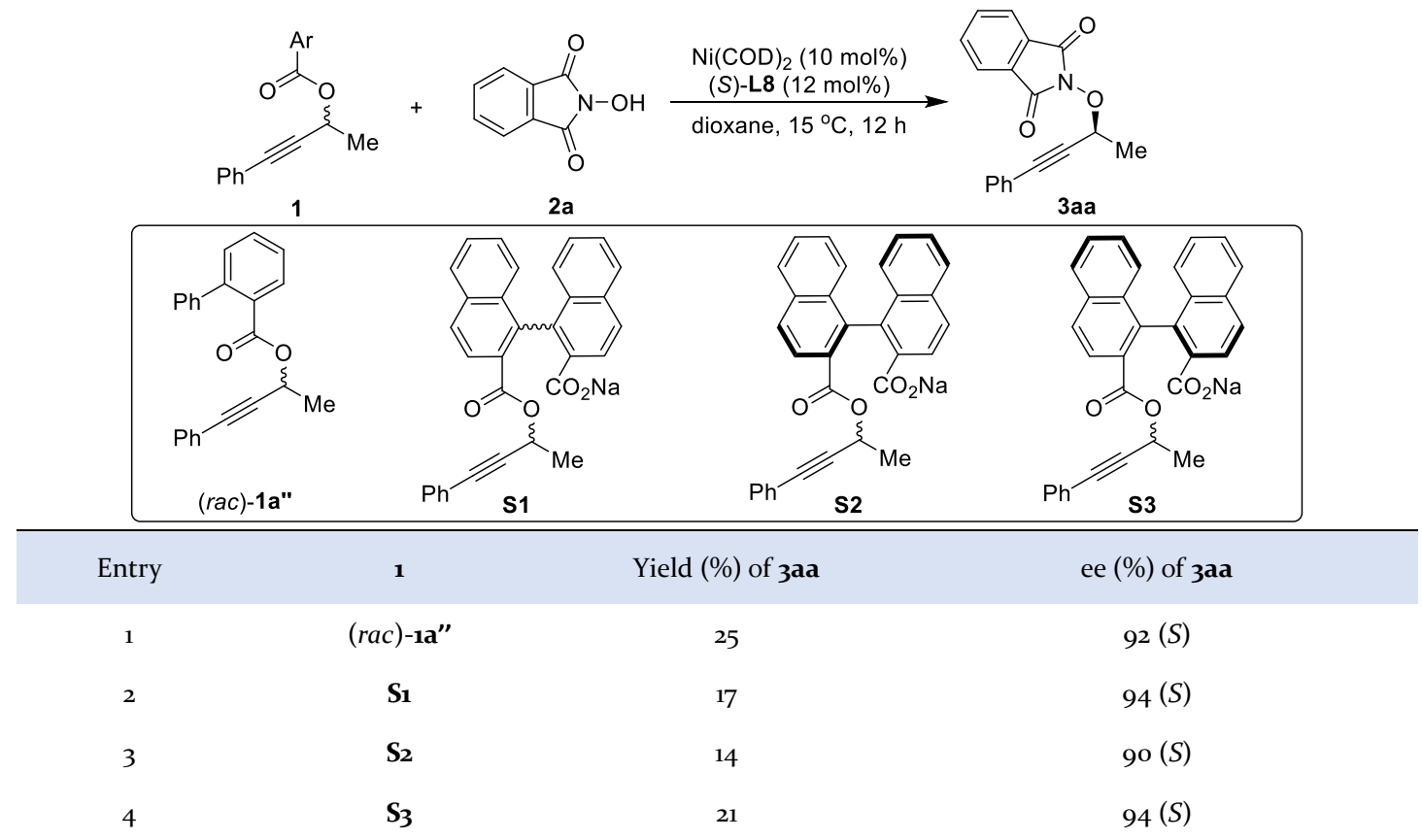

$\mathrm{Ni}(\mathrm{COD})_{2}(2.8 \mathrm{mg}, 0.01 \mathrm{mmol}, 10 \mathrm{~mol} \%),(S)-\mathrm{Cl}-\mathrm{MeO}-\mathrm{BIPHEP}(\mathbf{L 8})$ (7.8 mg, $0.012 \mathrm{mmol}, 12$ 
mol\%) were added into a $10 \mathrm{~mL}$ Schlenk flask equipped with a magnetic stirrer in a glove box. Then dry dioxane $(4.0 \mathrm{~mL})$ was added into the reaction system under argon and stirred for 30 minutes at room temperature. Then 1 ( $0.2 \mathrm{mmol}, 2.0$ equiv) and $\mathbf{2 a}(0.1 \mathrm{mmol}, 1.0$ equiv) were added into the reaction mixture under argon at $15{ }^{\circ} \mathrm{C}$. The reaction solution was stirred at $15{ }^{\circ} \mathrm{C}$ for $12 \mathrm{~h}$. The reaction mixture was filtered through a short pad of silica gel, and the solvent was evaporated under vacuum. The residue was purified by silica gel chromatography to afford the desired products 3aa.

The use of (rac)-1a" and S1-S3 as the substrates led to the product 3aa in low yields for $12 \mathrm{~h}$ (Table S1, entries 1-4). In addition, the use of monosodium carboxylate ( $R$ )-A10 instead of $\mathbf{A 3}$ furnished the target product in 58\% yield and $92 \%$ ee (Table 2, entry 13). Considering the possibility that S1-S3 would also serve as a monosodium carboxylate additive, we did kinetic experiments with ( $\mathrm{rac}$-1a" to investigate the influence of leaving groups on the reactivity and enantioselectivity of the transformation.

3.2.3 The reaction with $(\mathrm{rac})-\mathbf{1 a "}$ monitored over different reaction times

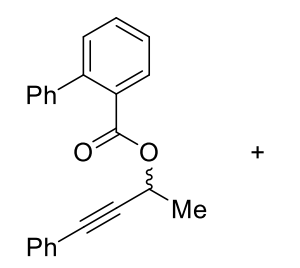

(rac)-1a"

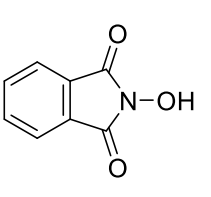

$2 \mathbf{a}$

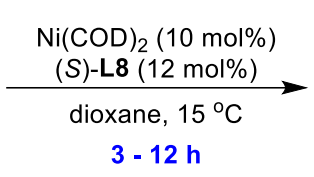

3 - $12 \mathrm{~h}$

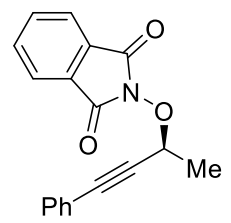

3aa

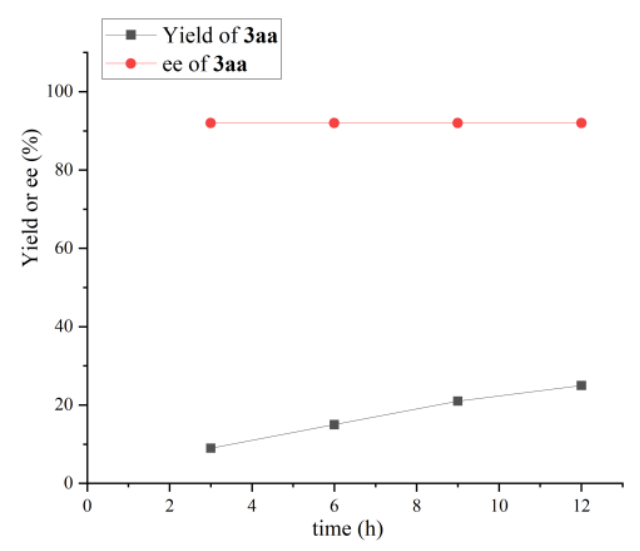

\begin{tabular}{ccc}
\hline time (h) & Yield of zaa & ee (\%) of zaa \\
3 & 9 & $92(S)$ \\
6 & 15 & $92(S)$ \\
9 & 21 & $92(S)$ \\
12 & 25 & $92(S)$ \\
\hline
\end{tabular}

Figure S5. The reaction with $(\mathrm{rac})$-1a" monitored over different reaction times

The enantioselectivity of $(S)$-3aa remains unchanged (92\% ee) throughout the reaction process.

3.2.4 Investigation of the carboxylate effect

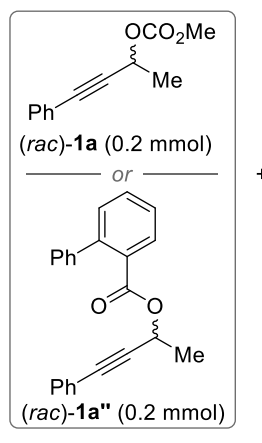

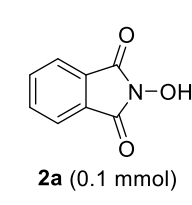

Condition A: with ( $(\mathrm{rac})$-1a as the substrate; without additive

Condition B: with ( $(\mathrm{rac})-\mathbf{1}$ a as the substrate; with $\mathbf{A} 1$ as additive Condition C: with ( $\mathrm{rac}$-1a" as the substrate; without additive 

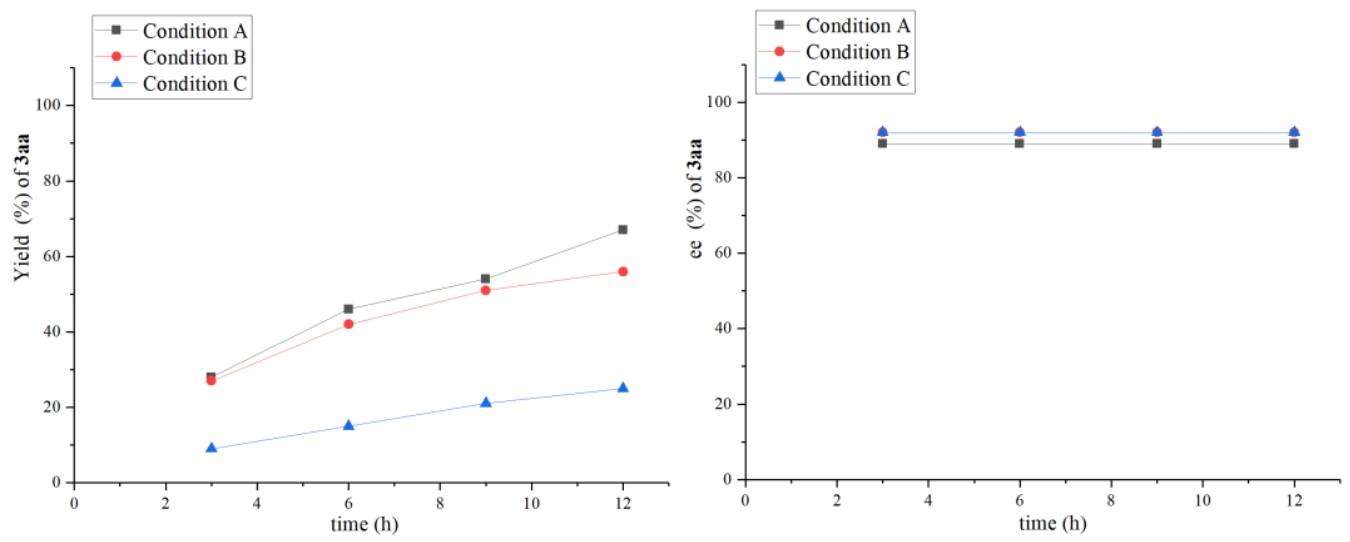

Figure S6. Carboxylate effect on the Ni-catalyzed $O$-APS reactions

The rate of product formation from the propargylic carbonate ( $\mathrm{rac}$ )-1a is faster than the rate from propargylic ester ( $\mathrm{rac}$ )-1a". In addition, the reaction of propargylic carbonate ( $\mathrm{rac}$ )-1a with sodium carboxylate A1 under the standard reaction condition led to 1a" in very low yield (3.2.1). Upon careful consideration of the proposed mechanism, we assumed the primary role of the carboxylate additive that served as a counterion for the nickel catalysis. Although the propargylic ester 1a" was not detected in Condition B, we cannot ruled out the possibility that the leaving group is replaced by the carboxylate group.

\subsection{Control reactions}

$\mathrm{Ni}(\mathrm{COD})_{2}$ (2.8 mg, $\left.0.01 \mathrm{mmol}, 10 \mathrm{~mol} \%\right),(S)$-Cl-MeO-BIPHEP (L8) (7.8 mg, $0.012 \mathrm{mmol}, 12$ mol\%) were added into a $10 \mathrm{~mL}$ Schlenk flask equipped with a magnetic stirrer in a glove box. Then dry dioxane $(4.0 \mathrm{~mL})$ was added into the reaction system under argon and stirred for 30 minutes at room temperature, after which the $(R)-\mathbf{A} \mathbf{3}(3.9 \mathrm{mg}, 0.01 \mathrm{mmol}, 10 \mathrm{~mol} \%)$ was added into the reaction system and stirred for 30 minutes under argon. Then propargylic carbonate $(\mathrm{rac})$-3af $(0.2$ mmol, 2.0 equiv) and $N$-hydroxyphthalimide $\mathbf{2 a}(0.1 \mathrm{mmol}, 1.0$ equiv) were added into the reaction mixture under argon at $15{ }^{\circ} \mathrm{C}$. The reaction solution was stirred at $15^{\circ} \mathrm{C}$ for $72 \mathrm{~h}$. The recovered substrate 3af and the corresponding product 3aa were analyzed by HPLC.

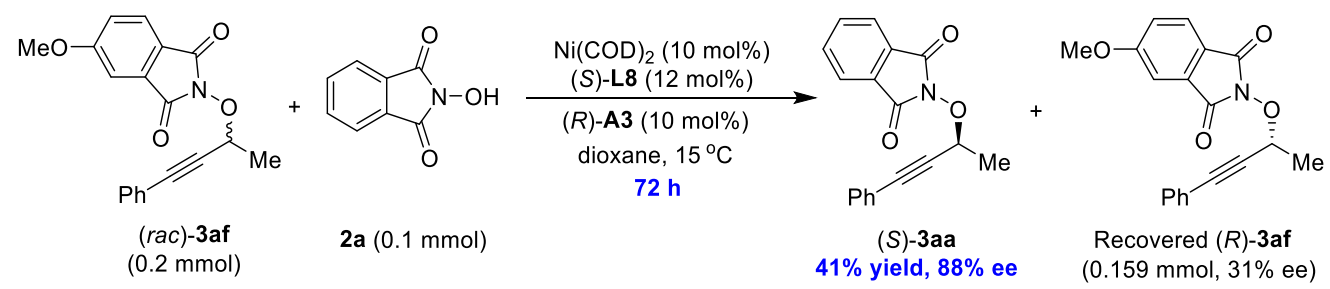

A cross-over experiment involving racemic 3af with $\mathbf{2 a}$ under the standard reaction condition led to 3aa in $41 \%$ yield and $88 \%$ ee with $(R)$-3af recovered in $31 \%$ ee, suggesting that the cleavage of the $\mathrm{C}-\mathrm{O}$ bond of the propargylated product $3 \mathrm{af}$ and a kinetic resolution process are involved in this reaction.

3.3.1 The reaction with $(\mathrm{rac})$-3af monitored over different reaction time

$\mathrm{Ni}(\mathrm{COD})_{2}$ (2.8 mg, $\left.0.01 \mathrm{mmol}, 10 \mathrm{~mol} \%\right)$, (S)-Cl-MeO-BIPHEP (L8) $(7.8 \mathrm{mg}, 0.012 \mathrm{mmol}, 12$ mol\%) were added into a $10 \mathrm{~mL}$ Schlenk flask equipped with a magnetic stirrer in a glove box. Then dry dioxane $(4.0 \mathrm{~mL})$ was added into the reaction system under argon and stirred for 30 minutes at room temperature, after which the $(R)-\mathbf{A 3}(3.9 \mathrm{mg}, 0.01 \mathrm{mmol}, 10 \mathrm{~mol} \%)$ was added into the 
reaction system and stirred for 30 minutes under argon. Then propargylic carbonate $(\mathrm{rac})$-3af $(0.2$ mmol, 2.0 equiv) and compound $2 \mathrm{a}(0.1 \mathrm{mmol}, 1.0$ equiv) were added into the reaction mixture under argon at $15{ }^{\circ} \mathrm{C}$. The reaction of (rac)-3af with $2 \mathbf{a}$ was monitored at $3 \mathrm{~h}, 6 \mathrm{~h}, 9 \mathrm{~h}$, and $12 \mathrm{~h}$. The recovered substrate $\mathbf{3} \mathbf{a f}$ and the corresponding products $\mathbf{3 a a}$ were analyzed by HPLC.<smiles>COc1ccc2c(c1)C(=O)N(OC(C)C#Cc1ccccc1)C2=O</smiles>

(rac)-3af $(0.2 \mathrm{mmol})$

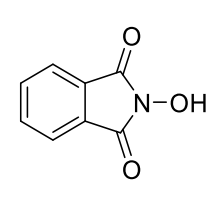

2a $(0.1 \mathrm{mmol})$

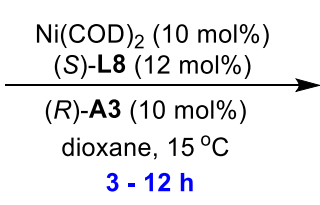

$3-12 \mathrm{~h}$

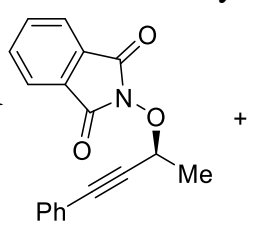

3aa

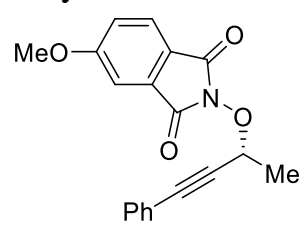

Recovered $(R)-\mathbf{3 a f}$

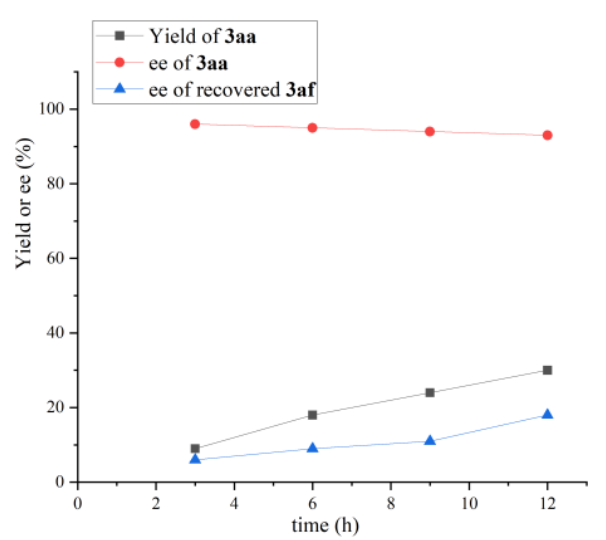

\begin{tabular}{cccc}
\hline time (h) & Yield of zaa & ee (\%) of zaa & ee (\%) of recovered zaf \\
3 & 9 & $96(S)$ & $6(R)$ \\
6 & 18 & $95(S)$ & $9(R)$ \\
9 & 24 & $94(S)$ & $11(R)$ \\
12 & 30 & $93(S)$ & $18(R)$ \\
\hline
\end{tabular}

Figure S7. The reaction with ( $\mathrm{rac}$-3af monitored over different reaction time

The ee value of 3aa versus reaction time was monitored, and a time-dependent change was found ranging from $96 \%$ ee $(S)$ at $3 \mathrm{~h}$ to $93 \%$ ee $(S)$ at $12 \mathrm{~h}$. In addition, the recovered 3af was resolved to its $R$-enantiomer.

3.3.2 The reaction with $(R)$-3af monitored over different reaction times

$\mathrm{Ni}(\mathrm{COD})_{2}(2.8 \mathrm{mg}, 0.01 \mathrm{mmol}, 10 \mathrm{~mol} \%),(S)$-Cl-MeO-BIPHEP (L8) $(7.8 \mathrm{mg}, 0.012 \mathrm{mmol}, 12$ mol\%) were added into a $10 \mathrm{~mL}$ Schlenk flask equipped with a magnetic stirrer in a glove box. Then dry dioxane $(4.0 \mathrm{~mL})$ was added into the reaction system under argon and stirred for 30 minutes at room temperature, after which the $(R)-\mathbf{A 3}(3.9 \mathrm{mg}, 0.01 \mathrm{mmol}, 10 \mathrm{~mol} \%)$ was added into the reaction system and stirred for 30 minutes under argon. Then propargylic carbonate $(R)$-3af $(0.2$ mmol, 2.0 equiv) and compound $\mathbf{2 a}(0.1 \mathrm{mmol}, 1.0$ equiv) were added into the reaction mixture under argon at $15^{\circ} \mathrm{C}$. The reaction of $(R)$-3af with $2 \mathbf{a}$ was monitored at $3 \mathrm{~h}, 6 \mathrm{~h}, 9 \mathrm{~h}$, and $12 \mathrm{~h}$. The recovered substrate $\mathbf{3 a f}$ and the corresponding products 3aa were analyzed by HPLC.

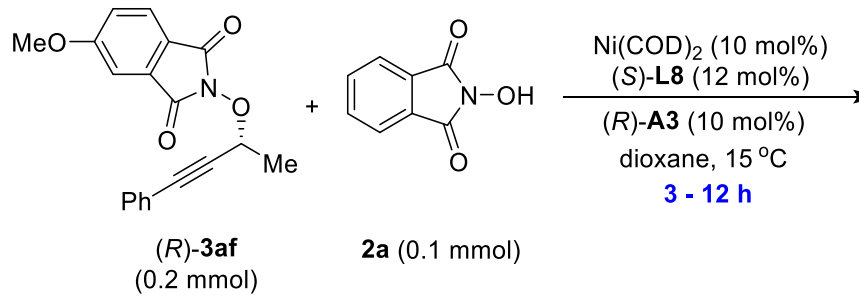

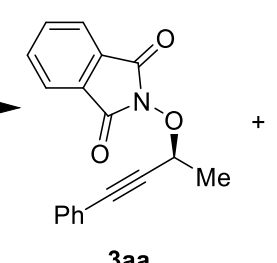

3 aа

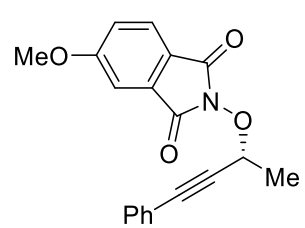

Recovered (R)-3af 


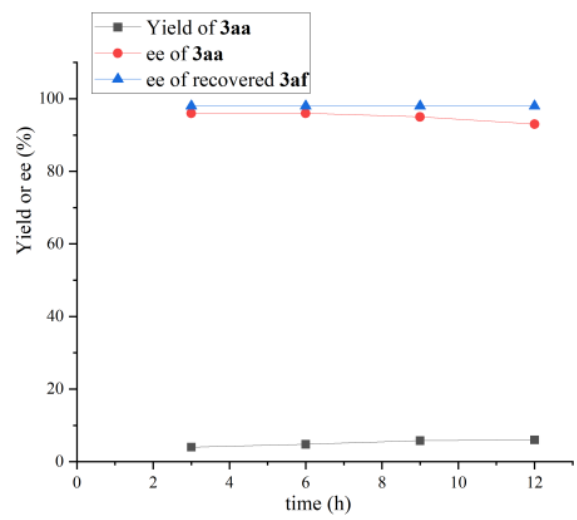

\begin{tabular}{cccc}
\hline time (h) & Yield of zaa & ee (\%) of zaa & ee (\%) of recovered zaf \\
3 & 4 & $96(S)$ & $98(R)$ \\
6 & 4.8 & $96(S)$ & $98(R)$ \\
9 & 5.8 & $95(S)$ & $98(R)$ \\
12 & 6 & $93(S)$ & $98(R)$ \\
\hline
\end{tabular}

Figure S8. The reaction with $(R)$-3af monitored over different reaction time

The ee value of 3aa versus reaction time was monitored, and a time-dependent change was found ranging from $96 \%$ ee $(S)$ at $3 \mathrm{~h}$ to $93 \%$ ee $(S)$ at $12 \mathrm{~h}$. The enantioselectivity of $(R)$-3af remains unchanged ( $98 \%$ ee) throughout the reaction process.

3.3.3 The reaction with $(S)$-3af monitored over different reaction time

$\mathrm{Ni}(\mathrm{COD})_{2}$ (2.8 mg, $0.01 \mathrm{mmol}, 10 \mathrm{~mol} \%$ ), (S)-Cl-MeO-BIPHEP (L8) (7.8 mg, $0.012 \mathrm{mmol}, 12$ mol\%) were added into a $10 \mathrm{~mL}$ Schlenk flask equipped with a magnetic stirrer in a glove box. Then dry dioxane $(4.0 \mathrm{~mL})$ was added into the reaction system under argon and stirred for 30 minutes at room temperature, after which the $(R)-\mathbf{A 3}(3.9 \mathrm{mg}, 0.01 \mathrm{mmol}, 10 \mathrm{~mol} \%)$ was added into the reaction system and stirred for 30 minutes under argon. Then propargylic carbonate $(S)$-3af $(0.2$ mmol, 2.0 equiv) and compound $2 \mathbf{a}(0.1 \mathrm{mmol}, 1.0$ equiv) were added into the reaction mixture under argon at $15{ }^{\circ} \mathrm{C}$. The reaction of $(S)$-3af with $2 \mathbf{a}$ was monitored at $3 \mathrm{~h}, 6 \mathrm{~h}, 9 \mathrm{~h}$, and $12 \mathrm{~h}$. The recovered substrate 3af and the corresponding products 3aa were analyzed by HPLC.

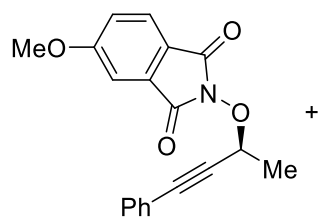

(S)-3af $(0.2 \mathrm{mmol})$

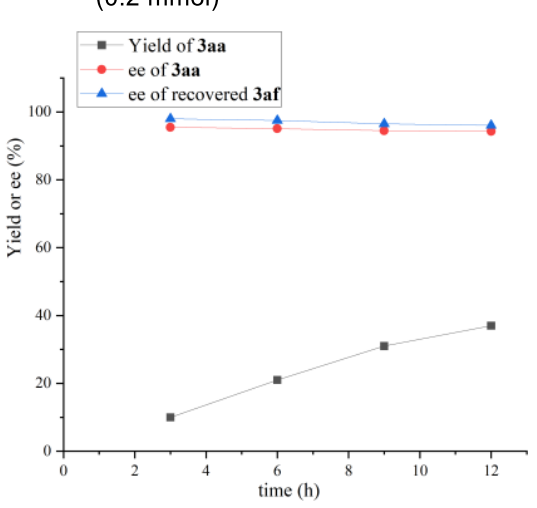

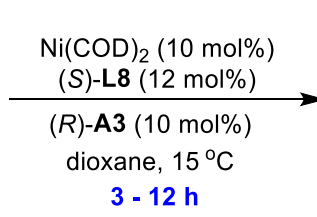

3 - $12 \mathrm{~h}$

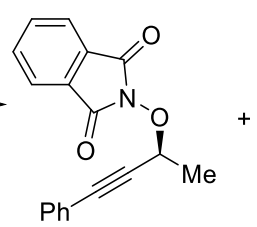

$3 a a$

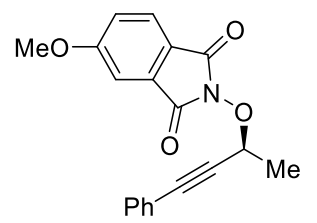

Recovered (S)-3af

\begin{tabular}{cccc}
\hline time (h) & Yield of zaa & ee (\%) of zaa & ee (\%) of recovered zaf \\
3 & 10 & $95.5(S)$ & $98(S)$ \\
6 & 21 & $95.1(S)$ & $97.5(S)$ \\
9 & 31 & $94.5(S)$ & $96.5(S)$ \\
12 & 37 & $94.3(S)$ & $96.1(S)$ \\
\hline
\end{tabular}

Figure S9. The reaction with $(S)$-3af monitored over different reaction time

The ee value of 3aa versus reaction time was monitored, and a time-dependent change was found ranging from $95.5 \%$ ee $(S)$ at $3 \mathrm{~h}$ to $94.3 \%$ ee $(S)$ at $12 \mathrm{~h}$. Furthermore, a slight loss of enantiomeric purity of recovered 3af was observed. 
3.3.5 The reaction with $(\mathrm{rac})$-3af, $(R)$-3af, or $(S)$-3af monitored over different reaction time<smiles>COc1ccc2c(c1)C(=O)N(OC(C)C#Cc1ccccc1)C2=O</smiles>

3af $(0.2 \mathrm{mmol})$

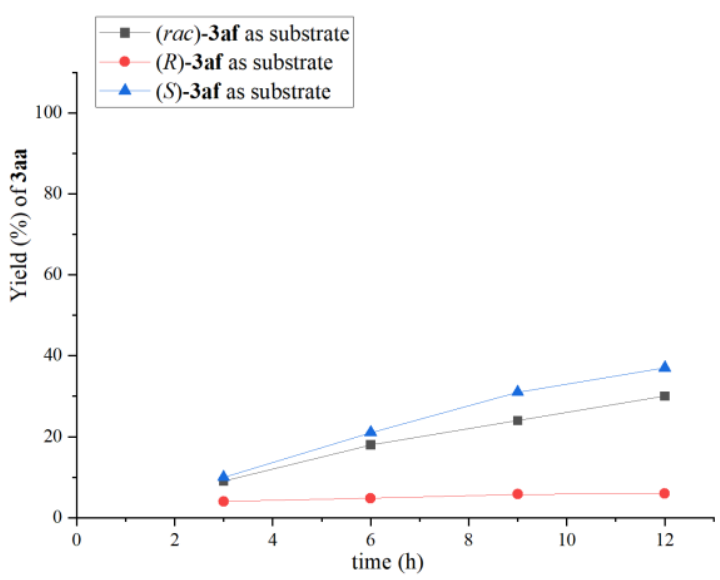

(a)

2a $(0.1 \mathrm{mmol})$<smiles>O=C1c2ccccc2C(=O)N1O</smiles>

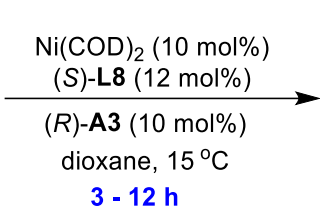

$3-12 \mathrm{~h}$

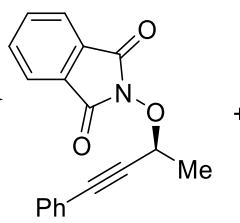

3aa

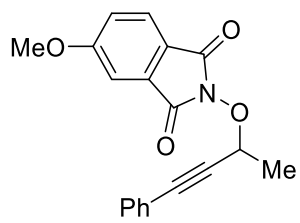

Recovered 3af

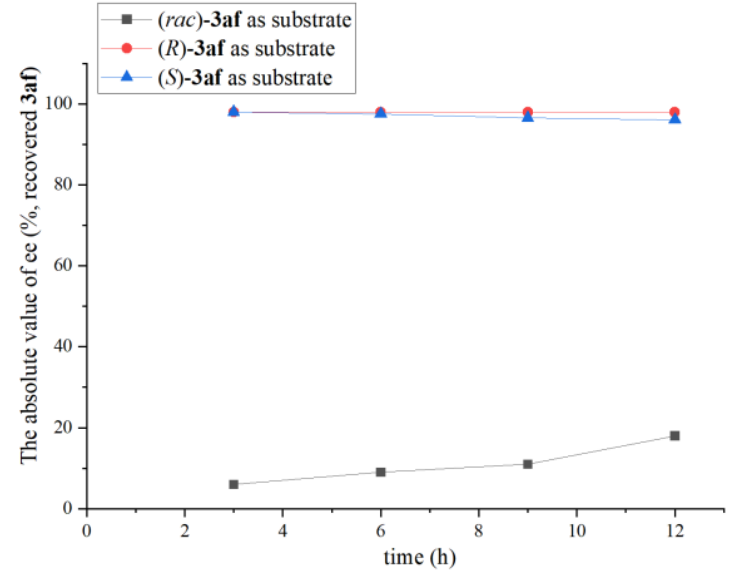

Figure S10. Time-course studies of the Ni-catalyzed $O$-APS reactions of 3 af

The reaction rate with $(S)$-3af was faster than that with $(R)$-3af. In addition, the propargylated product ( $r a c$ )-3af was successfully resolved to its $R$-enantiomer. As the major $(S)$-3af is removed more rapidly than $(R)$-3af, the ee of $(S)$-3af slowly decreases.

3.3.6 Leaving group effect
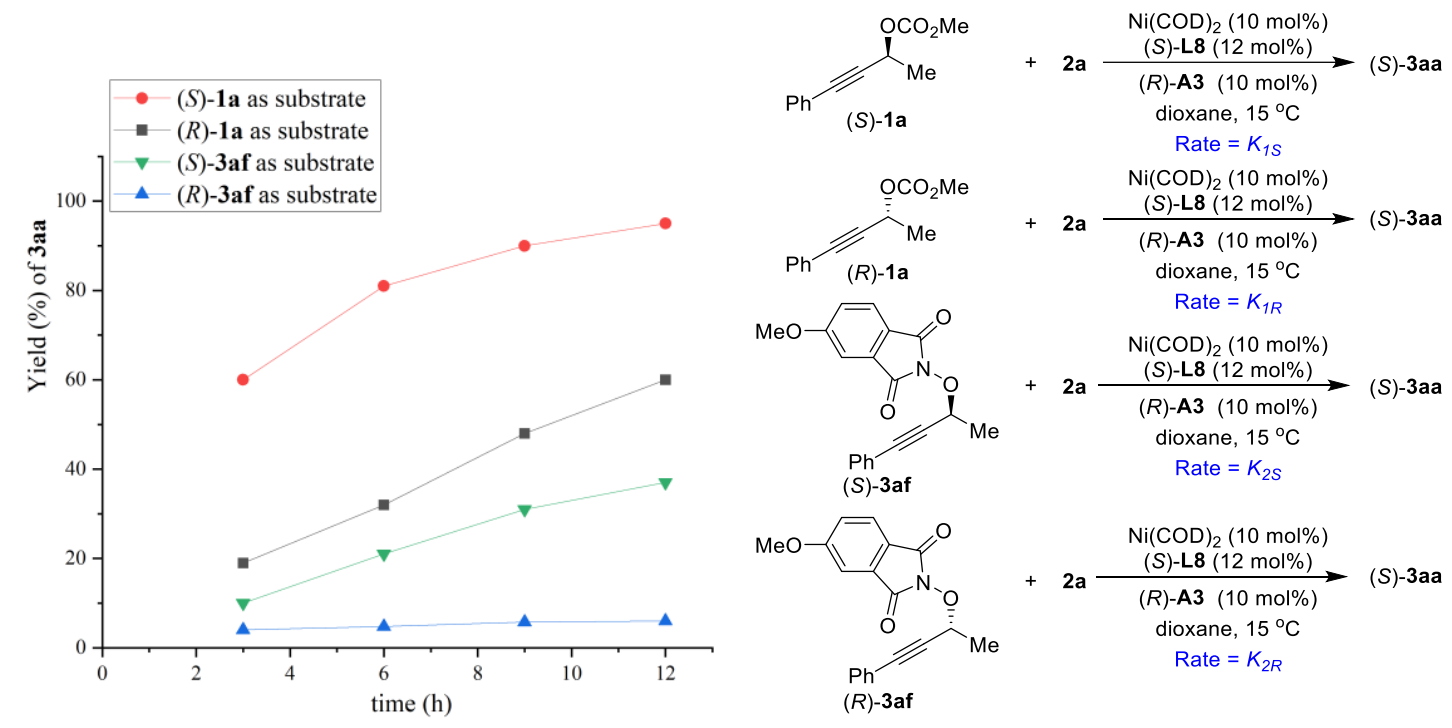

Figure S11. Leaving group effect 
3.3.7 Mechanistic rationale of a combined asymmetric propargylic substitution/kinetic resolution kinetic resolution

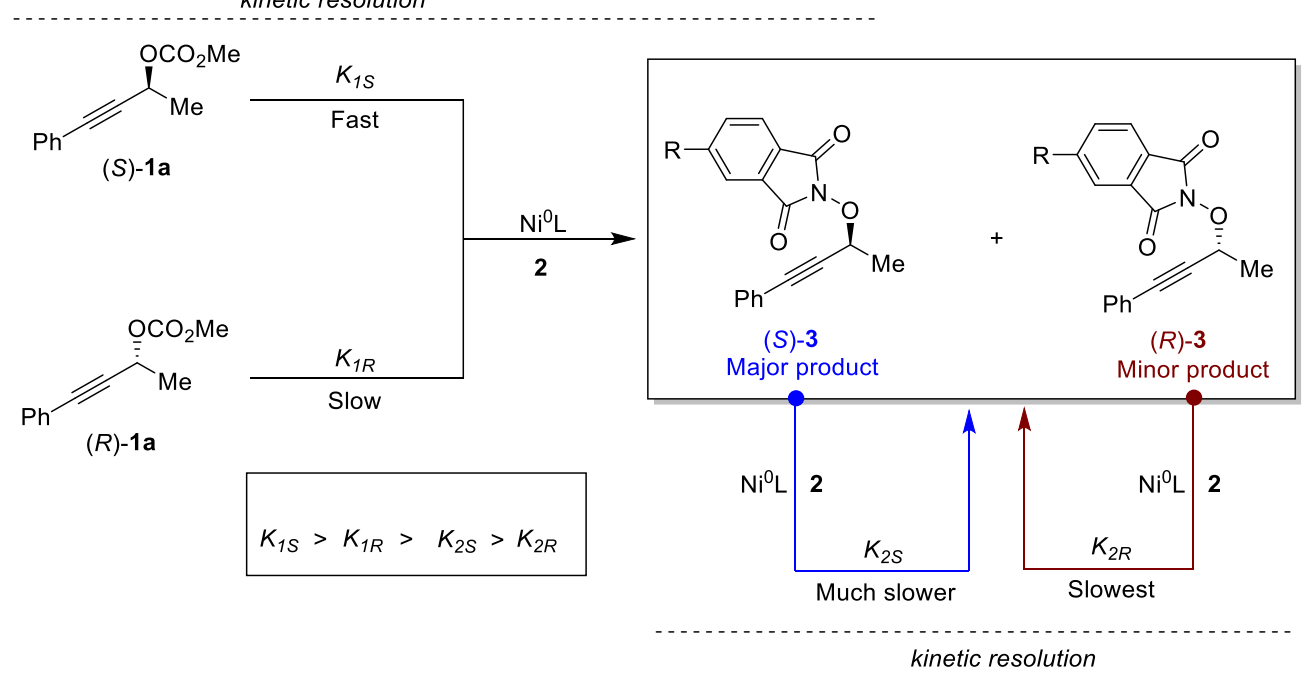

3.3.8 Control reactions

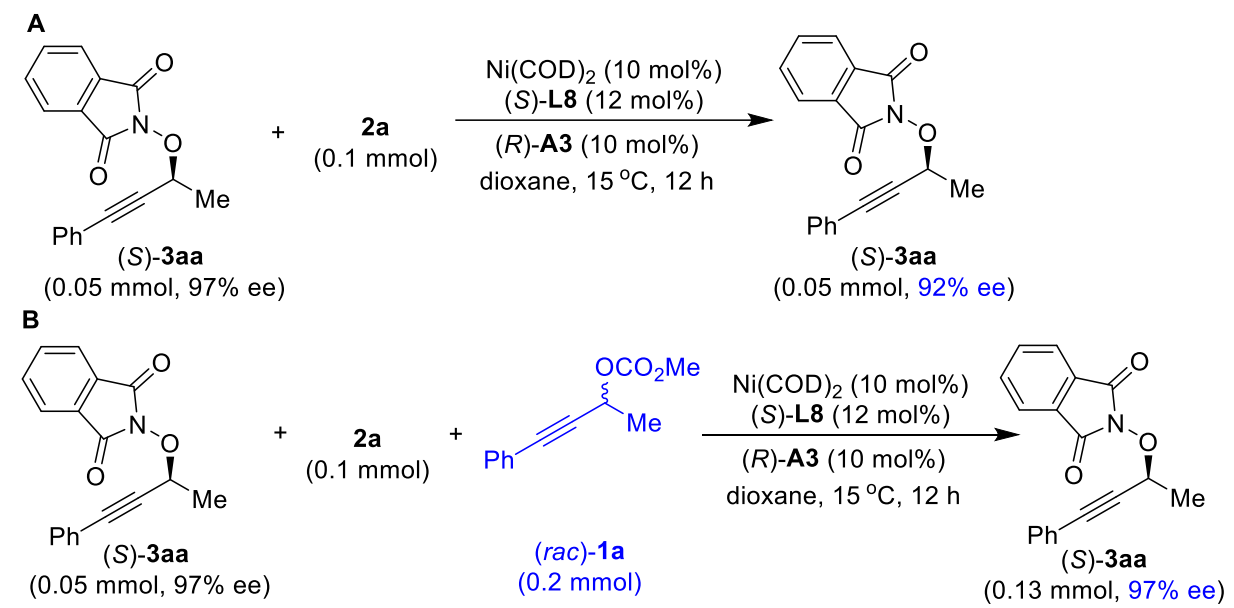

Figure S12. Control reactions. (A) Partial racemization of (S)-3aa. (B) Prevention of racemization in the $O$-APS reactions.

Upon the treatment of the (S)-3aa (97\% ee) with $\mathbf{2 a}$ under the standard catalytic conditions, a partial loss of enantiomeric purity was observed in $12 \mathrm{~h}$ (Figure S12A, from $97 \%$ ee to $92 \%$ ee). In contrast, the racemization of ( $S$ )-3aa was completely inhibited by the addition of ( $\mathrm{rac}$ )-1a (Figure S12B). Consequently, the maintained enantioselectivity likely reflects the discrimination by the $\mathrm{Ni}$ catalyst toward propargylic carbonate 1a and propargylated product 3aa in an oxidative-addition process of the catalytic cycle. Therefore, the racemization of (S)-3aa can be suppressed with the addition of excess propargylic carbonates 1a, and excellent stereocontrol was ensured in the $O$-APS reactions.

3.4. Cross-over experiments with racemic or achiral phosphine ligands 


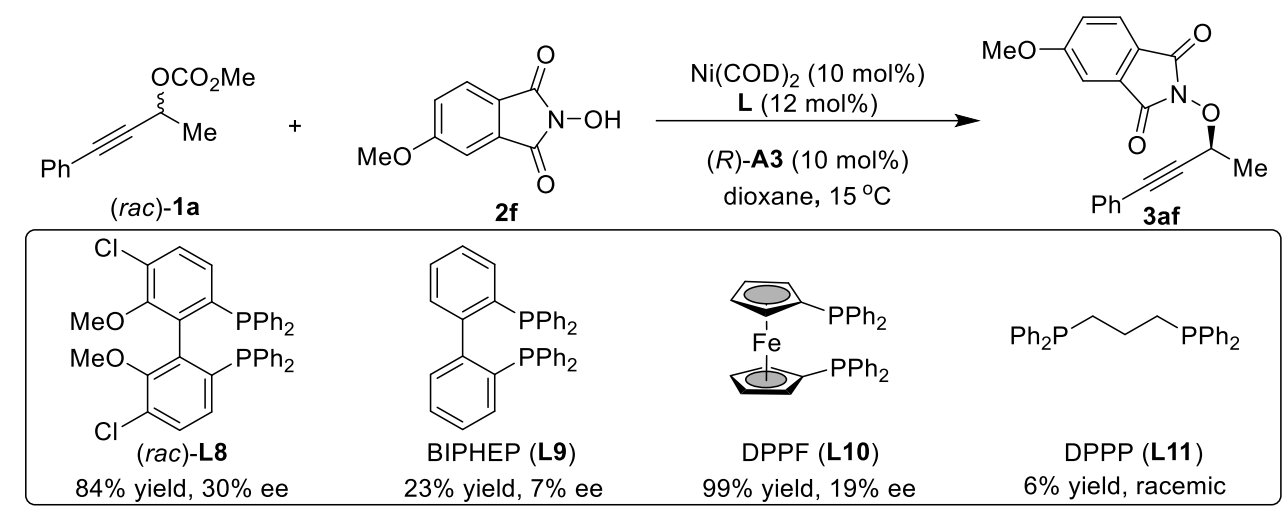

The use of $(R)$-A3 in combination with ( $\mathrm{rac}$ )-L8 indeed resulted in considerable enantioselection (30\% ee) under otherwise identical conditions. We next evaluated different achiral phosphine ligands (L9-L11), and the use of DFFP (L10) led to the propargylated product 3af in 99\% yield with $19 \%$ ee.

3.5 NMR studies

\subsection{1 ${ }^{31} \mathrm{P}$ NMR studies}

The ${ }^{31} \mathrm{P}$ NMR spectra at $20{ }^{\circ} \mathrm{C}$ were recorded with a Bruker AV $400 \mathrm{MHz}$ spectrometer. The ${ }^{31} \mathrm{P}$ NMR spectra in Figure S13 were done in a J. Young tube, to which were added Ni(COD) 2 (5.5 mg, $0.02 \mathrm{mmol}, 1.0$ equiv), (S)-Cl-MeO-BIPHEP (L8) (13 mg, $0.02 \mathrm{mmol}, 1.0$ equiv), and 1,4-dioxane $/ \mathrm{CD}_{3} \mathrm{CN}(0.1 \mathrm{~mL} / 0.5 \mathrm{~mL})$. The solution was shaken for 30 minutes at room temperature. Triphenyl phosphate $(2 \mathrm{mg})$ was added as the reference and the sample was analyzed by ${ }^{31} \mathrm{P}$ NMR. The addition of $(R)$-A3 is suggested to generate an active carboxylate nickel intermediate via anion exchange, which might be responsible for the high stereoselectivity of this system.

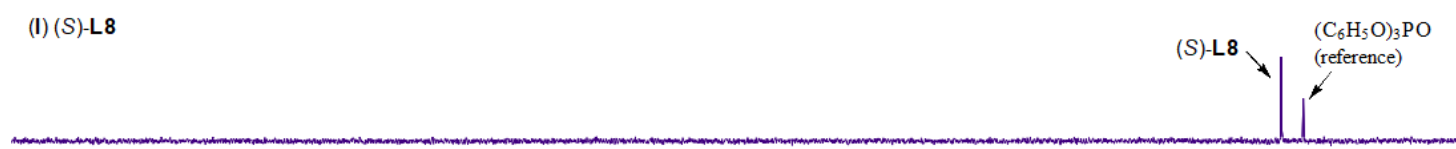

(II) $\mathrm{Ni}(\mathrm{COD})_{2}+(\mathrm{S})-\mathrm{L} \mathbf{8}$

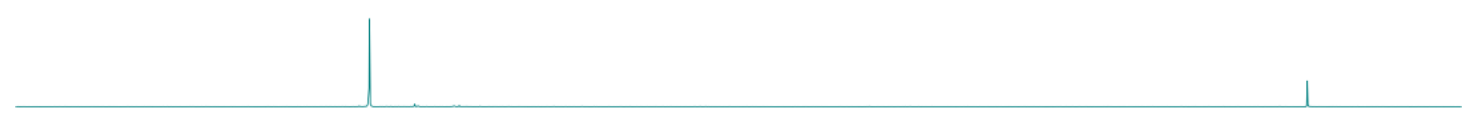

(III) $\mathrm{Ni}(\mathrm{COD})_{2}+(\mathrm{S})-\mathbf{L} 8+(\mathrm{rac})-\mathbf{1 a}$

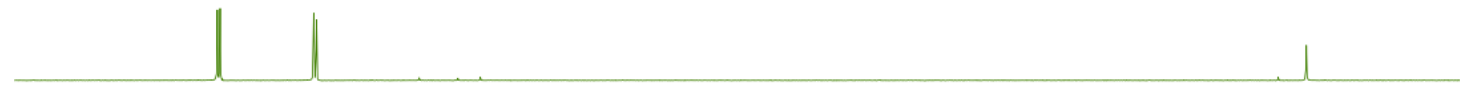

(IV) $\mathrm{Ni}(\mathrm{COD})_{2}+(\mathrm{S})-\mathbf{L} \mathbf{8}+(\mathrm{rac})-\mathbf{1} \mathbf{a}+(R)-\mathbf{A} \mathbf{3}$

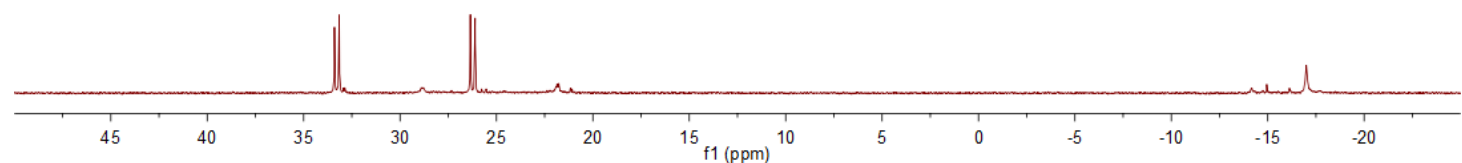

Figure S13. ${ }^{31} \mathrm{P}$ NMR spectra. (I) (S)-L8 (13 mg, $0.02 \mathrm{mmol}$ ), triphenyl phosphate (2 mg, reference), and 1,4-dioxane/CD ${ }_{3} \mathrm{CN}(0.1 \mathrm{~mL} / 0.5 \mathrm{~mL})$ were added to a J. Young tube, and the sample was analyzed by ${ }^{31} \mathrm{P} \mathrm{NMR}$; (II) Ni(COD) 2 (5.5 mg, $0.02 \mathrm{mmol}, 1.0$ equiv), ( $S$ )-L8 (13 mg, $0.02 \mathrm{mmol}$, 1.0 equiv), and 1,4-dioxane/ $\mathrm{CD}_{3} \mathrm{CN}(0.1 \mathrm{~mL} / 0.5 \mathrm{~mL})$ were added to a J. Young tube in the glove box. The solution was shaken for 30 minutes at room temperature, then triphenyl phosphate $(2 \mathrm{mg})$ 
was added and the sample was analyzed by ${ }^{31} \mathrm{P} \mathrm{NMR}$; (III) Ni(COD) 2 (5.5 mg, $0.02 \mathrm{mmol}, 1.0$ equiv), (S)-L8 (13 mg, $0.02 \mathrm{mmol}, 1.0$ equiv), and 1,4-dioxane/CD ${ }_{3} \mathrm{CN}(0.1 \mathrm{~mL} / 0.5 \mathrm{~mL})$ were added to a J. Young tube in the glove box. After the solution was shaken for 30 minutes, $(\mathrm{rac})-\mathbf{1 a}(4 \mathrm{mg}$, $0.02 \mathrm{mmol}, 1.0$ equiv) was added, and the solution was shaken for 30 minutes. Then triphenyl phosphate ( $2 \mathrm{mg}$ ) was added and the sample was analyzed by ${ }^{31} \mathrm{P} \mathrm{NMR}$; (IV) $\mathrm{Ni}(\mathrm{COD})_{2}(5.5 \mathrm{mg}$, $0.02 \mathrm{mmol}, 1.0$ equiv), (S)-L8 (13 mg, $0.02 \mathrm{mmol}, 1.0$ equiv), and 1,4-dioxane/CD ${ }_{3} \mathrm{CN}(0.1 \mathrm{~mL} / 0.5$ $\mathrm{mL}$ ) were added to a J. Young tube in the glove box. After the solution was shaken for 30 minutes, (rac)-1a (4 mg, $0.02 \mathrm{mmol}, 1.0$ equiv) was added and shaken for 30 minutes. Then $(R)$-A3 (7.8 mg, $0.02 \mathrm{mmol}, 1.0$ equiv) was added into the reaction system and shaken for 30 minutes, and the sample was analyzed by ${ }^{31} \mathrm{P}$ NMR.

We carried out the reaction with the intermediate detected in the ${ }^{31} \mathrm{P}$ NMR. The addition of $\mathbf{2 a}$ to the formed intermediate led to the corresponding product 3aa.

$\mathrm{Ni}(\mathrm{COD})_{2}$ (5.5 mg, $0.02 \mathrm{mmol}, 1.0$ equiv), (S)-L8 (15.6 mg, $0.024 \mathrm{mmol}, 1.2$ equiv), and 1,4dioxane/ $\mathrm{CD}_{3} \mathrm{CN}(0.1 \mathrm{~mL} / 0.5 \mathrm{~mL})$ were added to a J. Young tube in the glove box. Then $(\mathrm{rac})-1 \mathrm{a}$ (4.0 mg, $0.02 \mathrm{mmol}, 1.0$ equiv) and ( $R$ )-A3 (7.8 mg, $0.02 \mathrm{mmol}, 1.0$ equiv) were added. After the sample was analyzed by ${ }^{31} \mathrm{P}$ NMR, the solution was transferred to a dried and argon-filled Schlenk tube. Then ( $r a c)-1 \mathbf{a}(77.7 \mathrm{mg}, 0.38 \mathrm{mmol})$ and $\mathbf{2 a}(32.9 \mathrm{mg}, 0.20 \mathrm{mmol})$ in 1,4-dioxane $(4.9 \mathrm{~mL})$ were subsequently added to the mixture. The mixture was stirred at $15^{\circ} \mathrm{C}$ for 12 hours. The reaction mixture was filtered through a short pad of silica gel, and the solvent was evaporated under vacuum. The residue was purified by silica gel chromatography to afford the desired product 3aa (52\% yield, $86 \%$ ee).

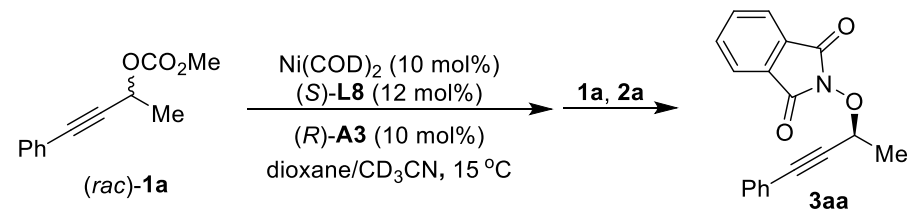

The yield and enantiomeric excess are comparable to the results obtained with the solvent mixture used in NMR.

To a Schlenk tube was added $\mathrm{Ni}(\mathrm{COD})_{2}(5.5 \mathrm{mg}, 0.02 \mathrm{mmol}, 1.0$ equiv), $(S)-\mathbf{L 8}$ (15.6 mg, 0.024 mmol, 1.2 equiv), $(R)-\mathbf{A 3}$ (7.8 mg, $0.02 \mathrm{mmol}, 1.0$ equiv) and 1,4-dioxane $(4 \mathrm{~mL})$. The resulting solution was stirred for $30 \mathrm{~min}$. Then propargylic carbonate $(\mathrm{rac})-\mathbf{1 a}(81.7 \mathrm{mg}, 0.4 \mathrm{mmol})$ and $\mathbf{2 a}$ (32.6 mg, $0.20 \mathrm{mmol})$ in 1,4-dioxane $/ \mathrm{CD}_{3} \mathrm{CN}(1.0 \mathrm{~mL} / 0.5 \mathrm{~mL}$ ) were added into the reaction mixture under argon at $15^{\circ} \mathrm{C}$. The reaction solution was stirred at $15{ }^{\circ} \mathrm{C}$ for 12 hours. The reaction mixture was filtered through a short pad of silica gel, and the solvent was evaporated under vacuum. The residue was purified by silica gel chromatography to afford the desired product $\mathbf{3 a}$ ( $60 \%$ yield, $87 \%$ ee).
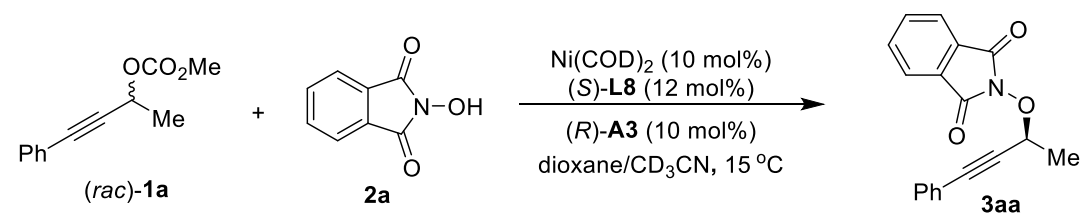

3.5.3. ${ }^{1} \mathrm{H}$ NMR, ${ }^{13} \mathrm{C}$ NMR and $2 \mathrm{D}$ NMR studies of allenylnickel species

${ }^{13} \mathrm{C}$ NMR of allenylnickel species could not be obtained in $\mathrm{CD}_{3} \mathrm{CN}$ due to the low solubility. Thus, we used $\mathrm{C}_{6} \mathrm{D}_{6}$ solvent for ${ }^{1} \mathrm{H}$ NMR and ${ }^{13} \mathrm{C}$ NMR studies. 


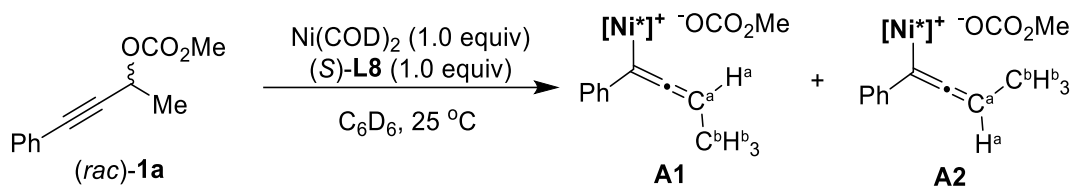

$\mathrm{Ni}(\mathrm{COD})_{2}$ (13.8 mg, $0.05 \mathrm{mmol}, 1.0$ equiv), $(S)-\mathbf{L 8}\left(32.6 \mathrm{mg}, 0.05 \mathrm{mmol}, 1.0\right.$ equiv), and $\mathrm{C}_{6} \mathrm{D}_{6}(0.6$ $\mathrm{mL}$ ) were added to a J. Young tube in the glove box. After the solution was shaken for 30 minutes at room temperature, $(\mathrm{rac})-\mathbf{1 a}(10.2 \mathrm{mg}, 0.05 \mathrm{mmol}, 1.0$ equiv) was added, and the solution was shaken for 30 minutes. The sample was analyzed by ${ }^{31} \mathrm{P}$ NMR, ${ }^{1} \mathrm{H}$ NMR, ${ }^{13} \mathrm{C}$ NMR, COSY NMR, and HSQC NMR (Two isomers A1 and $\mathbf{A 2}$ were observed under this condition).

${ }^{31} \mathrm{P}$ NMR NMR spectrum of species $\mathbf{A}$

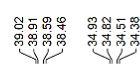
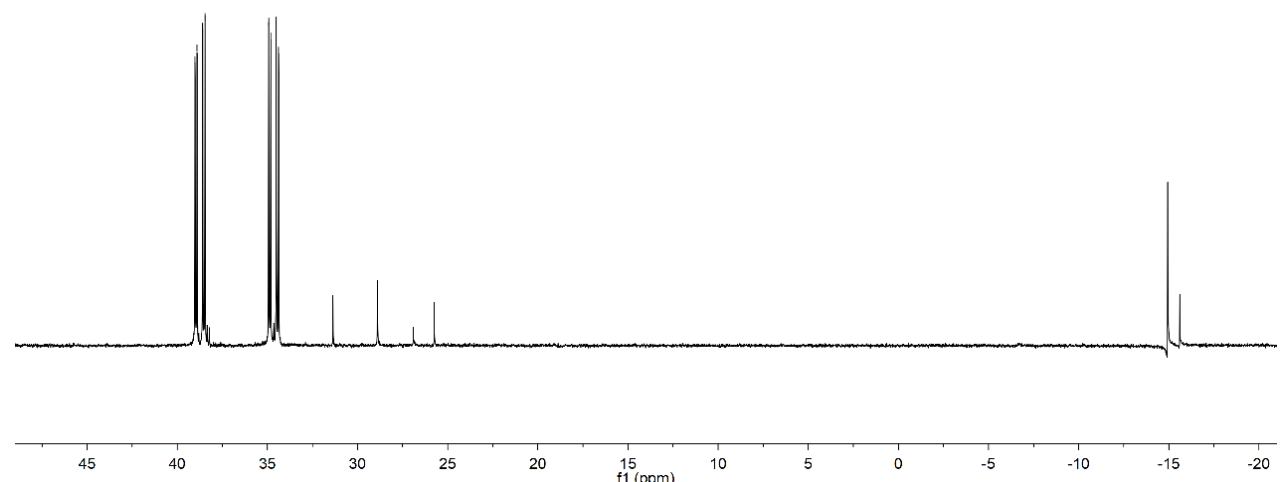

${ }^{1} \mathrm{H}$ NMR of species $\mathbf{A}$

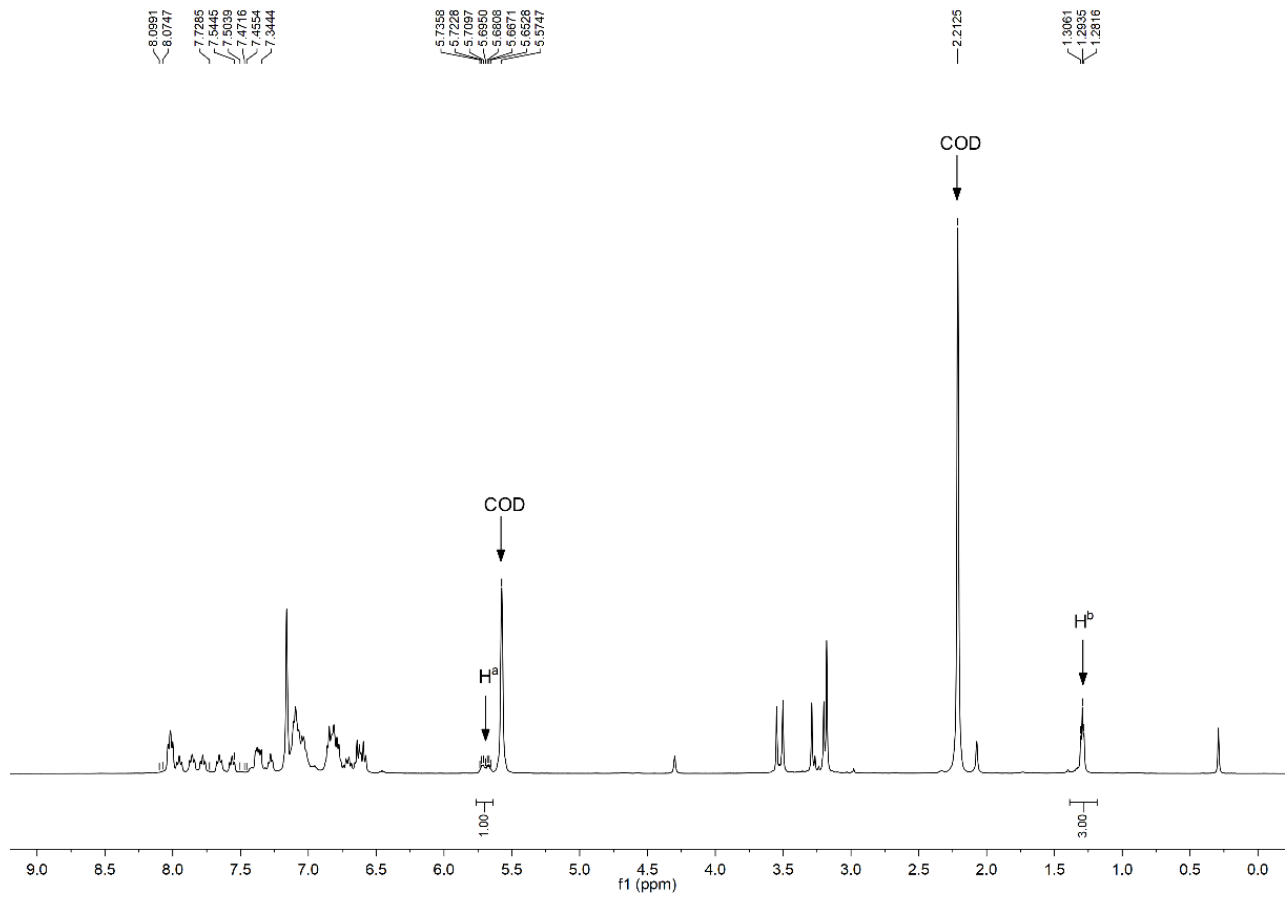


${ }^{13} \mathrm{C}$ NMR spectrum of species $\mathbf{A}$

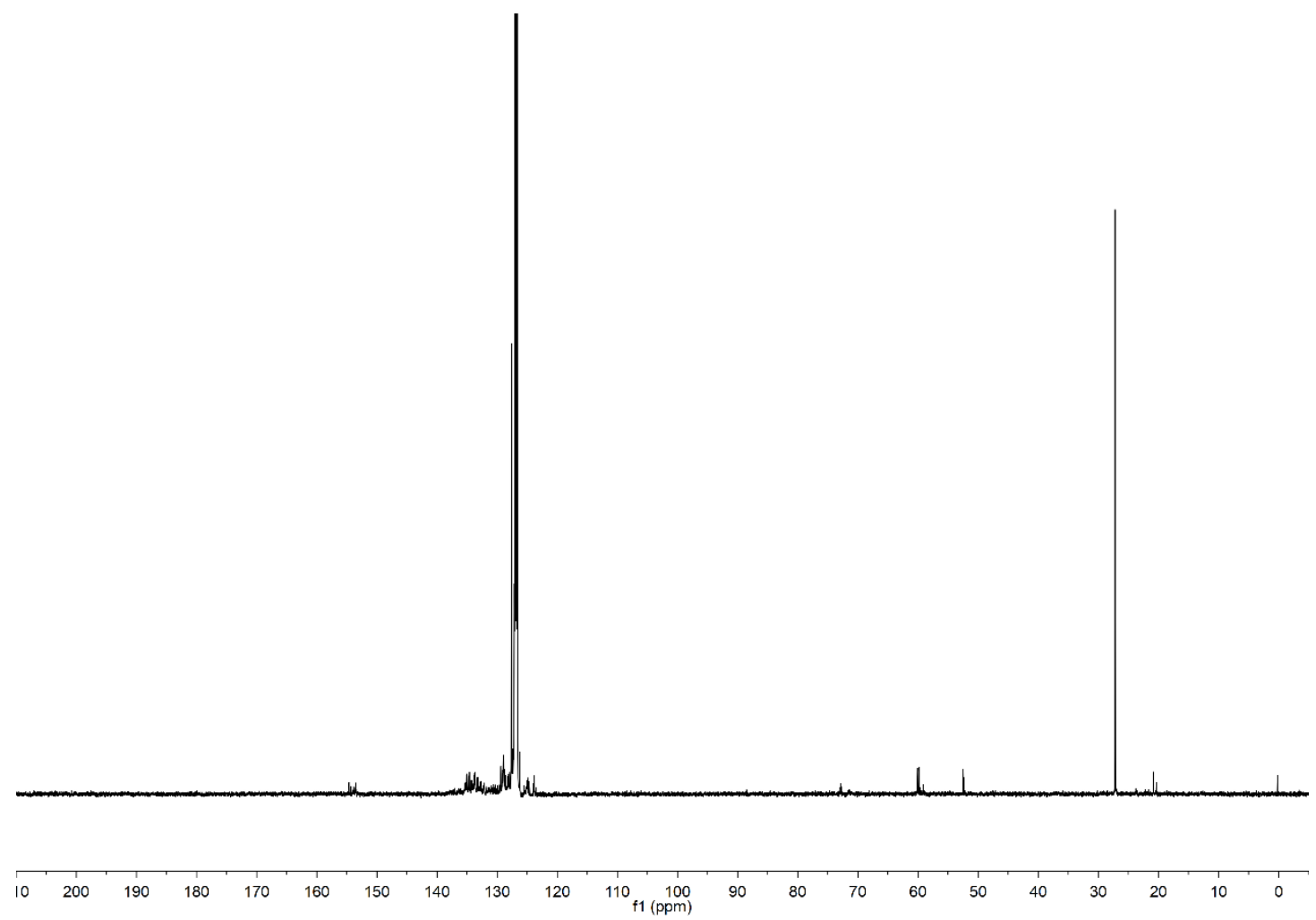

COSY NMR spectrum of species $\mathbf{A}$

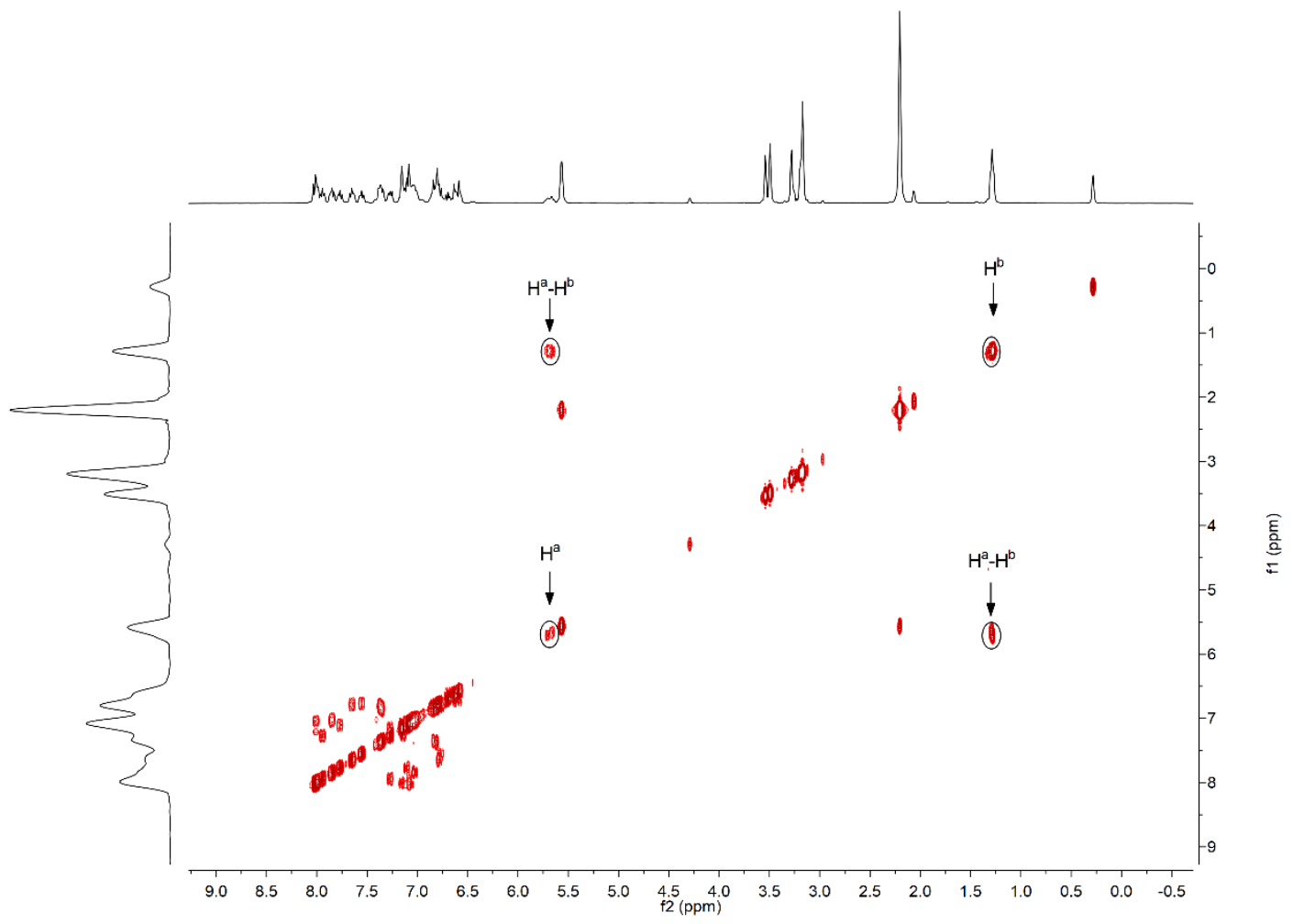


HSQC NMR spectrum of species A

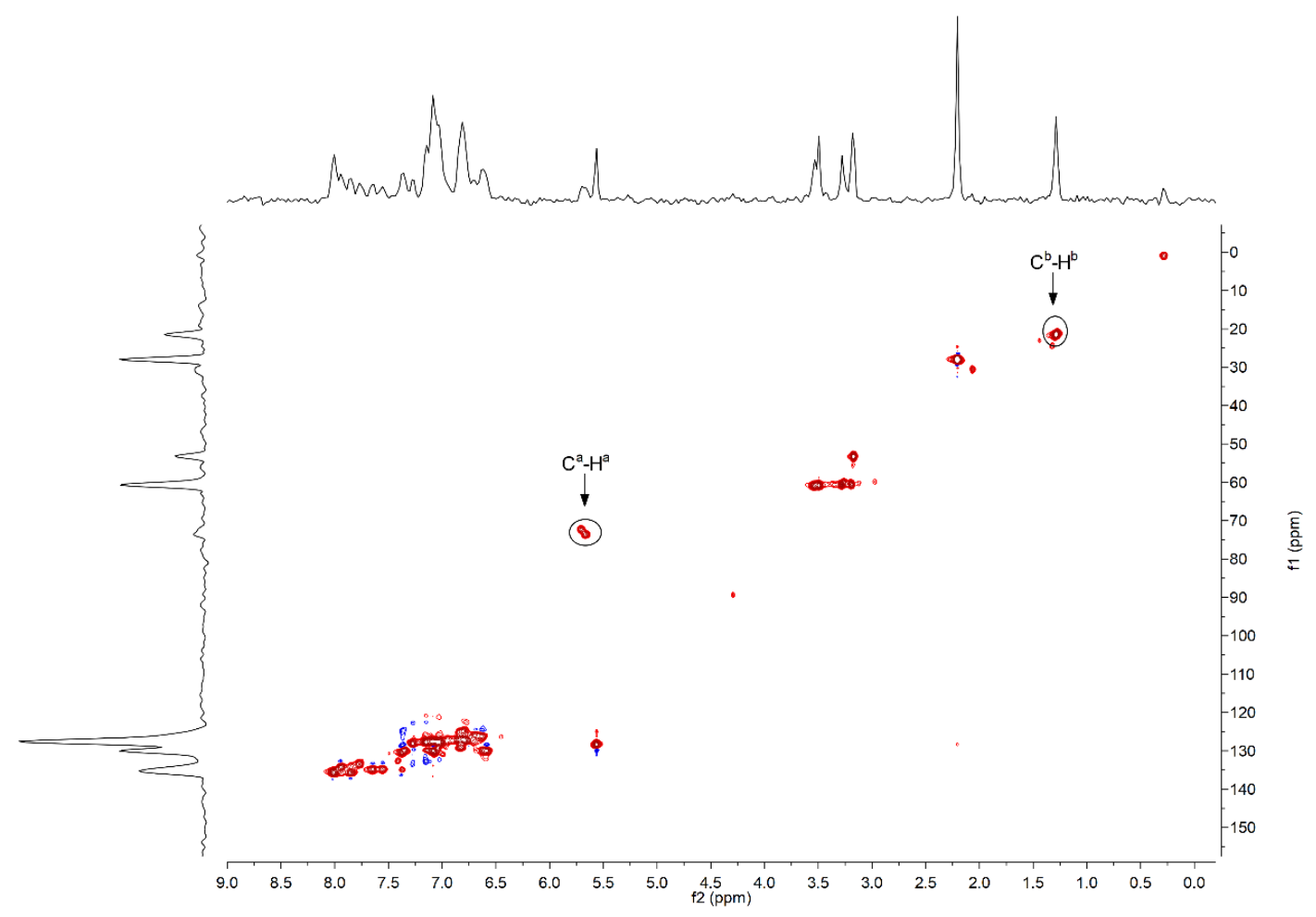

\section{Characterization of Products.}

(S)-2-((4-phenylbut-3-yn-2-yl)oxy)isoindoline-1,3-dione (3aa)

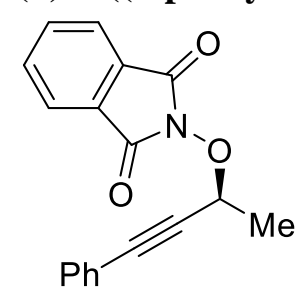

Yield: $28.5 \mathrm{mg}(98 \%) ;{ }^{1} \mathbf{H}$ NMR (500 $\left.\mathbf{~ M H z}, \mathbf{C D C l}_{\mathbf{3}}\right) \delta 7.87-7.83(\mathrm{~m}$, $2 \mathrm{H}), 7.76-7.72(\mathrm{~m}, 2 \mathrm{H}), 7.37-7.35(\mathrm{~m}, 2 \mathrm{H}), 7.31-7.24(\mathrm{~m}, 3 \mathrm{H}), 5.39$

$(\mathrm{q}, J=6.6 \mathrm{~Hz}, 1 \mathrm{H}), 1.76(\mathrm{~d}, J=6.7 \mathrm{~Hz}, 3 \mathrm{H}) .{ }^{\mathbf{1 3}} \mathbf{C ~ N M R}\left(\mathbf{1 2 5} \mathbf{~ M H z}, \mathbf{C D C l}_{3}\right)$ $\delta 163.74,134.48,131.65,128.91,128.70,128.24,123.57,121.90,87.72$, 85.95, 74.03, 20.21. ESI-MS: calculated $\left[\mathrm{C}_{18} \mathrm{H}_{18} \mathrm{NO}_{3}+\mathrm{Na}\right]^{+}:$314.0788, found: $314.0786 .[\alpha]_{\mathrm{D}}^{20}=-201.0\left(\mathrm{c}=0.97, \mathrm{CH}_{2} \mathrm{Cl}_{2}\right)$. The product was analyzed by HPLC to determine the enantiomeric excess: 97\% ee (CHIRALPAK IC, hexane $/ i$ PrOH $=70 / 30$, detector: $254 \mathrm{~nm}, \mathrm{~T}=25^{\circ} \mathrm{C}$, flow rate: $1 \mathrm{~mL} / \mathrm{min}$ ), $\mathrm{t}_{1}$ (major) $=6.71 \mathrm{~min}, \mathrm{t}_{2}$ (minor) $=8.44 \mathrm{~min}$.

(S)-5-chloro-2-((4-phenylbut-3-yn-2-yl)oxy)isoindoline-1,3-dione (3ab)<smiles>CC(C#Cc1ccccc1)ON1C(=O)c2ccc(Cl)cc2C1=O</smiles>

Yield: 14.8 mg (46\%); ${ }^{1} \mathbf{H}$ NMR (500 MHz, $\left.\mathbf{C D C l}_{3}\right) \delta 7.82(\mathrm{~s}, 1 \mathrm{H})$, $7.79(\mathrm{~d}, J=8.0 \mathrm{~Hz}, 1 \mathrm{H}), 7.71-7.69(\mathrm{~m}, 1 \mathrm{H}), 7.36-7.34(\mathrm{~m}, 2 \mathrm{H})$, $7.31-7.26(\mathrm{~m}, 3 \mathrm{H}), 5.37(\mathrm{q}, J=6.6 \mathrm{~Hz}, 2 \mathrm{H}), 1.76(\mathrm{~d}, J=6.7 \mathrm{~Hz}$, $5 \mathrm{H}) .{ }^{13} \mathbf{C}$ NMR (125 MHz, CDCl 3 ) $\delta 13 \mathrm{C} \mathrm{NMR} \mathrm{(126} \mathrm{MHz,} \mathrm{CDCl3)}$ $\delta 162.86,162.52,141.23,134.51,131.65,130.52,128.80,128.29$, 
126.90, 124.93, 124.08, 121.78, 87.88, 85.73, 74.21, 20.19. ESI-MS: calculated $\left[\mathrm{C}_{18} \mathrm{H}_{12} \mathrm{ClNO}_{3}\right.$ $+\mathrm{Na}]^{+}:$348.0398, found: $348.0402 .[\alpha]_{\mathrm{D}}^{20}=-164.1\left(\mathrm{c}=0.49, \mathrm{CH}_{2} \mathrm{Cl}_{2}\right)$. The product was analyzed by HPLC to determine the enantiomeric excess: $90 \%$ ee (CHIRALPAK IC, hexane $/ i-\mathrm{PrOH}$ $=70 / 30$, detector: $254 \mathrm{~nm}, \mathrm{~T}=25^{\circ} \mathrm{C}$, flow rate: $\left.1 \mathrm{~mL} / \mathrm{min}\right), \mathrm{t}_{1}$ (major) $=7.03 \mathrm{~min}, \mathrm{t}_{2}$ (minor) $=$ $9.54 \mathrm{~min}$.

(S)-5-bromo-2-((4-phenylbut-3-yn-2-yl)oxy)isoindoline-1,3-dione (3ac)

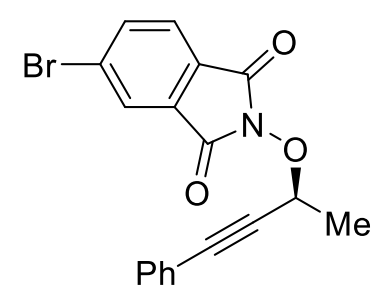

Yield: 15.5 mg (42\%); ${ }^{1} \mathbf{H}$ NMR (500 MHz, $\left.\mathbf{C D C l}_{3}\right) \delta 7.98(\mathrm{~s}, 1 \mathrm{H})$, $7.89-7.87(\mathrm{~m}, 1 \mathrm{H}), 7.71(\mathrm{~d}, J=7.9 \mathrm{~Hz}, 2 \mathrm{H}), 7.37-7.35(\mathrm{~m}, 2 \mathrm{H})$, $7.31-7.26(\mathrm{~m}, 4 \mathrm{H}), 5.37(\mathrm{q}, J=6.6 \mathrm{~Hz}, 2 \mathrm{H}), 1.76(\mathrm{~d}, J=6.7 \mathrm{~Hz}$, 4H). ${ }^{13} \mathbf{C}$ NMR (125 MHz, $\left.\mathbf{C D C l}_{3}\right) \delta 163.00,162.42,137.48,131.65$, $130.45,129.43,128.81,128.29,127.35,126.94,125.00,121.78$, 87.89, 85.72, 74.21, 20.20. ESI-MS: calculated $\left[\mathrm{C}_{18} \mathrm{H}_{12} \mathrm{BrNO}_{3}+\right.$ $\mathrm{Na}]^{+}:$391.9893, found: 391.9898. $[\alpha]_{\mathrm{D}}^{20}=-129.5\left(\mathrm{c}=0.28, \mathrm{CH}_{2} \mathrm{Cl}_{2}\right)$. The product was analyzed by HPLC to determine the enantiomeric excess: $90 \%$ ee (CHIRALPAK IC, hexane/i-PrOH $=70 / 30$, detector: $254 \mathrm{~nm}, \mathrm{~T}=25^{\circ} \mathrm{C}$, flow rate: $\left.1 \mathrm{~mL} / \mathrm{min}\right), \mathrm{t}_{1}$ (major) $=8.41 \mathrm{~min}, \mathrm{t}_{2}$ (minor) $=$ $11.19 \mathrm{~min}$.

(S)-5-phenyl-2-((4-phenylbut-3-yn-2-yl)oxy)isoindoline-1,3-dione (3ad)

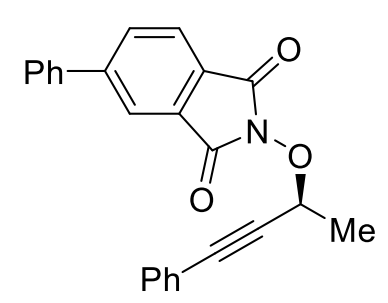

Yield: 34.9 mg (95\%). ${ }^{1} \mathbf{H}$ NMR (500 MHz, $\left.\mathbf{C D C l}_{3}\right) \delta 8.05-8.04$ (m, 1H), $7.93-7.88(\mathrm{~m}, 2 \mathrm{H}), 7.61-7.59(\mathrm{~m}, 2 \mathrm{H}), 7.50-7.46(\mathrm{~m}$, $2 \mathrm{H}), 7.45-7.42(\mathrm{~m}, 1 \mathrm{H}), 7.37-7.37(\mathrm{~m}, 2 \mathrm{H}), 7.29-7.24(\mathrm{~m}, 3 \mathrm{H})$, $5.40(\mathrm{q}, J=6.6 \mathrm{~Hz}, 1 \mathrm{H}), 1.77(\mathrm{~d}, J=6.7 \mathrm{~Hz}, 3 \mathrm{H}) .{ }^{13} \mathbf{C}$ NMR $(\mathbf{1 2 5}$ MHz, $\left.\mathbf{C D C l}_{3}\right) \delta 163.77,163.70,147.93,138.82,132.90,131.68$, $129.71,129.25,129.03,128.73,128.27,127.29,127.22,124.10$, 122.18, 121.94, 87.75, 86.04, 74.08, 20.24. ESI-MS: calculated $\left[\mathrm{C}_{24} \mathrm{H}_{17} \mathrm{NO}_{3}+\mathrm{Na}\right]^{+}:$390.1101, found: $390.1099 .[\alpha]_{\mathrm{D}}^{20}=-221.0\left(\mathrm{c}=0.83, \mathrm{CH}_{2} \mathrm{Cl}_{2}\right)$. The product was analyzed by HPLC to determine the enantiomeric excess: $95 \%$ ee (CHIRALPAK IC, hexane $/ i-\mathrm{PrOH}=70 / 30$, detector: $254 \mathrm{~nm}, \mathrm{~T}=25^{\circ} \mathrm{C}$, flow rate: $\left.1 \mathrm{~mL} / \mathrm{min}\right), \mathrm{t}_{1}($ major $)=10.19 \mathrm{~min}, \mathrm{t}_{2}($ minor $)=15.21 \mathrm{~min}$.

(S)-5-methyl-2-((4-phenylbut-3-yn-2-yl)oxy)isoindoline-1,3-dione (3ae)

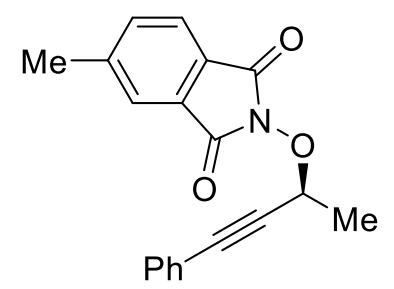

Yield: $25.8 \mathrm{mg}(85 \%) ;{ }^{\mathbf{1}} \mathbf{H}$ NMR (500 $\left.\mathbf{~ M H z}, \mathbf{C D C l}_{\mathbf{3}}\right) \delta 7.72(\mathrm{~d}, J=$ $7.6 \mathrm{~Hz}, 1 \mathrm{H}), 7.65(\mathrm{~s}, 1 \mathrm{H}), 7.52(\mathrm{~d}, J=7.7 \mathrm{~Hz}, 1 \mathrm{H}), 7.36(\mathrm{~d}, 2 \mathrm{H})$, $7.31-7.25(\mathrm{~m}, 3 \mathrm{H}), 5.37(\mathrm{q}, J=6.7 \mathrm{~Hz}, 1 \mathrm{H}), 2.50(\mathrm{~s}, 3 \mathrm{H}), 1.75(\mathrm{~d}$, $J=6.6 \mathrm{~Hz}, 3 \mathrm{H}) .{ }^{13} \mathbf{C}$ NMR (125 MHz, $\left.\mathbf{C D C l}_{3}\right) \delta 163.97,163.89$, $145.85,134.92,131.66,129.20,128.66,128.22,126.20,124.14$, 123.53, 121.97, 87.61, 86.05, 73.93, 22.12, 20.20. ESI-MS: calculated $\left[\mathrm{C}_{19} \mathrm{H}_{15} \mathrm{NO}_{3}+\mathrm{Na}\right]^{+}: 328.0944$, found: $328.0948[\alpha]_{\mathrm{D}}^{20}=-277.4\left(\mathrm{c}=0.53, \mathrm{CH}_{2} \mathrm{Cl}_{2}\right)$. The product was analyzed by HPLC to determine the enantiomeric excess: $97 \%$ ee (CHIRALPAK IC, hexane $/ i-\mathrm{PrOH}=70 / 30$, detector: $254 \mathrm{~nm}, \mathrm{~T}=25^{\circ} \mathrm{C}$, flow rate: $1 \mathrm{~mL} / \mathrm{min}$ ), $\mathrm{t}_{1}($ major $)=8.51$ min, $\mathrm{t}_{2}($ minor $)=12.79 \min$.

(S)-5-methoxy-2-((4-phenylbut-3-yn-2-yl)oxy)isoindoline-1,3-dione (3af) 


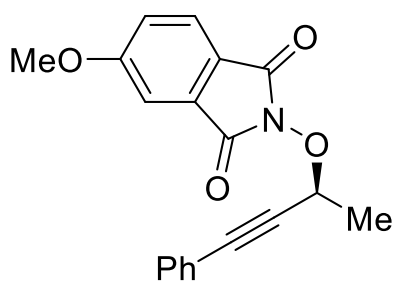

Yield: 30.7 mg (96\%). ${ }^{\mathbf{1}} \mathbf{H}$ NMR (500 MHz, $\left.\mathbf{C D C l}_{3}\right) \delta 7.76(\mathrm{~d}, J=$ $8.3 \mathrm{~Hz}, 1 \mathrm{H}), 7.37$ (d, $J=7.2 \mathrm{~Hz}, 2 \mathrm{H}), 7.33$ (s, 1H), $7.31-7.25$ (m, $3 \mathrm{H}), 7.17(\mathrm{~d}, J=8.2 \mathrm{~Hz}, 2 \mathrm{H}), 5.36$ (q, $J=6.6 \mathrm{~Hz}, 1 \mathrm{H}), 3.91(\mathrm{~s}, 3 \mathrm{H})$, $1.75(\mathrm{~d}, J=6.7 \mathrm{~Hz}, 3 \mathrm{H}) .{ }^{13} \mathbf{C}$ NMR (125 MHz, $\left.\mathbf{C D C l}_{3}\right) \delta 164.96$, $163.74,163.70,131.67,131.45,128.66,128.23,125.48,121.98$, $120.61,119.99,108.62,87.60,86.09,73.95,56.12,20.20$. ESI-MS: calculated $\left[\mathrm{C}_{19} \mathrm{H}_{15} \mathrm{NO}_{4}+\mathrm{H}\right]^{+}: 322.1074$, found: $322.1083 .[\alpha]_{D}^{20}=-168.7(\mathrm{c}=1.03$, $\mathrm{CH}_{2} \mathrm{Cl}_{2}$ ). The product was analyzed by HPLC to determine the enantiomeric excess: $99 \%$ ee (CHIRALPAK IC, hexane $/ i-\mathrm{PrOH}=70 / 30$, detector: $254 \mathrm{~nm}, \mathrm{~T}=25^{\circ} \mathrm{C}$, flow rate: $1 \mathrm{~mL} / \mathrm{min}$ ), $\mathrm{t}_{1}($ major $)=10.81 \mathrm{~min}, \mathrm{t}_{2}($ minor $)=16.69 \mathrm{~min}$

\section{(S)-5,6-dimethoxy-2-((4-phenylbut-3-yn-2-yl)oxy)isoindoline-1,3-dione (3ag)}

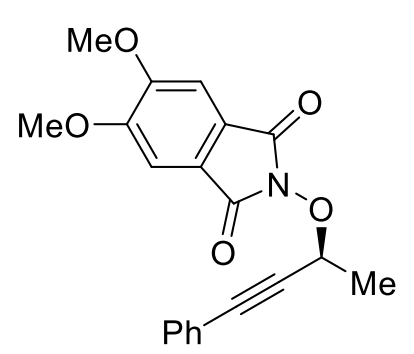

Yield: 28.6 mg (81\%). ${ }^{\mathbf{1}} \mathbf{H}$ NMR (500 $\left.\mathbf{~ M H z}, \mathbf{C D C l}_{\mathbf{3}}\right) \delta 7.39(\mathrm{~d}, J$ $=1.6 \mathrm{~Hz}, 1 \mathrm{H}), 7.38(\mathrm{~d}, J=2.0 \mathrm{~Hz}, 1 \mathrm{H}), 7.26-7.31(\mathrm{~m}, 5 \mathrm{H}), 5.34$ $(\mathrm{q}, J=6.6 \mathrm{~Hz}, 1 \mathrm{H}), 3.99(\mathrm{~s}, 3 \mathrm{H}), 1.75(\mathrm{~d}, J=6.6 \mathrm{~Hz}, 2 \mathrm{H}) .{ }^{13} \mathbf{C}$ NMR (125 MHz, $\left.\mathbf{C D C l}_{3}\right) \delta 164.29,154.20,131.71,128.66$, 128.23, 122.26, 122.03, 105.78, 87.49, 86.22, 73.96, 56.70, 20.19. ESI-MS: calculated $\left[\mathrm{C}_{20} \mathrm{H}_{17} \mathrm{NO}_{5}+\mathrm{Na}^{+}:\right.$374.0999, found: 374.1004. $[\alpha]_{\mathrm{D}}^{20}=-168.9\left(\mathrm{c}=0.95, \mathrm{CH}_{2} \mathrm{Cl}_{2}\right)$. The product was analyzed by HPLC to determine the enantiomeric excess: 97\% ee (CHIRALPAK IC, hexane $/ i$ PrOH $=70 / 30$, detector: $254 \mathrm{~nm}, \mathrm{~T}=25{ }^{\circ} \mathrm{C}$, flow rate: $\left.1 \mathrm{~mL} / \mathrm{min}\right), \mathrm{t}_{1}$ (major) $=19.97 \mathrm{~min}$, $\mathrm{t}_{2}($ minor $)=44.59 \mathrm{~min}$.

\section{(S)-2-((4-phenylbut-3-yn-2-yl)oxy)-1H-benzo[f]isoindole-1,3(2H)-dione (3ah)}

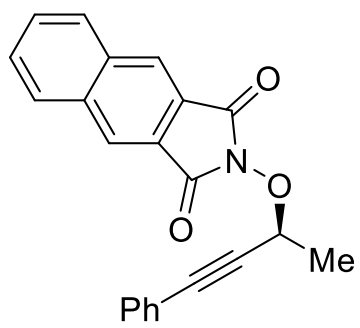

Yield: 19.3 mg (57\%). ${ }^{\mathbf{1}} \mathbf{H}$ NMR (500 MHz, $\left.\mathbf{C D C l}_{3}\right) \delta 8.28(\mathrm{~s}, 2 \mathrm{H})$, $7.97-8.00(\mathrm{~m}, 2 \mathrm{H}), 7.69-7.65(\mathrm{~m}, 2 \mathrm{H}), 7.38-7.36(\mathrm{~m}, 2 \mathrm{H}), 7.27$ $-7.22(\mathrm{~m}, 3 \mathrm{H}), 5.47(\mathrm{q}, J=6.6 \mathrm{~Hz}, 1 \mathrm{H}), 1.80(\mathrm{~d}, J=6.7 \mathrm{~Hz}, 3 \mathrm{H}) .{ }^{13} \mathrm{C}$ NMR (125 MHz, $\left.\mathbf{C D C l}_{3}\right) \delta 163.32,135.39,131.67,130.29,129.42$, $128.70,128.25,125.18,124.53,121.91,87.76,86.01,73.98,20.25$. ESI-MS: calculated $\left[\mathrm{C}_{22} \mathrm{H}_{15} \mathrm{NO}_{3}+\mathrm{Na}\right]^{+}: 364.0944$, found: 364.0949 . $[\alpha]_{\mathrm{D}}^{20}=-255.5\left(\mathrm{c}=0.64, \mathrm{CH}_{2} \mathrm{Cl}_{2}\right)$. The product was analyzed by HPLC to determine the enantiomeric excess: $94 \%$ ee (CHIRALPAK IC, hexane/ $i-\mathrm{PrOH}=70 / 30$, detector: $254 \mathrm{~nm}, \mathrm{~T}=25{ }^{\circ} \mathrm{C}$, flow rate: $\left.1 \mathrm{~mL} / \mathrm{min}\right), \mathrm{t}_{1}($ major $)=13.36 \mathrm{~min}, \mathrm{t}_{2}($ minor $)=23.62$ $\min$.

(S)-2-((4-(4-fluorophenyl)but-3-yn-2-yl)oxy)-5-methoxyisoindoline-1,3-dione (3bf)

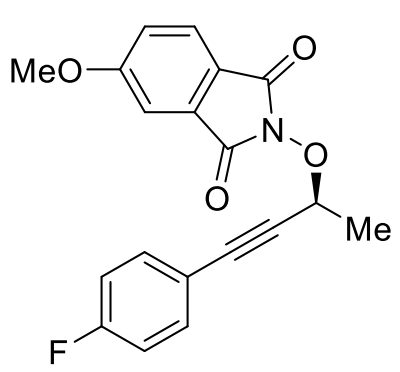
Yield: 33.2 mg (98\%). ${ }^{\mathbf{1}} \mathbf{H}$ NMR (500 $\left.\mathbf{~ M H z , ~} \mathbf{C D C l}_{3}\right) \delta 7.76(\mathrm{~d}, J=$ $8.3 \mathrm{~Hz}, 1 \mathrm{H}), 7.38-7.33(\mathrm{~m}, 3 \mathrm{H}), 7.19$ (dd, $J=8.4,2.3 \mathrm{~Hz}, 1 \mathrm{H})$, $6.99-6.95(\mathrm{~m}, 2 \mathrm{H}), 5.33(\mathrm{q}, J=6.6 \mathrm{~Hz}, 1 \mathrm{H}), 3.92(\mathrm{~s}, 3 \mathrm{H}), 1.75(\mathrm{~d}$, $J=6.7 \mathrm{~Hz}, 3 \mathrm{H}) .{ }^{13} \mathbf{C}$ NMR (125 MHz, $\left.\mathbf{C D C l}_{3}\right) \delta 165.07,163.81$, $163.76,134.85,132.98,131.47,128.68,125.59,120.60,120.52$, $120.10,108.72,87.19,86.49,73.95,56.21,20.16$. ESI-MS: calculated $\left[\mathrm{C}_{19} \mathrm{H}_{15} \mathrm{FNO}_{4}+\mathrm{Na}\right]^{+}: 362.0799$, found:362.0800. $[\alpha]_{\mathrm{D}}^{20}=$ $-148.1\left(\mathrm{c}=1.23, \mathrm{CH}_{2} \mathrm{Cl}_{2}\right)$. The product was analyzed by HPLC to determine the enantiomeric 
excess: $97 \%$ ee (CHIRALPAK IC, hexane $/ i-\mathrm{PrOH}=70 / 30$, detector: $254 \mathrm{~nm}, \mathrm{~T}=25^{\circ} \mathrm{C}$, flow rate: $1 \mathrm{~mL} / \mathrm{min}), \mathrm{t}_{1}($ major $)=9.44 \mathrm{~min}, \mathrm{t}_{2}($ minor $)=14.38 \mathrm{~min}$.

(S)-2-((4-(4-chlorophenyl)but-3-yn-2-yl)oxy)-5-methoxyisoindoline-1,3-dione (3cf)<smiles>COc1ccc2c(c1)C(=O)N(OC(C)C#Cc1ccc(Cl)cc1)C2=O</smiles>
Yield: 33.5 mg (94\%). ${ }^{1} \mathbf{H}$ NMR (500 $\left.\mathbf{~ M H z , ~} \mathbf{C D C l}_{3}\right) \delta 7.78-7.75$ (m, 1H), $7.34-7.30(\mathrm{~m}, 3 \mathrm{H}), 7.27-7.24(\mathrm{~m}, 2 \mathrm{H}), 7.20-7.17$ $(\mathrm{m}, 1 \mathrm{H}), 5.33(\mathrm{q}, J=6.5 \mathrm{~Hz}, 1 \mathrm{H}), 3.92(\mathrm{~s}, 2 \mathrm{H}), 1.75(\mathrm{~d}, J=6.6 \mathrm{~Hz}$, 3H). ${ }^{13} \mathbf{C}$ NMR (125 MHz, $\left.\mathbf{C D C l}_{3}\right) \delta$ 165.01, 163.75, 163.70, $134.79,132.92,131.41,128.62,125.53,120.54,120.46,120.04$, 108.65, 87.13, 86.43, 73.89, 56.15, 20.10. ESI-MS: calculated $\left[\mathrm{C}_{19} \mathrm{H}_{14} \mathrm{ClNO}_{4}+\mathrm{Na}\right]^{+}: 378.0504$, found: $378.0502 .[\alpha]_{\mathrm{D}}^{20}=-222.0(\mathrm{c}$ $\left.=0.97, \mathrm{CH}_{2} \mathrm{Cl}_{2}\right)$. The product was analyzed by HPLC to determine the enantiomeric excess: 98\% ee (CHIRALPAK IC, hexane $/ i-\mathrm{PrOH}=70 / 30$, detector: $254 \mathrm{~nm}, \mathrm{~T}=25^{\circ} \mathrm{C}$, flow rate: 1 $\mathrm{mL} / \mathrm{min}), \mathrm{t}_{1}($ major $)=10.57 \mathrm{~min}, \mathrm{t}_{2}($ minor $)=16.11 \mathrm{~min}$.

(S)-2-((4-(4-bromophenyl)but-3-yn-2-yl)oxy)-5-methoxyisoindoline-1,3-dione (3df)<smiles>COc1ccc2c(c1)C(=O)N(OC(C)C#Cc1ccc(Br)cc1)C2=O</smiles>
Yield: 33.2 mg (83\%). ${ }^{\mathbf{1}} \mathbf{H}$ NMR (500 $\left.\mathbf{~ M H z}, \mathbf{C D C l}_{\mathbf{3}}\right) \delta 7.77-7.75$ $(\mathrm{d}, J=8.3 \mathrm{~Hz}, 1 \mathrm{H}), 7.41(\mathrm{~d}, J=8.2 \mathrm{~Hz}, 2 \mathrm{H}), 7.34(\mathrm{~d}, J=2.3 \mathrm{~Hz}$, $1 \mathrm{H}), 7.26-7.23(\mathrm{~m}, 2 \mathrm{H}), 7.18(\mathrm{dd}, J=8.2,2.3 \mathrm{~Hz}, 1 \mathrm{H}), 5.32(\mathrm{q}, J$ = $6.6 \mathrm{~Hz}, 1 \mathrm{H}), 3.92(\mathrm{~s}, 3 \mathrm{H}), 1.74(\mathrm{~d}, J=6.7 \mathrm{~Hz}, 3 \mathrm{H}) .{ }^{13} \mathbf{C} \mathbf{~ N M R}$ (125 MHz, $\left.\mathbf{C D C l}_{3}\right) \delta 165.01,163.74,163.69,133.11,131.54$, $131.41,125.53,123.04,120.92,120.54,120.05,108.65,87.31$, 86.48, 77.28, 77.03, 76.78, 73.90, 56.14, 20.07. ESI-MS: calculated $\left[\mathrm{C}_{19} \mathrm{H}_{15} \mathrm{BrNO}_{4}+\mathrm{H}\right]^{+}$: 400.0179, found: 400.0183. $[\alpha]_{\mathrm{D}}^{20}=-152.4\left(\mathrm{c}=0.97, \mathrm{CH}_{2} \mathrm{Cl}_{2}\right)$. The product was analyzed by HPLC to determine the enantiomeric excess: $98 \%$ ee (CHIRALPAK IC, hexane $/ i-\mathrm{PrOH}=70 / 30$, detector: $254 \mathrm{~nm}, \mathrm{~T}=25^{\circ} \mathrm{C}$, flow rate: $1 \mathrm{~mL} / \mathrm{min}$ ), $\mathrm{t}_{1}($ major $)=10.33 \mathrm{~min}, \mathrm{t}_{2}($ minor $)=15.89 \mathrm{~min}$.

(S)-5-methoxy-2-((4-(p-tolyl)but-3-yn-2-yl)oxy)isoindoline-1,3-dione (3ef)

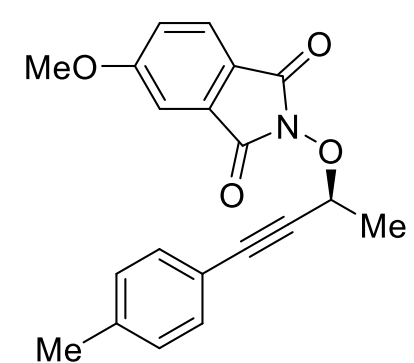
Yield: $32.8 \mathrm{mg}$ (98\%). ${ }^{\mathbf{1}} \mathbf{H}$ NMR (500 $\left.\mathbf{M H z}, \mathbf{C D C l}_{\mathbf{3}}\right) \delta 7.75(\mathrm{~d}, J=$ $8.3 \mathrm{~Hz}, 1 \mathrm{H}), 7.32(\mathrm{~d}, J=2.4 \mathrm{~Hz}, 1 \mathrm{H}), 7.27-7.25(\mathrm{~m}, 2 \mathrm{H}), 7.16$ $(\mathrm{dd}, J=8.3,2.4 \mathrm{~Hz}, 1 \mathrm{H}), 7.07(\mathrm{~d}, J=7.9 \mathrm{~Hz}, 2 \mathrm{H}), 5.36(\mathrm{q}, J=6.6$ $\mathrm{Hz}, 1 \mathrm{H}), 3.91(\mathrm{~s}, 3 \mathrm{H}), 2.31$ (s, 3H), $1.74(\mathrm{~d}, J=6.7 \mathrm{~Hz}, 3 \mathrm{H}) .{ }^{13} \mathrm{C}$ NMR (125 MHz, $\left.\mathbf{C D C l}_{3}\right) \delta$ 164.93, 163.74, 163.71, 138.84, $131.57,131.44,128.99,125.46,120.59,119.95,118.89,108.61$, 87.81, 85.37, 74.02, 56.12, 21.49, 20.24. ESI-MS: calculated $\left[\mathrm{C}_{20} \mathrm{H}_{17} \mathrm{NO}_{4}+\mathrm{Na}\right]^{+}: 358.1050$, found: 358.1057. $[\alpha]_{\mathrm{D}}^{20}=-181.6\left(\mathrm{c}=1.12, \mathrm{CH}_{2} \mathrm{Cl}_{2}\right)$. The product was analyzed by HPLC to determine the enantiomeric excess: $98 \%$ ee (CHIRALPAK IC, hexane $/ i-\mathrm{PrOH}=70 / 30$, detector: $254 \mathrm{~nm}, \mathrm{~T}=25{ }^{\circ} \mathrm{C}$, flow rate: $1 \mathrm{~mL} / \mathrm{min}$ ), $\mathrm{t}_{1}$ (major) $=11.29$ $\min , \mathrm{t}_{2}($ minor $)=18.56 \min$.

(S)-5-methoxy-2-((4-(4-methoxyphenyl)but-3-yn-2-yl)oxy)isoindoline-1,3-dione (3ff) 


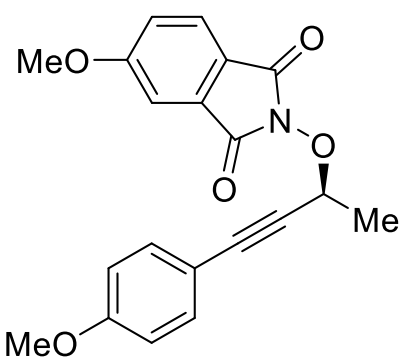

Yield: 34.4 mg (98\%); ${ }^{1} \mathbf{H}$ NMR (500 MHz, $\left.\mathbf{C D C l}_{3}\right) \delta 7.75(\mathrm{~d}, J$ $=8.3 \mathrm{~Hz}, 1 \mathrm{H}), 7.32-7.29(\mathrm{~m}, 3 \mathrm{H}), 7.16(\mathrm{dd}, J=8.3,2.3 \mathrm{~Hz}, 1 \mathrm{H})$, $6.79(\mathrm{~d}, J=8.8 \mathrm{~Hz}, 2 \mathrm{H}), 5.35(\mathrm{q}, J=6.6 \mathrm{~Hz}, 1 \mathrm{H}), 3.91(\mathrm{~s}, 3 \mathrm{H})$, $3.78(\mathrm{~s}, 3 \mathrm{H}), 1.74(\mathrm{~d}, J=6.6 \mathrm{~Hz}, 3 \mathrm{H}) .{ }^{13} \mathrm{C}$ NMR (125 MHz, $\left.\mathbf{C D C l}_{3}\right) \delta 164.92,163.76,163.73,159.87,133.21,131.44,125.45$, $120.59,119.93,114.03,113.88,113.86,108.61,87.65,84.71$, 74.08, 56.12, 55.27, 20.29. ESI-MS: calculated $\left[\mathrm{C}_{20} \mathrm{H}_{17} \mathrm{NO}_{5}+\mathrm{H}\right]^{+}$: 352.1179, found: $352.1180 .[\alpha]_{\mathrm{D}}^{20}=-176.5\left(\mathrm{c}=1.21, \mathrm{CH}_{2} \mathrm{Cl}_{2}\right)$. The product was analyzed by HPLC to determine the enantiomeric excess: 97\% ee (CHIRALPAK IC, hexane/ $i$-PrOH $=70 / 30$, detector: $254 \mathrm{~nm}, \mathrm{~T}=25^{\circ} \mathrm{C}$, flow rate: $\left.1 \mathrm{~mL} / \mathrm{min}\right), \mathrm{t}_{1}($ major $)=15.85 \mathrm{~min}, \mathrm{t}_{2}($ minor $)=26.83$ $\min$.

\section{(S)-2-((4-(3-chlorophenyl)but-3-yn-2-yl)oxy)-5-methoxyisoindoline-1,3-dione (3gf)}

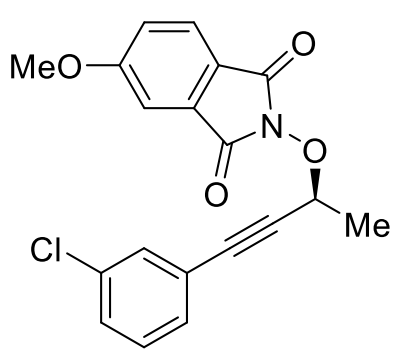

Yield: $30.6 \mathrm{mg}$ (86\%). ${ }^{\mathbf{1}} \mathbf{H}$ NMR (500 $\left.\mathbf{M H z}, \mathbf{C D C l}_{3}\right) \delta 7.77$ (d, $J=$ $8.3 \mathrm{~Hz}, 1 \mathrm{H}), 7.35-7.33(\mathrm{~m}, 2 \mathrm{H}), 7.29-7.26(\mathrm{~m}, 2 \mathrm{H}), 7.22-7.18$ $(\mathrm{m}, 2 \mathrm{H}), 5.34(\mathrm{q}, J=6.7 \mathrm{~Hz}, 1 \mathrm{H}), 3.93(\mathrm{~s}, 3 \mathrm{H}), 1.75(\mathrm{~d}, J=6.6 \mathrm{~Hz}$, 3H). ${ }^{13} \mathbf{C}$ NMR (125 MHz, $\left.\mathbf{C D C l}_{3}\right) \delta 165.03,163.74,163.68$, $134.08,131.51,131.40,129.83,129.51,129.00,125.55,123.65$, $120.53,120.11,108.64,87.34,86.15,73.81,56.14,20.06$. ESI-MS: calculated $\left[\mathrm{C}_{19} \mathrm{H}_{14} \mathrm{ClNO}_{4}+\mathrm{Na}\right]^{+}: 378.0504$, found: $378.0509 .[\alpha]_{\mathrm{D}}^{20}$ $=-111.5\left(\mathrm{c}=0.79, \mathrm{CH}_{2} \mathrm{Cl}_{2}\right)$. The product was analyzed by HPLC to determine the enantiomeric excess: $98 \%$ ee (CHIRALPAK IC, hexane $/ i-\mathrm{PrOH}=70 / 30$, detector: $254 \mathrm{~nm}, \mathrm{~T}=25{ }^{\circ} \mathrm{C}$, flow rate: $1 \mathrm{~mL} / \mathrm{min}), \mathrm{t}_{1}($ major $)=9.38 \mathrm{~min}, \mathrm{t}_{2}($ minor $)=14.27 \mathrm{~min}$.

(S)-5-methoxy-2-((4-(m-tolyl)but-3-yn-2-yl)oxy)isoindoline-1,3-dione (3hf)<smiles>COc1ccc2c(c1)C(=O)N(OC(C)C#Cc1cccc(C)c1)C2=O</smiles>
Yield: $28.3 \mathrm{mg}$ (98\%). ${ }^{\mathbf{1}} \mathbf{H}$ NMR (500 $\left.\mathbf{~ M H z}, \mathbf{C D C l}_{3}\right) \delta 7.75(\mathrm{~d}, J=$ $8.3 \mathrm{~Hz}, 1 \mathrm{H}), 7.33$ (d, $J=2.3 \mathrm{~Hz}, 1 \mathrm{H}), 7.18-7.09$ (m, 4H), 7.10 (d, $J=6.6 \mathrm{~Hz}, 1 \mathrm{H}), 5.36(\mathrm{q}, J=6.5 \mathrm{~Hz}, 1 \mathrm{H}), 3.91(\mathrm{~s}, 3 \mathrm{H}), 2.28(\mathrm{~s}, 3 \mathrm{H})$, $1.75(\mathrm{~d}, J=6.7 \mathrm{~Hz}, 3 \mathrm{H}) .{ }^{\mathbf{1 3}} \mathbf{C} \mathbf{N M R}\left(\mathbf{1 2 5} \mathbf{~ M H z}, \mathbf{C D C l}_{3}\right) \delta 164.94$, $163.74,163.70,137.90,132.22,131.45,129.57,128.76,128.13$, $125.48,121.75,120.60,119.96,108.62,87.85,85.67,73.97,56.12$, 21.18, 20.23. ESI-MS: calculated $\left[\mathrm{C}_{20} \mathrm{H}_{17} \mathrm{NO}_{4}+\mathrm{Na}\right]^{+}: 358.1050$, found:358.1051. $[\alpha]_{\mathrm{D}}^{20}=-194.4\left(\mathrm{c}=0.41, \mathrm{CH}_{2} \mathrm{Cl}_{2}\right)$. The product was analyzed by HPLC to determine the enantiomeric excess: $98 \%$ ee (CHIRALPAK IC, hexane $/ i-\mathrm{PrOH}=70 / 30$, detector: $254 \mathrm{~nm}, \mathrm{~T}=25^{\circ} \mathrm{C}$, flow rate: $\left.1 \mathrm{~mL} / \mathrm{min}\right), \mathrm{t}_{1}($ major $)=9.99 \mathrm{~min}, \mathrm{t}_{2}($ minor $)=15.49 \mathrm{~min}$.

\section{(S)-2-((4-(2-chlorophenyl)but-3-yn-2-yl)oxy)-5-methoxyisoindoline-1,3-dione (3if)}

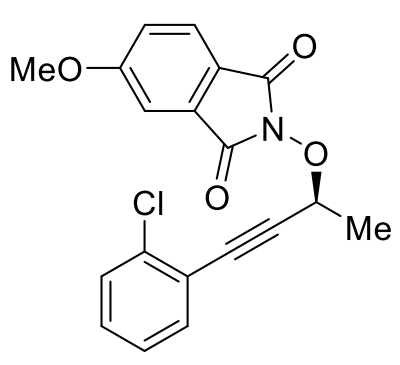
Yield: 29.5 mg (83\%); ${ }^{1} \mathbf{H}$ NMR (500 MHz, $\left.\mathbf{C D C l}_{3}\right) \delta 7.75(\mathrm{~d}, J=$ $8.2 \mathrm{~Hz}, 1 \mathrm{H}), 7.43(\mathrm{~d}, J=7.5 \mathrm{~Hz}, 1 \mathrm{H}), 7.35-7.29(\mathrm{~m}, 2 \mathrm{H}), 7.22(\mathrm{t}$, $J=7.7 \mathrm{~Hz}, 1 \mathrm{H}), 7.20-7.14(\mathrm{~m}, 2 \mathrm{H}), 5.46(\mathrm{q}, J=6.6 \mathrm{~Hz}, 1 \mathrm{H}), 3.92$ $(\mathrm{s}, 3 \mathrm{H}), 1.78(\mathrm{~d}, J=6.6 \mathrm{~Hz}, 3 \mathrm{H}) .{ }^{13} \mathbf{C ~ N M R}\left(\mathbf{1 2 5} \mathbf{M H z}, \mathbf{C D C l}_{3}\right) \delta$ $164.94,163.65,163.61,135.87,133.61,131.49,129.74,129.13$, $126.40,125.46,121.96,120.65,119.96,108.61,91.11,84.31$, 73.78, 56.12, 20.10. ESI-MS: calculated $\left[\mathrm{C}_{19} \mathrm{H}_{14} \mathrm{ClNO}_{4}+\mathrm{Na}\right]^{+}$: 
378.0504, found: $378.0509 .[\alpha]_{\mathrm{D}}^{20}=-118.8\left(\mathrm{c}=0.66, \mathrm{CH}_{2} \mathrm{Cl}_{2}\right)$. The product was analyzed by HPLC to determine the enantiomeric excess: $96 \%$ ee (CHIRALPAK IC, hexane $/ i-\mathrm{PrOH}=70 / 30$, detector: $254 \mathrm{~nm}, \mathrm{~T}=25{ }^{\circ} \mathrm{C}$, flow rate: $\left.1 \mathrm{~mL} / \mathrm{min}\right), \mathrm{t}_{1}($ major $)=10.39$ min, $\mathrm{t}_{2}($ minor $)=16.46$ $\min$.

(S)-5-methoxy-2-((4-(o-tolyl)but-3-yn-2-yl)oxy)isoindoline-1,3-dione (3jf)

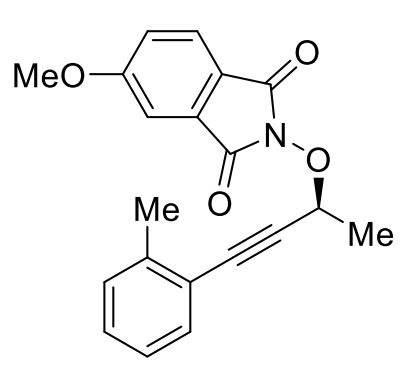

Yield: $32.8 \mathrm{mg}(98 \%)$; ${ }^{1} \mathbf{H}$ NMR (500 $\left.\mathbf{~ M H z}, \mathbf{C D C l}_{3}\right) \delta 7.75(\mathrm{~d}, J=$ $8.3 \mathrm{~Hz}, 1 \mathrm{H}), 7.34-7.32(\mathrm{~m}, 2 \mathrm{H}), 7.20-7.13(\mathrm{~m}, 3 \mathrm{H}), 7.08$ (td, $J$ $=7.5,1.4 \mathrm{~Hz}, 1 \mathrm{H}), 5.45(\mathrm{q}, J=6.6 \mathrm{~Hz}, 1 \mathrm{H}), 3.91(\mathrm{~s}, 3 \mathrm{H}), 2.32(\mathrm{~s}$, $3 \mathrm{H}), 1.77(\mathrm{~d}, J=6.6 \mathrm{~Hz}, 3 \mathrm{H}) .{ }^{13} \mathbf{C}$ NMR $\left(125 \mathbf{~ M H z}, \mathbf{C D C l}_{3}\right) \delta$ $164.94,163.70,163.67,140.35,132.20,131.44,129.36,128.71$, $125.46,121.73,120.58,119.96,108.61,89.79,86.60,73.94,56.12$, 20.51, 20.45. ESI-MS: calculated $\left[\mathrm{C}_{20} \mathrm{H}_{17} \mathrm{NO}_{4}+\mathrm{Na}\right]^{+}: 358.1050$, found: $358.1053 .[\alpha]_{D}^{20}=-136.3\left(\mathrm{c}=1.12, \mathrm{CH}_{2} \mathrm{Cl}_{2}\right)$. The product was analyzed by HPLC to determine the enantiomeric excess: $92 \%$ ee (CHIRALPAK IC, hexane $/ i$-PrOH $=70 / 30$, detector: $254 \mathrm{~nm}, \mathrm{~T}=25{ }^{\circ} \mathrm{C}$, flow rate: $\left.1 \mathrm{~mL} / \mathrm{min}\right), \mathrm{t}_{1}($ major $)=9.36 \mathrm{~min}, \mathrm{t}_{2}$ (minor) $=13.66 \mathrm{~min}$.

\section{(S)-2-((1,4-diphenylbut-3-yn-2-yl)oxy)-5-methoxyisoindoline-1,3-dione (3kf)}

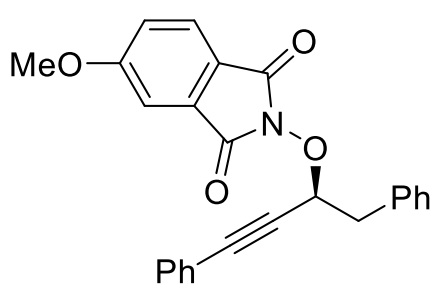

Yield: 32.5 mg (82\%). ${ }^{1} \mathbf{H}$ NMR (500 $\left.\mathbf{~ M H z , ~} \mathbf{C D C l}_{\mathbf{3}}\right) \delta 7.74(\mathrm{~d}$, $J=8.3 \mathrm{~Hz}, 1 \mathrm{H}), 7.43-7.41(\mathrm{~m}, 2 \mathrm{H}), 7.36-7.32(\mathrm{~m}, 3 \mathrm{H}), 7.31-$ $7.28(\mathrm{~m}, 3 \mathrm{H}), 7.27-7.24(\mathrm{~m}, 2 \mathrm{H}), 7.16(\mathrm{dd}, J=8.4,2.4 \mathrm{~Hz}, 1 \mathrm{H})$, $5.46(\mathrm{dd}, J=8.0,6.2 \mathrm{~Hz}, 1 \mathrm{H}), 3.90(\mathrm{~s}, 3 \mathrm{H}), 3.46(\mathrm{dd}, J=13.7$, $6.2 \mathrm{~Hz}, 1 \mathrm{H}), 3.31(\mathrm{dd}, J=13.7,8.0 \mathrm{~Hz}, 1 \mathrm{H}) .{ }^{13} \mathbf{C}$ NMR $(\mathbf{1 2 5}$

MHz, $\left.\mathbf{C D C l}_{3}\right) \delta 164.97,163.65,163.61,135.58,131.63,131.43$, 129.70, 128.72, 128.41, 128.22, 127.02, 125.49, 121.91, 120.59, 119.99, 108.64, 89.37, 84.78, 78.54, 56.12, 40.31. ESI-MS: calculated $\left[\mathrm{C}_{25} \mathrm{H}_{19} \mathrm{NO}_{4}+\mathrm{Na}\right]^{+}: 420.1206$, found: $420.1295 .[\alpha]_{\mathrm{D}}^{20}$ $=-58.7\left(\mathrm{c}=0.42, \mathrm{CH}_{2} \mathrm{Cl}_{2}\right)$. The product was analyzed by HPLC to determine the enantiomeric excess: $97 \%$ ee (CHIRALPAK IC, hexane $/ i-\mathrm{PrOH}=95 / 5$, detector: $254 \mathrm{~nm}, \mathrm{~T}=25{ }^{\circ} \mathrm{C}$, flow rate: $1 \mathrm{~mL} / \mathrm{min}), \mathrm{t}_{1}($ major $)=38.85 \mathrm{~min}, \mathrm{t}_{2}($ minor $)=44.63 \mathrm{~min}$.

(S)-2-((1,5-diphenylpent-1-yn-3-yl)oxy)-5-methoxyisoindoline-1,3-dione (3lf)

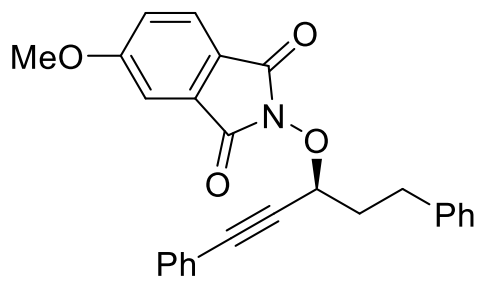

Yield: $38.4 \mathrm{mg}(87 \%) .{ }^{1} \mathbf{H}$ NMR (500 $\left.\mathbf{~ M H z}, \mathbf{C D C l}_{\mathbf{3}}\right) \delta 7.74(\mathrm{~d}$, $J=8.3 \mathrm{~Hz}, 1 \mathrm{H}), 7.39-7.35(\mathrm{~m}, 2 \mathrm{H}), 7.33-7.29(\mathrm{~m}, 5 \mathrm{H})$, $7.29-7.24(\mathrm{~m}, 3 \mathrm{H}), 7.23-7.19(\mathrm{~m}, 1 \mathrm{H}), 7.15(\mathrm{dd}, J=8.3$, $2.4 \mathrm{~Hz}, 1 \mathrm{H}), 5.27(\mathrm{t}, J=6.5 \mathrm{~Hz}, 1 \mathrm{H}), 3.89(\mathrm{~s}, 3 \mathrm{H}), 3.02(\mathrm{~m}$, 2H), $2.43(\mathrm{~m}, 1 \mathrm{H}), 2.31(\mathrm{~m}, 1 \mathrm{H}) .{ }^{13} \mathbf{C}$ NMR $(\mathbf{1 2 5} \mathbf{~ M H z}$,

CDCl $\left._{3}\right) \delta 164.96,163.74,163.70,140.89,131.72,131.46$, $128.75,128.65,128.58,128.53,128.29,126.17,125.49,121.96,120.60,119.99,108.64,88.81$, 85.04, 77.40, 56.13, 35.69, 31.38. ESI-MS: calculated $\left[\mathrm{C}_{26} \mathrm{H}_{21} \mathrm{NO}_{4}+\mathrm{Na}\right]^{+}:$434.1363, found: 434.1363. $[\alpha]_{\mathrm{D}}^{20}=-88.5\left(\mathrm{c}=0.33, \mathrm{CH}_{2} \mathrm{Cl}_{2}\right)$. The product was analyzed by HPLC to determine the enantiomeric excess: $96 \%$ ee (CHIRALPAK IC, hexane $/ i-\mathrm{PrOH}=85 / 15$, detector: $254 \mathrm{~nm}, \mathrm{~T}=$ $25^{\circ} \mathrm{C}$, flow rate: $\left.1 \mathrm{~mL} / \mathrm{min}\right), 1 \mathrm{~mL} / \mathrm{min}$ ), $\mathrm{t}_{1}$ (major) $=13.53 \mathrm{~min}, \mathrm{t}_{2}($ minor $)=17.07 \mathrm{~min}$.

(S)-5-methoxy-2-(pent-3-yn-2-yloxy)isoindoline-1,3-dione (3mf) 


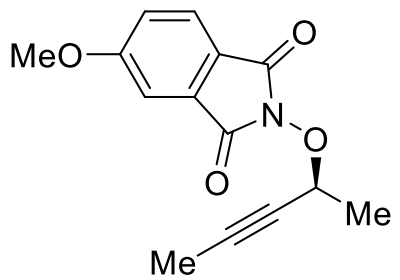

Yield: $21.5 \mathrm{mg}(83 \%) .{ }^{\mathbf{1}} \mathbf{H}$ NMR (500 $\left.\mathbf{~ M H z}, \mathbf{C D C l}_{\mathbf{3}}\right) \delta 7.76(\mathrm{~d}, J=$ $8.3 \mathrm{~Hz}, 1 \mathrm{H}), 7.34(\mathrm{~d}, J=2.3 \mathrm{~Hz}, 1 \mathrm{H}), 7.19(\mathrm{dd}, J=8.3,2.4 \mathrm{~Hz}$, $1 \mathrm{H}), 5.09$ (qq, $J=6.6,2.1 \mathrm{~Hz}, 1 \mathrm{H}), 3.94(\mathrm{~s}, 3 \mathrm{H}), 1.80(\mathrm{~d}, J=2.1$ $\mathrm{Hz}, 3 \mathrm{H}), 1.62(\mathrm{~d}, J=6.6 \mathrm{~Hz}, 3 \mathrm{H}) .{ }^{13} \mathbf{C}$ NMR $\left(\mathbf{1 2 5} \mathbf{M H z}, \mathbf{C D C l}_{3}\right) \delta$ 164.90, 163.71, 163.68, 131.43, 125.40, 120.58, 119.85, 108.58, 84.48, 76.48, 73.76, 56.11, 20.43, 3.64. ESI-MS: calculated $\left[\mathrm{C}_{14} \mathrm{H}_{13} \mathrm{NO}_{4}+\mathrm{Na}\right]^{+}:$282.0737, found: 282.0736. $[\alpha]_{\mathrm{D}}^{20}=-110.1\left(\mathrm{c}=0.54, \mathrm{CH}_{2} \mathrm{Cl}_{2}\right)$. The product was analyzed by HPLC to determine the enantiomeric excess: $91 \%$ ee (CHIRALPAK IC, hexane $/ i-\mathrm{PrOH}=70 / 30$, detector: $254 \mathrm{~nm}, \mathrm{~T}=25{ }^{\circ} \mathrm{C}$, flow rate: $\left.1 \mathrm{~mL} / \mathrm{min}\right), \mathrm{t}_{1}$ (major) $=15.56$ $\min , \mathrm{t}_{2}(\operatorname{minor})=34.01 \mathrm{~min}$.

(S)-5-methoxy-2-((6-phenylhex-3-yn-2-yl)oxy)isoindoline-1,3-dione (3nf)

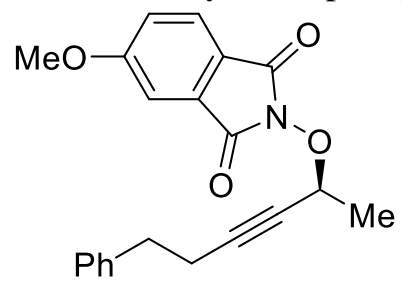

Yield: $31.8 \mathrm{mg}(91 \%) .{ }^{\mathbf{1}} \mathbf{H}$ NMR (500 $\left.\mathbf{~ M H z}, \mathbf{C D C l}_{3}\right) \delta 7.74(\mathrm{~d}, J$ $=8.3 \mathrm{~Hz}, 1 \mathrm{H}), 7.31(\mathrm{~d}, J=2.4 \mathrm{~Hz}, 1 \mathrm{H}), 7.22-7.16(\mathrm{~m}, 3 \mathrm{H}), 7.15$ $-7.11(\mathrm{~m}, 3 \mathrm{H}), 5.16-5.07(\mathrm{~m}, 1 \mathrm{H}), 3.92(\mathrm{~s}, 3 \mathrm{H}), 2.72(\mathrm{~m}, 2 \mathrm{H})$, $2.43(\mathrm{~m}, 2 \mathrm{H}), 1.61(\mathrm{~d}, J=6.6 \mathrm{~Hz}, 3 \mathrm{H}) .{ }^{\mathbf{1 3}} \mathrm{C}$ NMR (125 MHz, $\left.\mathbf{C D C l}_{3}\right) \delta 164.92,163.71,163.69,140.39,131.43,128.33,128.28$, $126.24,125.42,120.59,119.88,108.61,88.12,78.05,73.73,56.14$ 34.55, 20.88, 20.43. ESI-MS: calculated $\left[\mathrm{C}_{21} \mathrm{H}_{19} \mathrm{NO}_{4}+\mathrm{Na}\right]^{+}: 372.1206$, found: $372.1212 .[\alpha]_{\mathrm{D}}^{20}$ $=-69.6\left(\mathrm{c}=0.46, \mathrm{CH}_{2} \mathrm{Cl}_{2}\right)$. The product was analyzed by HPLC to determine the enantiomeric excess: $92 \%$ ee (CHIRALPAK IC, hexane $/ i-\mathrm{PrOH}=70 / 30$, detector: $254 \mathrm{~nm}, \mathrm{~T}=25^{\circ} \mathrm{C}$, flow rate: $1 \mathrm{~mL} / \mathrm{min}), \mathrm{t}_{1}($ major $)=12.47 \mathrm{~min}, \mathrm{t}_{2}(\operatorname{minor})=27.19 \mathrm{~min}$.

\section{(S)-5-methoxy-2-((1-phenylhex-4-yn-3-yl)oxy)isoindoline-1,3-dione (3of)}

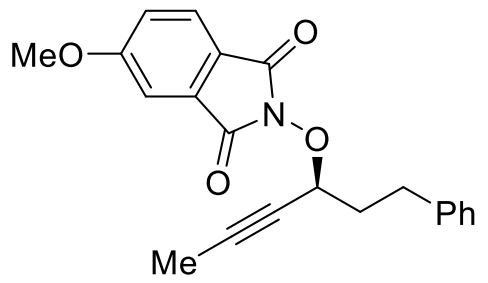

Yield: $29.3 \mathrm{mg}$ (87\%). ${ }^{1} \mathbf{H}$ NMR (500 $\left.\mathbf{~ M H z}, \mathbf{C D C l}_{3}\right) \delta 7.75$ (d, $J=8.3 \mathrm{~Hz}, 1 \mathrm{H}), 7.33-7.30(\mathrm{~m}, 2 \mathrm{H}), 7.29-7.26(\mathrm{~m}, 3 \mathrm{H})$, $7.21-7.16(\mathrm{~m}, 2 \mathrm{H}), 5.01(\mathrm{~m}, 1 \mathrm{H}), 3.92(\mathrm{~s}, 3 \mathrm{H}), 2.93(\mathrm{~m}, 2 \mathrm{H})$, $2.29(\mathrm{~m}, 1 \mathrm{H}), 2.17(\mathrm{~m}, 1 \mathrm{H}), 1.81(\mathrm{~d}, J=2.2 \mathrm{~Hz}, 3 \mathrm{H}) .{ }^{13} \mathrm{C}$ NMR (125 MHz, CDCl $) \delta 164.92,163.75,163.71,141.06$, $131.48,128.57,128.44,126.05,125.43,120.64,119.91$, 108.58, 85.72, 77.29, 75.36, 56.12, 35.82, 31.27, 3.76. ESI-MS: calculated $\left[\mathrm{C}_{21} \mathrm{H}_{19} \mathrm{NO}_{4}+\mathrm{Na}\right]^{+}$: 372.1206, found: $372.1213 .[\alpha]_{\mathrm{D}}^{20}=-42.4\left(\mathrm{c}=0.82, \mathrm{CH}_{2} \mathrm{Cl}_{2}\right)$. The product was analyzed by HPLC to determine the enantiomeric excess: $91 \%$ ee (CHIRALPAK IC, hexane/ $i-\mathrm{PrOH}=70 / 30$, detector: $254 \mathrm{~nm}, \mathrm{~T}=25^{\circ} \mathrm{C}$, flow rate: $\left.1 \mathrm{~mL} / \mathrm{min}\right), \mathrm{t}_{1}($ major $)=12.13 \mathrm{~min}, \mathrm{t}_{2}($ minor $)=17.59$ $\min$.

\section{Limitations of the $O$-APS reactions.}

We have tried propargylic carbonate bearing aryl groups at the propargylic position and terminal propargylic carbonate for the reaction. However, no product was detected with different types of phosphine ligands. 


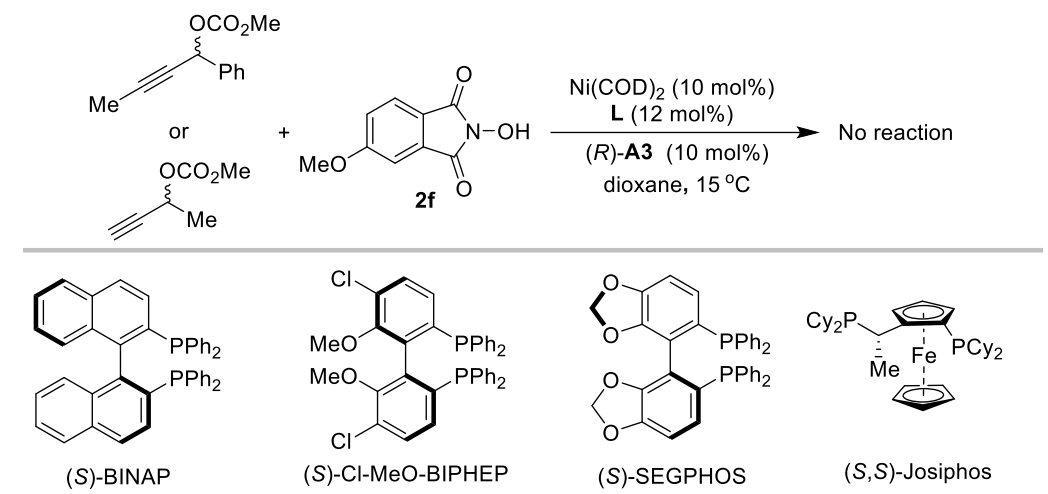

The use of propargylic carbonate bearing dialkyl groups at the propargylic position produced desired product. We evaluated different types of chiral phosphine ligands and solvents to determine their influence on the reactivity and enantioselectivity of the transformation. The use of $(S)$-ClMeO-BIPHEP as the phosphine ligand in toluene led to the desired adduct in $79 \%$ yield with $31 \%$ ee.

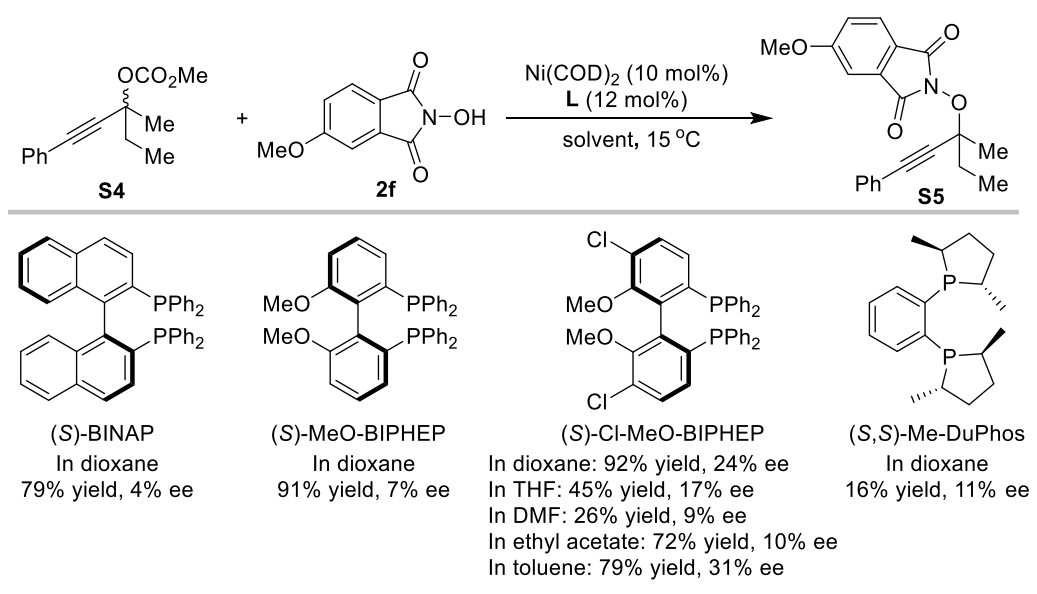




\section{Synthesis procedure of derivatization reaction}

5.1 Procedure for synthesis of potent firefly luciferase inhibitor and related isoxazolines ${ }^{3}$.
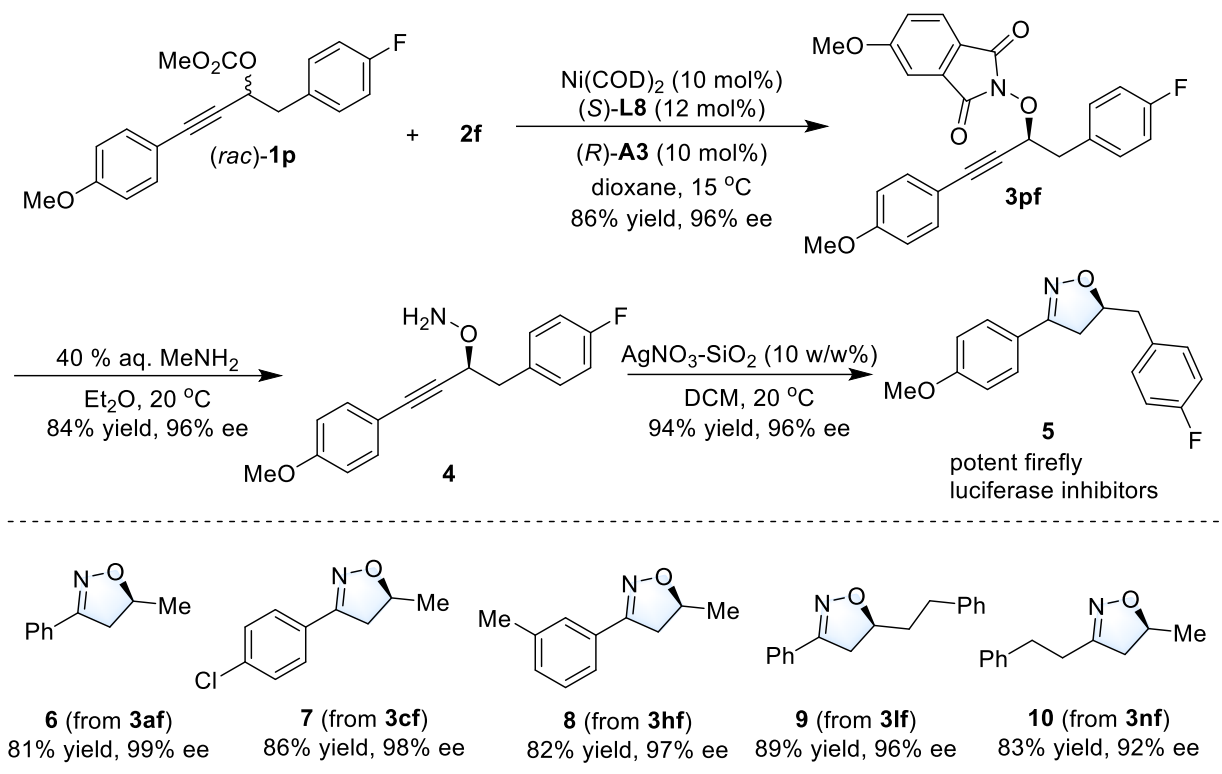

$\mathrm{Ni}(\mathrm{COD})_{2}$ (11 mg, $\left.0.04 \mathrm{mmol}, 10 \mathrm{~mol} \%\right),(S)$-Cl-MeO-BIPHEP (L8) (31.3 mg, $0.048 \mathrm{mmol}, 12$ mol\%) were added into a $50 \mathrm{~mL}$ Schlenk flask equipped with a magnetic stirrer in a glove box. Then dry 1,4-dioxane $(16 \mathrm{~mL})$ was added into the reaction system under argon and stirred for 30 minutes at room temperature, after which the $(R)-\mathbf{A 3}(15.5 \mathrm{mg}, 0.04 \mathrm{mmol}, 10 \mathrm{~mol} \%)$ was added into the reaction system and continued stirring for 30 minutes at room temperature under argon. Then propargylic carbonate ( $\mathrm{rac}) \mathbf{- 1 p}(262.7 \mathrm{mg}, 0.8 \mathrm{mmol}, 2.0$ equiv) and compound $\mathbf{2 f}(77.2 \mathrm{mg}, 0.4$ mmol, 1.0 equiv) were added into the reaction mixture under argon at $15^{\circ} \mathrm{C}$. The final solution was stirred at $15{ }^{\circ} \mathrm{C}$ until complete consumption of the substrate (monitored by TLC). The reaction mixture was then filtered through a short pad of silica gel, and the solvent was removed under reduced pressure. The residue was purified by flash column chromatography to give the desired product 3pf (144.5 mg, $86 \%$ yield).

3pf (144 mg, $0.32 \mathrm{mmol}, 1.0$ equiv) and ether $(15 \mathrm{~mL})$ were added to the reaction flask and stirred at $20{ }^{\circ} \mathrm{C}$ for 10 minutes. Then the $40 \%$ aqueous methylamine $(52 \mathrm{mg}, 0.67 \mathrm{mmol}, 2.1$ equiv) was added, and the reaction mixture was stirred at this temperature for 5 hours. After 3pf was consumed (monitored by TLC), the reaction mixture was then filtered through a short pad of silica gel. Then the filtrate was evaporated under vacuum. The residue was purified by flash column chromatography to give the desired product 4 (76.7 $\mathrm{mg}, 84 \%$ yield).

$4(76 \mathrm{mg}, 0.27 \mathrm{mmol}, 1.0$ equiv) and dichloromethane $(10 \mathrm{~mL})$ were added to the reaction flask and stirred at $20{ }^{\circ} \mathrm{C}$ for 10 minutes. Then the $10 \% \mathrm{w} / \mathrm{w} \mathrm{AgNO}_{3}-\mathrm{SiO}_{2}(2.3 \mathrm{mg} / 20.7 \mathrm{mg}$, $0.014 \mathrm{mmol}, 0.05$ equiv) was added, and the reaction mixture was stirred at this temperature for 20 minutes. When 5 was consumed (monitored by TLC), the reaction mixture was then filtered through a short pad of silica gel. Then the filtrate was evaporated under vacuum. Finally, the residue was purified by flash column chromatography to give the desired product 5 (71.4 mg, 94\% yield).

(S)-2-((1-(4-fluorophenyl)-4-(4-methoxyphenyl)but-3-yn-2-yl)oxy)-5-methoxyisoindoline- 


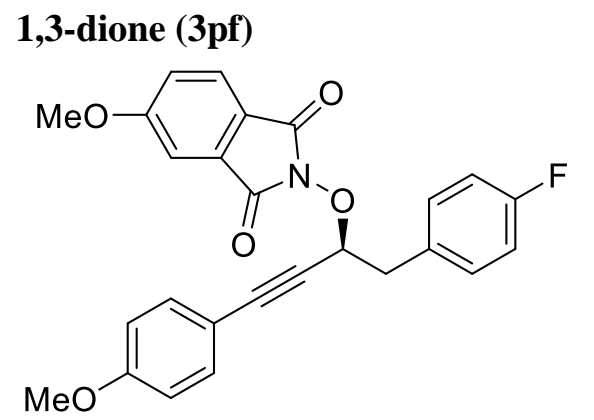

Yield: $144.5 \mathrm{mg}$ (86\%); ${ }^{1} \mathbf{H}$ NMR (500 $\left.\mathbf{~ M H z}, \mathbf{C D C l}_{3}\right) \delta$ $7.74(\mathrm{~d}, J=8.3 \mathrm{~Hz}, 1 \mathrm{H}), 7.39-7.36(\mathrm{~m}, 2 \mathrm{H}), 7.31(\mathrm{~d}, J$ $=2.3 \mathrm{~Hz}, 1 \mathrm{H}), 7.24-7.22(\mathrm{~m}, 2 \mathrm{H}), 7.17-7.15(\mathrm{~m}, 1 \mathrm{H})$, $7.04-7.00(\mathrm{~m}, 2 \mathrm{H}), 6.77(\mathrm{~d}, J=8.7 \mathrm{~Hz}, 2 \mathrm{H}), 5.40(\mathrm{t}, J$ $=6.9 \mathrm{~Hz}, 1 \mathrm{H}), 3.91(\mathrm{~s}, 3 \mathrm{H}), 3.77(\mathrm{~s}, 3 \mathrm{H}), 3.40(\mathrm{dd}, J=$ 13.9, $6.3 \mathrm{~Hz}, 1 \mathrm{H}), 3.27(\mathrm{dd}, J=13.9,7.5 \mathrm{~Hz}, 1 \mathrm{H}) .{ }^{13} \mathbf{C}$ NMR (125 MHz, $\left.\mathbf{C D C l}_{3}\right) \delta 164.96,163.64,163.61$, $162.97,161.03,159.98,133.17,131.44,131.41,131.28,131.21,125.47,120.56,119.95,115.27$, 115.10, 113.89, 113.86, 113.82, 108.64, 89.66, 83.14, 78.50, 56.12, 55.28, 39.53. ESI-MS: calculated $\left[\mathrm{C}_{26} \mathrm{H}_{20} \mathrm{FNO}_{5} \mathrm{~S}+\mathrm{H}\right]^{+}:$446.1398, found: 446.1403. $[\alpha]_{\mathrm{D}}^{20}=-88.6\left(\mathrm{c}=1.57, \mathrm{CH}_{2} \mathrm{Cl}_{2}\right)$. The product was analyzed by HPLC to determine the enantiomeric excess: $96 \%$ ee (CHIRALPAK IC, hexane $/ i-\mathrm{PrOH}=85 / 15$, detector: $254 \mathrm{~nm}, \mathrm{~T}=25^{\circ} \mathrm{C}$, flow rate: $1 \mathrm{~mL} / \mathrm{min}$ ), $\mathrm{t}_{1}($ major $)=21.92 \mathrm{~min}, \mathrm{t}_{2}(\operatorname{minor})=26.43 \mathrm{~min}$.

(S)-O-(1-(4-fluorophenyl)-4-(4-methoxyphenyl)but-3-yn-2-yl)hydroxylamine (4)

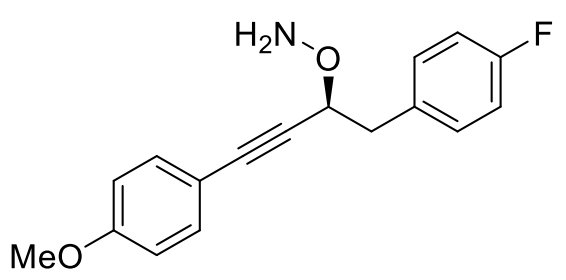

Yield: $76.7 \mathrm{mg}(84 \%) ;{ }^{1} \mathbf{H}$ NMR (500 $\left.\mathbf{M H z}, \mathbf{C D C l}_{\mathbf{3}}\right) \delta$ $7.36-7.32(\mathrm{~m}, 2 \mathrm{H}), 7.28-7.25(\mathrm{~m}, 2 \mathrm{H}), 7.01-6.96(\mathrm{~m}$, $2 \mathrm{H}), 6.84-6.79$ (m, 2H), 5.51 (brs, 2H), 4.64 (t, $J=6.6$ $\mathrm{Hz}, 1 \mathrm{H}), 3.81$ (s, 3H), $3.13-3.02(\mathrm{~m}, 2 \mathrm{H}) .{ }^{13} \mathbf{C}$ NMR (125 MHz, $\left.\mathbf{C D C l}_{\mathbf{3}}\right) \delta$ 162.79, 160.85, 159.79, 133.25, $132.75,132.73,131.17,131.11,115.10,114.93,114.47$,

113.92, 86.76, 85.67, 76.24, 55.29, 39.73. ESI-MS: calculated $\left[\mathrm{C}_{17} \mathrm{H}_{16} \mathrm{FNO}_{2}+\mathrm{H}\right]^{+}:$: 286.1238, found: $286.1246 .[\alpha]_{\mathrm{D}}^{20}=-28.2\left(\mathrm{c}=1.77, \mathrm{CH}_{2} \mathrm{Cl}_{2}\right)$. The product was analyzed by HPLC to determine the enantiomeric excess: $96 \%$ ee (CHIRALPAK IC, hexane $/ i$-PrOH $=85 / 15$, detector: $254 \mathrm{~nm}, \mathrm{~T}=25^{\circ} \mathrm{C}$, flow rate: $\left.1 \mathrm{~mL} / \mathrm{min}\right), \mathrm{t}_{1}($ minor $)=9.48 \mathrm{~min}, \mathrm{t}_{2}$ (major) $=11.19 \mathrm{~min}$.

(S)-5-(4-fluorobenzyl)-3-(4-methoxyphenyl)-4,5-dihydroisoxazole (5)

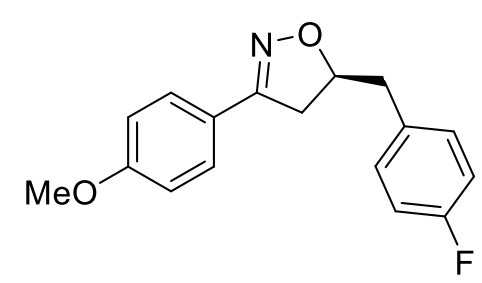

Yield: $71.4 \mathrm{mg}(94 \%) ;{ }^{1} \mathbf{H}$ NMR (500 $\left.\mathbf{~ M H z}, \mathbf{C D C l}_{\mathbf{3}}\right) \delta 7.59-$ $7.56(\mathrm{~m}, 2 \mathrm{H}), 7.25-7.21(\mathrm{~m}, 2 \mathrm{H}), 7.02-6.98(\mathrm{~m}, 2 \mathrm{H}), 6.92$ $-6.89(\mathrm{~m}, 2 \mathrm{H}), 4.95-4.89(\mathrm{~m}, 1 \mathrm{H}), 3.83(\mathrm{~s}, 3 \mathrm{H}), 3.31(\mathrm{dd}, J$ $=16.4,10.2 \mathrm{~Hz}, 1 \mathrm{H}), 3.09(\mathrm{dd}, J=14.1,6.4 \mathrm{~Hz}, 1 \mathrm{H}), 3.00(\mathrm{dd}$, $J=16.4,7.7 \mathrm{~Hz}, 1 \mathrm{H}), 2.88(\mathrm{dd}, J=14.1,6.5 \mathrm{~Hz}, 1 \mathrm{H}) .{ }^{13} \mathrm{C}$ NMR (125 MHz, $\left.\mathbf{C D C l}_{\mathbf{3}}\right) \delta 162.79,161.04,160.85,156.01$, 132.74, 132.72, 130.89, 130.82, 128.14, 122.17, 115.49, 115.32, 114.11, 81.40, 55.36, 40.21, 39.64. ESI-MS: calculated $\left[\mathrm{C}_{17} \mathrm{H}_{16} \mathrm{FNO}_{2}+\mathrm{H}\right]^{+}: 286.1238$, found: $286.1244 .[\alpha]_{\mathrm{D}}^{20}=-71.2(\mathrm{c}=$ $\left.0.25, \mathrm{CH}_{2} \mathrm{Cl}_{2}\right)$. The product was analyzed by HPLC to determine the enantiomeric excess: $96 \%$ ee (CHIRALPAK IA, hexane $/ i$-PrOH $=90 / 10$, detector: $254 \mathrm{~nm}, \mathrm{~T}=25^{\circ} \mathrm{C}$, flow rate: $1 \mathrm{~mL} / \mathrm{min}$ ), $\mathrm{t}_{1}($ minor $)=18.36 \mathrm{~min}, \mathrm{t}_{2}($ major $)=19.69 \mathrm{~min}$.

\section{(S)-5-methyl-3-phenyl-4,5-dihydroisoxazole (6)}

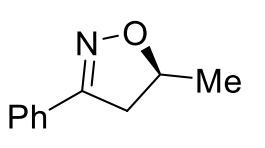

Yield: $13.1 \mathrm{mg}$ (81\%); ${ }^{1} \mathbf{H}$ NMR (500 MHz, $\left.\mathbf{C D C l}_{3}\right) \delta 7.68-7.64(\mathrm{~m}, 2 \mathrm{H})$,

$7.41-7.38(\mathrm{~m}, 3 \mathrm{H}), 4.92-4.84(\mathrm{~m}, 1 \mathrm{H}), 3.43(\mathrm{dd}, J=16.4,10.1 \mathrm{~Hz}, 1 \mathrm{H})$, $2.93(\mathrm{dd}, J=16.4,8.0 \mathrm{~Hz}, 1 \mathrm{H}), 1.43(\mathrm{~d}, J=6.2 \mathrm{~Hz}, 3 \mathrm{H}) .{ }^{13} \mathbf{C}$ NMR (125 
MHz, $\left.\mathbf{C D C l}_{3}\right) \delta 156.51,129.96,129.88,128.70,126.61,77.56,41.58,21.08$. ESI-MS: calculated $\left[\mathrm{C}_{10} \mathrm{H}_{11} \mathrm{NO}+\mathrm{H}\right]^{+}:$162.0913, found: $162.0916 .[\alpha]_{\mathrm{D}}^{20}=-84.8\left(\mathrm{c}=0.21, \mathrm{CH}_{2} \mathrm{Cl}_{2}\right)$. The product was analyzed by HPLC to determine the enantiomeric excess: $99 \%$ ee (CHIRALPAK OJ-H, hexane $/ i-\mathrm{PrOH}=95 / 5$, detector: $254 \mathrm{~nm}, \mathrm{~T}=25^{\circ} \mathrm{C}$, flow rate: $\left.1 \mathrm{~mL} / \mathrm{min}\right), \mathrm{t}_{1}($ major$)=$ 17.24 min, $\mathrm{t}_{2}($ minor $)=18.96 \min$.

\section{(S)-3-(4-chlorophenyl)-5-methyl-4,5-dihydroisoxazole (7)}<smiles>C[C@@H]1CC(c2ccc(Cl)cc2)=NO1</smiles>

Yield: $16.8 \mathrm{mg}$ (86\%); ${ }^{1} \mathbf{H}$ NMR (500 MHz, $\left.\mathbf{C D C l}_{3}\right) \delta 7.59(\mathrm{~d}, J=$ $8.5 \mathrm{~Hz}, 2 \mathrm{H}), 7.37(\mathrm{~d}, J=8.5 \mathrm{~Hz}, 2 \mathrm{H}), 4.93-4.86(\mathrm{~m}, 1 \mathrm{H}), 3.40(\mathrm{dd}$, $J=16.3,10.1 \mathrm{~Hz}, 1 \mathrm{H}), 2.90(\mathrm{dd}, J=16.3,8.1 \mathrm{~Hz}, 1 \mathrm{H}), 1.44(\mathrm{~d}, J=$ $6.3 \mathrm{~Hz}, 3 \mathrm{H}) .{ }^{13} \mathbf{C}$ NMR $\left(\mathbf{1 2 5} \mathbf{~ M H z}, \mathbf{C D C l}_{3}\right) \delta 155.53,135.83,128.94$ 128.43, 127.81, 77.83, 41.42, 21.00. ESI-MS: calculated $\left[\mathrm{C}_{10} \mathrm{H}_{10} \mathrm{ClNO}+\mathrm{H}\right]^{+}:$196.0524, found: 196.0531. $[\alpha]_{\mathrm{D}}^{20}=-79.8\left(\mathrm{c}=0.62, \mathrm{CH}_{2} \mathrm{Cl}_{2}\right)$. The product was analyzed by HPLC to determine the enantiomeric excess: $98 \%$ ee (CHIRALPAK OJ-H, hexane $/ i-\mathrm{PrOH}=95 / 5$, detector: $254 \mathrm{~nm}, \mathrm{~T}$ $=25^{\circ} \mathrm{C}$, flow rate: $\left.1 \mathrm{~mL} / \mathrm{min}\right), \mathrm{t}_{1}($ major $)=18.62 \mathrm{~min}, \mathrm{t}_{2}($ minor $)=20.55 \mathrm{~min}$.

(S)-5-methyl-3-(m-tolyl)-4,5-dihydroisoxazole (8)<smiles>Cc1cccc(C2=NOC(C)C2)c1</smiles>

Yield: 14.4 mg (82\%); ${ }^{1} \mathbf{H}$ NMR (500 MHz, $\left.\mathbf{C D C l}_{\mathbf{3}}\right) \delta 7.51$ (s, 1H), $7.44(\mathrm{~d}, J=7.6 \mathrm{~Hz}, 1 \mathrm{H}), 7.28(\mathrm{t}, J=7.6 \mathrm{~Hz}, 1 \mathrm{H}), 7.21(\mathrm{~d}, J=7.6$ $\mathrm{Hz}, 1 \mathrm{H}), 4.90-4.82(\mathrm{~m}, 1 \mathrm{H}), 3.41(\mathrm{dd}, J=16.3,10.1 \mathrm{~Hz}, 1 \mathrm{H}), 2.92$ (dd, $J=16.3,7.9 \mathrm{~Hz}, 1 \mathrm{H}), 2.37$ (s, 3H), $1.42(\mathrm{~d}, J=6.3 \mathrm{~Hz}, 3 \mathrm{H})$. ${ }^{13}$ C NMR (125 MHz, CDCl $) \delta 156.58,138.36,130.72,129.80,128.56,127.17,123.80,77.43$, 41.67, 21.36, 21.02. ESI-MS: calculated $\left[\mathrm{C}_{11} \mathrm{H}_{13} \mathrm{NO}+\mathrm{H}\right]^{+}: 176.1070$, found: $176.1078 .[\alpha]_{\mathrm{D}}^{20}=$ $-86.0\left(\mathrm{c}=0.15, \mathrm{CH}_{2} \mathrm{Cl}_{2}\right)$. The product was analyzed by HPLC to determine the enantiomeric excess: $97 \%$ ee (CHIRALPAK IB N-5, hexane $/ i-\mathrm{PrOH}=95 / 5$ detector: $254 \mathrm{~nm}, \mathrm{~T}=25^{\circ} \mathrm{C}$, flow rate: $1 \mathrm{~mL} / \mathrm{min}), \mathrm{t}_{1}($ minor $)=14.96 \mathrm{~min}, \mathrm{t}_{2}($ major $)=17.61 \mathrm{~min}$.

(S)-5-phenethyl-3-phenyl-4,5-dihydroisoxazole (9)

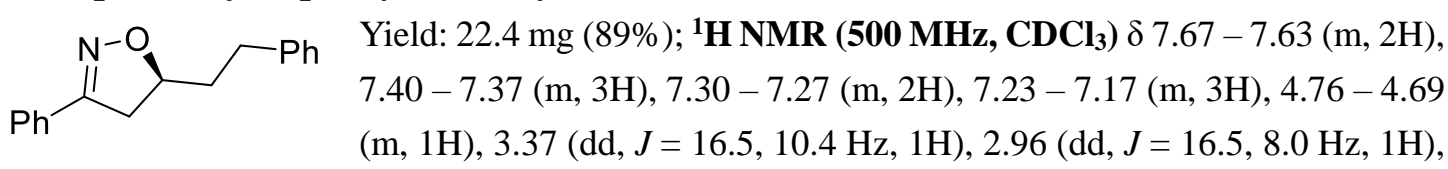
$2.89-2.73(\mathrm{~m}, 2 \mathrm{H}), 2.14-2.06(\mathrm{~m}, 1 \mathrm{H}), 1.96-1.88(\mathrm{~m}, 1 \mathrm{H}) .{ }^{\mathbf{1 3}} \mathbf{C}$ NMR (125 MHz, $\left.\mathbf{C D C l}_{\mathbf{3}}\right) \delta$ 156.46, 141.19, 129.99, 129.81, 128.71, 128.52, 126.63, 126.09, 80.46, 40.03, 37.14, 31.86. ESI-MS: calculated $\left[\mathrm{C}_{17} \mathrm{H}_{17} \mathrm{NO}+\mathrm{Na}\right]^{+}: 274.1202$, found: $274.1211 .[\alpha]_{\mathrm{D}}^{20}=-94.0\left(\mathrm{c}=0.12, \mathrm{CH}_{2} \mathrm{Cl}_{2}\right)$. The product was analyzed by HPLC to determine the enantiomeric excess: $96 \%$ ee (CHIRALPAK IB N-5, hexane $/ i-\mathrm{PrOH}=95 / 5$, detector: $254 \mathrm{~nm}, \mathrm{~T}=25^{\circ} \mathrm{C}$, flow rate: $\left.1 \mathrm{~mL} / \mathrm{min}\right), \mathrm{t}_{1}($ minor $)=13.59$ $\min , \mathrm{t}_{2}($ major $)=14.56 \mathrm{~min}$.

(S)-5-methyl-3-phenethyl-4,5-dihydroisoxazole (10)

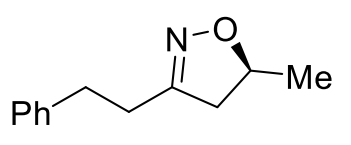

Yield: $15.7 \mathrm{mg}$ (83\%); ${ }^{1} \mathbf{H}$ NMR (500 MHz, $\left.\mathbf{C D C l}_{\mathbf{3}}\right) \delta 7.30$ (t, $J=7.5 \mathrm{~Hz}$,

$2 \mathrm{H}), 7.22-7.20(\mathrm{~m}, 3 \mathrm{H}), 4.69-4.61(\mathrm{~m}, 1 \mathrm{H}), 2.96(\mathrm{dd}, J=16.7,7.9 \mathrm{~Hz}$, $1 \mathrm{H}), 2.91(\mathrm{t}, 2 \mathrm{H}), 2.66(\mathrm{t}, J=8.0 \mathrm{~Hz}, 2 \mathrm{H}), 2.46(\mathrm{dd}, J=16.7,7.9 \mathrm{~Hz}, 1 \mathrm{H})$, $1.29(\mathrm{~d}, J=6.2 \mathrm{~Hz}, 3 \mathrm{H}) .{ }^{13} \mathbf{C}$ NMR $\left(\mathbf{1 2 5} \mathbf{~ M H z}, \mathbf{C D C l}_{3}\right) \delta 158.27,140.62,128.57,128.29,126.34$, 76.28, 43.99, 32.72, 29.66, 20.84. ESI-MS: calculated $\left[\mathrm{C}_{11} \mathrm{H}_{13} \mathrm{NO}+\mathrm{H}\right]^{+}:$190.1226, found: 
190.1232. $[\alpha]_{\mathrm{D}}^{20}=-117.6\left(\mathrm{c}=0.1, \mathrm{CH}_{2} \mathrm{Cl}_{2}\right)$. The product was analyzed by HPLC to determine the enantiomeric excess: 92\% ee (CHIRALPAK IB N-5, hexane/i-PrOH $=98 / 2$, detector: $220 \mathrm{~nm}, \mathrm{~T}=$ $25{ }^{\circ} \mathrm{C}$, flow rate: $\left.1 \mathrm{~mL} / \mathrm{min}\right), \mathrm{t}_{1}($ minor $)=20.49 \mathrm{~min}, \mathrm{t}_{2}($ major $)=21.89 \mathrm{~min}$.

\subsection{Procedure for synthesis of (S)-dihydroyashabushiketol ${ }^{4}$.}

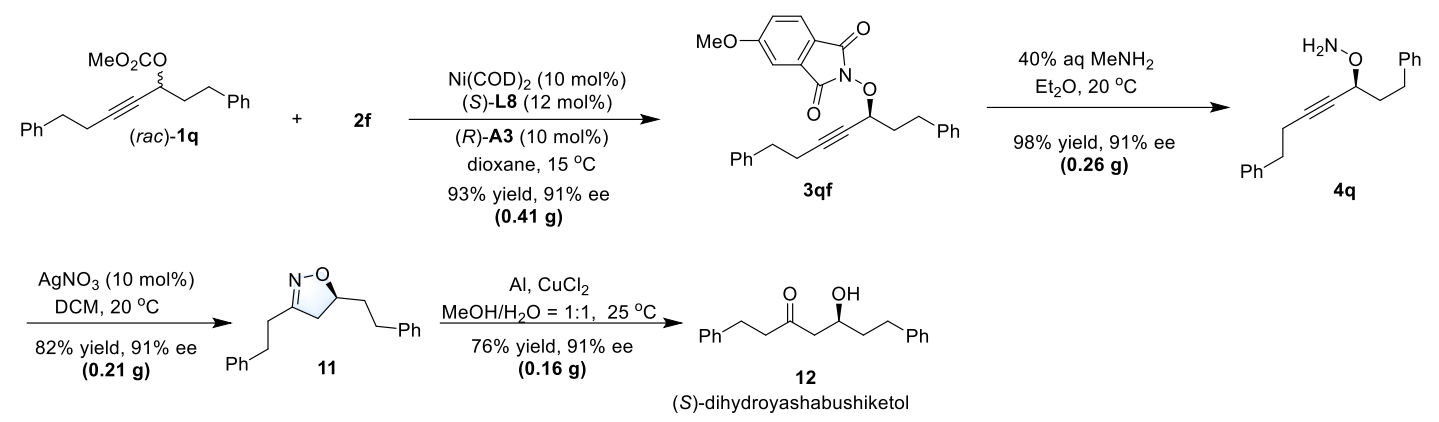

$\mathrm{Ni}(\mathrm{COD})_{2}$ (27.5 mg, $\left.0.1 \mathrm{mmol}, 10 \mathrm{~mol} \%\right)$, (S)-Cl-MeO-BIPHEP (L8) (78.1 mg, $0.12 \mathrm{mmol}, 12$ mol\%) were added into a $100 \mathrm{~mL}$ Schlenk flask equipped with a magnetic stirrer in a glove box. Then dry 1,4-dioxane $(40 \mathrm{~mL})$ was added into the reaction system under argon and stirred for 30 minutes at room temperature, after which the $(R)-\mathbf{A 3}(38.6 \mathrm{mg}, 0.1 \mathrm{mmol}, 10 \mathrm{~mol} \%)$ was added into the reaction system and continued stirring for 30 minutes at room temperature under argon. Then propargylic carbonate ( $\mathrm{rac}$ )-1q (408.4 mg, $2.0 \mathrm{mmol}, 2.0$ equiv) and compound $\mathbf{2 f}$ (193.1 $\mathrm{mg}, 1.0$ mmol, 1.0 equiv) were added into the reaction mixture under argon at $15^{\circ} \mathrm{C}$. The final solution was stirred at $15^{\circ} \mathrm{C}$ until complete consumption of the substrate (monitored by TLC). The reaction mixture was then filtered through a short pad of silica gel, and then the filtrate was evaporated under vacuum. The residue was purified by flash column chromatography to give the desired product 3qf ( $409 \mathrm{mg}, 93 \%$ yield).

3qf (409 $\mathrm{mg}, 0.93 \mathrm{mmol}, 1.0$ equiv) and ether $(15 \mathrm{~mL})$ were added to the reaction flask and stirred at $20{ }^{\circ} \mathrm{C}$ for 10 minutes. Then the $40 \%$ aqueous methylamine $(151 \mathrm{mg}, 1.95 \mathrm{mmol}, 2.1$ equiv) was added, and the reaction was continued stirring at this temperature for 5 hours (monitored by TLC). The reaction mixture was then filtered through a short pad of silica gel, and then the filtrate was evaporated under vacuum. The residue was purified by flash column chromatography to give the desired product $\mathbf{4 q}$ ( $255 \mathrm{mg}, 98 \%$ yield).

$\mathbf{4 q}(255 \mathrm{mg}, 0.91 \mathrm{mmol}, 1.0$ equiv) and dichloromethane $(15 \mathrm{~mL})$ were added to the reaction flask and stirred at $20^{\circ} \mathrm{C}$ for 10 minutes. Then the $10 \%$ w/w $\mathrm{AgNO}_{3}-\mathrm{SiO}_{2}(7.7 \mathrm{mg} / 69 \mathrm{mg}, 0.05$ equiv) was added, and the reaction was continued at this temperature for 20 minutes (monitored by TLC). The reaction mixture was then filtered through a short pad of silica gel, and then the filtrate was evaporated under vacuum. Finally, the residue was purified by flash column chromatography to give the desired product $\mathbf{1 1}$ (209 $\mathrm{mg}, 82 \%$ yield).

Compound 11 (209 mg, $0.75 \mathrm{mmol}, 1.0$ equiv) and aluminum powder $(607.1 \mathrm{mg}, 22.5 \mathrm{mmol}$, 30 equiv) were added to methanol $(5 \mathrm{~mL})$ at $20{ }^{\circ} \mathrm{C} . \mathrm{CuCl}_{2} \cdot 2 \mathrm{H}_{2} \mathrm{O}(1.28 \mathrm{~g}, 7.5 \mathrm{mmol}, 10$ equiv $)$ was dissolved in water $(5 \mathrm{~mL})$ and then slowly added to the reaction. The final solution was stirred at $25{ }^{\circ} \mathrm{C}$ until complete consumption of the substrate (monitored by TLC). The reaction mixture was then filtered through a short pad of silica gel, and then the filtrate was evaporated under vacuum. The residue was purified by flash column chromatography to give the desired product 12 (160 $\mathrm{mg}, 76 \%$ yield). 
(S)-2-((1,7-diphenylhept-4-yn-3-yl)oxy)-5-methoxyisoindoline-1,3-dione (3qf)

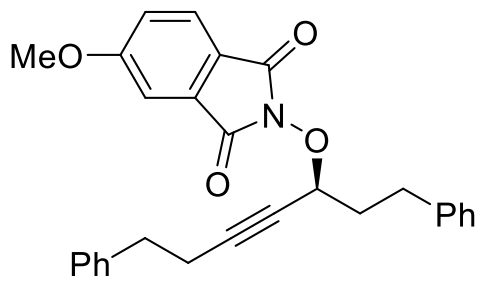

Yield: 409 mg (93\%); ${ }^{1} \mathbf{H}$ NMR (500 MHz, $\left.\mathbf{C D C l}_{3}\right) \delta 7.74$ (d, $J=8.3 \mathrm{~Hz}, 1 \mathrm{H}), 7.31-7.27(\mathrm{~m}, 3 \mathrm{H}), 7.24-7.11(\mathrm{~m}, 9 \mathrm{H})$, $5.02(\mathrm{~m}, 1 \mathrm{H}), 3.90(\mathrm{~s}, 3 \mathrm{H}), 2.94-2.81(\mathrm{~m}, 2 \mathrm{H}), 2.79-2.69$ $(\mathrm{m}, 2 \mathrm{H}), 2.48-2.44(\mathrm{~m}, 2 \mathrm{H}), 2.30-2.23(\mathrm{~m}, 1 \mathrm{H}), 2.11-2.18$ (m, 1H). ${ }^{13} \mathbf{C}$ NMR (125 MHz, $\left.\mathbf{C D C l}_{3}\right) \delta$ 164.94, 163.73, $163.70,141.04,140.35,131.48,128.60,128.46,128.36$, $128.34,126.30,126.08,125.43,120.63,119.91,108.61,89.36,76.90,76.87,56.14,35.78$, 34.55, 31.24, 20.89. ESI-MS: calculated $\left[\mathrm{C}_{28} \mathrm{H}_{25} \mathrm{NO}_{4}+\mathrm{Na}\right]^{+}: 462.1676$, found: 462.1685 . $[\alpha]_{\mathrm{D}}^{20}$ $=-46.8\left(\mathrm{c}=0.25, \mathrm{CH}_{2} \mathrm{Cl}_{2}\right)$. The product was analyzed by HPLC to determine the enantiomeric excess: $91 \%$ ee (CHIRALPAK IC, hexane $/ i-\mathrm{PrOH}=70 / 30$, detector: $254 \mathrm{~nm}, \mathrm{~T}=25^{\circ} \mathrm{C}$, flow rate: $1 \mathrm{~mL} / \mathrm{min}), \mathrm{t}_{1}($ major $)=8.27 \mathrm{~min}, \mathrm{t}_{2}($ minor $)=11.66 \mathrm{~min}$.

(S)-O-(1,7-diphenylhept-4-yn-3-yl)hydroxylamine (4q)

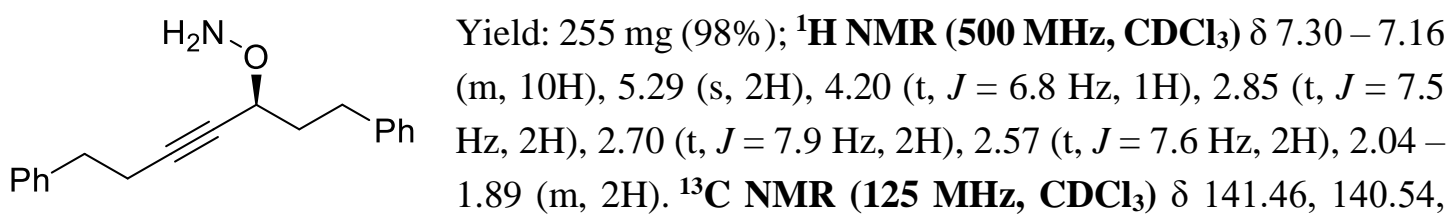
128.51, 128.50, 128.42, 128.40, 126.38, 125.94, 86.20, 79.29, 74.72, 35.70, 35.02, 31.41, 20.93. ESI-MS: calculated $\left[\mathrm{C}_{19} \mathrm{H}_{21} \mathrm{NO}+\mathrm{Na}\right]^{+}: 302.1515$, found: $302.1516 .[\alpha]_{\mathrm{D}}^{20}=-40.7(\mathrm{c}=0.63$, $\mathrm{CH}_{2} \mathrm{Cl}_{2}$ ). The product was analyzed by HPLC to determine the enantiomeric excess: $91 \%$ ee (CHIRALPAK OD-H, hexane $/ i-\mathrm{PrOH}=70 / 30$, detector: $212 \mathrm{~nm}, \mathrm{~T}=25^{\circ} \mathrm{C}$, flow rate: 1 $\mathrm{mL} / \mathrm{min}), \mathrm{t}_{1}($ major $)=10.27 \mathrm{~min}, \mathrm{t}_{2}($ minor $)=13.89 \mathrm{~min}$.

(S)-3,5-diphenethyl-4,5-dihydroisoxazole (11)

Yield: $209 \mathrm{mg}(82 \%) ;{ }^{1} \mathbf{H ~ N M R}\left(\mathbf{5 0 0 ~ M H z}, \mathbf{C D C l}_{\mathbf{3}}\right) \delta 7.31-7.26$
$(\mathrm{~m}, 4 \mathrm{H}), 7.22-7.17(\mathrm{~m}, 6 \mathrm{H}), 4.51(\mathrm{~m}, 1 \mathrm{H}), 2.93-2.88(\mathrm{~m}, 3 \mathrm{H})$,
$2.78-2.72(\mathrm{~m}, 1 \mathrm{H}), 2.69-2.63(\mathrm{~m}, 3 \mathrm{H}), 2.51-2.46(\mathrm{~m}, 1 \mathrm{H})$, $2.00-1.93(\mathrm{~m}, 1 \mathrm{H}), 1.81-1.74(\mathrm{~m}, 1 \mathrm{H}) .{ }^{\mathbf{1 3}} \mathbf{C}$ NMR (125 MHz, CDCl $) \delta$ 158.24, 141.30, $140.59,128.59$, 128.49, 128.47, 128.31, 126.38, 126.02, 79.25, 42.45, 36.98, 32.71, 31.86, 29.62. ESI-MS: calculated $\left[\mathrm{C}_{19} \mathrm{H}_{21} \mathrm{NO}+\mathrm{Na}\right]^{+}: 302.1515$, found: $302.1522 .[\alpha]_{\mathrm{D}}^{20}=-81.4(\mathrm{c}=$ $1.87, \mathrm{CH}_{2} \mathrm{Cl}_{2}$ ). The product was analyzed by HPLC to determine the enantiomeric excess: $91 \%$ ee (CHIRALPAK IC hexane $/ i$-PrOH $=98 / 2$, detector: $254 \mathrm{~nm}, \mathrm{~T}=25^{\circ} \mathrm{C}$, flow rate: $1 \mathrm{~mL} / \mathrm{min}$ ), $\mathrm{t}_{1}($ major $)=47.21 \mathrm{~min}, \mathrm{t}_{2}($ minor $)=52.29 \mathrm{~min}$.

\section{(S)-5-hydroxy-1,7-diphenylheptan-3-one (12)}<smiles>O=C(CCc1ccccc1)CC(O)CCc1ccccc1</smiles>

Yield: $160 \mathrm{mg}$ (76\%); ${ }^{\mathbf{1}} \mathbf{H}$ NMR (500 MHz, $\left.\mathbf{C D C l}_{3}\right) \delta 7.29-7.24$ $(\mathrm{m}, 4 \mathrm{H}), 7.21-7.15(\mathrm{~m}, 6 \mathrm{H}), 4.06-4.02(\mathrm{~m}, 1 \mathrm{H}), 3.07(\mathrm{~d}, J=$ $3.5 \mathrm{~Hz}, 1 \mathrm{H}), 2.89(\mathrm{t}, J=7.6 \mathrm{~Hz}, 2 \mathrm{H}), 2.82-2.72(\mathrm{~m}, 3 \mathrm{H}), 2.69-$ $2.63(\mathrm{~m}, 1 \mathrm{H}), 2.58-2.49(\mathrm{~m}, 2 \mathrm{H}), 1.83-1.76(\mathrm{~m}, 1 \mathrm{H}), 1.69-1.62(\mathrm{~m}, 1 \mathrm{H}) .{ }^{13} \mathbf{C}$ NMR $(\mathbf{1 2 5}$ MHz, $\left.\mathbf{C D C l}_{3}\right) \delta 211.13,141.82,140.67,128.58,128.48,128.44,128.29,126.25,125.90,66.86$, 49.29, 45.04, 38.05, 31.76, 29.51. ESI-MS: calculated $\left[\mathrm{C}_{19} \mathrm{H}_{22} \mathrm{O}_{2}+\mathrm{Na}\right]^{+}: 305.1512$, found: 305.1513. $[\alpha]_{\mathrm{D}}^{20}=+12.8\left(\mathrm{c}=1.76, \mathrm{CH}_{2} \mathrm{Cl}_{2}\right)$. The product was analyzed by HPLC to determine 
the enantiomeric excess: $91 \%$ ee (CHIRALPAK IC, hexane $/ i$-PrOH $=85 / 15$, detector: $254 \mathrm{~nm}$, $\mathrm{T}=25{ }^{\circ} \mathrm{C}$, flow rate: $\left.1 \mathrm{~mL} / \mathrm{min}\right), \mathrm{t}_{1}($ minor $)=10.39 \mathrm{~min}, \mathrm{t}_{2}$ (major $)=11.73 \mathrm{~min}$.

\subsection{Procedure for synthetic applications ${ }^{3,5,6,7}$}

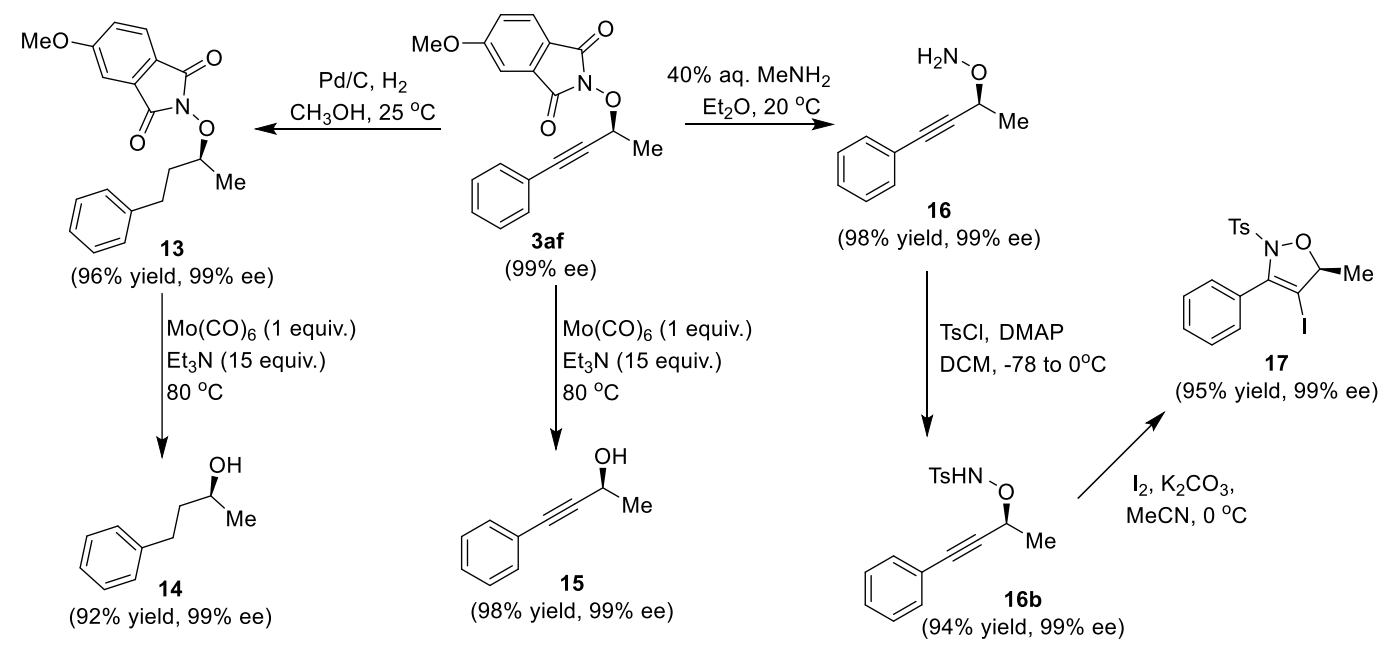

Compounds 3af (10 mg, $0.03 \mathrm{mmol}, 1.0$ equiv), $\mathrm{Mo}(\mathrm{CO})_{6}(8.2 \mathrm{mg}, 0.03 \mathrm{mmol}, 1.0$ equiv), and $\mathrm{CH}_{3} \mathrm{CN}-\mathrm{H}_{2} \mathrm{O}(15: 1,1 \mathrm{~mL})$ were added into the reaction flask. Then trimethylamine $(47.2 \mathrm{mg}, 65$ $\mu \mathrm{L}, 15$ equiv) was added to the reaction mixture, which was stirred at $80{ }^{\circ} \mathrm{C}$ for 12 hours. Then the reaction solution was concentrated, and the residue was purified by flash column chromatography to give the desired product $\mathbf{1 5}$ ( $4.4 \mathrm{mg}, 96 \%$ yield).

\section{(S)-5-methoxy-2-((4-phenylbutan-2-yl)oxy)isoindoline-1,3-dione (13)}

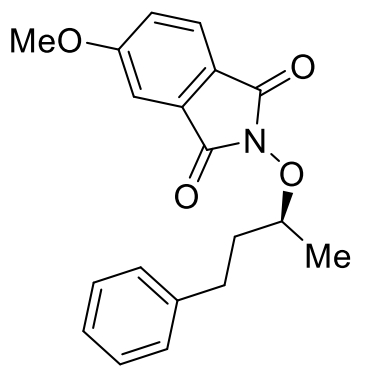

Yield: 48.6 mg (96\%); ${ }^{1} \mathbf{H}$ NMR (500 $\left.\mathbf{~ M H z , ~} \mathbf{C D C l}_{3}\right) \delta 7.72(\mathrm{~d}, J=8.2$ $\mathrm{Hz}, 1 \mathrm{H}), 7.30-7.24$ (m, 5H), $7.19-7.14(\mathrm{~m}, 2 \mathrm{H}), 4.43-4.37$ (m, 1H), $3.90(\mathrm{~s}, 3 \mathrm{H}), 2.87(\mathrm{dd}, J=9.2,6.9 \mathrm{~Hz}, 2 \mathrm{H}), 2.15-2.07(\mathrm{~m}, 1 \mathrm{H}), 1.94-$ $1.86(\mathrm{~m}, 1 \mathrm{H}), 1.37(\mathrm{~d}, J=6.3 \mathrm{~Hz}, 3 \mathrm{H}) .{ }^{13} \mathbf{C} \mathbf{N M R}\left(\mathbf{1 2 5} \mathbf{~ M H z}, \mathbf{C D C l}_{3}\right) \delta$ $164.94,164.39,164.33,141.76,131.51,128.52,128.41,125.90,125.38$, $120.66,119.85,108.60,83.67,56.13,36.82,31.46,18.99$. ESI-MS: calculated $\left[\mathrm{C}_{19} \mathrm{H}_{19} \mathrm{NO}_{4}+\mathrm{H}\right]^{+}: 326.1387$, found: 326.1395 . $[\alpha]_{D}^{20}=-4.1(\mathrm{c}$ $\left.=0.38, \mathrm{CH}_{2} \mathrm{Cl}_{2}\right)$. The product was analyzed by HPLC to determine the enantiomeric excess: $99 \%$ ee (CHIRALPAK IC, hexane $/ i-\mathrm{PrOH}=70 / 30$, detector: $254 \mathrm{~nm}, \mathrm{~T}=25^{\circ} \mathrm{C}$, flow rate: $1 \mathrm{~mL} / \mathrm{min}$ ), $\mathrm{t}_{1}($ minor $)=11.09 \mathrm{~min}, \mathrm{t}_{2}($ major $)=12.00 \mathrm{~min}$.

Compounds 13 (30 mg, 0.093mmol, 1.0 equiv), $\mathrm{Mo}(\mathrm{CO})_{6}(24.4 \mathrm{mg}, 0.093 \mathrm{mmol}, 1.0$ equiv), $\mathrm{CH}_{3} \mathrm{CN}-\mathrm{H}_{2} \mathrm{O}(15: 1,2 \mathrm{~mL})$ were added into the reaction flask. Then trimethylamine (140 mg, 0.2 $\mathrm{mL}, 15$ equiv) was added to the reaction mixture, which was stirred at $80^{\circ} \mathrm{C}$ for 12 hours. Then the reaction solution was concentrated, and the residue was purified by flash column chromatography to give the desired product $\mathbf{1 4}$ (12.8 $\mathrm{mg}, 98 \%$ yield).

\section{(S)-4-phenylbutan-2-ol (14)}




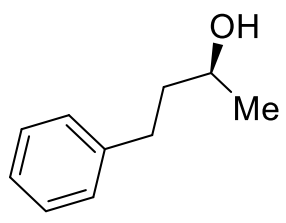

Yield: $12.8 \mathrm{mg}(92 \%) ;{ }^{1} \mathbf{H}$ NMR (500 MHz, $\left.\mathbf{C D C l}_{3}\right) \delta 7.28(\mathrm{t}, J=7.5 \mathrm{~Hz}$, 2H), $7.21-7.16(\mathrm{~m}, 3 \mathrm{H}), 3.83(\mathrm{q}, J=6.3 \mathrm{~Hz}, 1 \mathrm{H}), 2.79-2.64(\mathrm{~m}, 2 \mathrm{H}), 1.82$ $-1.71(\mathrm{~m}, 2 \mathrm{H}), 1.40(\mathrm{~s}, 1 \mathrm{H}), 1.23(\mathrm{~d}, J=6.4 \mathrm{~Hz}, 3 \mathrm{H}) .{ }^{13} \mathbf{C}$ NMR $(\mathbf{1 2 5} \mathbf{~ M H z}$, $\left.\mathbf{C D C l}_{3}\right) \delta 142.09,128.42,125.84,67.52,40.87,32.17,23.65$. ESI-MS: calculated $\left[\mathrm{C}_{10} \mathrm{H}_{14} \mathrm{O}+\mathrm{H}\right]^{+}: 151.1117$, found: $151.1120 .[\alpha]_{\mathrm{D}}^{20}=+42.3(\mathrm{c}=0.01$,

$\mathrm{CH}_{2} \mathrm{Cl}_{2}$ ). The product was analyzed by HPLC to determine the enantiomeric excess: $99 \%$ ee (CHIRALPAK OD-H, hexane $/ i$-PrOH $=85 / 15$, detector: $212 \mathrm{~nm}, \mathrm{~T}=25^{\circ} \mathrm{C}$, flow rate: $1 \mathrm{~mL} / \mathrm{min}$ ), $\mathrm{t}_{1}($ minor $)=6.26 \mathrm{~min}, \mathrm{t}_{2}($ major $)=8.05 \mathrm{~min}$.

3af $(25.2 \mathrm{mg}, 0.078 \mathrm{mmol}, 1.0$ equiv) and ether $(10 \mathrm{~mL})$ were added to the reaction flask, and the reaction mixture was stirred at $20^{\circ} \mathrm{C}$ for 10 minutes. Then the $40 \%$ aqueous methylamine $(12.7 \mathrm{mg}$, $0.16 \mathrm{mmol}, 2.1$ equiv) was added, and the final solution was stirred at $20{ }^{\circ} \mathrm{C}$ until complete consumption of the substrate (monitored by TLC). The reaction mixture was then filtered through a short pad of silica gel, and then the filtrate was evaporated under vacuum. Finally, the filtrate was evaporated under vacuum, and the residue was purified by flash column chromatography to give the desired product $\mathbf{1 6}(12.4 \mathrm{mg}, 84 \%$ yield $)$.

\section{(S)-4-phenylbut-3-yn-2-ol (15)}

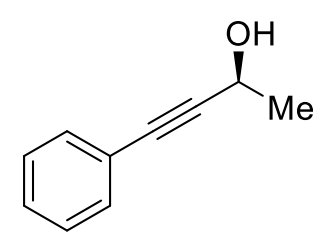

Yield: 4.4 mg (96\%); ${ }^{1} \mathbf{H}$ NMR (500 MHz, CDCl $) \delta 7.43-7.41(\mathrm{~m}, 2 \mathrm{H})$, $7.30-7.27(\mathrm{~m}, 3 \mathrm{H}), 4.76(\mathrm{q}, J=6.3 \mathrm{~Hz}, 1 \mathrm{H}), 2.08(\mathrm{~d}, J=5.1 \mathrm{~Hz}, 1 \mathrm{H}), 1.55$ $(\mathrm{d}, J=6.5 \mathrm{~Hz}, 3 \mathrm{H}) .{ }^{13} \mathbf{C} \mathbf{N M R}\left(\mathbf{1 2 5} \mathbf{~ M H z}, \mathbf{C D C l}_{3}\right) \delta 131.67,128.39,128.29$, 122.60, 90.98, 84.03, 58.88, 24.41. ESI-MS: calculated $\left[\mathrm{C}_{10} \mathrm{H}_{10} \mathrm{O}+\mathrm{H}\right]^{+}$: 147.0804, found: $147.0807 .[\alpha]_{\mathrm{D}}^{20}=-4.5\left(\mathrm{c}=0.11, \mathrm{CH}_{2} \mathrm{Cl}_{2}\right)$. The product was analyzed by HPLC to determine the enantiomeric excess: $99 \%$ ee (CHIRALPAK OJ-H, hexane $/ i$ $\mathrm{PrOH}=98 / 2$, detector: $254 \mathrm{~nm}, \mathrm{~T}=25^{\circ} \mathrm{C}$, flow rate: $1 \mathrm{~mL} / \mathrm{min}$ ), $\mathrm{t}_{1}($ minor $)=33.79 \mathrm{~min}, \mathrm{t}_{2}$ (major) $=$ $39.25 \mathrm{~min}$.

3af (50 mg, $0.16 \mathrm{mmol}, 1.0$ equiv), $10 \mathrm{wt} \% \mathrm{Pd} / \mathrm{C}(3.3 \mathrm{mg}, 0.003 \mathrm{mmol}, 2 \mathrm{~mol} \%)$ were added into a $10 \mathrm{~mL}$ Schlenk flask, then $\mathrm{CH}_{3} \mathrm{OH}(2 \mathrm{~mL})$ was added into the Schlenk flask. The mixture was stirred under the $\mathrm{H}_{2}$ atmosphere (a $42 \mathrm{~L}$ oxygen bag) at room temperature for 4 hours. The final solution was stirred at $15{ }^{\circ} \mathrm{C}$ until complete consumption of the substrate (monitored by TLC). The reaction mixture was then filtered through a short pad of silica gel, and then the filtrate was evaporated under vacuum. The residue was purified by flash column chromatography to give the desired product 13 (48.6mg, $96 \%$ yield).

\section{(S)-O-(4-phenylbut-3-yn-2-yl)hydroxylamine (16)}

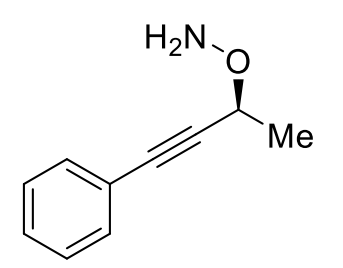

Yield: $12.4 \mathrm{mg}(98 \%) ;{ }^{1} \mathbf{H}$ NMR (500 $\left.\mathbf{~ M H z}, \mathbf{C D C l}_{3}\right) \delta 7.47-7.45(\mathrm{~m}, 2 \mathrm{H})$, $7.33-7.29(\mathrm{~m}, 3 \mathrm{H}), 5.53(\mathrm{~s}, 2 \mathrm{H}), 4.61(\mathrm{q}, J=6.6 \mathrm{~Hz}, 1 \mathrm{H}), 1.50(\mathrm{~d}, J=6.6$ $\mathrm{Hz}, 3 \mathrm{H}) .{ }^{13} \mathbf{C}$ NMR (125 MHz, $\left.\mathbf{C D C l}_{\mathbf{3}}\right) \delta 131.85,128.45,128.29,122.55$, 88.65, 84.96, 71.27, 20.40. ESI-MS: calculated $\left[\mathrm{C}_{10} \mathrm{H}_{11} \mathrm{NO}+\mathrm{H}\right]^{+}:$162.0913, found: $162.0914 .[\alpha]_{D}^{20}=-34.3\left(\mathrm{c}=0.55, \mathrm{CH}_{2} \mathrm{Cl}_{2}\right)$. The product was analyzed by HPLC to determine the enantiomeric excess: $99 \%$ ee (CHIRALPAK OJ-H, hexane/ $i$-PrOH $=85 / 15$, detector: $254 \mathrm{~nm}, \mathrm{~T}=25^{\circ} \mathrm{C}$, flow rate: $\left.1 \mathrm{~mL} / \mathrm{min}\right), \mathrm{t}_{1}$ (major) $=19.34$ min, $\mathrm{t}_{2}($ minor $)=22.69$ $\min$. 
Compound 16 (10 mg, 0.062 mmol, 1.0 equiv), pyridine (4.9 mg, 0.062mmol, 1.0 equiv), 4dimethylaminopyridine $(7.6 \mathrm{mg}, 0.0062 \mathrm{mmol}, 10 \mathrm{~mol} \%)$, and dichloromethane $(2 \mathrm{~mL})$ were added into the flask and stirred at $-78^{\circ} \mathrm{C}$ for 10 minutes. 4-Tosyl chloride ( $11.8 \mathrm{mg}, 0.062 \mathrm{mmol}, 1.0$ equiv) was added to the reaction mixture. Then the reaction mixture was stirred at $0^{\circ} \mathrm{C}$ for 16 hours. After the reaction solution was concentrated, the residue was purified by flash column chromatography to give the desired product $\mathbf{1 6 b}$ (18.4 $\mathrm{mg}, 94 \%$ yield).

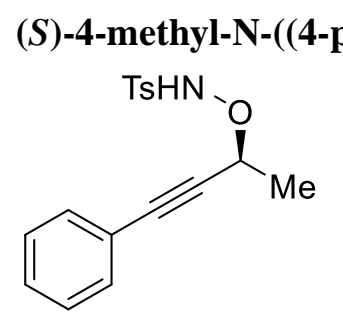

ESI-MS: calculated $\left[\mathrm{C}_{17} \mathrm{H}_{17} \mathrm{NO}_{3} \mathrm{~S}+\mathrm{Na}\right]^{+}: 338.0821$, found: $338.0827 .[\alpha]_{\mathrm{D}}^{20}=-30.4(\mathrm{c}=1.33$, $\mathrm{CH}_{2} \mathrm{Cl}_{2}$ ). The product was analyzed by HPLC to determine the enantiomeric excess: $99 \%$ ee (CHIRALPAK OJ-H, hexane $/ i$-PrOH $=95 / 5$, detector: $220 \mathrm{~nm}, \mathrm{~T}=25^{\circ} \mathrm{C}$, flow rate: $1 \mathrm{~mL} / \mathrm{min}$ ), $\mathrm{t}_{1}($ major $)=56.32 \mathrm{~min}, \mathrm{t}_{2}($ minor $)=68.78 \mathrm{~min}$.

Compound $16 \mathbf{b}$ ( $9 \mathrm{mg}, 0.029 \mathrm{mmol}, 1.0$ equiv) was added into acetonitrile $(2 \mathrm{~mL})$ and the reaction mixture was stirred for 10 minutes at $0{ }^{\circ} \mathrm{C}$, then potassium carbonate $(12 \mathrm{mg}, 0.087 \mathrm{mmol}, 3.0$ equiv) and iodine ( $22.1 \mathrm{mg}, 0.087 \mathrm{mmol}, 3.0$ equiv) were added. After the reaction was completed, the excess iodine was consumed by adding sodium sulfite saturated aqueous solution. The solution was extracted by ethyl acetate three times, dried by anhydrous sodium sulfate, and filtered to obtain the filtrate. Then the solution was concentrated, and the residue was purified by flash column chromatography to give the desired product $\mathbf{1 7}$ (11.8 $\mathrm{mg}, 95 \%$ yield).

\section{(S)-4-iodo-5-methyl-3-phenyl-2-tosyl-2,5-dihydroisoxazole (17)}<smiles>CC1ON([TeH])C(c2ccccc2)=C1I</smiles>

Yield: $11.8 \mathrm{mg}(95 \%) ;{ }^{1} \mathbf{H}$ NMR (500 $\left.\mathbf{~ M H z}, \mathbf{C D C l}_{3}\right) \delta 7.74-7.71(\mathrm{~m}, 4 \mathrm{H})$, $7.46-7.43(\mathrm{~m}, 3 \mathrm{H}), 7.36(\mathrm{~d}, J=7.9 \mathrm{~Hz}, 2 \mathrm{H}), 4.65(\mathrm{q}, J=6.2 \mathrm{~Hz}, 1 \mathrm{H}), 2.48$ $(\mathrm{s}, 3 \mathrm{H}), 1.27(\mathrm{~d}, J=6.2 \mathrm{~Hz}, 3 \mathrm{H}) .{ }^{13} \mathbf{C}$ NMR $\left(\mathbf{1 2 5} \mathbf{~ M H z}, \mathbf{C D C l}_{3}\right) \delta 145.86$, $141.04,131.05,129.97,129.89,129.66,129.64,129.31,128.22,87.64$, 81.70, 21.88, 20.58. ESI-MS: calculated $\left[\mathrm{C}_{17} \mathrm{H}_{16} \mathrm{INO}_{3} \mathrm{~S}+\mathrm{H}\right]^{+}: 441.9968$, found: 441.9970. $[\alpha]_{\mathrm{D}}^{20}=-71.5\left(\mathrm{c}=0.39, \mathrm{CH}_{2} \mathrm{Cl}_{2}\right)$. The product was analyzed by HPLC to determine the enantiomeric excess: $99 \%$ ee (CHIRALPAK AD-H, hexane $/ i-\operatorname{PrOH}=70 / 30$, detector: $220 \mathrm{~nm}$, $\mathrm{T}=25^{\circ} \mathrm{C}$, flow rate: $\left.1 \mathrm{~mL} / \mathrm{min}\right), \mathrm{t}_{1}($ minor $)=6.96 \mathrm{~min}, \mathrm{t}_{2}$ (major) $=8.05 \mathrm{~min}$. 


\section{X-ray Single Crystal Data for 3ah and 3gf}

\section{Stereochemistry determination of $3 \mathrm{ah}$ via $\mathrm{X}$-ray crystallographic analysis.}

Product 3ah was crystallized as a colorless crystal via vaporization of a $\mathrm{CDCl}_{3}$ solution. The structure of $\mathbf{3 a h}$ was determined by $\mathrm{x}$-ray structure analysis. CCDC 2076782 (3ah) contain the supplementary crystallographic data that can be obtained free of charge from the Cambridge Crystallographic Data Centre via www.ccdc.cam.ac.uk/data_request/cif. The absolute structure refinement of 3af was tested using single-crystal X-ray diffraction data collected using $\mathrm{Cu} K \alpha$ radiation. The Flack $x$ parameter is suited in particular to absolute configuration determination. The value of the Flack parameter is close to zero, therefore, the absolute structure given by the structure refinement is likely correct. ${ }^{8}$

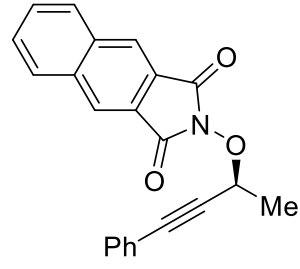

$3 a h$

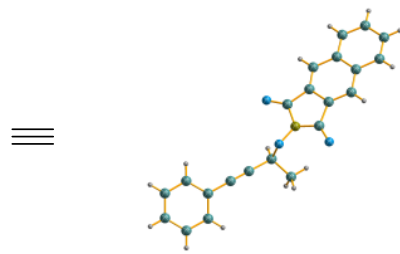

3ah, CCDC (2076782)

\begin{tabular}{cc}
\hline Chemical formula & $\mathrm{C}_{22} \mathrm{H}_{15} \mathrm{NO}_{3}$ \\
\hline Formula weight & 341.35 \\
Space group & $\mathrm{P} 2{ }_{2} 2_{1} 2_{1}$ \\
$\mathrm{Z}$ & 4 \\
$\mathrm{a}, \AA$ & $7.2766(2)$ \\
$\mathrm{b}, \AA$ & $12.2387(3)$ \\
$\mathrm{c}, \AA$ & $18.8863(4)$ \\
$\alpha,^{\circ}$ & 90 \\
$\beta,{ }^{\circ}$ & 90 \\
$\gamma,{ }^{\circ}$ & 90 \\
$\mathrm{~V}, \AA^{3}$ & $1681.94(7)$ \\
Flack parameter & $0.03(14)$ \\
\hline
\end{tabular}




\section{Stereochemistry determination of $3 g f$ via $X$-ray crystallographic analysis.}

CCDC 2110208 (3gf) contain the supplementary crystallographic data that can be obtained free of charge from the Cambridge Crystallographic Data Centre via www.ccdc.cam.ac.uk/data_request/cif. In addition, the absolute configuration of propargylic adduct $\mathbf{3 g f}$ with heavy atom $(\mathrm{Cl}$ atom) was also assigned by $\mathrm{X}$-ray analysis, which is consistent with the results of $\mathbf{3} \mathbf{a h}$.

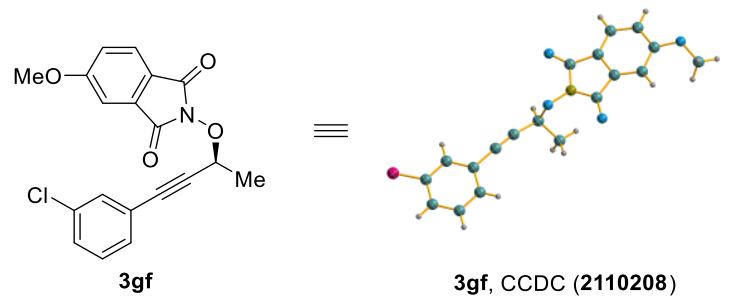

\begin{tabular}{cc}
\hline Chemical formula & $\mathrm{C}_{19} \mathrm{H}_{14} \mathrm{ClNO}_{4}$ \\
\hline Formula weight & 355.76 \\
Space group & $\mathrm{P} 21$ \\
$\mathrm{Z}$ & 2 \\
$\mathrm{a}, \AA$ & $4.124(4)$ \\
$\mathrm{b}, \AA$ & $12.036(11)$ \\
$\mathrm{c}, \AA$ & $17.161(16)$ \\
$\alpha,{ }^{\circ}$ & 90 \\
$\beta,{ }^{\circ}$ & $93.651(13)$ \\
$\gamma,{ }^{\circ}$ & 90 \\
$\mathrm{~V}, \AA^{3}$ & $850.1(13)$ \\
Flack parameter & $0.02(17)$ \\
\hline
\end{tabular}




\section{References}

1. Peng, J.; Gao, Y.; Hu, W.; Gao, Y.; Hu, M.; Wu, W.; Ren, Y.; Jiang, H. Palladium-catalyzed multicomponent reaction (MCR) of propargylic carbonates with isocyanides. Org. Lett. 2016, 18, 5924-5927.

2. Krylov, I. B.; Kompanets, M. O.; Novikova, K. V.; Opeida, I. O.; Kushch, O. V.; Shelimov, B. N.; Nikishin, G. I.; Levitsky, D. O.; Terent'ev, A. O. Well-known mediators of selective oxidation with unknown electronic structure: metal-free generation and EPR study of imide- $N$-oxyl radicals. J. Phys. Chem. A 2016, 120, 68-73.

3. Knight, D. W.; Proctor, A. J.; Clough. J. M. New regiospecific catalytic approaches to 4,5dihydroisoxazoles and 2,5-dihydroisoxazoles from $O$-propargylic hydroxylamines. Synlett 2010, 4, 628-632.

4. Karpaviciene, I.; Lapinskaite, R.; Brukstus, A.; Cikotiene, I. Reductive ring cleavage of nonconjugated $\Delta^{2}$-isoxazolines to $\beta$-hydroxy ketones with aluminum and copper(II) chloride. Synlett 2012, 23, 381-384.

5. Hashimoto, T.; Sakata, K.; Maruoka, K. Phase-transfer-catalyzed olefin isomerization/ $\alpha-$ alkylation of $\alpha$-alkynylcrotonates as a route for 1,4-enynes. Adv. Synth. Catal. 2010, 352, $1653-$ 1656.

6. Cicchi, S.; Goti, A.; Brandi, A.; Guarna, A.; Sarlo, F. D. 1,3-Aminoalcohols by reductive cleavage of isoxazolidines with molybdenum hexacarbonyl. Tetrahedron Lett. 1990, 31, 3351-3354.

7. Foot, O. F.; Knight, D. W.; Low, A.; Li, Y. On the viability of 5-endo-dig cyclisations of $O$ propargylic hydroxylamine derivatives, leading to 2,5-dihydroisoxazoles (3-isoxazolines). Tetrahedron Lett. 2007, 48, 647-650.

8. Parsons, S.; Flack, H. D.; Wagner, T. Use of intensity quotients and differences in absolute structure refinement. Acta Cryst. 2013, B69, 249-259. 


\section{8. ${ }^{1} \mathrm{H}$ and ${ }^{13} \mathrm{C}$ NMR spectra}

${ }^{1} \mathrm{H}$ NMR spectrum of $(R)$-A3

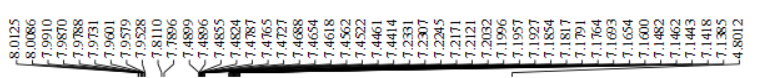
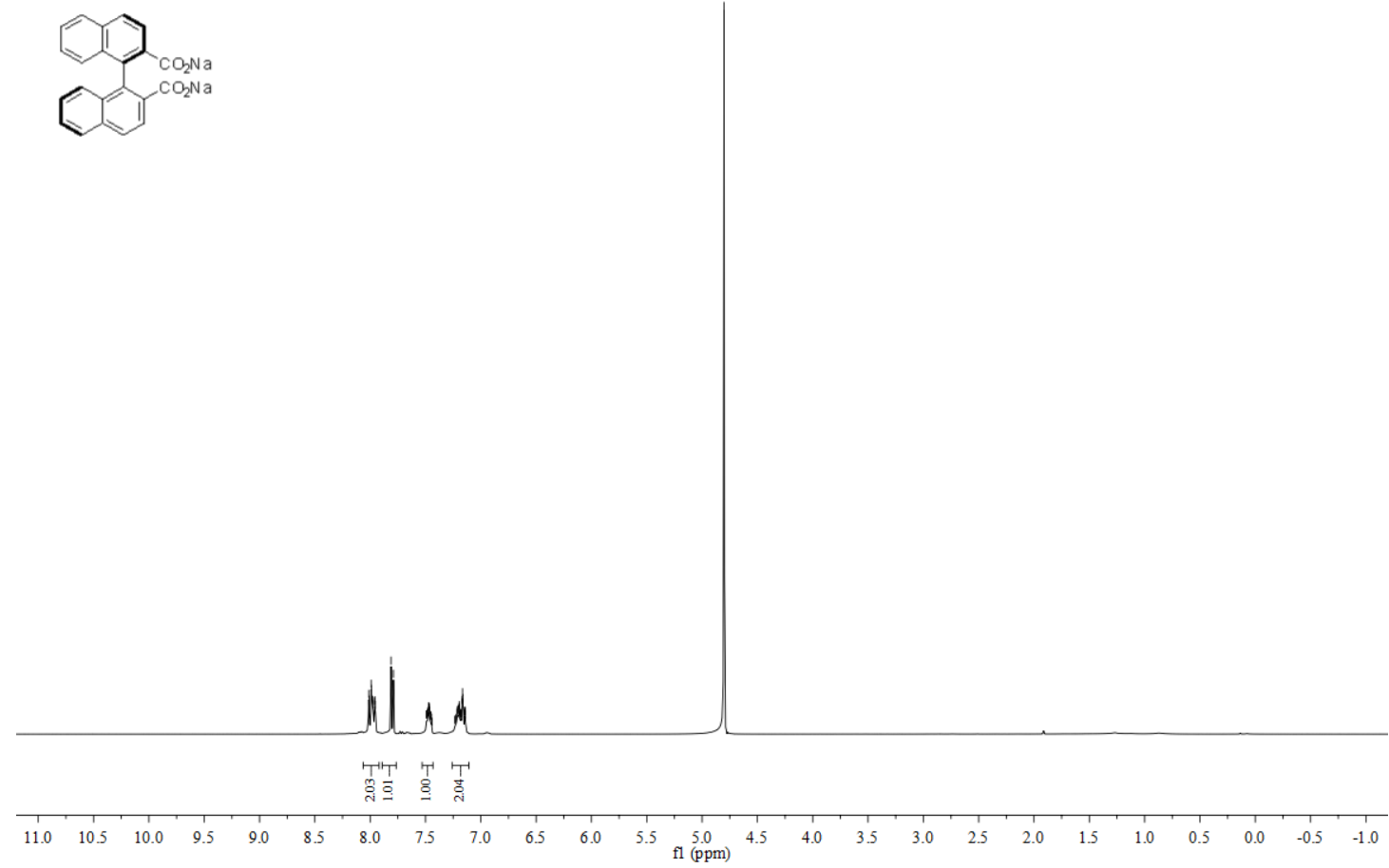

${ }^{13} \mathrm{C}$ NMR spectrum of $(R)-\mathbf{A 3}$

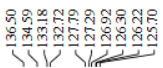
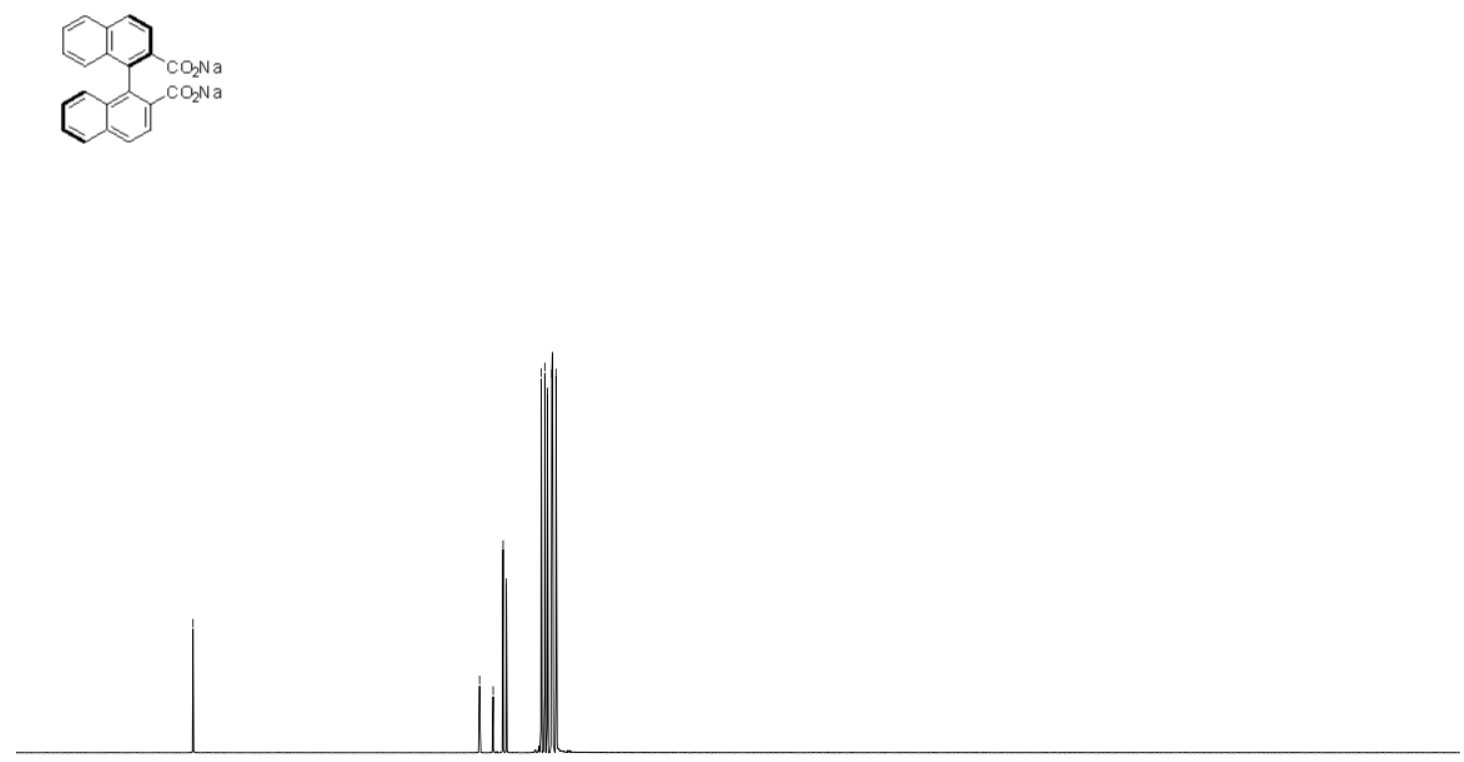

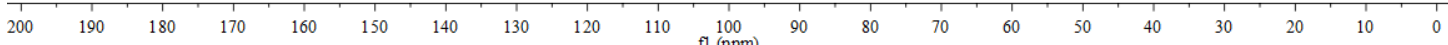


${ }^{1} \mathrm{H}$ NMR spectrum of $\mathbf{3 a a}$

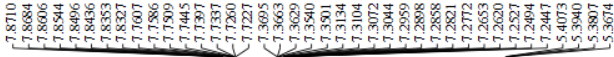

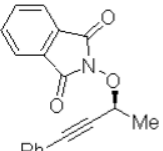

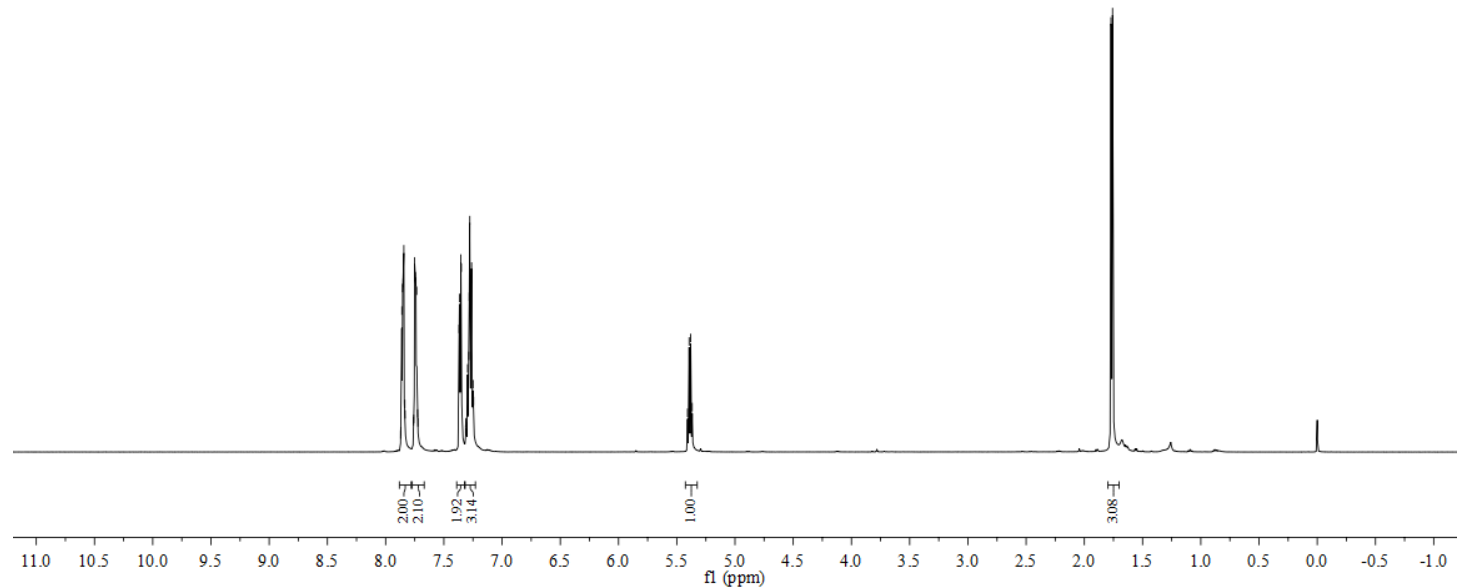

${ }^{13} \mathrm{C}$ NMR spectrum of $\mathbf{3 a a}$

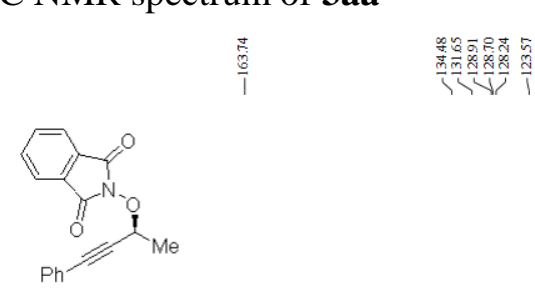

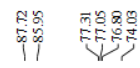

잼

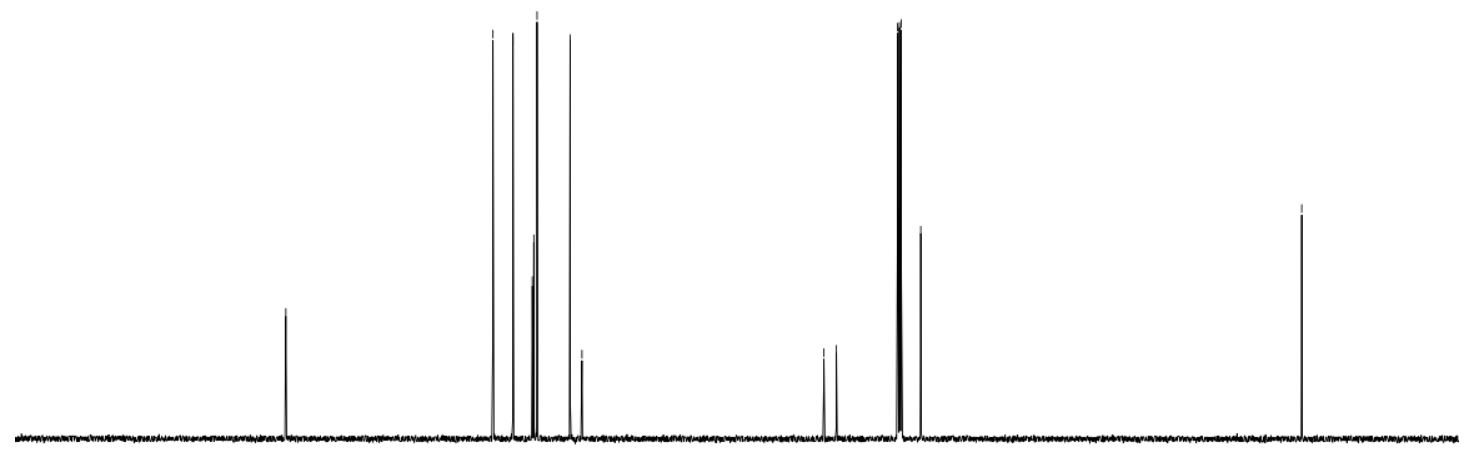

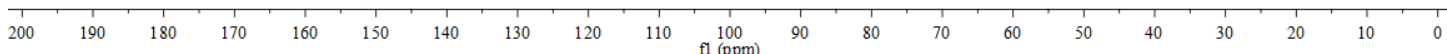


${ }^{1} \mathrm{H}$ NMR spectrum of $\mathbf{3 a b}$

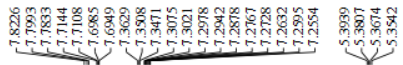

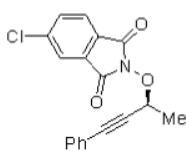

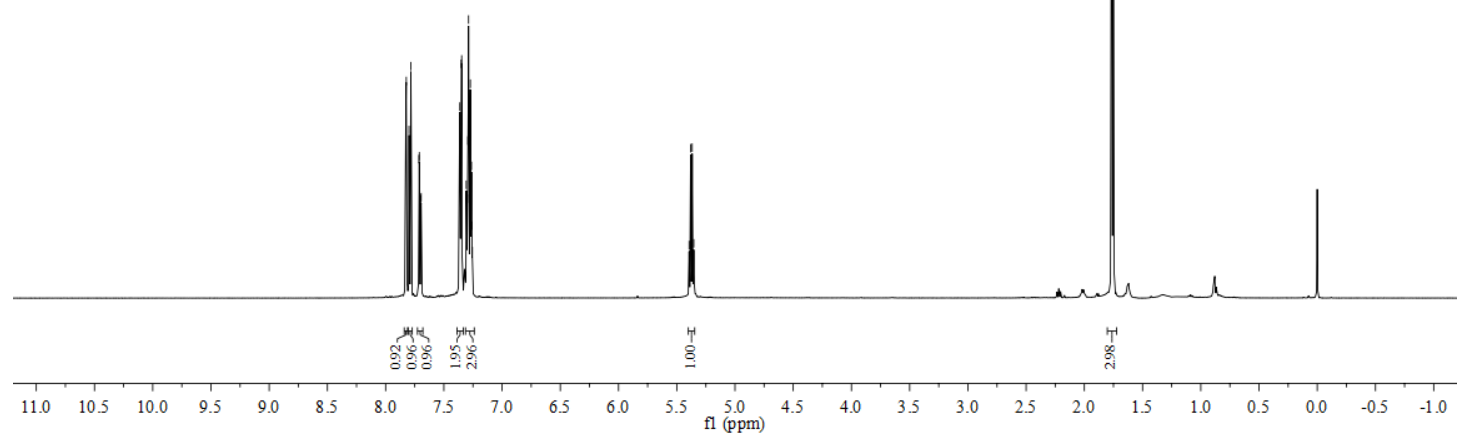

${ }^{13} \mathrm{C}$ NMR spectrum of $\mathbf{3 a b}$
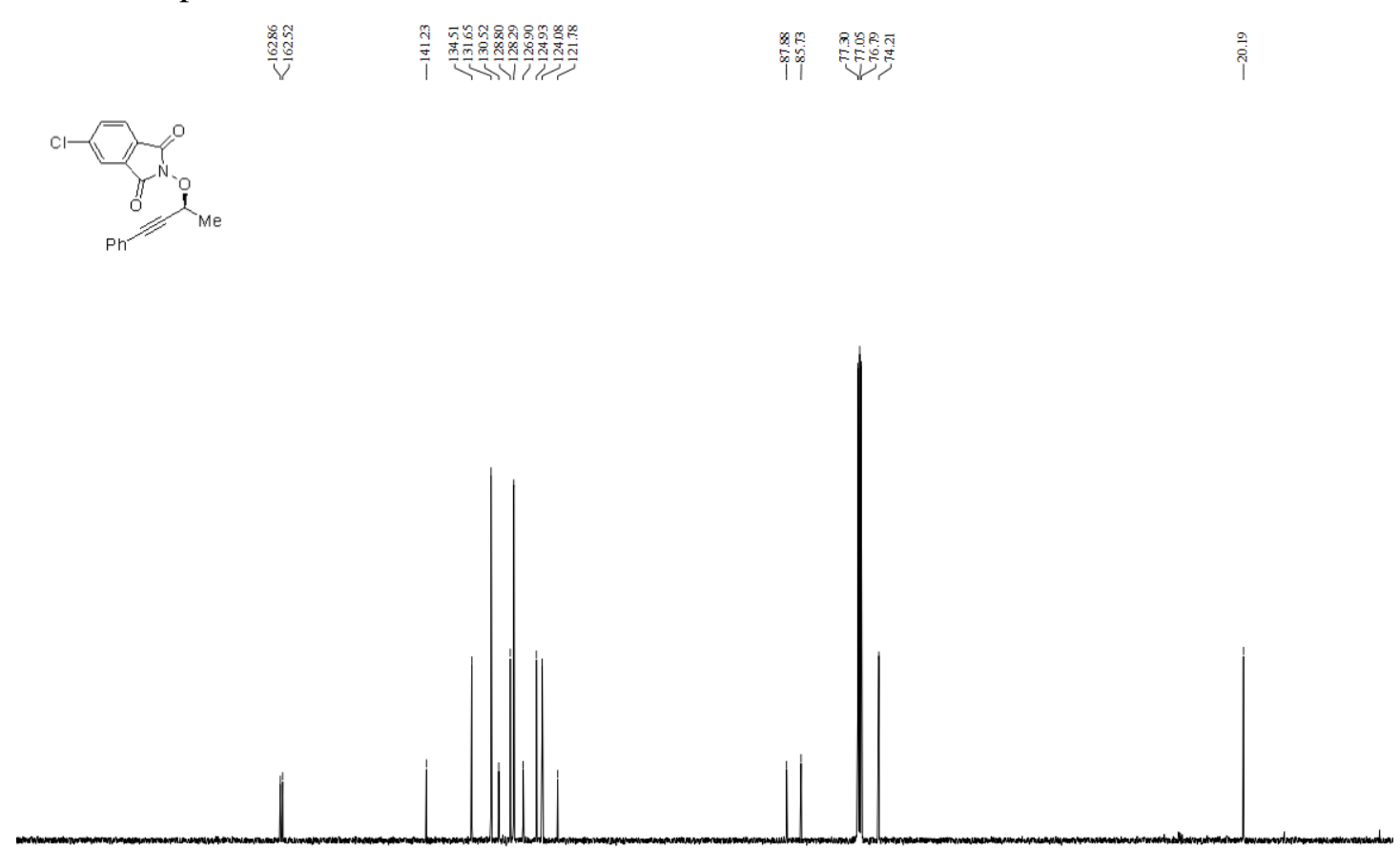

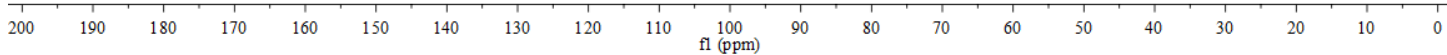


${ }^{1} \mathrm{H}$ NMR spectrum of $\mathbf{3 a c}$

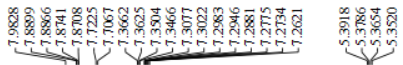

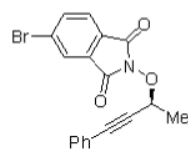

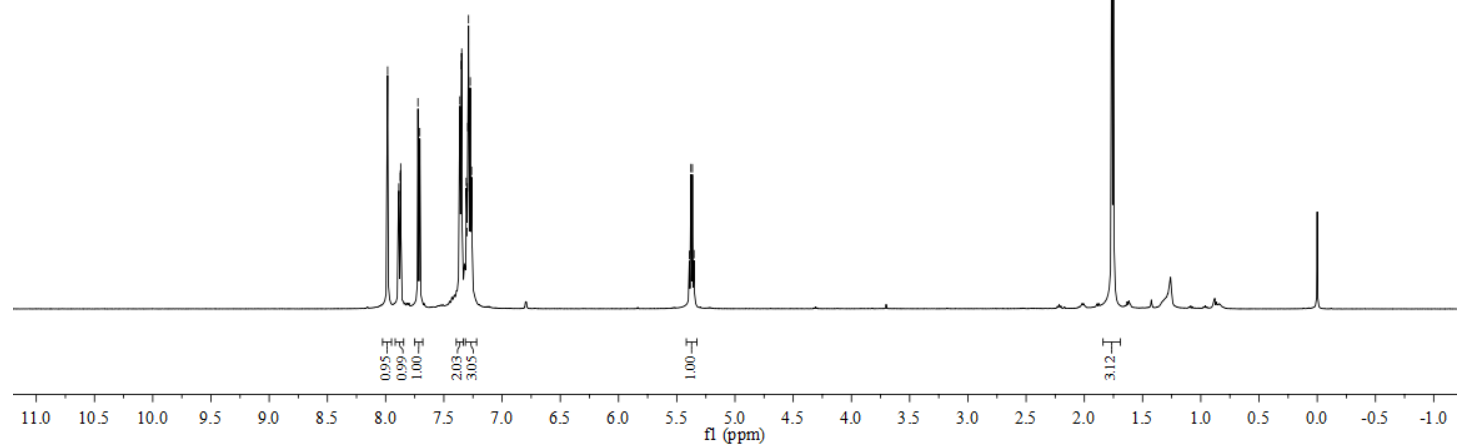

${ }^{13} \mathrm{C}$ NMR spectrum of $\mathbf{3 a c}$
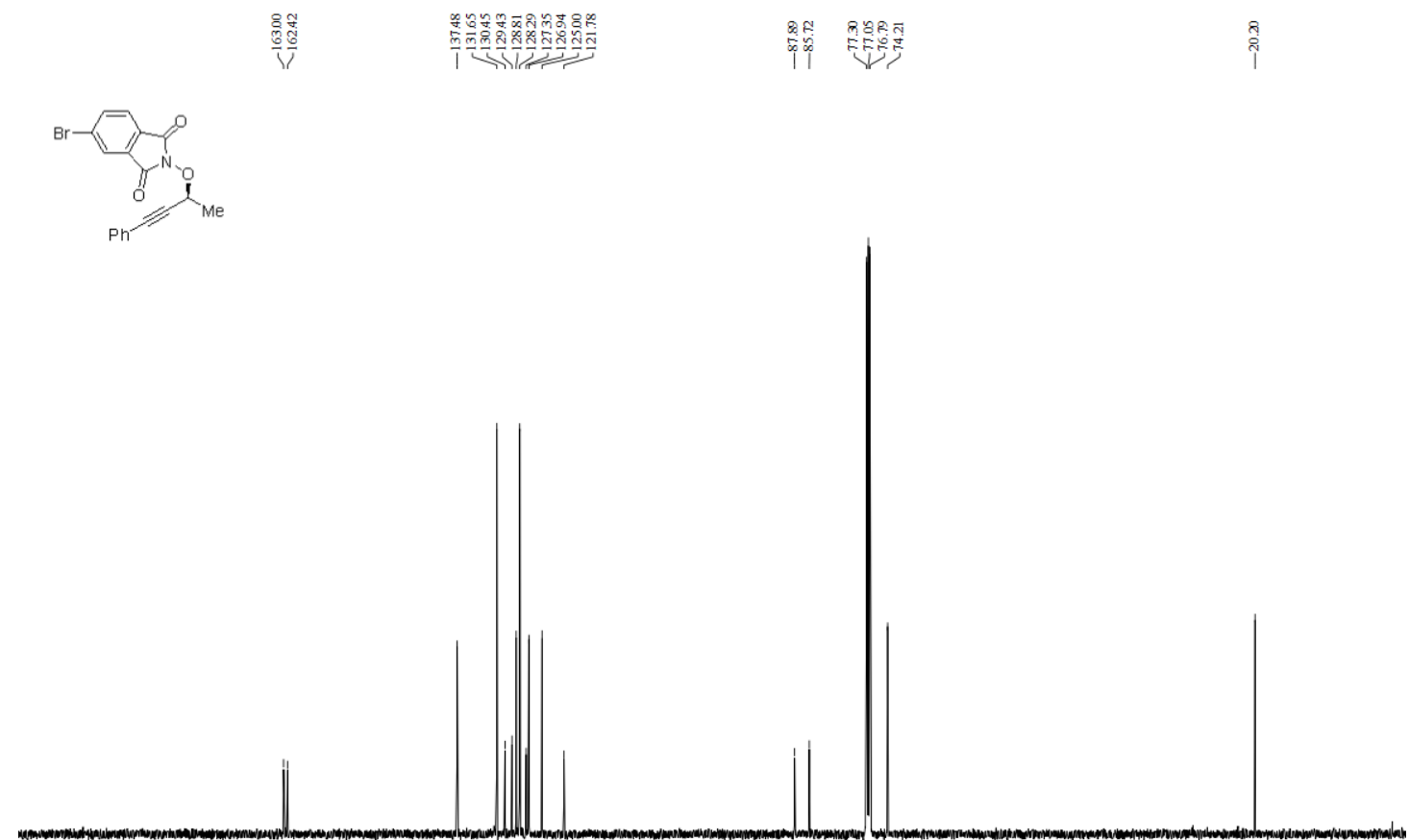

$$
200
$$
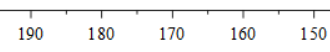

$30 \quad 120 \quad 110 \begin{array}{cc}100 & 1 \\ \mathrm{fl}(\mathrm{ppm}) & 90\end{array}$

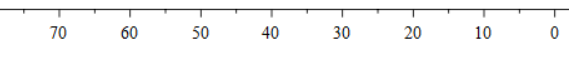


${ }^{1} \mathrm{H}$ NMR spectrum of $\mathbf{3 a d}$

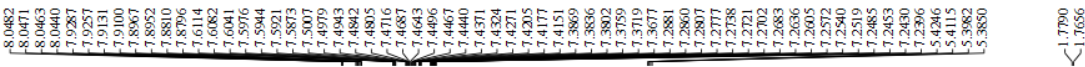

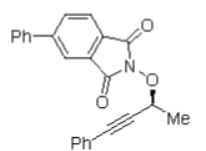

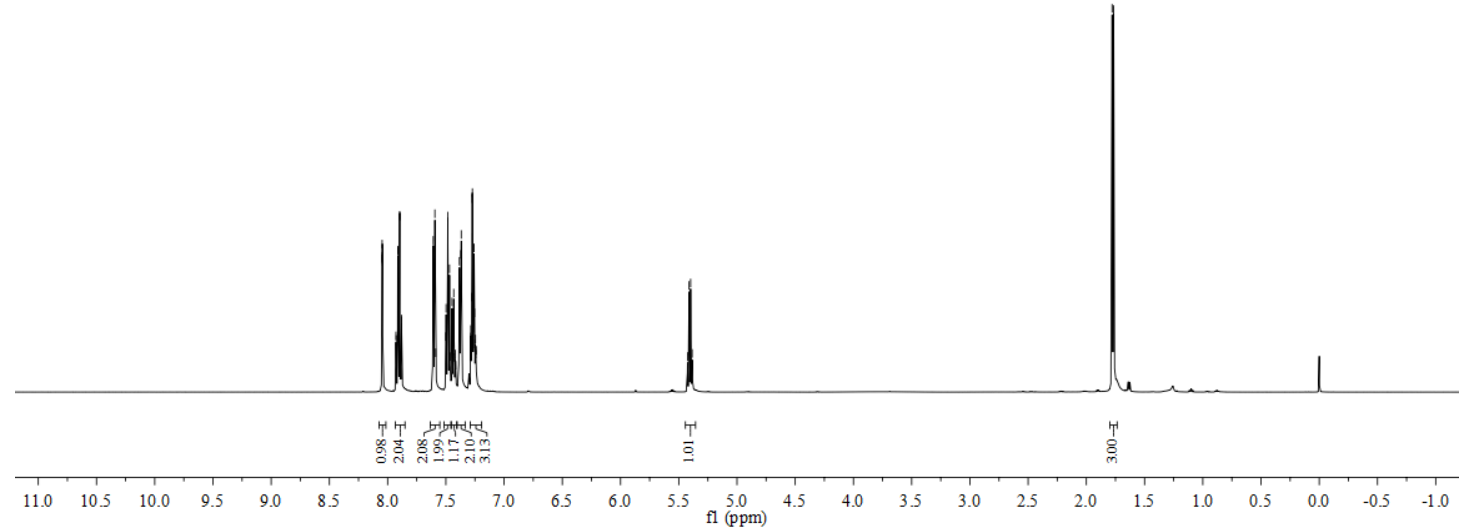

${ }^{13} \mathrm{C}$ NMR spectrum of $\mathbf{3 a d}$
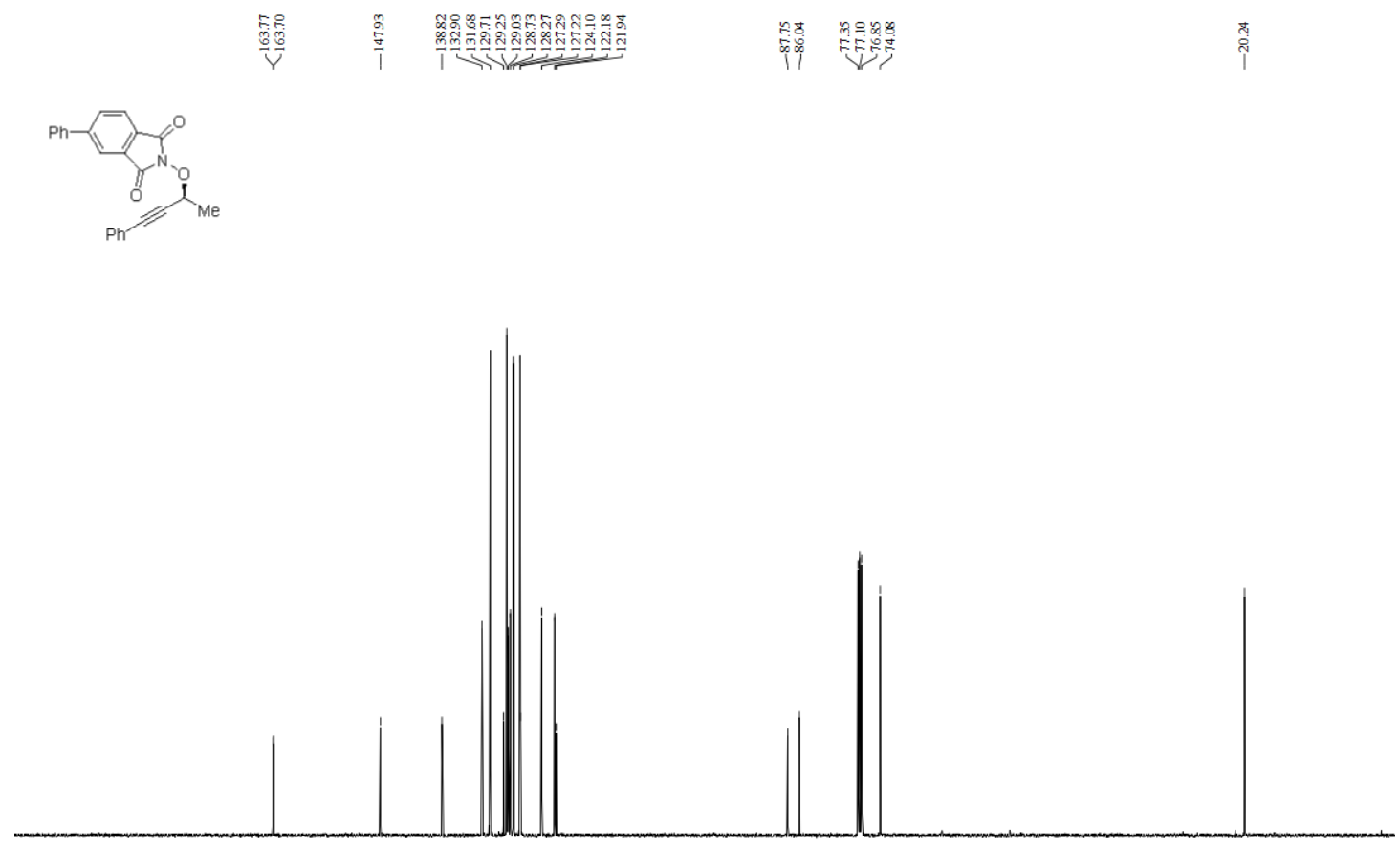

$\begin{array}{lllllllllllllllllllll}1 & 1 & 1 & 1 & 1 & 1 & 1 & 1 & 1 & 1 & 1 & 1 & 1 \\ 200 & 190 & 180 & 170 & 160 & 150 & 140 & 130 & 120 & 110 & \begin{array}{c}100 \\ \mathrm{fl}(\mathrm{ppm})\end{array} & 90 & 80 & 70 & 60 & 50 & 40 & 30 & 20 & 10 & 0\end{array}$ 
${ }^{1} \mathrm{H}$ NMR spectrum of 3ae

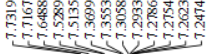

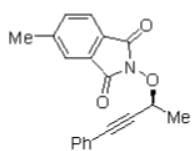

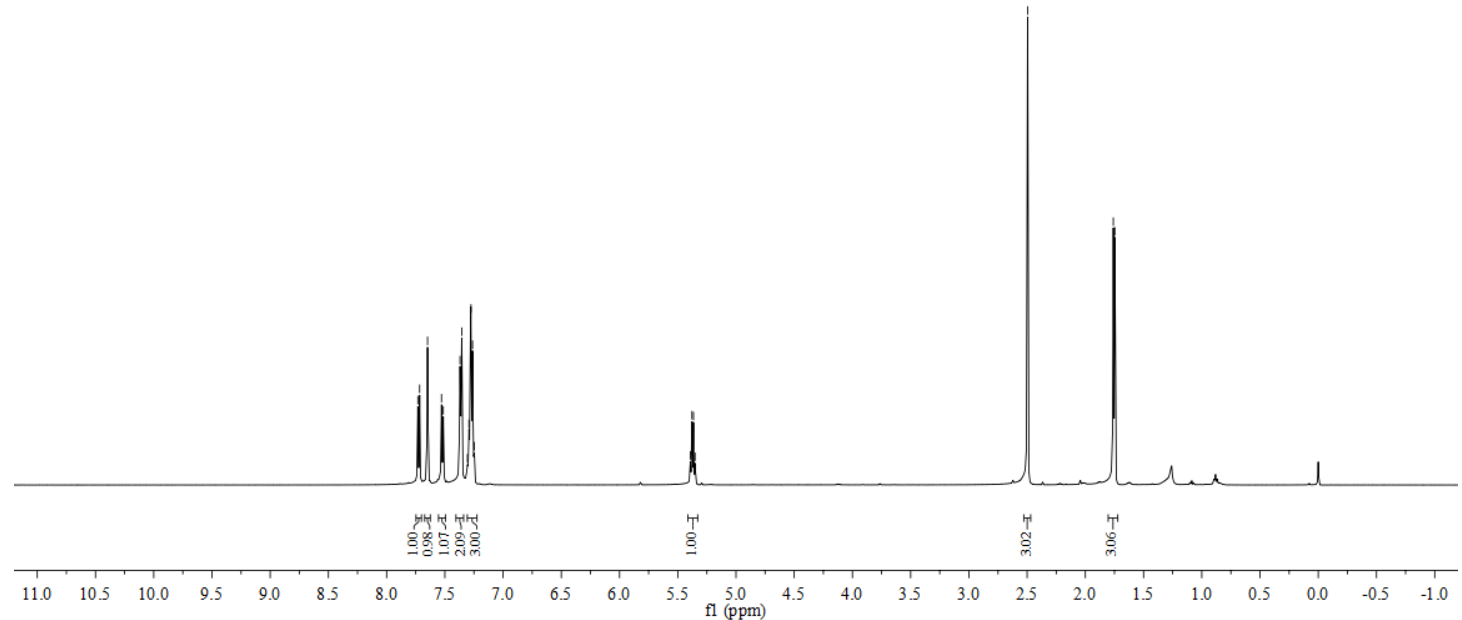

${ }^{13} \mathrm{C}$ NMR spectrum of $\mathbf{3 a e}$

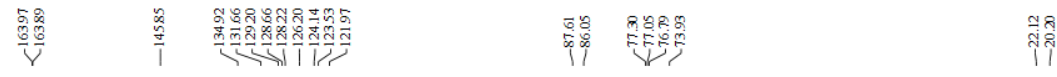

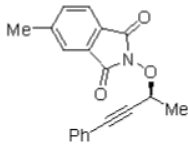
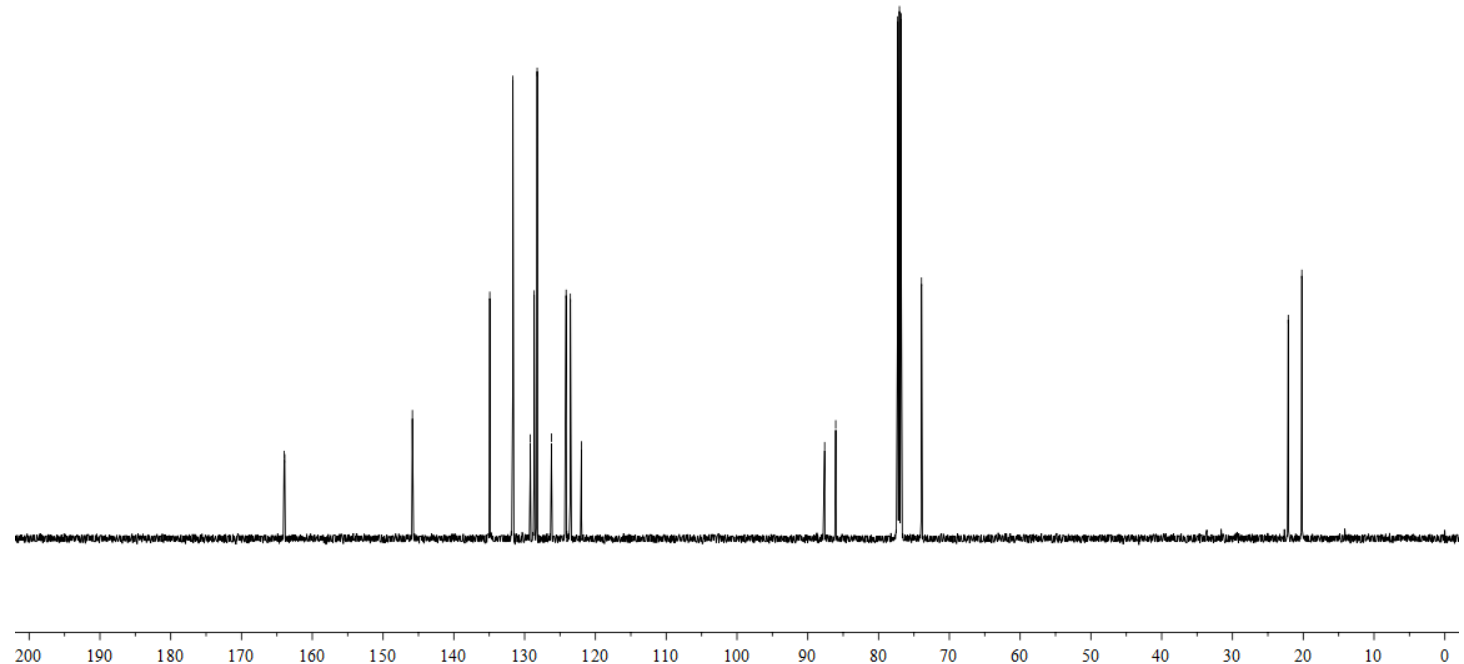

100
$\mathrm{fl}(\mathrm{ppr})$ 
${ }^{1} \mathrm{H}$ NMR spectrum of $\mathbf{3 a f}$

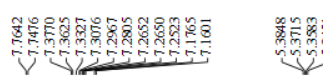

祼
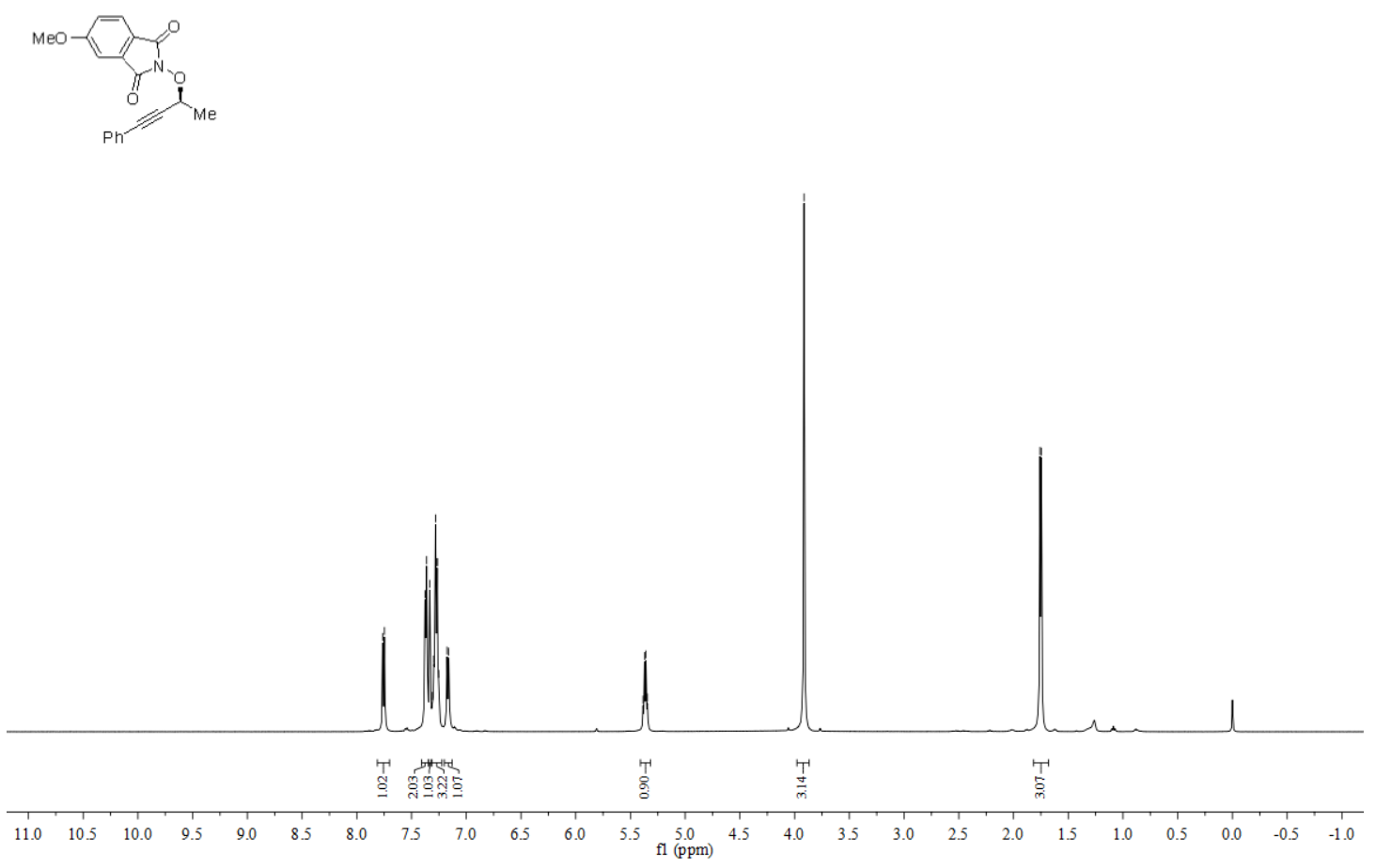

${ }^{13} \mathrm{C}$ NMR spectrum of $\mathbf{3 a f}$

琶鰝

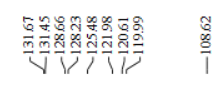

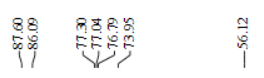

จุ
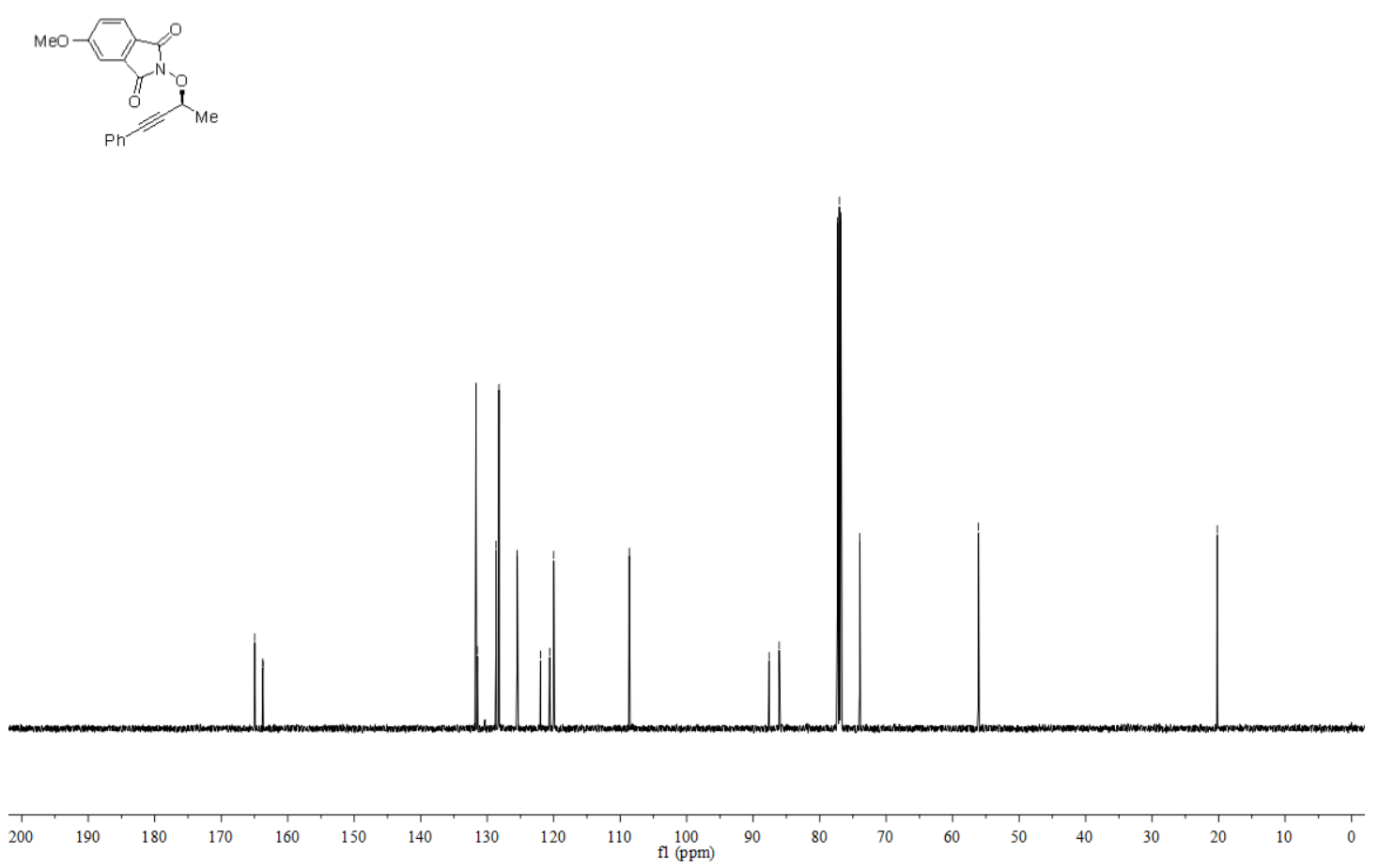

49 
${ }^{1} \mathrm{H}$ NMR spectrum of $\mathbf{3 a g}$

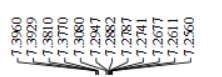

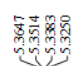

繁
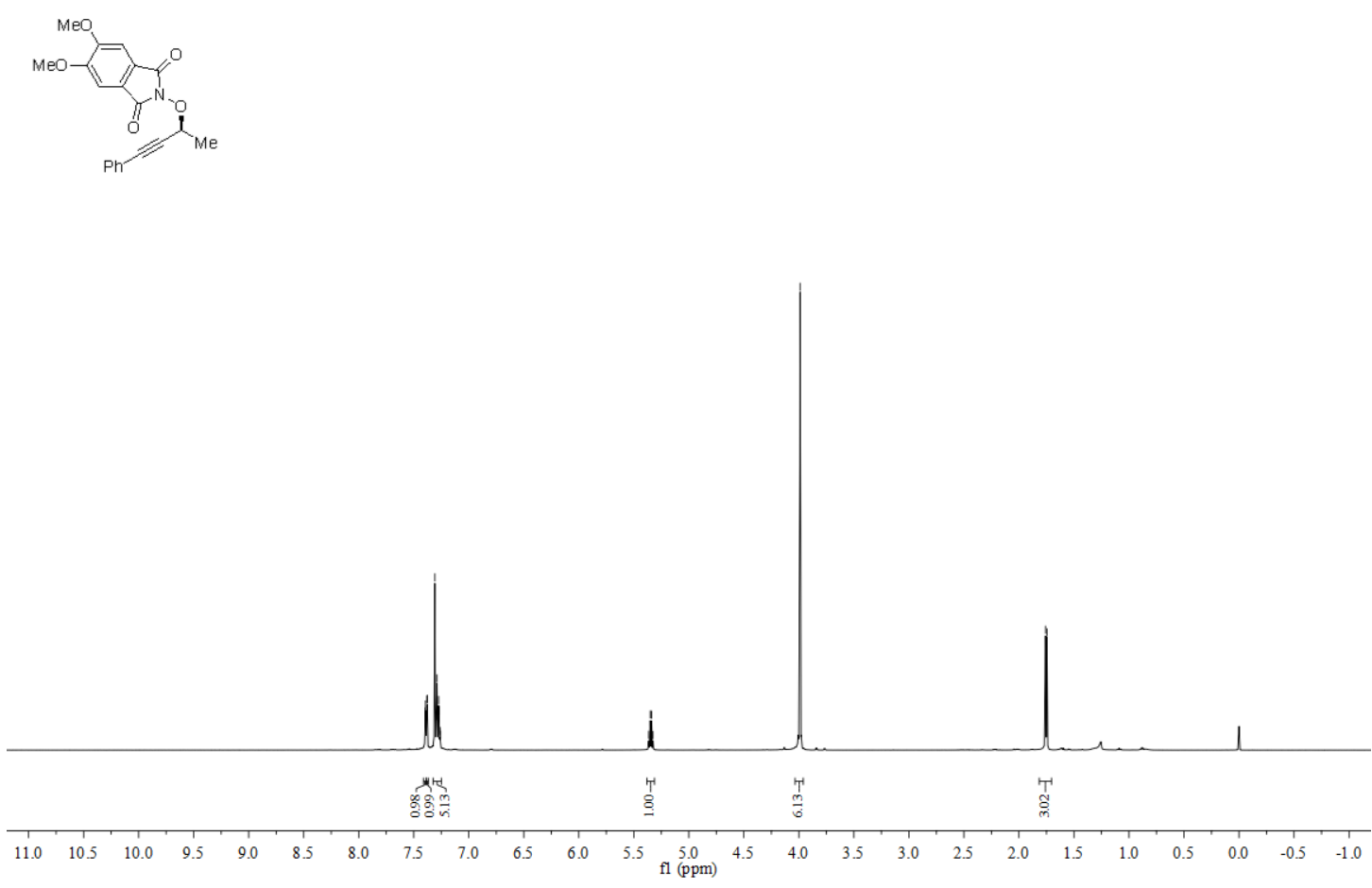

${ }^{13} \mathrm{C}$ NMR spectrum of $\mathbf{3 a g}$
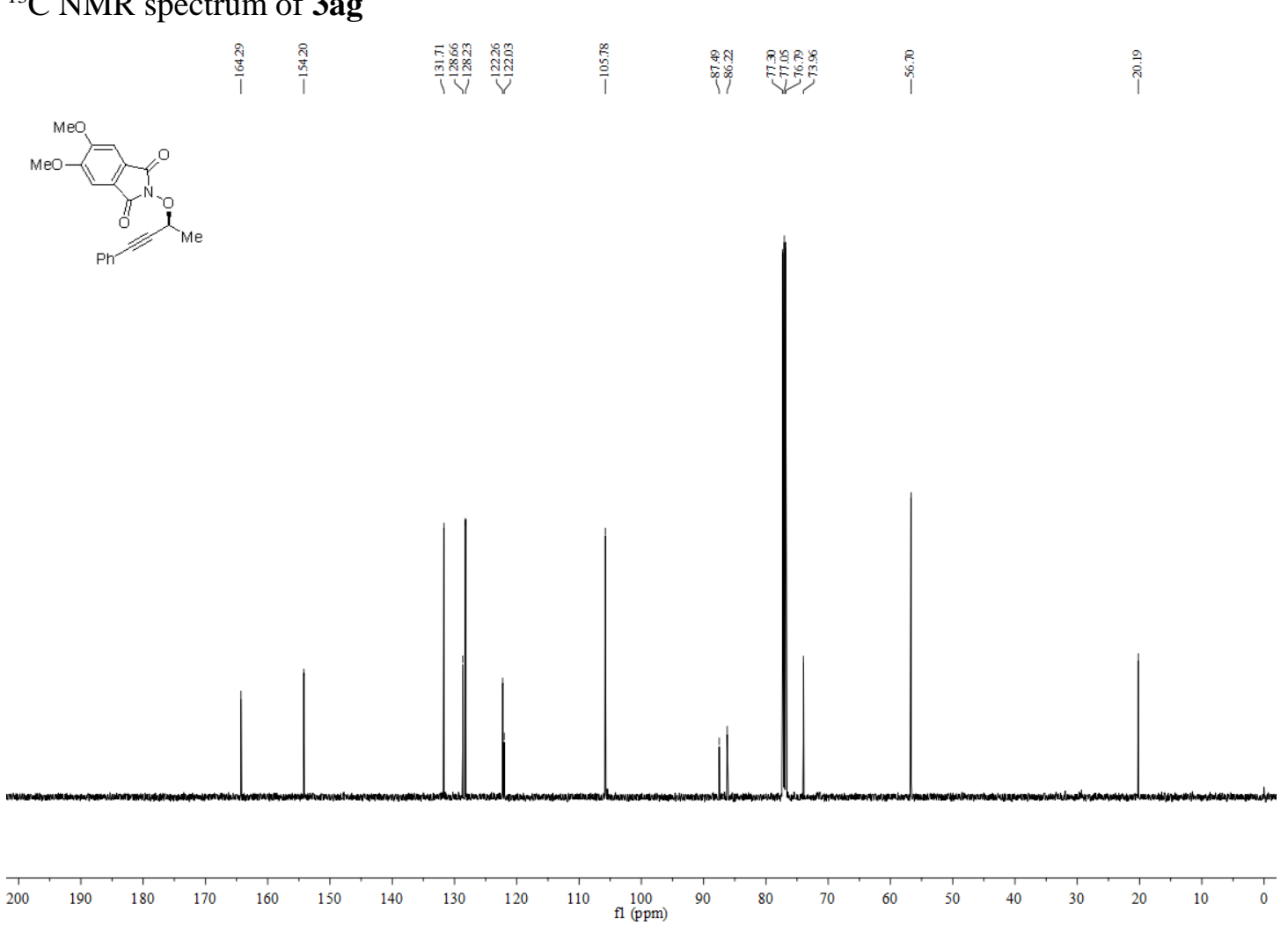

50 
${ }^{1} \mathrm{H}$ NMR spectrum of $\mathbf{3 a h}$

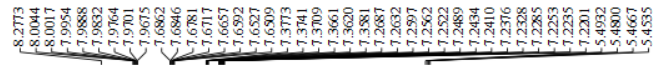
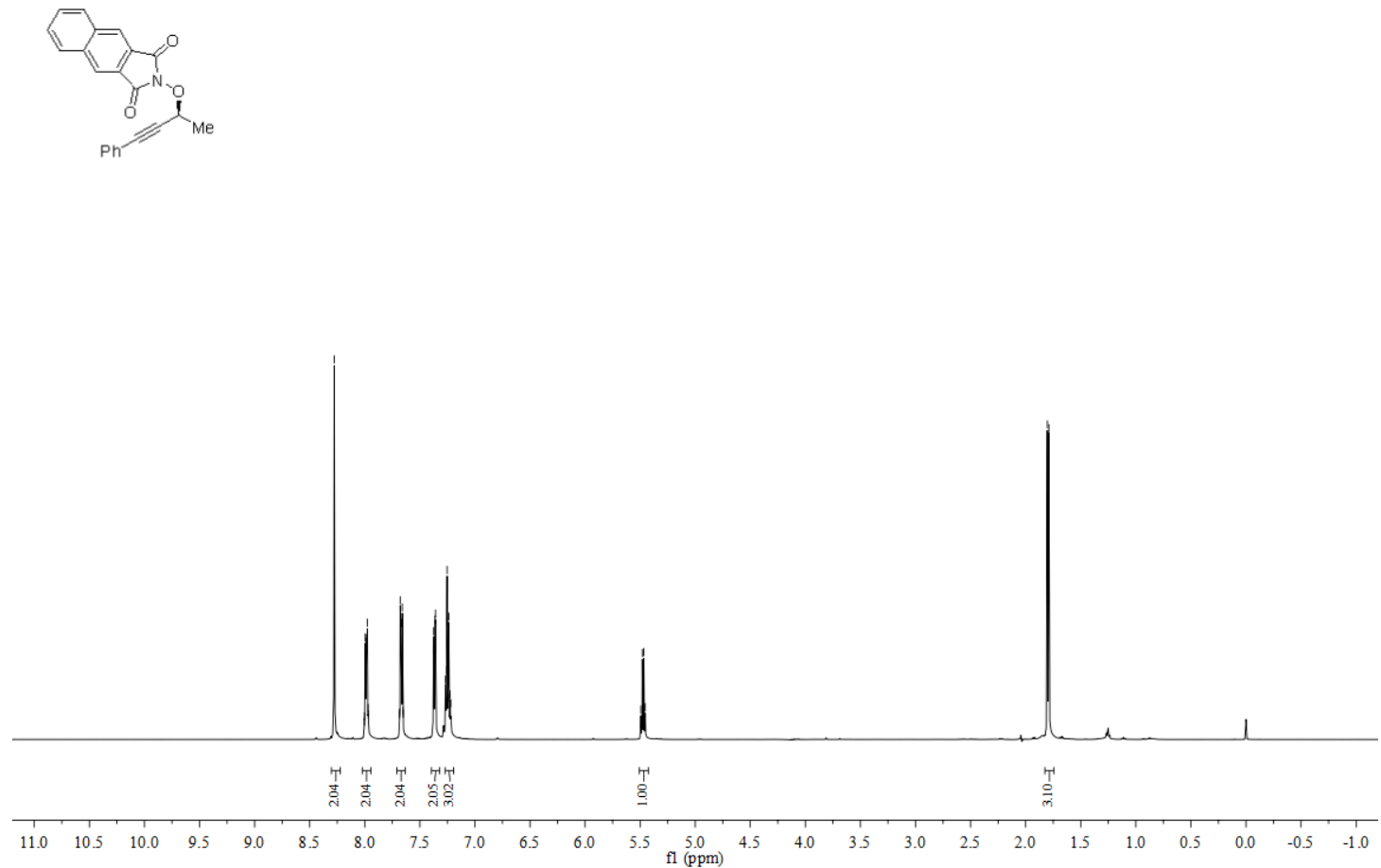

${ }^{13} \mathrm{C}$ NMR spectrum of $\mathbf{3 a h}$
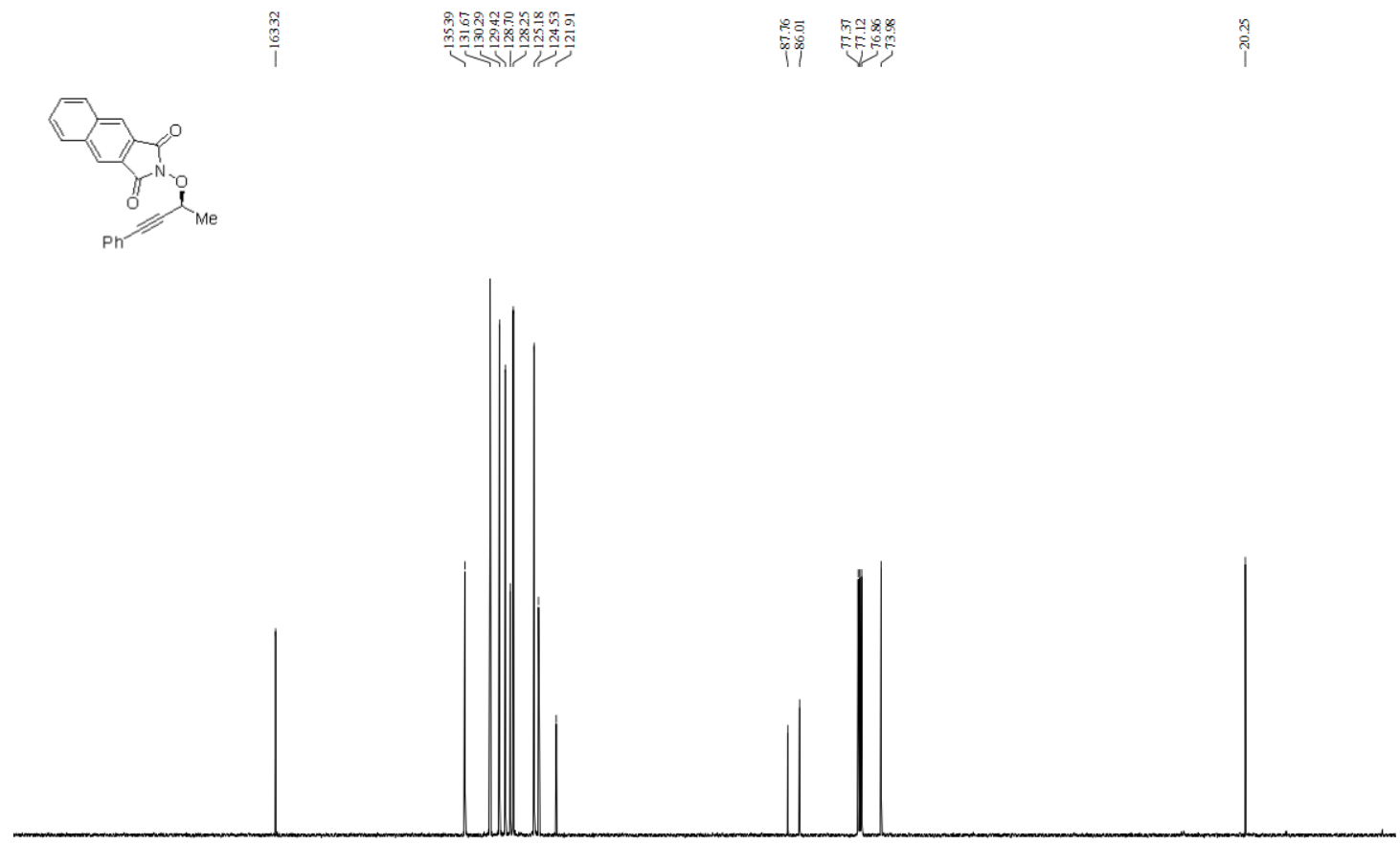

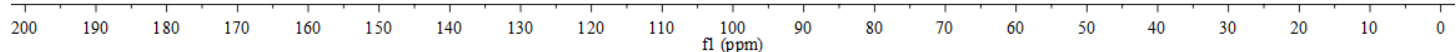


${ }^{1} \mathrm{H}$ NMR spectrum of $\mathbf{3 b f}$

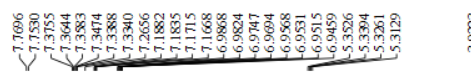

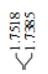
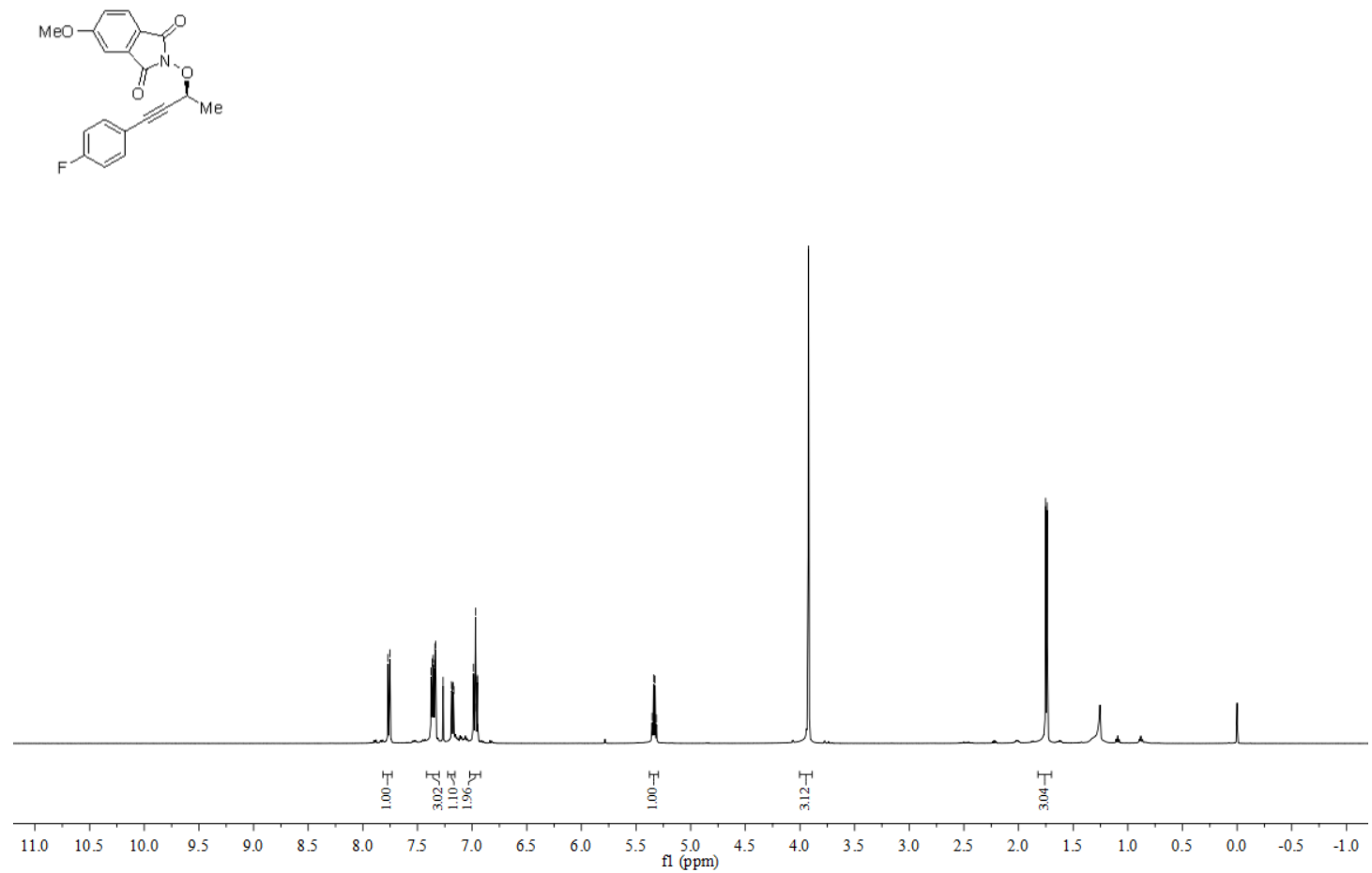

${ }^{13} \mathrm{C}$ NMR spectrum of $\mathbf{3 b f}$

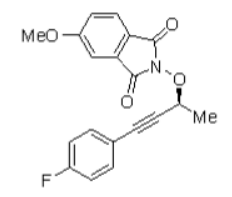

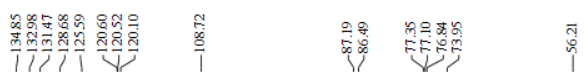

ำำ

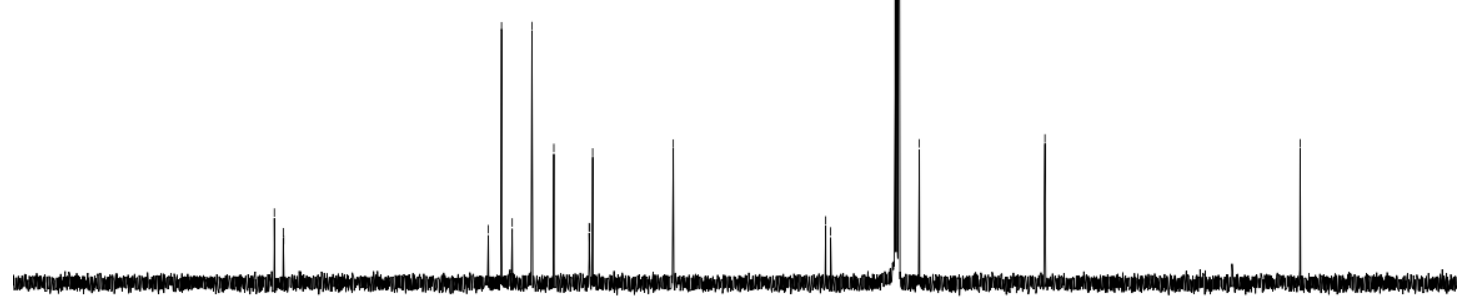

200

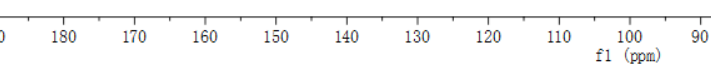

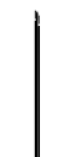


${ }^{1} \mathrm{H}$ NMR spectrum of $\mathbf{3 c f}$

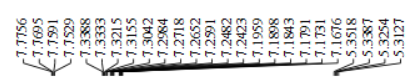

$\frac{2}{\mathrm{~V}}$

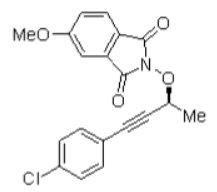

疍

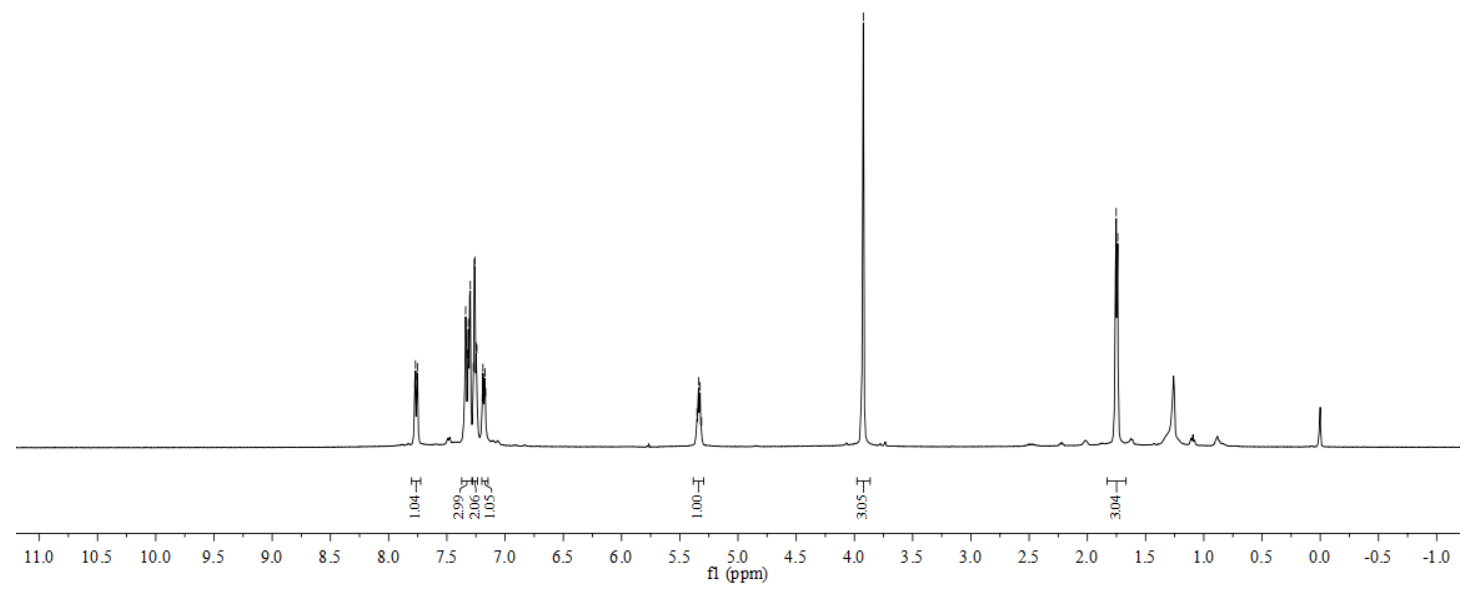

${ }^{13} \mathrm{C}$ NMR spectrum of $\mathbf{3 c f}$

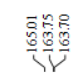

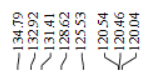

0.0
$\stackrel{0}{1}$

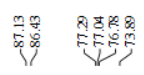

$\stackrel{n}{0}$

$\stackrel{9}{\stackrel{1}{1}}$
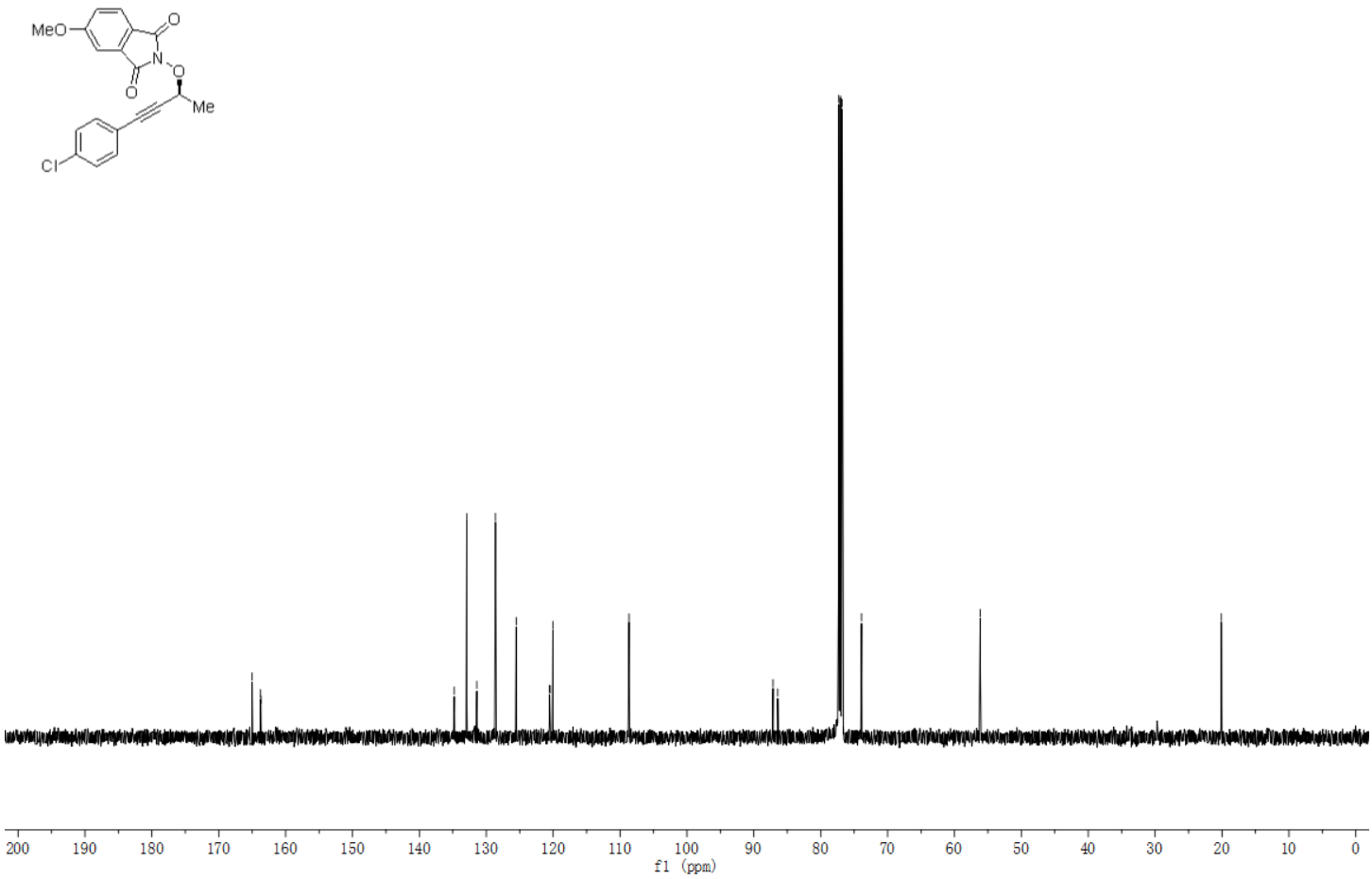

53 
${ }^{1} \mathrm{H}$ NMR spectrum of $\mathbf{3 d f}$

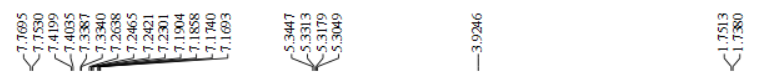
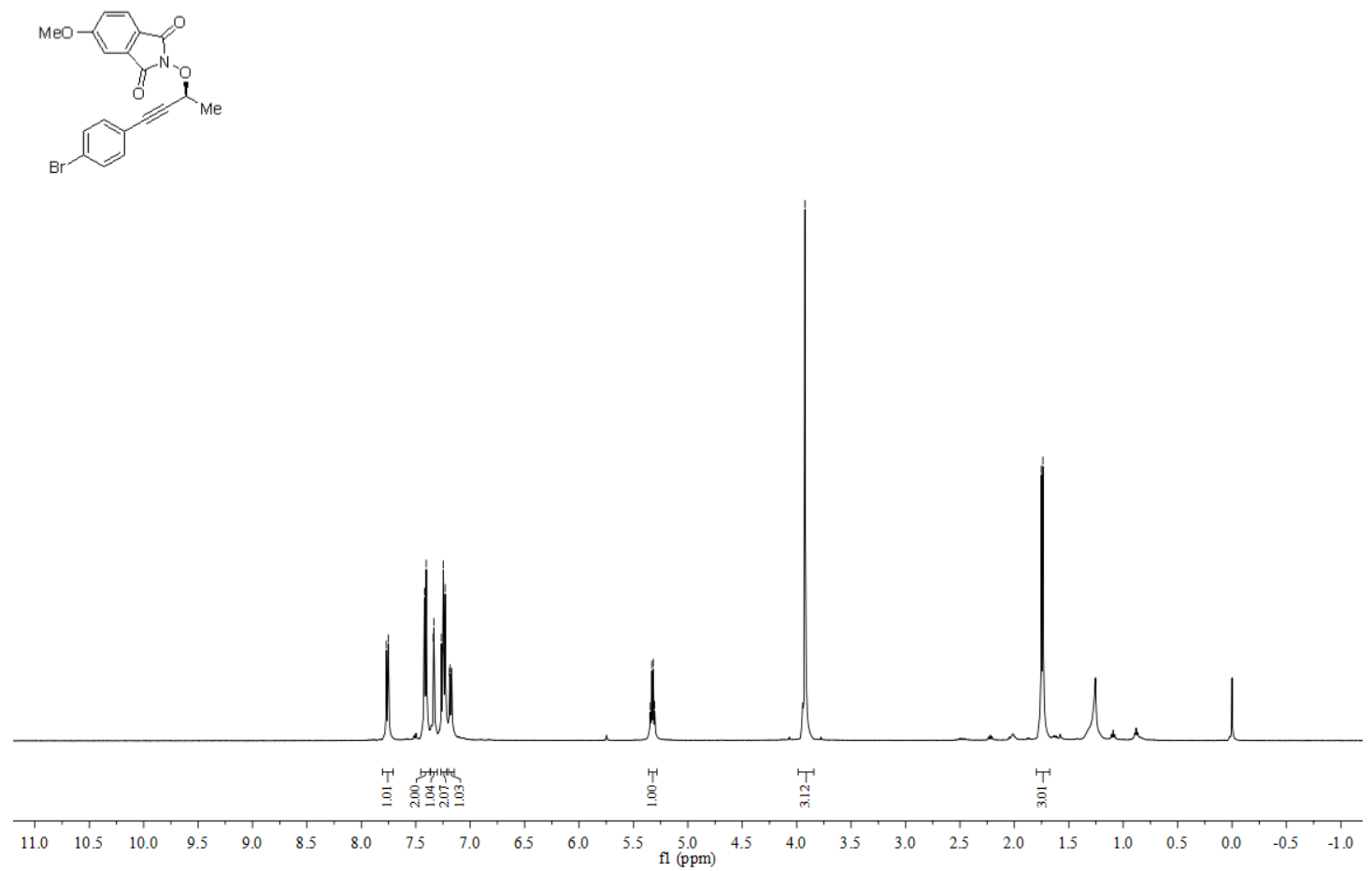

${ }^{13} \mathrm{C}$ NMR spectrum of $\mathbf{3 d f}$
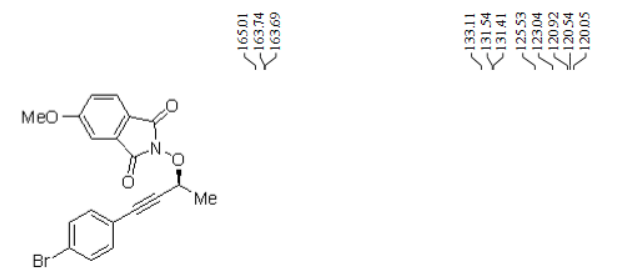

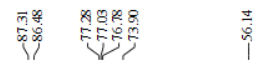

iิ
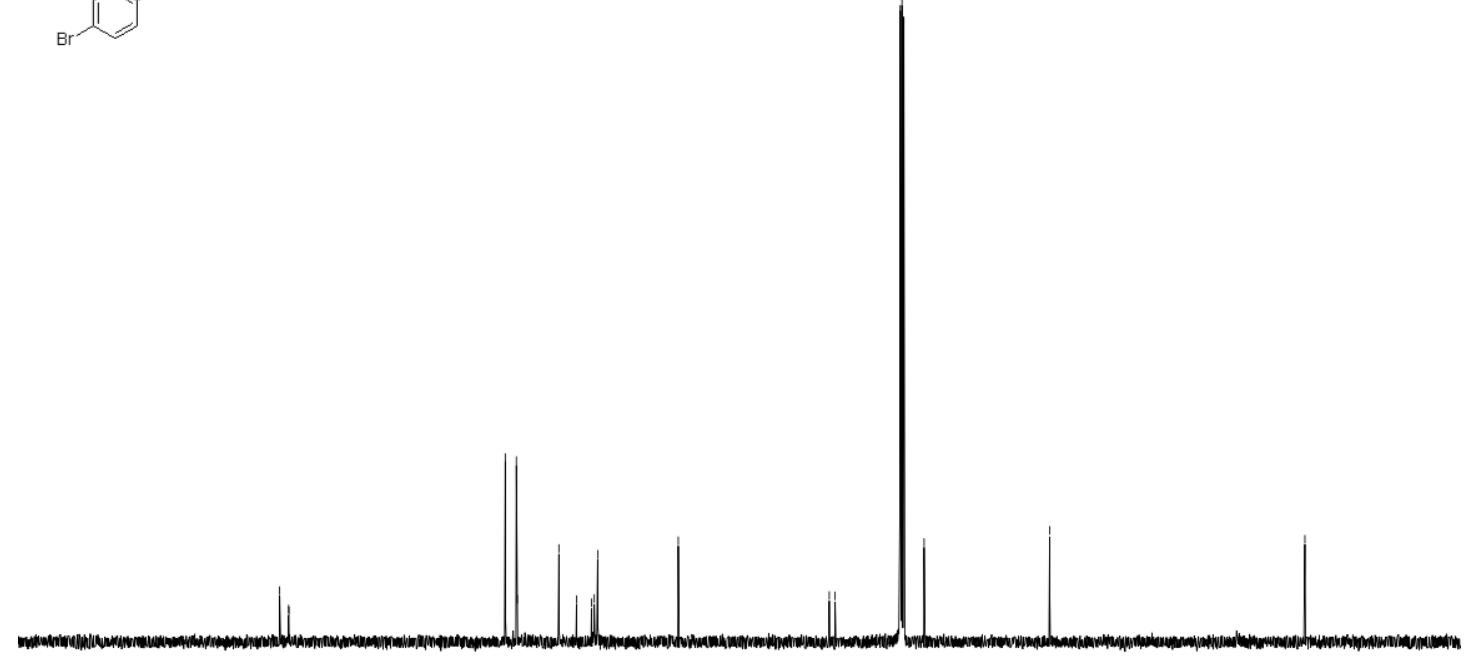

200

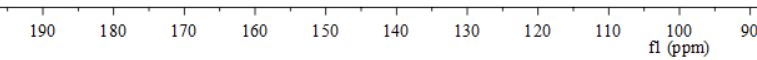


${ }^{1} \mathrm{H}$ NMR spectrum of $3 \mathbf{e f}$
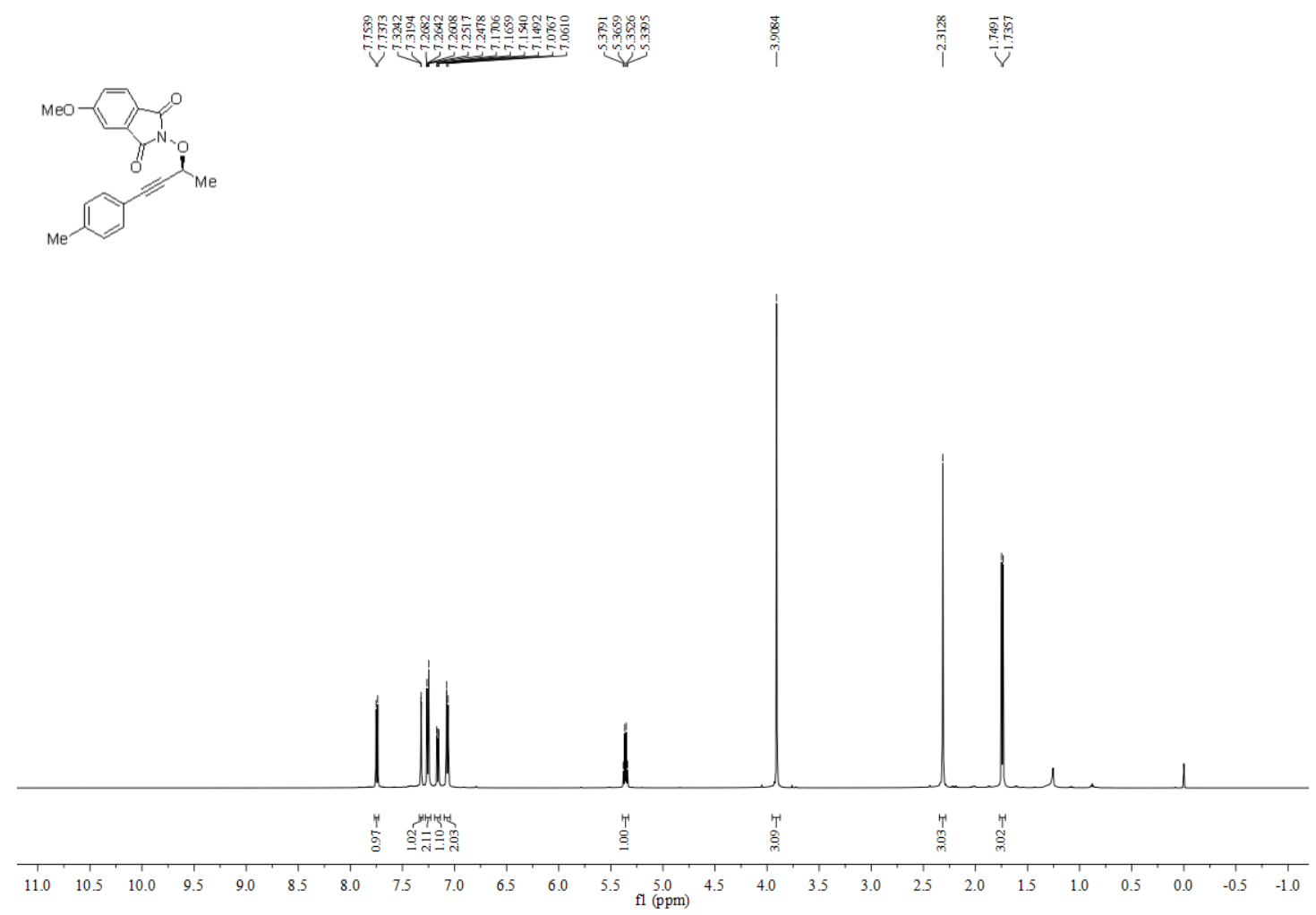

${ }^{13} \mathrm{C}$ NMR spectrum of $3 \mathbf{e f}$
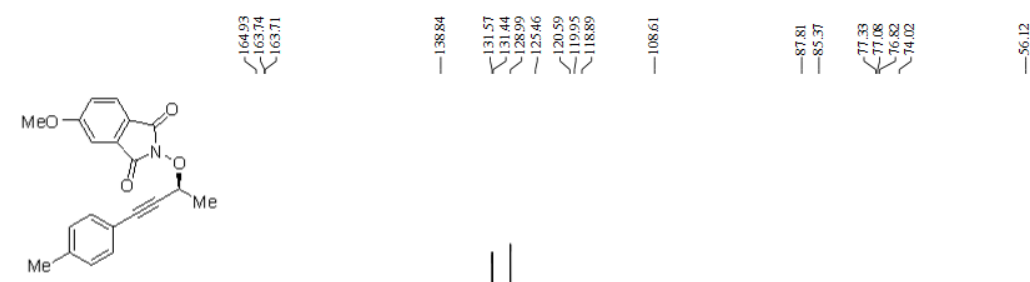

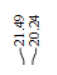

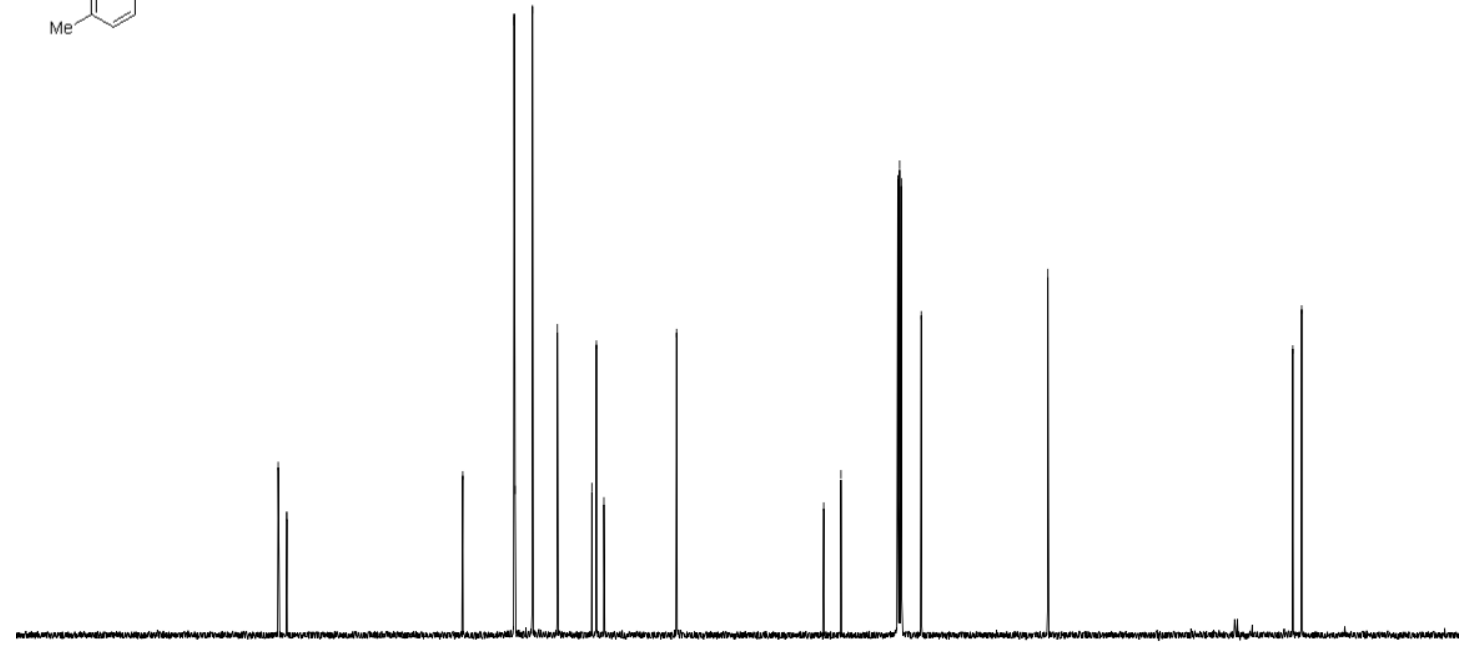

200
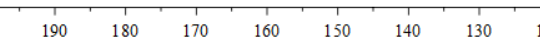

$10 \quad \begin{gathered}100 \\ \mathrm{fl}(\mathrm{ppm})\end{gathered}$ 
${ }^{1} \mathrm{H}$ NMR spectrum of $\mathbf{3 f f}$

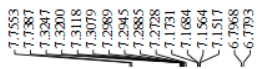

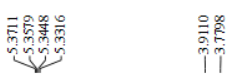

$\frac{\infty}{200}$
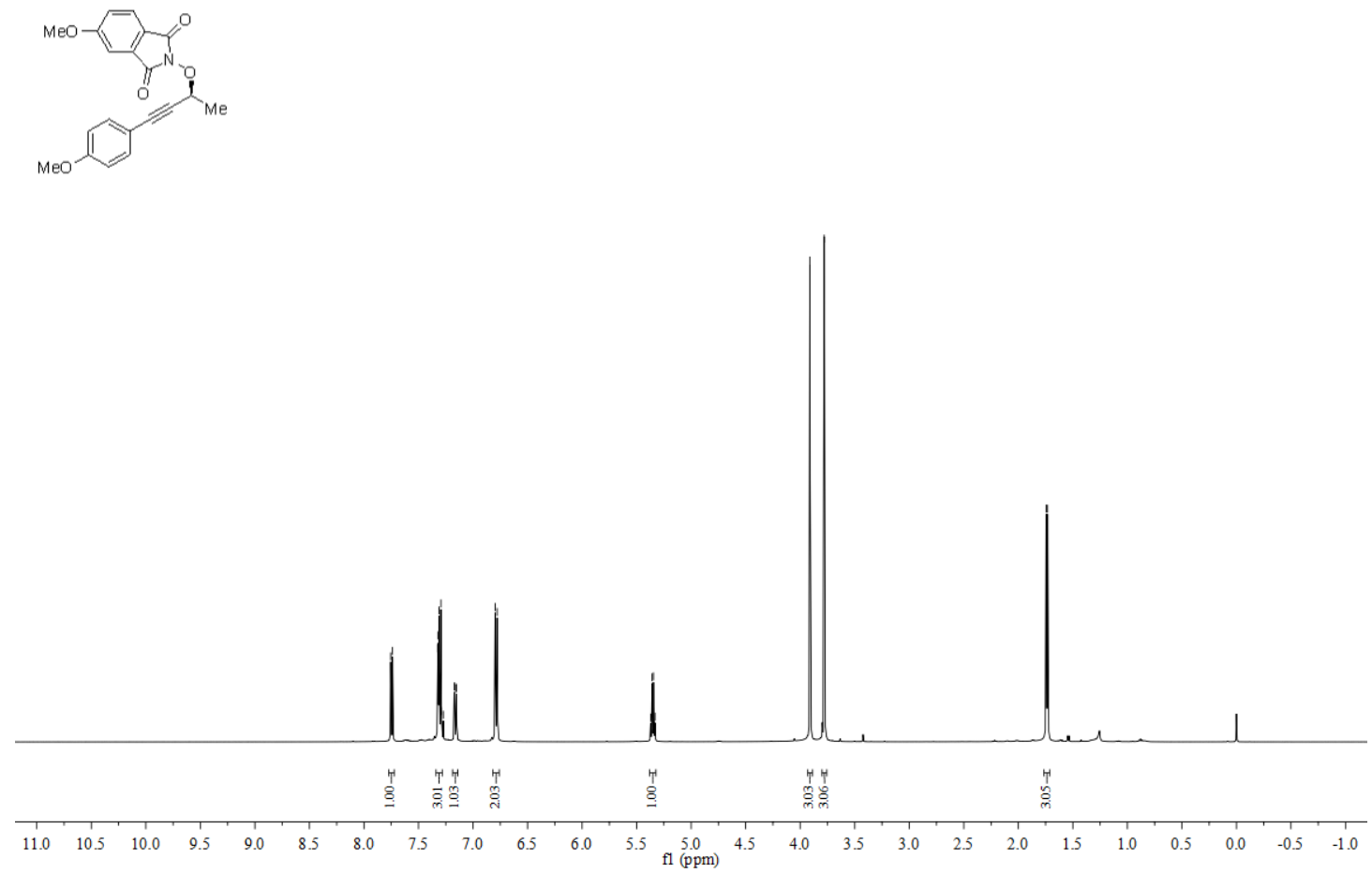

${ }^{13} \mathrm{C}$ NMR spectrum of $\mathbf{3 f f}$
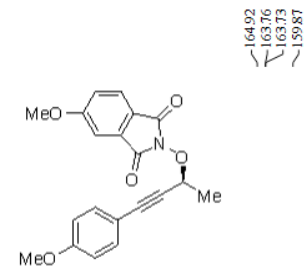

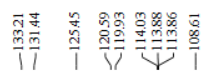

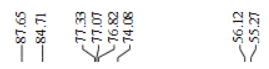

ㅇำ

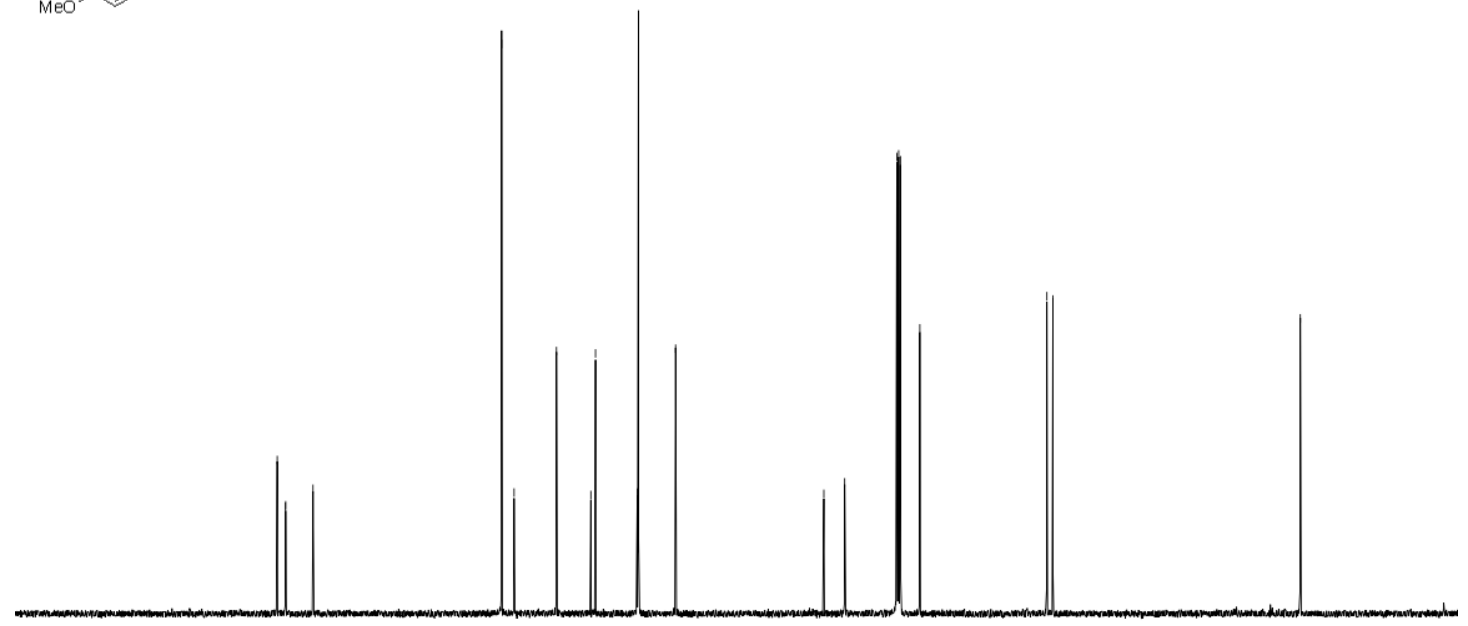

200
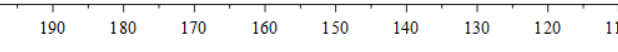

100
$\mathrm{f}(\mathrm{ppm})$ 
${ }^{1} \mathrm{H}$ NMR spectrum of $\mathbf{3 g f}$

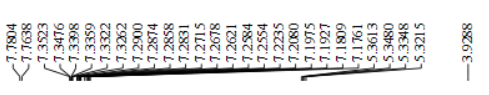

袈
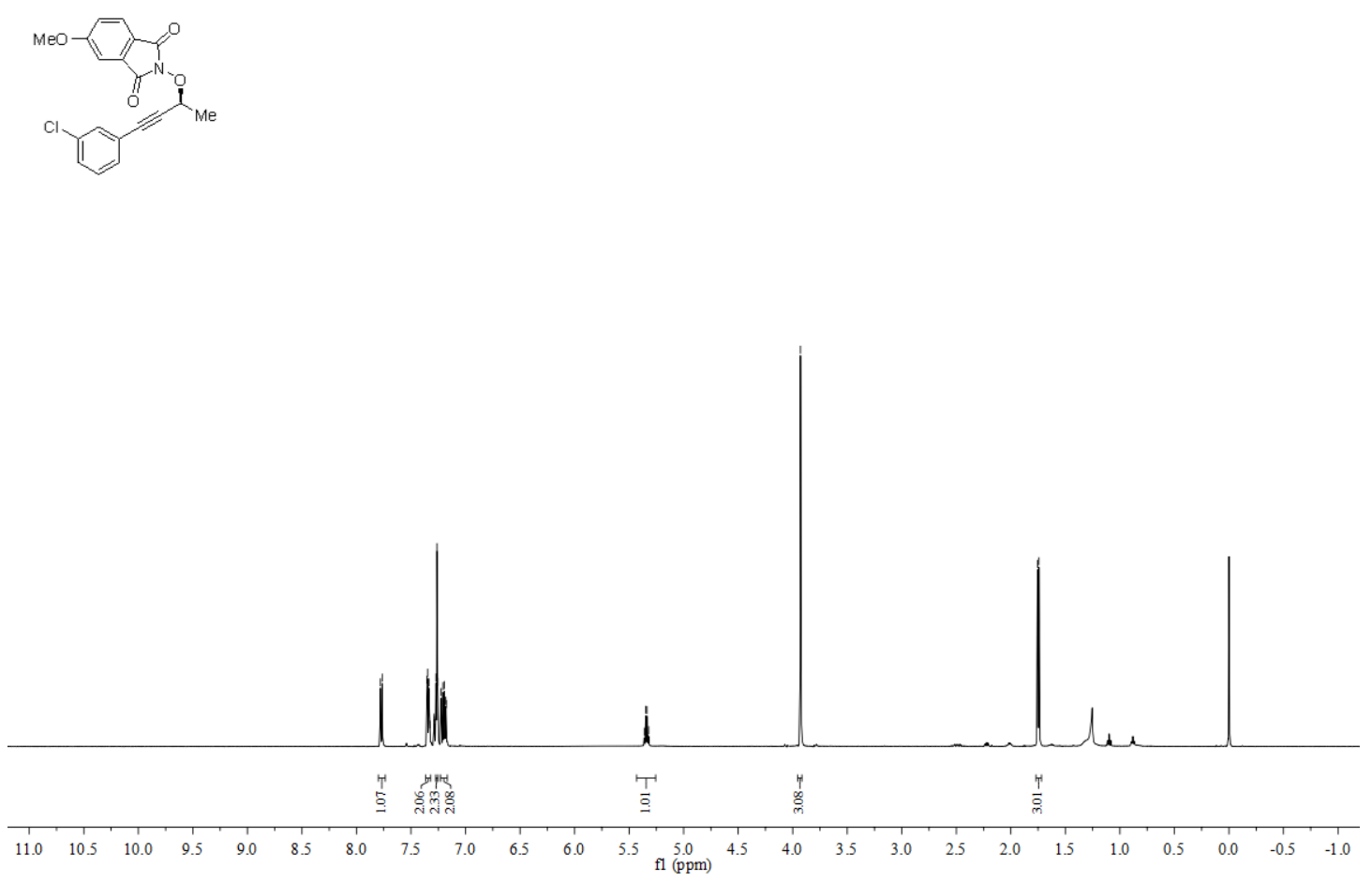

${ }^{13} \mathrm{C}$ NMR spectrum of $\mathbf{3 g f}$

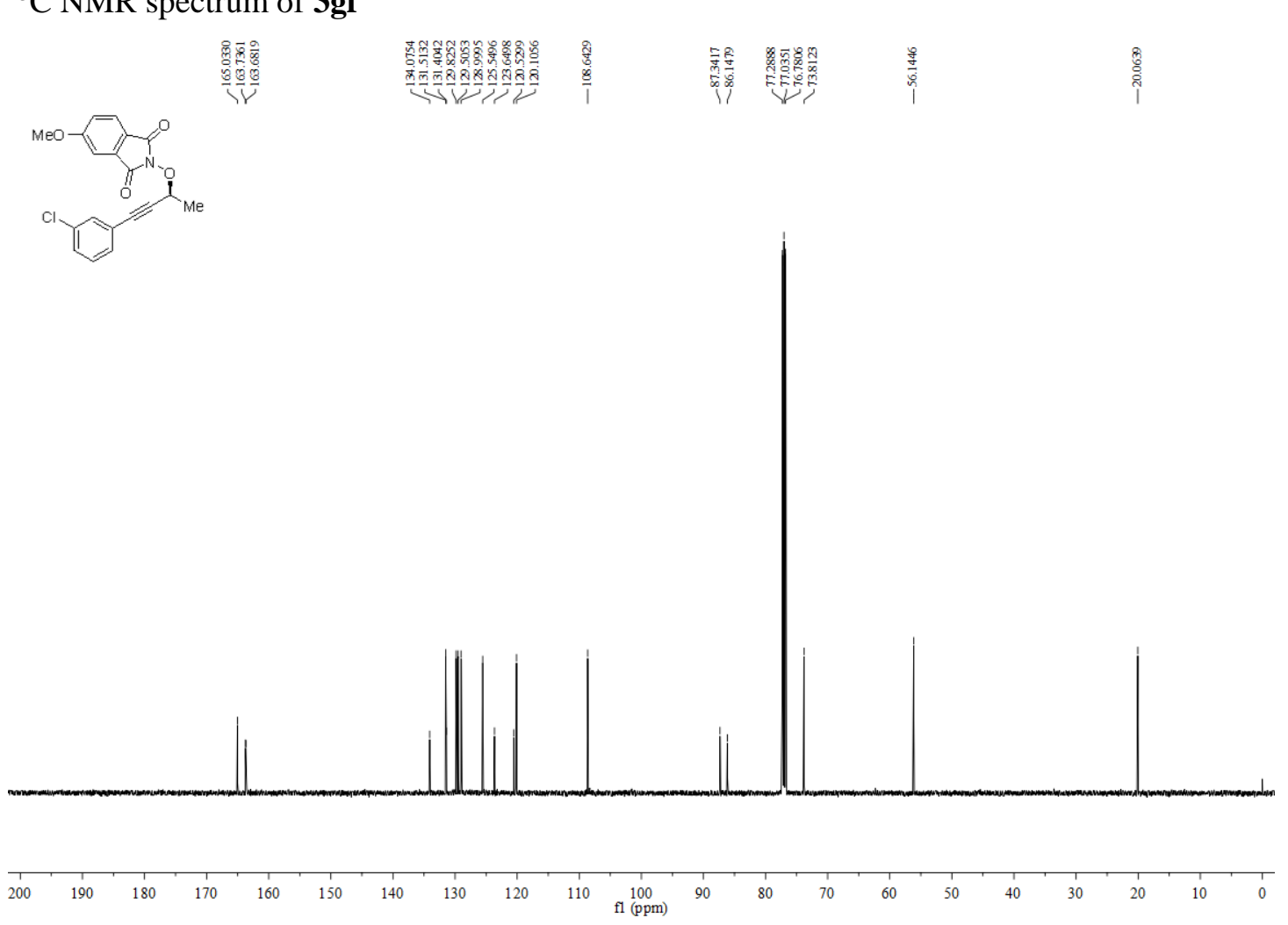

57 
${ }^{1} \mathrm{H}$ NMR spectrum of $\mathbf{3 h f}$

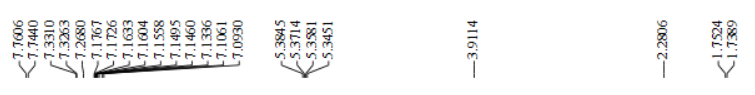
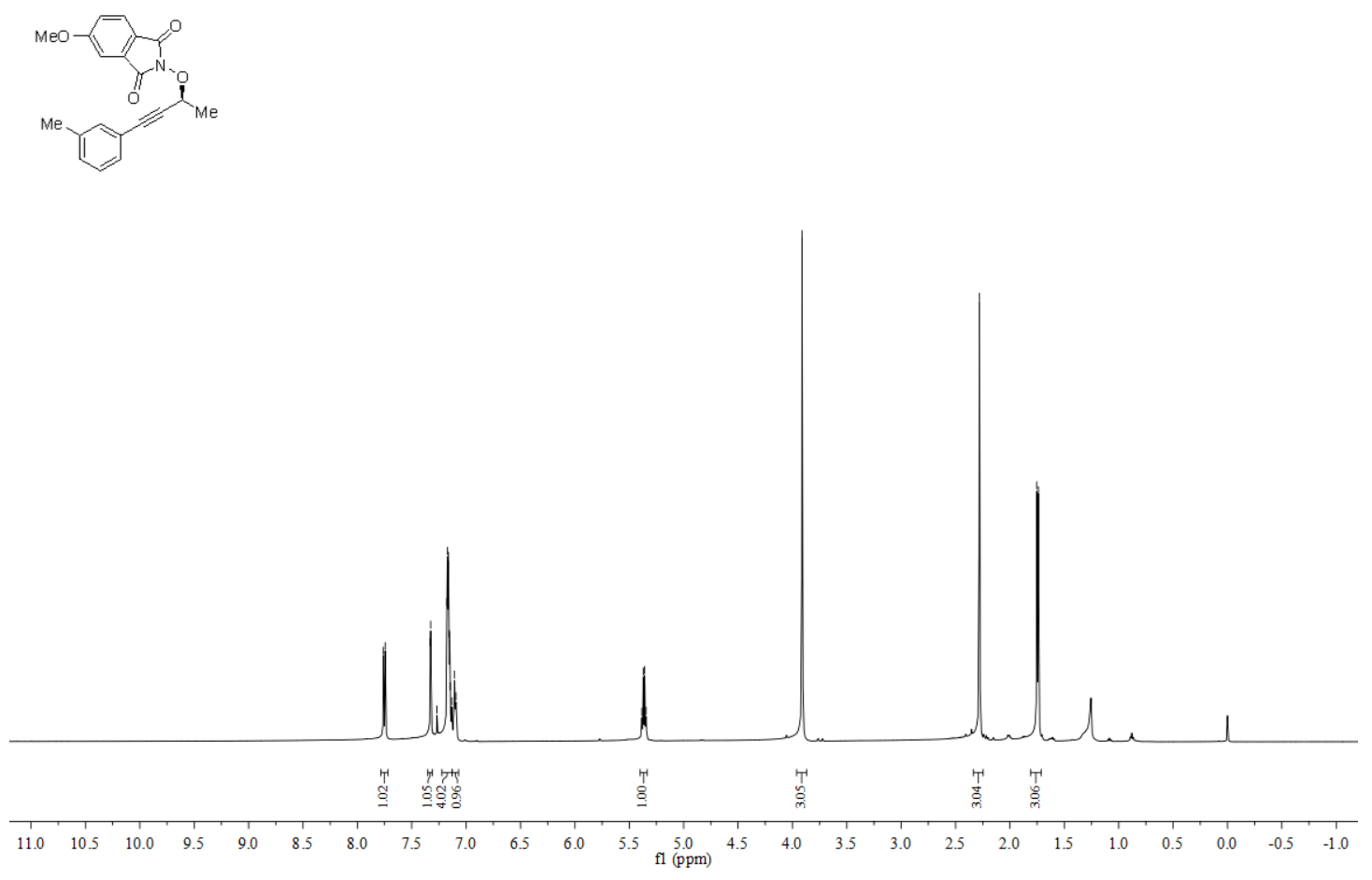

${ }^{13} \mathrm{C}$ NMR spectrum of $\mathbf{3 h f}$
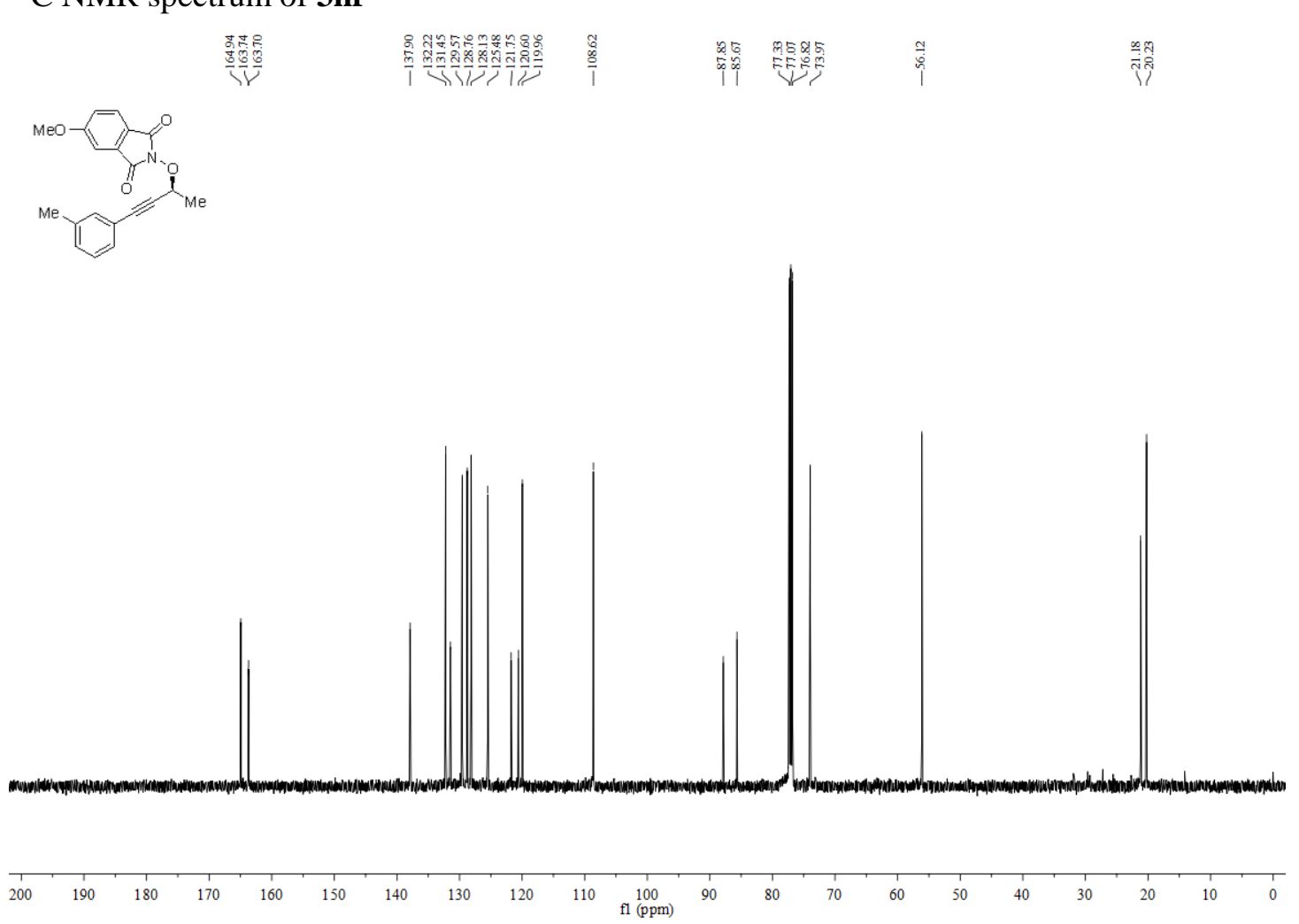

58 
${ }^{1} \mathrm{H}$ NMR spectrum of $\mathbf{3 i f}$

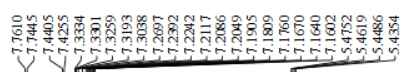

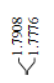
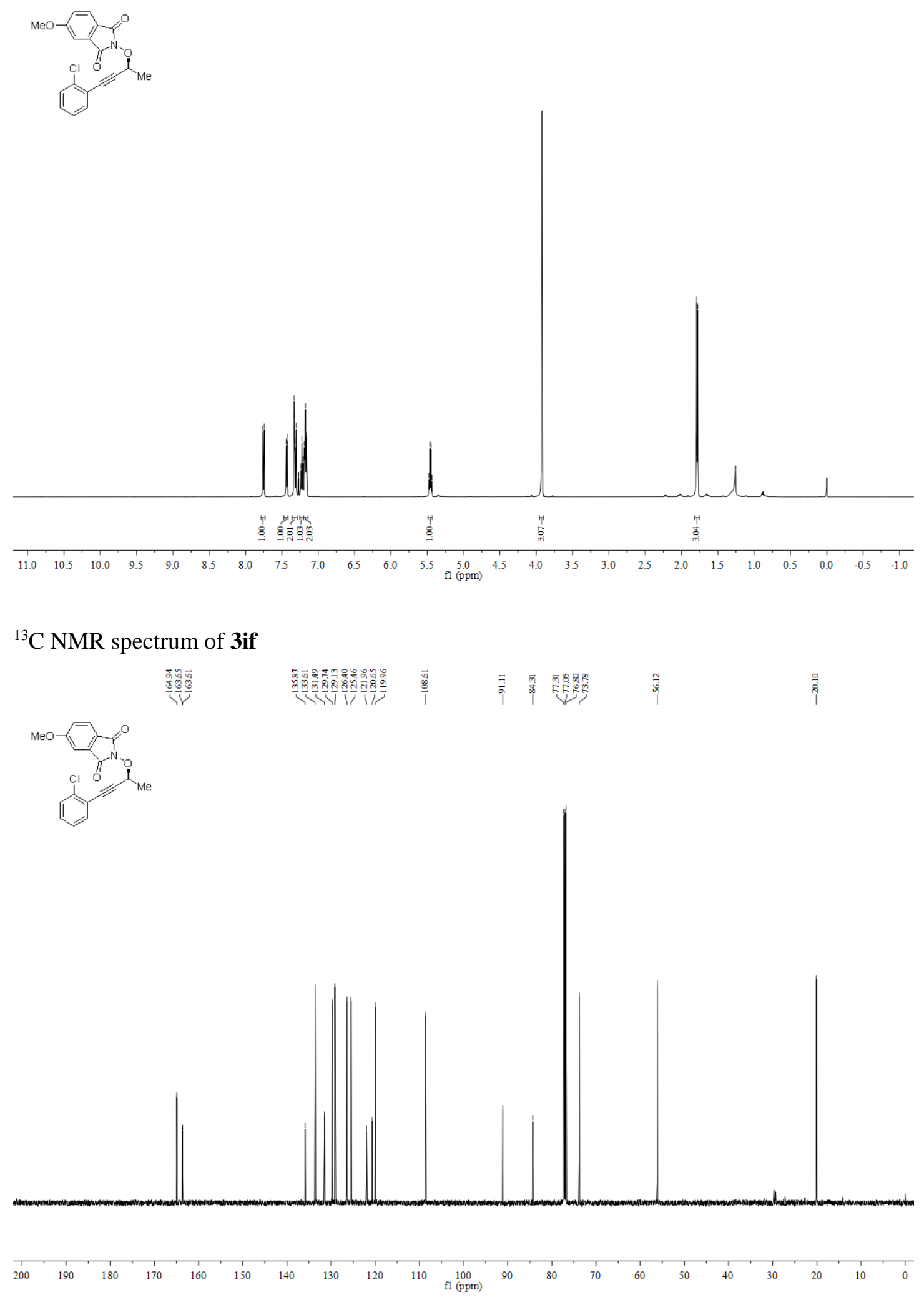

59 
${ }^{1} \mathrm{H}$ NMR spectrum of $\mathbf{3 j f}$

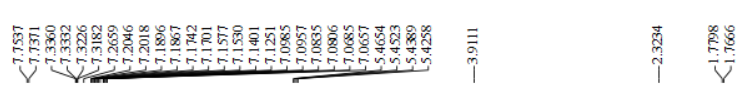
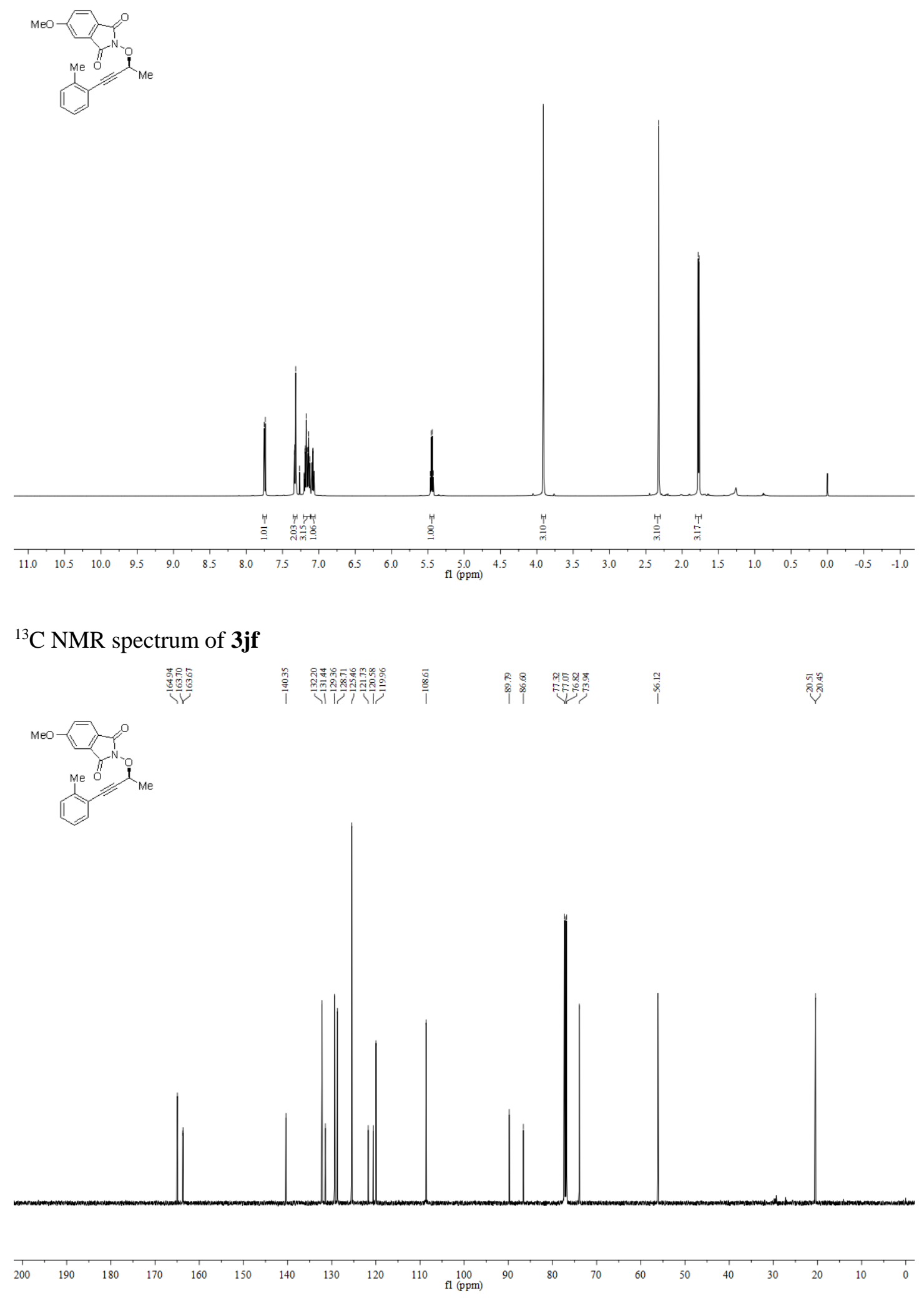

60 
${ }^{1} \mathrm{H}$ NMR spectrum of $\mathbf{3 k f}$

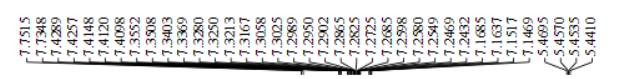

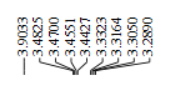
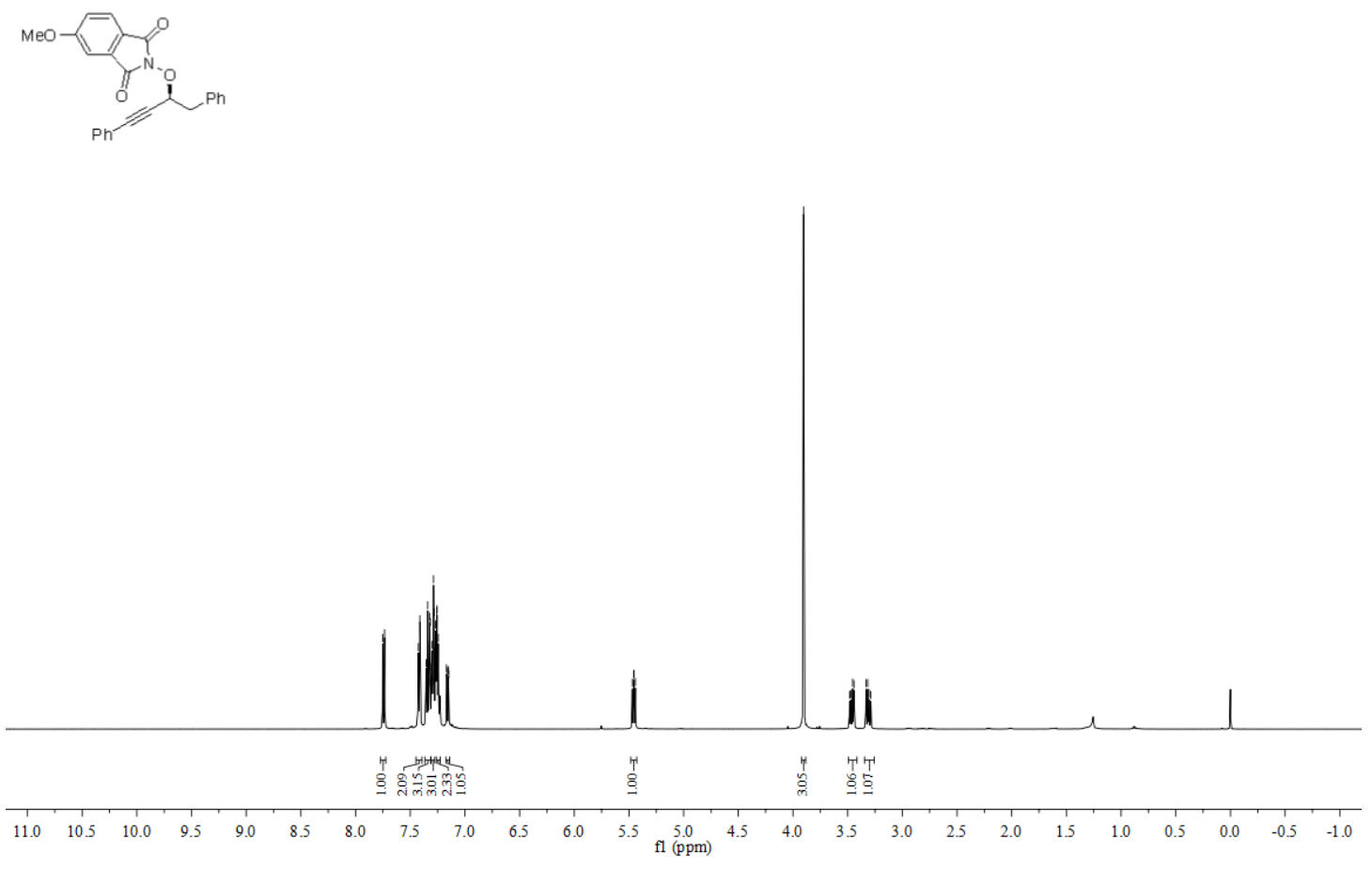

${ }^{13} \mathrm{C}$ NMR spectrum of $\mathbf{3 k f}$
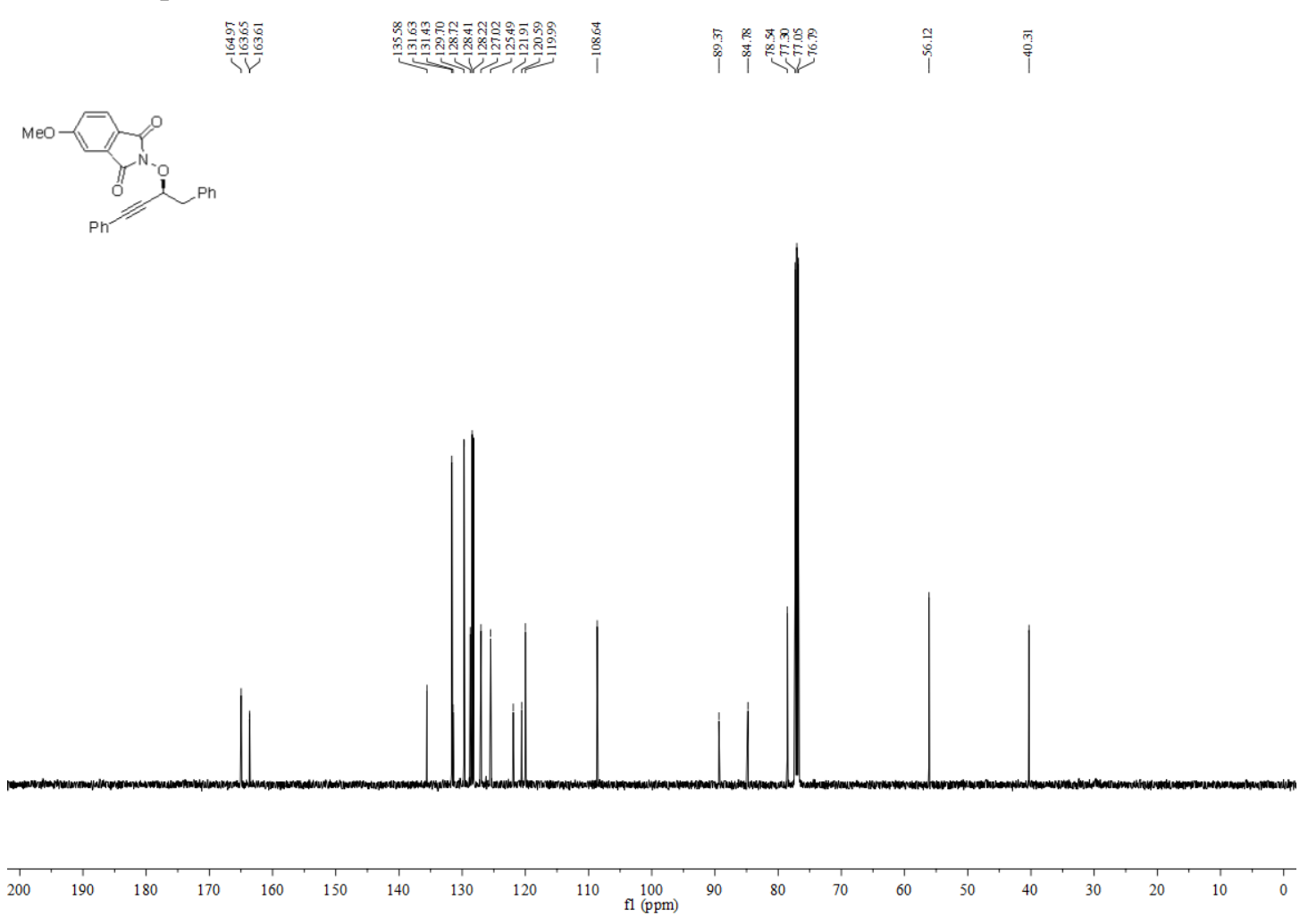

61 
${ }^{1} \mathrm{H}$ NMR spectrum of 3 lf

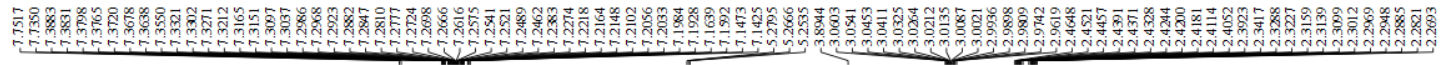

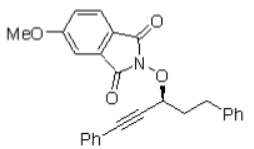

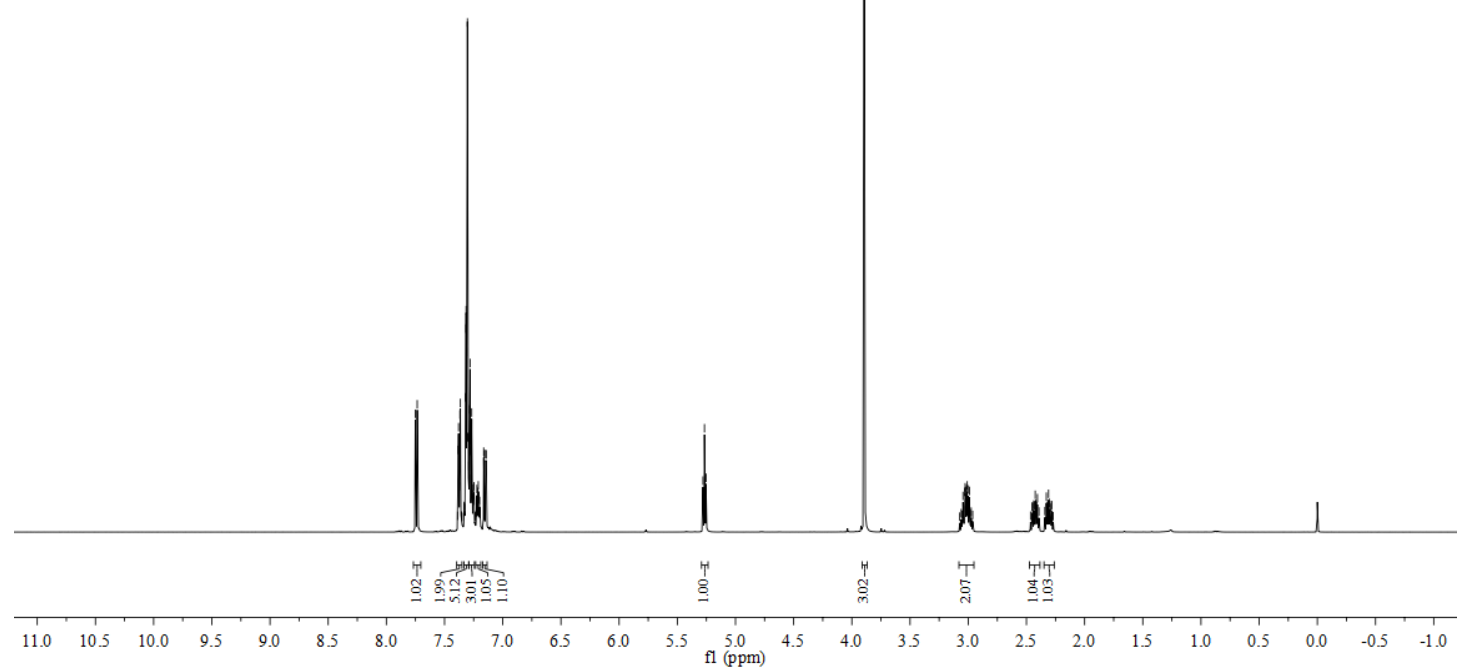

${ }^{13} \mathrm{C}$ NMR spectrum of $\mathbf{3 l f}$
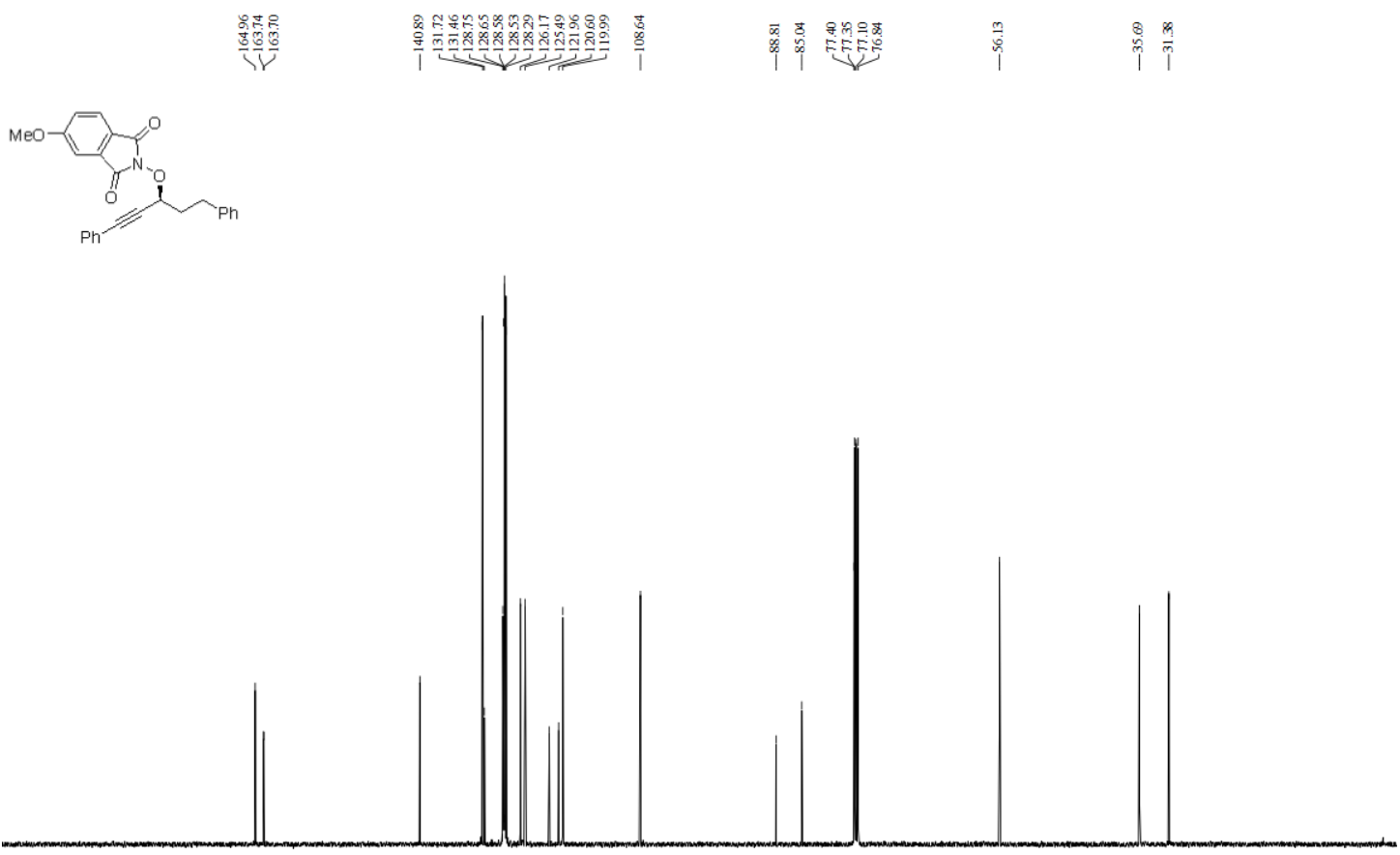

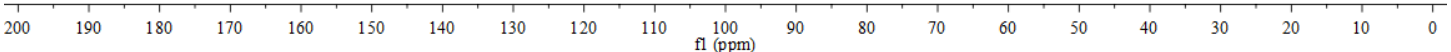


${ }^{1} \mathrm{H}$ NMR spectrum of $\mathbf{3} \mathbf{m f}$

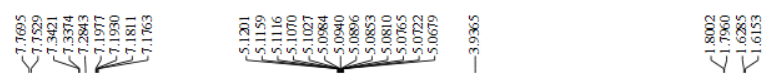
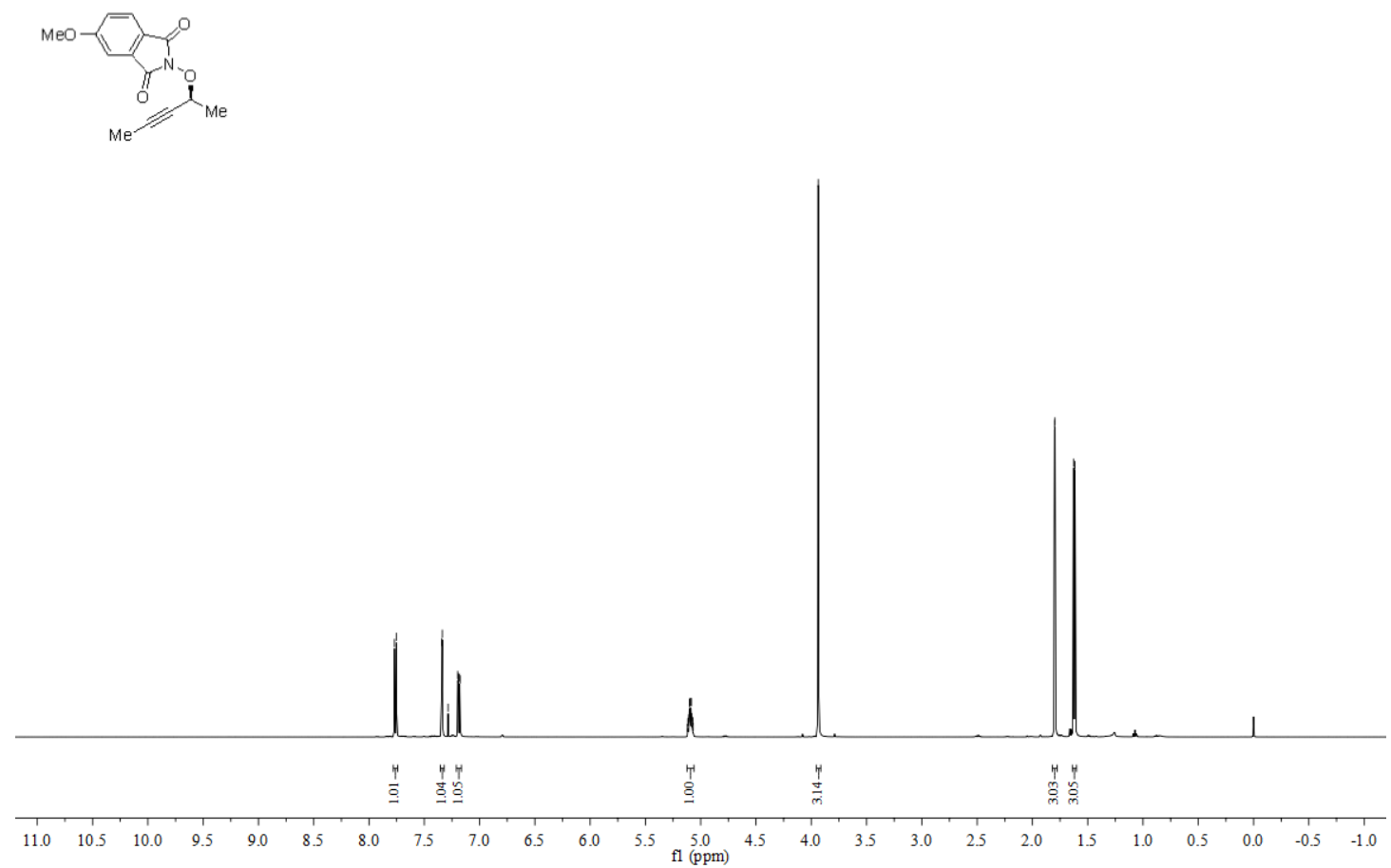

${ }^{13} \mathrm{C}$ NMR spectrum of $\mathbf{3} \mathbf{m f}$
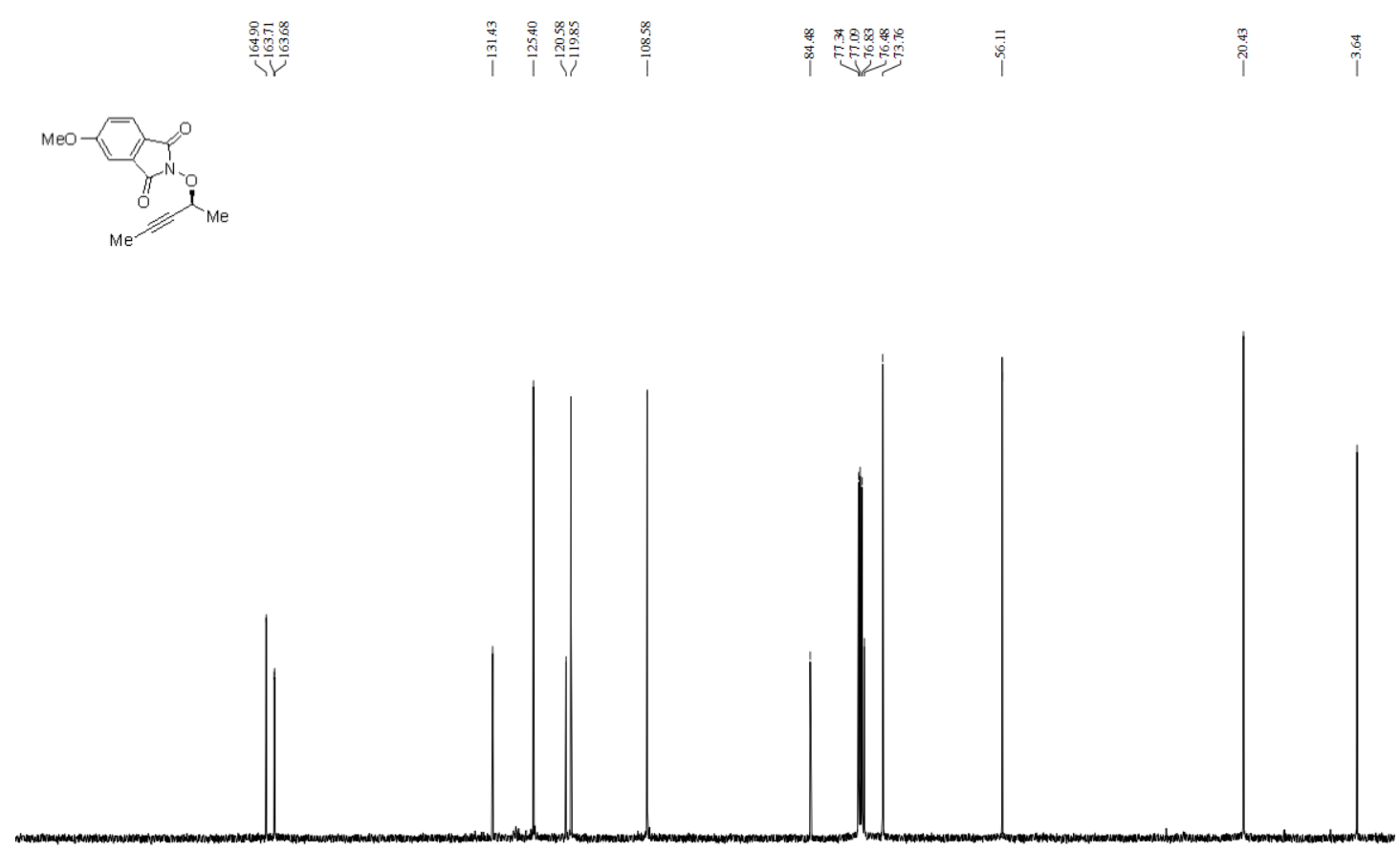

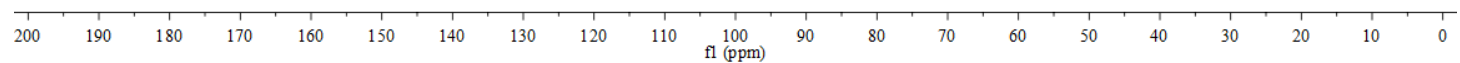


${ }^{1} \mathrm{H}$ NMR spectrum of $\mathbf{3 n f}$

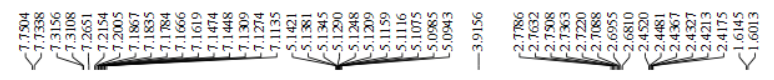
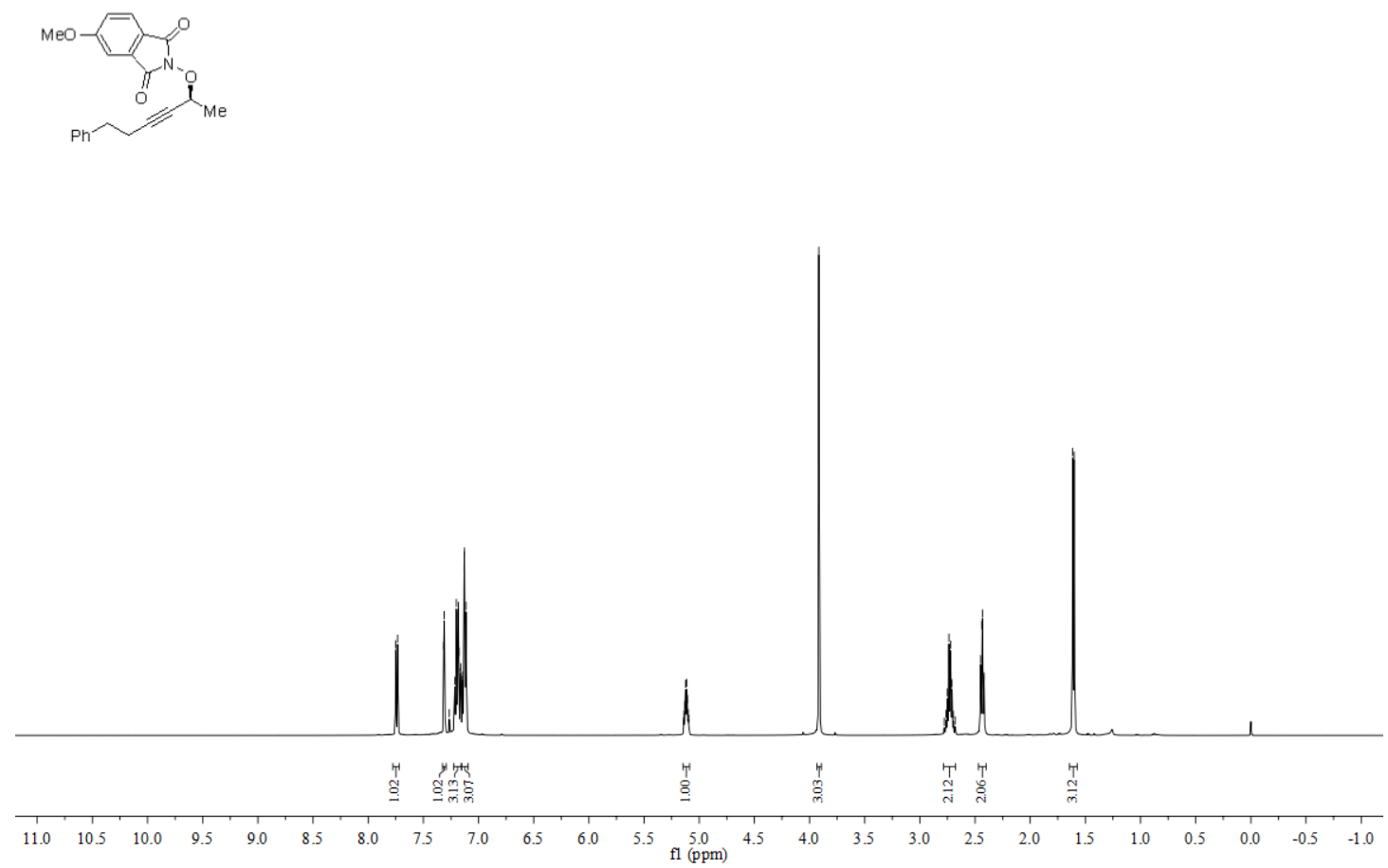

${ }^{13} \mathrm{C}$ NMR spectrum of $\mathbf{3 n f}$

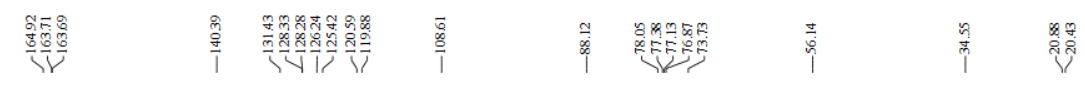
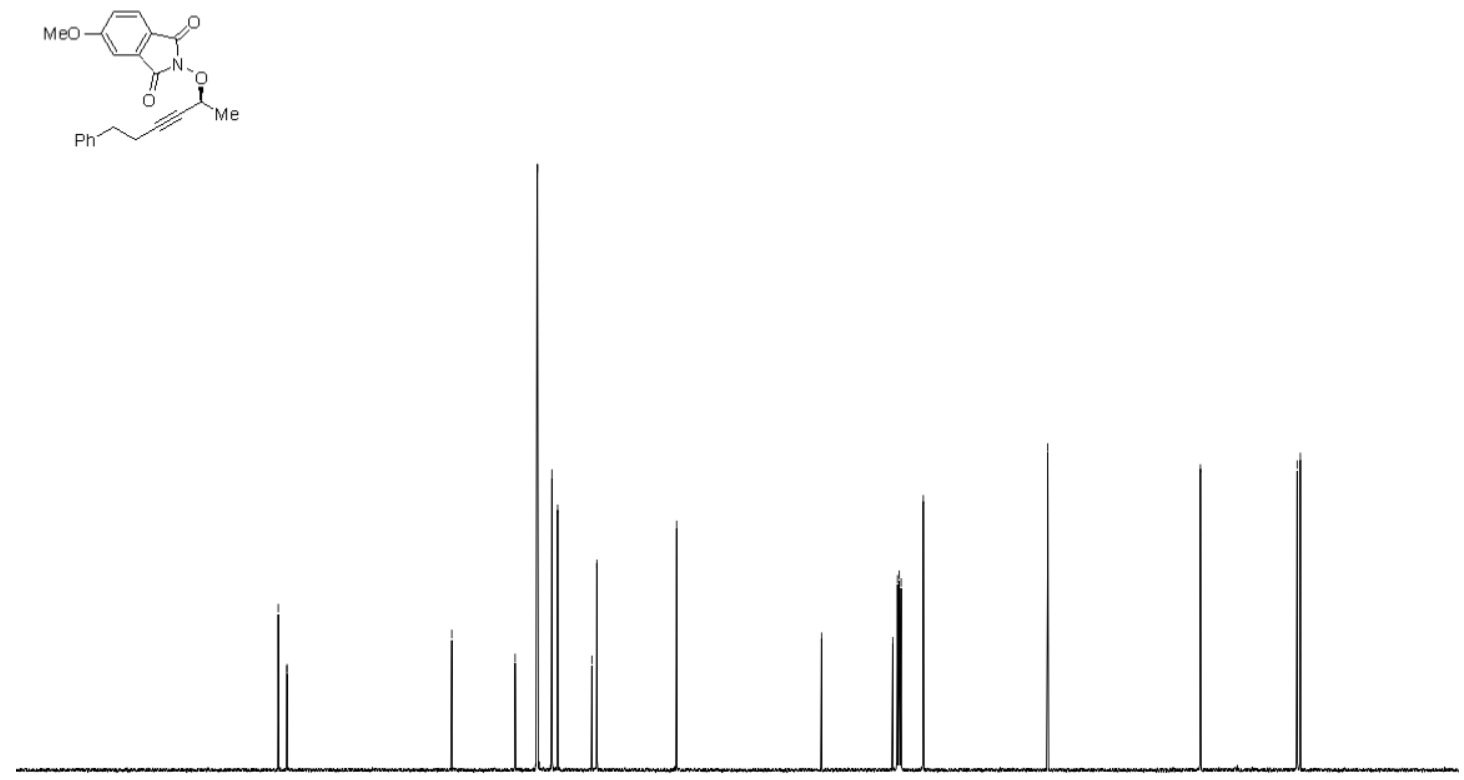

$\begin{array}{lllllllllllllllllllll}1 & 1 & 1 & 1 & 1 & 1 & 1 & 1 & 1 & 1 & 1 & 1 & 1 \\ 200 & 190 & 180 & 170 & 160 & 150 & 140 & 130 & 120 & 110 & \begin{array}{c}100 \\ \mathrm{fl}(\mathrm{ppm})\end{array} & 90 & 80 & 70 & 60 & 50 & 40 & 30 & 20 & 10 & 0\end{array}$ 
${ }^{1} \mathrm{H}$ NMR spectrum of $\mathbf{3 o f}$

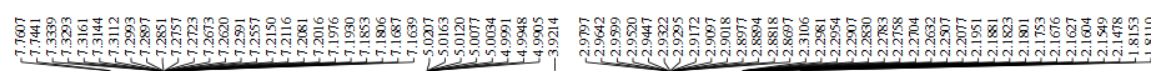
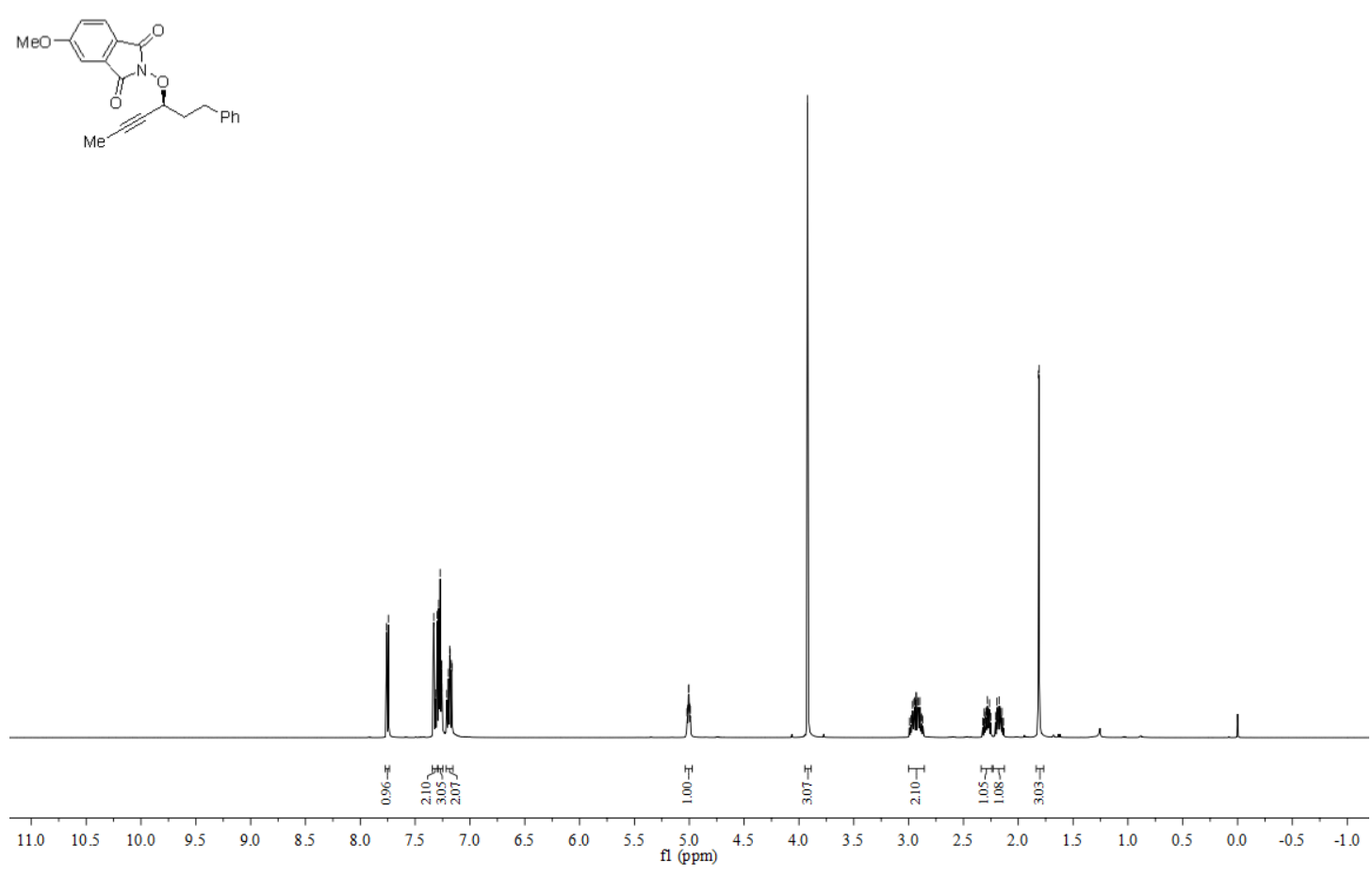

${ }^{13} \mathrm{C}$ NMR spectrum of $\mathbf{3 o f}$
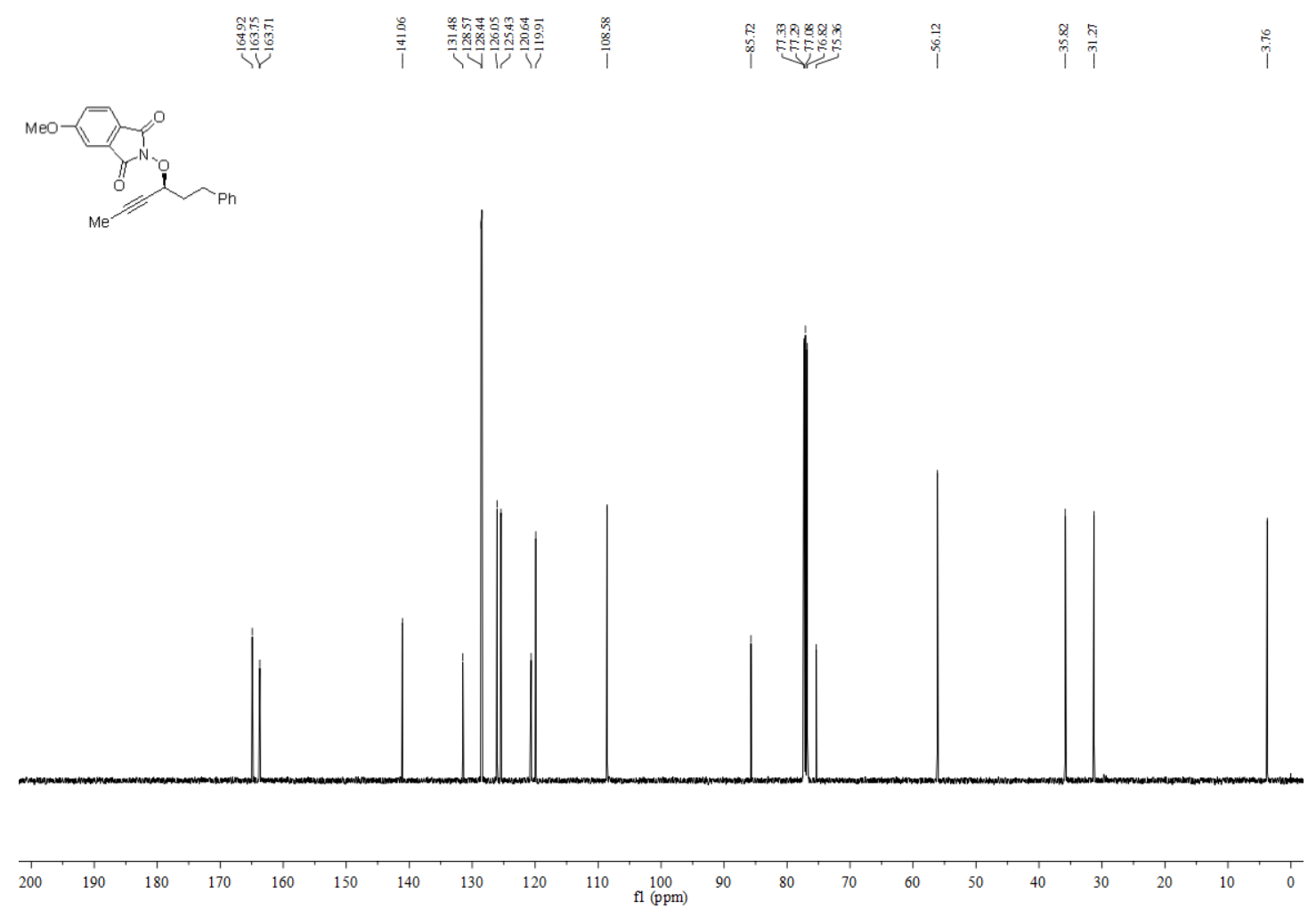

65 
${ }^{1} \mathrm{H}$ NMR spectrum of $\mathbf{3 p f}$

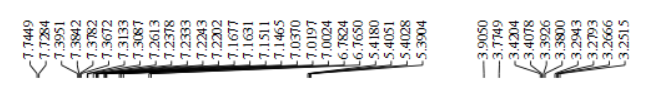
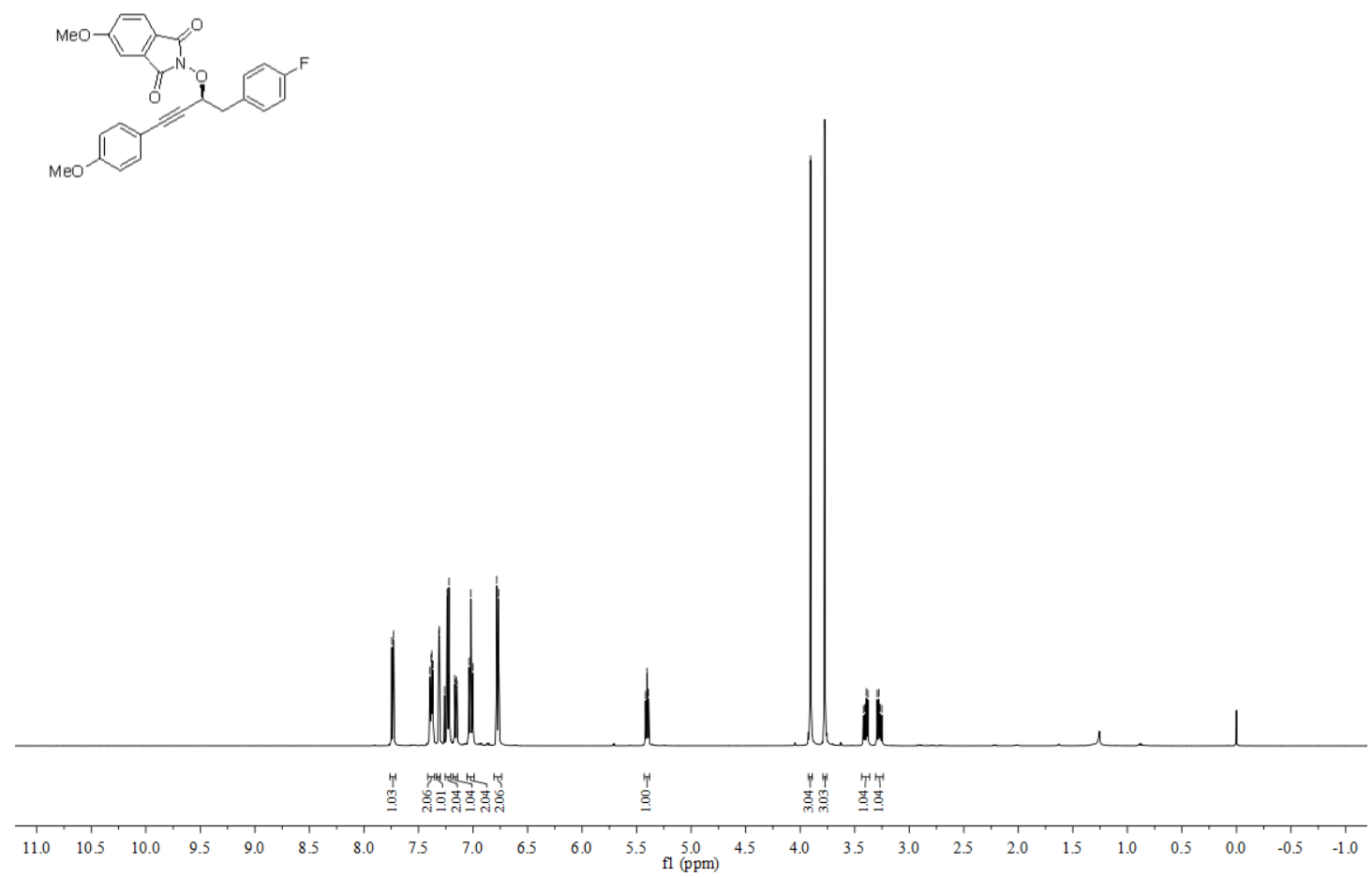

${ }^{13} \mathrm{C}$ NMR spectrum of $\mathbf{3 p f}$

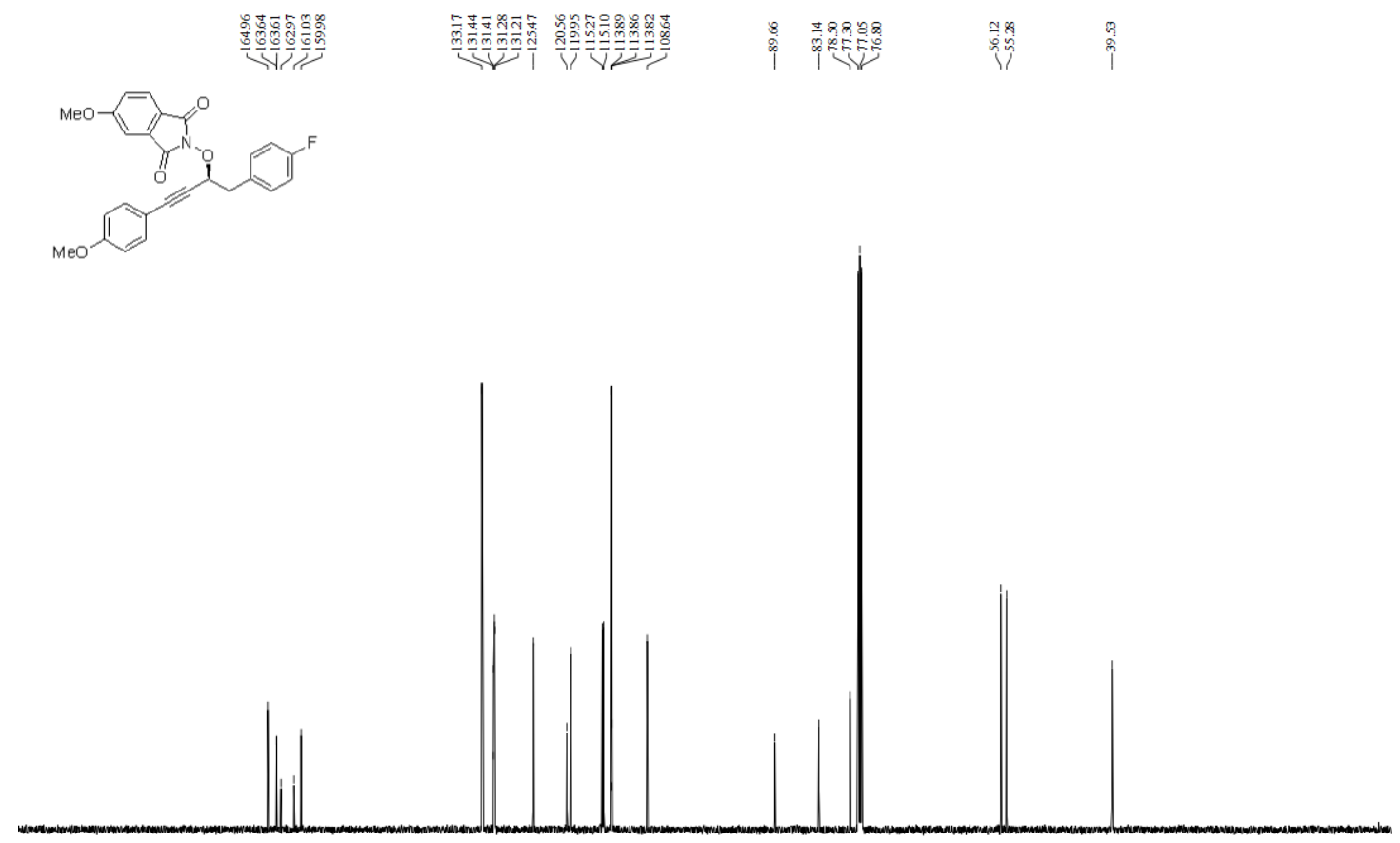

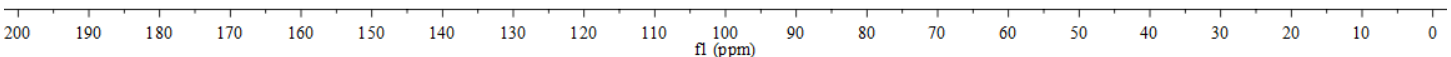


${ }^{1} \mathrm{H}$ NMR spectrum of 4

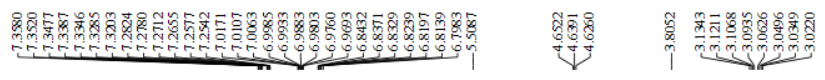
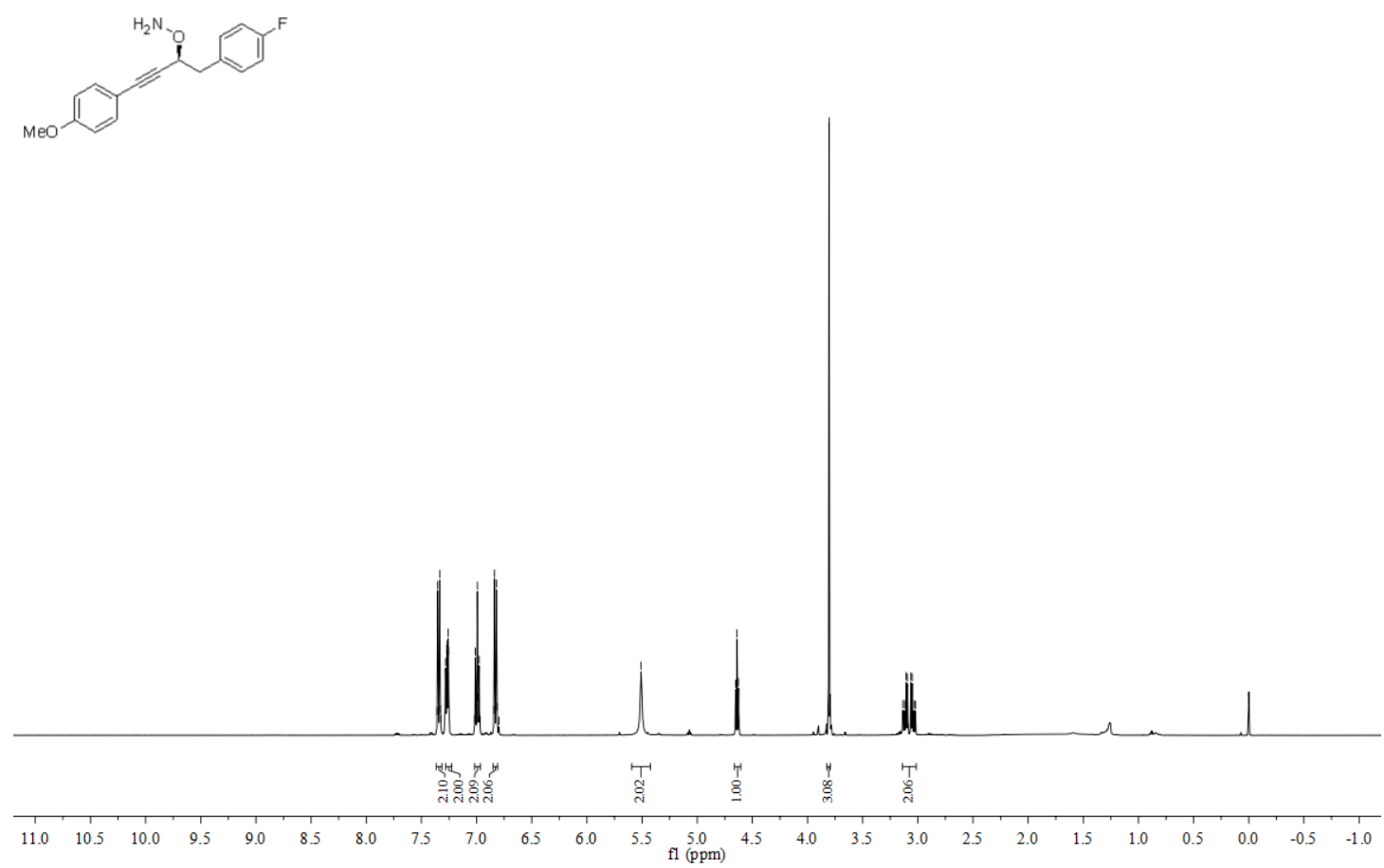

${ }^{13} \mathrm{C}$ NMR spectrum of $\mathbf{4}$

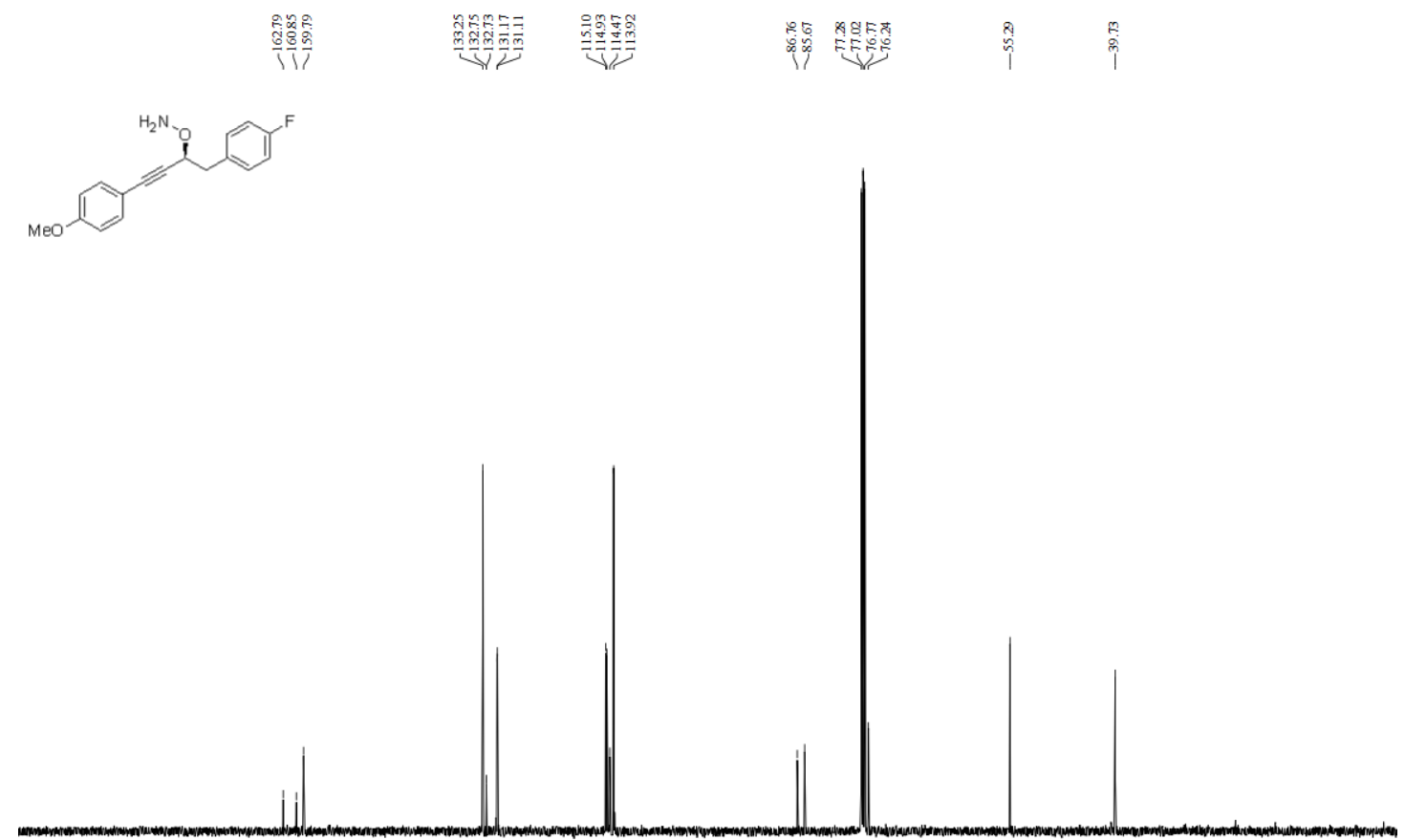

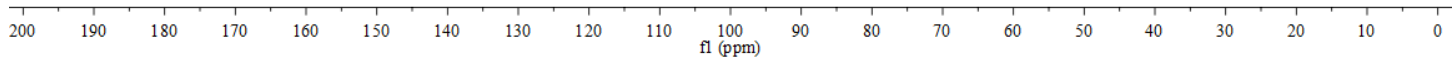


${ }^{1} \mathrm{H}$ NMR spectrum of 5

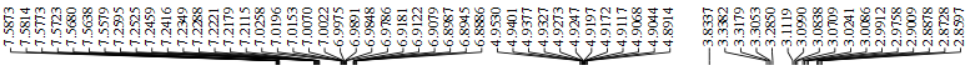
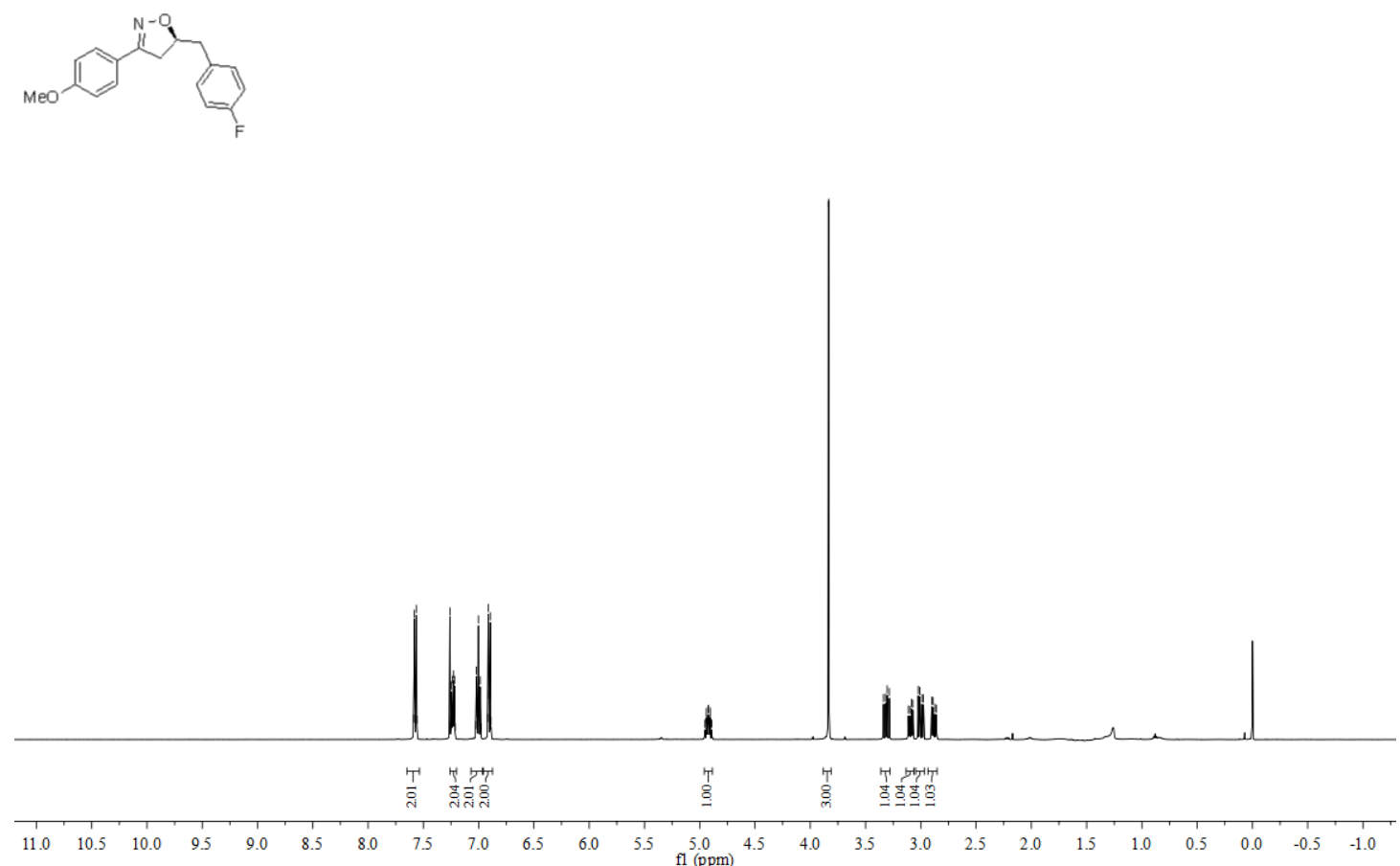

${ }^{13} \mathrm{C}$ NMR spectrum of $\mathbf{5}$

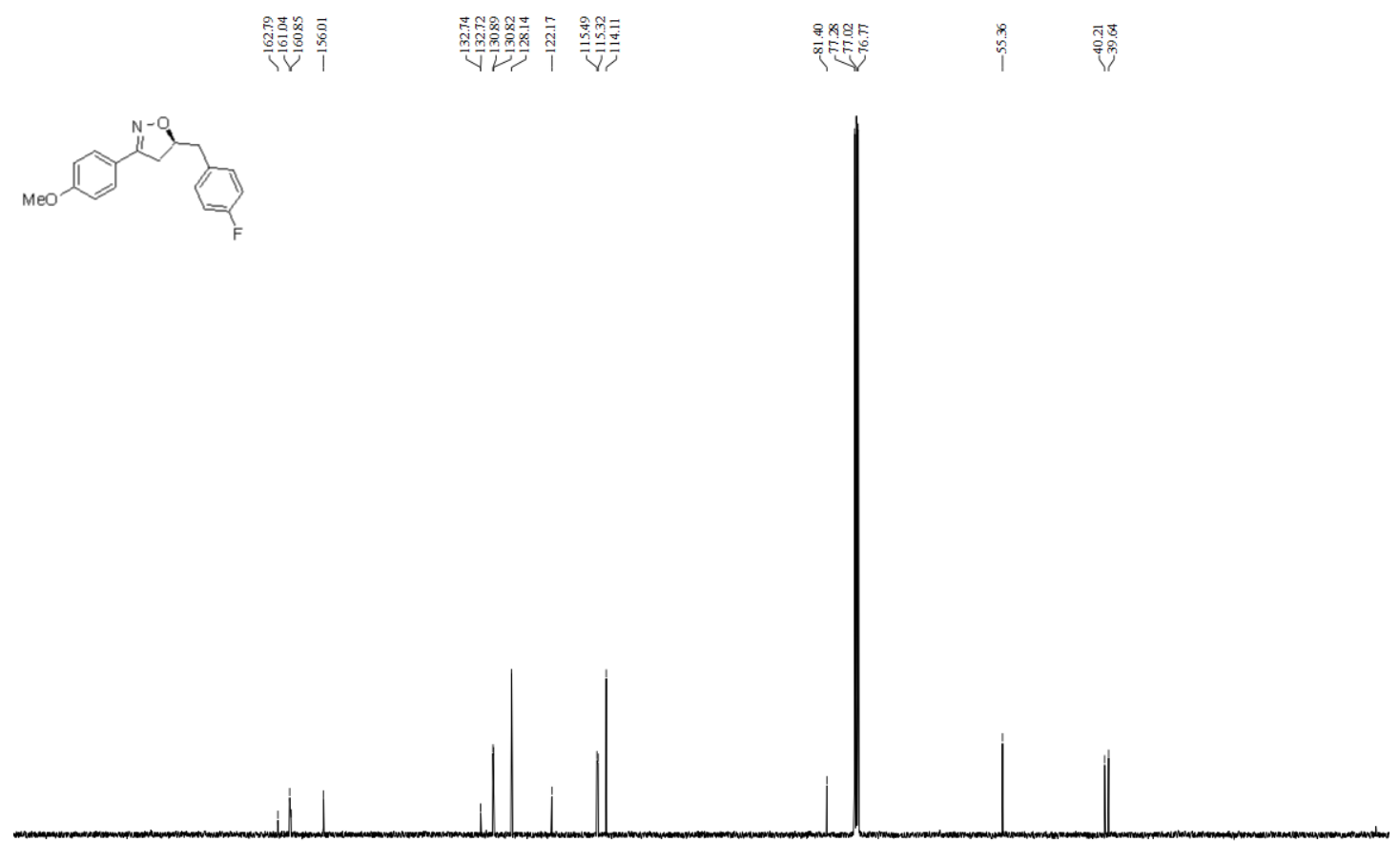

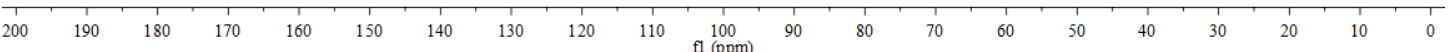


${ }^{1}$ H NMR spectrum of 6

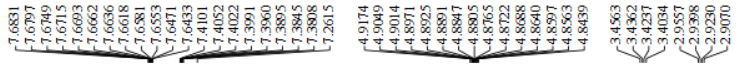

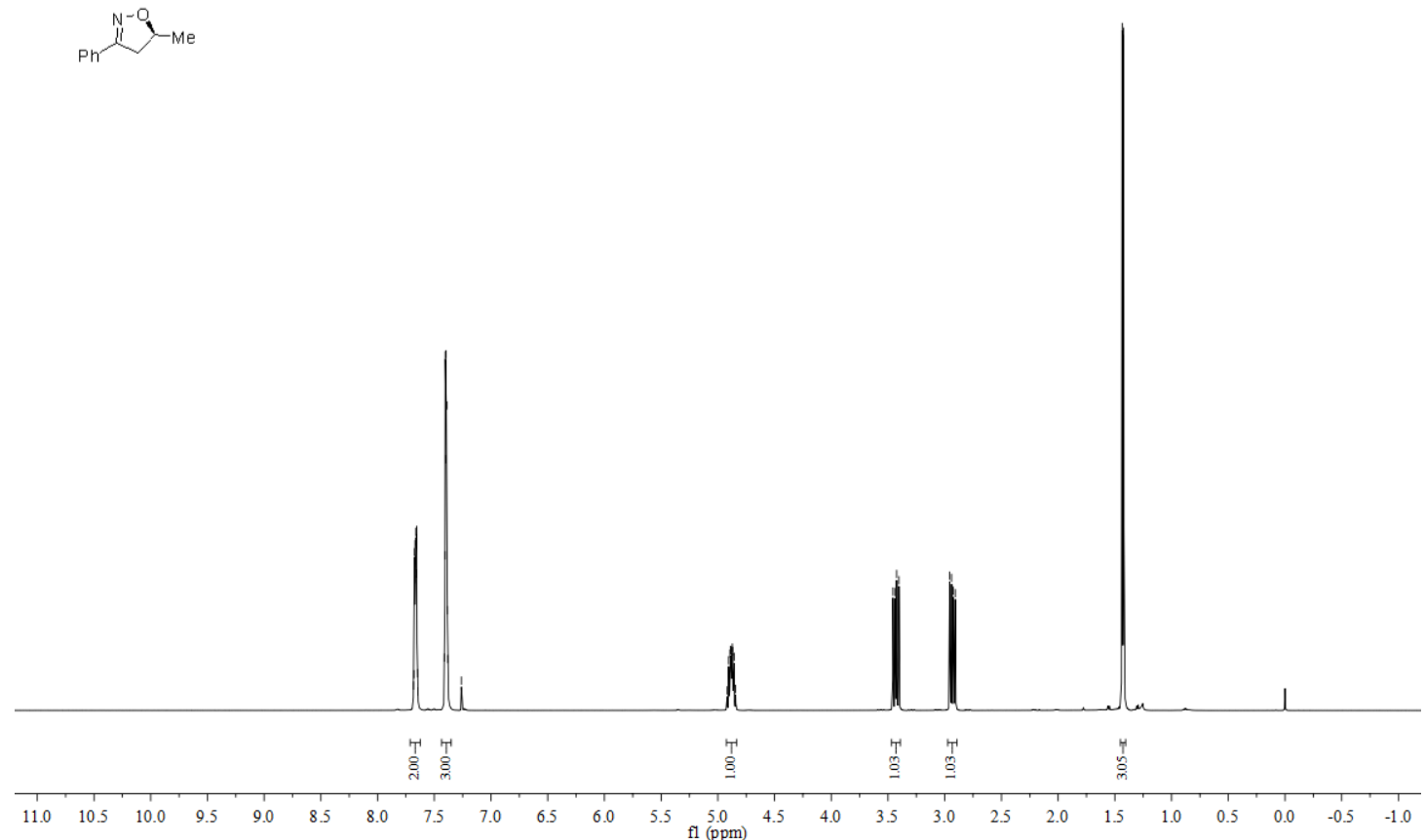

${ }^{13} \mathrm{C}$ NMR spectrum of $\mathbf{6}$
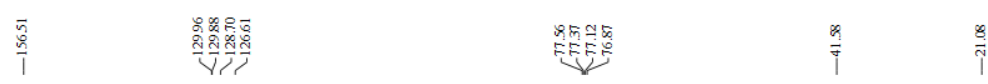

Ph

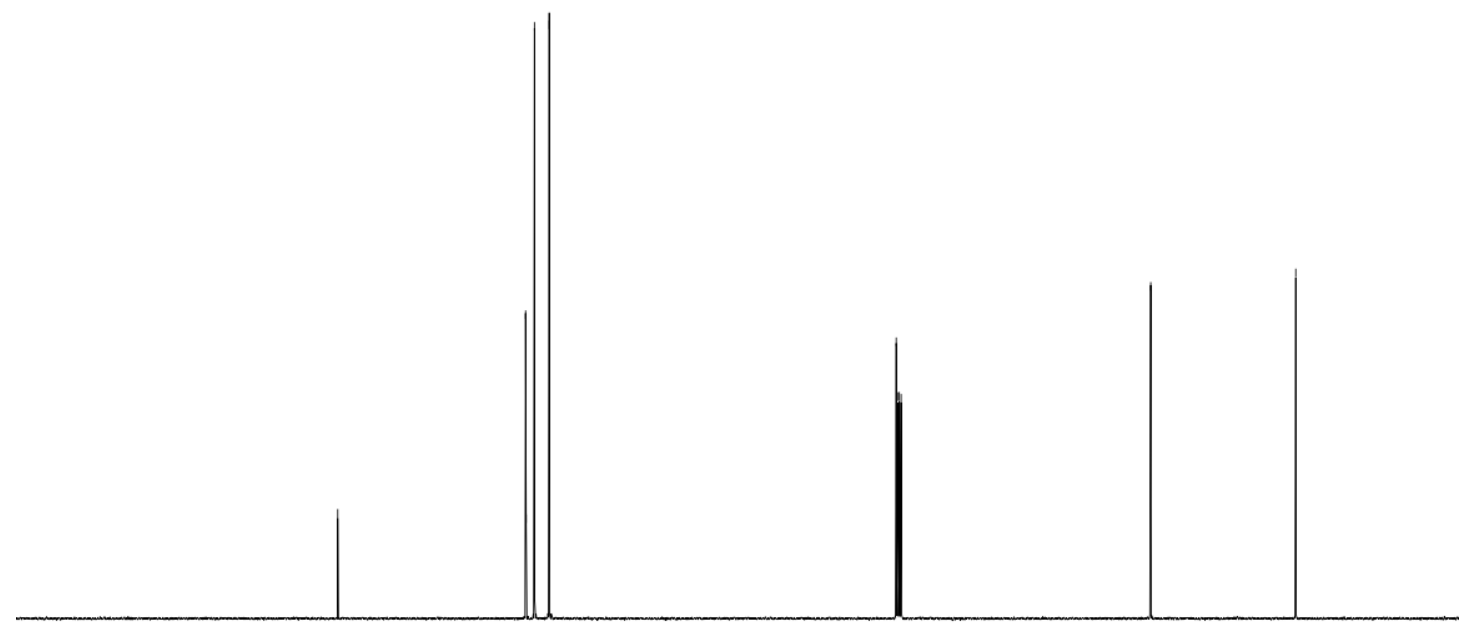

$200 \quad 190$
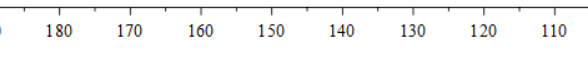

fl $(\mathrm{ppm})$ 
${ }^{1} \mathrm{H}$ NMR spectrum of 7

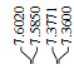

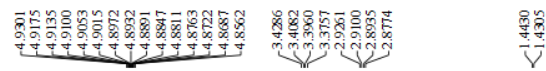
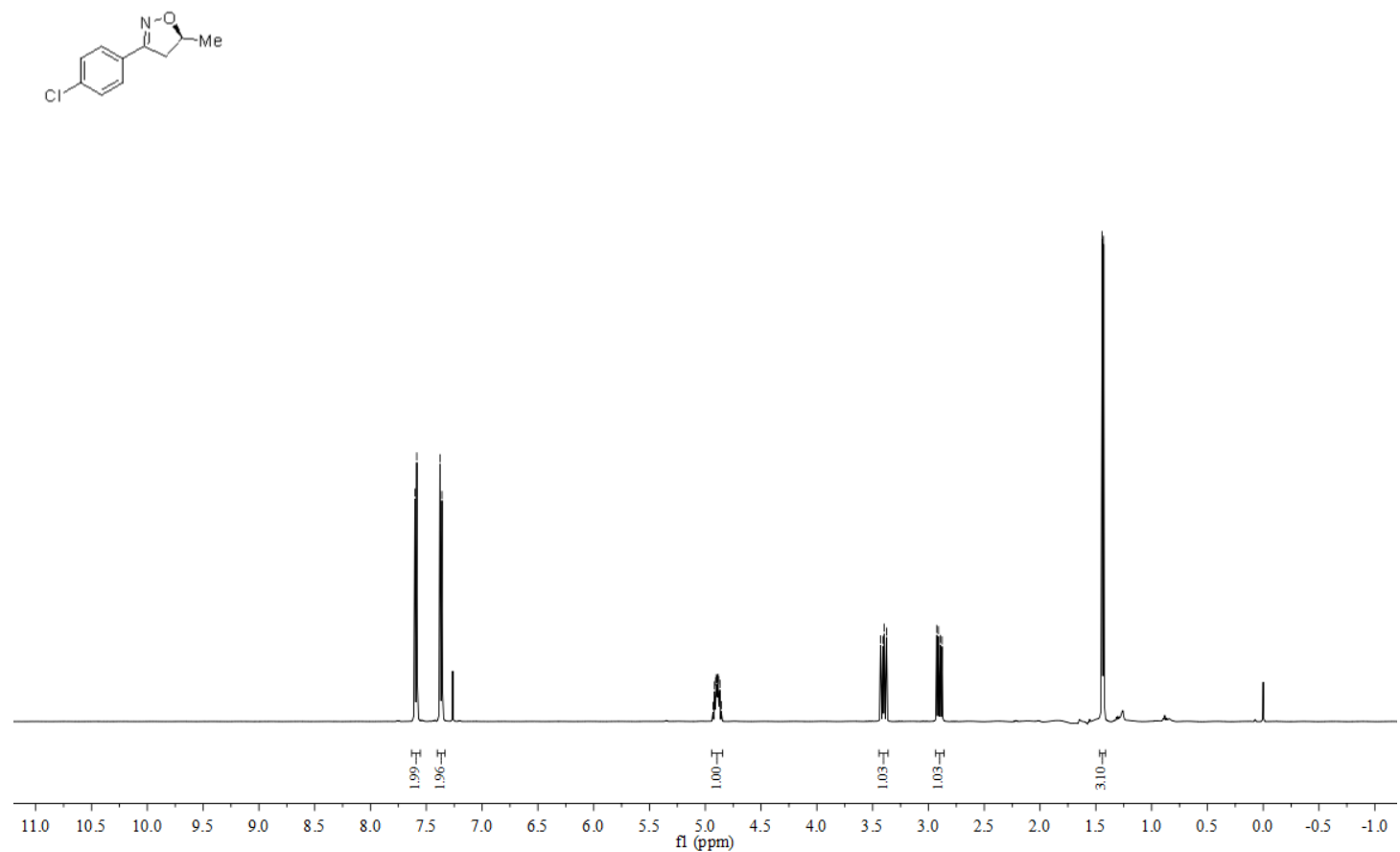

${ }^{13} \mathrm{C}$ NMR spectrum of 7

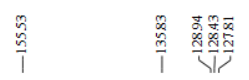

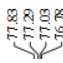

$\frac{8}{7} \quad \frac{8}{7}$
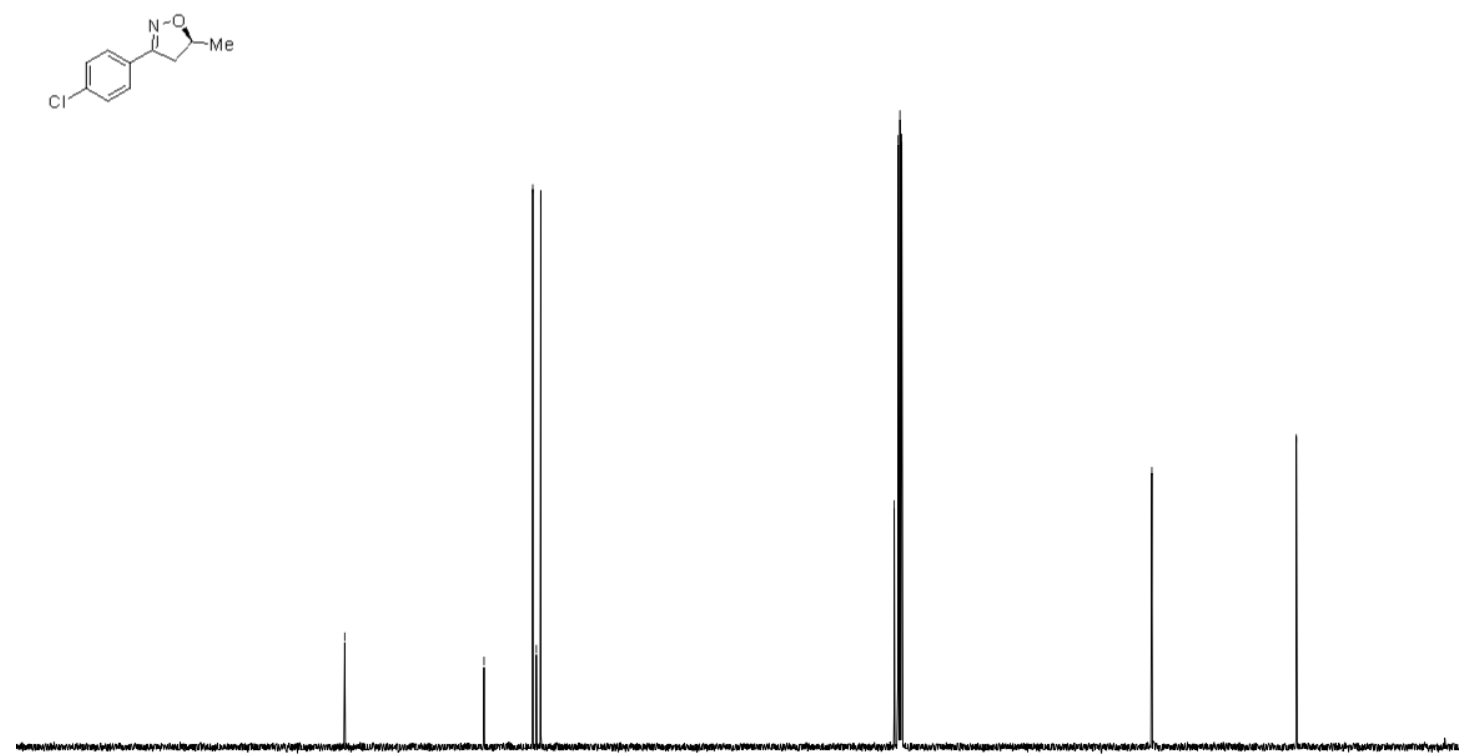

200

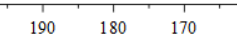

160

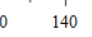

$30 \quad 120$

100
$\mathrm{fl}(\mathrm{ppm})$ 
${ }^{1}$ H NMR spectrum of 8

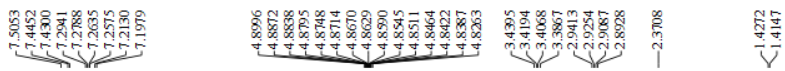

Me $(1)^{N-1}-$ -

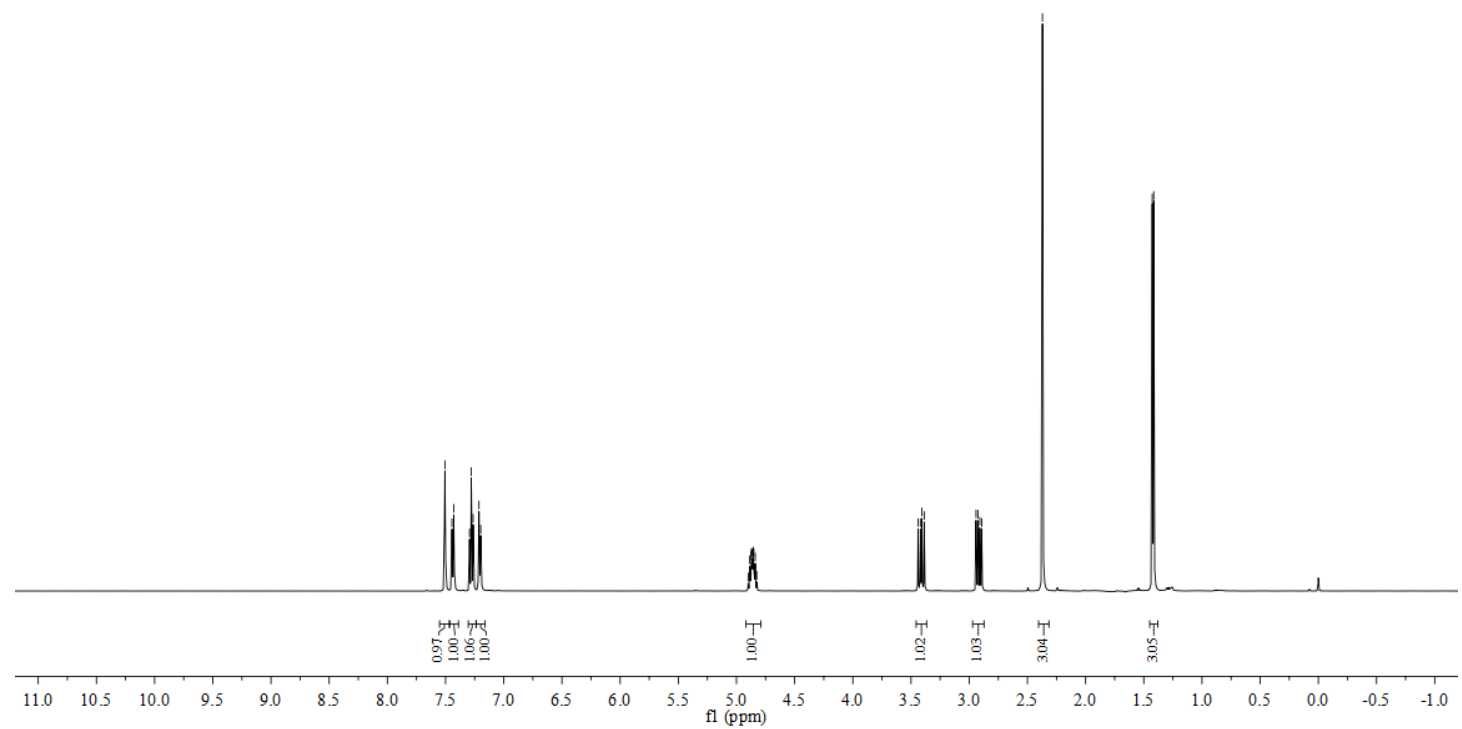

${ }^{13} \mathrm{C}$ NMR spectrum of $\mathbf{8}$

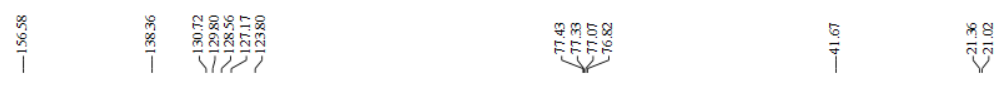<smiles>Cc1ccc(C2CON2)cc1</smiles>

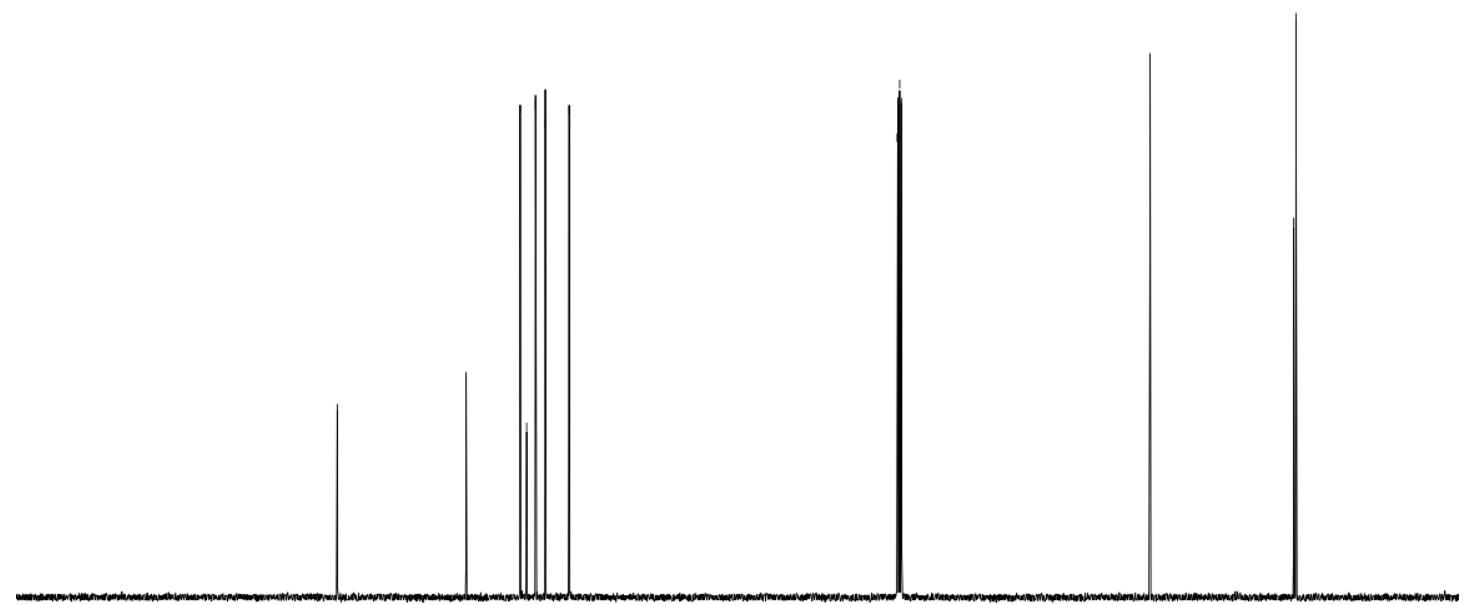

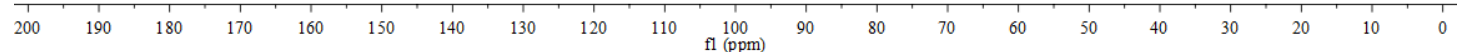


${ }^{1} \mathrm{H}$ NMR spectrum of 9

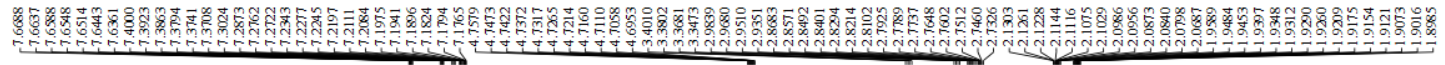

$\operatorname{Ph}^{N-\mathrm{N}}-\sim^{\mathrm{Ph}}$

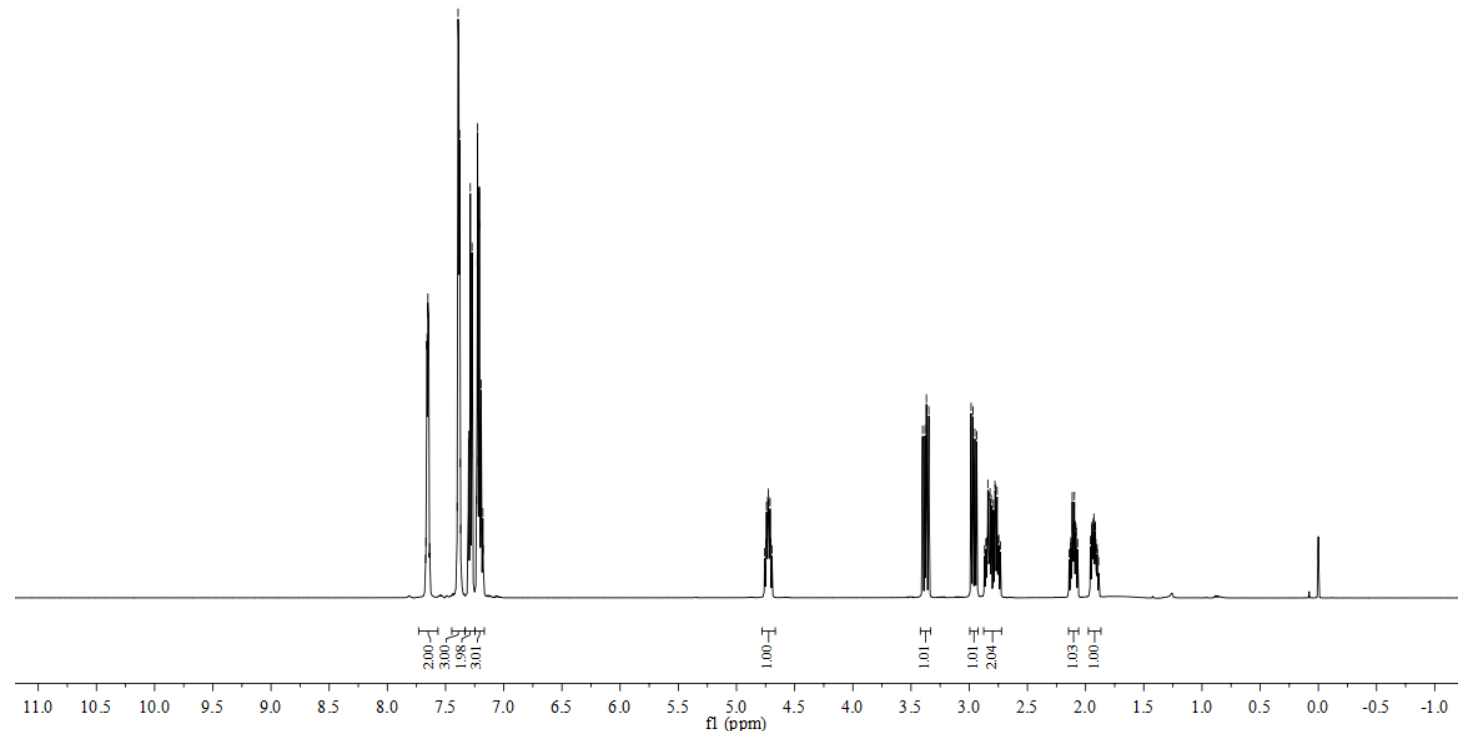

${ }^{13} \mathrm{C}$ NMR spectrum of 9

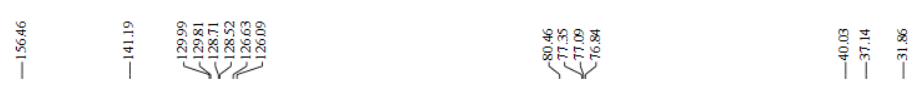

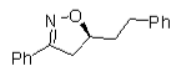

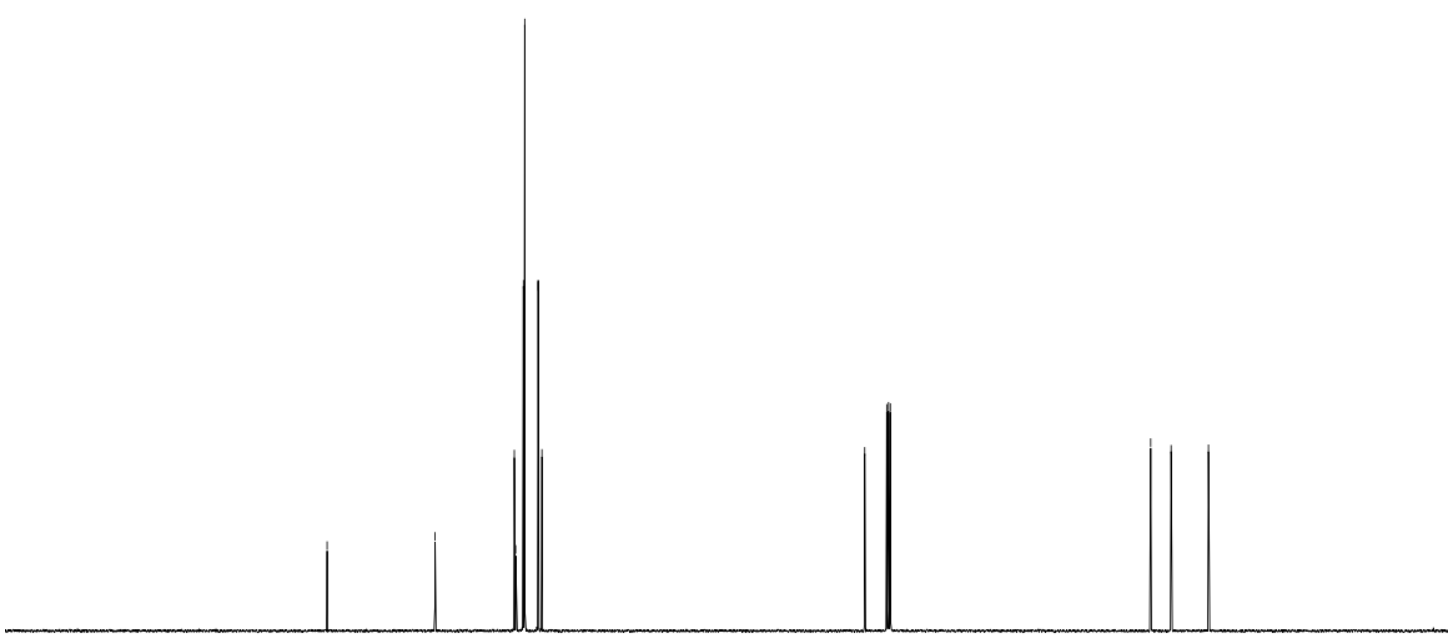

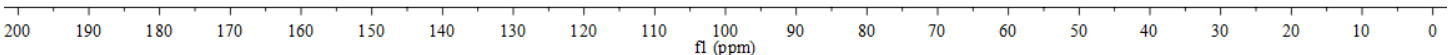


${ }^{1} \mathrm{H}$ NMR spectrum of $\mathbf{1 0}$

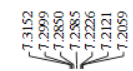

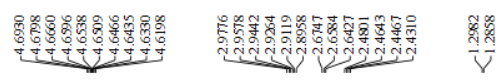
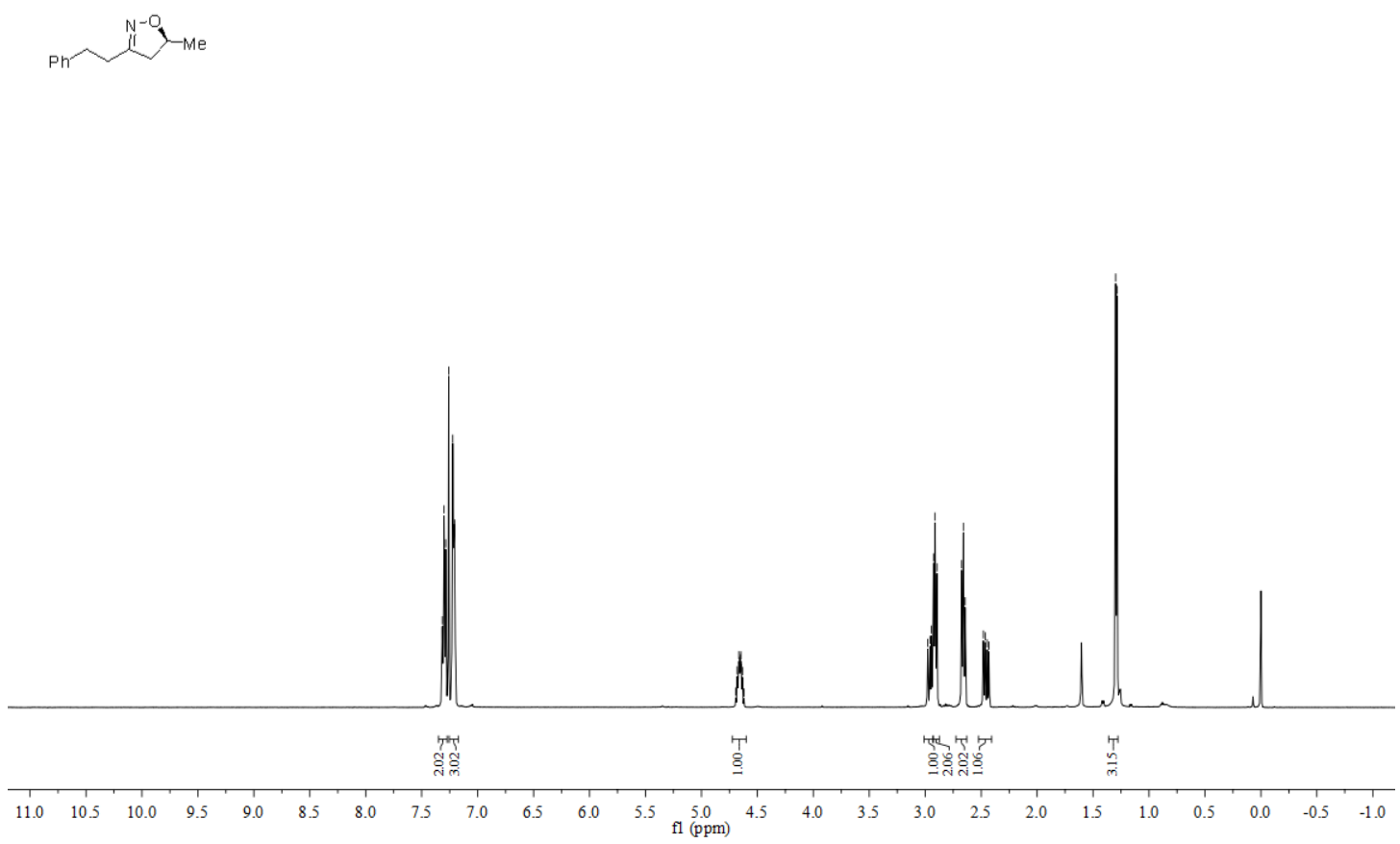

${ }^{13} \mathrm{C}$ NMR spectrum of $\mathbf{1 0}$

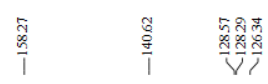

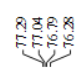

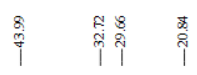
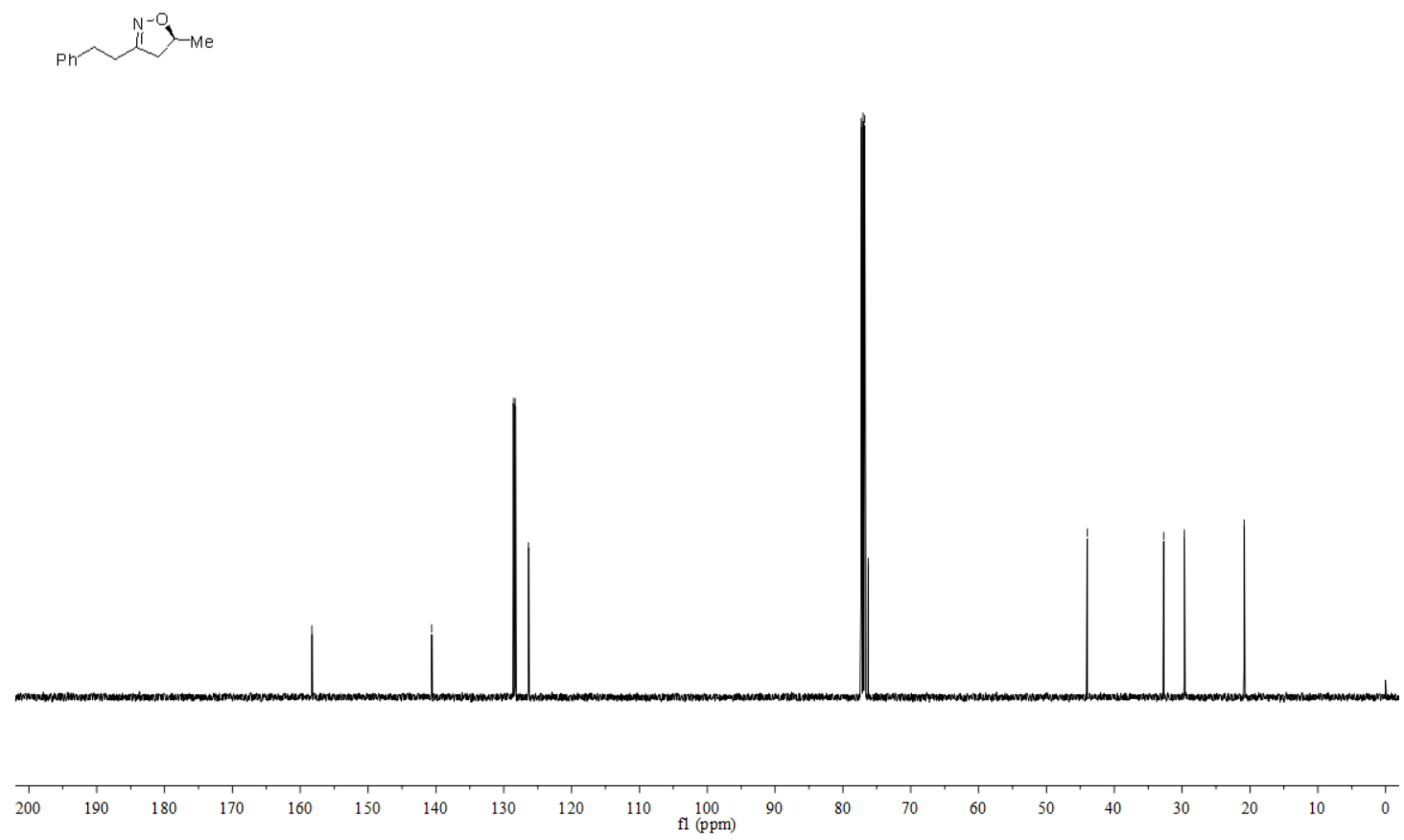

73 
${ }^{1} \mathrm{H}$ NMR spectrum of $\mathbf{3 q f}$

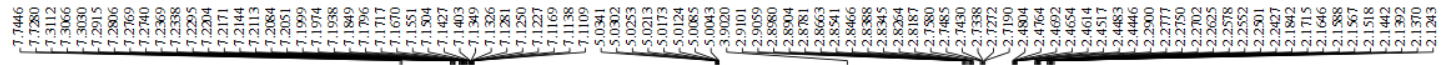
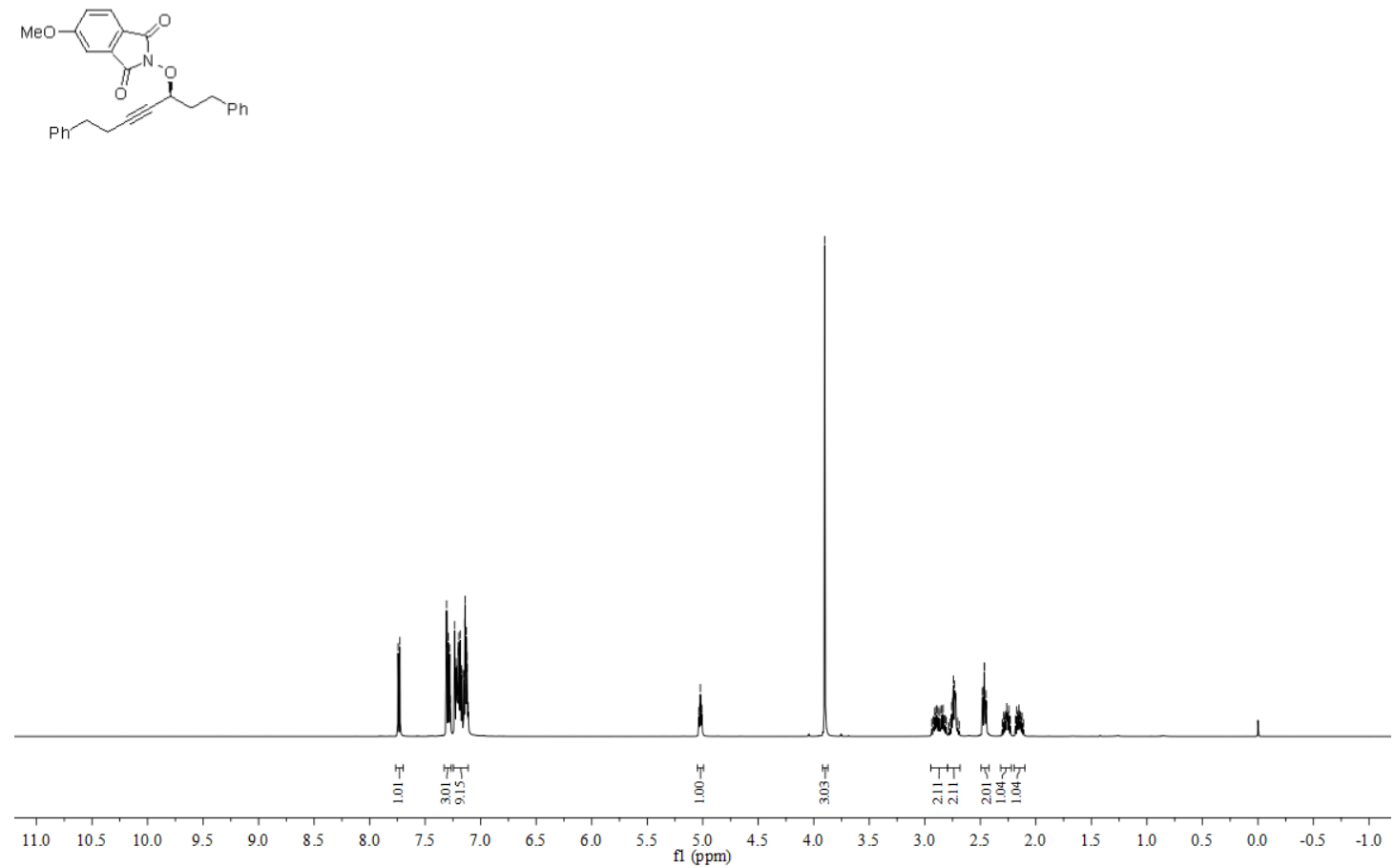

${ }^{13} \mathrm{C}$ NMR spectrum of $\mathbf{3 q f}$

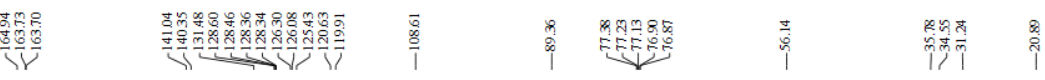
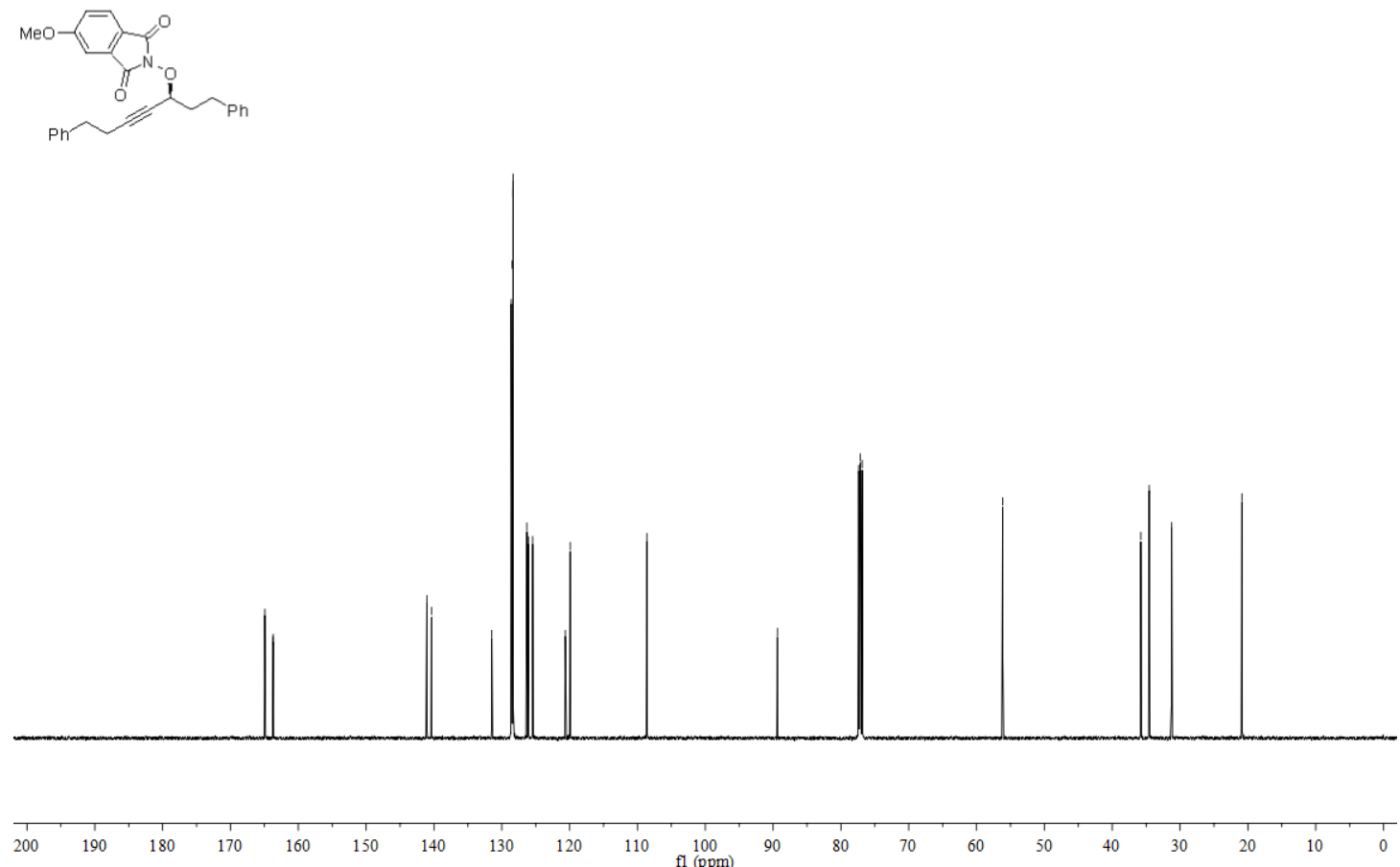
${ }^{1} \mathrm{H}$ NMR spectrum of $\mathbf{4 q}$

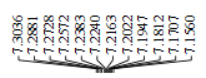

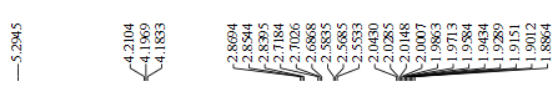
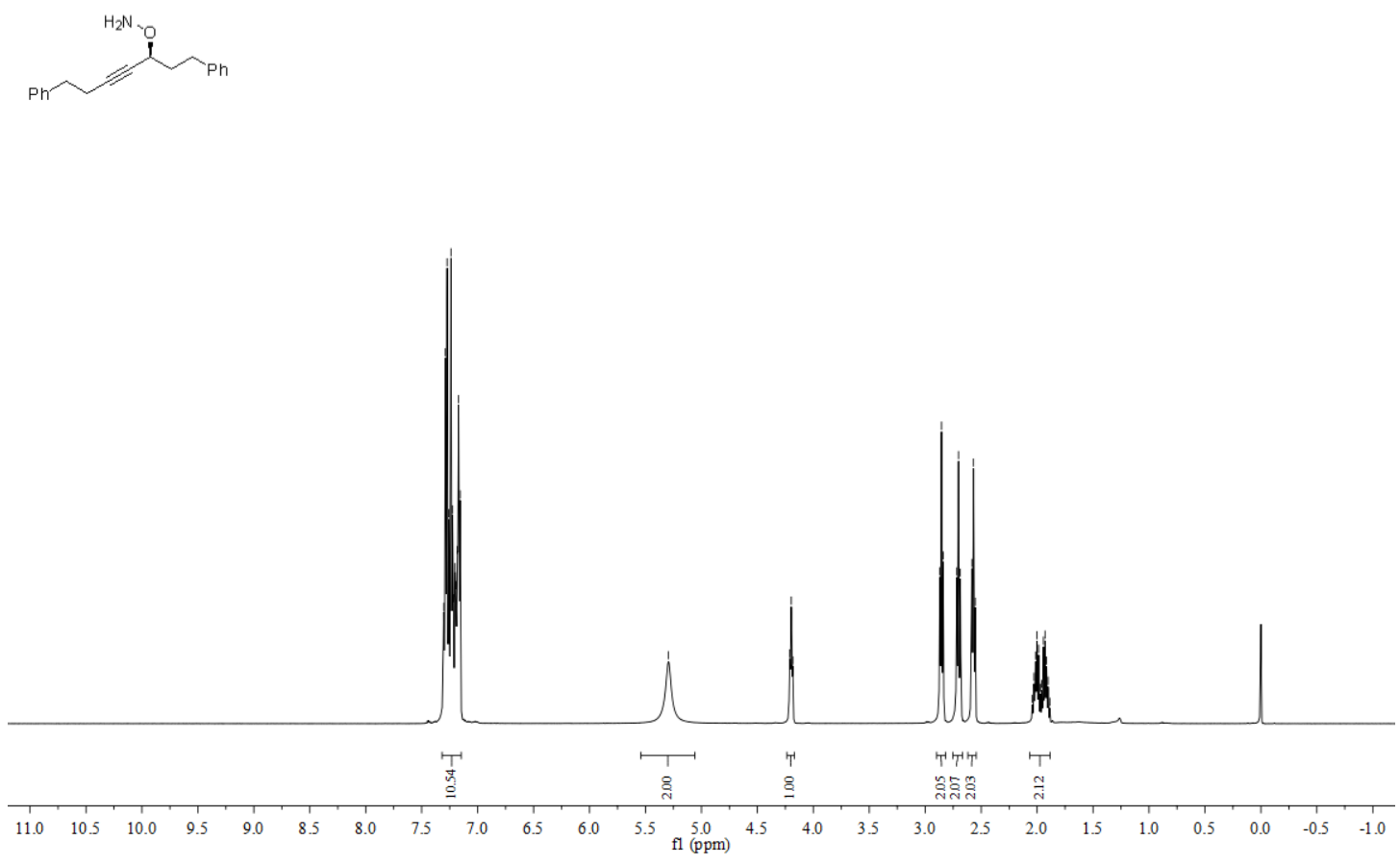

${ }^{13} \mathrm{C}$ NMR spectrum of $\mathbf{4 q}$

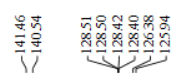

จำ

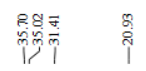
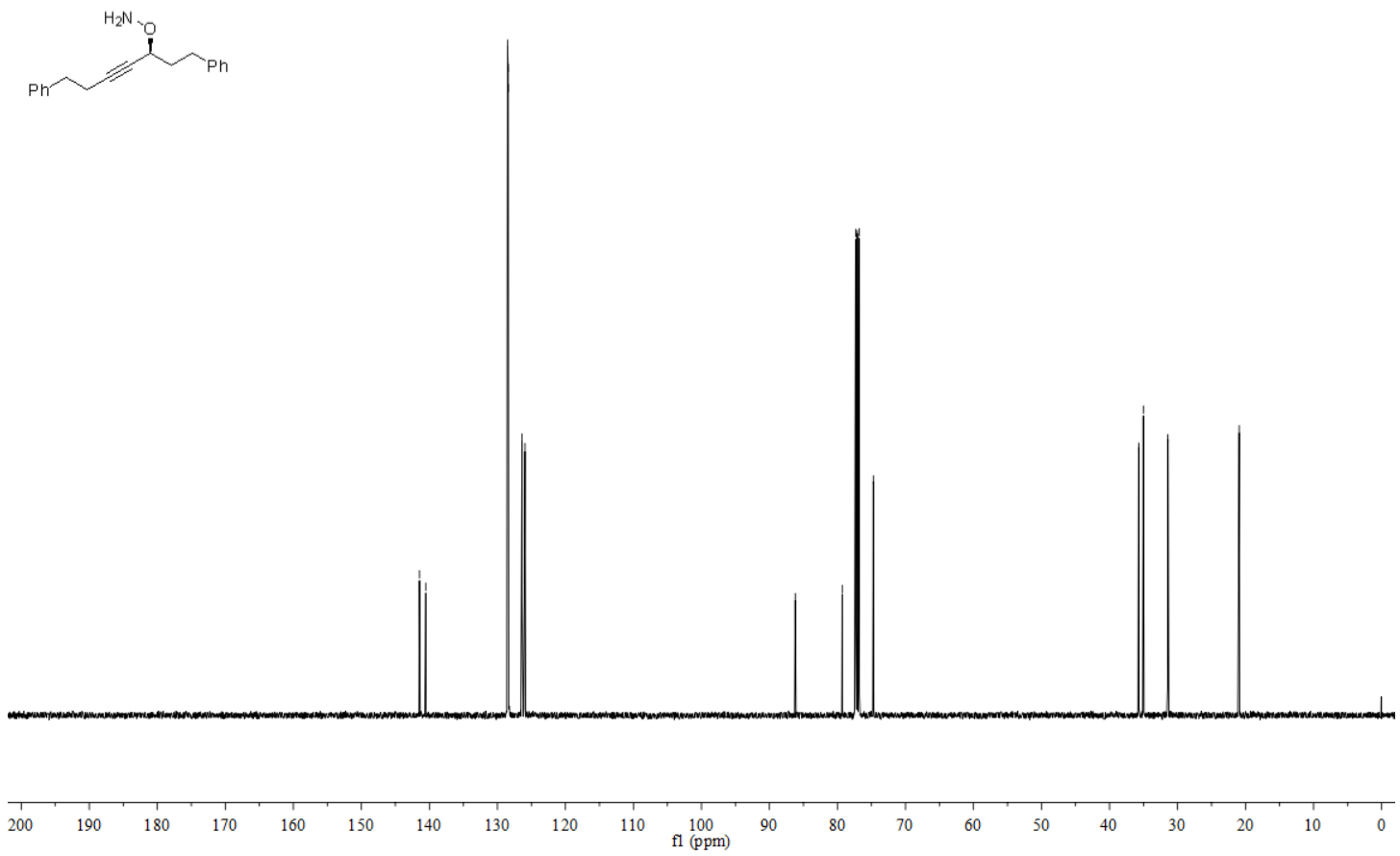

75 
${ }^{1} \mathrm{H}$ NMR spectrum of $\mathbf{1 1}$

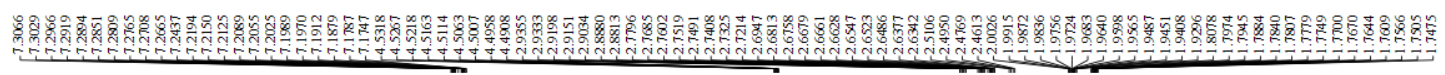

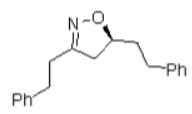

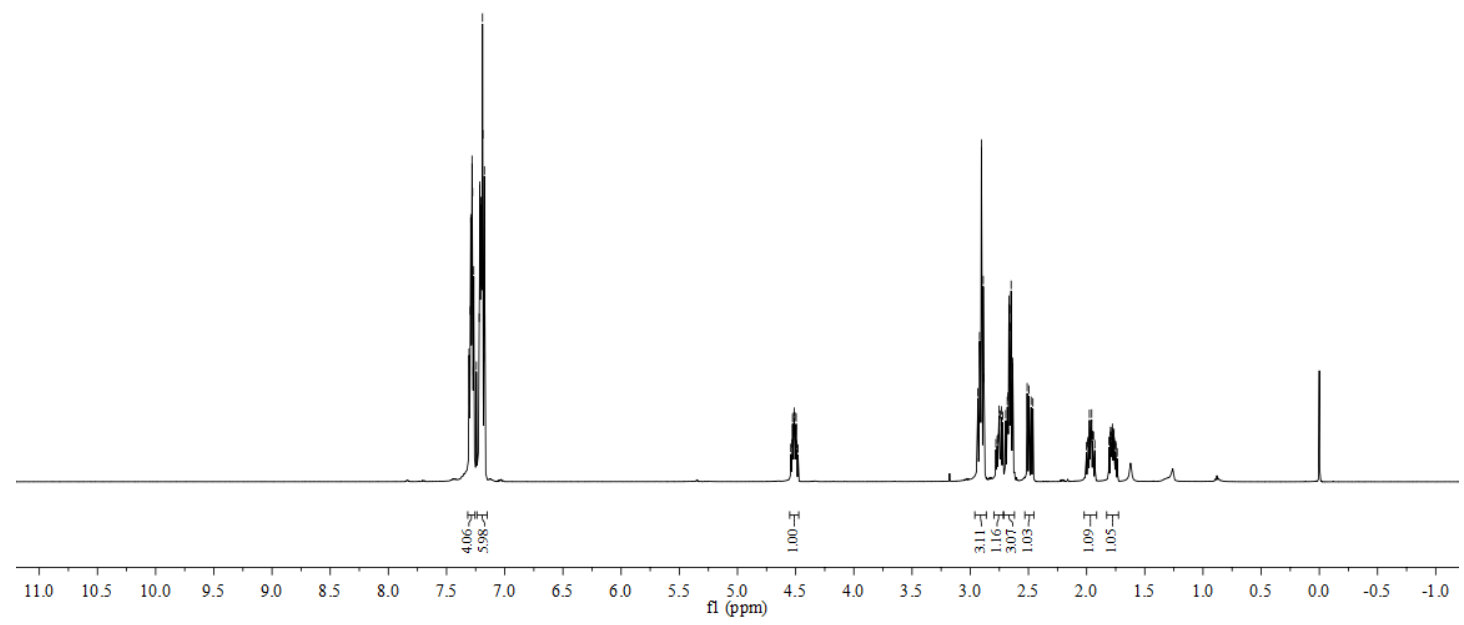

${ }^{13} \mathrm{C}$ NMR spectrum of $\mathbf{1 1}$

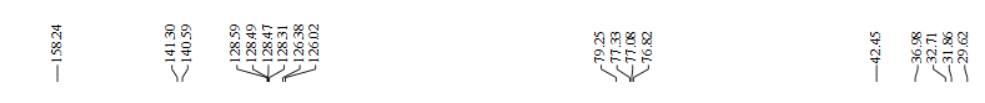<smiles>CCCC1CC(CCP)CO1</smiles>

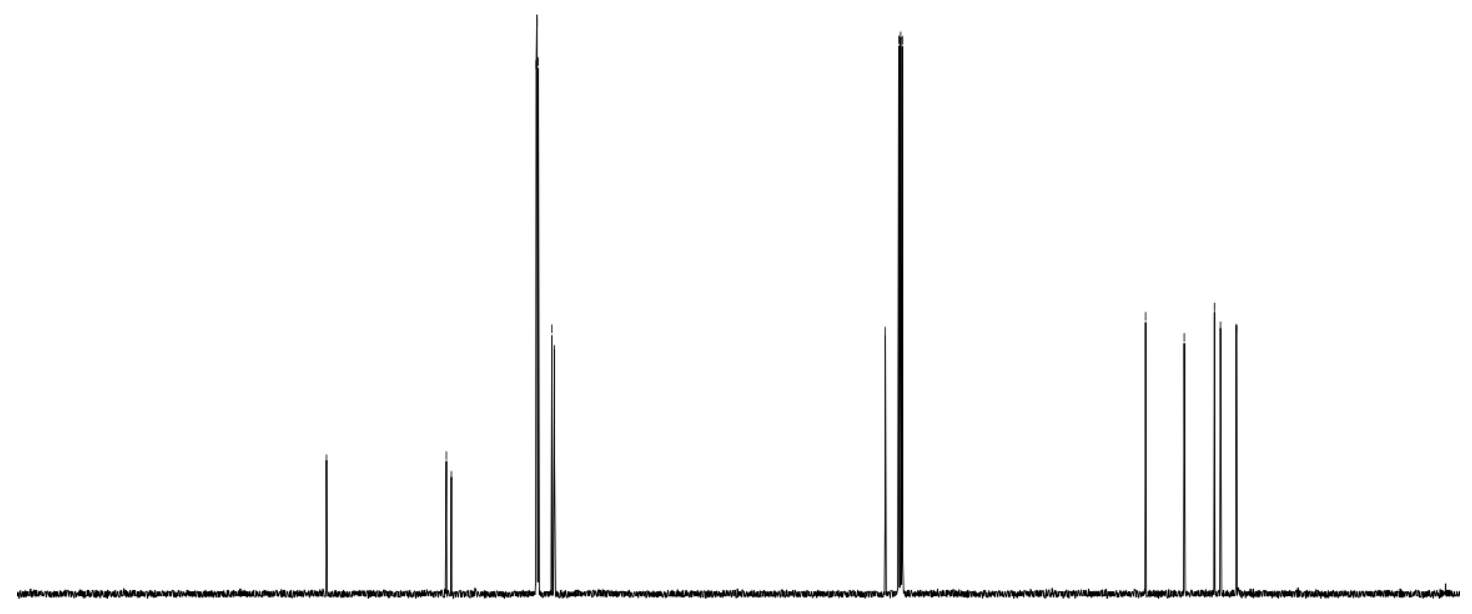

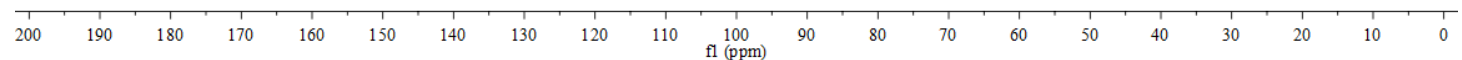


${ }^{1} \mathrm{H}$ NMR spectrum of $\mathbf{1 2}$

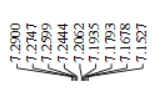

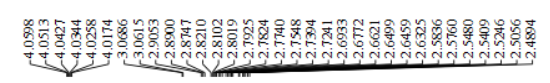

phr足㕵

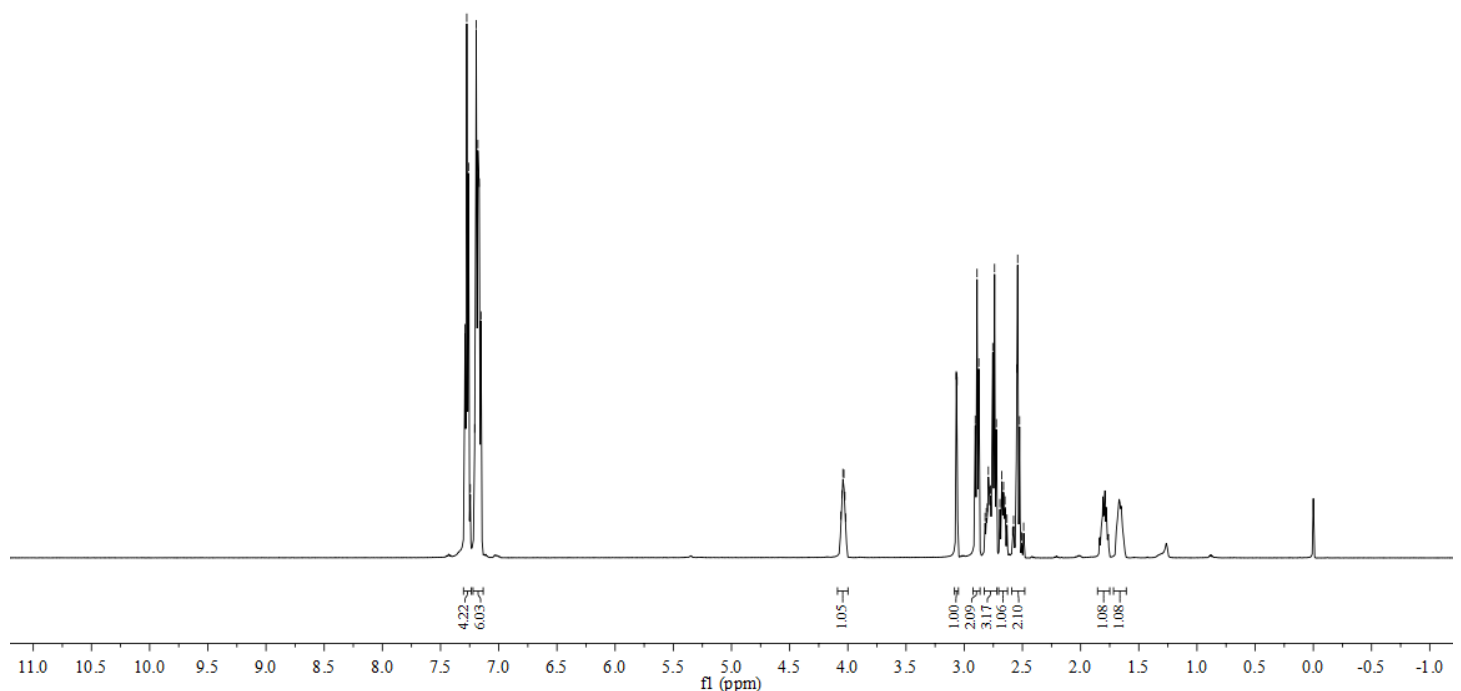

${ }^{13} \mathrm{C}$ NMR spectrum of $\mathbf{1 2}$

誉

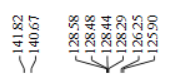

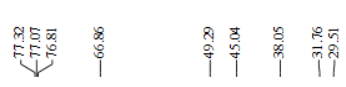

$\overbrace{\text { Ph }}^{\text {in }}$

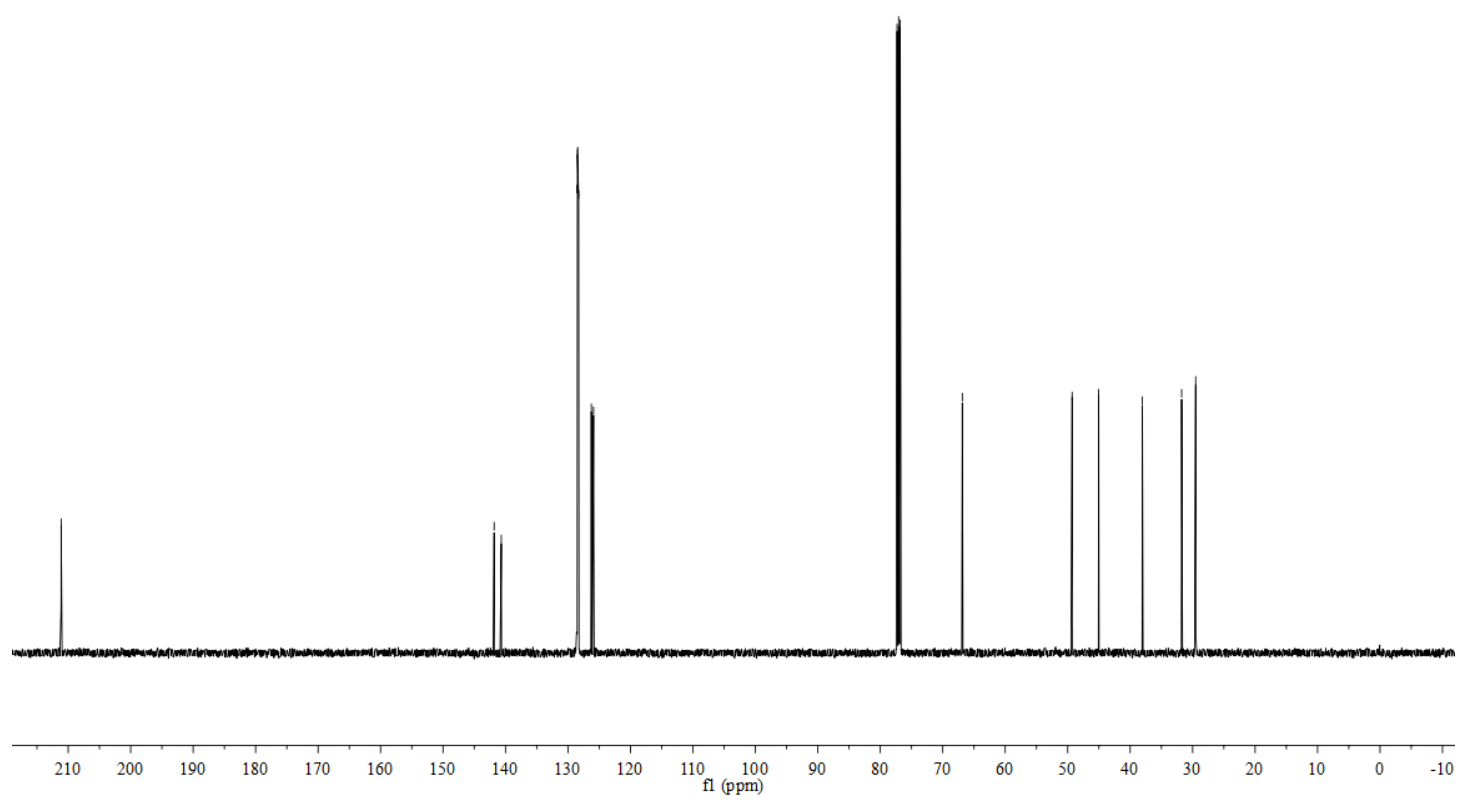

77 
${ }^{1} \mathrm{H}$ NMR spectrum of $\mathbf{1 3}$

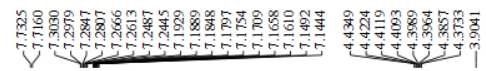

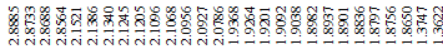

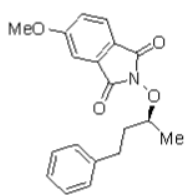

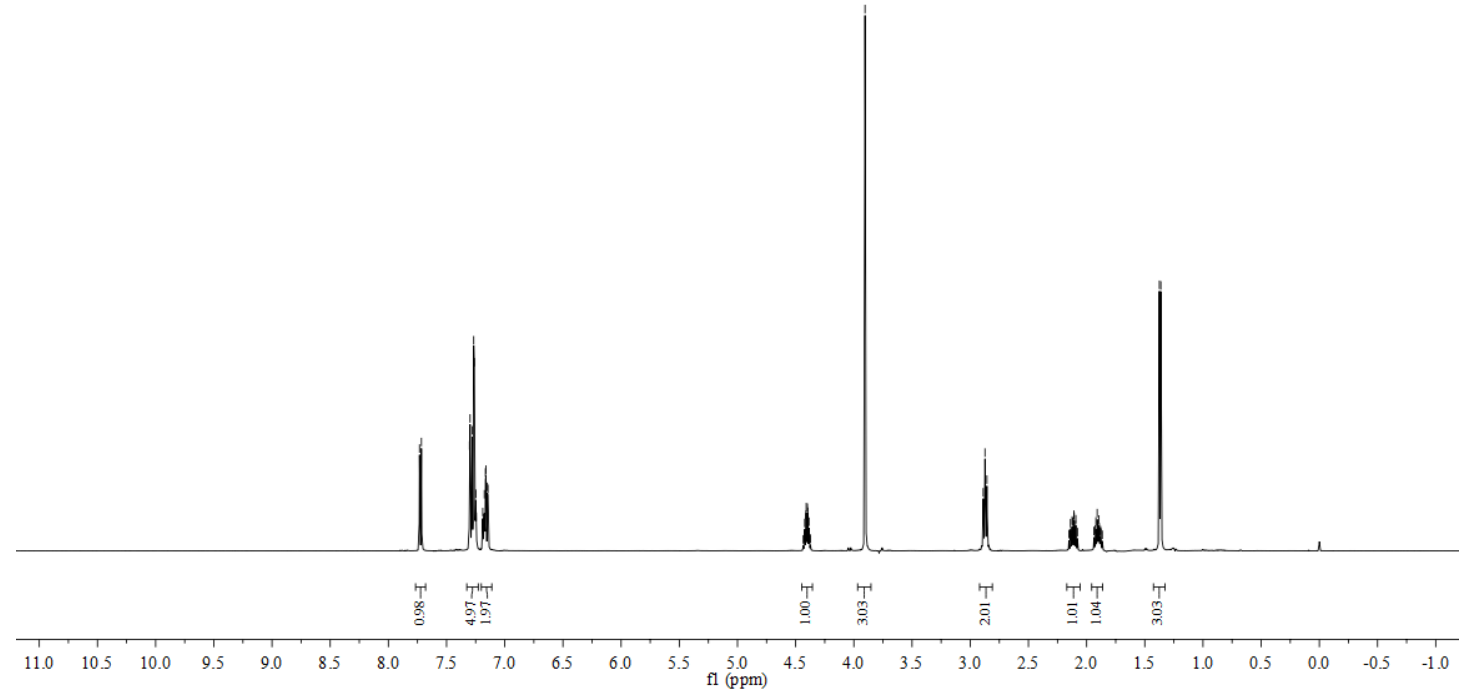

${ }^{13} \mathrm{C}$ NMR spectrum of $\mathbf{1 3}$

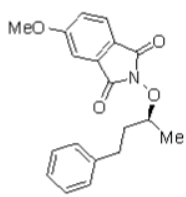

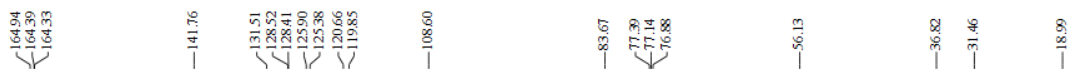

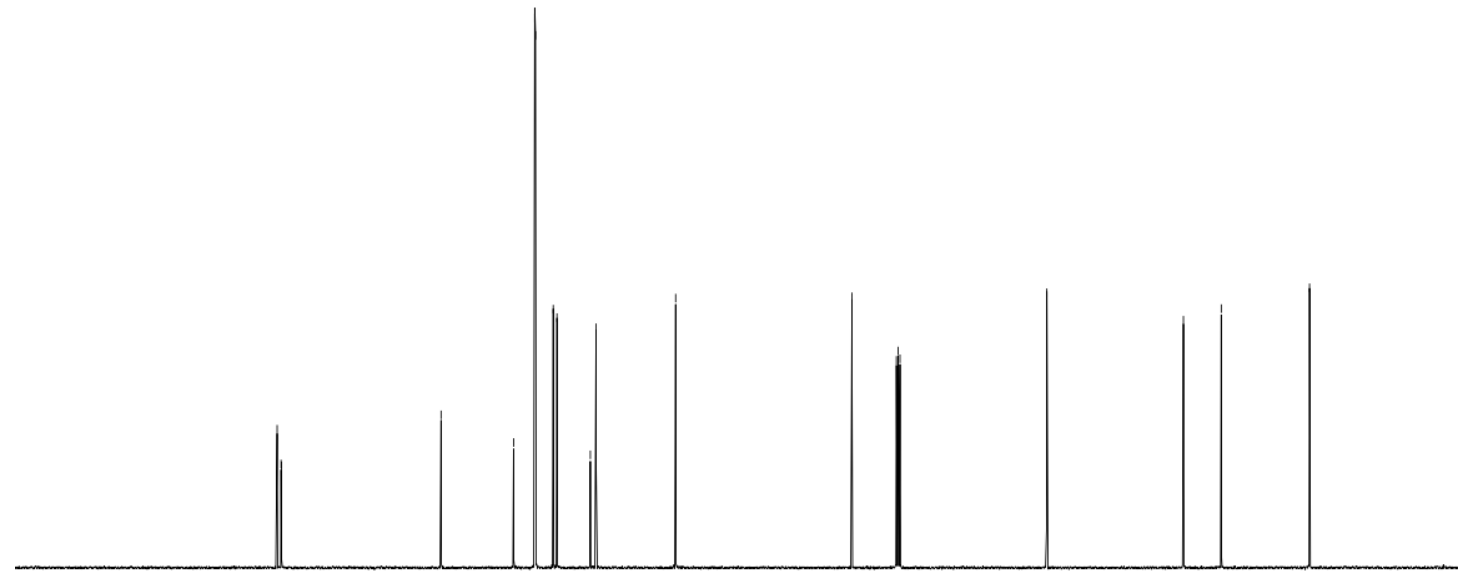

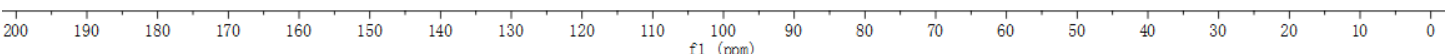


${ }^{1} \mathrm{H}$ NMR spectrum of $\mathbf{1 4}$

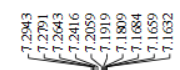

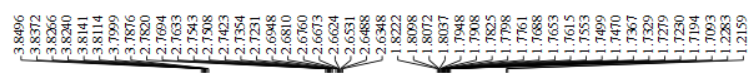

$a^{\prime}$

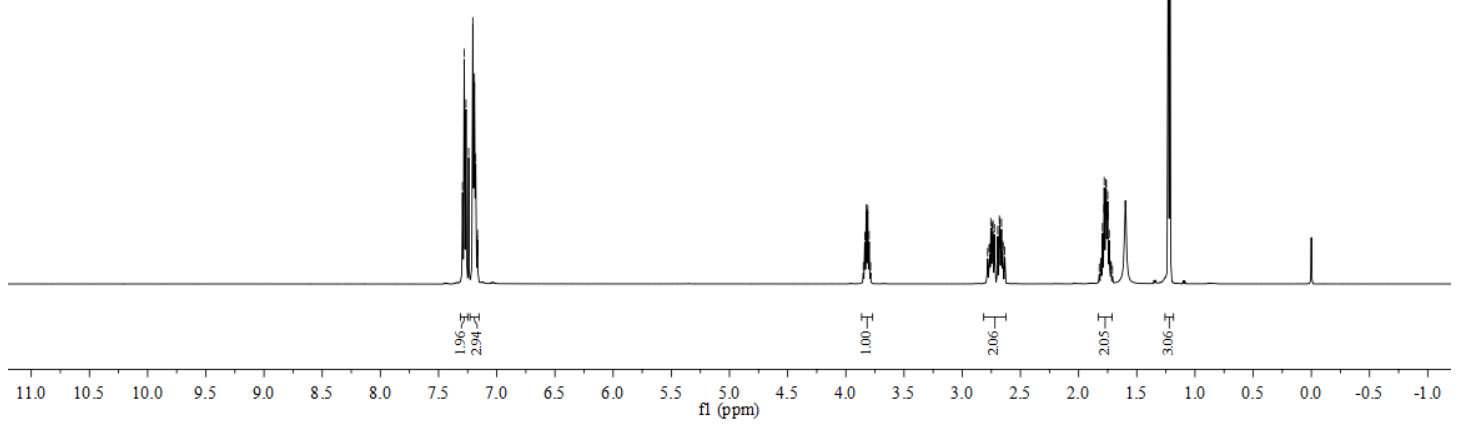

${ }^{13} \mathrm{C}$ NMR spectrum of $\mathbf{1 4}$

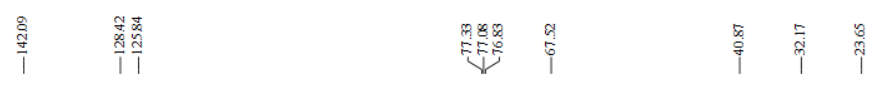
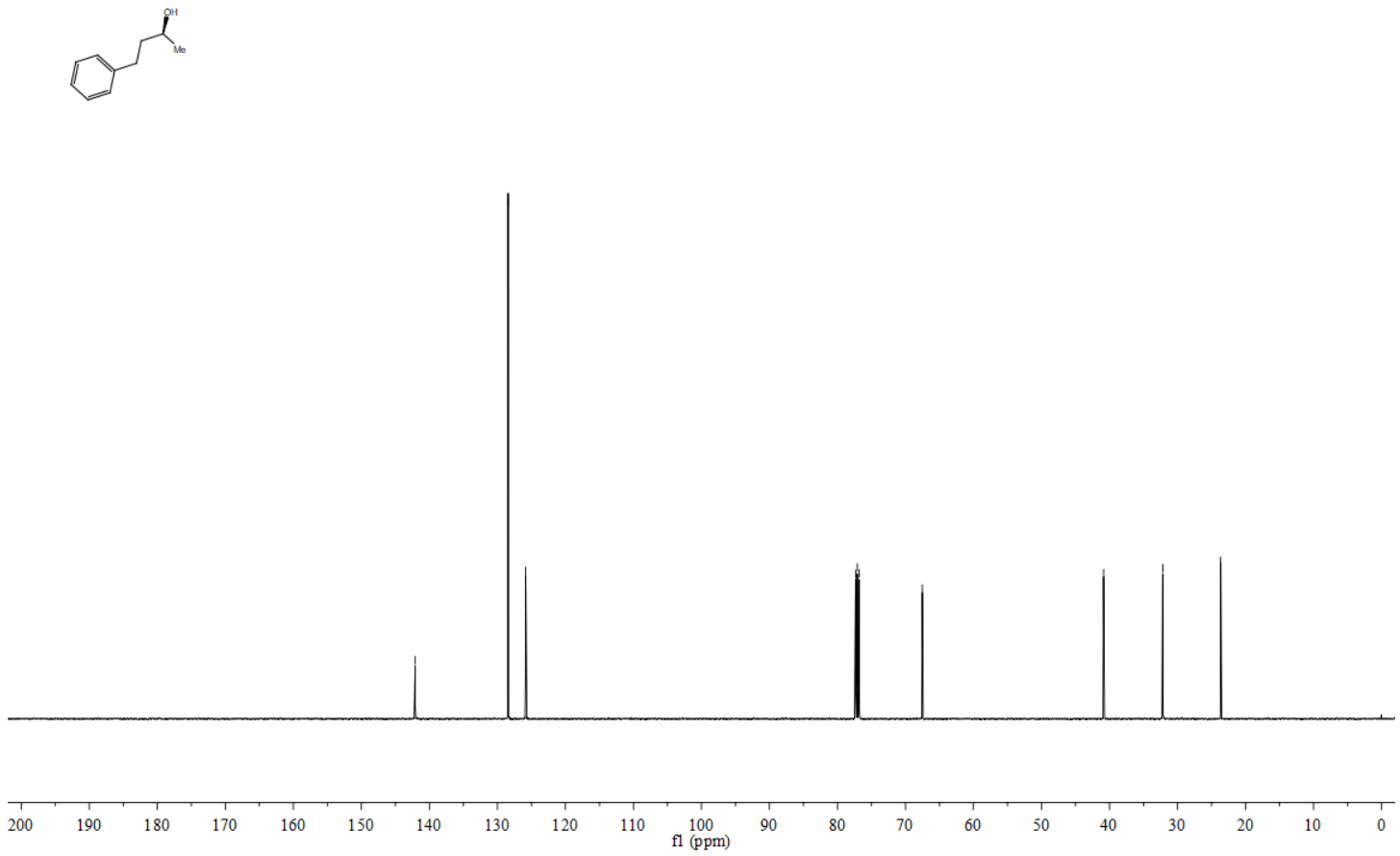

79 
${ }^{1} \mathrm{H}$ NMR spectrum of $\mathbf{1 5}$

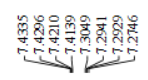

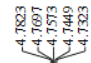

渻警<smiles>[3H][TeH]</smiles>

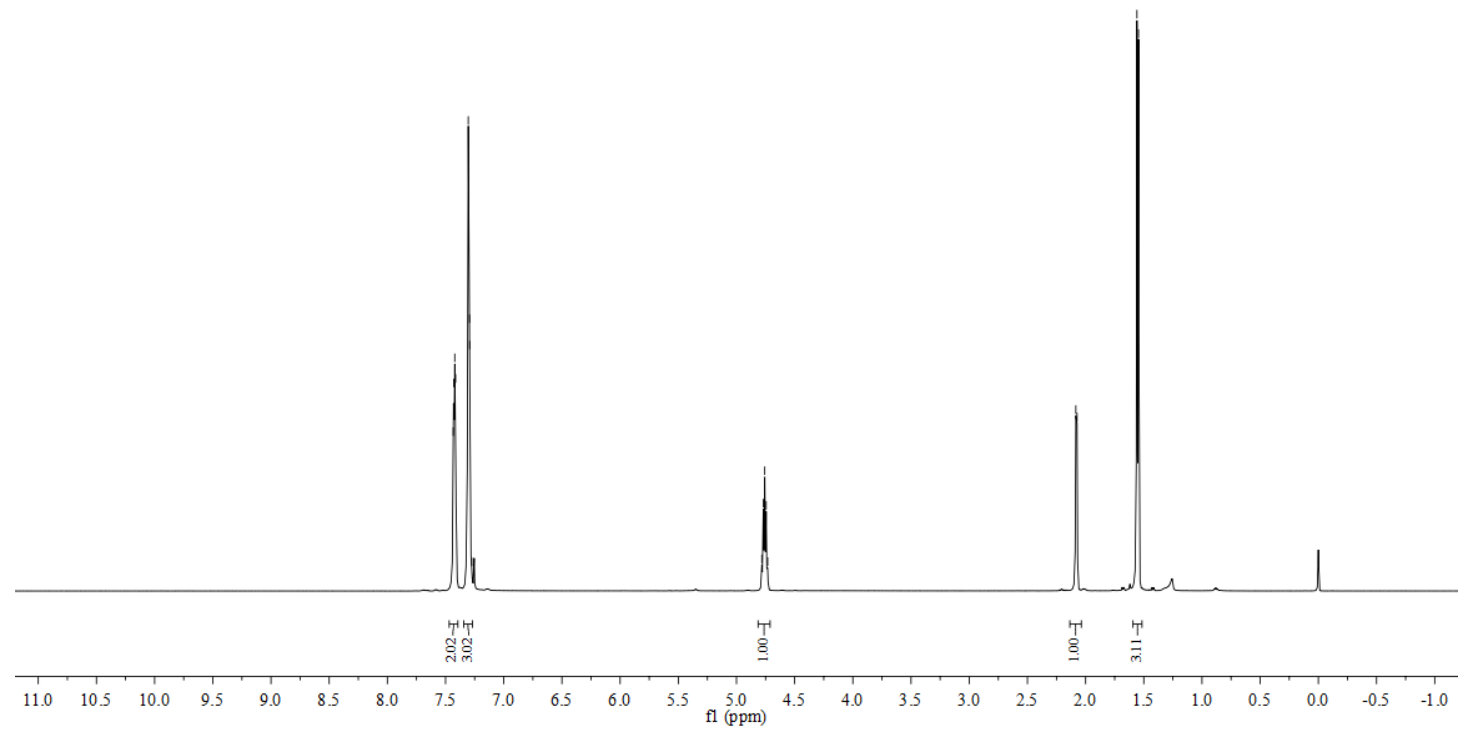

${ }^{13} \mathrm{C}$ NMR spectrum of $\mathbf{1 5}$

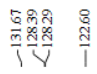
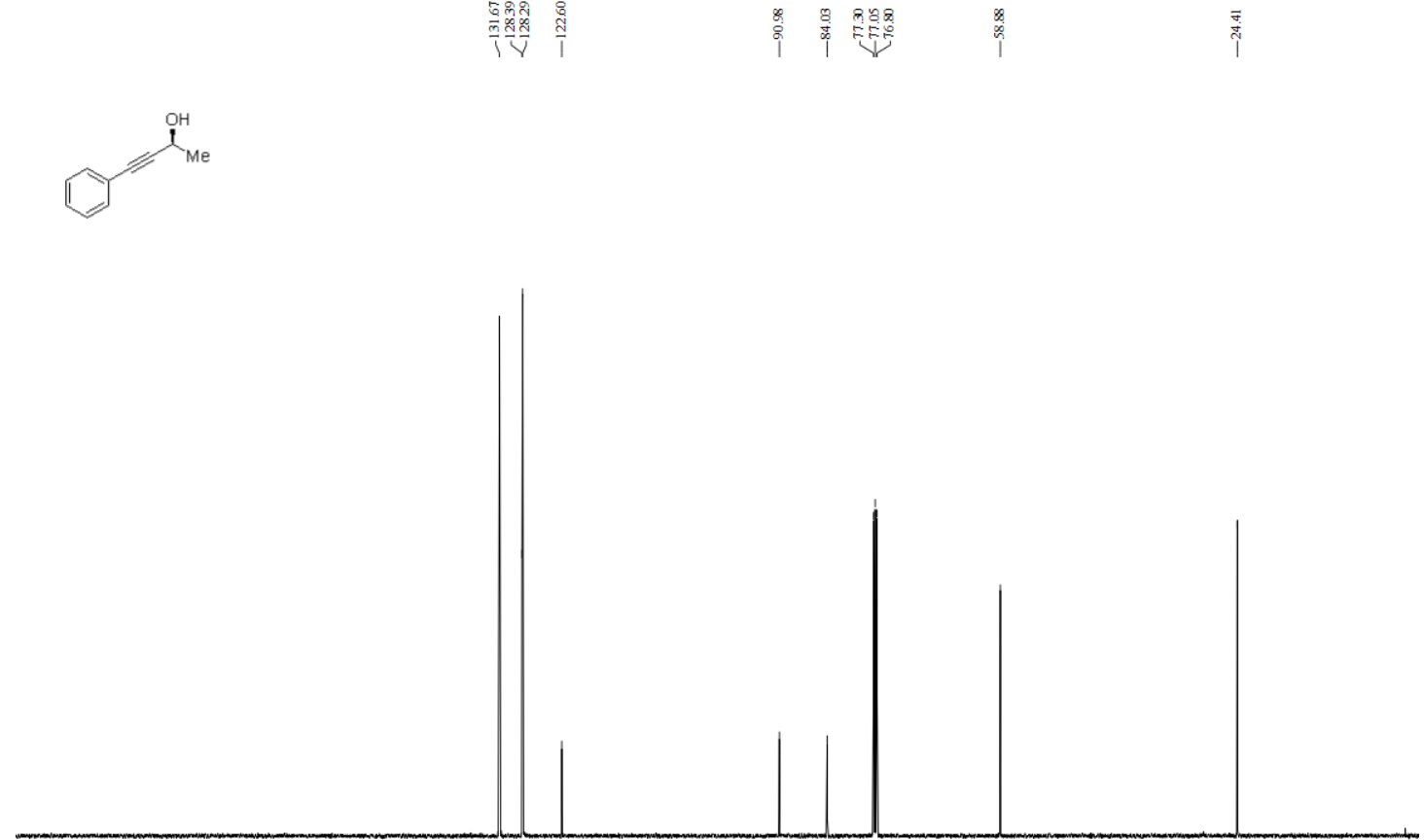

20
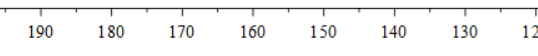

$10 \quad \begin{array}{r}100 \\ \mathrm{fl}(\mathrm{ppm})\end{array}$ 
${ }^{1} \mathrm{H}$ NMR spectrum of $\mathbf{1 6}$

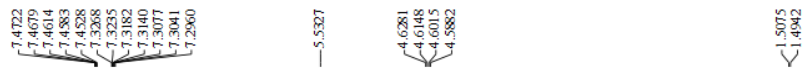<smiles>NC=Cc1ccccc1</smiles>

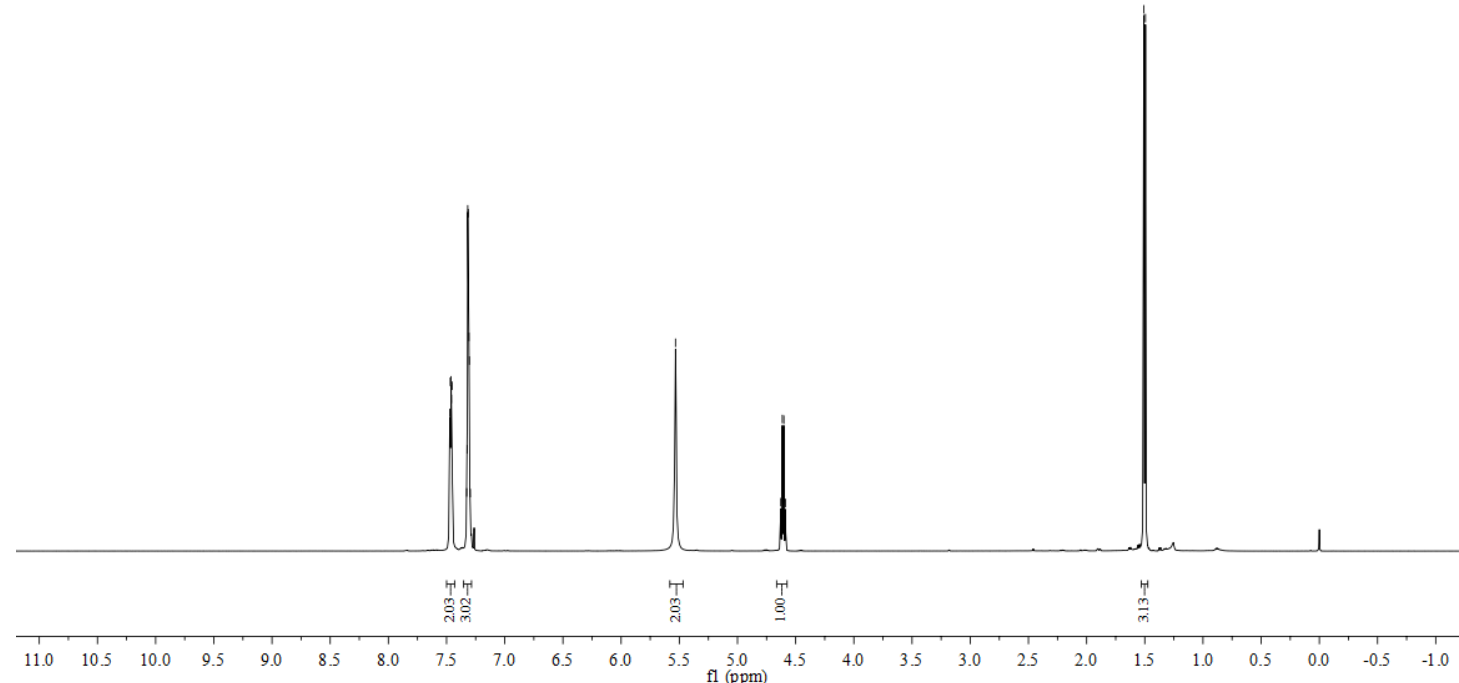

${ }^{13} \mathrm{C}$ NMR spectrum of $\mathbf{1 6}$

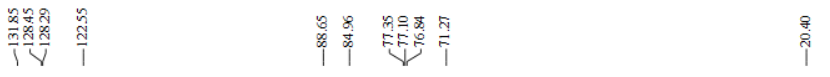<smiles>CC(C)=CC1CCC1</smiles>

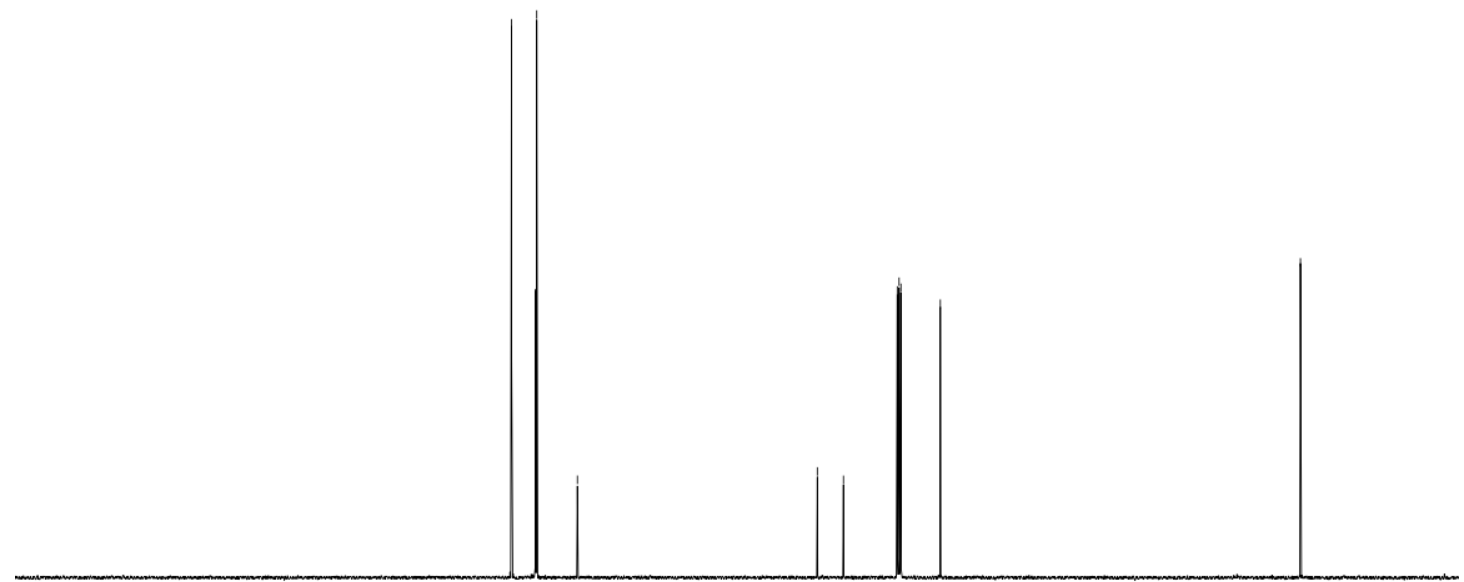

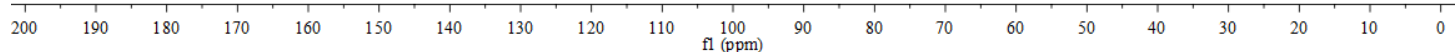


${ }^{1} \mathrm{H}$ NMR spectrum of $\mathbf{1 6 b}$

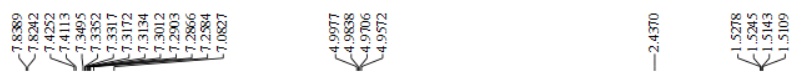

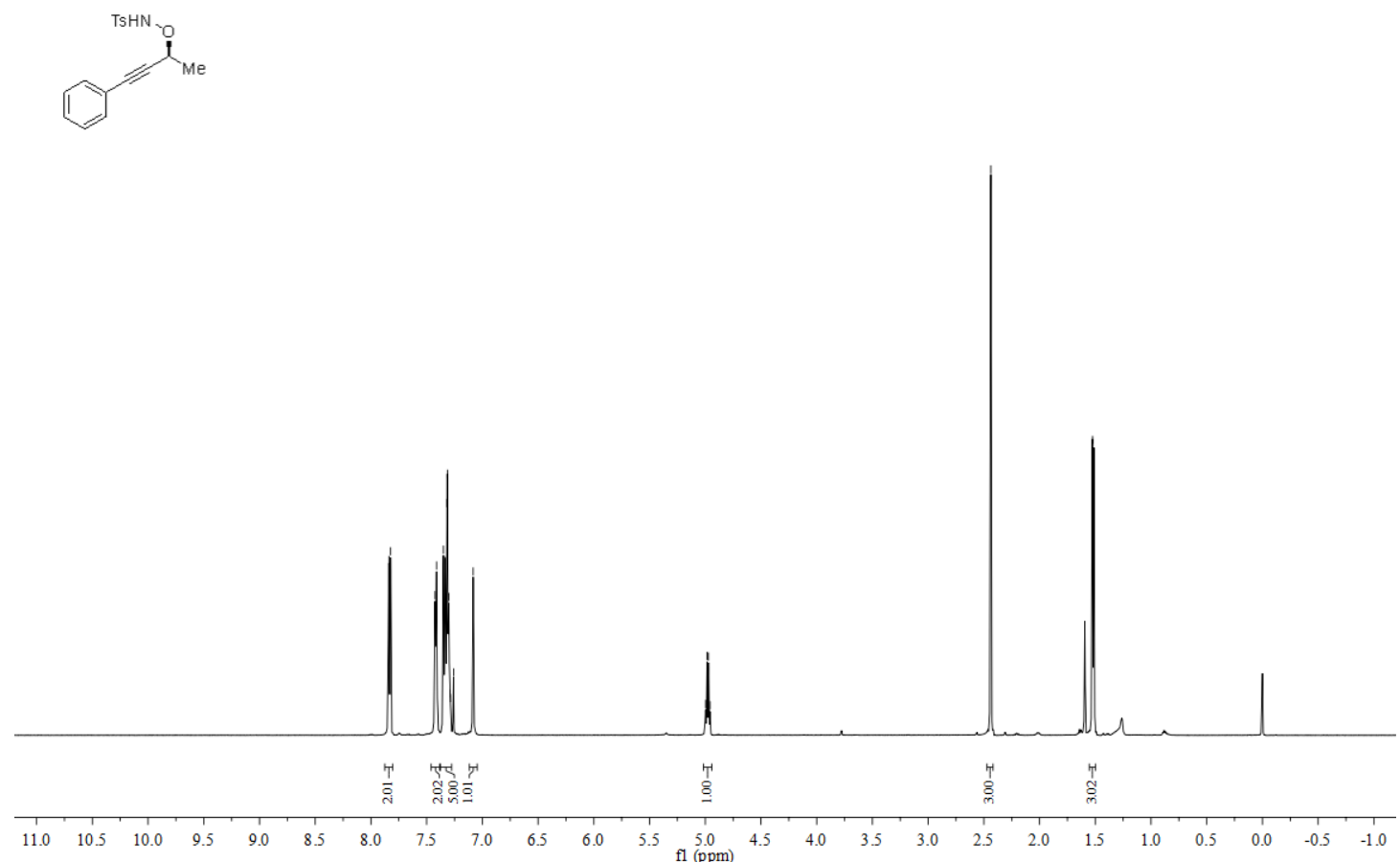

${ }^{13} \mathrm{C}$ NMR spectrum of $\mathbf{1 6 b}$

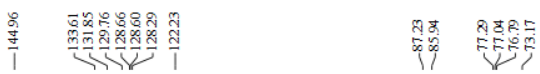

พิก
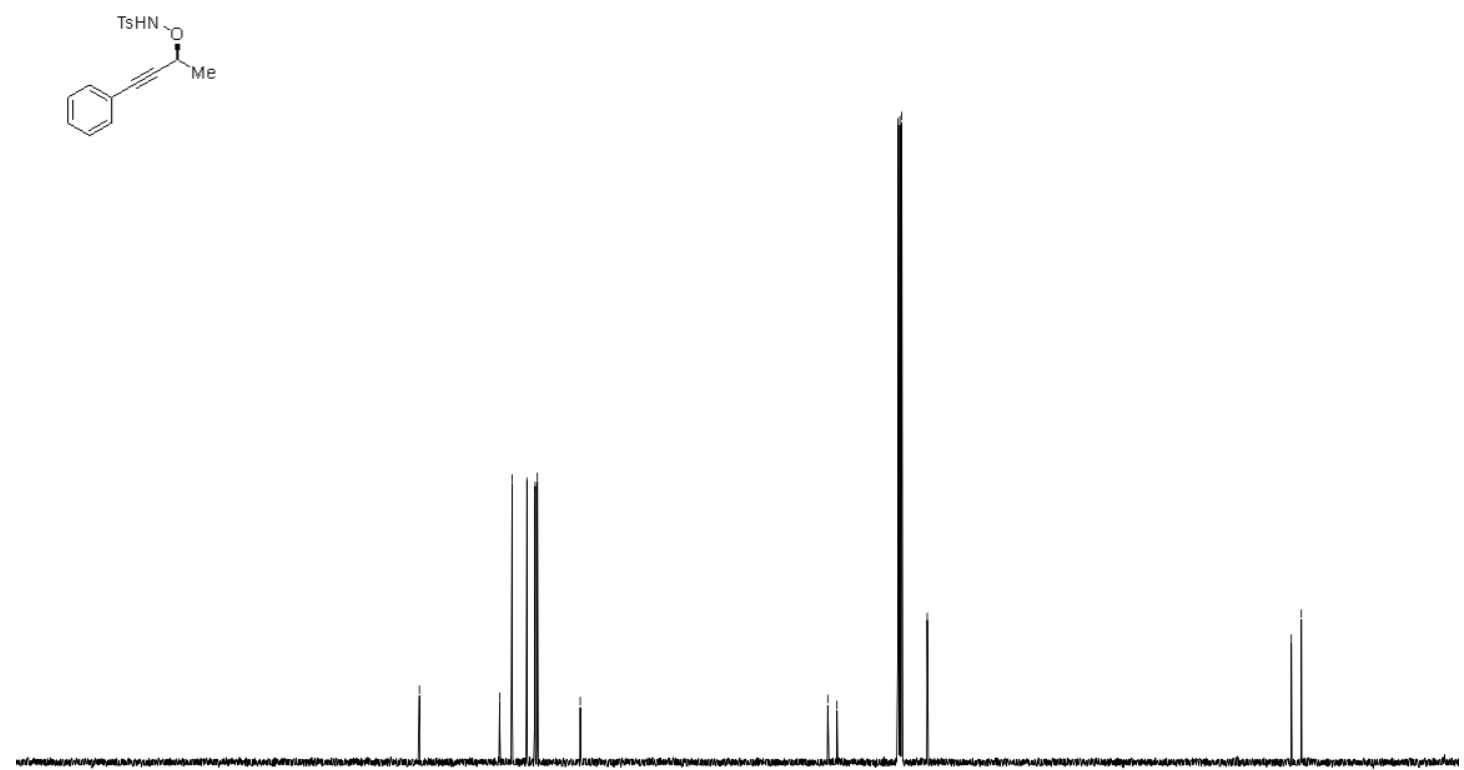

200
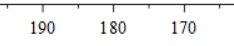

150

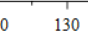

100 
${ }^{1} \mathrm{H}$ NMR spectrum of $\mathbf{1 7}$

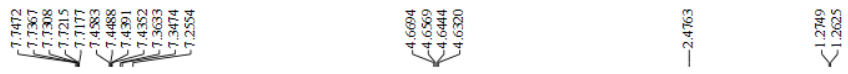
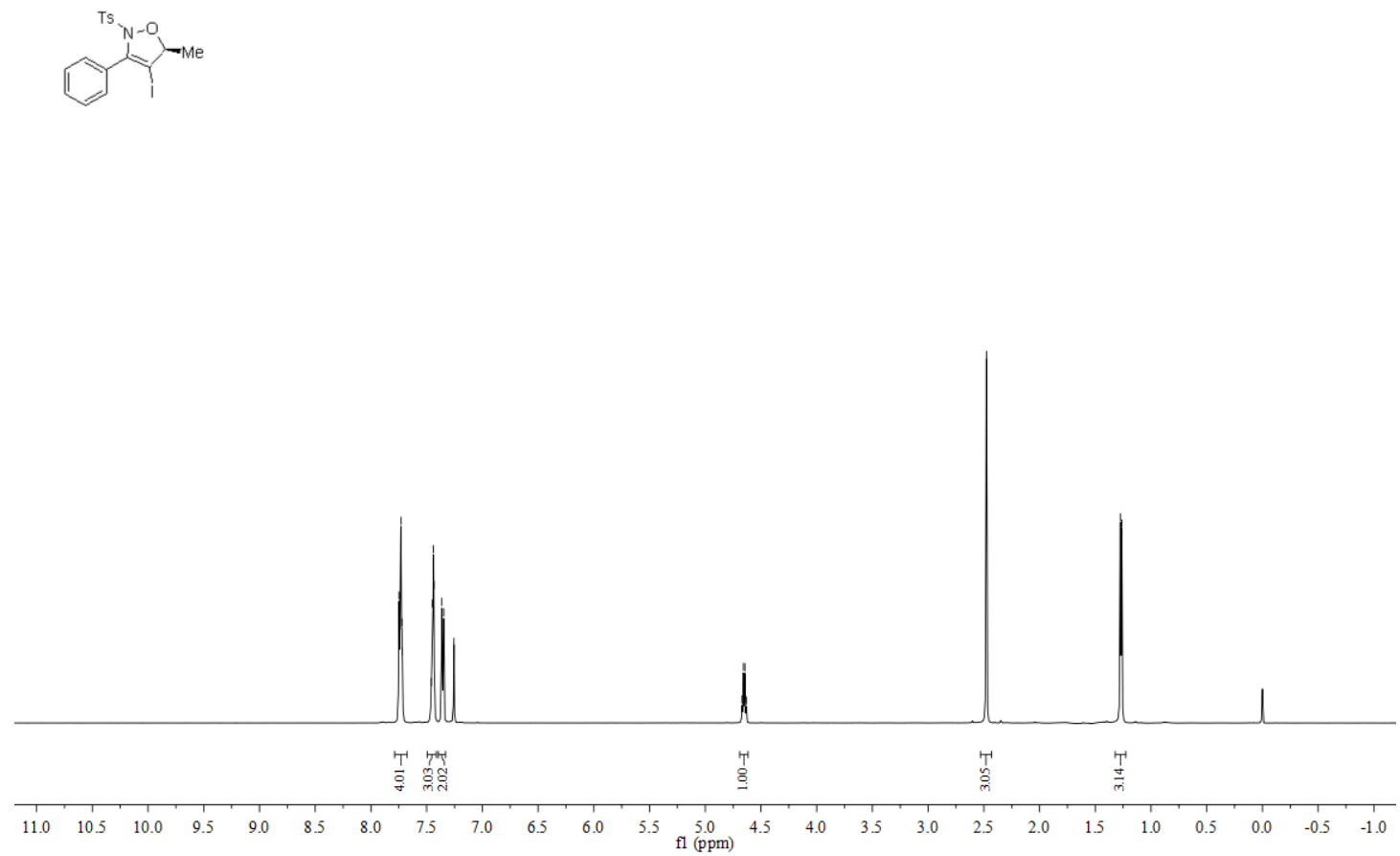

${ }^{13} \mathrm{C}$ NMR spectrum of $\mathbf{1 7}$
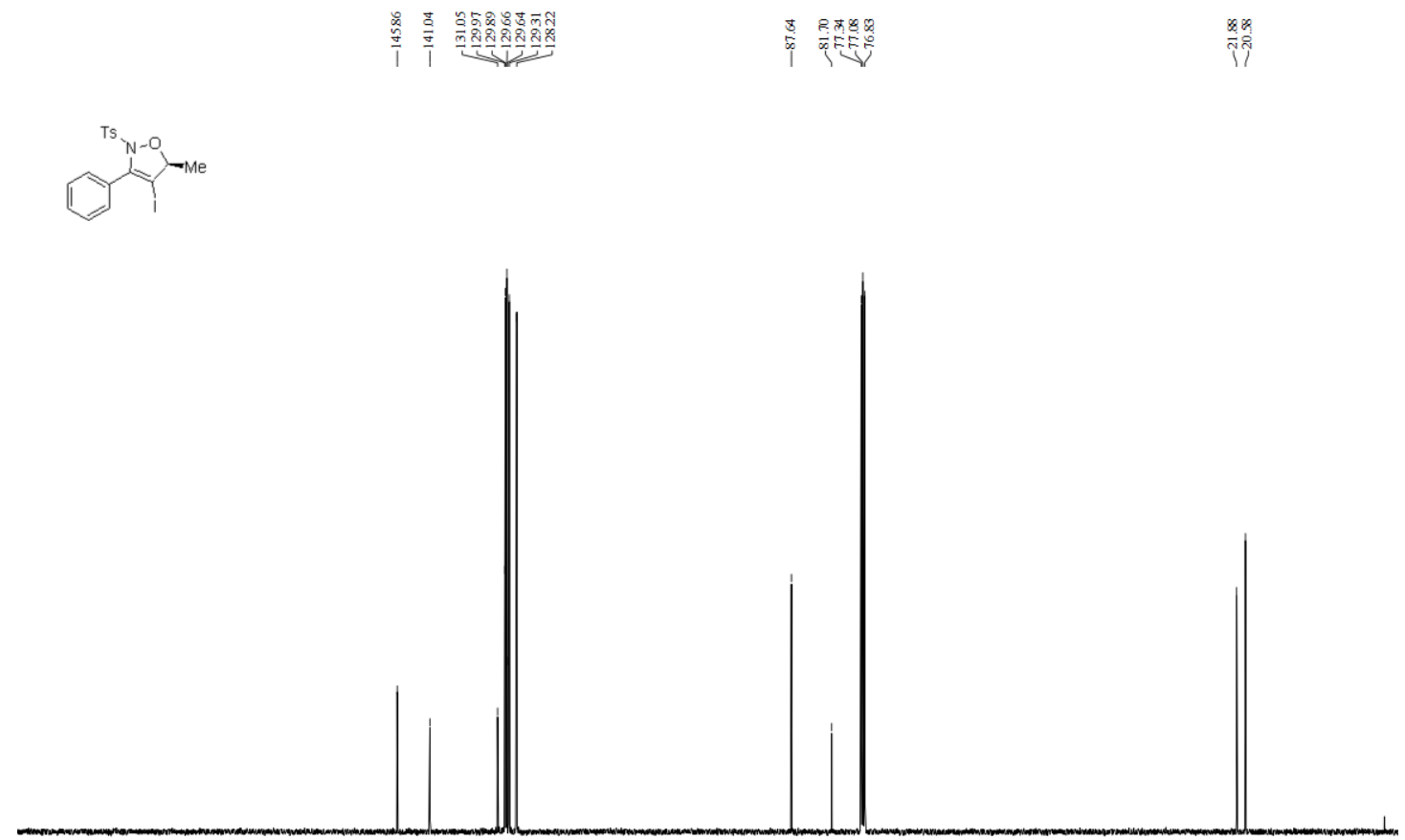

200

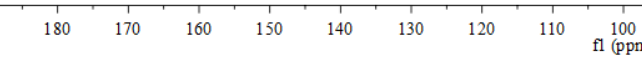

100
$(\mathrm{ppm})$ 


\section{HPLC of products}

\section{Rac-3aa}

\begin{tabular}{|llll|}
\hline \multicolumn{2}{c|}{ S A M P L E } & IN F O R M A T I O N \\
\hline \hline Sample Name: & xxh-3-124-rac-IC-30\% & Acquired By: & System \\
Sample Type: & Unknown & Sample Set Name & \\
Vial: & 50 & Acq. Method Set: & $30 \%$ quanbo \\
Injection \#: & 1 & Processing Method & 3124 \\
Injection Volume: & $10.00 \mathrm{ul}$ & Channel Name: & $254.0 \mathrm{~nm}$ \\
Run Time: & 100.0 Minutes & Proc. Chnl. Descr.: & 2998 PDA 254.0 nm (2998 \\
Date Acquired: & $1 / 2 / 20204: 31: 23$ PM CST & & \\
Date Processed: & $3 / 24 / 20218: 25: 54$ PM CST & & \\
\hline
\end{tabular}

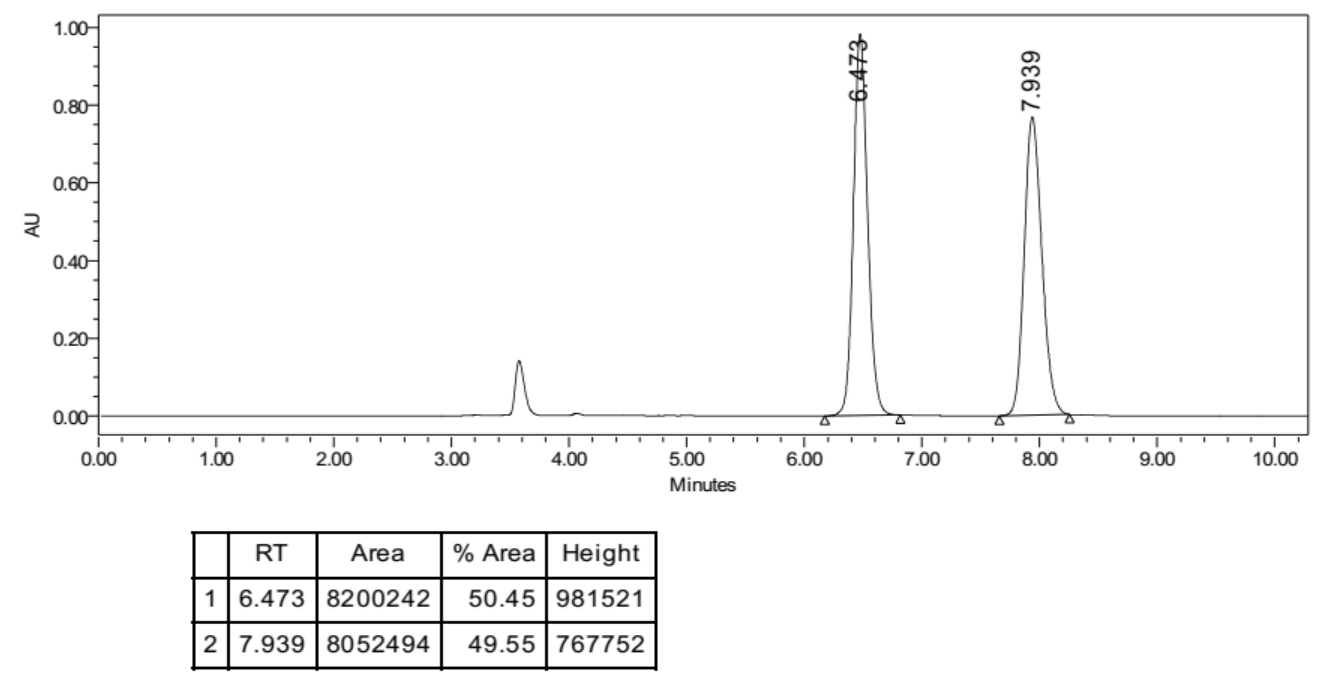

Asy-3aa

\begin{tabular}{|llll|}
\hline & \multicolumn{2}{c|}{ S A M P L E } & IN F O R M A T I O N \\
\hline \hline Sample Name: & xxh-6-189-IC-30\% & Acquired By: & System \\
Sample Type: & Unknown & Sample Set Name & 20200107 \\
Vial: & 81 & Acq. Method Set: & $30 \%$ quanbo \\
Injection \#: & 1 & Processing Method & 6189 \\
Injection Volume: & 10.00 ul & Channel Name: & $254.0 \mathrm{~nm}$ \\
Run Time: & 11.0 Minutes & Proc. Chnl. Descr.: & 2998 PDA 254.0 nm (2998 \\
& & & \\
Date Acquired: & $1 / 7 / 20219: 46: 18$ AM CST & & \\
Date Processed: & $3 / 24 / 20218: 18: 27$ PM CST & & \\
\hline
\end{tabular}

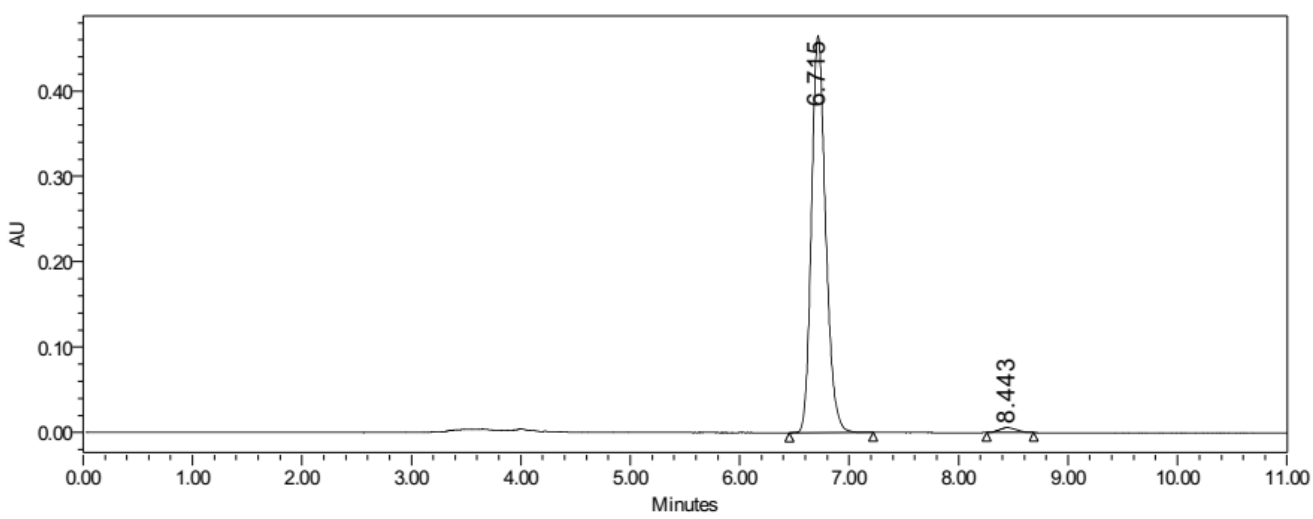

\begin{tabular}{|r|c|r|r|r|}
\hline & RT & \multicolumn{1}{|c|}{ Area } & $\%$ Area & Height \\
\hline 1 & 6.715 & 4167818 & 98.51 & 464892 \\
\hline 2 & 8.443 & 63239 & 1.49 & 5740 \\
\hline
\end{tabular}


Rac-3ab

\begin{tabular}{|llll|}
\hline & \multicolumn{2}{c|}{ S A M P L E } & IN F O R M A T I O N \\
\hline \hline Sample Name: & xxh-7-4-rac-IC-30\% & Acquired By: & System \\
Sample Type: & Unknown & Sample Set Name & 20200115 \\
Vial: & 82 & Acq. Method Set: & $30 \%$ quanbo \\
Injection \#: & 1 & Processing Method & 74 \\
Injection Volume: & 10.00 ul & Channel Name: & $254.0 \mathrm{~nm}$ \\
Run Time: & 13.0 Minutes & Proc. Chnl. Descr.: & 2998 PDA 254.0 nm (2998 \\
& & & \\
Date Acquired: & 1/15/2021 5:26:05 PM CST & & \\
Date Processed: & $3 / 24 / 20219: 46: 02$ PM CST & & \\
\hline
\end{tabular}

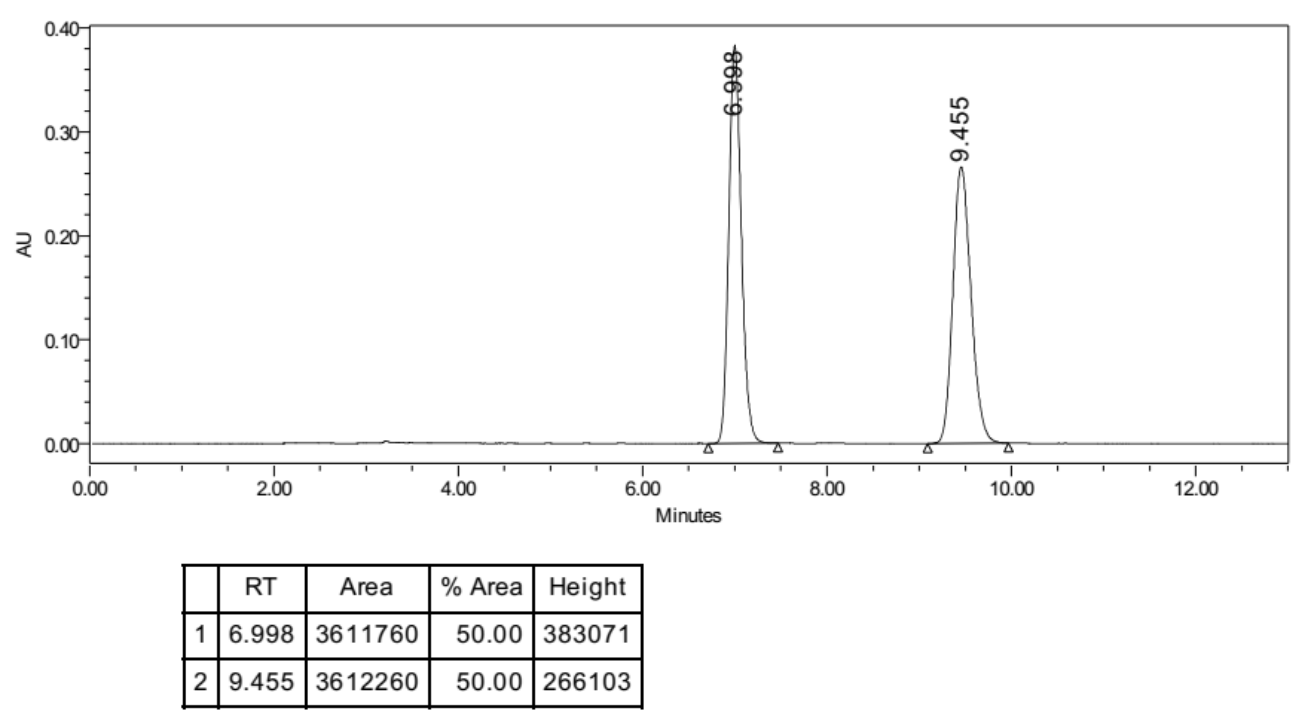

\section{Asy-3ab}

\begin{tabular}{|llll|}
\hline & \multicolumn{2}{c|}{ S A M P L E } & IN F O R M A T I O N \\
\hline \hline Sample Name: & xxh-6-196-IC-30\% & Acquired By: & System \\
Sample Type: & Unknown & Sample Set Name & 20200112 \\
Vial: & 82 & Acq. Method Set: & $30 \%$ quanbo \\
Injection \#: & 1 & Processing Method & 6196 \\
Injection Volume: & 10.00 ul & Channel Name: & $254.0 \mathrm{~nm}$ \\
Run Time: & 13.0 Minutes & Proc. Chnl. Descr.: & 2998 PDA 254.0 nm (2998 \\
& & & \\
Date Acquired: & $1 / 12 / 2021$ 9:54:57 AM CST & & \\
Date Processed: & $3 / 24 / 20219: 46: 58$ PM CST & & \\
\hline
\end{tabular}

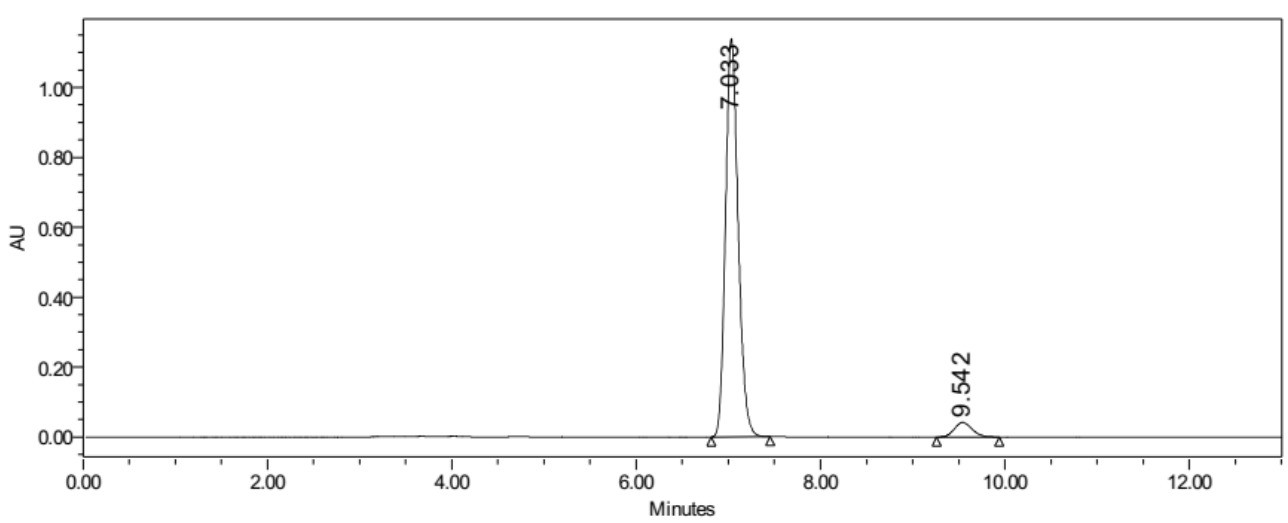

\begin{tabular}{|c|c|r|r|r|}
\hline & RT & \multicolumn{1}{|c|}{ Area } & \% Area & Height \\
\hline 1 & 7.033 & 10806344 & 95.05 & 1138077 \\
\hline 2 & 9.542 & 562730 & 4.95 & 41698 \\
\hline
\end{tabular}




\section{Rac-3ac}

\begin{tabular}{|llll|}
\hline & \multicolumn{2}{c|}{ S A M P L E } & IN F O R M A T I O N \\
\hline \hline Sample Name: & xxh-7-33-rac-IC-30\% & Acquired By: & System \\
Sample Type: & Unknown & Sample Set Name & 20210119 \\
Vial: & 61 & Acq. Method Set: & $30 \%$ quanbo \\
Injection \#: & 1 & Processing Method & 733 \\
Injection Volume: & 10.00 ul & Channel Name: & $254.0 \mathrm{~nm}$ \\
Run Time: & 14.0 Minutes & Proc. Chnl. Descr.: & 2998 PDA 254.0 nm (2998 \\
& & & \\
Date Acquired: & $1 / 20 / 20215: 33: 46$ AM CST & & \\
Date Processed: & $3 / 24 / 20219: 29: 04$ PM CST & & \\
\hline
\end{tabular}

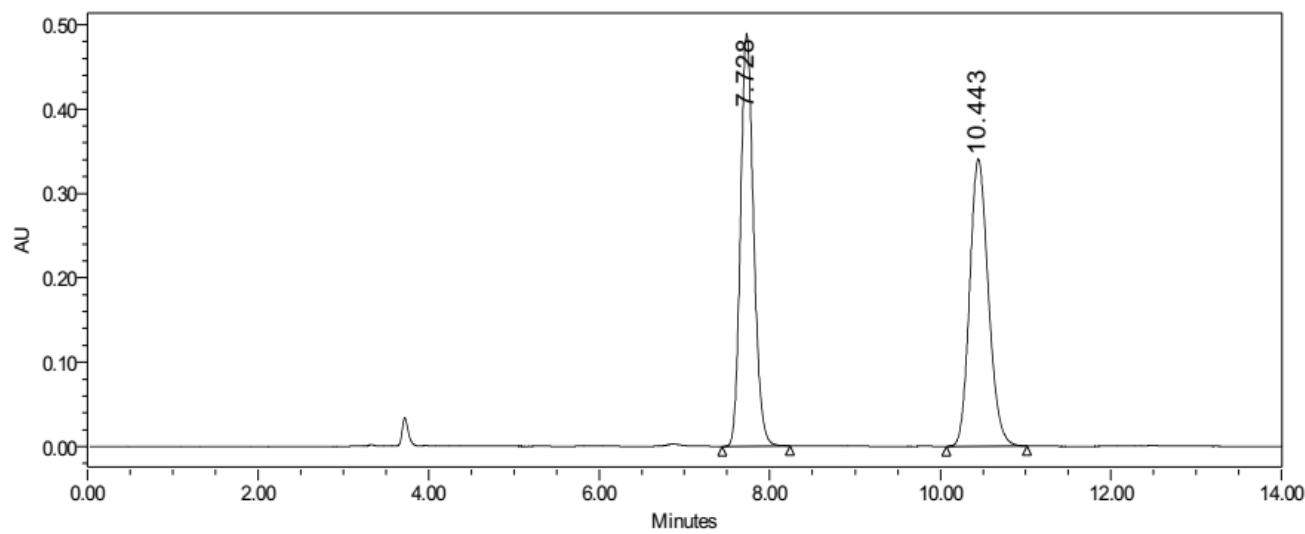

\begin{tabular}{|c|c|c|r|c|}
\hline & RT & Area & $\%$ Area & Height \\
\hline 1 & 7.728 & 5183430 & 50.00 & 488983 \\
\hline 2 & 10.443 & 5183434 & 50.00 & 340837 \\
\hline
\end{tabular}

\section{Asy-3ac}

\begin{tabular}{|llll|}
\hline & \multicolumn{2}{c|}{ S A M P L E } & IN F O R M A T I O N \\
\hline \hline Sample Name: & xxh-7-197-IC-30\% & Acquired By: & System \\
Sample Type: & Unknown & Sample Set Name & 20210318 \\
Vial: & 78 & Acq. Method Set: & $30 \%$ quanbo \\
Injection \#: & 1 & Processing Method & 6197 \\
Injection Volume: & 10.00 ul & Channel Name: & $254.0 \mathrm{~nm}$ \\
Run Time: & 15.0 Minutes & Proc. Chnl. Descr.: & 2998 PDA 254.0 nm (2998 \\
& & & \\
Date Acquired: & $3 / 18 / 20219: 11: 24$ AM CST & & \\
Date Processed: & $3 / 24 / 20219: 30: 24$ PM CST & & \\
\hline
\end{tabular}

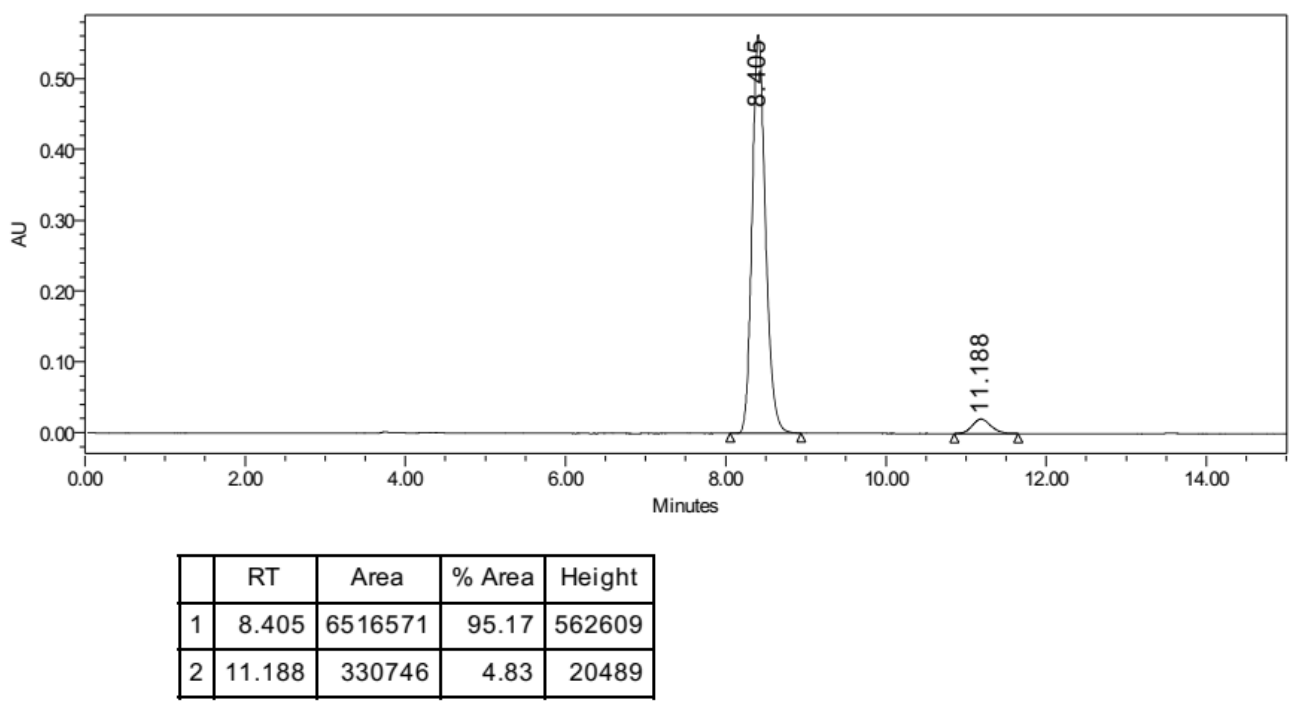


Rac-3ad

\begin{tabular}{|llll|}
\hline & \multicolumn{2}{c|}{ S A M P L E } & IN F O R M A T I O N \\
\hline \hline Sample Name: & xxh-7-32-rac-IC-30\% & Acquired By: & System \\
Sample Type: & Unknown & Sample Set Name & 20210119 \\
Vial: & 60 & Acq. Method Set: & $30 \%$ quanbo \\
Injection \#: & 1 & Processing Method & 732 \\
Injection Volume: & 10.00 ul & Channel Name: & $254.0 \mathrm{~nm}$ \\
Run Time: & 38.0 Minutes & Proc. Chnl. Descr.: & 2998 PDA 254.0 nm (2998 \\
& & & \\
Date Acquired: & $1 / 20 / 20214: 55: 03$ AM CST & & \\
Date Processed: & $3 / 24 / 20219: 20: 59$ PM CST & & \\
\hline
\end{tabular}

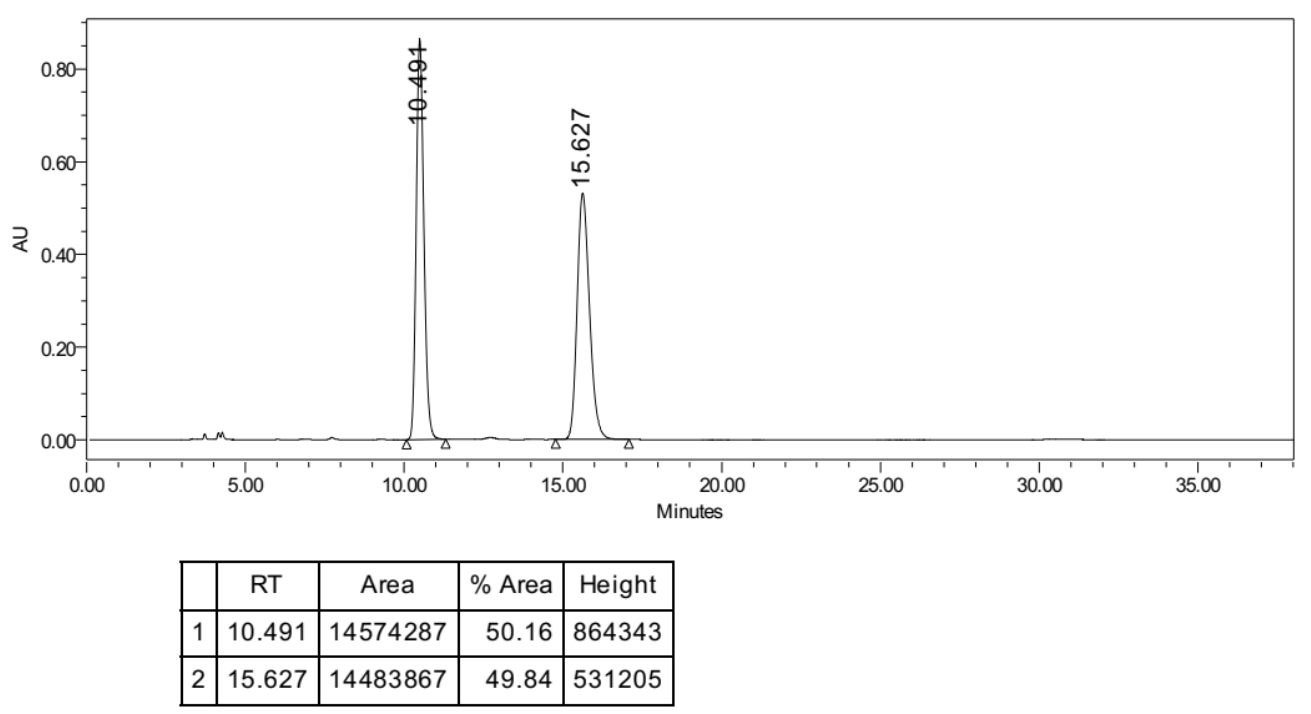

Asy-3ad

\begin{tabular}{|llll|}
\hline & \multicolumn{2}{c|}{ S A M P L E } & IN F O R M A T I O N \\
\hline \hline Sample Name: & xxh-6-194-IC-30\% & Acquired By: & System \\
Sample Type: & Unknown & Sample Set Name & 20200111 \\
Vial: & 83 & Acq. Method Set: & $30 \%$ quanbo \\
Injection \#: & 1 & Processing Method & 61942 \\
Injection Volume: & 10.00 ul & Channel Name: & $254.0 \mathrm{~nm}$ \\
Run Time: & 19.0 Minutes & Proc. Chnl. Descr.: & 2998 PDA 254.0 nm (2998 \\
& & & \\
Date Acquired: & $1 / 11 / 20218: 18: 23$ PM CST & & \\
Date Processed: & $5 / 20 / 202111: 29: 01$ PM CST & & \\
\hline
\end{tabular}

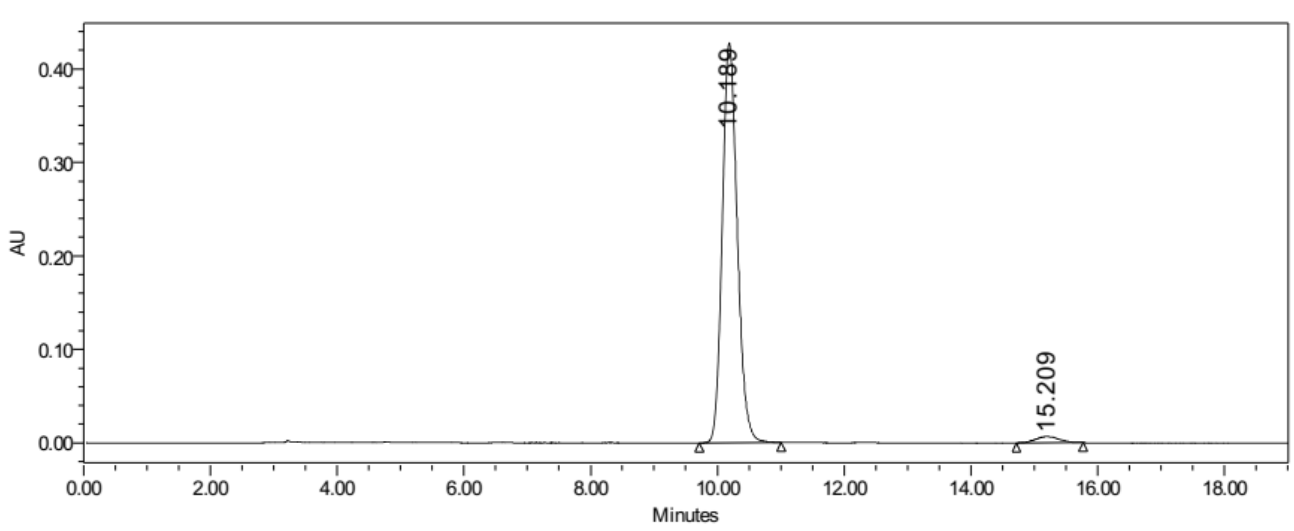

\begin{tabular}{|c|c|r|r|r|}
\hline & RT & Area & $\%$ Area & Height \\
\hline 1 & 10.189 & 7016376 & 97.57 & 427600 \\
\hline 2 & 15.209 & 174503 & 2.43 & 6681 \\
\hline
\end{tabular}




\section{Rac-3ae}

\begin{tabular}{|llll|}
\hline & \multicolumn{2}{c|}{ S A M P L E } & IN F O R M A T I O N \\
\hline \hline Sample Name: & xxh-7-3-rac-IC-30\% & Acquired By: & System \\
Sample Type: & Unknown & Sample Set Name & 20200115 \\
Vial: & 81 & Acq. Method Set: & $30 \%$ quanbo \\
Injection \#: & 1 & Processing Method & 73 \\
Injection Volume: & 10.00 ul & Channel Name: & $254.0 \mathrm{~nm}$ \\
Run Time: & 17.0 Minutes & Proc. Chnl. Descr.: & 2998 PDA 254.0 nm (2998 \\
& & & \\
Date Acquired: & $1 / 15 / 2021$ 5:08:24 PM CST & & \\
Date Processed: & $3 / 24 / 2021$ 8:29:01 PM CST & & \\
\hline
\end{tabular}

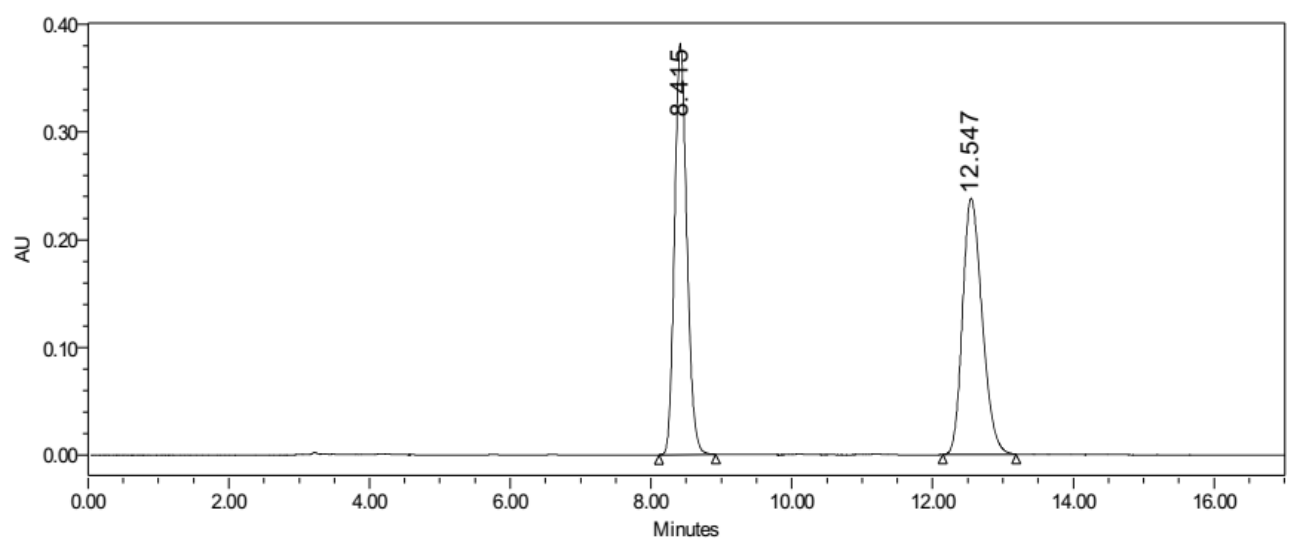

\begin{tabular}{|c|c|c|r|c|}
\hline & \multicolumn{1}{|c|}{ RT } & Area & $\%$ Area & Height \\
\hline 1 & 8.415 & 4576027 & 49.99 & 381871 \\
\hline 2 & 12.547 & 4577702 & 50.01 & 237887 \\
\hline
\end{tabular}

\section{Asy-3ae}

\begin{tabular}{|llll|}
\hline & \multicolumn{2}{c|}{ S A M P L E } & IN F O R M A T I O N \\
\hline \hline Sample Name: & xxh-6-195-IC-30\% & Acquired By: & System \\
Sample Type: & Unknown & Sample Set Name & 20200112 \\
Vial: & 81 & Acq. Method Set: & $30 \%$ quanbo \\
Injection \#: & 1 & Processing Method & 6195 \\
Injection Volume: & 10.00 ul & Channel Name: & $254.0 \mathrm{~nm}$ \\
Run Time: & 17.0 Minutes & Proc. Chnl. Descr.: & 2998 PDA 254.0 nm (2998 \\
& & & \\
Date Acquired: & $1 / 12 / 20219: 37: 15$ AM CST & & \\
Date Processed: & $3 / 24 / 20218: 30: 50$ PM CST & & \\
\hline
\end{tabular}

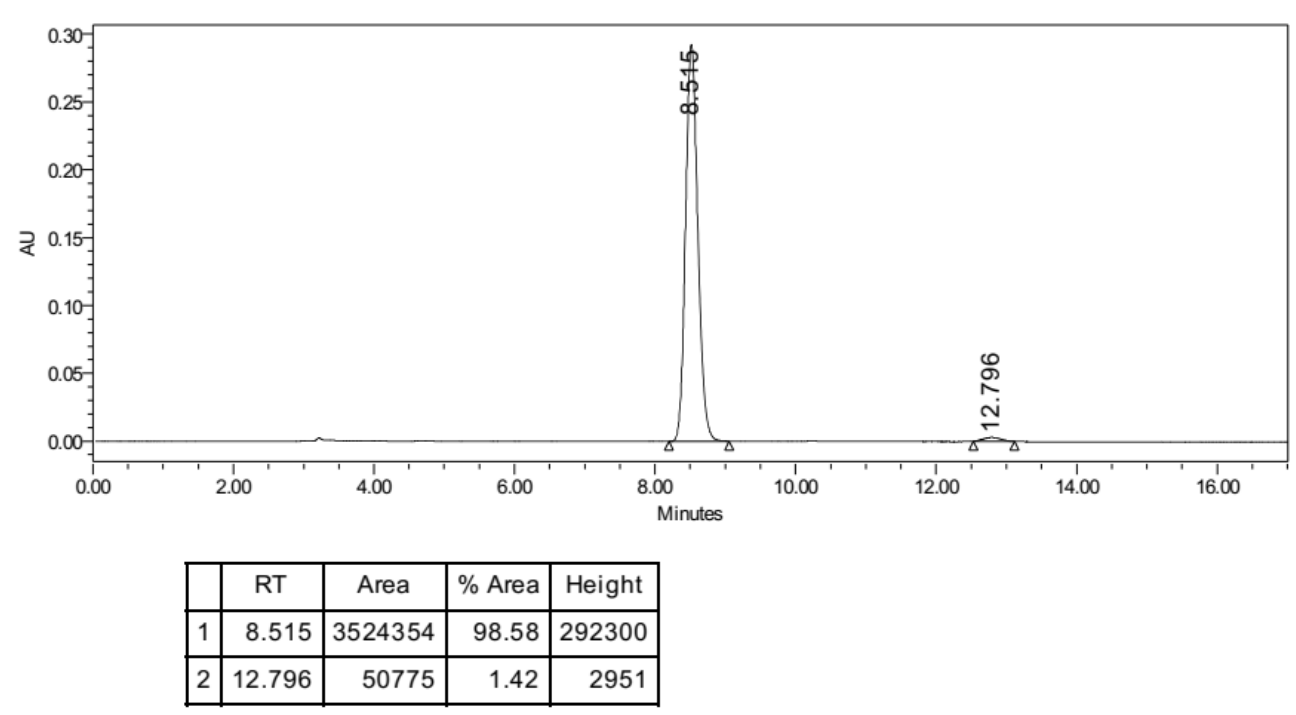


Rac-3af

\begin{tabular}{|llll|}
\hline & \multicolumn{2}{c|}{ S A M P L E } & IN F O R M A T I O N \\
\hline \hline Sample Name: & xxh-7-66-rac-IC-30\% & Acquired By: & System \\
Sample Type: & Unknown & Sample Set Name & 20210324 \\
Vial: & 13 & Acq. Method Set: & $30 \%$ quanbo \\
Injection \#: & 1 & Processing Method & 766 \\
Injection Volume: & 10.00 ul & Channel Name: & $254.0 \mathrm{~nm}$ \\
Run Time: & 20.0 Minutes & Proc. Chnl. Descr.: & 2998 PDA 254.0 nm (2998 \\
& & & \\
Date Acquired: & $3 / 24 / 20219: 12: 20$ PM CST & & \\
Date Processed: & $3 / 24 / 20219: 39: 37$ PM CST & & \\
\hline
\end{tabular}

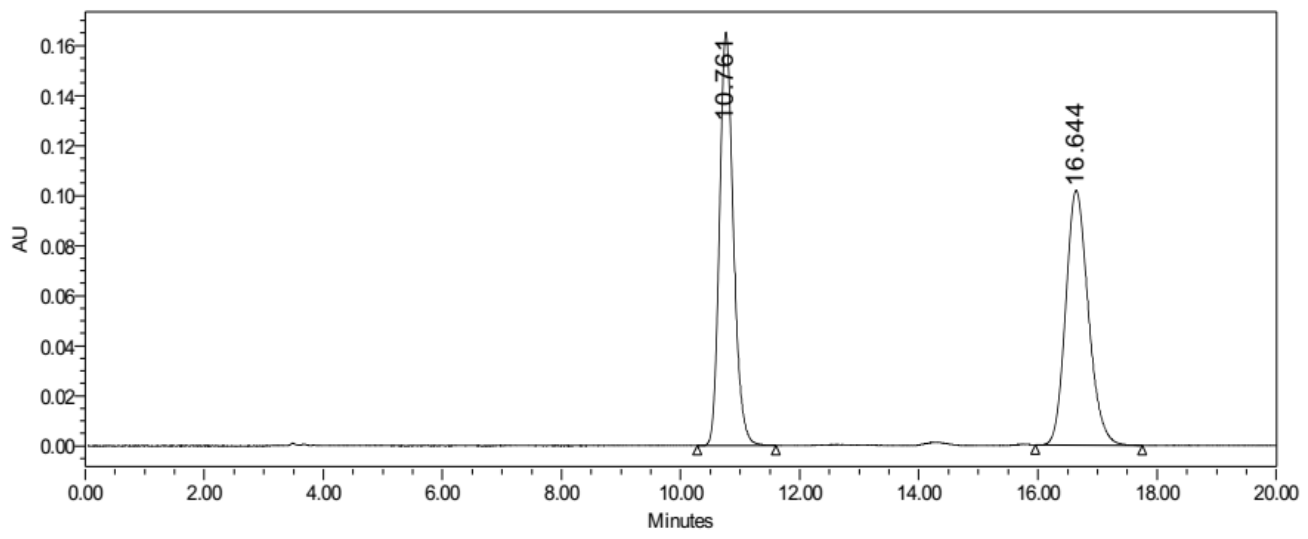

\begin{tabular}{|c|c|c|r|c|}
\hline & RT & Area & $\%$ Area & Height \\
\hline 1 & 10.761 & 2682149 & 50.00 & 165071 \\
\hline 2 & 16.644 & 2682530 & 50.00 & 101787 \\
\hline
\end{tabular}

\section{Asy-3af}

\begin{tabular}{|llll|}
\hline \multicolumn{2}{c|}{ S A M P L E } & I N F O R M A T I O N \\
\hline \hline Sample Name: & xxh-8-25-3-IC-30\% & Acquired By: & System \\
Sample Type: & Unknown & Sample Set Name & 20210323 \\
Vial: & 83 & Acq. Method Set: & $30 \%$ quanbo \\
Injection \#: & 1 & Processing Method 8253 \\
Injection Volume: & $10.00 \mathrm{ul}$ & Channel Name: & $254.0 \mathrm{~nm}$ \\
Run Time: & 22.0 Minutes & Proc. Chnl. Descr.: & 2998 PDA 254.0 nm (2998 \\
& & & \\
Date Acquired: & $3 / 23 / 2021$ 12:04:16 PM CST & & \\
Date Processed: & $3 / 24 / 20219: 41: 24$ PM CST & & \\
\hline
\end{tabular}

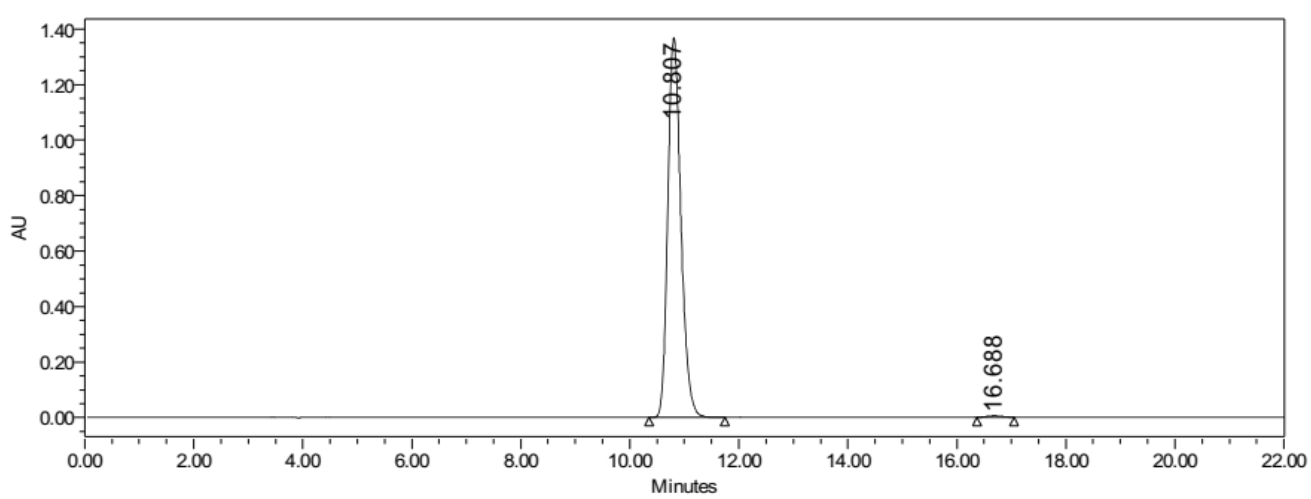

\begin{tabular}{|r|c|r|r|r|}
\hline & RT & \multicolumn{1}{|c|}{ Area } & $\%$ Area & Height \\
\hline 1 & 10.807 & 22502896 & 99.50 & 1368043 \\
\hline 2 & 16.688 & 112942 & 0.50 & 5204 \\
\hline
\end{tabular}




\section{Rac-3ag}

\begin{tabular}{|llll|}
\hline & \multicolumn{2}{c|}{ S A M P L E } & IN F O R M A T I O N \\
\hline \hline Sample Name: & xxh-6-170-rac-IC-30\% & Acquired By: & System \\
Sample Type: & Unknown & Sample Set Name & 20210324 \\
Vial: & 14 & Acq. Method Set: & $30 \%$ quanbo \\
Injection \#: & 1 & Processing Method & 6170 \\
Injection Volume: & 10.00 ul & Channel Name: & $254.0 \mathrm{~nm}$ \\
Run Time: & 66.0 Minutes & Proc. Chnl. Descr.: & 2998 PDA 254.0 nm (2998 \\
& & & \\
Date Acquired: & $3 / 24 / 2021$ 10:53:22 PM CST & & \\
Date Processed: & $3 / 25 / 20219: 20: 27$ AM CST & & \\
\hline
\end{tabular}

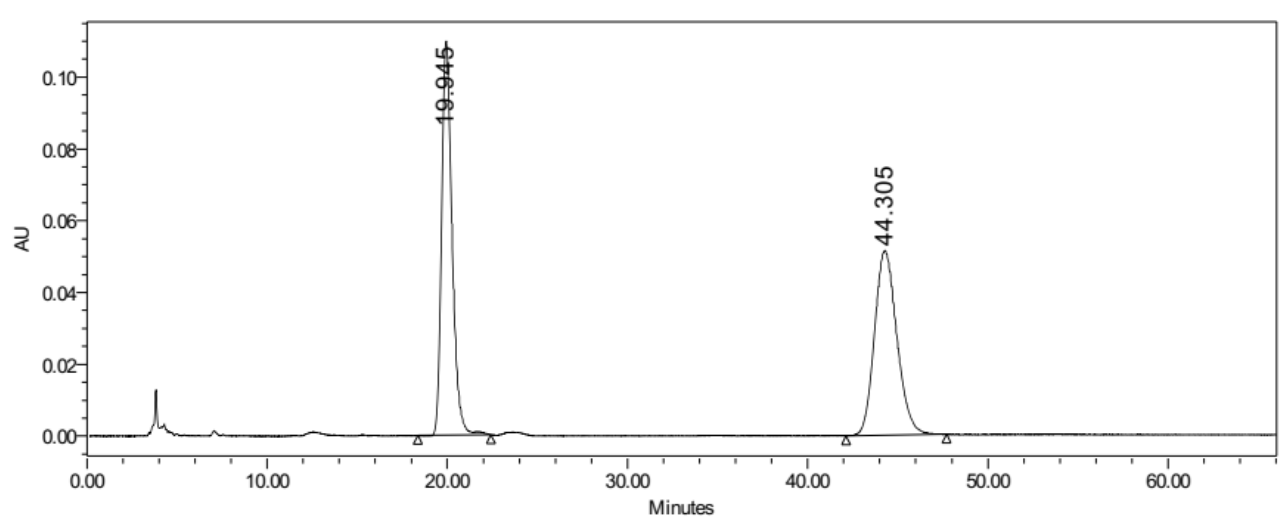

\begin{tabular}{|c|c|c|r|r|}
\hline & RT & Area & $\%$ Area & Height \\
\hline 1 & 19.945 & 4415856 & 50.00 & 109678 \\
\hline 2 & 44.305 & 4416611 & 50.00 & 51441 \\
\hline
\end{tabular}

\section{Asy-3ag}

\begin{tabular}{|llll|}
\hline & \multicolumn{2}{c|}{ S A M P L E } & IN F O R M A T I O N \\
\hline \hline Sample Name: & xxh-6-200-IC-30\% & Acquired By: & System \\
Sample Type: & Unknown & Sample Set Name & 20210324 \\
Vial: & 13 & Acq. Method Set: & $30 \%$ quanbo \\
Injection \#: & 1 & Processing Method & 6200 \\
Injection Volume: & 10.00 ul & Channel Name: & $254.0 \mathrm{~nm}$ \\
Run Time: & 66.0 Minutes & Proc. Chnl. Descr.: & 2998 PDA 254.0 nm (2998 \\
& & & \\
Date Acquired: & $3 / 24 / 20219: 46: 43$ PM CST & & \\
Date Processed: & $3 / 25 / 20219: 21: 44$ AM CST & & \\
\hline
\end{tabular}

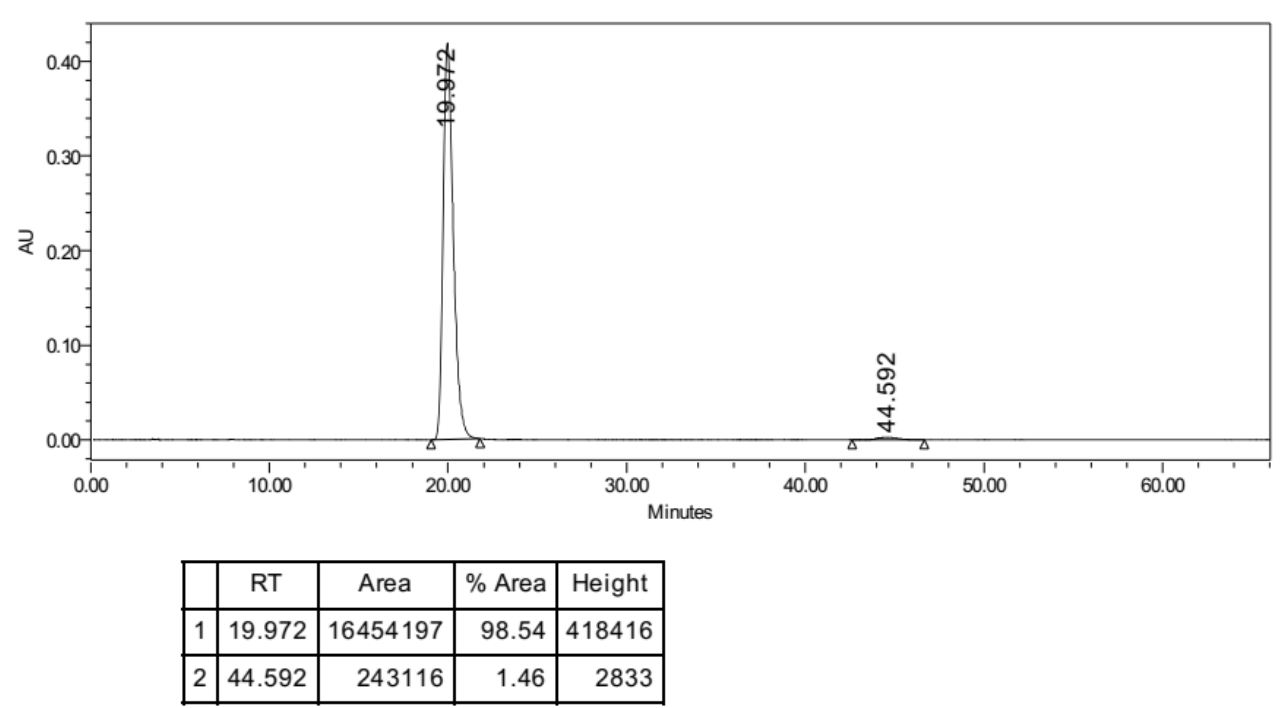




\section{Rac-3ah}

\begin{tabular}{|llll|}
\hline & \multicolumn{2}{c|}{ S A M P LE } & IN F O R M A T I O N \\
\hline \hline Sample Name: & xxh-7-34-rac-IC-30\% & Acquired By: & System \\
Sample Type: & Unknown & Sample Set Name & 20210119 \\
Vial: & 62 & Acq. Method Set: & $30 \%$ quanbo \\
Injection \#: & 1 & Processing Method & 734 \\
Injection Volume: & 10.00 ul & Channel Name: & $254.0 \mathrm{~nm}$ \\
Run Time: & 28.0 Minutes & Proc. Chnl. Descr.: & 2998 PDA 254.0 nm (2998 \\
& & & \\
Date Acquired: & $1 / 20 / 20215: 48: 27$ AM CST & & \\
Date Processed: & $3 / 24 / 2021$ 9:49:53 PM CST & & \\
\hline
\end{tabular}

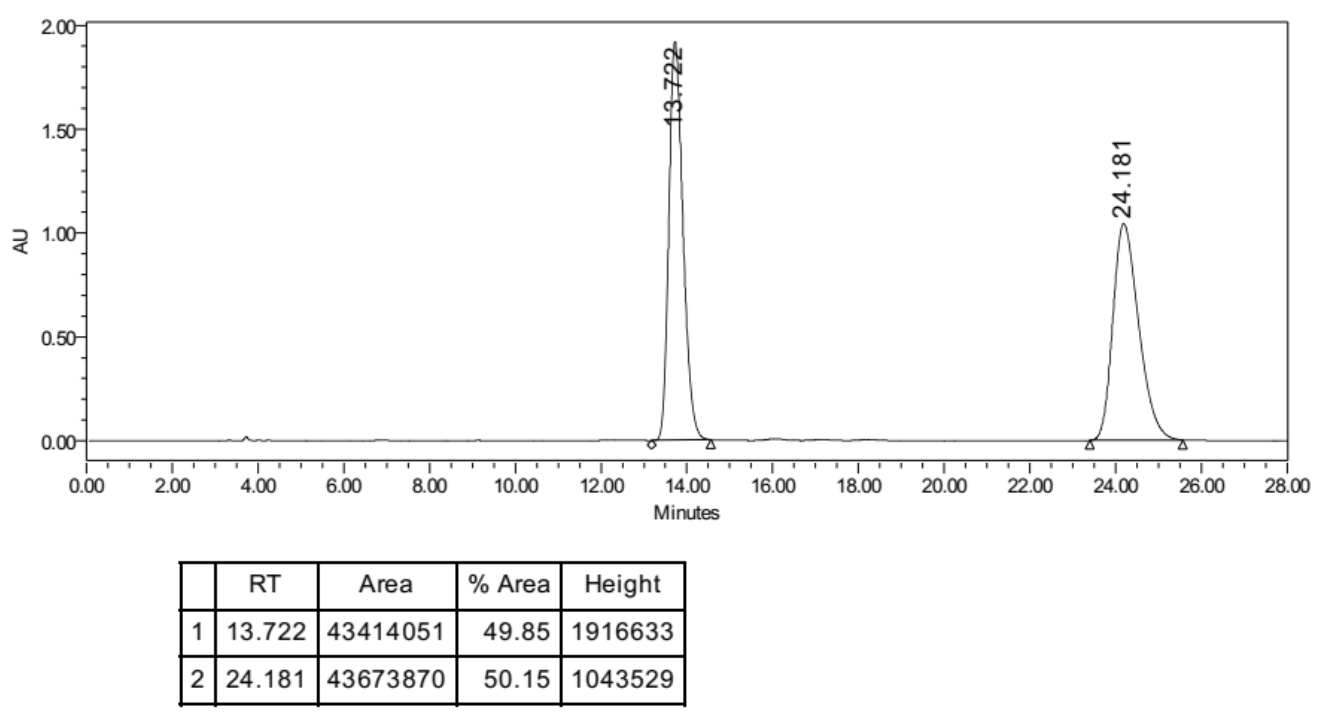

\section{Asy-3ah}

\begin{tabular}{|llll|}
\hline & \multicolumn{2}{c|}{ S A M P L E } & IN F O R M A T I O N \\
\hline \hline Sample Name: & xxh-6-199-IC-30\% & Acquired By: & System \\
Sample Type: & Unknown & Sample Set Name & 20200111 \\
Vial: & 86 & Acq. Method Set: & $30 \%$ quanbo \\
Injection \#: & 1 & Processing Method & 6199 \\
Injection Volume: & 10.00 ul & Channel Name: & $254.0 \mathrm{~nm}$ \\
Run Time: & 26.0 Minutes & Proc. Chnl. Descr.: & 2998 PDA 254.0 nm (2998 \\
& & & \\
Date Acquired: & $1 / 11 / 20219: 12: 25$ PM CST & & \\
Date Processed: & $3 / 24 / 20219: 50: 55$ PM CST & & \\
\hline
\end{tabular}

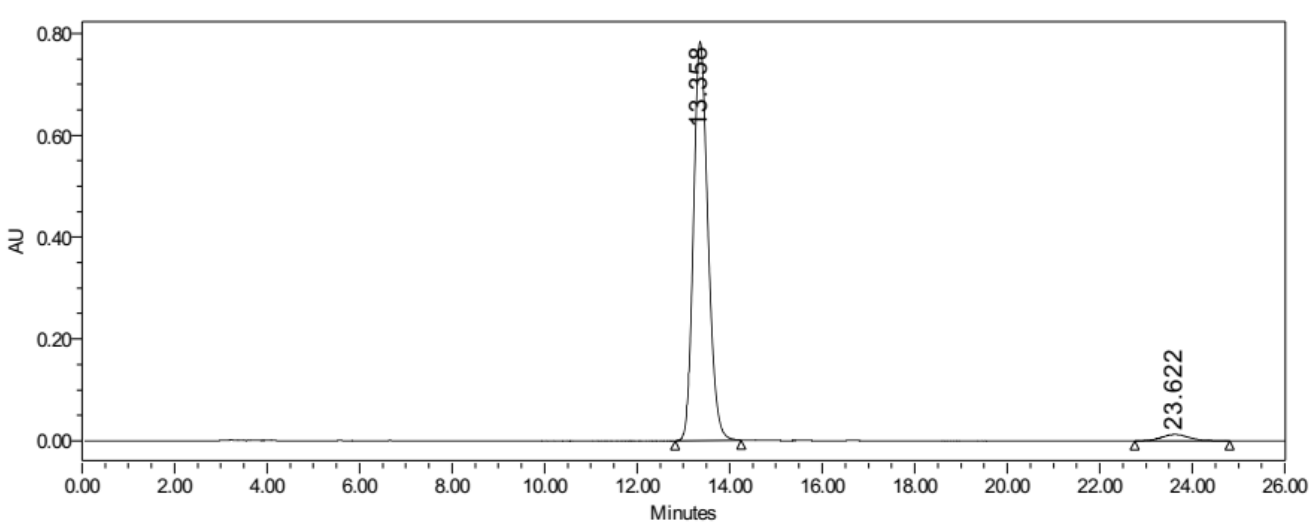

\begin{tabular}{|r|c|r|r|r|}
\hline & RT & \multicolumn{1}{|c|}{ Area } & $\%$ Area & Height \\
\hline 1 & 13.358 & 17114495 & 97.09 & 783435 \\
\hline 2 & 23.622 & 513595 & 2.91 & 12546 \\
\hline
\end{tabular}


Rac-3bf

\begin{tabular}{|llll|}
\hline & \multicolumn{2}{c|}{ S A M P L E } & IN F O R M A T I O N \\
\hline \hline Sample Name: & xxh-7-7-rac-IC-30\% & Acquired By: & System \\
Sample Type: & Unknown & Sample Set Name & 20200115 \\
Vial: & 84 & Acq. Method Set: & $30 \%$ quanbo \\
Injection \#: & 1 & Processing Method & 77 \\
Injection Volume: & 10.00 ul & Channel Name: & $254.0 \mathrm{~nm}$ \\
Run Time: & 19.0 Minutes & Proc. Chnl. Descr.: & 2998 PDA 254.0 nm (2998 \\
& & & \\
Date Acquired: & 1/15/2021 5:59:27 PM CST & & \\
Date Processed: & 3/24/2021 9:59:55 PM CST & & \\
\hline
\end{tabular}

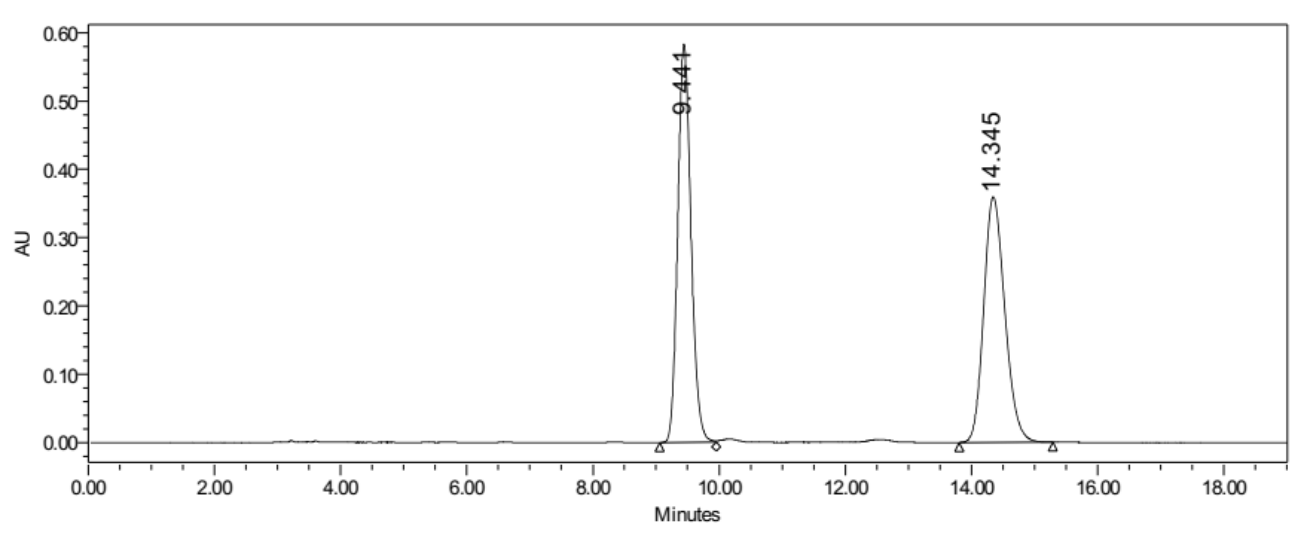

\begin{tabular}{|c|c|c|r|c|}
\hline & \multicolumn{1}{|c|}{ RT } & Area & $\%$ Area & Height \\
\hline 1 & 9.441 & 8425164 & 50.03 & 582830 \\
\hline 2 & 14.345 & 8416585 & 49.97 & 359703 \\
\hline
\end{tabular}

\section{Asy-3bf}

\begin{tabular}{|llll|}
\hline & \multicolumn{2}{c|}{ S A M P L E } & IN F O R M A T I O N \\
\hline \hline Sample Name: & xxh-7-21-IC-30\% & Acquired By: & System \\
Sample Type: & Unknown & Sample Set Name & 20200115 \\
Vial: & 86 & Acq. Method Set: & $30 \%$ quanbo \\
Injection \#: & 1 & Processing Method & 721 \\
Injection Volume: & 10.00 ul & Channel Name: & $254.0 \mathrm{~nm}$ \\
Run Time: & 19.0 Minutes & Proc. Chnl. Descr.: & 2998 PDA 254.0 nm (2998 \\
& & \\
Date Acquired: & $1 / 15 / 20216: 38: 51$ PM CST & & \\
Date Processed: & $3 / 24 / 2021$ 10:02:08 PM CST & & \\
\hline
\end{tabular}

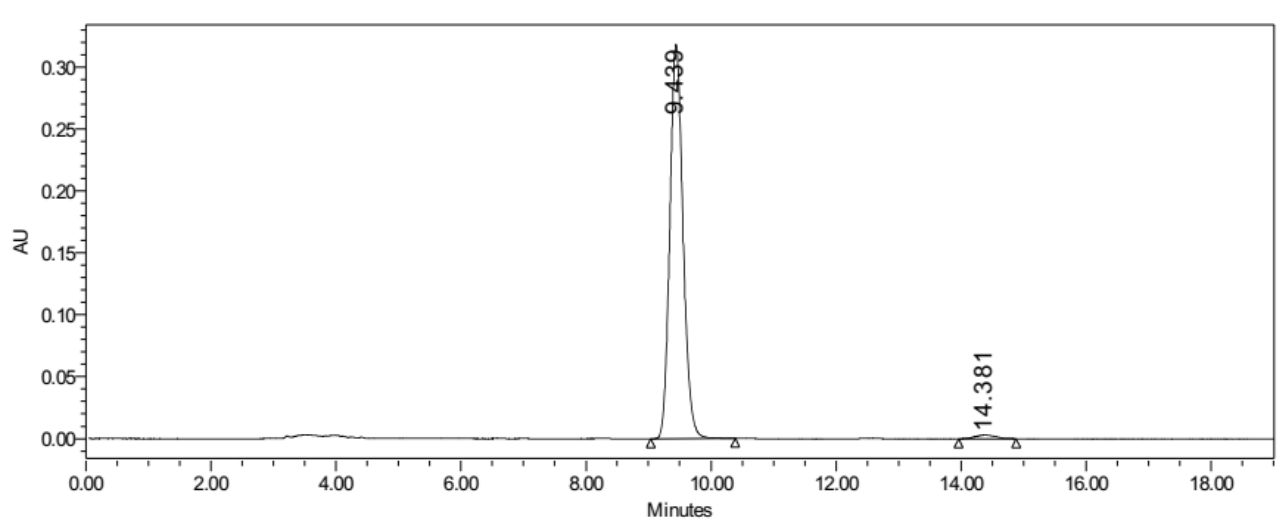

\begin{tabular}{|l|r|r|r|r|}
\hline & \multicolumn{1}{|c|}{ RT } & \multicolumn{1}{c|}{ Area } & $\%$ Area & Height \\
\hline 1 & 9.439 & 4607295 & 98.52 & 318137 \\
\hline 2 & 14.381 & 69114 & 1.48 & 3026 \\
\hline
\end{tabular}




\section{Rac-3cf}

\begin{tabular}{|llll|}
\hline & \multicolumn{2}{c|}{ S A M P L E } & IN F O R M A T I O N \\
\hline \hline Sample Name: & xxh-7-8-rac-IC-30\% & Acquired By: & System \\
Sample Type: & Unknown & Sample Set Name & 20200115 \\
Vial: & 85 & Acq. Method Set: & $30 \%$ quanbo \\
Injection \#: & 1 & Processing Method & 78 \\
Injection Volume: & $10.00 \mathrm{ul}$ & Channel Name: & $254.0 \mathrm{~nm}$ \\
Run Time: & 19.0 Minutes & Proc. Chnl. Descr.: & 2998 PDA 254.0 nm (2998 \\
& & & \\
Date Acquired: & 1/15/2021 6:19:07 PM CST & & \\
Date Processed: & $3 / 24 / 2021$ 10:05:45 PM CST & & \\
\hline
\end{tabular}

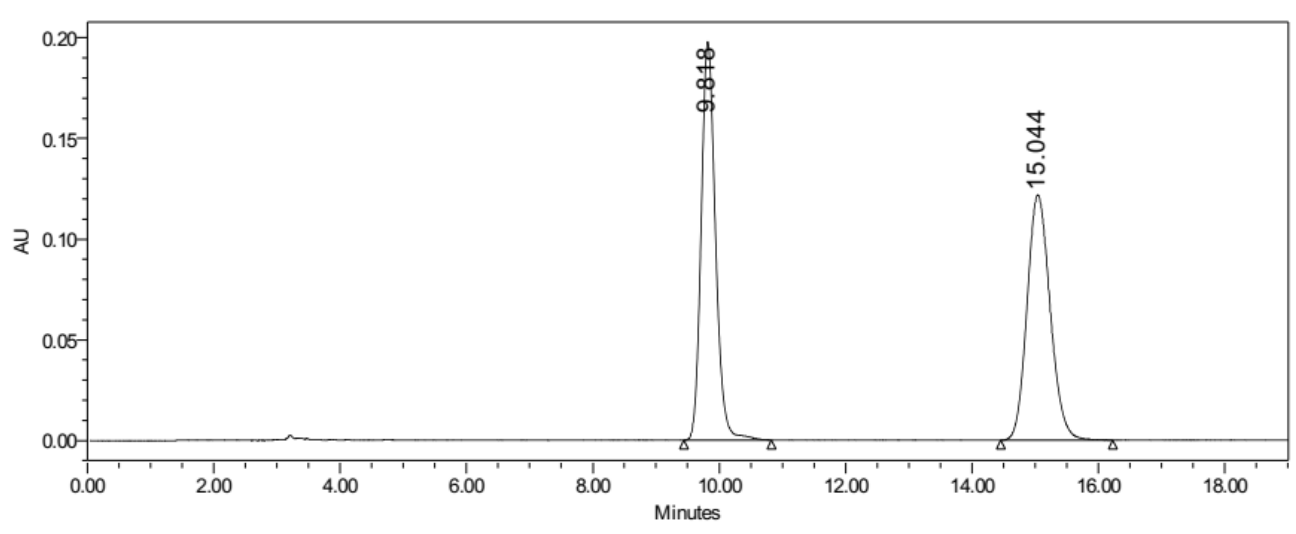

\begin{tabular}{|l|r|c|r|c|}
\hline & \multicolumn{1}{|c|}{ RT } & Area & $\%$ Area & Height \\
\hline 1 & 9.818 & 3127145 & 50.51 & 197679 \\
\hline 2 & 15.044 & 3064482 & 49.49 & 121745 \\
\hline
\end{tabular}

\section{Asy-3cf}

\begin{tabular}{|llll|}
\hline & \multicolumn{2}{c|}{ S A M P LE } & IN F O R M A T I O N \\
\hline \hline Sample Name: & xxh-7-154-IC-30\% & Acquired By: & System \\
Sample Type: & Unknown & Sample Set Name & 20210301 \\
Vial: & 76 & Acq. Method Set: & $30 \%$ quanbo \\
Injection \#: & 1 & Processing Method & 7154 \\
Injection Volume: & 10.00 ul & Channel Name: & $254.0 \mathrm{~nm}$ \\
Run Time: & 24.0 Minutes & Proc. Chnl. Descr.: & 2998 PDA 254.0 nm (2998 \\
& & & \\
Date Acquired: & $3 / 1 / 202111: 11: 02$ PM CST & & \\
Date Processed: & $3 / 24 / 202110: 07: 11$ PM CST & & \\
\hline
\end{tabular}

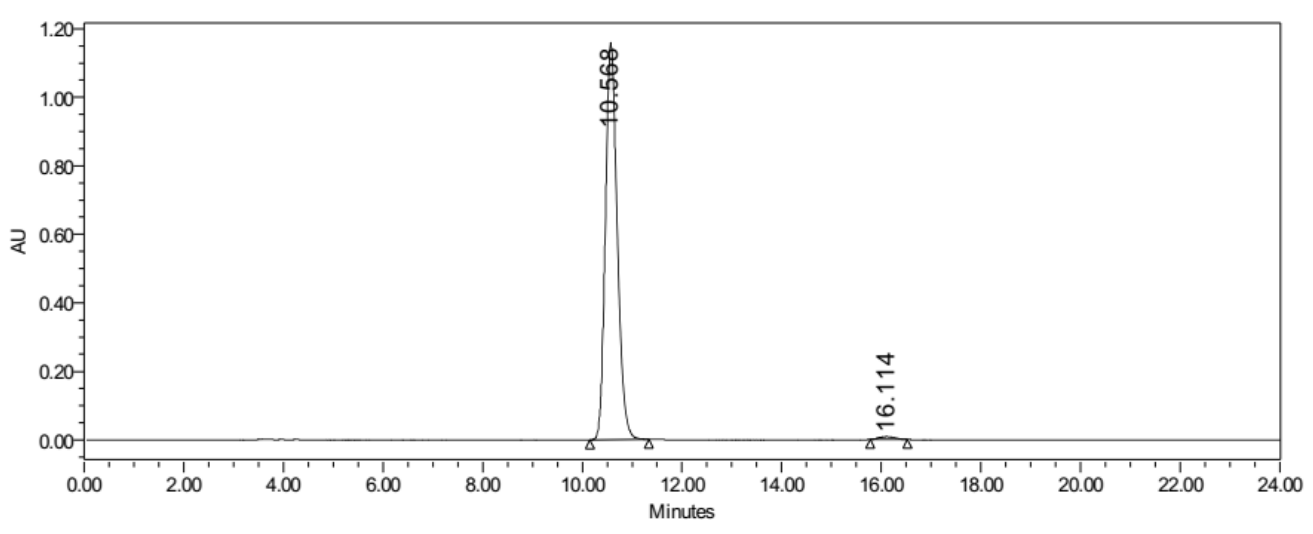

\begin{tabular}{|l|c|r|r|r|}
\hline & RT & \multicolumn{1}{|c|}{ Area } & $\%$ Area & \multicolumn{1}{c|}{ Height } \\
\hline 1 & 10.568 & 18957503 & 99.00 & 1158283 \\
\hline 2 & 16.114 & 190637 & 1.00 & 8360 \\
\hline
\end{tabular}


Rac-3df

\begin{tabular}{llll}
\hline & \multicolumn{2}{c}{ S A M P L E } & IN F O R M A T I O N \\
\hline \hline Sample Name: & xxh-7-9-rac-IC-30\% & Acquired By: & System \\
Sample Type: & Unknown & Sample Set Name & \\
Vial: & 81 & Acq. Method Set: & $30 \%$ quanbo \\
Injection \#: & 1 & Processing Method & 79 \\
Injection Volume: & 10.00 ul & Channel Name: & $254.0 \mathrm{~nm}$ \\
Run Time: & 100.0 Minutes & Proc. Chnl. Descr.: & 2998 PDA 254.0 nm (2998 \\
& & & \\
Date Acquired: & $1 / 15 / 2021$ 7:27:25 PM CST & & \\
Date Processed: & $3 / 24 / 2021$ 10:10:07 PM CST & & \\
\hline
\end{tabular}

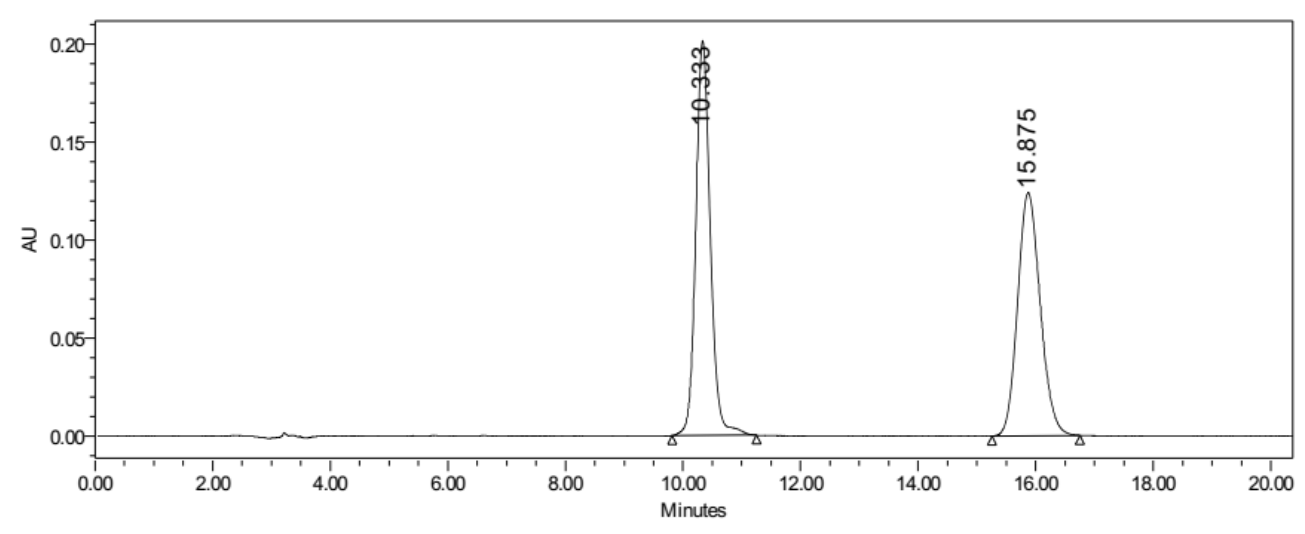

\begin{tabular}{|c|c|c|r|c|}
\hline & RT & Area & $\%$ Area & Height \\
\hline 1 & 10.333 & 3448878 & 50.96 & 201229 \\
\hline 2 & 15.875 & 3319209 & 49.04 & 123953 \\
\hline
\end{tabular}

\section{Asy-3df}

\begin{tabular}{|llll|}
\hline & \multicolumn{2}{c|}{ S A M P L E } & IN F O R M A T I O N \\
\hline \hline Sample Name: & xxh-7-23-IC-30\% & Acquired By: & System \\
Sample Type: & Unknown & Sample Set Name & 20200115 \\
Vial: & 81 & Acq. Method Set: & $30 \%$ quanbo \\
Injection \#: & 1 & Processing Method & 723 \\
Injection Volume: & $10.00 \mathrm{ul}$ & Channel Name: & $254.0 \mathrm{~nm}$ \\
Run Time: & 20.0 Minutes & Proc. Chnl. Descr.: & 2998 PDA 254.0 nm (2998 \\
& & & \\
Date Acquired: & $1 / 15 / 2021$ 9:17:39 PM CST & & \\
Date Processed: & $3 / 24 / 2021$ 10:11:56 PM CST & & \\
\hline
\end{tabular}

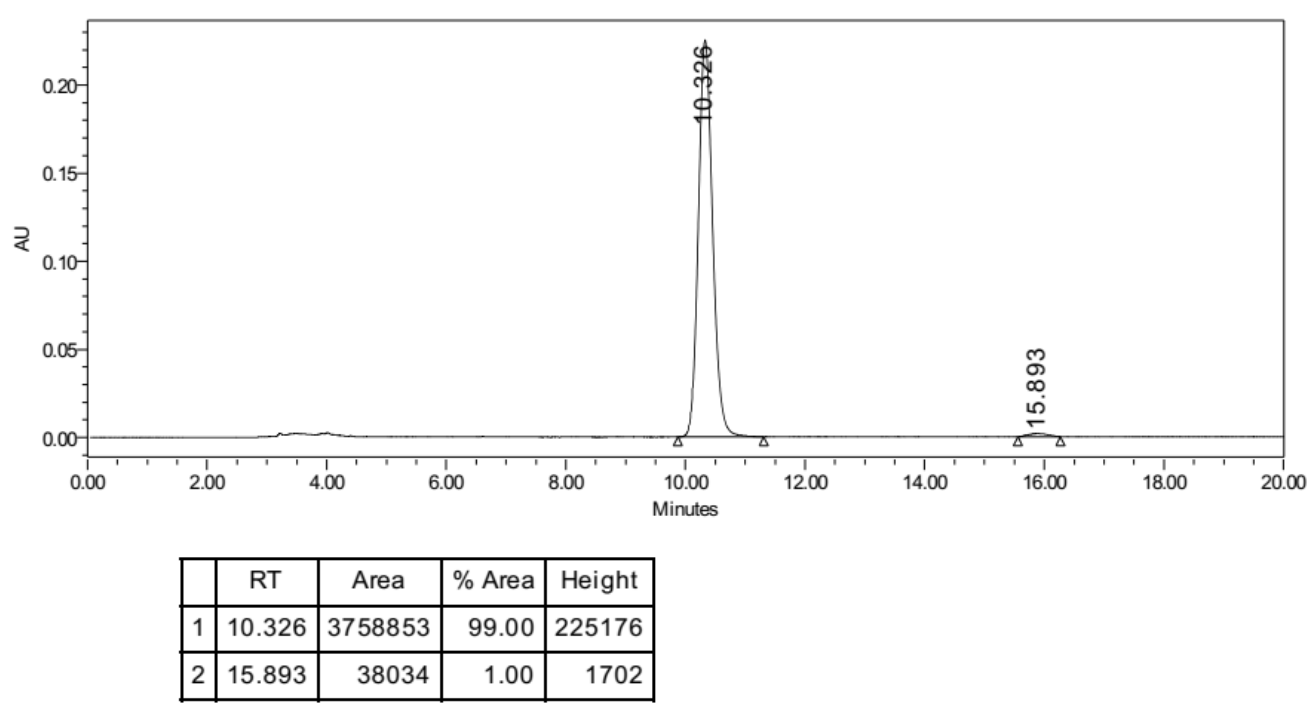




\section{Rac-3ef}

\begin{tabular}{|llll|}
\hline & \multicolumn{2}{c|}{ S A M P L E } & IN F O R M A T I O N \\
\hline \hline Sample Name: & xxh-7-10-rac-lC-30\% & Acquired By: & System \\
Sample Type: & Unknown & Sample Set Name: & \\
Vial: & 82 & Acq. Method Set: & $30 \%$ quanbo \\
Injection \#: & 1 & Processing Method & 710 \\
Injection Volume: & $10.00 \mathrm{ul}$ & Channel Name: & $254.0 \mathrm{~nm}$ \\
Run Time: & 100.0 Minutes & Proc. Chnl. Descr.: & 2998 PDA 254.0 nm (2998 \\
& & & \\
Date Acquired: & 1/15/2021 7:49:11 PM CST & & \\
Date Processed: & $3 / 24 / 2021$ 10:14:29 PM CST & & \\
\hline
\end{tabular}

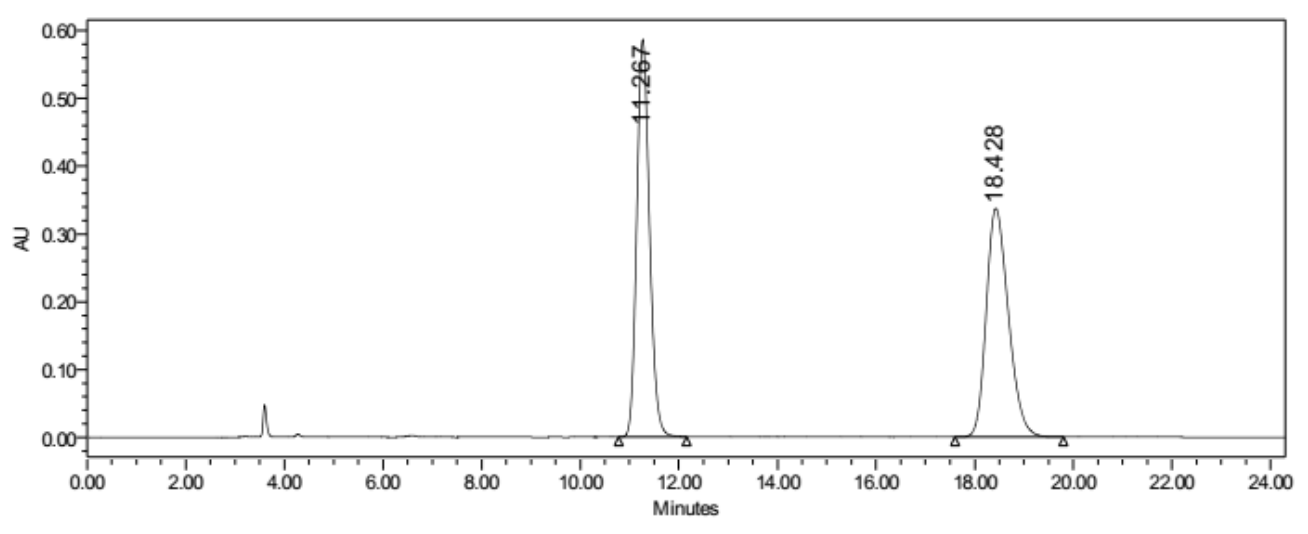

\begin{tabular}{|c|c|c|r|c|}
\hline & RT & Area & $\%$ Area & Height \\
\hline 1 & 11.267 & 10596224 & 50.00 & 586131 \\
\hline 2 & 18.428 & 10598197 & 50.00 & 337172 \\
\hline
\end{tabular}

\section{Asy-3ef}

\begin{tabular}{|llll|}
\hline & \multicolumn{2}{c|}{ S A M P L E } & IN F O R M A T I O N \\
\hline \hline Sample Name: & xxh-7-24-IC-30\% & Acquired By: & System \\
Sample Type: & Unknown & Sample Set Name & 20200115 \\
Vial: & 82 & Acq. Method Set: & $30 \%$ quanbo \\
Injection \#: & 1 & Processing Method & 724 \\
Injection Volume: & 10.00 ul & Channel Name: & $254.0 \mathrm{~nm}$ \\
Run Time: & 24.0 Minutes & Proc. Chnl. Descr.: & 2998 PDA 254.0 nm (2998 \\
& & & \\
Date Acquired: & $1 / 15 / 20219: 38: 19$ PM CST & & \\
Date Processed: & $3 / 24 / 2021$ 10:15:36 PM CST & & \\
\hline
\end{tabular}

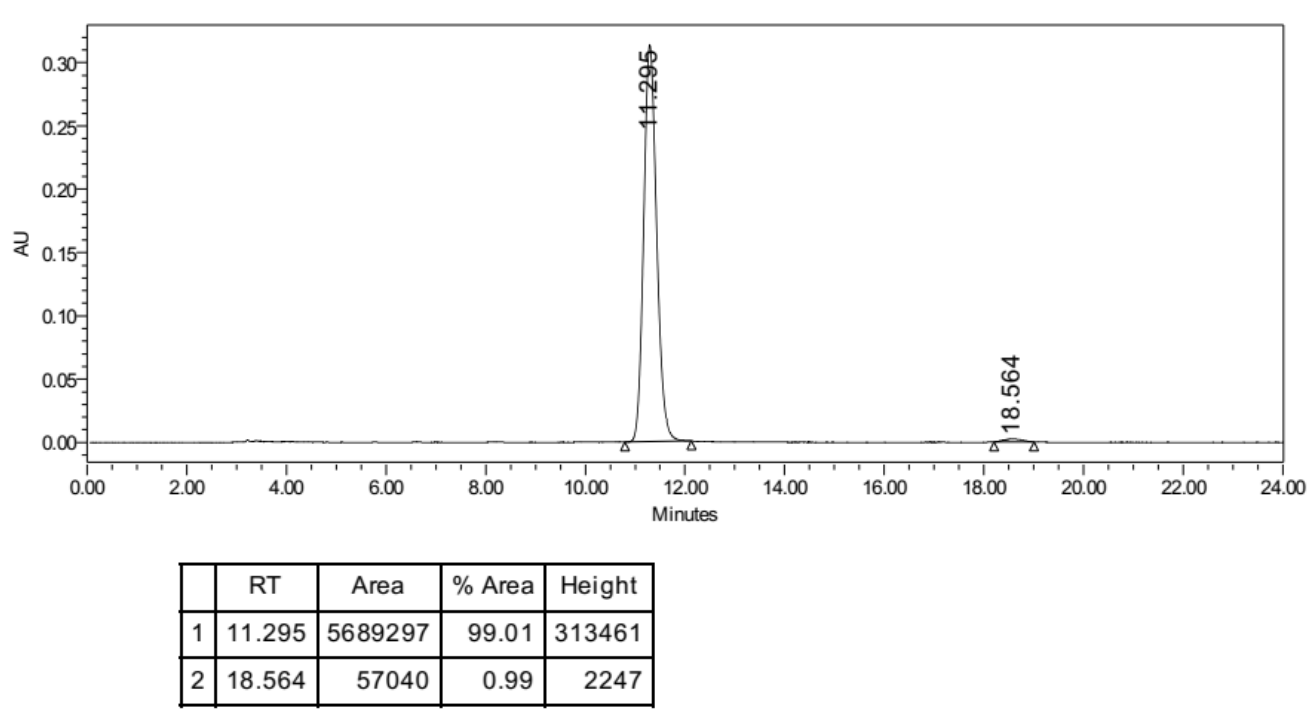


Rac-3ff

\begin{tabular}{|llll|}
\hline & \multicolumn{2}{c|}{ S A M P L E } & IN F O R M A T I O N \\
\hline \hline Sample Name: & xxh-6-107-rac-IC-30\% & Acquired By: & System \\
Sample Type: & Unknown & Sample Set Name & \\
Vial: & 1 & Acq. Method Set: & $30 \%$ quanbo \\
Injection \#: & 1 & Processing Method & 6107 \\
Injection Volume: & $10.00 \mathrm{ul}$ & Channel Name: & $254.0 \mathrm{~nm}$ \\
Run Time: & 100.0 Minutes & Proc. Chnl. Descr.: & 2998 PDA 254.0 nm (2998 \\
& & & \\
Date Acquired: & $12 / 15 / 2020$ 6:53:29 PM CST & & \\
Date Processed: & $3 / 24 / 2021$ 10:21:55 PM CST & & \\
\hline
\end{tabular}

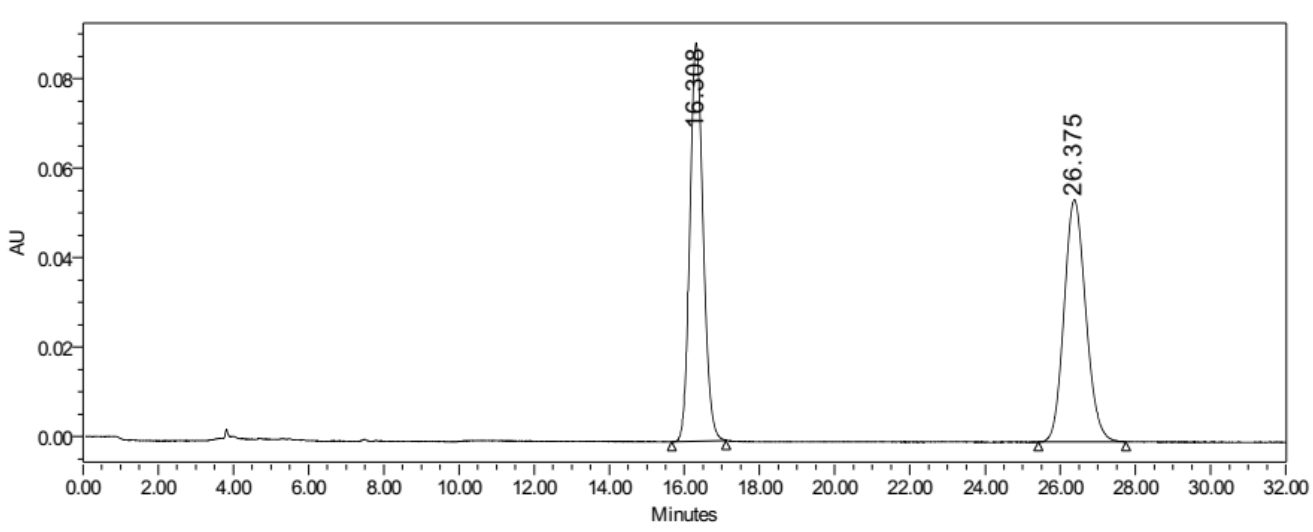

\begin{tabular}{|c|c|c|r|c|}
\hline & RT & Area & $\%$ Area & Height \\
\hline 1 & 16.308 & 2184253 & 50.00 & 88981 \\
\hline 2 & 26.375 & 2184604 & 50.00 & 54119 \\
\hline
\end{tabular}

Asy-3ff

\begin{tabular}{|llll|}
\hline & \multicolumn{2}{c|}{ S A M P E } & IN F O R M A T I O N \\
\hline \hline Sample Name: & xxh-7-40-IC-30\% & Acquired By: & System \\
Sample Type: & Unknown & Sample Set Name & 20210119 \\
Vial: & 50 & Acq. Method Set: & $30 \%$ quanbo \\
Injection \#: & 1 & Processing Method & 740 \\
Injection Volume: & 10.00 ul & Channel Name: & $254.0 \mathrm{~nm}$ \\
Run Time: & 32.0 Minutes & Proc. Chnl. Descr.: & 2998 PDA 254.0 nm (2998 \\
& & & \\
Date Acquired: & 1/19/2021 11:07:46 PM CST & & \\
Date Processed: & $3 / 24 / 202110: 23: 02$ PM CST & & \\
\hline
\end{tabular}

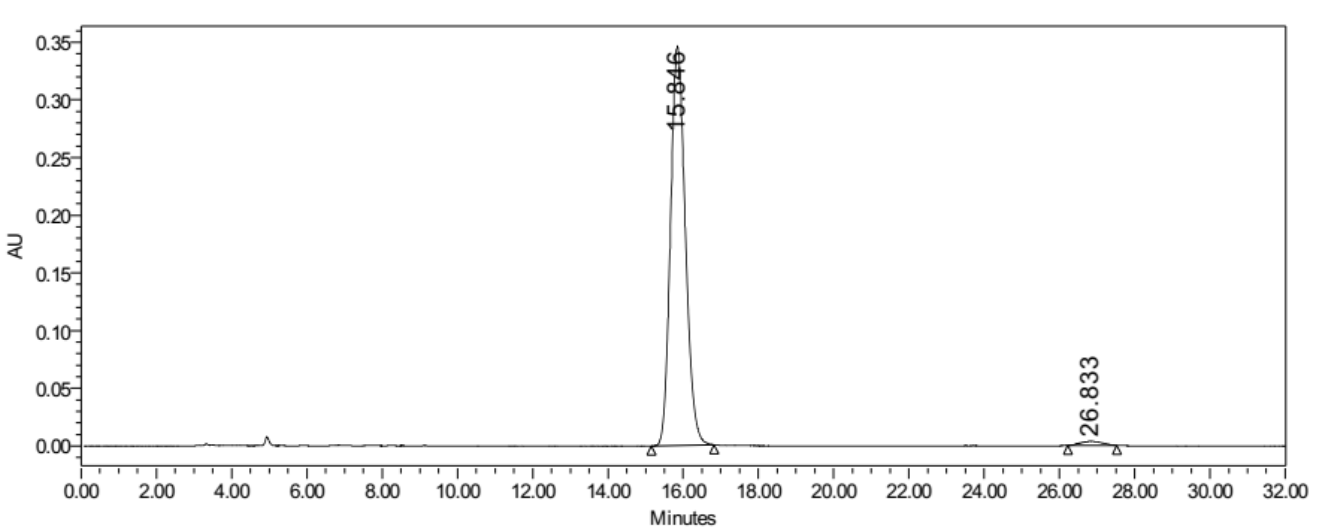

\begin{tabular}{|r|c|r|r|r|}
\hline & RT & Area & $\%$ Area & Height \\
\hline 1 & 15.846 & 9324075 & 98.50 & 346178 \\
\hline 2 & 26.833 & 142125 & 1.50 & 3547 \\
\hline
\end{tabular}


Rac-3gf

\begin{tabular}{|llll|}
\hline & \multicolumn{2}{c|}{ S A M P L E } & IN F O R M A T I O N \\
\hline \hline Sample Name: & xxh-7-14-rac-lC-30\% & Acquired By: & System \\
Sample Type: & Unknown & Sample Set Name: & 20200116 \\
Vial: & 86 & Acq. Method Set: & $30 \%$ quanbo \\
Injection \#: & 1 & Processing Method & 714 \\
Injection Volume: & 10.00 ul & Channel Name: & $254.0 \mathrm{~nm}$ \\
Run Time: & 18.0 Minutes & Proc. Chnl. Descr.: & 2998 PDA 254.0 nm (2998 \\
& & & \\
Date Acquired: & 1/16/2021 4:23:30 PM CST & & \\
Date Processed: & 3/25/2021 9:49:40 AM CST & & \\
\hline
\end{tabular}

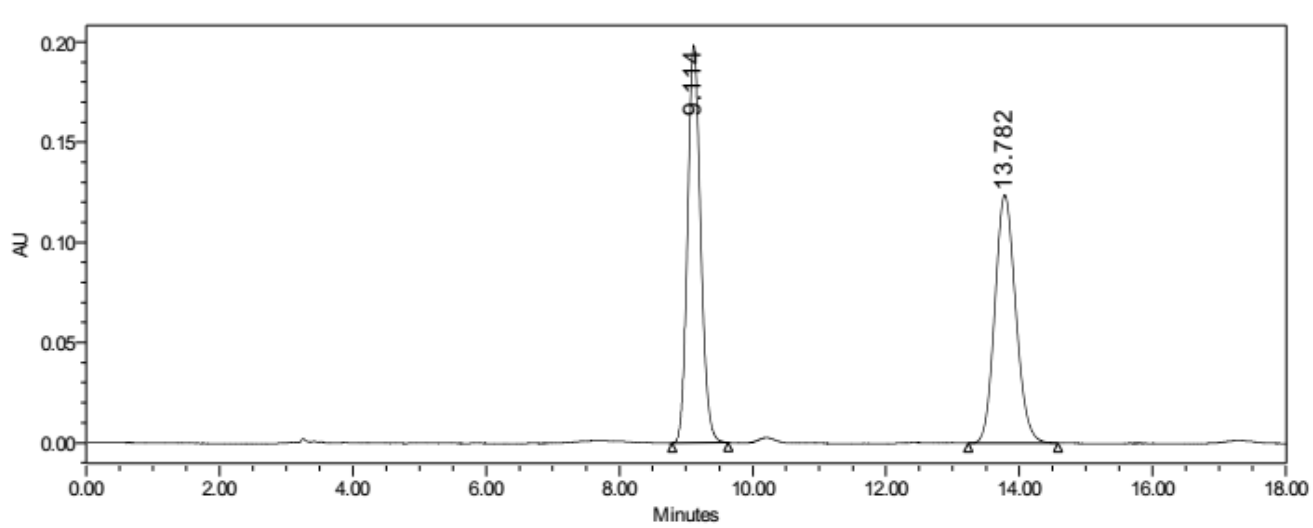

\begin{tabular}{|c|c|c|r|c|}
\hline & RT & Area & $\%$ Area & Height \\
\hline 1 & 9.114 & 2715926 & 50.00 & 198541 \\
\hline 2 & 13.782 & 2716159 & 50.00 & 124032 \\
\hline
\end{tabular}

\section{Asy-3gf}

\begin{tabular}{|llll|}
\hline & \multicolumn{2}{c|}{ S A M P L E } & IN F O R M A T I O N \\
\hline \hline Sample Name: & xxh-7-41-IC-30\% & Acquired By: & System \\
Sample Type: & Unknown & Sample Set Name & 20210119 \\
Vial: & 51 & Acq. Method Set: & $30 \%$ quanbo \\
Injection \#: & 1 & Processing Method & 741 \\
Injection Volume: & 10.00 ul & Channel Name: & $254.0 \mathrm{~nm}$ \\
Run Time: & 20.0 Minutes & Proc. Chnl. Descr.: & 2998 PDA 254.0 nm (2998 \\
& & & \\
Date Acquired: & $1 / 19 / 202111: 40: 28$ PM CST & & \\
Date Processed: & $3 / 25 / 20219: 51: 02$ AM CST & & \\
\hline
\end{tabular}

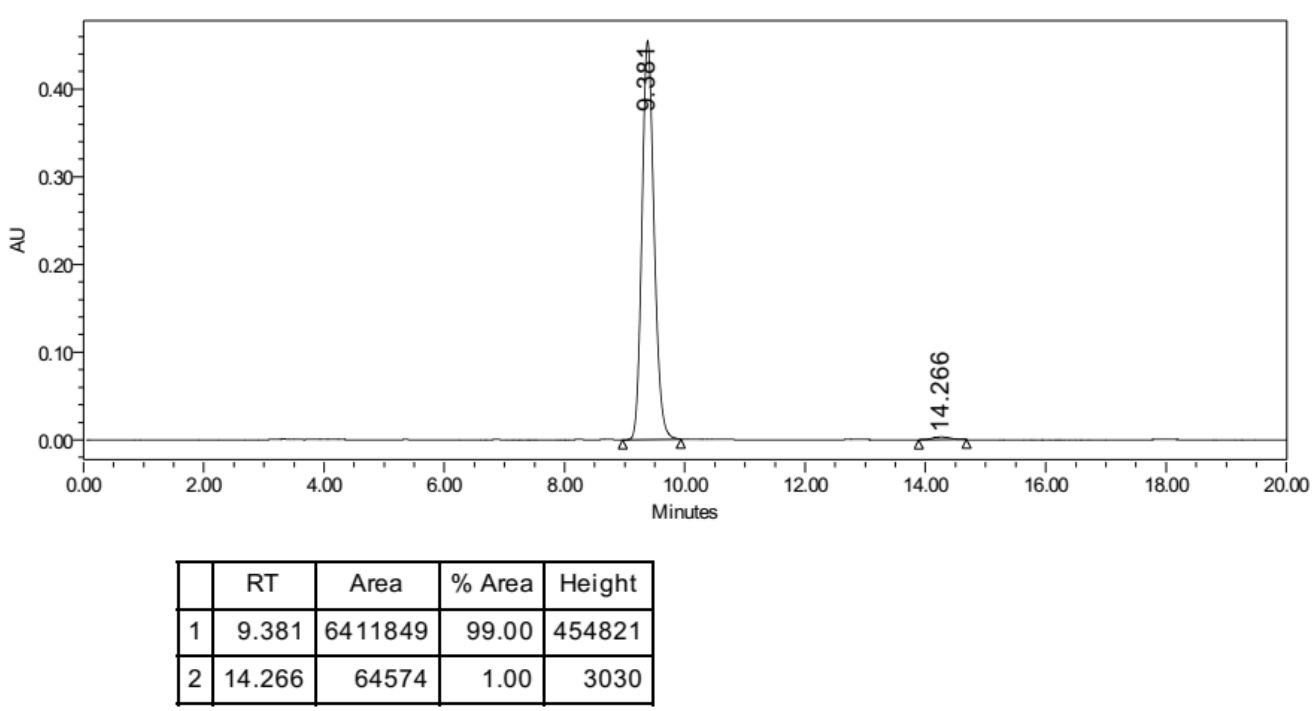


Rac-3hf

\begin{tabular}{|llll|}
\hline & \multicolumn{2}{c|}{ S A M P L E } & IN F O R M A T I O N \\
\hline \hline Sample Name: & xxh-7-13-rac-IC-30\% & Acquired By: & System \\
Sample Type: & Unknown & Sample Set Name & \\
Vial: & 85 & Acq. Method Set: & $30 \%$ quanbo \\
Injection \#: & 1 & Processing Method & 713 \\
Injection Volume: & 10.00 ul & Channel Name: & $254.0 \mathrm{~nm}$ \\
Run Time: & 100.0 Minutes & Proc. Chnl. Descr.: & 2998 PDA 254.0 nm (2998 \\
& & & \\
Date Acquired: & 1/15/2021 8:54:39 PM CST & & \\
Date Processed: & $3 / 25 / 20219: 25: 31$ AM CST & & \\
\hline
\end{tabular}

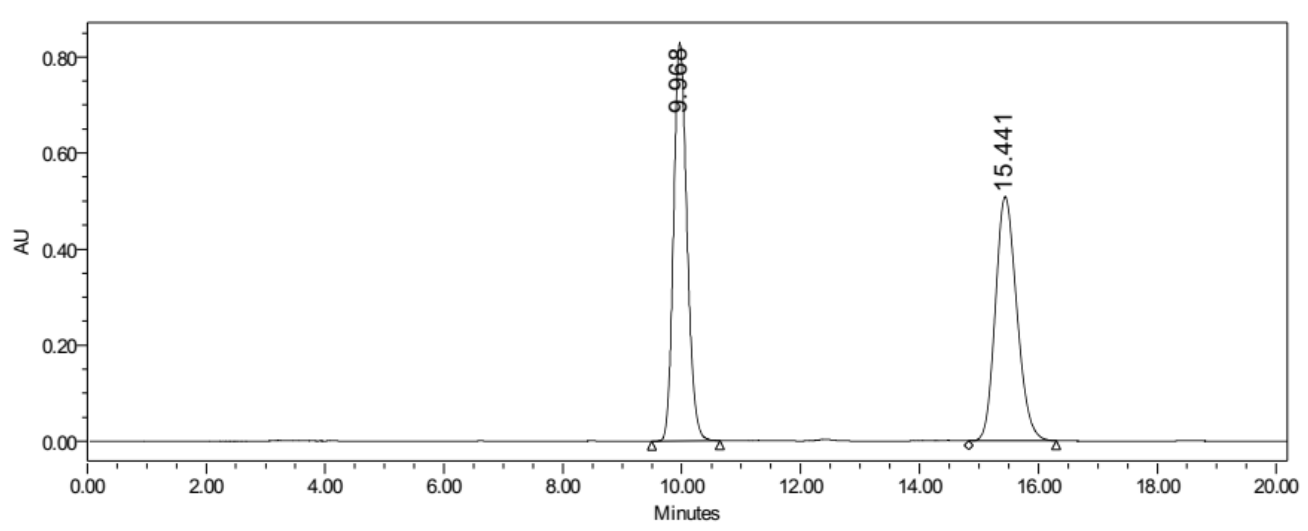

\begin{tabular}{|c|c|c|c|c|}
\hline & \multicolumn{1}{|c|}{ RT } & Area & $\%$ Area & Height \\
\hline 1 & 9.968 & 12831314 & 50.00 & 829511 \\
\hline 2 & 15.441 & 12832837 & 50.00 & 508345 \\
\hline
\end{tabular}

\section{Asy-3hf}

\begin{tabular}{|llll|}
\hline & \multicolumn{2}{c|}{ S A M P LE } & IN F O R M A T I O N \\
\hline \hline Sample Name: & xxh-7-29-IC-30\% & Acquired By: & System \\
Sample Type: & Unknown & Sample Set Name & 20200115 \\
Vial: & 85 & Acq. Method Set: & $30 \%$ quanbo \\
Injection \#: & 1 & Processing Method & 729 \\
Injection Volume: & 10.00 ul & Channel Name: & $254.0 \mathrm{~nm}$ \\
Run Time: & 20.0 Minutes & Proc. Chnl. Descr.: & 2998 PDA 254.0 nm (2998 \\
& & & \\
Date Acquired: & $1 / 15 / 202110: 42: 21$ PM CST & & \\
Date Processed: & $3 / 25 / 20219: 35: 37$ AM CST & & \\
\hline
\end{tabular}

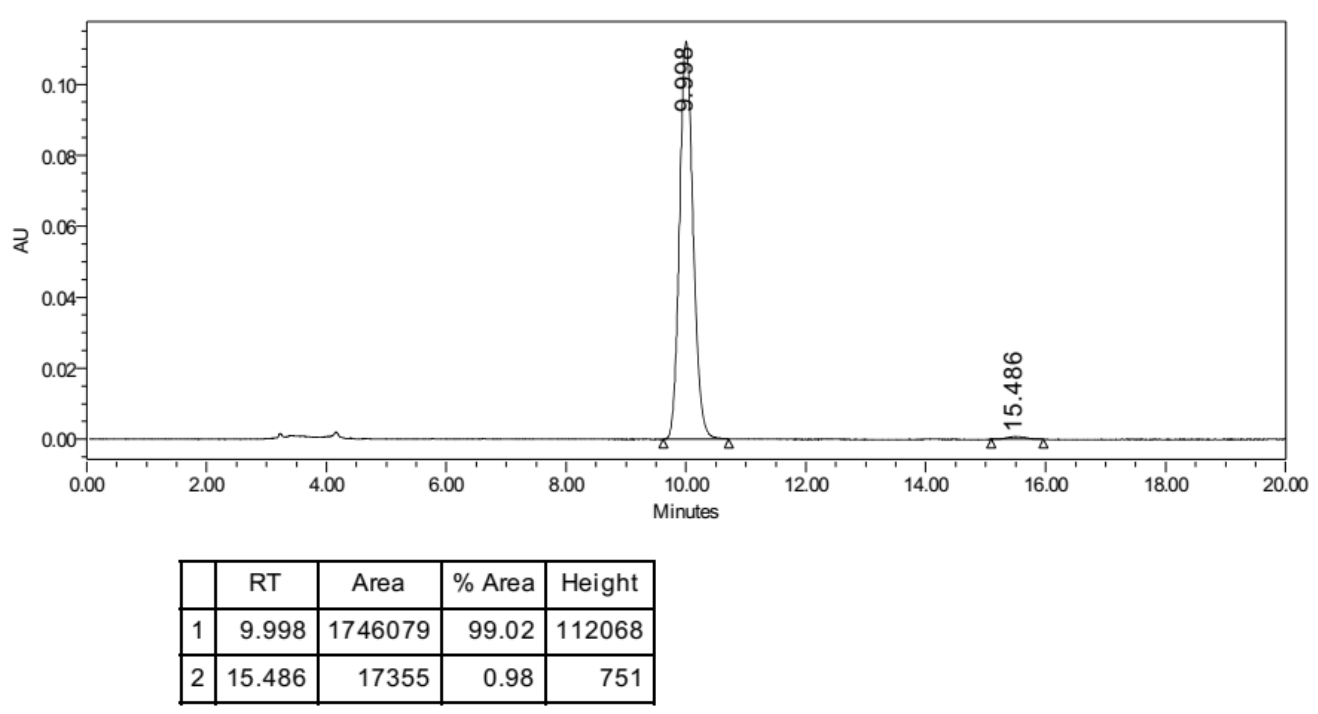


Rac-3if

\begin{tabular}{|llll|}
\hline & \multicolumn{2}{c|}{ S A M P L E } & IN F O R M A T I O N \\
\hline \hline Sample Name: & xxh-7-11-rac-IC-30\% & Acquired By: & System \\
Sample Type: & Unknown & Sample Set Name & \\
Vial: & 83 & Acq. Method Set: & $30 \%$ quanbo \\
Injection \#: & 1 & Processing Method & 711 \\
Injection Volume: & $10.00 \mathrm{ul}$ & Channel Name: & $254.0 \mathrm{~nm}$ \\
Run Time: & 100.0 Minutes & Proc. Chnl. Descr.: & 2998 PDA 254.0 nm (2998 \\
& & & \\
Date Acquired: & 1/15/2021 8:14:19 PM CST & & \\
Date Processed: & $3 / 24 / 2021$ 10:28:55 PM CST & & \\
\hline
\end{tabular}

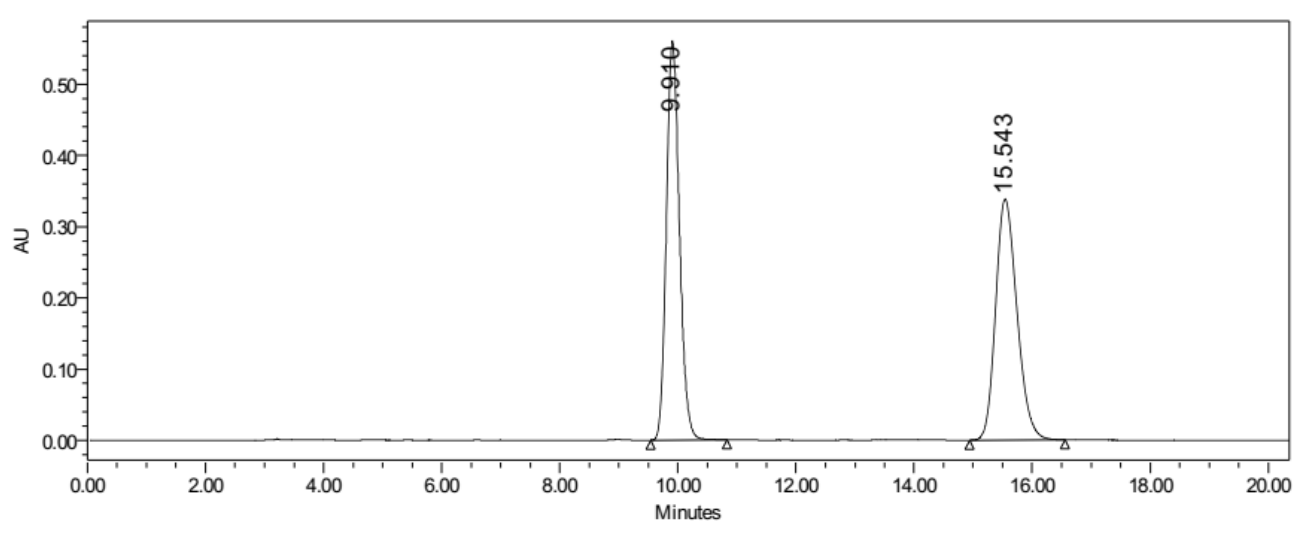

\begin{tabular}{|c|c|c|r|c|}
\hline & \multicolumn{1}{|c|}{ RT } & Area & $\%$ Area & Height \\
\hline 1 & 9.910 & 8554216 & 50.00 & 560163 \\
\hline 2 & 15.543 & 8554435 & 50.00 & 338225 \\
\hline
\end{tabular}

\section{Asy-3if}

\begin{tabular}{|llll|}
\hline & \multicolumn{2}{c|}{ S A M P L E } & IN F O R M A T I O N \\
\hline \hline Sample Name: & xxh-7-48-IC-30\% & Acquired By: & System \\
Sample Type: & Unknown & Sample Set Name & 20210119 \\
Vial: & 58 & Acq. Method Set: & $30 \%$ quanbo \\
Injection \#: & 1 & Processing Method & 748 \\
Injection Volume: & 10.00 ul & Channel Name: & $254.0 \mathrm{~nm}$ \\
Run Time: & 20.0 Minutes & Proc. Chnl. Descr.: & 2998 PDA 254.0 nm (2998 \\
& & & \\
Date Acquired: & $1 / 20 / 20213: 55: 39$ AM CST & & \\
Date Processed: & $3 / 24 / 202110: 28: 16$ PM CST & & \\
\hline
\end{tabular}

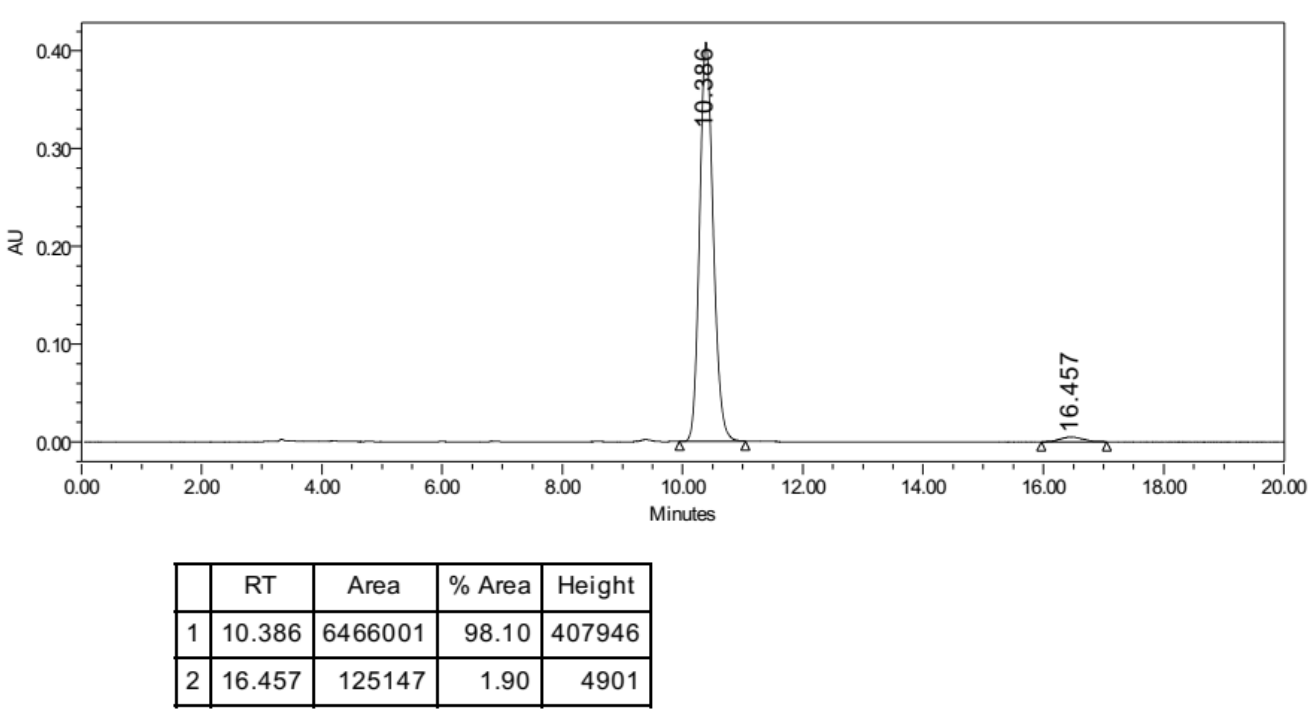




\section{Rac-3jf}

\begin{tabular}{|llll|}
\hline & \multicolumn{2}{c|}{ S A M P L E } & IN F O R M A T I O N \\
\hline \hline Sample Name: & xxh-7-12-rac-IC-30\% & Acquired By: & System \\
Sample Type: & Unknown & Sample Set Name & \\
Vial: & 84 & Acq. Method Set: & $30 \%$ quanbo \\
Injection \#: & 1 & Processing Method & 712 \\
Injection Volume: & $10.00 \mathrm{ul}$ & Channel Name: & $254.0 \mathrm{~nm}$ \\
Run Time: & 100.0 Minutes & Proc. Chnl. Descr.: & 2998 PDA 254.0 nm (2998 \\
& & & \\
Date Acquired: & 1/15/2021 8:35:38 PM CST & & \\
Date Processed: & $3 / 24 / 2021$ 10:31:48 PM CST & & \\
\hline
\end{tabular}

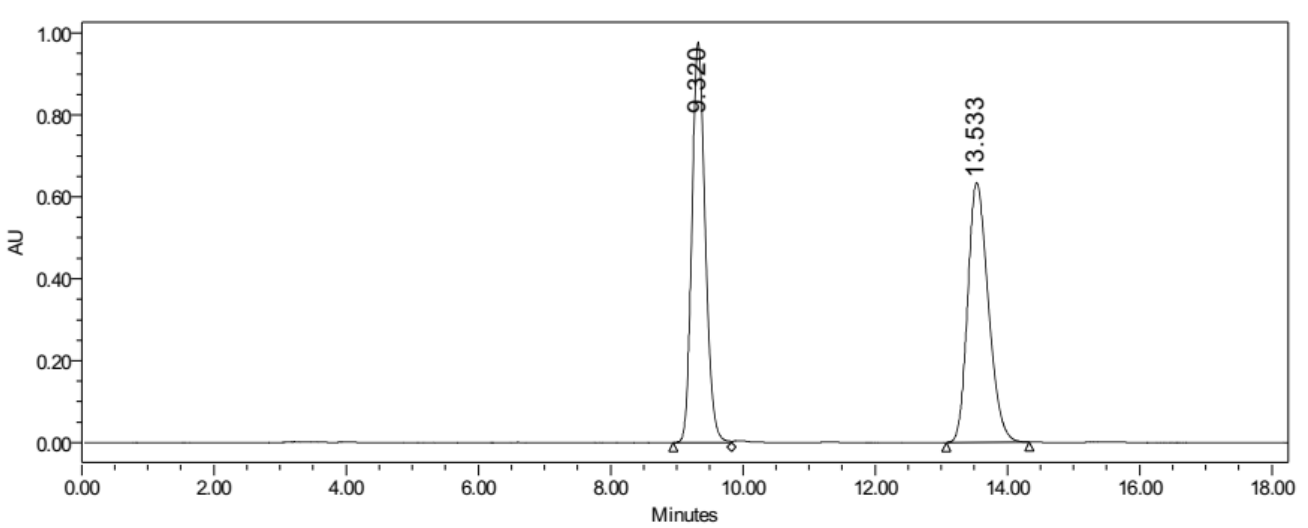

\begin{tabular}{|l|c|c|r|c|}
\hline & RT & Area & $\%$ Area & Height \\
\hline 1 & 9.320 & 13720110 & 50.00 & 977637 \\
\hline 2 & 13.533 & 13722185 & 50.00 & 634107 \\
\hline
\end{tabular}

\section{Asy-3jf}

\begin{tabular}{|llll|}
\hline & \multicolumn{2}{c|}{ S A M P LE } & IN F O R M A T I O N \\
\hline \hline Sample Name: & xxh-7-28-IC-30\% & Acquired By: & System \\
Sample Type: & Unknown & Sample Set Name & 20200115 \\
Vial: & 84 & Acq. Method Set: & $30 \%$ quanbo \\
Injection \#: & 1 & Processing Method & 728 \\
Injection Volume: & $10.00 \mathrm{ul}$ & Channel Name: & $254.0 \mathrm{~nm}$ \\
Run Time: & 18.0 Minutes & Proc. Chnl. Descr.: & 2998 PDA 254.0 nm (2998 \\
& & & \\
Date Acquired: & $1 / 15 / 2021$ 10:23:42 PM CST & & \\
Date Processed: & $3 / 24 / 2021$ 10:32:51 PM CST & & \\
\hline
\end{tabular}

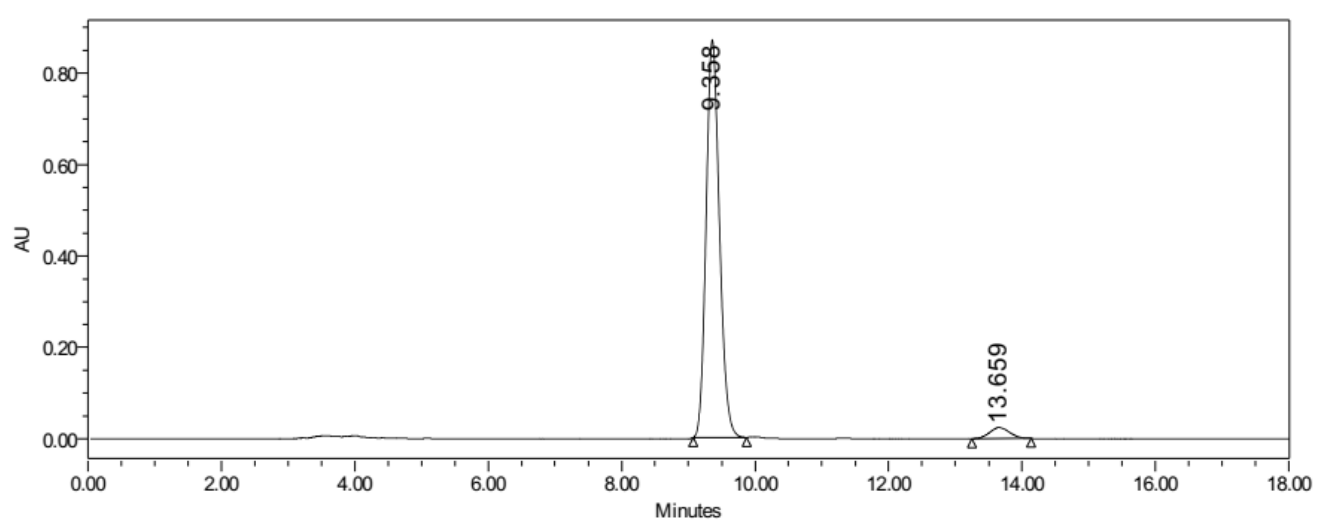

\begin{tabular}{|l|r|r|r|r|}
\hline & \multicolumn{1}{|c|}{ RT } & \multicolumn{1}{c|}{ Area } & $\%$ Area & Height \\
\hline 1 & 9.358 & 12160460 & 96.04 & 870289 \\
\hline 2 & 13.659 & 501959 & 3.96 & 23894 \\
\hline
\end{tabular}




\section{Rac-3kf}

\begin{tabular}{|llll|}
\hline & \multicolumn{2}{c|}{ S A M P E } & IN F O R M A T I O N \\
\hline \hline Sample Name: & xxh-7-15-rac-IC-5\% & Acquired By: & System \\
Sample Type: & Unknown & Sample Set Name & 20200116 \\
Vial: & 87 & Acq. Method Set: & $5 \%$ quanbo \\
Injection \#: & 1 & Processing Method & 715 \\
Injection Volume: & $10.00 \mathrm{ul}$ & Channel Name: & $254.0 \mathrm{~nm}$ \\
Run Time: & 55.0 Minutes & Proc. Chnl. Descr.: & 2998 PDA 254.0 nm (2998 \\
& & & \\
Date Acquired: & $1 / 16 / 20214: 52: 20$ PM CST & & \\
Date Processed: & $3 / 25 / 20219: 54: 15$ AM CST & & \\
\hline
\end{tabular}

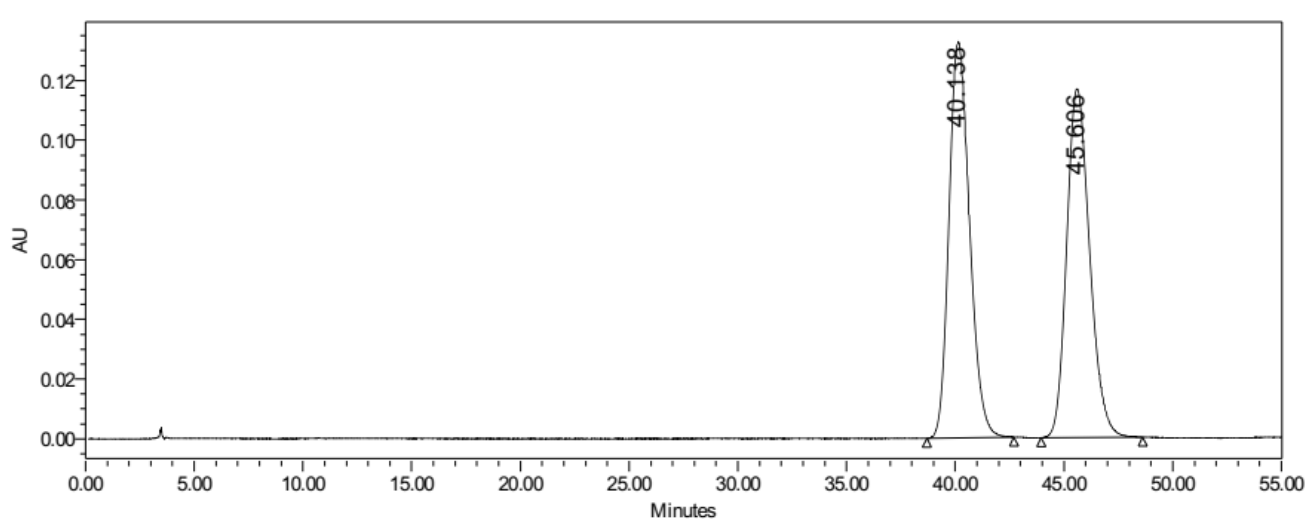

\begin{tabular}{|c|c|c|r|c|}
\hline & RT & Area & $\%$ Area & Height \\
\hline 1 & 40.138 & 8616407 & 50.00 & 132716 \\
\hline 2 & 45.606 & 8617890 & 50.00 & 116833 \\
\hline
\end{tabular}

\section{Asy-3kf}

\begin{tabular}{|llll|}
\hline & \multicolumn{2}{c|}{ S A M P L E } & IN F O R M A T I O N \\
\hline \hline Sample Name: & xxh-7-42-IC-30\% & Acquired By: & System \\
Sample Type: & Unknown & Sample Set Name & 20200119 \\
Vial: & 86 & Acq. Method Set: & $5 \%$ quanbo \\
Injection \#: & 1 & Processing Method & 742 \\
Injection Volume: & 10.00 ul & Channel Name: & $254.0 \mathrm{~nm}$ \\
Run Time: & 56.0 Minutes & Proc. Chnl. Descr.: & 2998 PDA 254.0 nm (2998 \\
& & & \\
Date Acquired: & $1 / 19 / 20216: 24: 20$ PM CST & & \\
Date Processed: & $3 / 25 / 20219: 54: 59$ AM CST & & \\
\hline
\end{tabular}

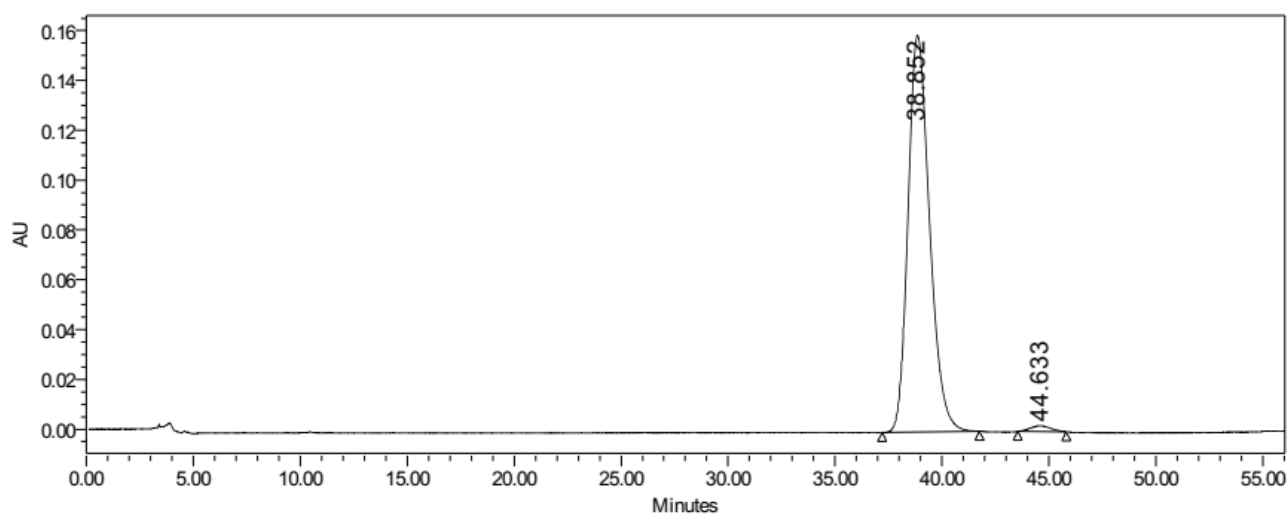

\begin{tabular}{|r|c|r|r|r|}
\hline & RT & \multicolumn{1}{|c|}{ Area } & $\%$ Area & Height \\
\hline 1 & 38.852 & 11202741 & 98.59 & 159288 \\
\hline 2 & 44.633 & 159763 & 1.41 & 2319 \\
\hline
\end{tabular}


Rac-3lf

\begin{tabular}{|llll|}
\hline & \multicolumn{2}{c|}{ S A M P L E } & IN F O R M A T I O N \\
\hline \hline Sample Name: & xxh-7-16-rac-IC-15\% & Acquired By: & System \\
Sample Type: & Unknown & Sample Set Name & 20200116 \\
Vial: & 88 & Acq. Method Set: & $15 \%$ quanbo \\
Injection \#: & 1 & Processing Method & 716 \\
Injection Volume: & 10.00 ul & Channel Name: & $254.0 \mathrm{~nm}$ \\
Run Time: & 21.0 Minutes & Proc. Chnl. Descr.: & 2998 PDA 254.0 nm (2998 \\
& & & \\
Date Acquired: & $1 / 16 / 20215: 58: 09$ PM CST & & \\
Date Processed: & $3 / 25 / 20219: 59: 55$ AM CST & & \\
\hline
\end{tabular}

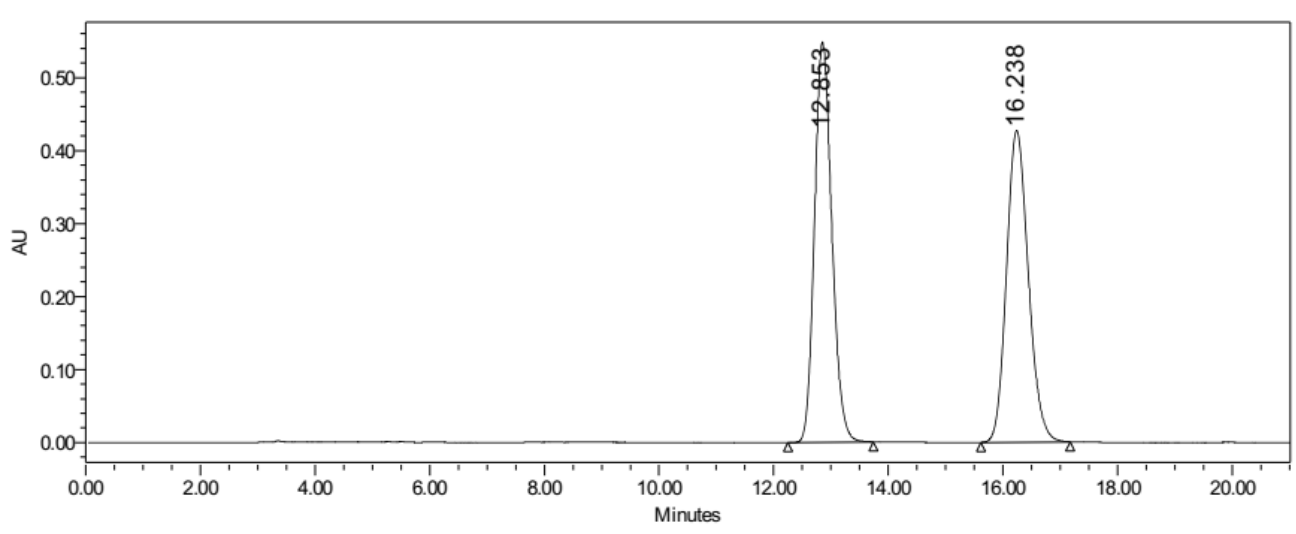

\begin{tabular}{|c|c|c|r|c|}
\hline & RT & Area & $\%$ Area & Height \\
\hline 1 & 12.853 & 11358506 & 50.00 & 548125 \\
\hline 2 & 16.238 & 11360482 & 50.00 & 427085 \\
\hline
\end{tabular}

\section{Asy-3lf}

\begin{tabular}{|llll|}
\hline & \multicolumn{2}{c|}{ S A M P L E } & IN F O R M A T I O N \\
\hline \hline Sample Name: & xxh-7-43-IC-30\% & Acquired By: & System \\
Sample Type: & Unknown & Sample Set Name & 20200119 \\
Vial: & 87 & Acq. Method Set: & $15 \%$ quanbo \\
Injection \#: & 1 & Processing Method & 743 \\
Injection Volume: & 10.00 ul & Channel Name: & $254.0 \mathrm{~nm}$ \\
Run Time: & 21.0 Minutes & Proc. Chnl. Descr.: & 2998 PDA 254.0 nm (2998 \\
& & & \\
Date Acquired: & $1 / 19 / 20217: 21: 01$ PM CST & & \\
Date Processed: & $3 / 25 / 20219: 58: 10$ AM CST & & \\
\hline
\end{tabular}

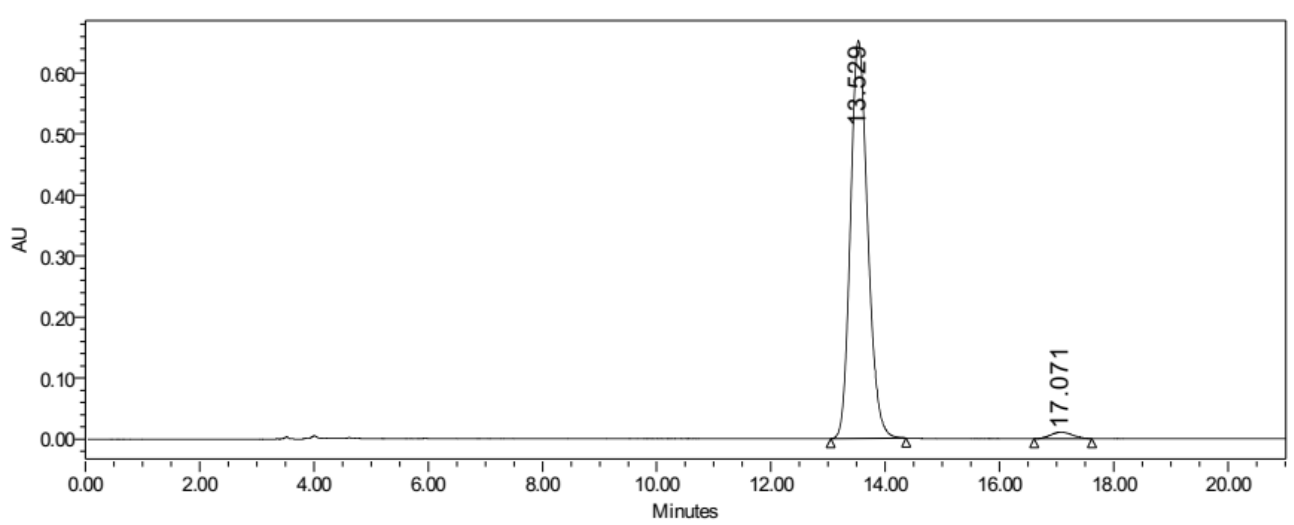

\begin{tabular}{|r|c|r|r|r|}
\hline & RT & \multicolumn{1}{c|}{ Area } & $\%$ Area & Height \\
\hline 1 & 13.529 & 13732293 & 98.00 & 651996 \\
\hline 2 & 17.071 & 280115 & 2.00 & 10708 \\
\hline
\end{tabular}




\section{Rac-3mf}

\begin{tabular}{|llll|}
\hline & \multicolumn{2}{c|}{ S A M P L E } & IN F O R M A T I O N \\
\hline \hline Sample Name: & xxh-7-50-rac-IC-30\% & Acquired By: & System \\
Sample Type: & Unknown & Sample Set Name & 20210308 \\
Vial: & 76 & Acq. Method Set: & $30 \%$ quanbo \\
Injection \#: & 1 & Processing Method & 750 \\
Injection Volume: & 10.00 ul & Channel Name: & $254.0 \mathrm{~nm}$ \\
Run Time: & 40.0 Minutes & Proc. Chnl. Descr.: & 2998 PDA 254.0 nm (2998 \\
& & & \\
Date Acquired: & $3 / 8 / 20219: 02: 57$ PM CST & & \\
Date Processed: & $3 / 25 / 2021$ 10:02:23 AM CST & & \\
\hline
\end{tabular}

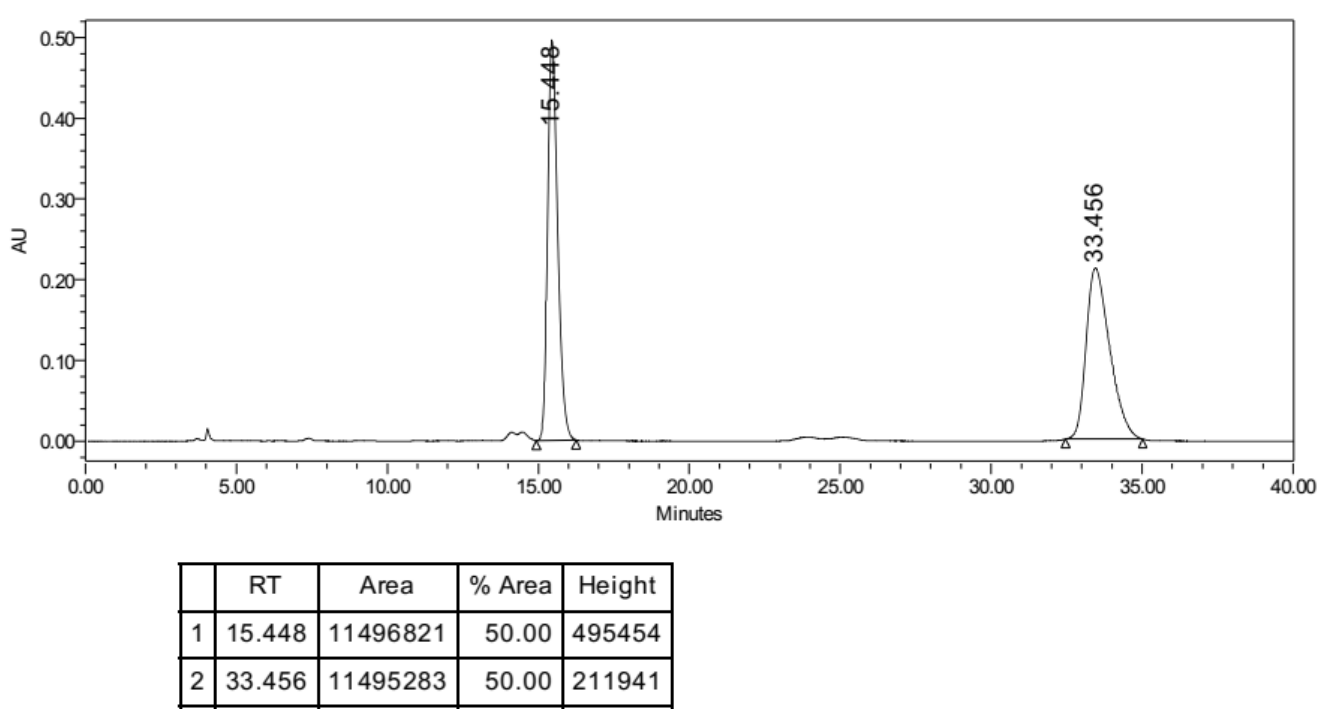

\section{Asy-3mf}

\begin{tabular}{|llll|}
\hline & \multicolumn{2}{c|}{ S A M P L E } & IN F O R M A T I O N \\
\hline \hline Sample Name: & xxh-7-178-IC-30\% & Acquired By: & System \\
Sample Type: & Unknown & Sample Set Name & 20210308 \\
Vial: & 77 & Acq. Method Set: & $30 \%$ quanbo \\
Injection \#: & 1 & Processing Method & 7178 \\
Injection Volume: & $10.00 \mathrm{ul}$ & Channel Name: & $254.0 \mathrm{~nm}$ \\
Run Time: & 40.0 Minutes & Proc. Chnl. Descr.: & 2998 PDA 254.0 nm (2998 \\
& & & \\
Date Acquired: & $3 / 8 / 20217: 54: 29$ PM CST & & \\
Date Processed: & $3 / 25 / 202110: 03: 22$ AM CST & & \\
\hline
\end{tabular}

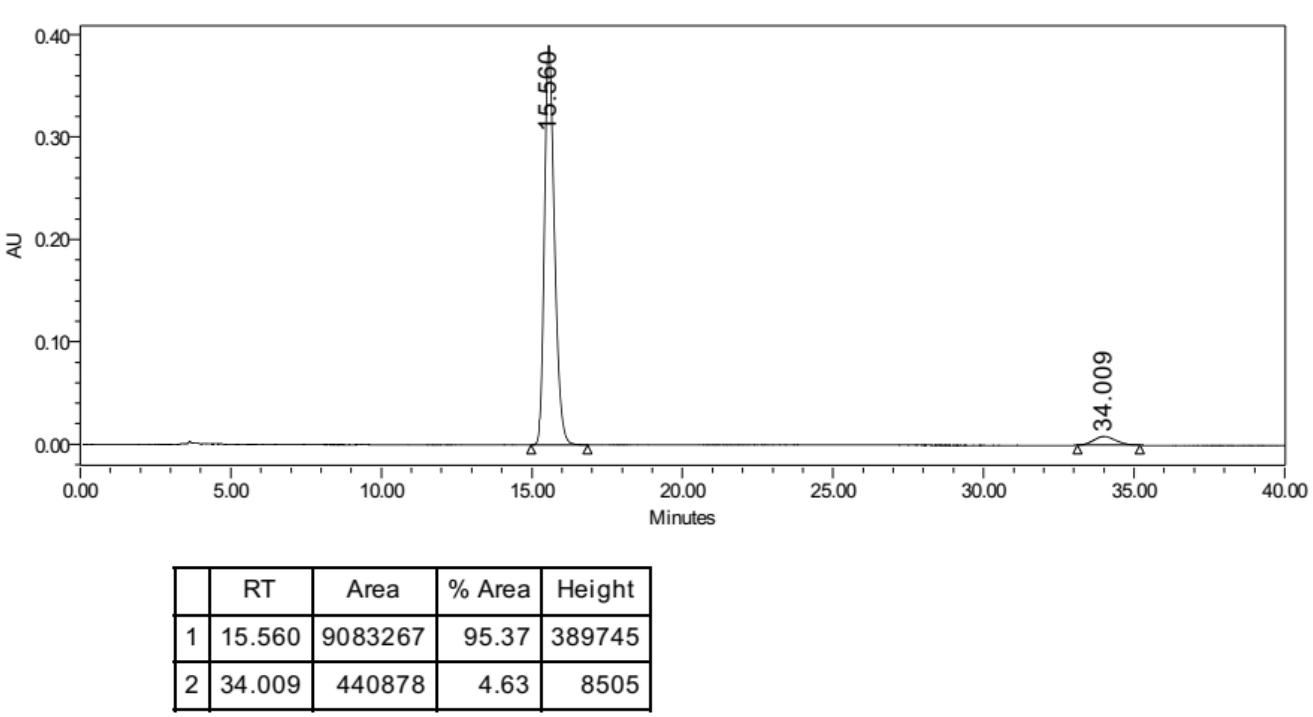


Rac-3nf

\begin{tabular}{|llll|}
\hline & \multicolumn{2}{c|}{ S A M P L E } & IN F O R M A T I O N \\
\hline \hline Sample Name: & xxh-7-51-rac-IC-30\% & Acquired By: & System \\
Sample Type: & Unknown & Sample Set Name & \\
Vial: & 49 & Acq. Method Set: & $30 \%$ quanbo \\
Injection \#: & 1 & Processing Method & 751 \\
Injection Volume: & $10.00 \mathrm{ul}$ & Channel Name: & $254.0 \mathrm{~nm}$ \\
Run Time: & 100.0 Minutes & Proc. Chnl. Descr.: & 2998 PDA 254.0 nm (2998 \\
& & & \\
Date Acquired: & 1/19/2021 10:16:56 PM CST & & \\
Date Processed: & $3 / 25 / 2021$ 10:09:41 AM CST & & \\
\hline
\end{tabular}

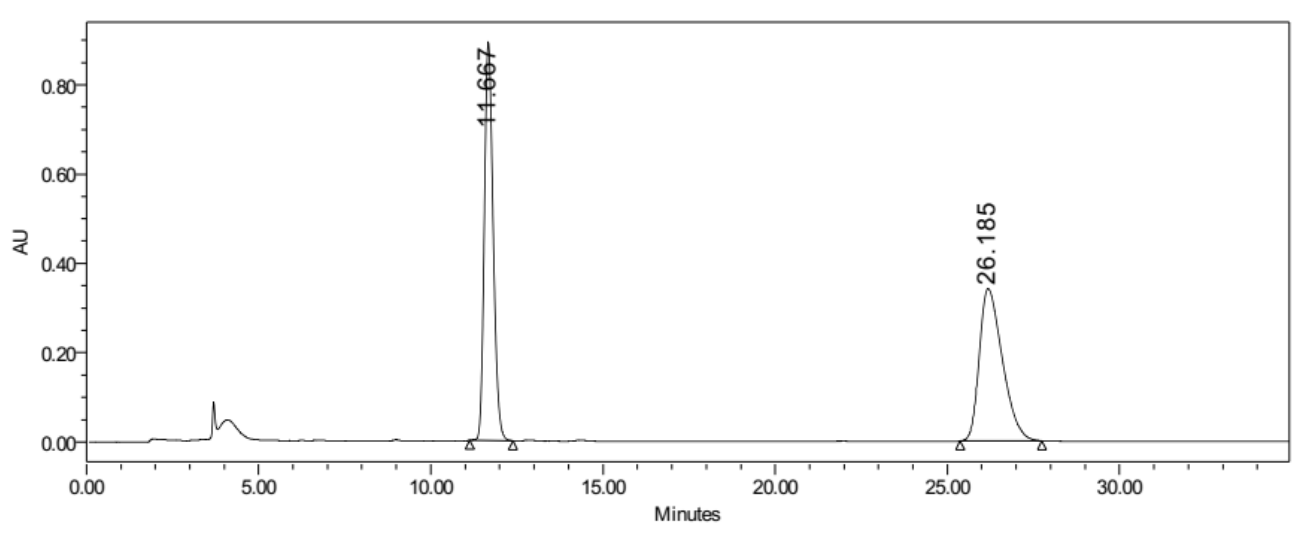

\begin{tabular}{|c|c|c|r|c|}
\hline & RT & Area & $\%$ Area & Height \\
\hline 1 & 11.667 & 15956829 & 50.00 & 891988 \\
\hline 2 & 26.185 & 15958669 & 50.00 & 340908 \\
\hline
\end{tabular}

\section{Asy-3nf}

\begin{tabular}{|llll|}
\hline & \multicolumn{2}{c|}{ S A M P L E } & IN F O R M A T I O N \\
\hline \hline Sample Name: & xxh-8-1-IC-30\% & Acquired By: & System \\
Sample Type: & Unknown & Sample Set Name & 20210316 \\
Vial: & 81 & Acq. Method Set: & $30 \%$ quanbo \\
Injection \#: & 1 & Processing Method & 81 \\
Injection Volume: & 10.00 ul & Channel Name: & $254.0 \mathrm{~nm} @ 1$ \\
Run Time: & 40.0 Minutes & Proc. Chnl. Descr.: & 2998 PDA 254.0 nm (2998 \\
& & & \\
Date Acquired: & $3 / 16 / 20217: 00: 26$ PM CST & & \\
Date Processed: & $3 / 25 / 202110: 11: 32$ AM CST & & \\
\hline
\end{tabular}

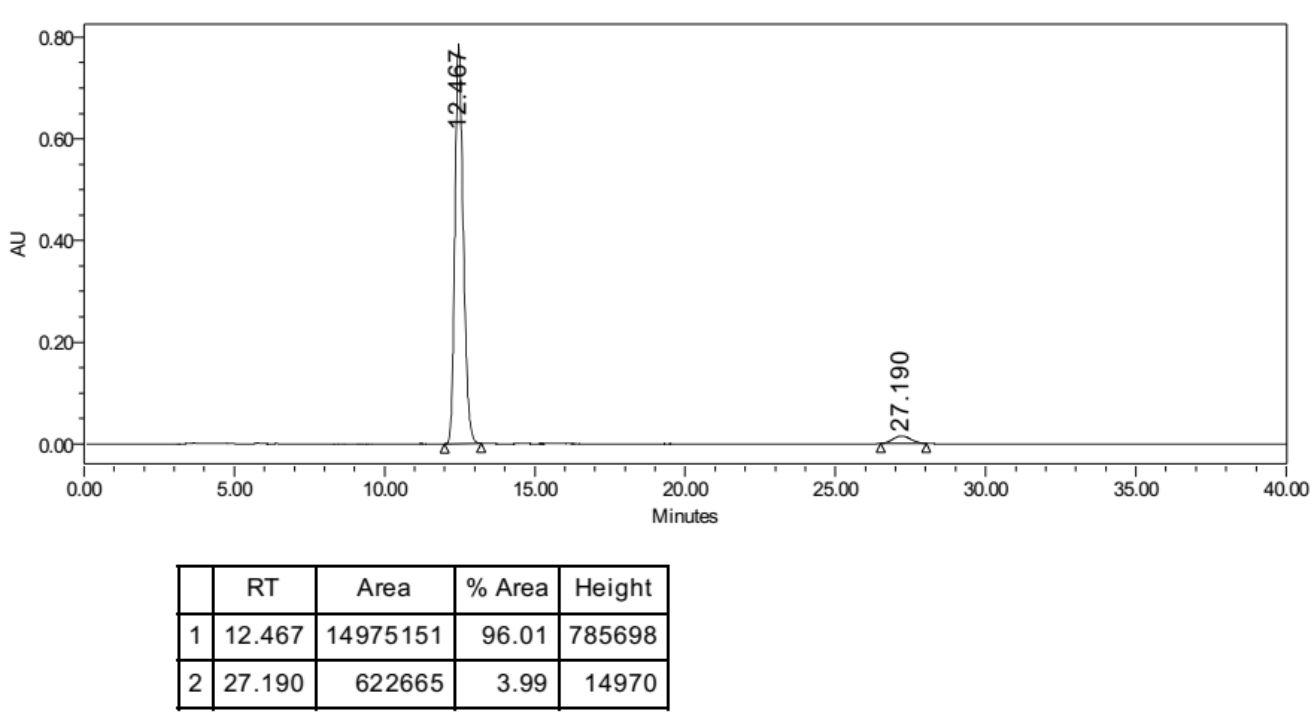


Rac-3of

\begin{tabular}{|llll|}
\hline & \multicolumn{2}{c|}{ S A M P L E } & IN F O R M A T I O N \\
\hline \hline Sample Name: & xxh-6-145-rac-IC-30\% & Acquired By: & System \\
Sample Type: & Unknown & Sample Set Name & 20210305 \\
Vial: & 79 & Acq. Method Set: & $30 \%$ quanbo \\
Injection \#: & 1 & Processing Method & 6145 \\
Injection Volume: & 10.00 ul & Channel Name: & $254.0 \mathrm{~nm}$ \\
Run Time: & 22.0 Minutes & Proc. Chnl. Descr.: & 2998 PDA 254.0 nm (2998 \\
& & & \\
Date Acquired: & $3 / 5 / 202110: 25: 55$ PM CST & & \\
Date Processed: & $3 / 25 / 202110: 15: 32$ AM CST & & \\
\hline
\end{tabular}

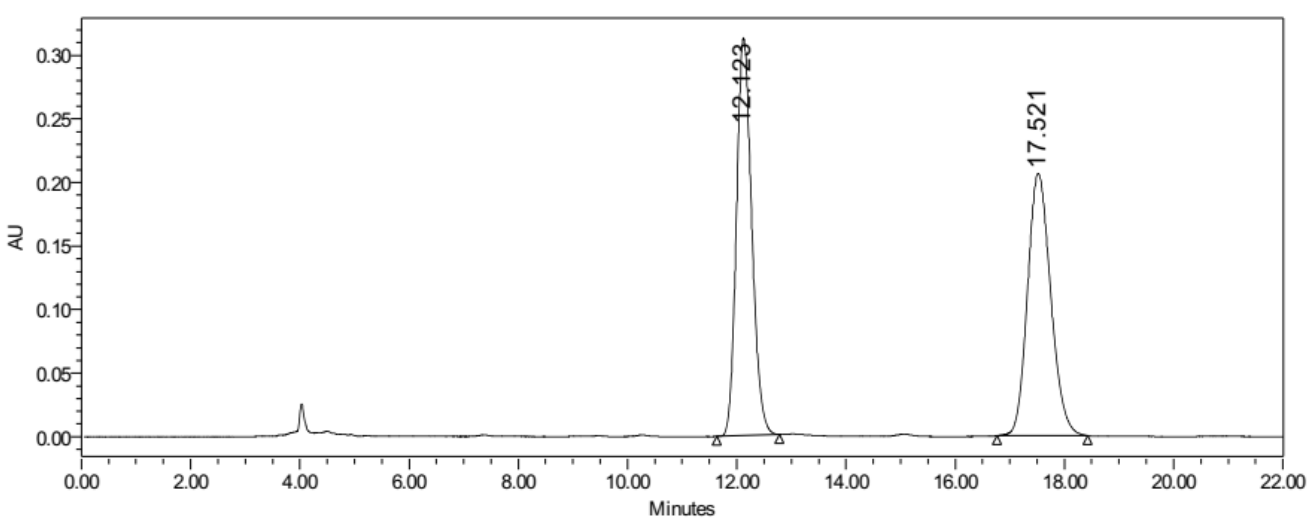

\begin{tabular}{|c|c|c|r|c|}
\hline & RT & Area & $\%$ Area & Height \\
\hline 1 & 12.123 & 6028343 & 50.00 & 312799 \\
\hline 2 & 17.521 & 6029438 & 50.00 & 206247 \\
\hline
\end{tabular}

\section{Asy-3of}

\begin{tabular}{|llll|}
\hline & \multicolumn{2}{c|}{ S A M P L E } & IN F O R M A T I O N \\
\hline \hline Sample Name: & xxh-7-173-IC-30\% & Acquired By: & System \\
Sample Type: & Unknown & Sample Set Name & 20210305 \\
Vial: & 78 & Acq. Method Set: & $30 \%$ quanbo \\
Injection \#: & 1 & Processing Method & 7173 \\
Injection Volume: & 10.00 ul & Channel Name: & $254.0 \mathrm{~nm}$ \\
Run Time: & 22.0 Minutes & Proc. Chnl. Descr.: & 2998 PDA 254.0 nm (2998 \\
& & & \\
Date Acquired: & $3 / 5 / 202110: 03: 15$ PM CST & & \\
Date Processed: & $3 / 25 / 202110: 17: 13$ AM CST & & \\
\hline
\end{tabular}

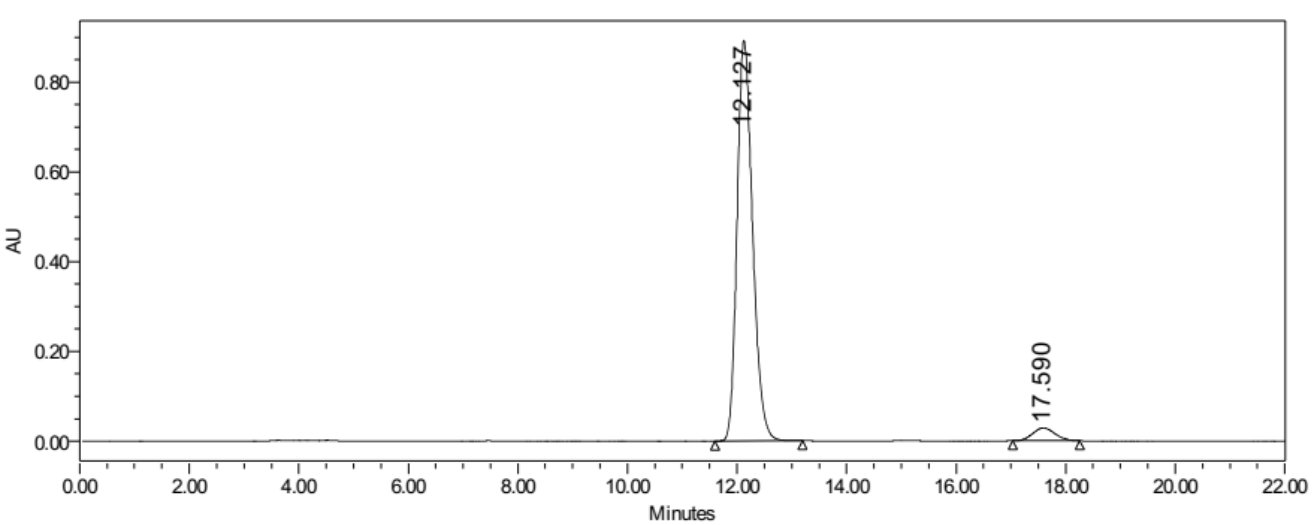

\begin{tabular}{|r|c|r|r|r|}
\hline & RT & \multicolumn{1}{|c|}{ Area } & $\%$ Area & Height \\
\hline 1 & 12.127 & 17360604 & 95.50 & 891952 \\
\hline 2 & 17.590 & 818768 & 4.50 & 28581 \\
\hline
\end{tabular}


Rac-3pf

\begin{tabular}{|llll|}
\hline & \multicolumn{2}{c|}{ S A M P L E } & IN F O R M A T I O N \\
\hline \hline Sample Name: & xxh-7-85-rac-IC-15\% & Acquired By: & System \\
Sample Type: & Unknown & Sample Set Name & 20200219 \\
Vial: & 2 & Acq. Method Set: & $15 \%$ quabbo \\
Injection \#: & 1 & Processing Method & 785 \\
Injection Volume: & $10.00 \mathrm{ul}$ & Channel Name: & $254.0 \mathrm{~nm}$ \\
Run Time: & 34.0 Minutes & Proc. Chnl. Descr.: & 2998 PDA 254.0 nm (2998 \\
& & & \\
Date Acquired: & $2 / 19 / 2021$ 11:10:11 AM CST & & \\
Date Processed: & $3 / 27 / 2021$ 8:52:25 AM CST & & \\
\hline
\end{tabular}

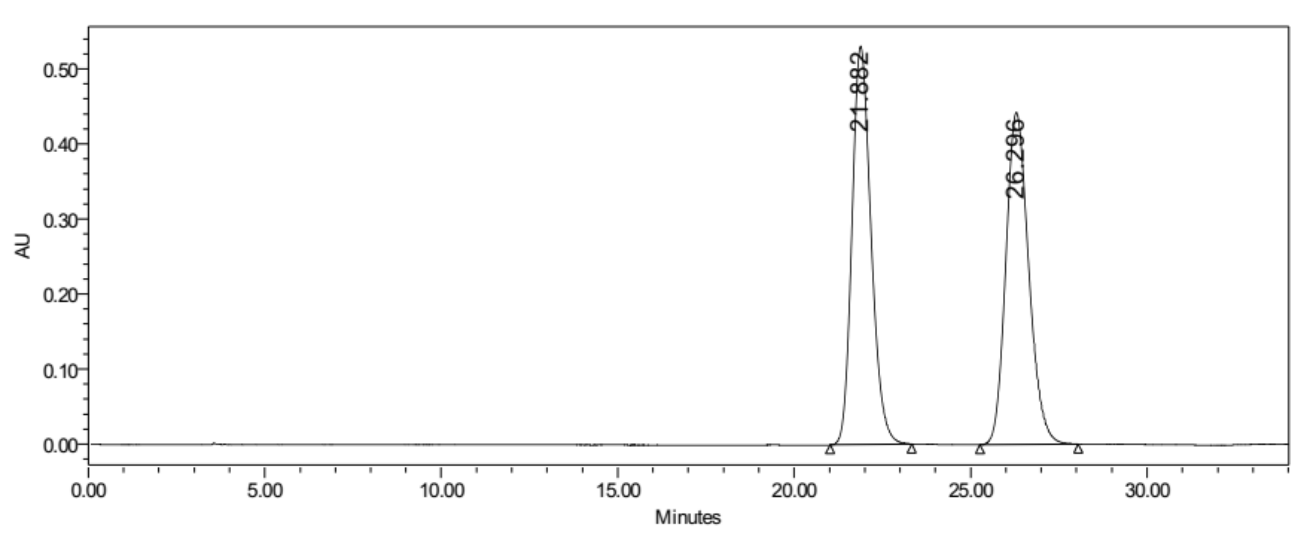

\begin{tabular}{|c|c|c|r|c|}
\hline & RT & Area & $\%$ Area & Height \\
\hline 1 & 21.882 & 20027973 & 50.00 & 530497 \\
\hline 2 & 26.296 & 20030080 & 50.00 & 442303 \\
\hline
\end{tabular}

\section{Asy-3pf}

\begin{tabular}{|llll|}
\hline & \multicolumn{2}{c|}{ S A M P L E } & IN F O R M A T I O N \\
\hline \hline Sample Name: & xxh-7-84-IC-15\% & Acquired By: & System \\
Sample Type: & Unknown & Sample Set Name & 20200219 \\
Vial: & 1 & Acq. Method Set: & $15 \%$ quabbo \\
Injection \#: & 1 & Processing Method & 784 \\
Injection Volume: & 10.00 ul & Channel Name: & $254.0 \mathrm{~nm}$ \\
Run Time: & 34.0 Minutes & Proc. Chnl. Descr.: & 2998 PDA 254.0 nm (2998 \\
& & & \\
Date Acquired: & $2 / 19 / 2021$ 10:35:30 AM CST & & \\
Date Processed: & $3 / 27 / 20218: 53: 33$ AM CST & & \\
\hline
\end{tabular}

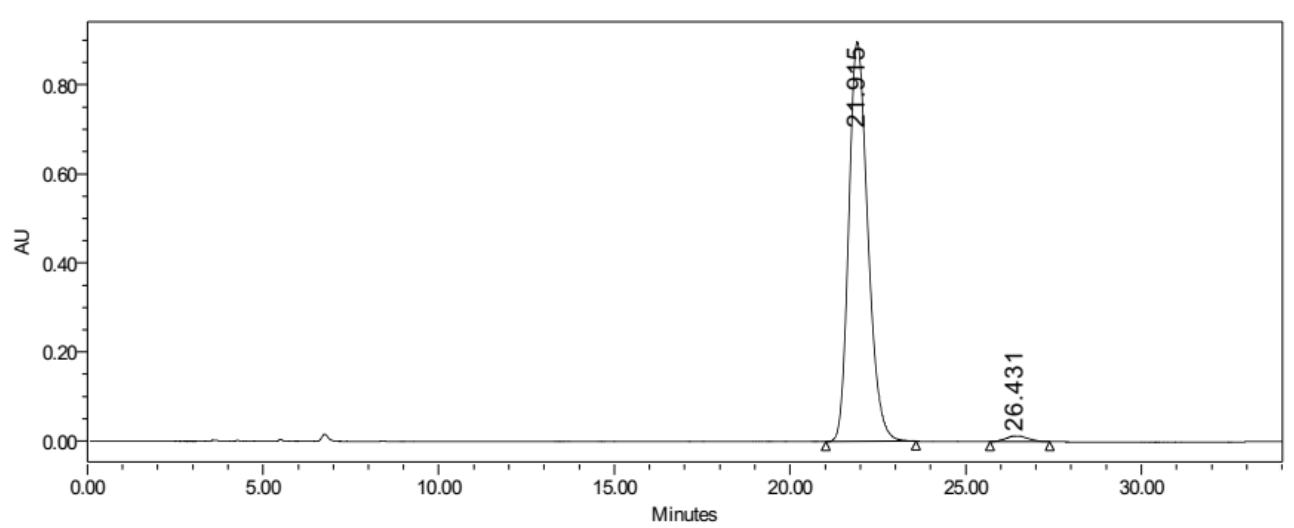

\begin{tabular}{|l|c|r|r|r|}
\hline & RT & \multicolumn{1}{|c|}{ Area } & $\%$ Area & Height \\
\hline 1 & 21.915 & 34087047 & 98.36 & 897853 \\
\hline 2 & 26.431 & 569172 & 1.64 & 12958 \\
\hline
\end{tabular}


Rac-4

\begin{tabular}{|llll|}
\hline & \multicolumn{2}{c|}{ S A M P L E } & IN F O R M A T I O N \\
\hline \hline Sample Name: & xxh-7-133-IC-rac-15\% & Acquired By: & System \\
Sample Type: & Unknown & Sample Set Name & \\
Vial: & 3 & Acq. Method Set: & $15 \%$ quabbo \\
Injection \#: & 1 & Processing Method & 7133 \\
Injection Volume: & $10.00 \mathrm{ul}$ & Channel Name: & $254.0 \mathrm{~nm}$ \\
Run Time: & 100.0 Minutes & Proc. Chnl. Descr.: & 2998 PDA 254.0 nm (2998 \\
& & & \\
Date Acquired: & $2 / 23 / 202111: 05: 21$ AM CST & & \\
Date Processed: & $3 / 27 / 20219: 00: 38$ AM CST & & \\
\hline
\end{tabular}

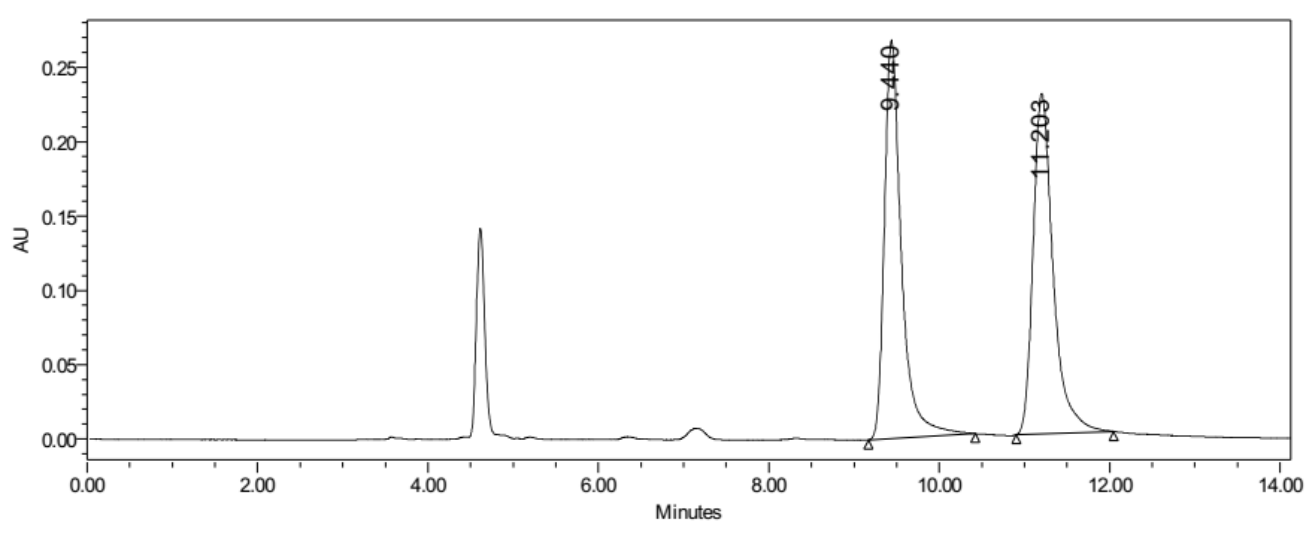

\begin{tabular}{|c|c|c|r|c|}
\hline & \multicolumn{1}{|c|}{ RT } & Area & $\%$ Area & Height \\
\hline 1 & 9.440 & 3713775 & 50.00 & 267959 \\
\hline 2 & 11.203 & 3713518 & 50.00 & 229061 \\
\hline
\end{tabular}

Asy-4

\begin{tabular}{|llll|}
\hline & \multicolumn{2}{c|}{ S A M P L E } & IN F O R M A T I O N \\
\hline \hline Sample Name: & xxh-7-139-IC-15\% & Acquired By: & System \\
Sample Type: & Unknown & Sample Set Name & 20200223 \\
Vial: & 1 & Acq. Method Set: & $15 \%$ quabbo \\
Injection \#: & 1 & Processing Method & 7139 \\
Injection Volume: & 10.00 ul & Channel Name: & $254.0 \mathrm{~nm}$ \\
Run Time: & 16.0 Minutes & Proc. Chnl. Descr.: & 2998 PDA 254.0 nm (2998 \\
& & & \\
Date Acquired: & $2 / 23 / 202111: 23: 07$ AM CST & & \\
Date Processed: & $3 / 27 / 20219: 01: 40$ AM CST & & \\
\hline
\end{tabular}

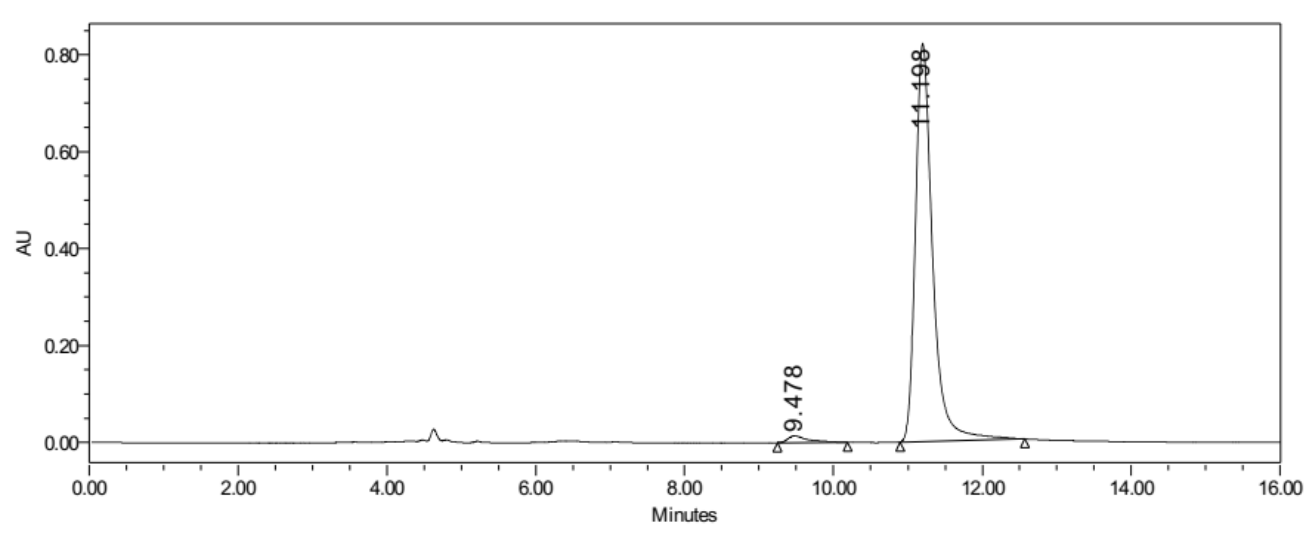

\begin{tabular}{|r|r|r|r|r|}
\hline & \multicolumn{1}{|c|}{ RT } & \multicolumn{1}{c|}{ Area } & \% Area & Height \\
\hline 1 & 9.478 & 266338 & 1.98 & 14073 \\
\hline 2 & 11.198 & 13217427 & 98.02 & 821034 \\
\hline
\end{tabular}


Rac-5

\begin{tabular}{|llll|}
\hline & \multicolumn{2}{c|}{ S A M P L E } & IN F O R M A T I O N \\
\hline \hline Sample Name: & xxh-7-141-IA-rac-10\% & Acquired By: & System \\
Sample Type: & Unknown & Sample Set Name & 20210223 \\
Vial: & 2 & Acq. Method Set: & $10 \%$ quanbo \\
Injection \#: & 1 & Processing Method & 7141 \\
Injection Volume: & 10.00 ul & Channel Name: & $254.0 \mathrm{~nm}$ \\
Run Time: & 25.0 Minutes & Proc. Chnl. Descr.: & 2998 PDA 254.0 nm (2998 \\
& & & \\
Date Acquired: & $2 / 23 / 2021$ 8:25:43 PM CST & & \\
Date Processed: & 3/27/2021 9:06:09 AM CST & & \\
\hline
\end{tabular}

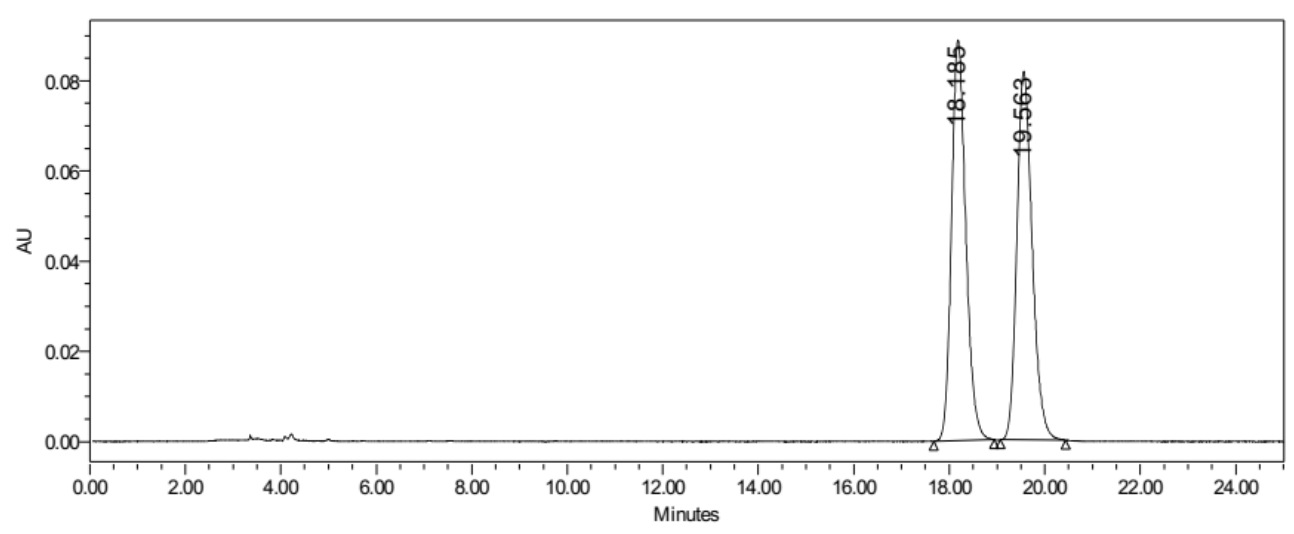

\begin{tabular}{|c|c|c|r|c|}
\hline & RT & Area & $\%$ Area & Height \\
\hline 1 & 18.185 & 1821373 & 50.00 & 88672 \\
\hline 2 & 19.563 & 1821503 & 50.00 & 81666 \\
\hline
\end{tabular}

\section{Asy-5}

\begin{tabular}{|llll|}
\hline & \multicolumn{2}{c|}{ S A M P L E } & IN F O R M A T I O N \\
\hline \hline Sample Name: & xxh-7-142-IA-10\% & Acquired By: & System \\
Sample Type: & Unknown & Sample Set Name & 20210224 \\
Vial: & 65 & Acq. Method Set: & $10 \% q u a n b o$ \\
Injection \#: & 1 & Processing Method & 7142 \\
Injection Volume: & 10.00 ul & Channel Name: & $254.0 \mathrm{~nm}$ \\
Run Time: & 25.0 Minutes & Proc. Chnl. Descr.: & 2998 PDA 254.0 nm (2998 \\
& & & \\
Date Acquired: & 2/24/2021 2:28:08 PM CST & & \\
Date Processed: & $3 / 27 / 20219: 07: 03$ AM CST & & \\
\hline
\end{tabular}

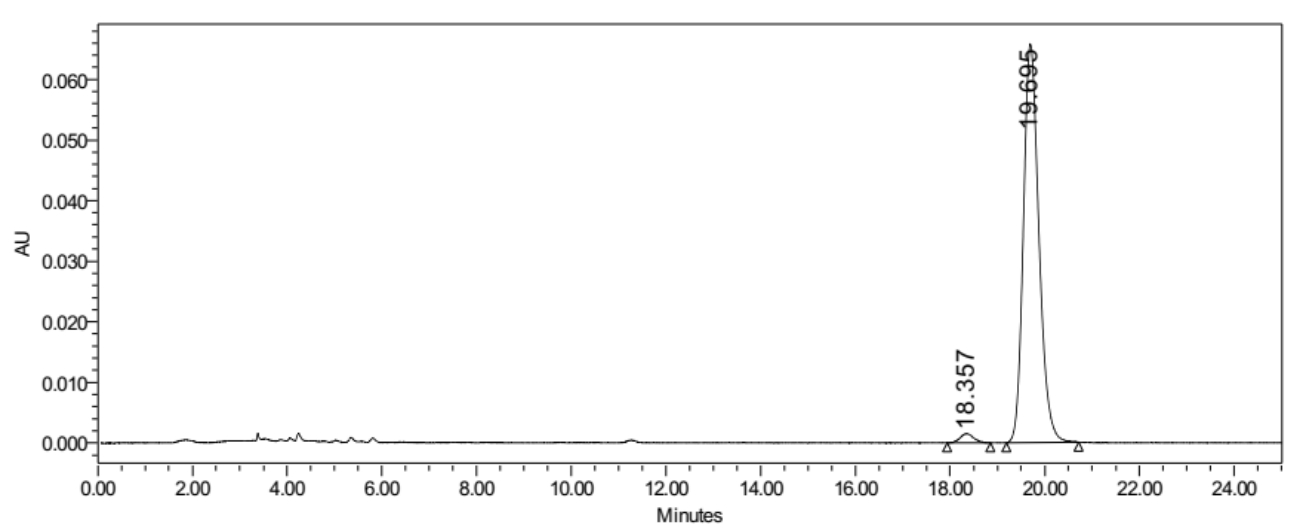

\begin{tabular}{|r|c|r|r|r|}
\hline & RT & Area & $\%$ Area & Height \\
\hline 1 & 18.357 & 29734 & 1.95 & 1485 \\
\hline 2 & 19.695 & 1494524 & 98.05 & 65768 \\
\hline
\end{tabular}


Rac-6

\begin{tabular}{|llll|}
\hline & \multicolumn{2}{c|}{ S A M P L E } & I N F O R M A T I O N \\
\hline \hline Sample Name: & xxh-6-160-rac-0J-5\% & Acquired By: & System \\
Sample Type: & Unknown & Sample Set Name: & 20210319 \\
Vial: & 84 & Acq. Method Set: & $5 \%$ quanbo \\
Injection \#: & 1 & Processing Method & 6160 \\
Injection Volume: & 10.00 ul & Channel Name: & $254.0 \mathrm{~nm}$ \\
Run Time: & 24.0 Minutes & Proc. Chnl. Descr.: & 2998 PDA 254.0 nm (2998 \\
& & & \\
Date Acquired: & $3 / 19 / 2021$ 10:56:06 PM CST & & \\
Date Processed: & $3 / 25 / 202110: 22: 47$ AM CST & & \\
\hline
\end{tabular}

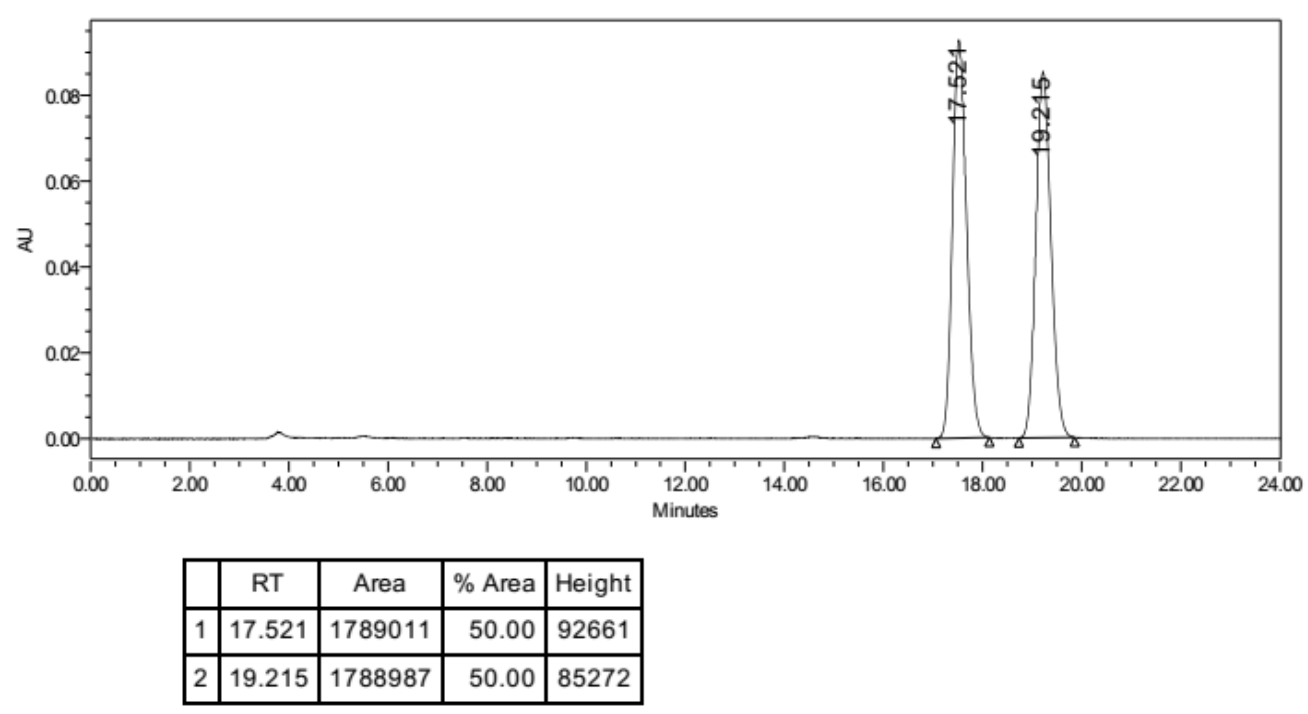

Asy-6

\begin{tabular}{|llll|}
\hline & \multicolumn{1}{c|}{ S A M P LE } & IN F O R M A T I O N \\
\hline \hline Sample Name: & xxh-8-34-OJ-5\% & Acquired By: & Sysem \\
Sample Type: & Unknown & Sample Set Name & 20210329 \\
Vial: & 1 & Acq. Method Set: & $5 \%$ quanbo \\
Injection \#: & 1 & Processina M ethoc & 8345 \\
Injection Volume: & 10.00 ul & Channel Name: & $254.0 \mathrm{~nm}$ \\
Run Time: & 24.0 M inutes & Proc. Chnl. Descr.: & 2998 PDA 254.0 nm (2998 \\
& & & \\
Date Acquired: & $3 / 29 / 20213: 49: 49$ PM CST & & \\
Date Processed: & $3 / 29 / 20214: 24: 18$ PM CST & & \\
\hline
\end{tabular}

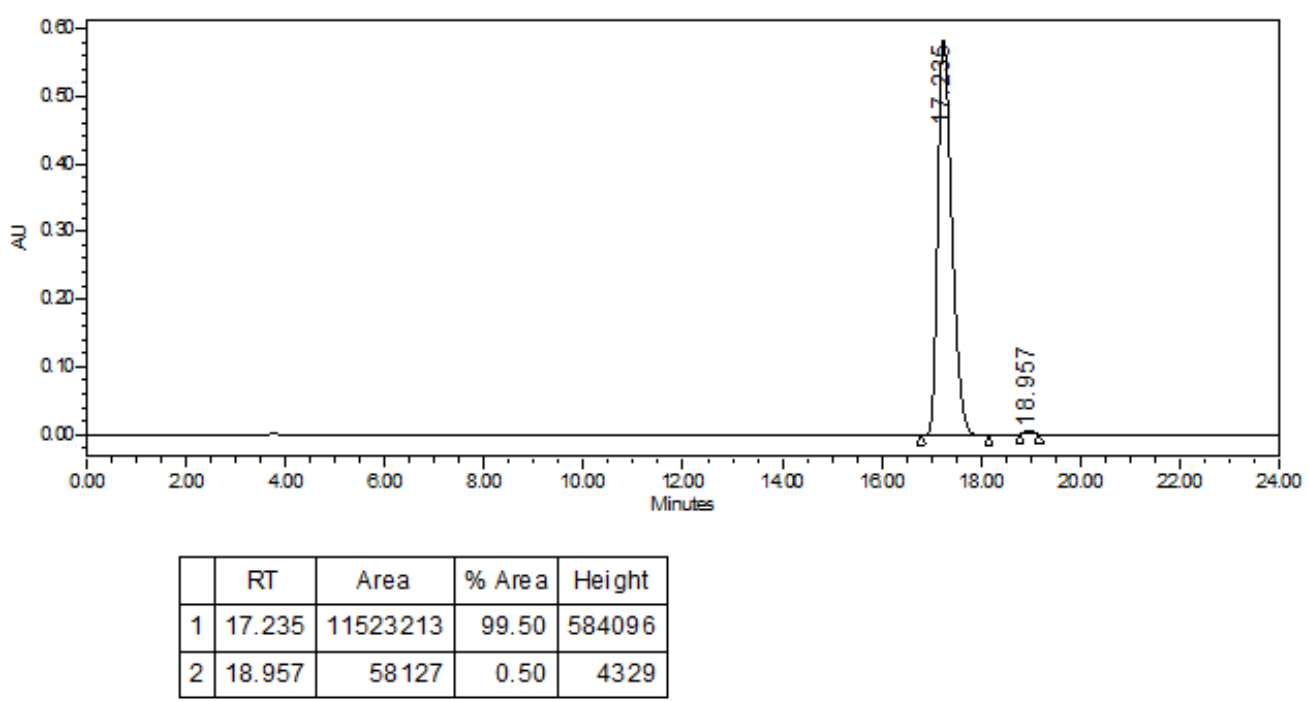


Rac-7

\begin{tabular}{|llll|}
\hline & \multicolumn{2}{c|}{ S A M P L E } & IN F O R M A T I O N \\
\hline \hline Sample Name: & xxh-7-149-rac-OJ-5\% & Acquired By: & System \\
Sample Type: & Unknown & Sample Set Name & 20210227 \\
Vial: & 77 & Acq. Method Set: & $5 \%$ quanbo \\
Injection \#: & 1 & Processing Method & 7149 \\
Injection Volume: & 10.00 ul & Channel Name: & $254.0 \mathrm{~nm}$ \\
Run Time: & 26.0 Minutes & Proc. Chnl. Descr.: & 2998 PDA 254.0 nm (2998 \\
& & & \\
Date Acquired: & $2 / 27 / 20215: 21: 48$ PM CST & & \\
Date Processed: & $3 / 25 / 2021$ 10:38:15 AM CST & & \\
\hline
\end{tabular}

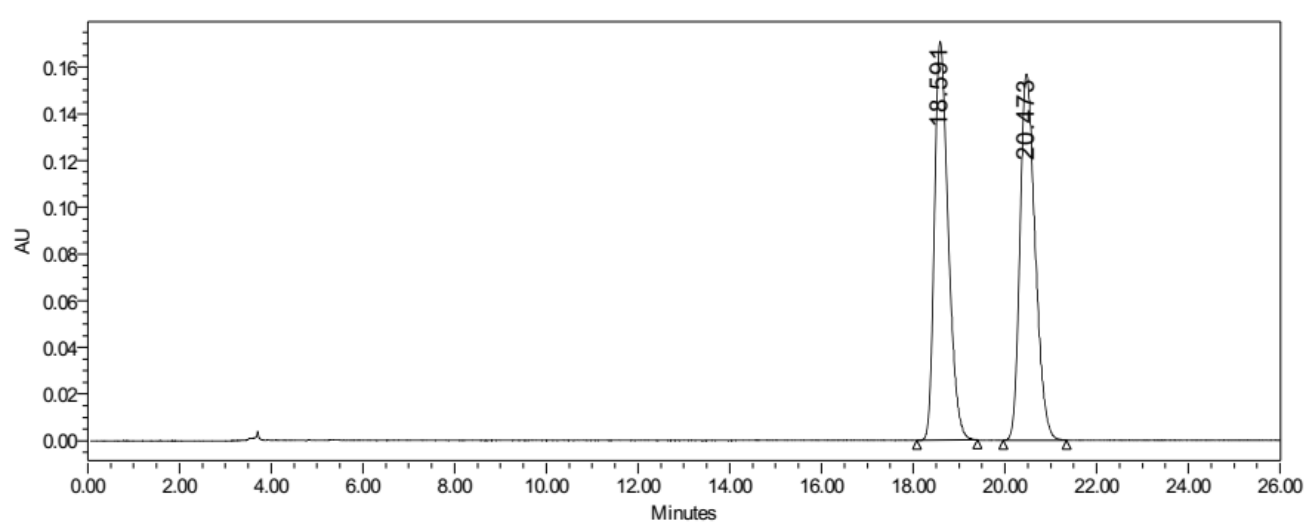

\begin{tabular}{|c|c|c|r|c|}
\hline & RT & Area & $\%$ Area & Height \\
\hline 1 & 18.591 & 3574289 & 50.00 & 170736 \\
\hline 2 & 20.473 & 3574472 & 50.00 & 156795 \\
\hline
\end{tabular}

\section{Asy-7}

\begin{tabular}{|llll|}
\hline & \multicolumn{2}{c|}{ S A M P L E } & IN F O R M A T I O N \\
\hline \hline Sample Name: & xxh-7-159-OJ-5\% & Acquired By: & System \\
Sample Type: & Unknown & Sample Set Name & 20210227 \\
Vial: & 78 & Acq. Method Set: & $5 \%$ quanbo \\
Injection \#: & 1 & Processing Method & 7159 \\
Injection Volume: & $10.00 \mathrm{ul}$ & Channel Name: & $254.0 \mathrm{~nm}$ \\
Run Time: & 26.0 Minutes & Proc. Chnl. Descr.: & 2998 PDA 254.0 nm (2998 \\
& & & \\
Date Acquired: & $2 / 27 / 20215: 48: 29$ PM CST & & \\
Date Processed: & $3 / 25 / 2021$ 10:42:45 AM CST & & \\
\hline
\end{tabular}

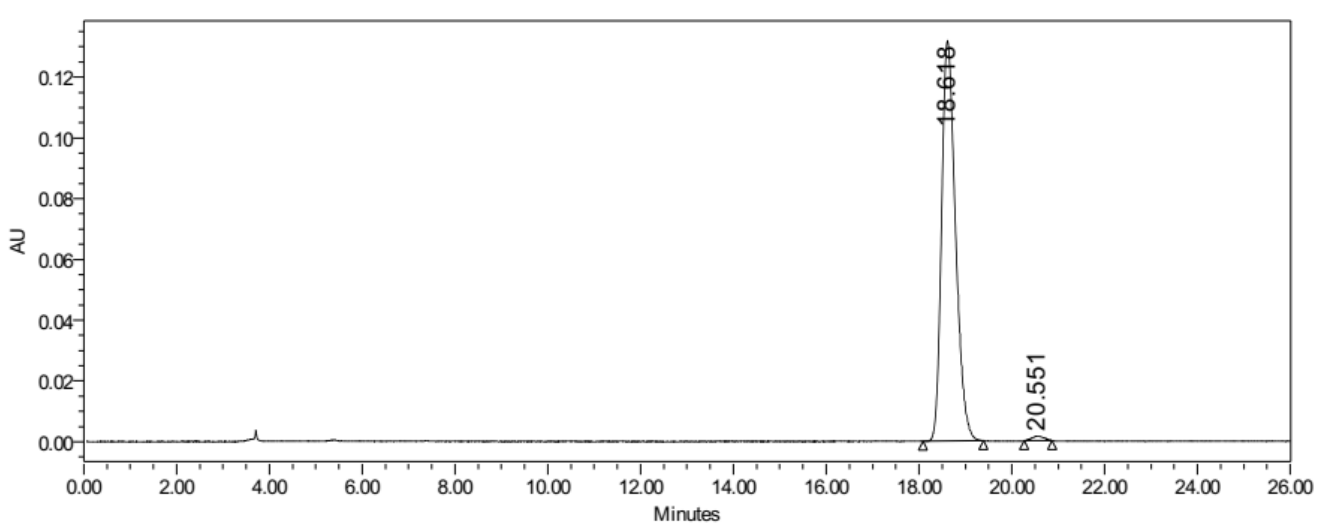

\begin{tabular}{|l|c|r|r|r|}
\hline & RT & \multicolumn{1}{|c|}{ Area } & $\%$ Area & Height \\
\hline 1 & 18.618 & 2750723 & 99.01 & 131745 \\
\hline 2 & 20.551 & 27526 & 0.99 & 1463 \\
\hline
\end{tabular}


Rac-8

\begin{tabular}{|llll|}
\hline & \multicolumn{2}{c|}{ S A M P L E } & IN F O R M A T I O N \\
\hline \hline Sample Name: & xxh-7-160-IB-rac-5\% & Acquired By: & System \\
Sample Type: & Unknown & Sample Set Name & 20210301 \\
Vial: & 77 & Acq. Method Set: & $5 \%$ quanbo \\
Injection \#: & 1 & Processing Method & 7160 \\
Injection Volume: & 10.00 ul & Channel Name: & $254.0 \mathrm{~nm}$ \\
Run Time: & 20.0 Minutes & Proc. Chnl. Descr.: & 2998 PDA 254.0 nm (2998 \\
& & & \\
Date Acquired: & $3 / 1 / 20218: 31: 35$ PM CST & & \\
Date Processed: & $3 / 25 / 2021$ 10:34:28 PM CST & & \\
\hline
\end{tabular}

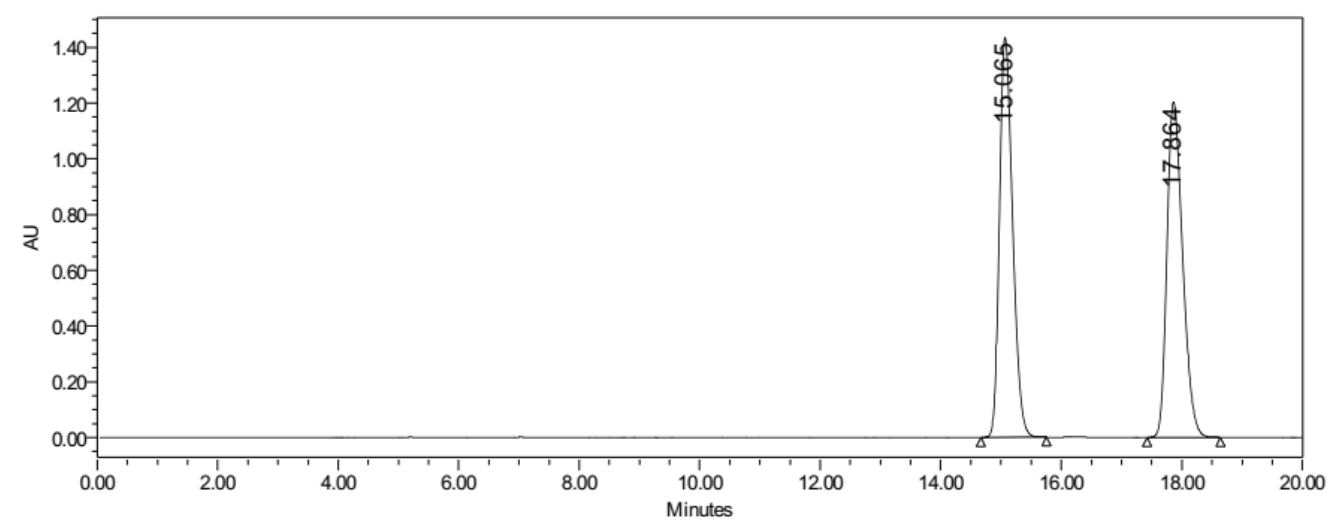

\begin{tabular}{|c|c|c|r|c|}
\hline & RT & Area & $\%$ Area & Height \\
\hline 1 & 15.065 & 21894771 & 50.00 & 1434477 \\
\hline 2 & 17.864 & 21898664 & 50.00 & 1204296 \\
\hline
\end{tabular}

\section{Asy-8}

\begin{tabular}{|llll|}
\hline & \multicolumn{2}{c|}{ S A M P LE } & IN F O R M A T I O N \\
\hline \hline Sample Name: & xxh-7-161-IB-5\% & Acquired By: & System \\
Sample Type: & Unknown & Sample Set Name & 20210301 \\
Vial: & 78 & Acq. Method Set: & $5 \%$ quanbo \\
Injection \#: & 1 & Processing Method & 7161 \\
Injection Volume: & $10.00 \mathrm{ul}$ & Channel Name: & $254.0 \mathrm{~nm}$ \\
Run Time: & 20.0 Minutes & Proc. Chnl. Descr.: & 2998 PDA 254.0 nm (2998 \\
& & & \\
Date Acquired: & $3 / 1 / 20219: 43: 47$ PM CST & & \\
Date Processed: & $3 / 25 / 202110: 37: 52$ PM CST & & \\
\hline
\end{tabular}

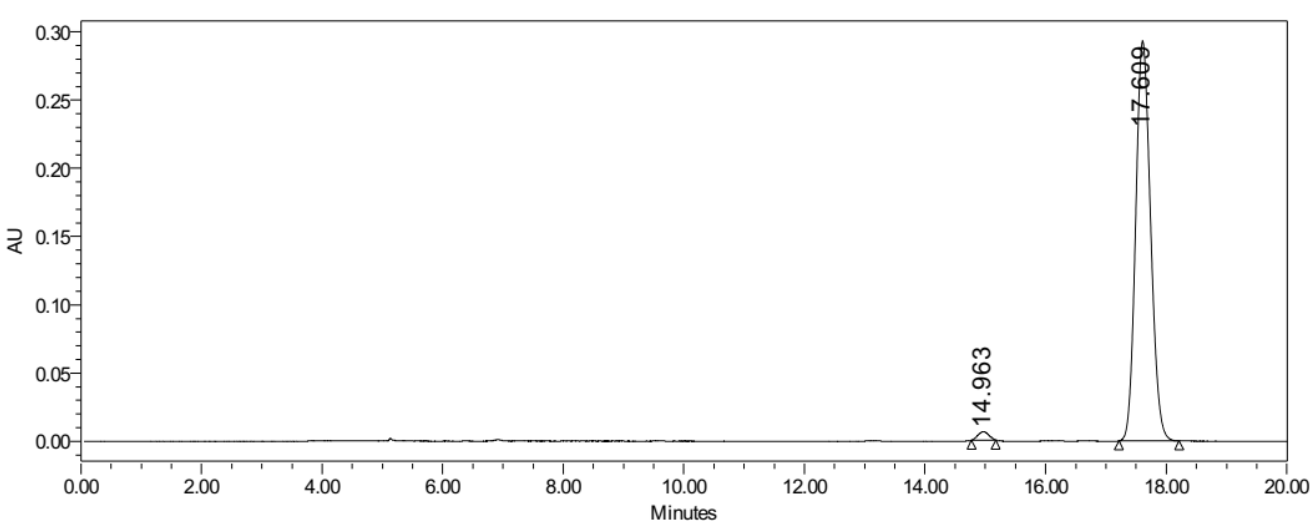

\begin{tabular}{|c|c|c|r|r|}
\hline & RT & Area & $\%$ Area & Height \\
\hline 1 & 14.963 & 77493 & 1.50 & 6220 \\
\hline 2 & 17.609 & 5075102 & 98.50 & 293271 \\
\hline
\end{tabular}


Rac-9

\begin{tabular}{|llll|}
\hline & \multicolumn{2}{c|}{ S A M P L E } & IN F O R M A T I O N \\
\hline \hline Sample Name: & xxh-7-163-rac-IB-5\% & Acquired By: & System \\
Sample Type: & Unknown & Sample Set Name & \\
Vial: & 78 & Acq. Method Set: & $5 \%$ quanbo \\
Injection \#: & 1 & Processing Method & 7163 \\
Injection Volume: & $10.00 \mathrm{ul}$ & Channel Name: & $254.0 \mathrm{~nm}$ \\
Run Time: & 100.0 Minutes & Proc. Chnl. Descr.: & 2998 PDA 254.0 nm (2998 \\
& & & \\
Date Acquired: & $3 / 2 / 20214: 01: 36$ PM CST & & \\
Date Processed: & $3 / 25 / 202110: 41: 40$ PM CST & & \\
\hline
\end{tabular}

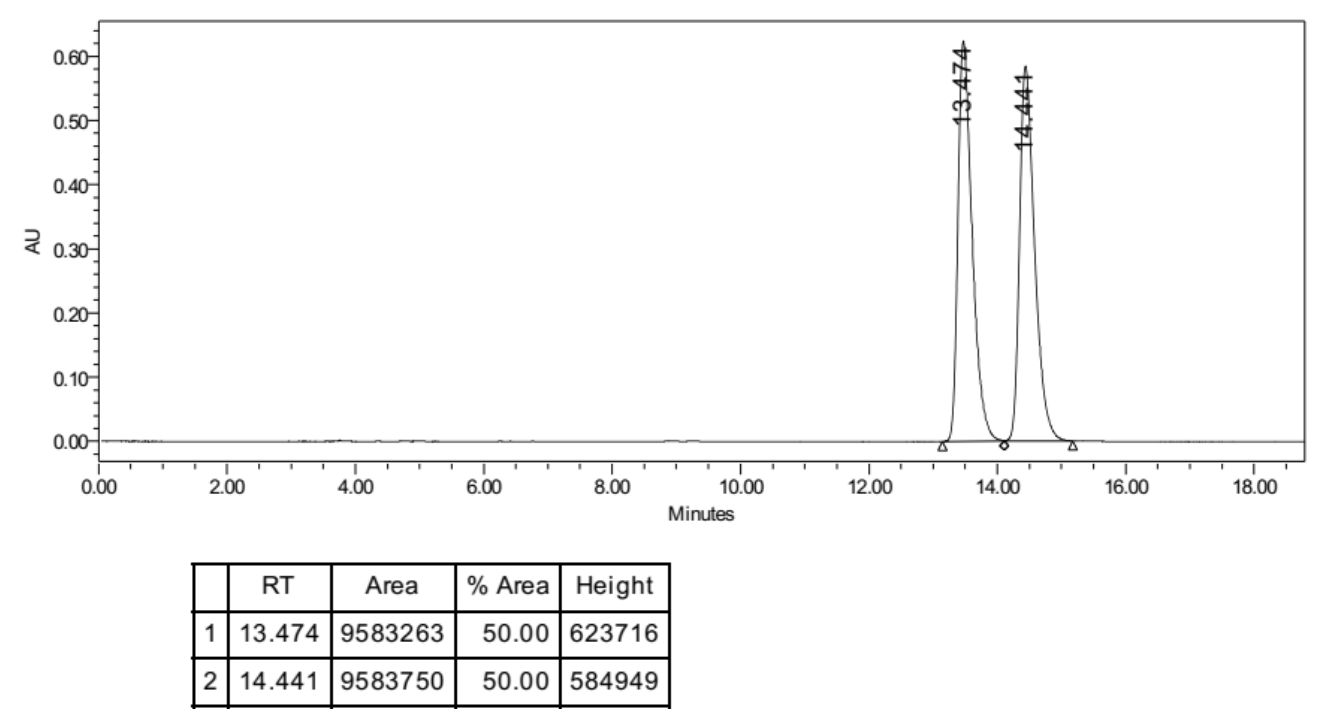

Asy-9

\begin{tabular}{|llll|}
\hline & \multicolumn{2}{c|}{ S A M P L E } & IN F O R M A T I O N \\
\hline \hline Sample Name: & xxh-7-168-IB-5\% & Acquired By: & System \\
Sample Type: & Unknown & Sample Set Name & \\
Vial: & 79 & Acq. Method Set: & $5 \%$ quanbo \\
Injection \#: & 1 & Processing Method & 7168 \\
Injection Volume: & 10.00 ul & Channel Name: & $254.0 \mathrm{~nm}$ \\
Run Time: & 100.0 Minutes & Proc. Chnl. Descr.: & 2998 PDA 254.0 nm (2998 \\
& & & \\
Date Acquired: & $3 / 2 / 20214: 22: 04$ PM CST & & \\
Date Processed: & $3 / 25 / 202110: 42: 34$ PM CST & & \\
\hline
\end{tabular}

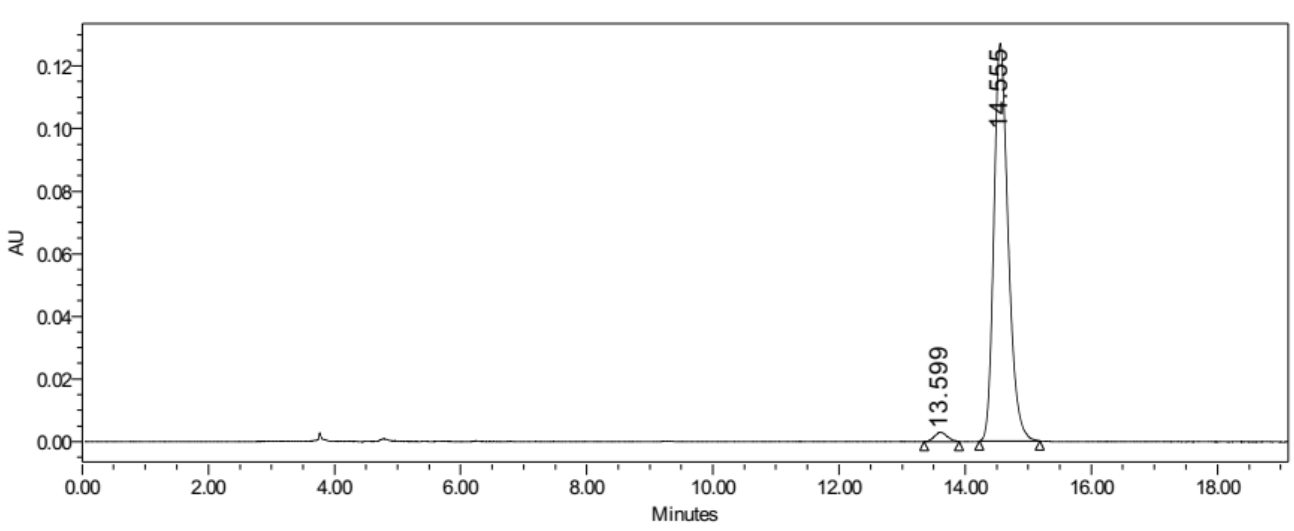

\begin{tabular}{|l|c|c|r|r|}
\hline & RT & Area & $\%$ Area & Height \\
\hline 1 & 13.599 & 41701 & 2.03 & 2978 \\
\hline 2 & 14.555 & 2015093 & 97.97 & 127003 \\
\hline
\end{tabular}


Rac-10

\begin{tabular}{|llll|}
\hline & \multicolumn{2}{c|}{ S A M P L E } & IN F O R M A T I O N \\
\hline \hline Sample Name: & xxh-7-170-IB-rac-2\% & Acquired By: & System \\
Sample Type: & Unknown & Sample Set Name & 20210317 \\
Vial: & 77 & Acq. Method Set: & $2 \%$ quanbo \\
Injection \#: & 1 & Processing Method & 7170 \\
Injection Volume: & 10.00 ul & Channel Name: & $220.0 \mathrm{~nm}$ \\
Run Time: & 30.0 Minutes & Proc. Chnl. Descr.: & 2998 PDA 220.0 nm (2998 \\
& & & \\
Date Acquired: & $3 / 17 / 2021$ 11:01:26 PM CST & & \\
Date Processed: & $3 / 25 / 2021$ 10:46:33 PM CST & & \\
\hline
\end{tabular}

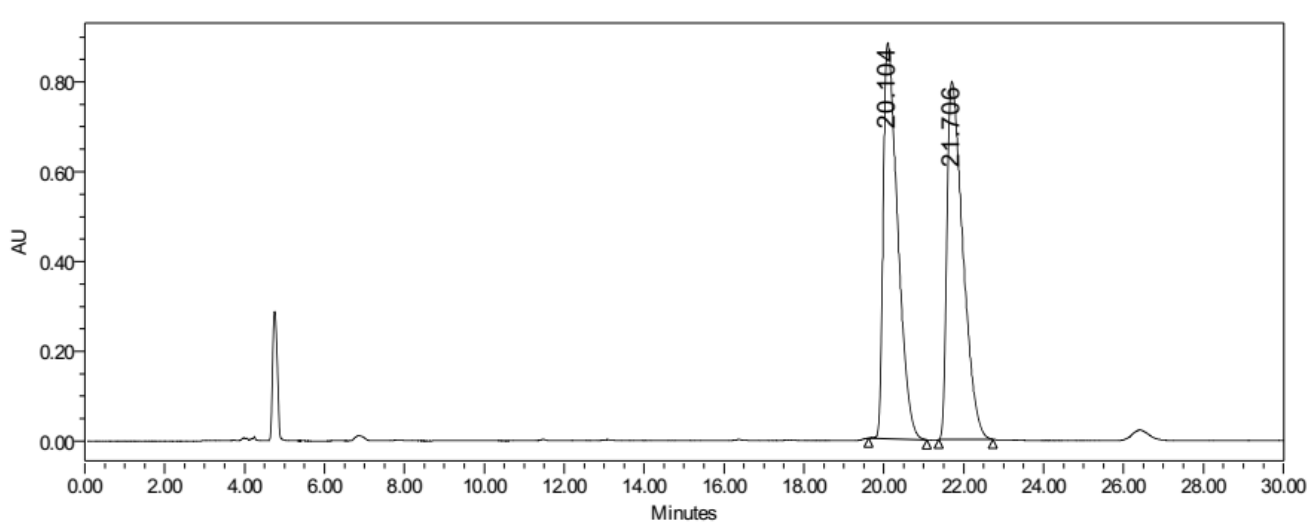

\begin{tabular}{|c|c|c|r|c|}
\hline & RT & Area & $\%$ Area & Height \\
\hline 1 & 20.104 & 21898159 & 50.00 & 881293 \\
\hline 2 & 21.706 & 21898985 & 50.00 & 797644 \\
\hline
\end{tabular}

Asy-10

\begin{tabular}{|llll|}
\hline & \multicolumn{2}{c|}{ S A M P L E } & IN F O R M A T I O N \\
\hline \hline Sample Name: & xxh-8-15-IB-2\% & Acquired By: & System \\
Sample Type: & Unknown & Sample Set Name & 20210317 \\
Vial: & 78 & Acq. Method Set: & $2 \%$ quanbo \\
Injection \#: & 1 & Processing Method & 815 \\
Injection Volume: & 10.00 ul & Channel Name: & $220.0 \mathrm{~nm}$ \\
Run Time: & 30.0 Minutes & Proc. Chnl. Descr.: & 2998 PDA 220.0 nm (2998 \\
& & & \\
Date Acquired: & $3 / 17 / 202111: 32: 07$ PM CST & & \\
Date Processed: & $3 / 25 / 202110: 47: 36$ PM CST & & \\
\hline
\end{tabular}

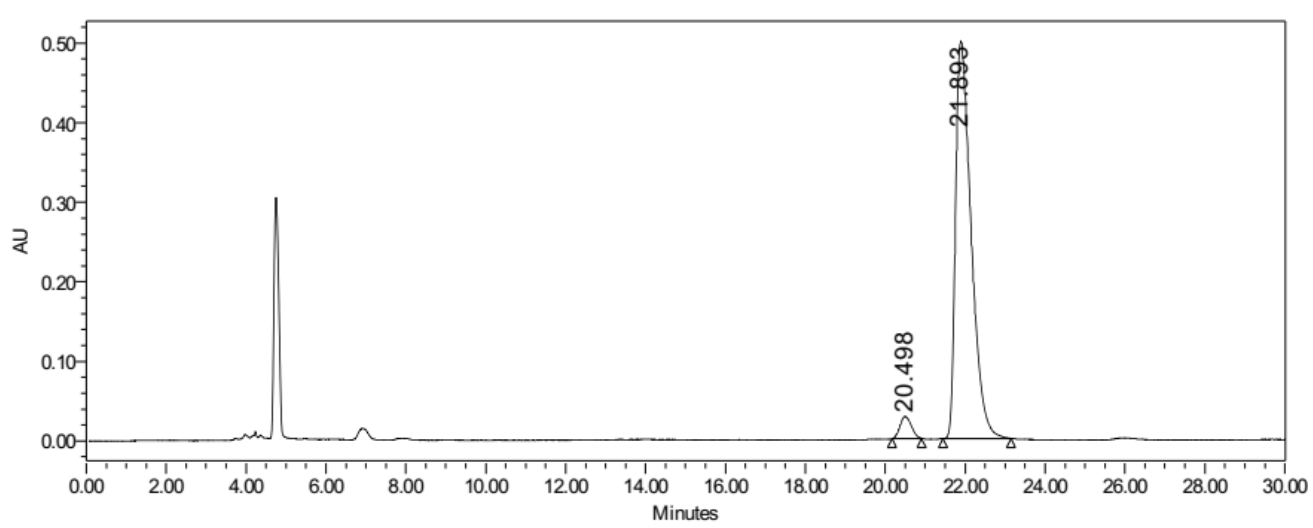

\begin{tabular}{|r|c|r|r|r|}
\hline & RT & \multicolumn{1}{|c|}{ Area } & $\%$ Area & Height \\
\hline 1 & 20.498 & 555594 & 4.00 & 27885 \\
\hline 2 & 21.893 & 13320475 & 96.00 & 499472 \\
\hline
\end{tabular}


Rac-3qf

\begin{tabular}{|llll|}
\hline & \multicolumn{2}{c|}{ S A M P L E } & I N F O R M A T I O N \\
\hline \hline Sample Name: & xxh-6-146-rac-30\% & Acquired By: & System \\
Sample Type: & Unknown & Sample Set Name: & 20210615 \\
Vial: & 92 & Acq. Method Set: & $30 \%$ quanbo \\
Injection \#: & 1 & Processing Method: & 6146 rac \\
Injection Volume: & 10.00 ul & Channel Name: & $254.0 \mathrm{~nm}$ \\
Run Time: & 20.0 Minutes & Proc. Chnl. Descr.: & 2998 PDA 254.0 nm (2998 \\
& & & \\
Date Acquired: & 6/15/2021 6:48:39 PM CST & & \\
Date Processed: & $6 / 15 / 20217: 31: 38$ PM CST & & \\
\hline
\end{tabular}

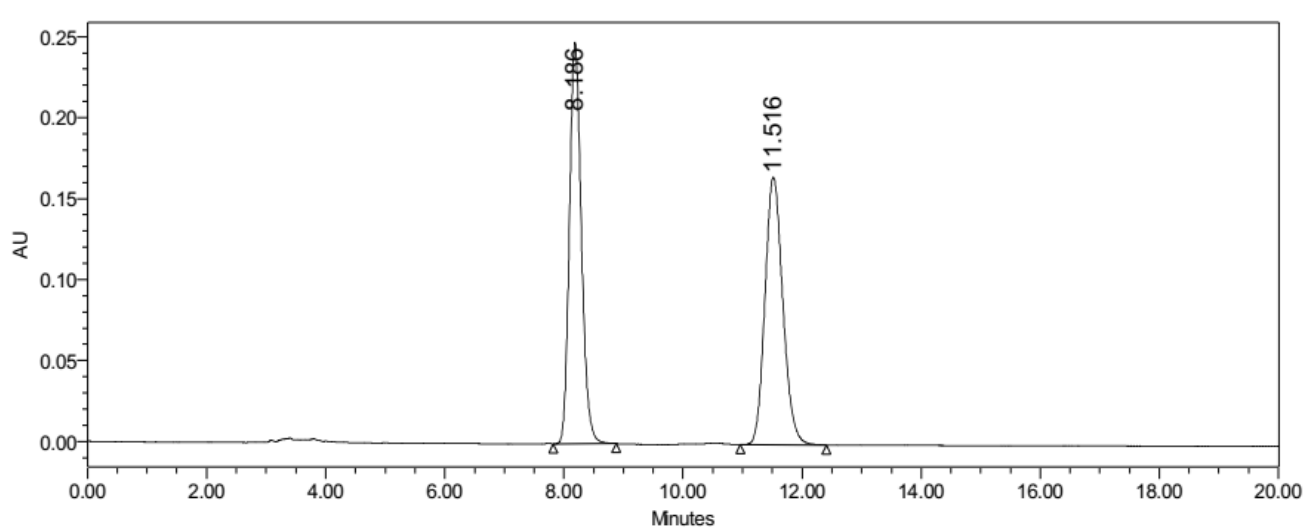

\begin{tabular}{|r|r|c|r|c|}
\hline & \multicolumn{1}{|c|}{ RT } & Area & $\%$ Area & Height \\
\hline 1 & 8.186 & 3414113 & 50.00 & 247852 \\
\hline 2 & 11.516 & 3413444 & 50.00 & 165212 \\
\hline
\end{tabular}

\section{Asy-3qf}

\begin{tabular}{|llll|}
\hline & \multicolumn{2}{c|}{ S A M P L E } & I N F O R M A T I O N \\
\hline \hline Sample Name: & xxh-7-131-30\% & Acquired By: & System \\
Sample Type: & Unknown & Sample Set Name: & 20210615 \\
Vial: & 93 & Acq. Method Set: & $30 \%$ quanbo \\
Injection \#: & 1 & Processing Method: & 71312 \\
Injection Volume: & $10.00 \mathrm{ul}$ & Channel Name: & $254.0 \mathrm{~nm}$ \\
Run Time: & 20.0 Minutes & Proc. Chnl. Descr.: & 2998 PDA 254.0 nm (2998 \\
& & & \\
Date Acquired: & $6 / 15 / 20217: 09: 21$ PM CST & & \\
Date Processed: & $6 / 15 / 20217: 38: 45$ PM CST & & \\
\hline
\end{tabular}

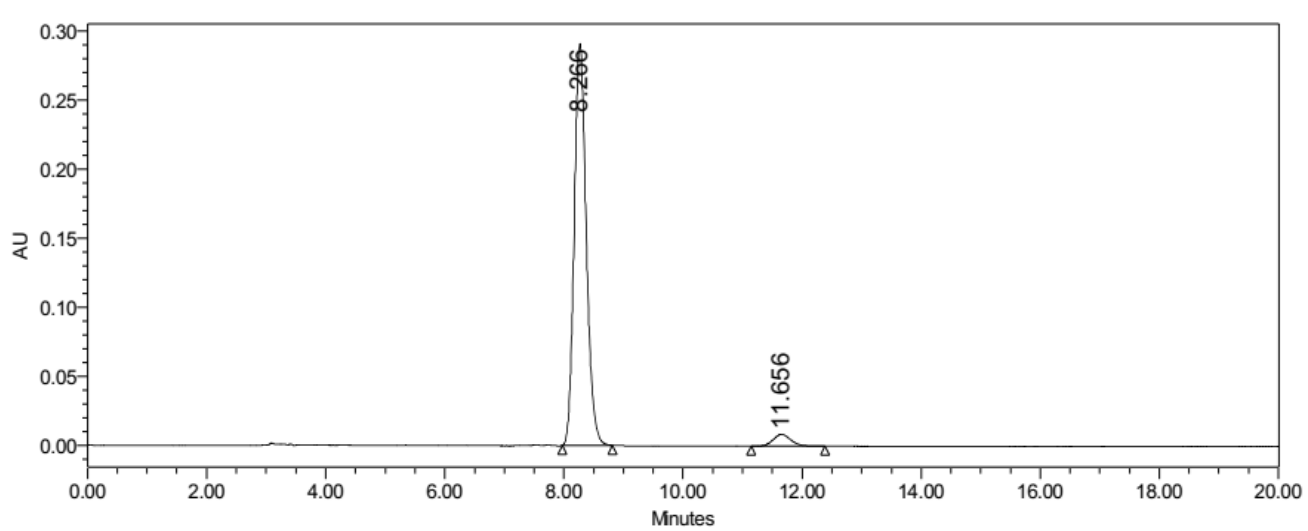

\begin{tabular}{|l|r|r|r|r|}
\hline & \multicolumn{1}{|c|}{ RT } & \multicolumn{1}{c|}{ Area } & \% Area & Height \\
\hline 1 & 8.266 & 4047210 & 95.75 & 290747 \\
\hline 2 & 11.656 & 179510 & 4.25 & 8510 \\
\hline
\end{tabular}




\begin{tabular}{|llll|}
\hline & \multicolumn{2}{c|}{ S A M P L E } & IN F O R M A T I O N \\
\hline \hline Sample Name: & xxh-10-186-rac-OD-30\% & Acquired By: & System \\
Sample Type: & Unknown & Sample Set Name & 20210901 \\
Vial: & 1 & Acq. Method Set: & $30 \%$ quanbo \\
Injection \#: & 1 & Processing Method & 101863 \\
Injection Volume: & 10.00 ul & Channel Name: & $254.0 \mathrm{~nm}$ \\
Run Time: & 18.0 Minutes & Proc. Chnl. Descr.: & 2998 PDA 254.0 nm (2998 \\
& & & \\
Date Acquired: & $9 / 1 / 20215: 52: 14$ PM CST & & \\
Date Processed: & $9 / 1 / 20217: 01: 04$ PM CST & & \\
\hline
\end{tabular}

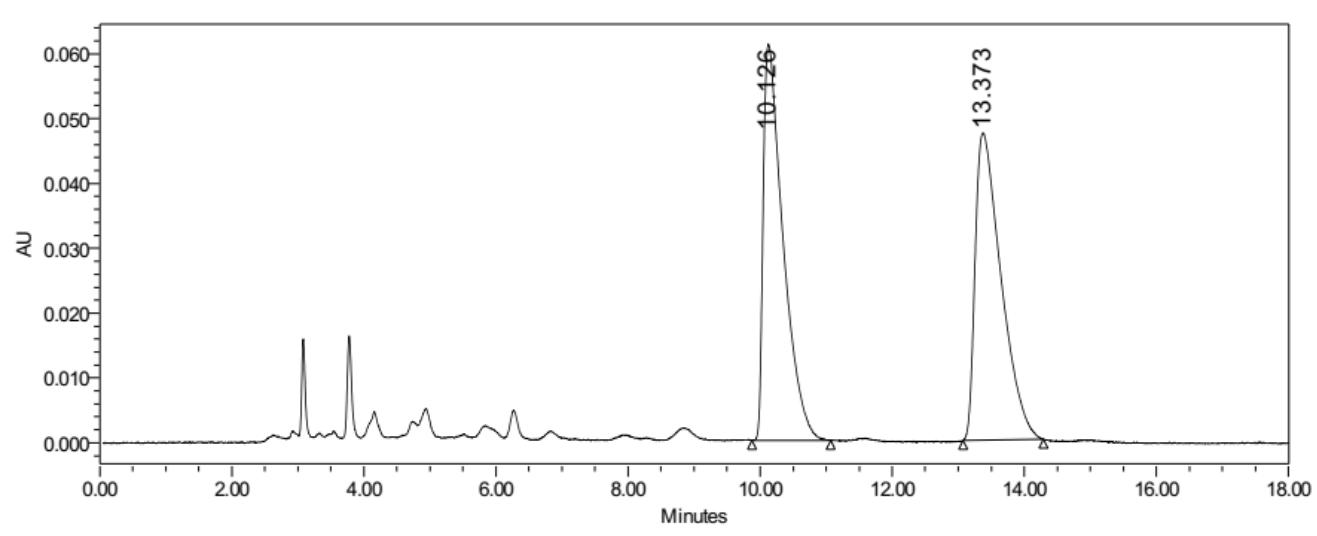

\begin{tabular}{|c|c|c|r|c|}
\hline & RT & Area & $\%$ Area & Height \\
\hline 1 & 10.126 & 1276817 & 49.70 & 61121 \\
\hline 2 & 13.373 & 1292230 & 50.30 & 47419 \\
\hline
\end{tabular}

\section{Asy-4q}

\begin{tabular}{|llll|}
\hline & \multicolumn{2}{c|}{ S A M P L E } & IN F O R M A T I O N \\
\hline \hline Sample Name: & xxh-10-187-OD-30\% & Acquired By: & System \\
Sample Type: & Unknown & Sample Set Name & 20210901 \\
Vial: & 2 & Acq. Method Set: & $30 \%$ quanbo \\
Injection \#: & 1 & Processing Method & 10773 \\
Injection Volume: & 10.00 ul & Channel Name: & $254.0 \mathrm{~nm}$ \\
Run Time: & 18.0 Minutes & Proc. Chnl. Descr.: & 2998 PDA 254.0 nm (2998 \\
& & & \\
Date Acquired: & $9 / 1 / 20216: 10: 53$ PM CST & & \\
Date Processed: & $9 / 1 / 20216: 59: 48$ PM CST & & \\
\hline
\end{tabular}

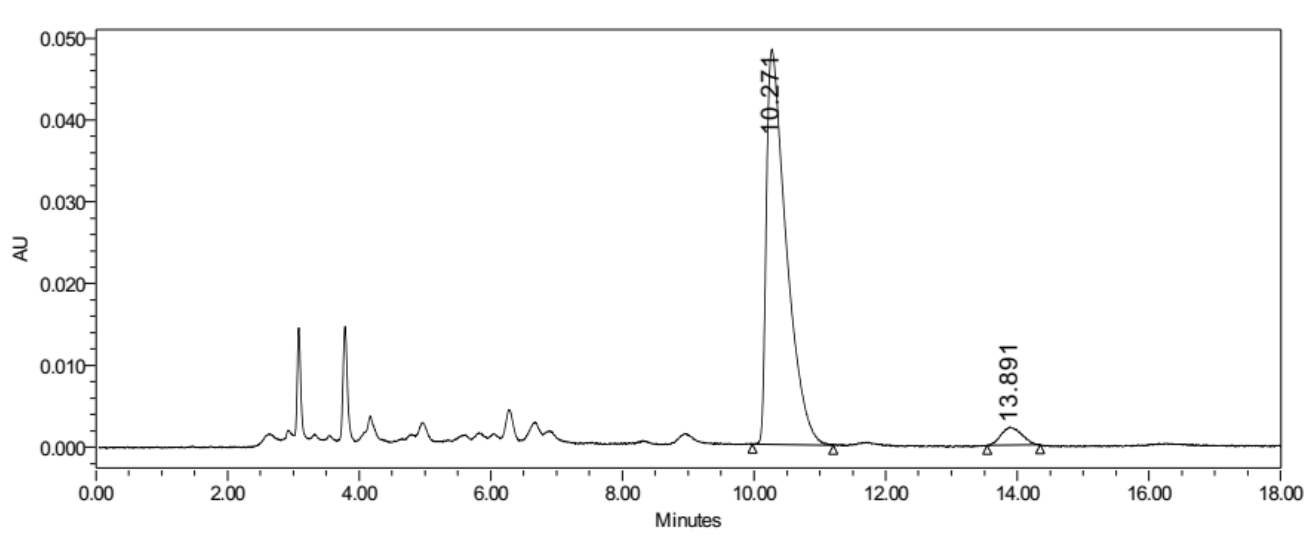

\begin{tabular}{|r|c|r|r|r|}
\hline & RT & \multicolumn{1}{|c|}{ Area } & $\%$ Area & Height \\
\hline 1 & 10.271 & 1012833 & 95.51 & 48277 \\
\hline 2 & 13.891 & 47643 & 4.49 & 2202 \\
\hline
\end{tabular}


Rac-11

\begin{tabular}{|llll|}
\hline & \multicolumn{2}{c|}{ S A M P L E } & IN F O R M A T I O N \\
\hline \hline Sample Name: & xxh-7-37-rac-IC-2\% & Acquired By: & System \\
Sample Type: & Unknown & Sample Set Name & 20210225 \\
Vial: & 77 & Acq. Method Set: & $2 \%$ quanbo \\
Injection \#: & 1 & Processing Method & 737 \\
Injection Volume: & 10.00 ul & Channel Name: & $254.0 \mathrm{~nm}$ \\
Run Time: & 60.0 Minutes & Proc. Chnl. Descr.: & 2998 PDA 254.0 nm (2998 \\
& & & \\
Date Acquired: & $2 / 26 / 20212: 42: 05$ PM CST & & \\
Date Processed: & $3 / 27 / 20213: 03: 23$ PM CST & & \\
\hline
\end{tabular}

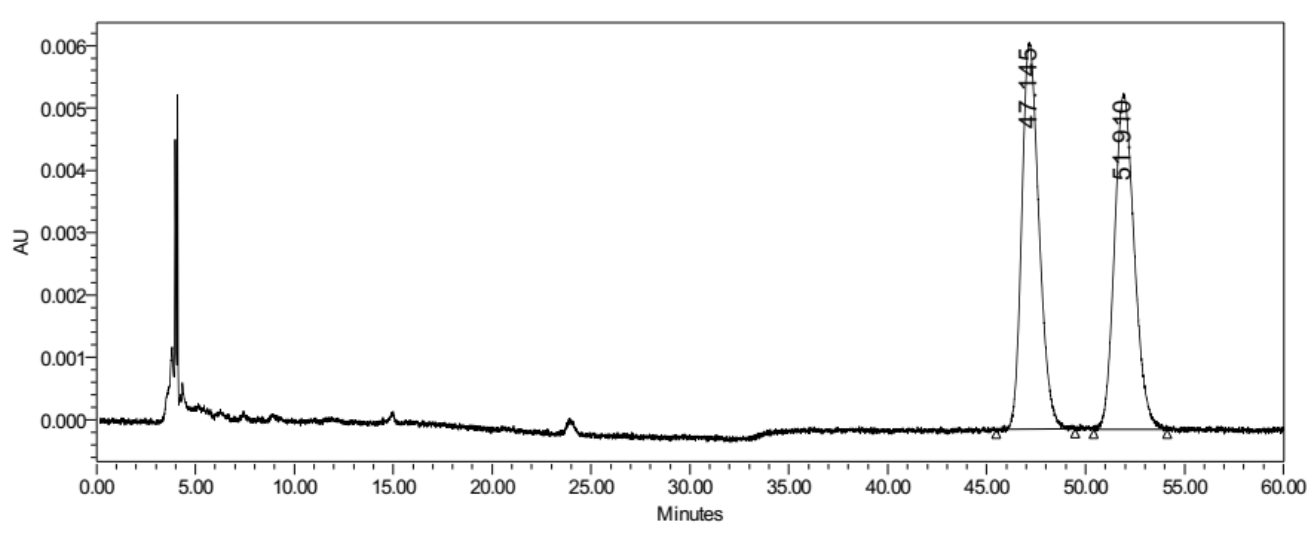

\begin{tabular}{|c|c|c|r|r|}
\hline & RT & Area & $\%$ Area & Height \\
\hline 1 & 47.145 & 375366 & 50.00 & 6198 \\
\hline 2 & 51.910 & 375336 & 50.00 & 5385 \\
\hline
\end{tabular}

Asy-11

\begin{tabular}{|llll|}
\hline & \multicolumn{2}{c|}{ S A M P E } & IN F O R M A T I O N \\
\hline \hline Sample Name: & xxh-7-150-IC-2\% & Acquired By: & System \\
Sample Type: & Unknown & Sample Set Name & 20210225 \\
Vial: & 77 & Acq. Method Set: & $2 \%$ quanbo \\
Injection \#: & 1 & Processing Method & 7150 \\
Injection Volume: & 10.00 ul & Channel Name: & $254.0 \mathrm{~nm}$ \\
Run Time: & 60.0 Minutes & Proc. Chnl. Descr.: & 2998 PDA 254.0 nm (2998 \\
& & & \\
Date Acquired: & $2 / 26 / 202111: 26: 57$ AM CST & & \\
Date Processed: & $3 / 27 / 20213: 04: 05$ PM CST & & \\
\hline
\end{tabular}

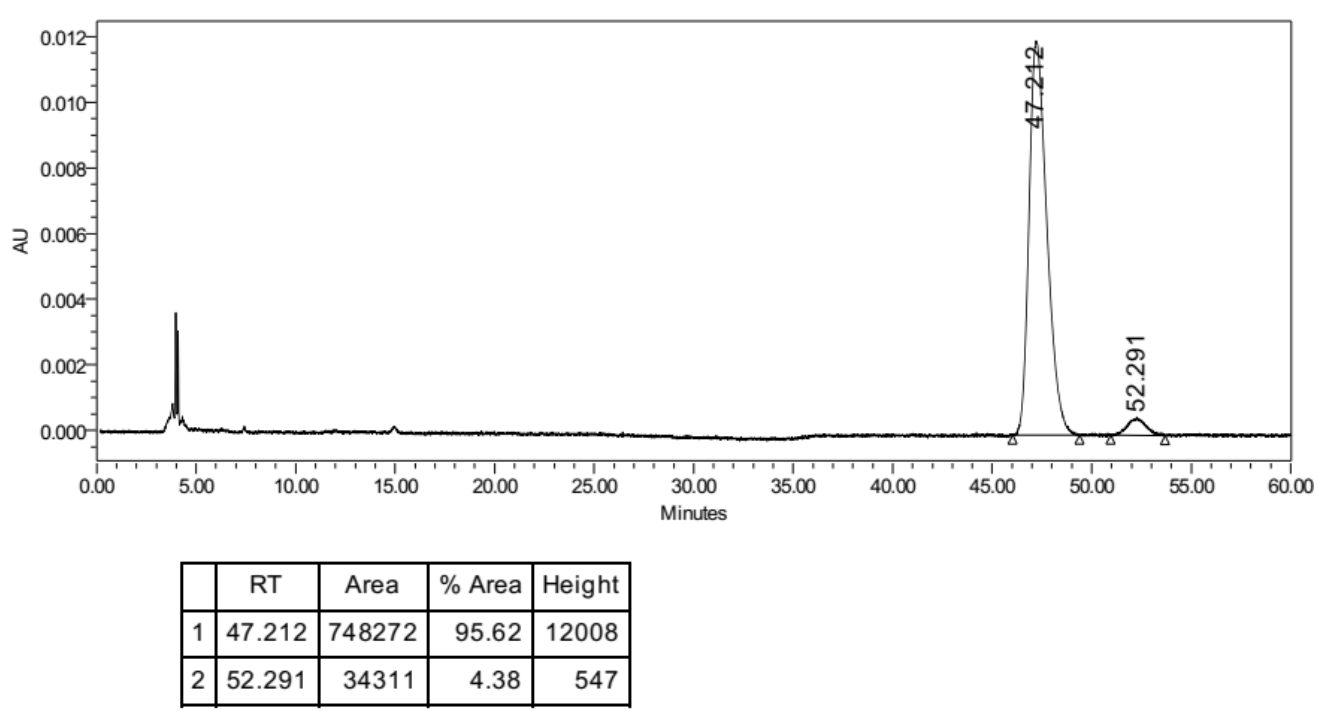


Rac-12

\begin{tabular}{|llll|}
\hline & \multicolumn{2}{c|}{ S A M P L E } & IN F O R M A T I O N \\
\hline \hline Sample Name: & xxh-7-38-rac-lC-15\% & Acquired By: & System \\
Sample Type: & Unknown & Sample Set Name: & 20210308 \\
Vial: & 78 & Acq. Method Set: & $15 \%$ quabbo \\
Injection \#: & 1 & Processing Method & 738 \\
Injection Volume: & $10.00 \mathrm{ul}$ & Channel Name: & $254.0 \mathrm{~nm}$ \\
Run Time: & 18.0 Minutes & Proc. Chnl. Descr.: & 2998 PDA 254.0 nm (2998 \\
& & & \\
Date Acquired: & $3 / 8 / 20214: 47: 27$ PM CST & & \\
Date Processed: & 3/27/2021 3:09:58 PM CST & & \\
\hline
\end{tabular}

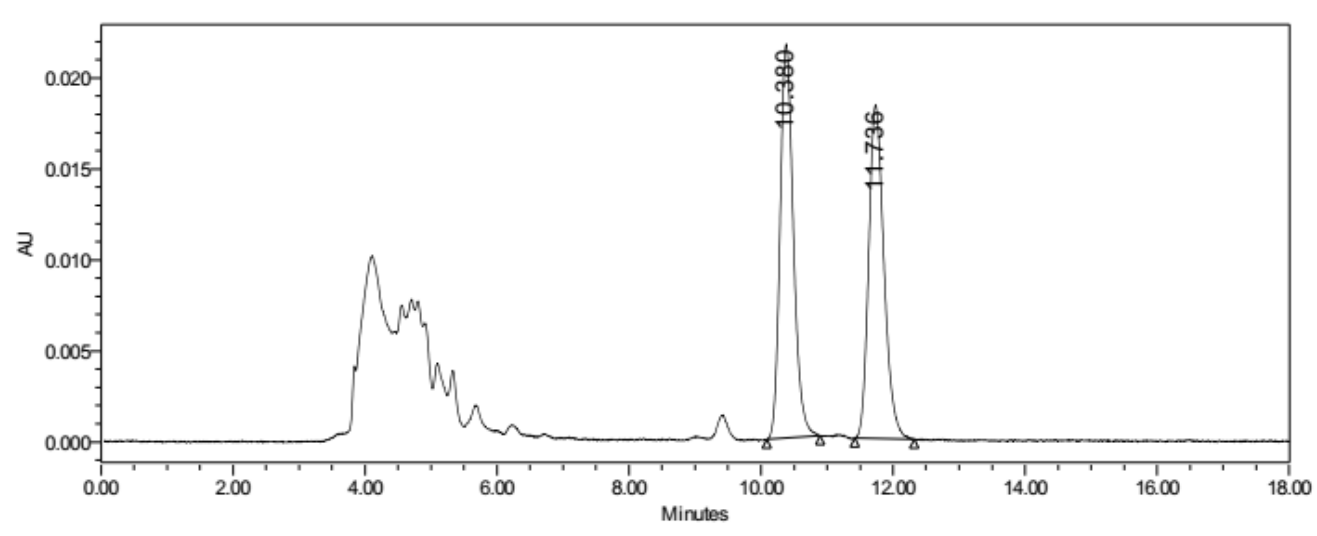

\begin{tabular}{|c|c|c|r|c|}
\hline & RT & Area & $\%$ Area & Height \\
\hline 1 & 10.380 & 302217 & 50.68 & 21637 \\
\hline 2 & 11.736 & 294061 & 49.32 & 18347 \\
\hline
\end{tabular}

Asy-12

\begin{tabular}{|llll|}
\hline & \multicolumn{2}{c|}{ S A M P L E } & IN F O R M A T I O N \\
\hline \hline Sample Name: & xxh-7-187-IC-15\% & Acquired By: & System \\
Sample Type: & Unknown & Sample Set Name & 20210308 \\
Vial: & 77 & Acq. Method Set: & $15 \%$ quabbo \\
Injection \#: & 1 & Processing Method & 7187 \\
Injection Volume: & $10.00 \mathrm{ul}$ & Channel Name: & $254.0 \mathrm{~nm}$ \\
Run Time: & 18.0 Minutes & Proc. Chnl. Descr.: & 2998 PDA 254.0 nm (2998 \\
& & & \\
Date Acquired: & $3 / 8 / 20214: 28: 47$ PM CST & & \\
Date Processed: & $3 / 27 / 20213: 13: 08$ PM CST & & \\
\hline
\end{tabular}

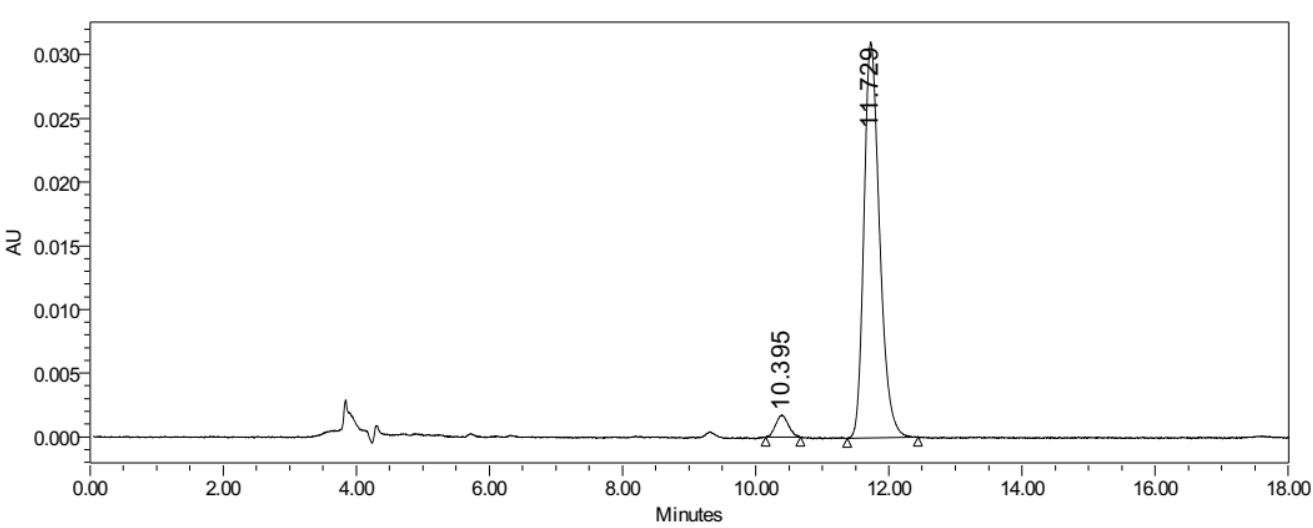

\begin{tabular}{|c|c|c|r|r|}
\hline & RT & Area & $\%$ Area & Height \\
\hline 1 & 10.395 & 23637 & 4.47 & 1743 \\
\hline 2 & 11.729 & 505634 & 95.53 & 31060 \\
\hline
\end{tabular}


Rac-13

\begin{tabular}{|llll|}
\hline \multicolumn{2}{c|}{ S A M P L E } & IN F O R M A T I O N \\
\hline \hline Sample Name: & xxh-7-110-rac-IC-30\% & Acquired By: & System \\
Sample Type: & Unknown & Sample Set Name & 20210309 \\
Vial: & 77 & Acq. Method Set: & $30 \%$ quanbo \\
Injection \#: & 1 & Processing Method & 7110 \\
Injection Volume: & 10.00 ul & Channel Name: & $254.0 \mathrm{~nm}$ \\
Run Time: & 15.0 Minutes & Proc. Chnl. Descr.: & 2998 PDA 254.0 nm (2998 \\
& & & \\
Date Acquired: & $3 / 9 / 20218: 51: 30$ AM CST & & \\
Date Processed: & $3 / 27 / 20213: 34: 11$ PM CST & & \\
\hline
\end{tabular}

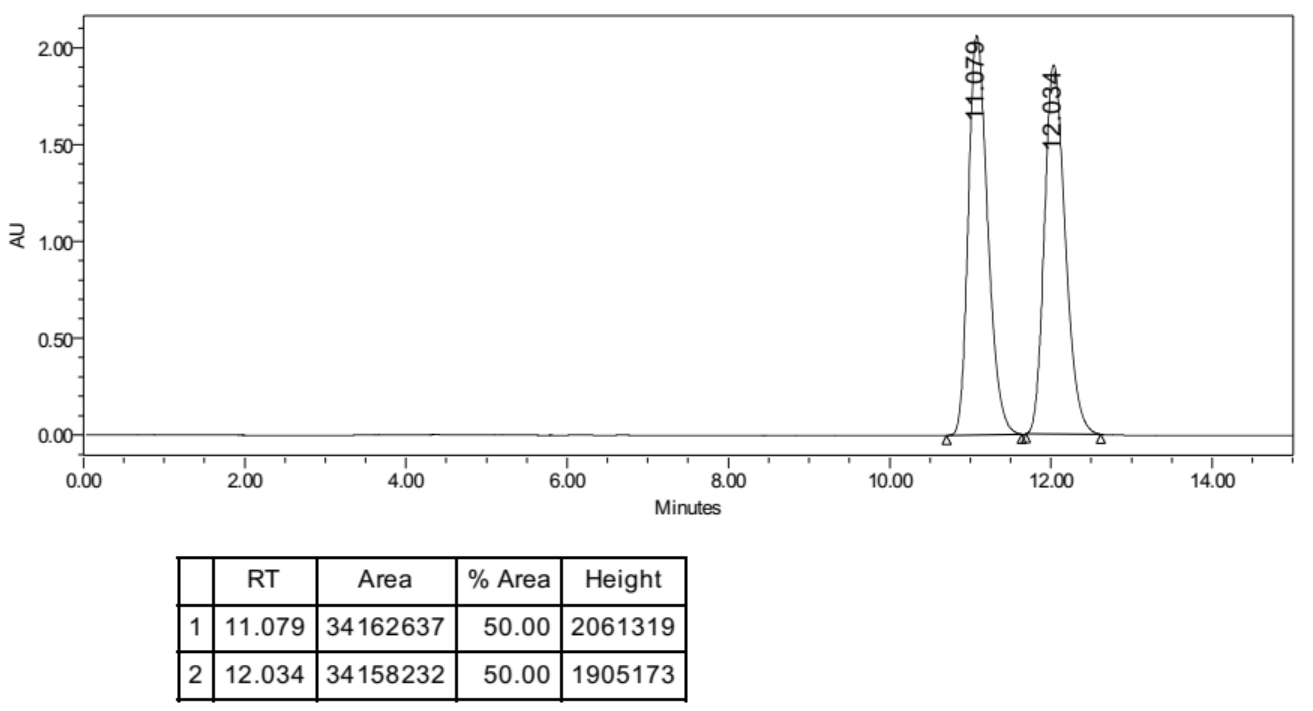

Asy-13

\begin{tabular}{|llll|}
\hline & \multicolumn{2}{c|}{ S A M P L E } & IN F O R M A T I O N \\
\hline \hline Sample Name: & xxh-7-185-IC-30\% & Acquired By: & System \\
Sample Type: & Unknown & Sample Set Name & 20210309 \\
Vial: & 78 & Acq. Method Set: & $30 \%$ quanbo \\
Injection \#: & 1 & Processing Method & 7185 \\
Injection Volume: & 10.00 ul & Channel Name: & $254.0 \mathrm{~nm}$ \\
Run Time: & 15.0 Minutes & Proc. Chnl. Descr.: & 2998 PDA 254.0 nm (2998 \\
& & & \\
Date Acquired: & $3 / 9 / 20219: 07: 08$ AM CST & & \\
Date Processed: & $3 / 27 / 20213: 36: 18$ PM CST & & \\
\hline
\end{tabular}

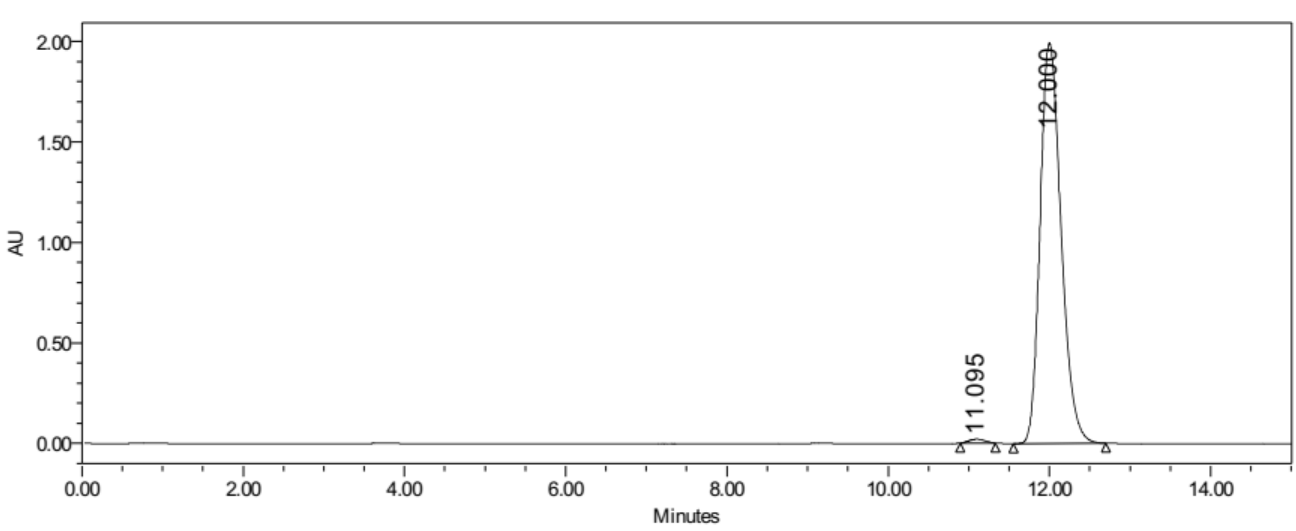

\begin{tabular}{|c|c|c|r|r|}
\hline & RT & Area & $\%$ Area & Height \\
\hline 1 & 11.095 & 270057 & 0.75 & 19731 \\
\hline 2 & 12.000 & 35824155 & 99.25 & 1993540 \\
\hline
\end{tabular}


Rac-14

\begin{tabular}{|llll|}
\hline & \multicolumn{2}{c|}{ S A M P L E } & IN F O R M A T I O N \\
\hline \hline Sample Name: & xxh-8-21-rac-OD-15\% & Acquired By: & System \\
Sample Type: & Unknown & Sample Set Name & 20210322 \\
Vial: & 82 & Acq. Method Set: & $15 \%$ quabbo \\
Injection \#: & 1 & Processing Method & 821 \\
Injection Volume: & 10.00 ul & Channel Name: & $212.0 \mathrm{~nm}$ \\
Run Time: & 12.0 Minutes & Proc. Chnl. Descr.: & 2998 PDA 212.0 nm (2998 \\
& & & \\
Date Acquired: & $3 / 22 / 2021$ 11:43:59 PM CST & & \\
Date Processed: & $3 / 27 / 20213: 42: 23$ PM CST & & \\
\hline
\end{tabular}

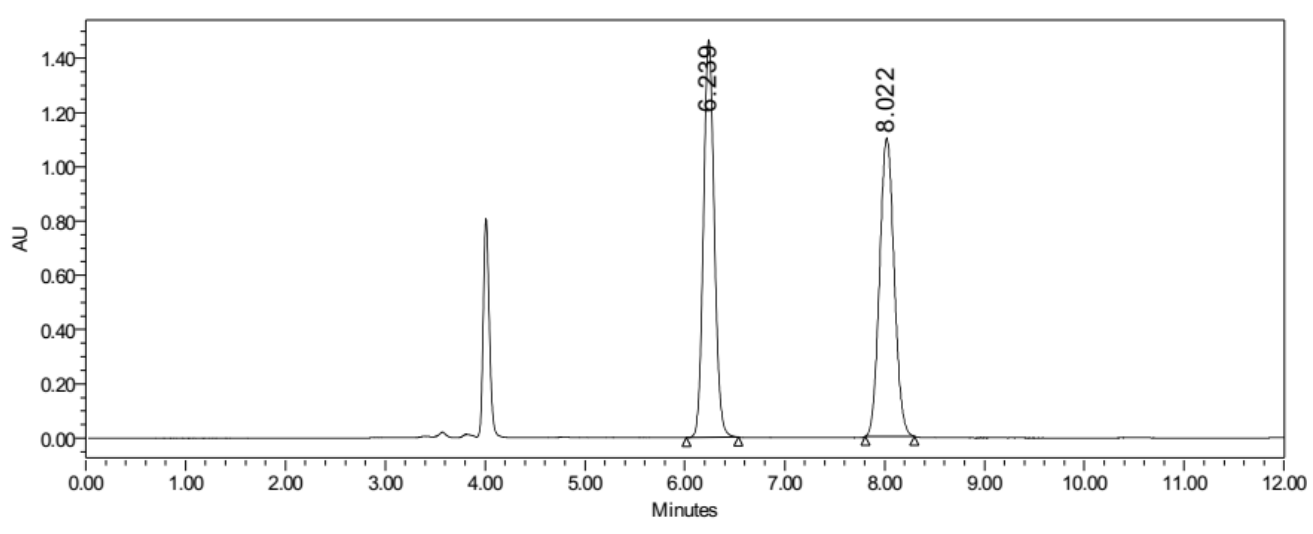

\begin{tabular}{|c|c|c|r|c|}
\hline & RT & Area & $\%$ Area & Height \\
\hline 1 & 6.239 & 11127010 & 50.00 & 1465021 \\
\hline 2 & 8.022 & 11126378 & 50.00 & 1099887 \\
\hline
\end{tabular}

\section{Asy-14}

\begin{tabular}{|llll|}
\hline & \multicolumn{2}{c|}{ S A M P L E } & IN F O R M A T I O N \\
\hline \hline Sample Name: & xxh-8-22-OD-15\% & Acquired By: & System \\
Sample Type: & Unknown & Sample Set Name & 20210322 \\
Vial: & 83 & Acq. Method Set: & $15 \%$ quabbo \\
Injection \#: & 1 & Processing Method & 8223 \\
Injection Volume: & 10.00 ul & Channel Name: & $212.0 \mathrm{~nm}$ \\
Run Time: & 12.0 Minutes & Proc. Chnl. Descr.: & 2998 PDA 212.0 nm (2998 \\
& & & \\
Date Acquired: & $3 / 22 / 202110: 45: 35$ PM CST & & \\
Date Processed: & $3 / 27 / 20214: 41: 45$ PM CST & & \\
\hline
\end{tabular}

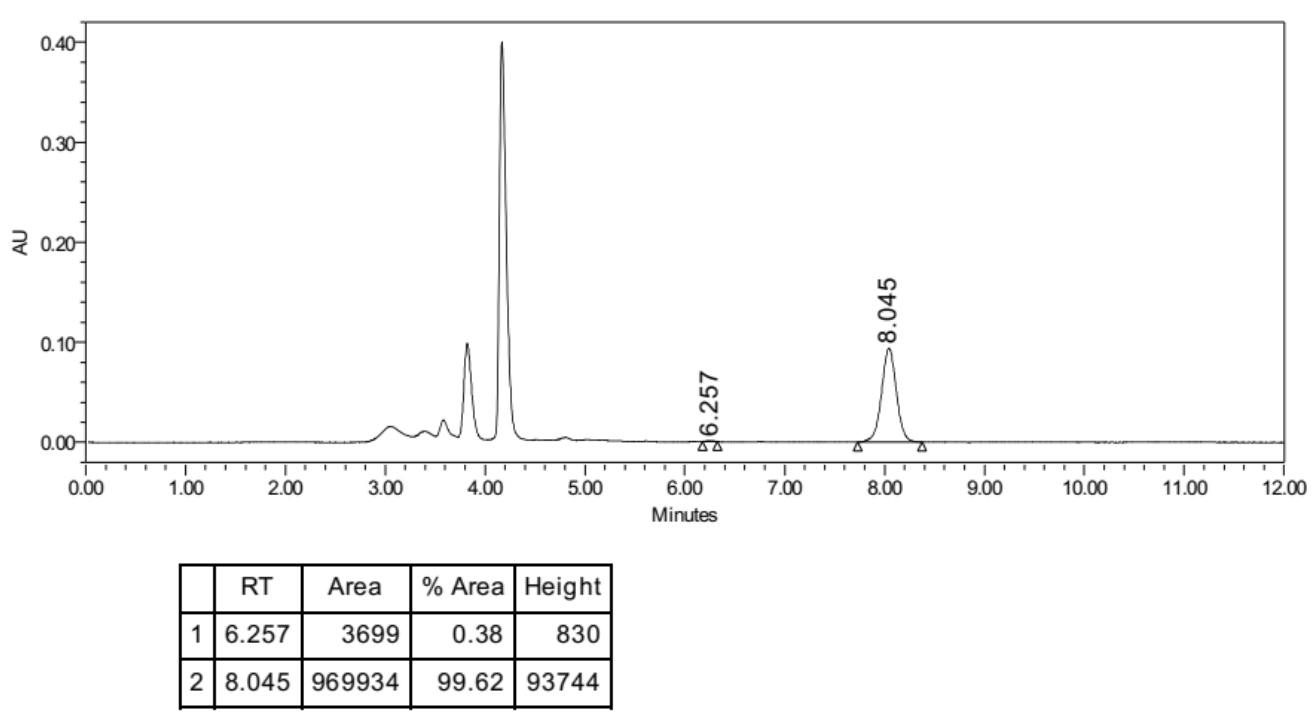


Rac-15

\begin{tabular}{|llll|}
\hline & \multicolumn{2}{c|}{ S A M P LE } & IN F O R M A T I O N \\
\hline \hline Sample Name: & xxh-7-67-rac-OJ-2\% & Acquired By: & System \\
Sample Type: & Unknown & Sample Set Name & 20210304 \\
Vial: & 80 & Acq. Method Set: & $2 \%$ quanbo \\
Injection \#: & 1 & Processing Method & 767 \\
Injection Volume: & 10.00 ul & Channel Name: & $254.0 \mathrm{~nm}$ \\
Run Time: & 50.0 Minutes & Proc. Chnl. Descr.: & 2998 PDA 254.0 nm (2998 \\
& & & \\
Date Acquired: & $3 / 4 / 202110: 31: 10$ AM CST & & \\
Date Processed: & $3 / 27 / 20213: 16: 47$ PM CST & & \\
\hline
\end{tabular}

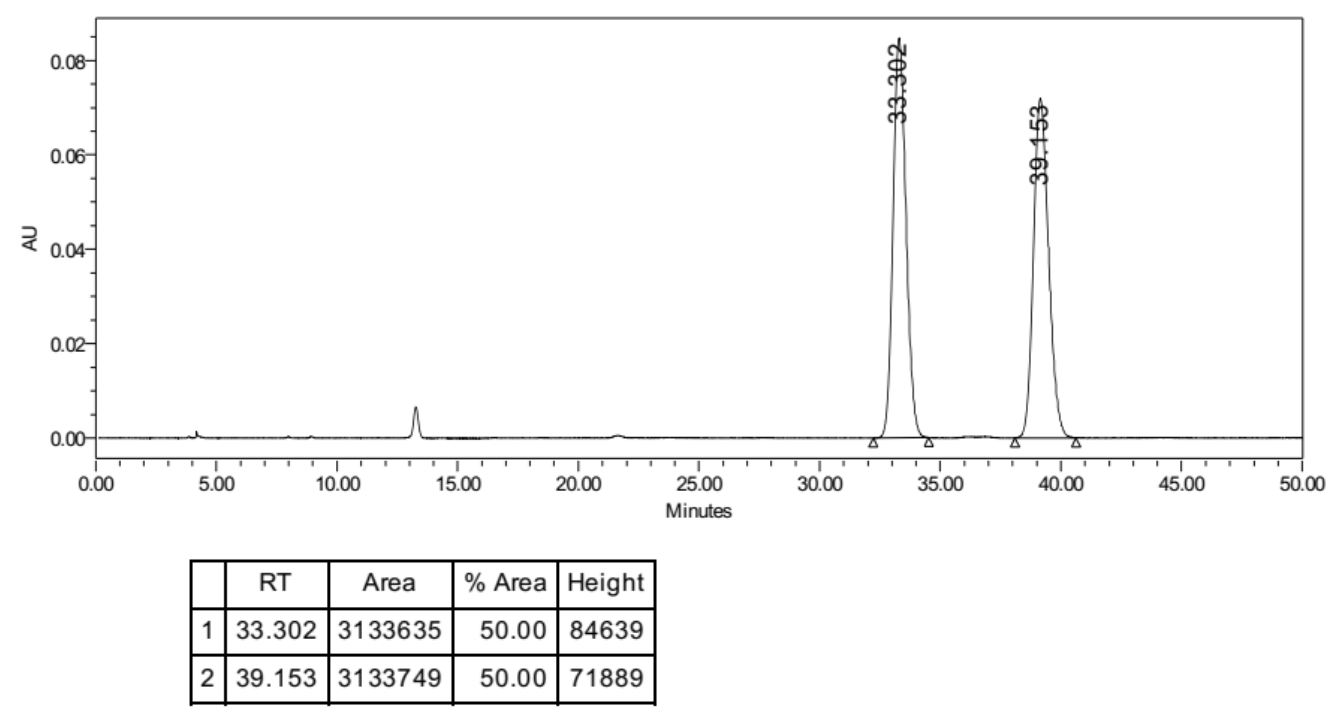

\section{Asy-15}

\begin{tabular}{|llll|}
\hline & \multicolumn{2}{c|}{ S A M P L E } & IN F O R M A T I O N \\
\hline \hline Sample Name: & xxh-7-181-OJ-2\% & Acquired By: & System \\
Sample Type: & Unknown & Sample Set Name & 20210306 \\
Vial: & 78 & Acq. Method Set: & $2 \%$ quanbo \\
Injection \#: & 1 & Processing Method & 7181 \\
Injection Volume: & $10.00 \mathrm{ul}$ & Channel Name: & $254.0 \mathrm{~nm}$ \\
Run Time: & 50.0 Minutes & Proc. Chnl. Descr.: & 2998 PDA 254.0 nm (2998 \\
& & & \\
Date Acquired: & $3 / 6 / 20218: 37: 23$ PM CST & & \\
Date Processed: & $3 / 27 / 20213: 29: 17$ PM CST & & \\
\hline
\end{tabular}

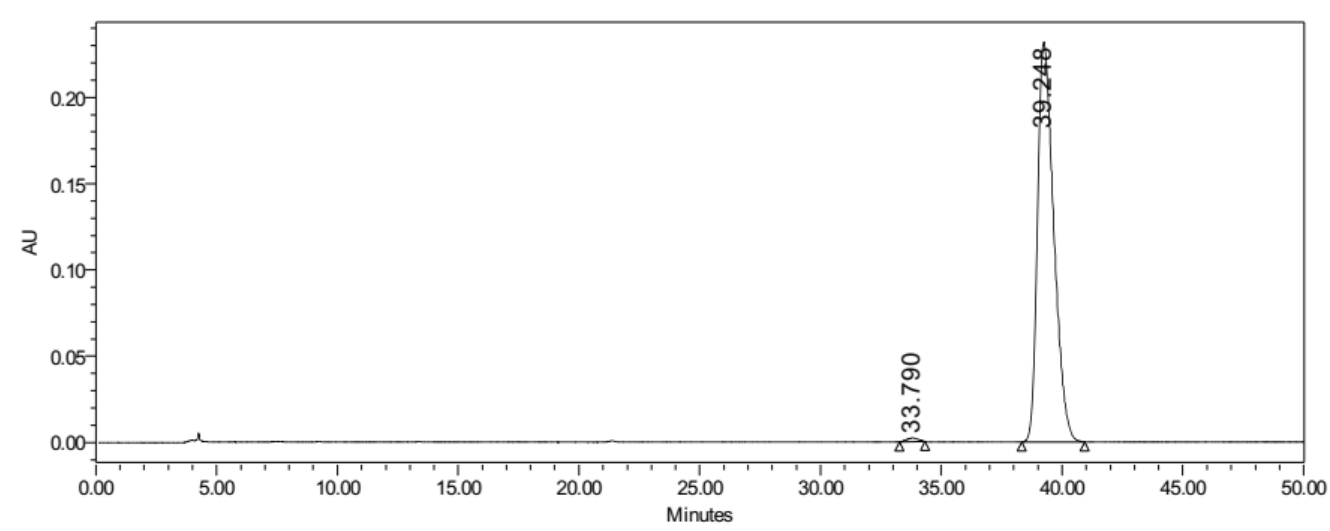

\begin{tabular}{|r|c|r|r|r|}
\hline & RT & \multicolumn{1}{|c|}{ Area } & $\%$ Area & Height \\
\hline 1 & 33.790 & 69786 & 0.64 & 2116 \\
\hline 2 & 39.248 & 10751299 & 99.36 & 231606 \\
\hline
\end{tabular}


Rac-16

\begin{tabular}{|llll|}
\hline & \multicolumn{2}{c|}{ S A M P L E } & IN F O R M A T I O N \\
\hline \hline Sample Name: & xxh-6-143-rac-OJ-15\% & Acquired By: & System \\
Sample Type: & Unknown & Sample Set Name & \\
Vial: & 81 & Acq. Method Set: & $15 \%$ quanbo \\
Injection \#: & 1 & Processing Method & 6143 \\
Injection Volume: & 10.00 ul & Channel Name: & $254.0 \mathrm{~nm}$ \\
Run Time: & 100.0 Minutes & Proc. Chnl. Descr.: & 2998 PDA 254.0 nm (2998 \\
& & & \\
Date Acquired: & 1/1/2021 11:08:47 PM CST & & \\
Date Processed: & $3 / 29 / 20218: 36: 29$ AM CST & & \\
\hline
\end{tabular}

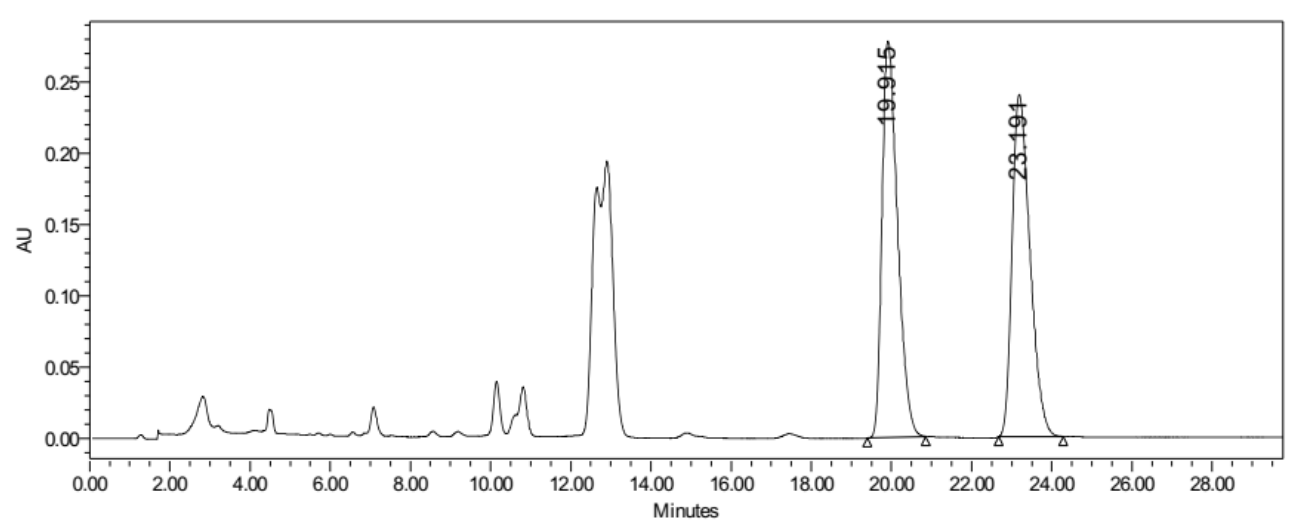

\begin{tabular}{|c|c|c|r|c|}
\hline & RT & Area & $\%$ Area & Height \\
\hline 1 & 19.915 & 7420822 & 50.00 & 277581 \\
\hline 2 & 23.191 & 7419737 & 50.00 & 239972 \\
\hline
\end{tabular}

\section{Asy-16}

\begin{tabular}{|llll|}
\hline & \multicolumn{2}{c|}{ S A M P L E } & I N F O R M A T I O N \\
\hline \hline Sample Name: & xxh-7-140-1-OJ-15\% & Acquired By: & System \\
Sample Type: & Unknown & Sample Set Name: & 20210329 \\
Vial: & 1 & Acq. Method Set: & $15 \%$ quanbo \\
Injection \#: & 1 & Processing Method: & 7140 \\
Injection Volume: & 10.00 ul & Channel Name: & $254.0 \mathrm{~nm}$ \\
Run Time: & 30.0 Minutes & Proc. Chnl. Descr.: & 2998 PDA 254.0 nm (2998 \\
& & & \\
Date Acquired: & $3 / 29 / 2021$ 1:22:57 AM CST & & \\
Date Processed: & $3 / 29 / 20218: 33: 48$ AM CST & & \\
\hline
\end{tabular}

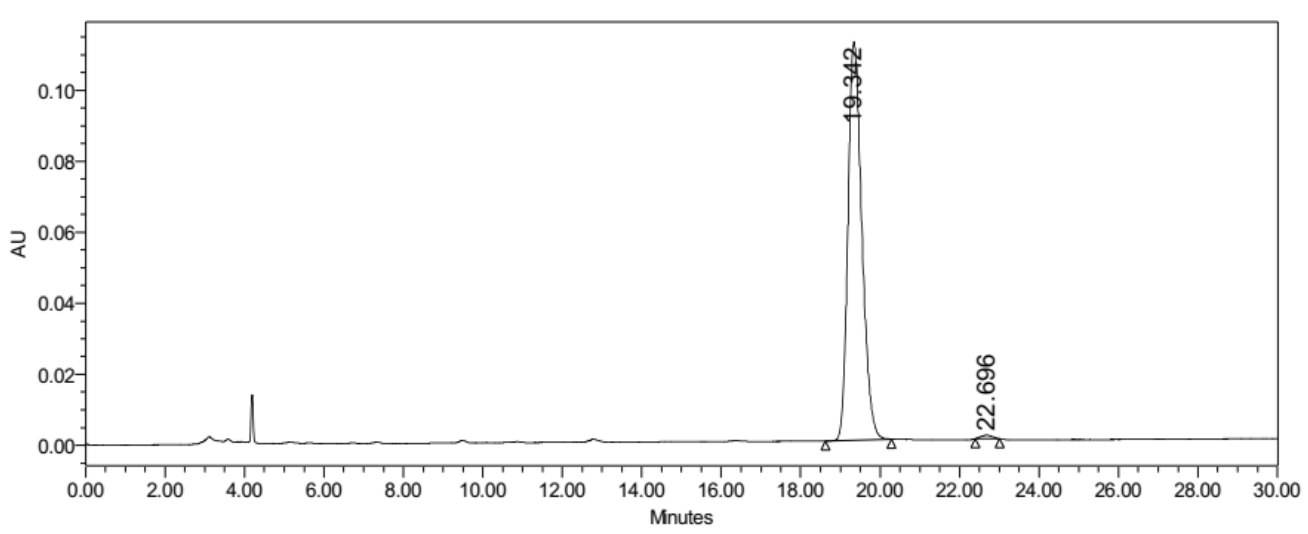

\begin{tabular}{|l|c|r|r|r|}
\hline & RT & \multicolumn{1}{c|}{ Area } & \% Area & Height \\
\hline 1 & 19.342 & 2689403 & 99.25 & 112200 \\
\hline 2 & 22.696 & 20329 & 0.75 & 989 \\
\hline
\end{tabular}


Rac-16b

\begin{tabular}{|llll|}
\hline & \multicolumn{2}{c|}{ S A M P L E } & IN F O R M A T I O N \\
\hline \hline Sample Name: & xxh-7-177-OJ-rac-5\% & Acquired By: & System \\
Sample Type: & Unknown & Sample Set Name & 20210318 \\
Vial: & 78 & Acq. Method Set: & $5 \%$ quanbo \\
Injection \#: & 1 & Processing Method & 71772 \\
Injection Volume: & 10.00 ul & Channel Name: & $220.0 \mathrm{~nm}$ \\
Run Time: & 84.0 Minutes & Proc. Chnl. Descr.: & 2998 PDA 220.0 nm (2998 \\
& & & \\
Date Acquired: & $3 / 18 / 202111: 11: 16$ PM CST & & \\
Date Processed: & $3 / 29 / 202112: 17: 36$ AM CST & & \\
\hline
\end{tabular}

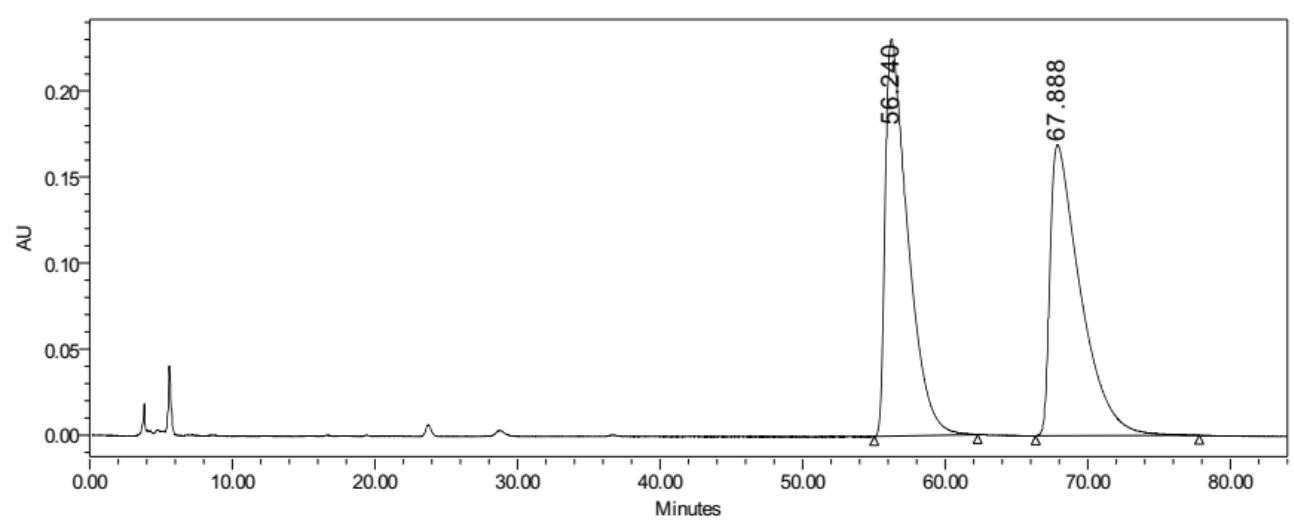

\begin{tabular}{|c|c|c|r|c|}
\hline & RT & Area & $\%$ Area & Height \\
\hline 1 & 56.240 & 25187883 & 50.00 & 230760 \\
\hline 2 & 67.888 & 25188575 & 50.00 & 169211 \\
\hline
\end{tabular}

\section{Asy-16b}

\begin{tabular}{|llll|}
\hline & \multicolumn{2}{c|}{ S A M P L E } & IN F O R M A T I O N \\
\hline \hline Sample Name: & xxh-8-33-OJ-5\% & Acquired By: & System \\
Sample Type: & Unknown & Sample Set Name & 20210325 \\
Vial: & 75 & Acq. Method Set: & $5 \%$ quanbo \\
Injection \#: & 1 & Processing Method & 8332 \\
Injection Volume: & 10.00 ul & Channel Name: & $220.0 \mathrm{~nm}$ \\
Run Time: & 90.0 Minutes & Proc. Chnl. Descr.: & 2998 PDA 220.0 nm (2998 \\
& & & \\
Date Acquired: & $3 / 25 / 202112: 48: 33$ PM CST & & \\
Date Processed: & $3 / 29 / 202112: 13: 48$ AM CST & & \\
\hline
\end{tabular}

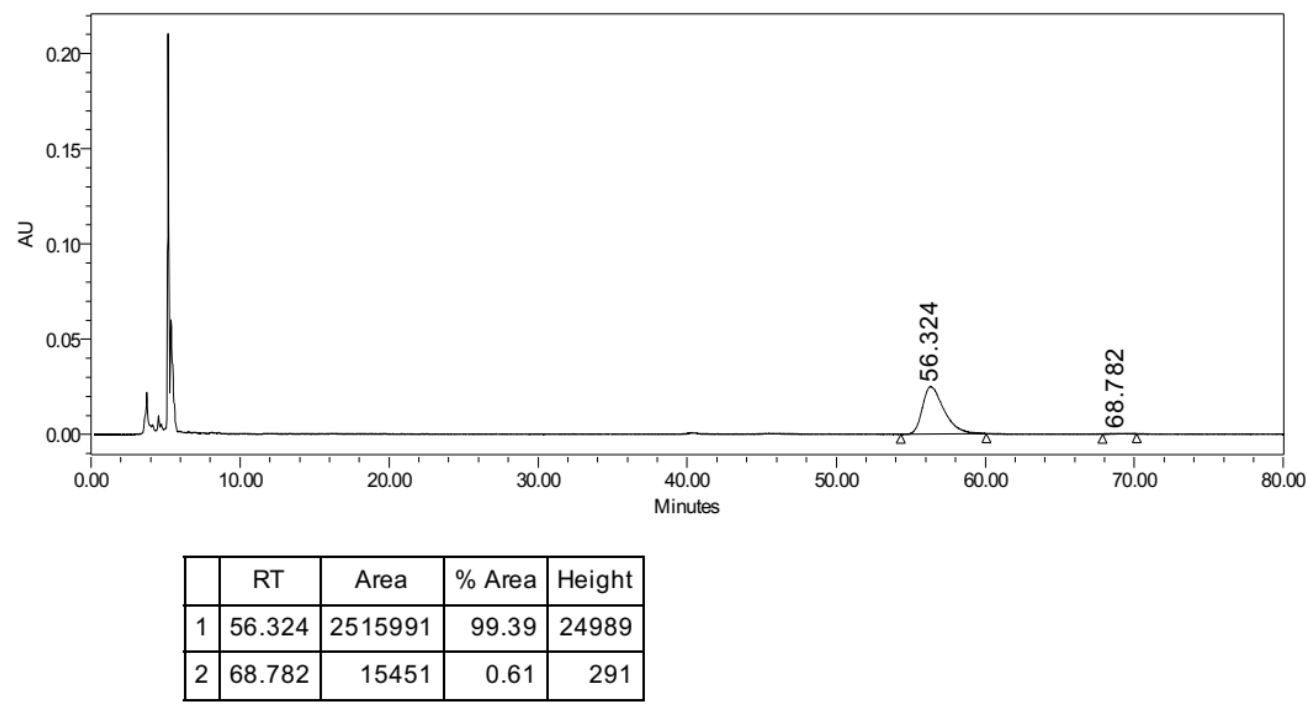


Rac-17

\begin{tabular}{|llll|}
\hline \multicolumn{2}{c|}{ S A M P L E } & IN F O R M A T I O N \\
\hline \hline Sample Name: & xxh-7-189-AD-rac-30\% & Acquired By: & System \\
Sample Type: & Unknown & Sample Set Name & \\
Vial: & 74 & Acq. Method Set: & $30 \%$ quanbo \\
Injection \#: & 1 & Processing Method & 718922 \\
Injection Volume: & 10.00 ul & Channel Name: & $220.0 \mathrm{~nm}$ \\
Run Time: & 100.0 Minutes & Proc. Chnl. Descr.: & 2998 PDA 220.0 nm (2998 \\
& & & \\
Date Acquired: & $3 / 25 / 20218: 11: 14$ PM CST & & \\
Date Processed: & $5 / 20 / 202111: 02: 58$ PM CST & & \\
\hline
\end{tabular}

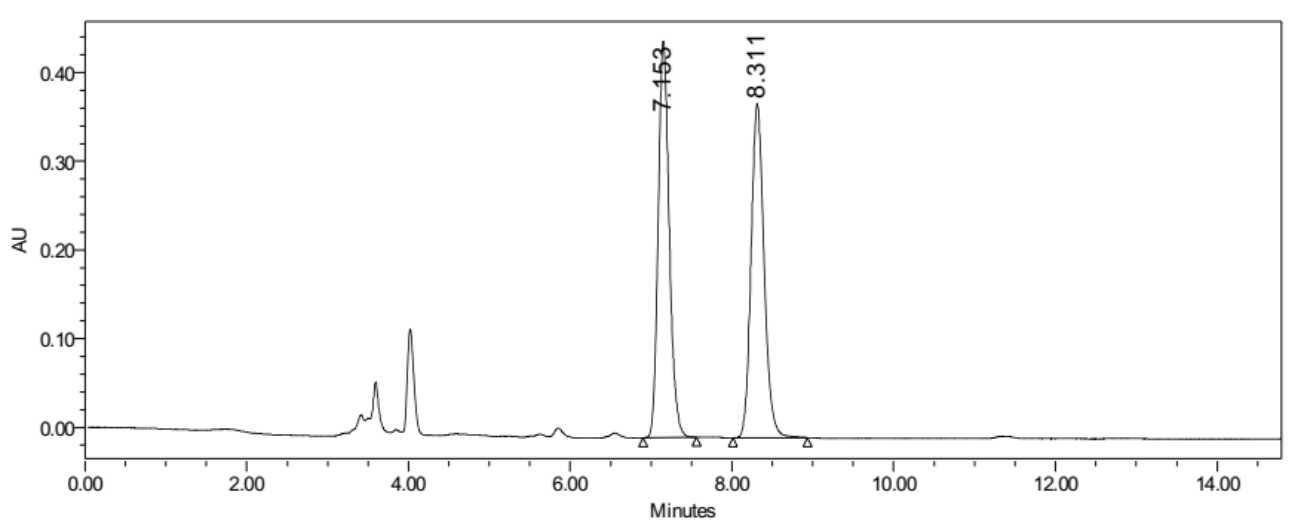

\begin{tabular}{|c|c|c|r|c|}
\hline & RT & Area & $\%$ Area & Height \\
\hline 1 & 7.153 & 4212810 & 50.08 & 446592 \\
\hline 2 & 8.311 & 4199568 & 49.92 & 377046 \\
\hline
\end{tabular}

Asy-17

\begin{tabular}{|llll|}
\hline & \multicolumn{2}{c|}{ S A M P LE } & \multicolumn{1}{c|}{ IN F O R M A T I O N } \\
\hline \hline Sample Name: & xxh-8-37-AD-30\% & Acquired By: & Sysem \\
Sample Type: & Unknown & Sample Set Name & 20210325 \\
Vial: & 74 & Acq. Method Set: & $30 \%$ quanbo \\
Injection \#: & 1 & Processina M ethoc & 83723 \\
Injection Volume: & 10.00 ul & Channel Name: & $220.0 \mathrm{~nm}$ \\
Run T ime: & 11.0 M inutes & Proc. Chnl. Descr.: & 2998 PDA 220.0 nm (2998 \\
& & & \\
Date Acquired: & $3 / 25 / 20219: 27: 52$ PM CST & & \\
Date Processed: & $3 / 29 / 20216: 59: 45$ PM CST & & \\
\hline
\end{tabular}

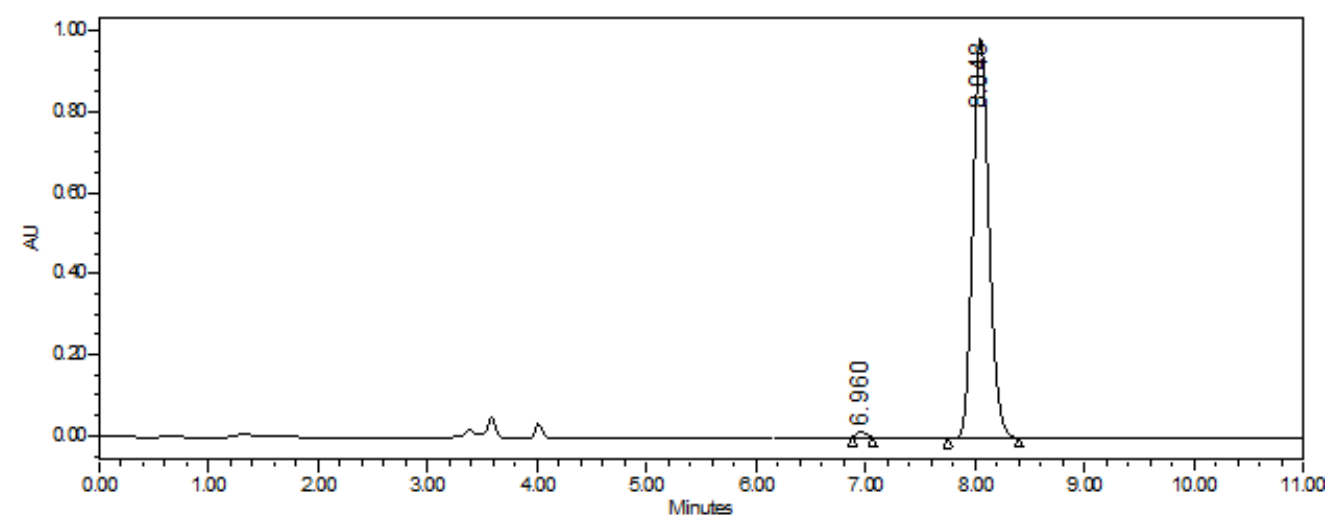

\begin{tabular}{|c|c|c|r|r|}
\hline & RT & \multicolumn{1}{|c|}{ Area } & \% Area & Height \\
\hline 1 & 6.960 & 76753 & 0.75 & 12024 \\
\hline 2 & 8.048 & 10169854 & 99.25 & 987467 \\
\hline
\end{tabular}

